\title{
THE OSTEOLOGY OF RHOMBOMYLUS \\ (MAMMALIA, GLIRES): IMPLICATIONS \\ FOR PHYLOGENY AND EVOLUTION \\ OF GLIRES
}

\author{
JIN MENG \\ Associate Curator, Division of Paleontology \\ American Museum of Natural History \\ e-mail:jmeng@amnh.org
}

\section{YAOMING HU}

Graduate Student, Division of Paleontology

American Museum of Natural History

e-mail:yhu@amnh.org

Institute of Vertebrate Paleontology and Paleoanthropology

Chinese Academy of Sciences

Biology Program (EEB), Graduate School and City College

City University of New York

\author{
CHUANKUI LI \\ Institute of Vertebrate Paleontology and Paleoanthropology \\ Chinese Academy of Sciences \\ e-mail: li.chuankui@pa.ivpp.ac.cn
}

BULLETIN OF THE AMERICAN MUSEUM OF NATURAL HISTORY

CENTRAL PARK WEST AT 79TH STREET, NEW YORK, NY 10024

Number 275, 247 pp., 81 figures, 5 tables

Issued February 28, 2003 


\section{CONTENTS}

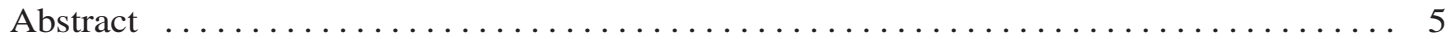

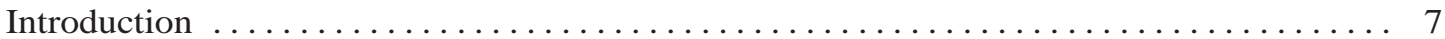

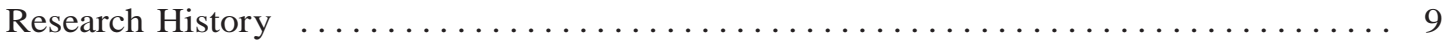

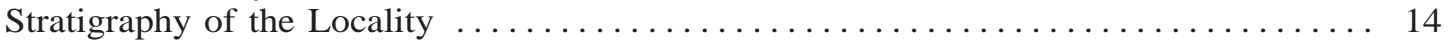

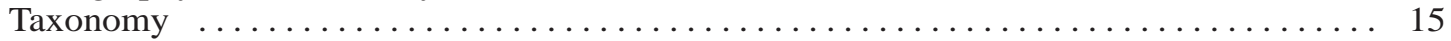

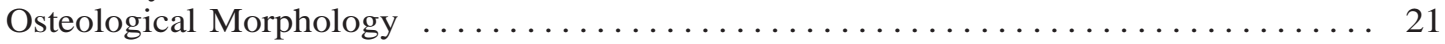

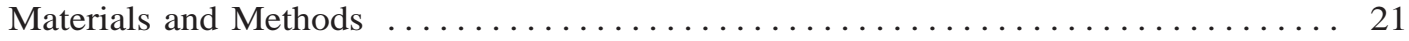

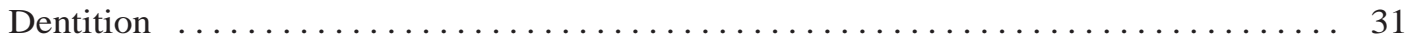

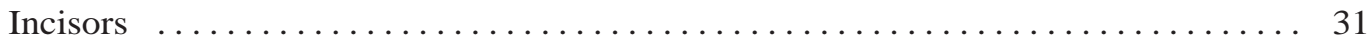

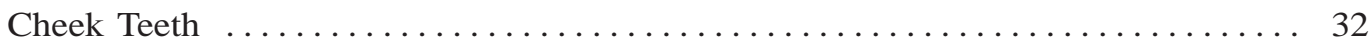

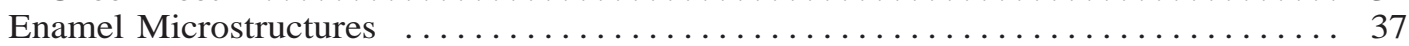

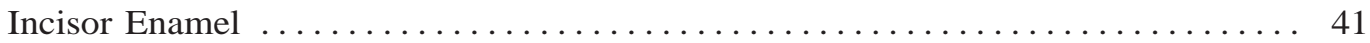

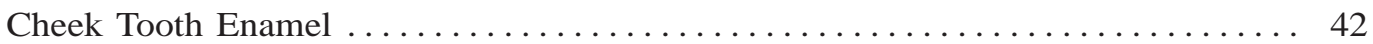

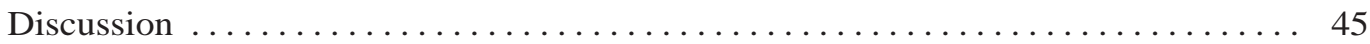

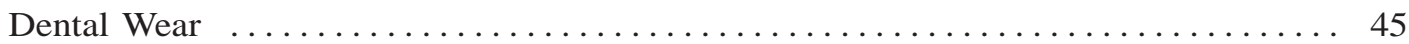

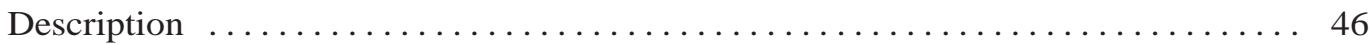

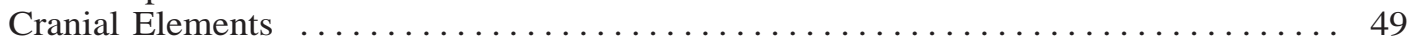

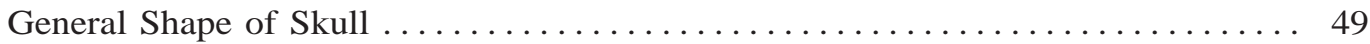

Nasal ........................................ 49

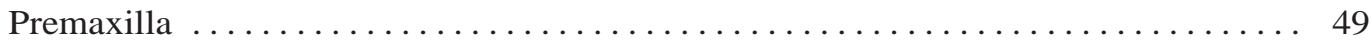

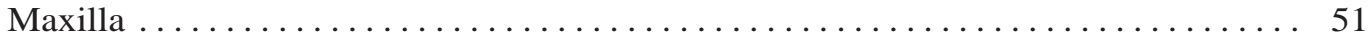

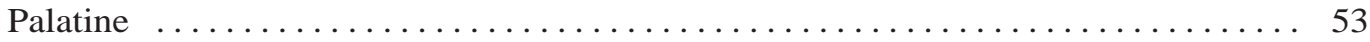

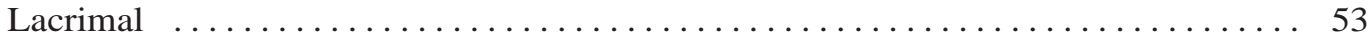

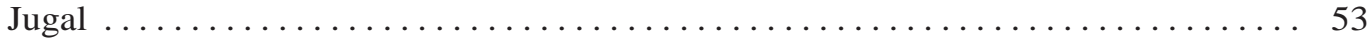

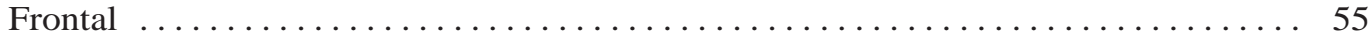

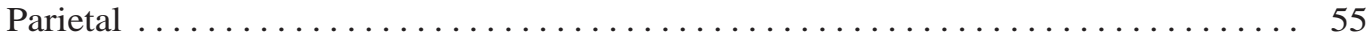

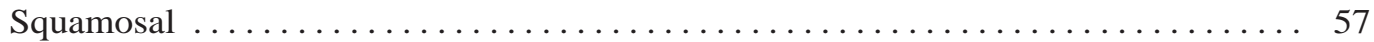

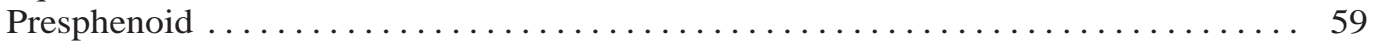

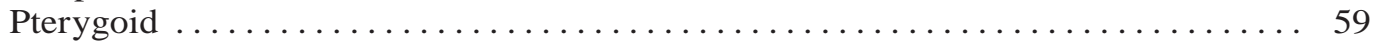

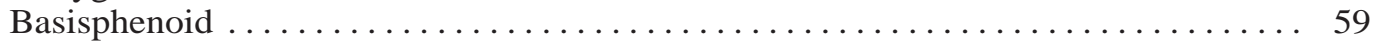

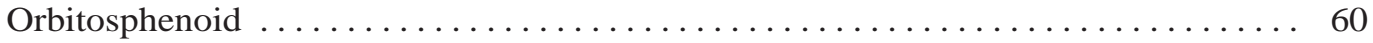

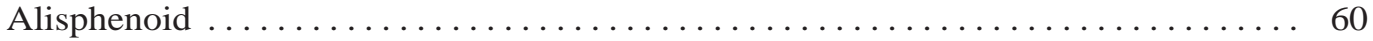

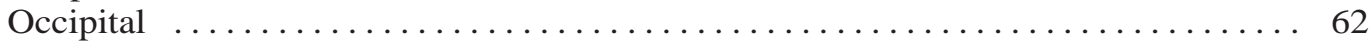

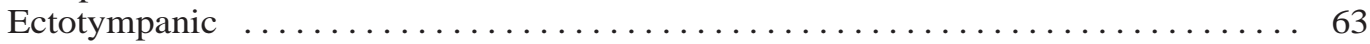

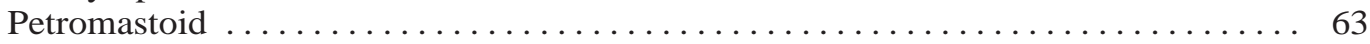

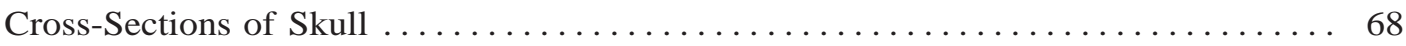

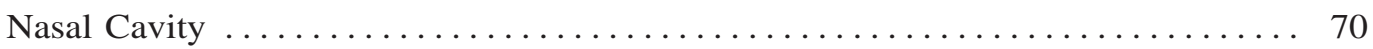

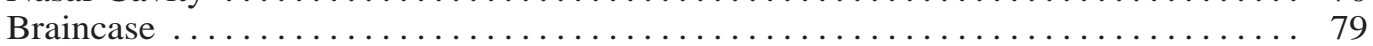

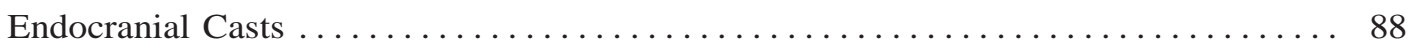

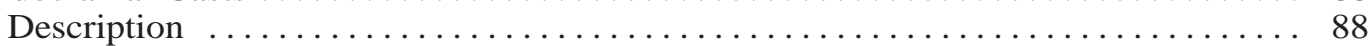

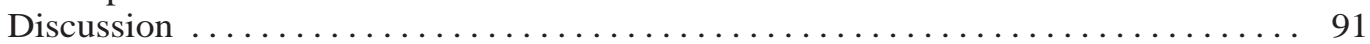

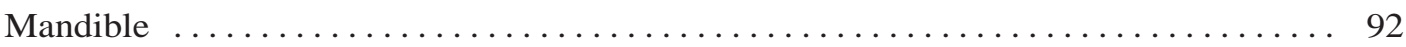

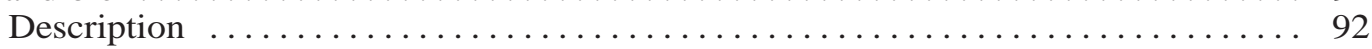

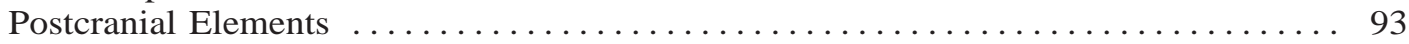

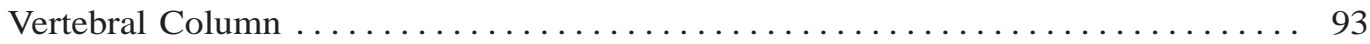

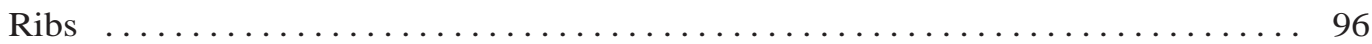

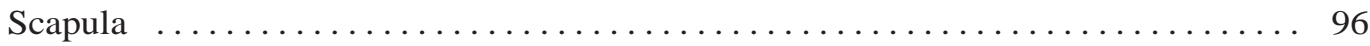

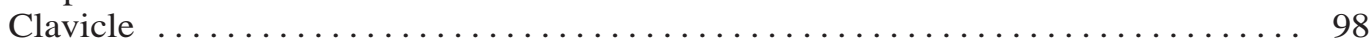

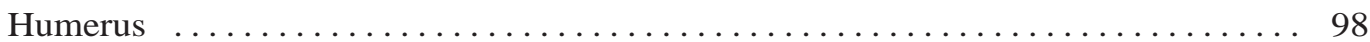

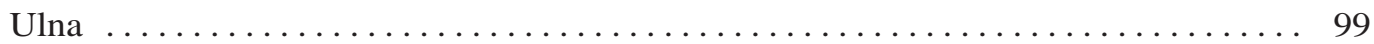

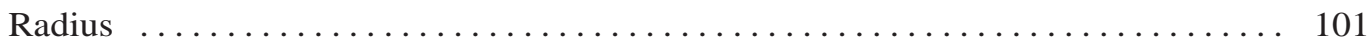




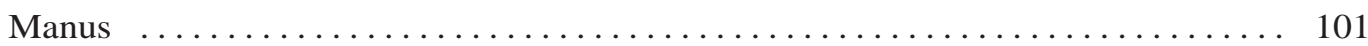

Pelvic Girdle ...................................... 103

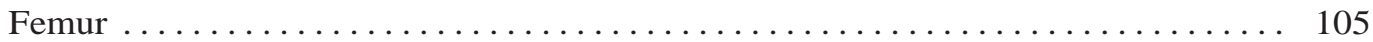

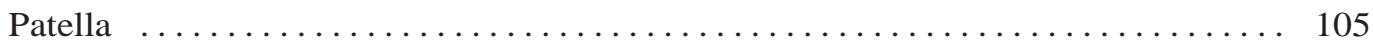

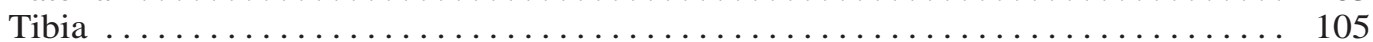

Fibula ......................................... 109

Astragalus ........................................... 109

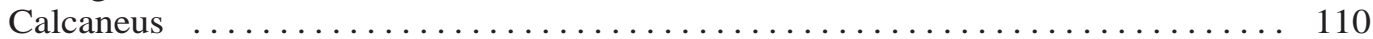

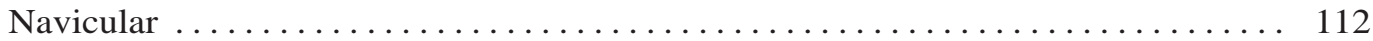

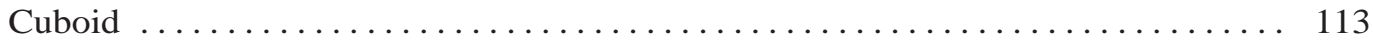

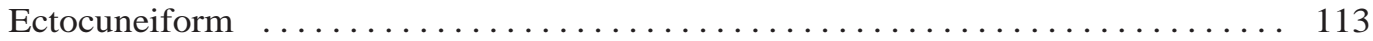

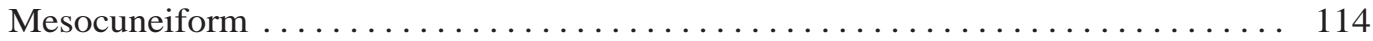

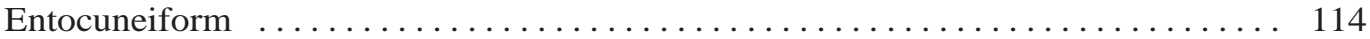

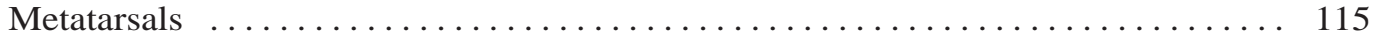

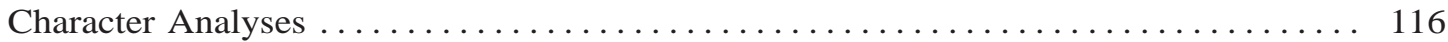

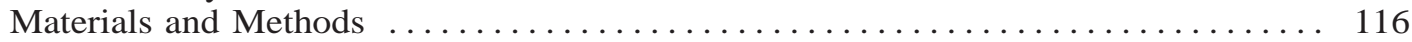

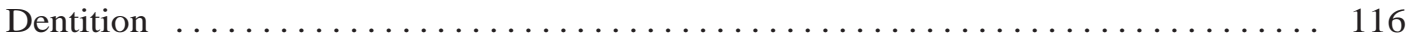

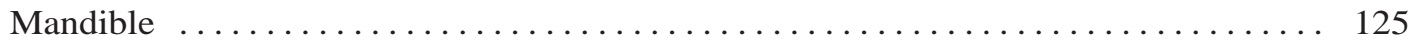

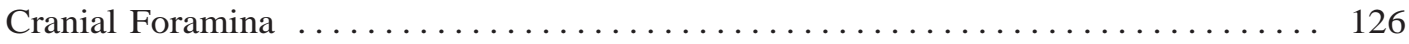

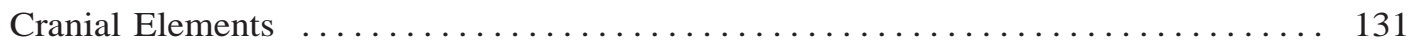

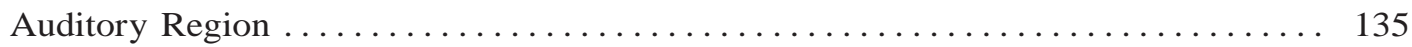

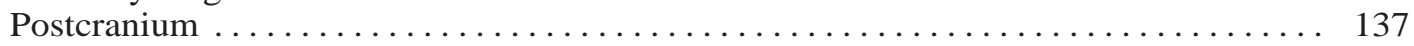

Phylogenetic Analyses . . . . . . . . . . . . . . . . . . . . . 144

Materials and Methods ................................. 144

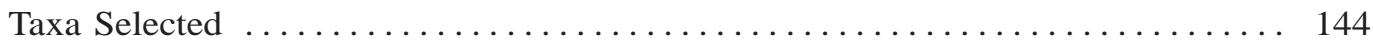

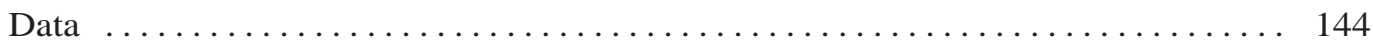

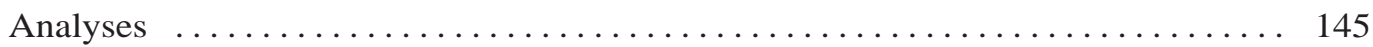

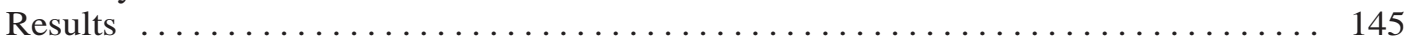

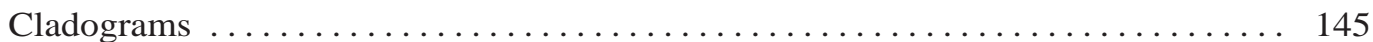

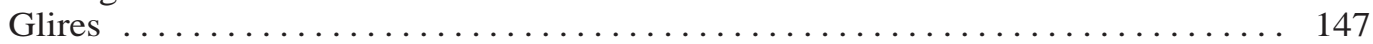

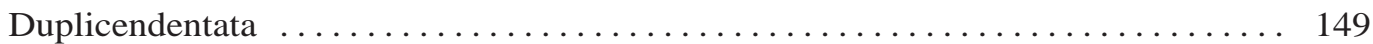

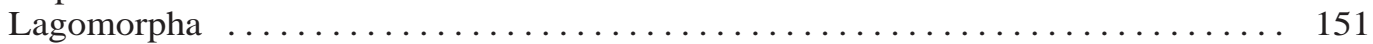

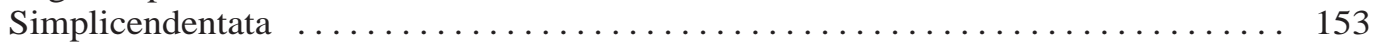

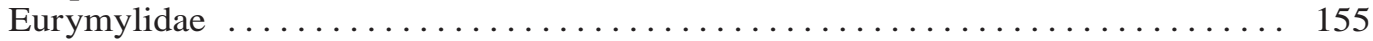

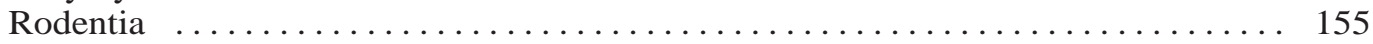

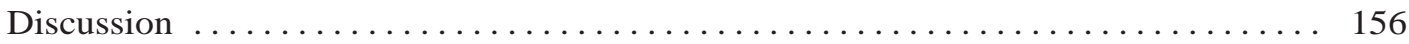

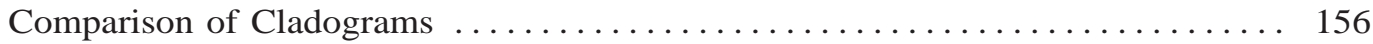

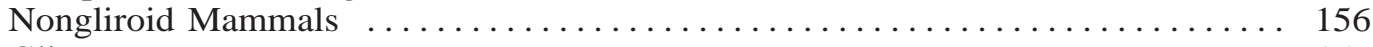

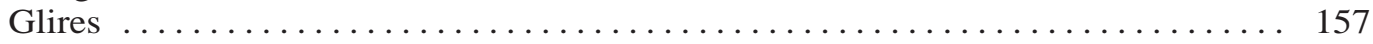

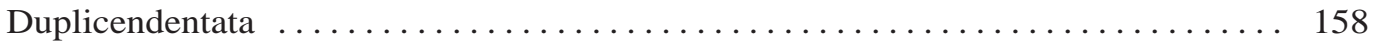

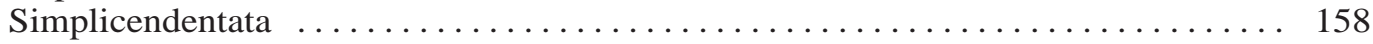

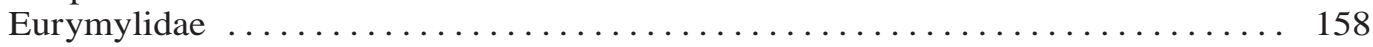

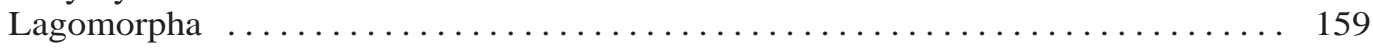

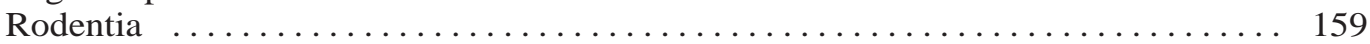

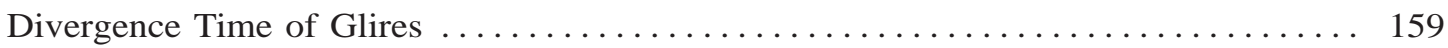

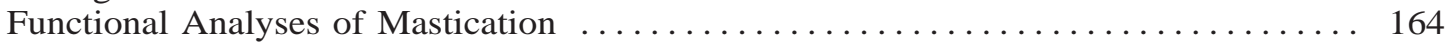

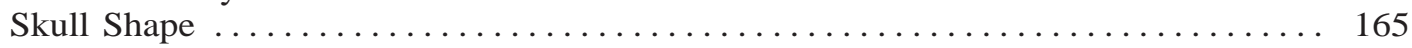

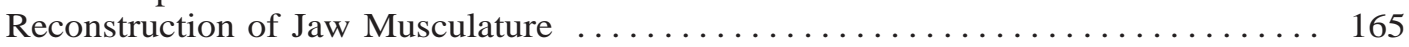

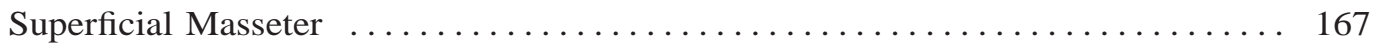

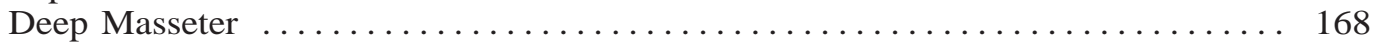

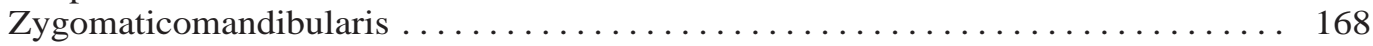

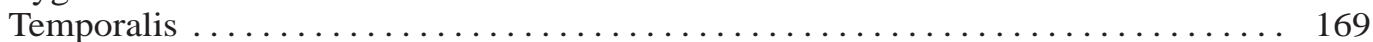

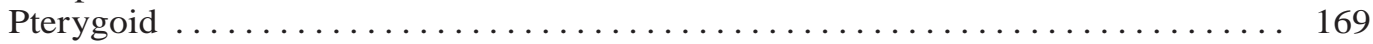




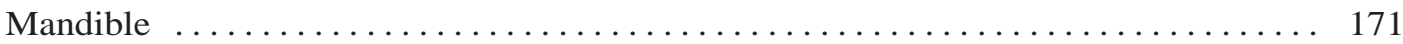

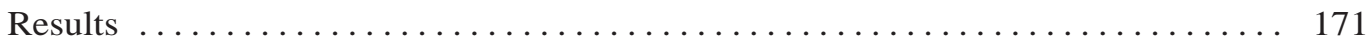

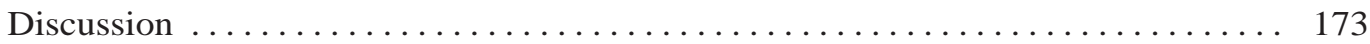

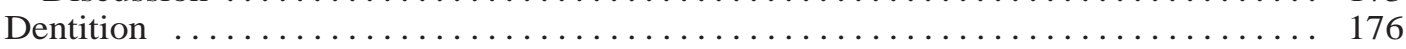

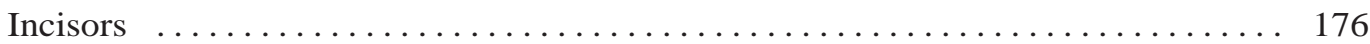

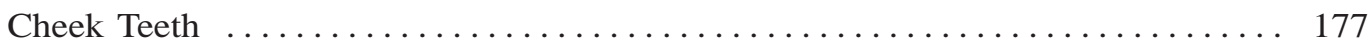

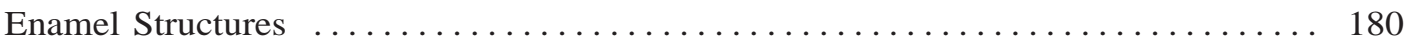

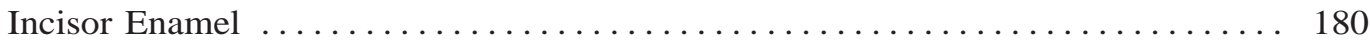

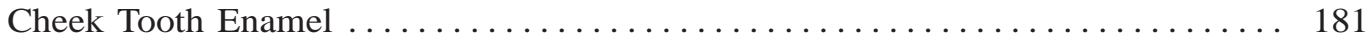

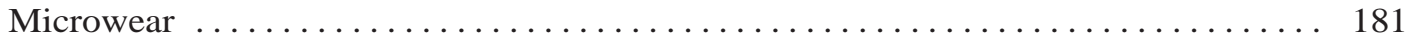

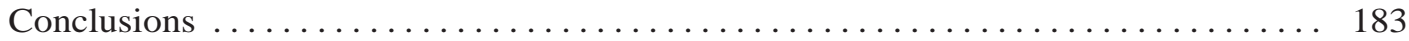

Functional Analysis of Postcranial Skeleton $\ldots \ldots \ldots \ldots \ldots \ldots \ldots \ldots \ldots \ldots \ldots \ldots \ldots . \ldots \ldots$

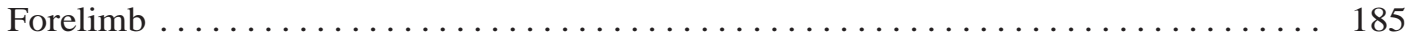

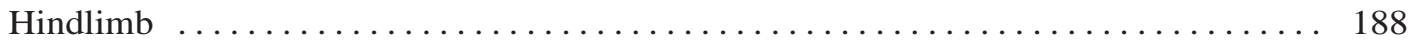

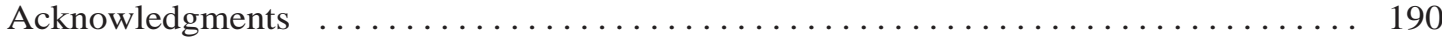

References .................................... 191

Appendix 1: Taxa Selected for Analysis and Source of Data $\ldots \ldots \ldots \ldots \ldots \ldots \ldots 212$

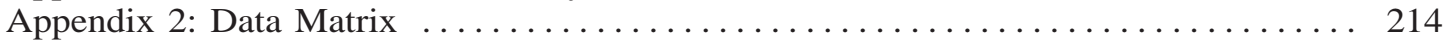

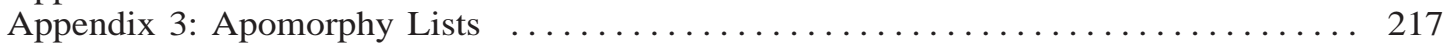

Appendix 4: Measurements of Mandibles ........................ 242

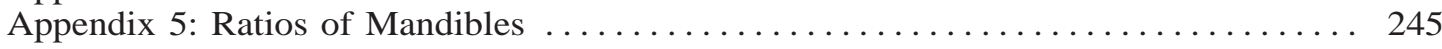




\section{ABSTRACT}

Rhombomylus is a gliroid mammal endemic to several early Eocene localities of central and eastern Asia. It is best represented by a collection of numerous jaws and teeth, dozens of juvenile and adult skulls, and associated postcranial specimens from the early Eocene $\mathrm{Yu}-$ huangding Formation at the Dajian village, Junxian County, Hubei Province, China. These specimens are the best preserved material of early nonrodent, nonlagomorph Glires known to date, and they provide a unique opportunity for us to understand anatomy, intraspecies variation, postnatal ontogeny, phylogeny, and evolution of basal gliroid mammals.

This study opens with an introduction to the current knowledge of the phylogeny of gliroid mammals, a brief review of the research history of Rhombomylus, and a description of the locality where the specimens were collected. The core of the study consists of a taxonomic revision of the genus, a detailed description of the osteological morphology, extensive analyses on morphological characters, analyses on phylogeny, discussions on divergence time of the Glires, and analyses on functional morphology of mastication and locomotion.

In comparing all known specimens assigned to the genus, we recognize only a single species, Rhombomylus turpanensis, for the genus and consider " $R$. laianensis" a junior synonym of $R$. turpanensis. This monotypic genus is rediagnosed. As a typical gliroid mammal, Rhombomylus possesses the following features: a single pair of enlarged, ever-growing incisors in both upper and lower jaws, the enamel band covering primarily the anterior and lateral surface of the incisor, a large diastema between the incisor and premolars, and a dental formula of dI2, C0, P3-P4, M1-M3/di2, c0, p3-p4, m1-m3. The single pair of upper incisors distinguishes Rhombomylus from duplicendentate Glires. Rhombomylus is more similar to Matutinia than to other simplicidentate Glires in having the following features: a broad hypocone shelf on the upper cheek tooth, a specialized zygomatic arch formed by the maxilla and jugal, an expanded mastoid of the petrosal that is exposed on the skull roof between the squamosal and parietal, an extensively interdigital suture between the premaxilla and frontal, a contribution of the ectotympanic to the medial wall of the glenoid fossa, and a distinct external meatus contacting the squamosal and blocking the postglenoid fossa posteriorly. Rhombomylus differs from Matutinia in being generally larger, in having higher tooth crowns, a stronger protocone and paracone, a complete vertical groove on the lingual surface of cheek teeth, and stronger crests on cheek teeth, and in lacking the carotid foramen in the auditory region. In general, Rhombomylus is more derived in morphology than is Matutinia.

Although the general skull morphology of Rhombomylus is rodentlike, the dental morphology and detailed anatomy of the skull display unique specialization of the genus. Upper tooth crowns are higher lingually than labially. Lophs are well developed, whereas cusps are not distinct. The incisor enamel is pauciserial and has two layers, with the outer layer being poorly differentiated. The enamel is thicker basally than apically in the same incisor and thicker in incisors of adult individuals than in juveniles. The basic enamel microstructure does not vary much at different sections of the same incisor, nor does it differ significantly in incisors from individuals of different ages. The thinnest enamel of the cheek teeth is on the occlusal surface, whereas the thickest is along the anterior surface of the upper tooth and the posterior wall of the lower tooth trigonid; these two regions are the main shearing surfaces of cheek teeth. Although the enamel microstructure varies from area to area on the same cheek tooth, there is always a region where the enamel microstructure is comparable to that of the incisor. The shearing facets on the cheek teeth bear numerous fine pits and striations. The orientation of the striations indicates a lateromedial movement of the mandible during mastication. Lack of enamel ridges on the occlusal surface and predominance of fine striations and pits on the wear facets of teeth suggest a dietary preference on soft vegetation. The skull in general is rodentlike, although the temporal region is primitively larger. Specialized cranial features include a strong zygomatic process of a complex shape, a complete bulla, expanded mastoid of the petrosal, exposure of the mastoid on the skull roof, loss of the internal carotid and stapedial arteries, the postglenoid foramen situated in the end of the glenoid fossa, and exclusion of the squamosal from the middle ear region. Cross sections show that the nasal cavity is simple and lacks any paranasal sinus except for possible existence of the maxillary sinus. The Jacobson's fossa is well developed. The ethmoid is similar to that of rodents in having four endoturbinals with the lamella of the second split to form two olfactory plates and a low number of ectoturbinals. Unlike other mammals in which the two olfactory plates of endoturbinal II inroll in 
the same direction, those of Rhombomylus inroll oppositely. In addition, entoturbinal III and IV fold dorsally instead of ventrally. The brain of Rhombomylus has relatively long olfactory lobes, small and unconvoluted cerebrum and cerebellum, and relatively narrow, long cerebrum.

The postcranial skeleton of Rhombomylus is relatively conservative compared to the dentition and skull. The scapula is triangular in shape with distinct anterior and posterior angles. The clavicle is slim. The humerus has a low but prominent greater tubercle, a moderate deltopectoral crest, a moderate lateral epicondylar crest, and a deep olecranon fossa. The ulna has a large olecranon process and a deep trochlear notch. The hindlimb is much longer than the forelimb. The femur has a high greater trochanter, a posteromedially directed lesser trochanter, a slender shaft, a deep patellar trochea, and an anteroposteriorly deep distal epiphysis. The tibia and fibula are also elongate and separate. The tibial crest is prominent. The upper ankle joint has the calcaneofibular contact.

Based on the morphology of Rhombomylus, the literature, and observation of specimens and casts of other mammals, we analyzed and tabulated 227 dental, cranial, and postcranial characters (608 states) among 50 terminal taxa. Characters were selected based on their use in previous studies, their amenability to description as discrete states, and a nonautapomorphic distribution. Terminal taxa include both fossil and extant genera and are representative of main Glires subgroups and of other eutherians that have been previously considered to be relatives of gliroid mammals. Fossil Glires chosen for the study are those represented by relatively complete material.

Phylogenetic analyses were conducted using MacClade and PAUP*. Five strict consensus cladograms resulted from heuristic searches based on three datasets, respectively: the dental, skeletal, and combined data matrix. Detailed information is provided for the preferred cladogram that was obtained from the combined data with all characters unordered. In all of the cladograms, the clade of Glires is identified. In the preferred cladogram the clade is supported by the highest Bremer index (5), branch length (45), and bootstrapping value (100). The sister group of the Glires is preferably the clade consisting of Anagale, Anagalopsis, and Pseudictops. In any case, zalambdalestids were only distantly related to the Glires. Within the Glires, clades of Duplicendentata, Simplicendentata, Eurymylidae, Lagomorpha, and Rodentia are identified. The clades of Duplicendentata and Lagomorpha are relatively robust, whereas that of the Simplicendentata is weakly supported. Our analyses identified a clade consisting of Eurymylus, Heomys, Rhombomylus, and Matutinia, which are the core members of the Eurymylidae. The phylogenetic position of Sinomylus is unstable. In Rodentia, the clade of the Hystricognathi is robust. Tataromys forms the sister group of the Hystricognathi-a clade named Hystricognathiformes. Sciurognath rodents are paraphyletic. Cavia is deeply nested in rodents; its removal from rodents and attachment to the primates or other clades significantly increases the steps of character transformations on the cladogram.

Phylogenetically constrained distributions of the selected taxa indicate that the divergences of the Glires from other eutherian clades, between Lagomorpha and Rodentia, and among the main subgroups of rodents do not predate the Cretaceous-Tertiary boundary. Phylogenies and distributions of gliroid mammals at various levels support the traditional view that modern placental orders evolved after the Cretaceous-Tertiary boundary in an explosive pattern and disagree with hypotheses that attempt to reconcile the discrepancies between molecular clock dating and fossil records of modern placental divergences. These hypotheses include low preservation rates of Cretaceous mammals, lack of morphologically diagnostic features in Cretaceous taxa, unknown Cretaceous fossil records in southern continents, and deep superordinal branching of eutherian mammals in the Cretaceous.

The masticatory apparatus and mastication of Rhombomylus are similar to those of lagomorphs in having upper cheek teeth wider than the lower ones, cheek teeth with transverse shearing ridges, main shearing facets on the anterior surface of the upper cheek tooth and the posterior wall of the trigonid of the lower, the thickest enamel forming the main shearing edges, and power strokes of chewing unilaterally and buccolingually directed with both shearing and crushing. The masticatory apparatus and mastication of Rhombomylus are similar to those of rodents in absence of I3 and P2, an anteroposteriorly elongate glenoid fossa, a relatively long minimum working distance of the mandible, upper diastema longer than the lower one, and similarity in the general morphology of the mandible. The masticatory apparatus of Rhombomylus shows several primitive conditions compared with more advanced gliroid mammals, including posteriorly positioned masseter muscles, larger coronoid process of the man- 
dible and temporal region of the skull, slender incisors, enamel pauciserial, and primarily transverse movement of the mandible during mastication. The chewing cycle of Rhombomylus can be divided into six phases: rest position of the anisognathic dentition; jaw opening and moving labially to the working side; vertical movement of the lower jaw to bring the upper and lower teeth into contact; transverse shearing between teeth; compression between the enlarged hypocone shelf and the trigonid and between the talonid basin and the protocone; and back to rest position.

Conclusions on evolution of the masticatory apparatus of gliroid mammals include: (1) The propalinal movement of the mandible that permits gnawing and incisor sharpening is a gliroid synapomorphy. (2) Although all gliroid mammals can move lower jaws anteroposteriorly for incisal biting and sharpening, only some advanced forms are capable of propalinal mastication. The propalinal mastication is a derived condition and has been independently evolved several times in rodents. (3) The evolution of the gnawing function is prior to and independent of that of the propalinal chewing and is prior to the anterior shift of the jaw muscles. (4) Anterior shifting of the jaw muscles was originally and primarily to emphasize the strength of the incisal bite. (5) Modifications in jaw musculature occur in several directions independently among various gliroid groups, particularly rodents. (6) Acquisition of some cranial features such as the anteroposteriorly elongate glenoid fossa is originally related to gnawing and incisor sharpening, not to mastication. (7) Reduction in number of teeth is a general evolutionary trend in rodents; however, number of enamel bands functioning as grinding edges commonly increases. (8) The mastication in gliroid mammals evolved from primarily crushing and shearing to grinding; the latter is more efficient for processing vegetable food with tough fabers. (9) The evolution of rodent mastication is a refining process that involves many aspects of the masticatory apparatus including the skull, mandible, jaw muscles, tooth morphologies, tooth size, enamel structures, and chewing rates. Refinement of various masticatory features evolved independently in different directions and in mosaic combinations. The heterochronical acquisition of masticatory features and the mosaic distributions of these features convey phylogenetic information at various levels for the Glires.

Morphological analog with extant mammals indicate that the forelimbs of Rhombomylus do not show specialization toward either arboreal, terrestrial cursorial, or fossorial behavior. The forearms are probably capable of extensive movement in anteroposterior directions, but with limited abduction and adduction. They may also have the capacity for supination and pronation to some extent in food manipulation. The hindlimb of Rhombomylus shows many characteristics of terrestrial mammals. Some features even suggest capability of running or jumping. The hindlimb is much longer than the forelimb, indicating asymmetrical gait. Rhombomylus is basically a terrestrial mammal, capable of walking or jumping with an asymmetrical gait.

\section{INTRODUCTION}

In a simplified picture Glires includes two mammalian orders, Lagomorpha and Rodentia. Lagomorpha (pikas, rabbits, and hares) comprises two living families (13 genera, 81 species) (Nowak, 1999) and 56 fossil genera (McKenna and Bell, 1997) that first appeared in the Paleocene of Asia (Dawson, 1977; McKenna, 1982), although it is debatable whether some of these fossils are lagomorphs. Rodentia consists of 29 Recent families (about 468 genera, 2052 species) (Nowak, 1999) and 743 extinct genera (McKenna and Bell, 1997) that date to the late Paleocene of North America (Wood, 1962; Dawson et al., 1984; Korth, 1984; Dawson and Beard, 1996). Lagomorphs and rodents constitute nearly half the species diversity of extant mammals, provide medical subjects, physiological models, and molecular resources, and are the subject of many biological experiments (Flynn, 1994).

Glires was first proposed by Linnaeus (1758), and at that time included known living rodents, lagomorphs, and one perissodactyl (Rhinoceros) mainly on the basis of a pair of enlarged incisors in both lower and upper jaws of these taxa. By the same token, the primate Daubentonia and diprotodontan marsupials were once classified with rodents at the beginning of the 19th century (Cuvier, 1800-1805). Rhinoceros, Daubentonia, and diprotodontan marsupials were afterward firmly removed from Glires, while the relationship between rodents and lagomorphs became doubtful (Illiger, 1811; de Blainville, 
1816; Gervais, 1848; Brandt, 1855; Tullberg, 1899; Weber, 1904; Gidley, 1912).

Although contemporary systematists had increasingly gained confidence to define rodents and to separate them from lagomorphs (Gidley, 1912; Simpson, 1945; Hartenberger, 1985; Wilson, 1989), Glires monophyly has proven one of the most controversial issues in higher level mammalian systematics of the 20th century (Wilson, 1949; Wood, 1957; Dawson, 1967; Landry, 1974, 1999; Luckett and Hartenberger, 1985; Li and Ting, 1985; Novacek, 1990; Jaeger, 1988; Luckett and Hartenberger, 1993). For many years Landry $(1970,1974)$ was the only active defender in North America for a special relationship of lagomorphs and rodents ( $\mathrm{Li}$ et al., 1987). In some studies, the Rodentia was even regarded as an incertae sedis group among eutherian mammals (Olson, 1971; McKenna, 1975; Szalay, 1977).

The advent of phylogenetic systematics and recognition that synapomorphy rather than degree of morphologic difference is the appropriate arbiter of evolutionary relationship have again brought the Glires question into focus. Discoveries of several early gliroid mammals from Asia further tilted the scale toward the Glires monophyly (Gingerich and Gunnell, 1979; Gingerich and McKenna, 1980; Hartenberger, 1980; Korth, 1984; Dawson et al., 1984; Wilson, 1989; Landry, 1999). Indeed, numerous investigations have been made in exploring relationships among gliroid mammals (Luckett, 1977, 1985; Hartenberger, 1977, 1980, 1985; Szalay, 1977, 1985; Li, 1977; Li and Ting, 1985, 1993; Li et al., 1987; McKenna, 1982; Novacek, 1982b, 1985; Dashzeveg and Russell, 1988; Dashzeveg, 1990a, 1990b; Luckett and Hartenberger, 1993; Meng et al., 1994). In most of these studies, however, characters have been cited frequently as selfevident or have been sampled across a limited number of Glires taxa. Morphological data from different parts of the organisms have never been fully integrated in a synthetic study using cladistic methodology.

In addition to the sister-group relationship of rodents and lagomorphs, each group has for a time been allied with other mammalian taxa. Rodents were related to multituberculates (Major, 1893; Hinton, 1926; Friant,
1932), mixodectids (Osborn, 1902), tillodonts and taeniodonts (Cope, 1888), primates (McKenna, 1961; Van Valen, 1966, 1971; Lillegraven, 1969; Wood, 1962, 1977; Patterson and Wood, 1982), leptictids (Szalay, 1977, 1985), and eurymylids (Li, 1977; Li and Yan, 1979; Gingerich and Gunnell, 1979; Gingerich and McKenna, 1980; Hartenberger, 1980; Korth, 1984; Dawson et al., 1984; Wilson, 1989; Meng et al., 1994; Meng and Wyss, 2001). Lagomorphs, on the other hand, were associated with triconodonts (Gidley, 1906; Ehik, 1926), artiodactyls (Gidley, 1912; Hürzeler, 1936; Moody et al., 1949), condylarths (Wood, 1957), zalambdodont insectivorans (L.S. Russell, 1959), pseudictopids (Van Valen, 1964), anagalids (Szalay and McKenna, 1971), macroscelidids (McKenna, 1975), eurymylids (Wood, 1942), zalambdalestids (Szalay and McKenna, 1971; McKenna, 1975, 1994), and mimotonids (Li and Yan, 1979; Li and Ting, 1985, 1993; Meng et al., 1994; Meng and Wyss, 2001). Of all the postulated relationships, the following ones are current.

RoDENT-PRIMATE RELATIONSHIP: McKenna (1961) recognized that an upper cheek tooth of Acritoparamys atavus resembles those of Plesiadapis and Phenacolemur, and that it probably suggests relationships near the base of rodents and primates. This idea was followed by others (Van Valen, 1966; Lillegraven, 1969; Wood, 1962, 1977; Patterson and Wood, 1982) and was most clearly addressed by Wood (1977), who proposed that southeastern North America became the most probable area of origin, with the order presumed to have arisen (most probably from primates) in the middle Paleocene. A rodentprimate relationship, although thought still unfalsified (Butler, 1985), is further complicated by recent recognition of relationships between plesiadapiformes and Dermoptera on the basis of the postcranial and cranial similarities (Beard, 1990, 1993a, 1993b; Kay et al., 1990), despite that the strength of the cranial evidence has been questioned by others (Wible and Martin, 1993).

Rodent-Leptictid Relationship: The North American fossil family Leptictidae provided the ancestral stock for Rodentia, because the two taxa share some similarities in postcranial skeletons and because, on the 
other hand, there is no postcranial evidence to support a rodent-lagomorph relationship (Szalay, 1977, 1985). The nature of those shared postcranial similarities (i.e., whether they are uniquely shared by the two taxa) has been questioned by several authors (Novacek, 1980; Luckett and Hartenberger, 1985). On the contrary, cranial characters indicate a sister group of rodents and lagomorphs, rejecting a leptictid-rodent clade (Novacek, 1986b).

LAGOMORPH-ZALAMBdAlESTID RELATIONSHIP: Lagomorphs are closely related to zalambdalestids to the exclusion of rodents (Szalay and McKenna, 1971; McKenna, 1975). Recently, McKenna (1994) argued that the Late Cretaceous Barunlestes ( $\mathrm{Za}$ lambdalestes as well) "seems to me to be a distant relative of lagomorphs-closer to them than to many other kinds of mammals because it seems to share at least a few derived features with them." A lagomorph-zalambdalestid relationship not only extends the known history of lagomorphs into the Cretaceous but also forces either rejection of Glires monophyly or a greatly expanded concept of Glires.

RodEnT-Eurymylid-LAGOMORPH-MiMOTONID RELATIONSHIP: Rodents and lagomorphs share an ancestor in common with basal gliroid mammals such as eurymylids and mimotonids, an assumption amounting to an expanded concept of Glires currently favored by many paleontologists (Li, 1977; Li and Yan, 1979; Gingerich and Gunnell, 1979; Gingerich and McKenna, 1980; Hartenberger, 1980, 1985; Korth, 1984; Dawson et al., 1984; Flynn et al., 1986; Li et al., 1987; Wilson, 1989; Meng et al., 1994; Meng and Wyss, 2001). In this relationship, rodents and eurymylids usually form Simplicidentata, a group characterized mainly by a single pair of incisors in both lower and upper jaws, whereas two pairs of upper incisors link lagomorphs and mimotonids as Duplicidentata. Despite the agreement among these paleontologists, there are still many problems existing in this scenario, such as the monophylies of Rodentia, Simplicidentata, Duplicidentata, Eurymylidae, and Mimotonidae; the placement of Heomys; and the interpretation of some character transformations (Li and Ting, 1985, 1993; Flynn et al., 1986; Flynn et al., 1987; Dashzeveg et al., 1987; Dashzeveg and Russell, 1988; Luckett and Hartenberger, 1993; Meng et al., 1994; McKenna and Meng, 2001; Meng and Wyss, 2001).

MoleCUlar Hypotheses: Molecular studies further indicate that the Glires problem partially interlocks with systematic problems of other eutherians. Several hypotheses concerning Glires have been proposed, including nonmonophyly of Rodentia (Graur et al., 1991, 1992; Li et al., 1992; D’Erchia et al., 1996), a primate-lagomorph clade (Easteal, 1990; Penny et al., 1991), a primate-rodentlagomorph relationship that involves tree shrews (Miyamoto and Goodman, 1986; Czelusniak et al., 1990), and a supraordinal clade comprised of Primates, Dermoptera, Scandentia, Lagomorpha, and Rodentia (Stanhope et al., 1993). However, more recent molecular studies (Madsen et al., 2001; Murphy et al., 2001a, 2001b) are congruent with the conventional rodent monophyly and Rodentia-Lagomorpha sister group relationship.

Rhombomylus is an eurymylid taxon from the early Eocene of Asia and was considered to be a close relative of rodents ( $\mathrm{Li}$ et al., 1987; Li and Ting, 1993; Meng et al., 1994; Meng and Wyss, 2001). It is represented by a collection of numerous jaws and teeth, juvenile and adult skulls, and associated postcranial specimens. These specimens provide a unique opportunity for us to understand anatomy, intraspecies variation, ontogeny, phylogeny, and evolution of basal gliroid mammals. This monograph is a study of these specimens and their implications to the phylogeny and evolution of the Glires.

\section{RESEARCH HISTORY}

Discoveries OF RHOMBOMYLUS: Two species have been named in Rhombomylus. The species published first was $R$. laianensis (Zhai et al., 1976) from early Eocene Zhangshanji Formation at the Laian locality, Laian District, Anhui Province, China. The second species, $R$. turpanesis, was described by Zhai (1978) based on specimens collected from the early Eocene Shisanjianfang Formation, east of Turpan Basin, Xinjiang Province, China. Zhai considered Rhombomylus a new 
genus in his 1978 paper and specified $R$. turpanesis the type species of the genus. In the 1976 paper Zhai et al. compared R. laianensis with $R$. turpanesis and had apparently submitted the manuscript about $R$. turpanesis for publication earlier than that about $R$. laianensis. The $R$. turpanesis paper, however, was published later than that on $R$. laianensis.

Additional and better specimens of Rhombomylus were discovered from the early Eocene Yuhuangding Formation, Dajian locality, Hubei Province, China, during a period of more than two decades from 1975 to 1998. Rhombomylus sp. was later reported from there (Xu et al., 1979; Li and Yan, 1979), but no specimen was described. A right maxilla with P2-M2 from Fangxian, Hubei, was identified as Rhombomylus cf. turpanensis by Lei (1984), but again there was no description of the specimens. A fragmentary lower jaw with p3-m2 (IVPP $\mathrm{V} 11833$ ) and three lower molars from the early Eocene Yuhuangding Fm. at Leibei, Danjiangkou City, Hubei, was recently referred to as Rhombomylus cf. turpanensis by Guo et al. (2000). Rhombomylus cf. turpanensis and Rhombomylus sp. were reported but not described by Ma and Cheng (1991) from the Yuhuangding Fm. of the Liguanqiao Basin. Preliminary studies have been made on some Dajian specimens (Ting and Li, 1984; Li and Ting, 1985, 1993). Postcranial specimens of Rhombomylus were poorly documented. Zhai (1978) mentioned a calcaneus of Rhombomylus turpanensis (V4366), but this specimen is now lost. Li and Ting (1993) briefly described some postcranial remains of Rhombomylus, but assignment of these specimens to the genus is uncertain (see below).

Dashzeveg et al. (1987) noted several specimens of Rhombomylus sp. from TsaganKhushu of Mongolia (PSS 20-3, left m2-m3; PSS 20-54, right $\mathrm{m} 1$; PSS 20-100, right jaw fragment with incisor and p4; PSS 20-117, right 1; PSS 20-118, right $\mathrm{m} 3$; PSS 20-165; PSS 20-220, right $\mathrm{m} 2$; PSS 20-125, right $\mathrm{m} 1$.), and recognized the similarity between those specimens and $R$. turpanensis. Those Mongolian specimens, however, have not been described up to this point. Dashzeveg and Russell (1988) reported additional spec- imens (PSS 20-164, a left maxilla with P4M3; PSS 20-169, p4-m3) from Tsagan-Khushu and PSS 20-165, which was in Dashzeveg et al. (1987), and referred those specimens to $R$. cf. turpanensis.

Enamel microstructure of the incisors assigned to Rhombomylus or cf. Rhombomylus has been documented by Martin (1992, 1993, 1999).

TAXONOMY OF RHombomylus: The classification and phylogenetic relationships of Rhombomylus with other basal Glires have been unstable (fig. 1; table 1). Originally, Zhai (1978) placed Rhombomylus in the family Eurymylidae under the order Anagalida; the latter at the time included Zalambdalestidae, Anagalidae, Pseudictopidae, and Eurymylidae (Szalay and McKenna, 1971). The content of Anagalida was expanded in McKenna and Bell (1997; table 1). Zhai (1978) recognized several similarities between Rhombomylus and Eurymylus, such as the dental formula and development of the hypocone shelf, as well as the prominent difference of the incisors between Eurymylidae and other families contained in Anagalida. Others (Li and Ting, 1985, 1993; McKenna and Bell, 1997) followed Zhai's assignment of Rhombomylus to Eurymylidae of Anagalida.

The type genus for the family Eurymylidae is Eurymylus Matthew and Granger, 1925. When Eurymylus laticeps was described, it was represented by an upper dentition and was placed in ?Plesiadapidae of Menotyphla (Matthew and Granger, 1925). In the same paper, a lower jaw with m1-m2 was considered as a different genus and species ("Baenomys ambiguus") and was placed in Glires. Based on additional specimens, Matthew et al. (1929) realized that the upper jaw of Eurymylus and the lower jaw bearing the name "Baenomys ambiguus" actually belonged to the same species, and they selected Eurymylus laticeps as the definitive name because it was based on a better specimen. Matthew et al. (1929) then proposed the family Eurymylidae. Because of the mixture of rodent and lagomorph traits recognized by the authors at that time, the family was referred to ?Glires. In a detailed comparison of Eurymylus with lagomorphs, Wood (1942) considered that the Eurymyli- 


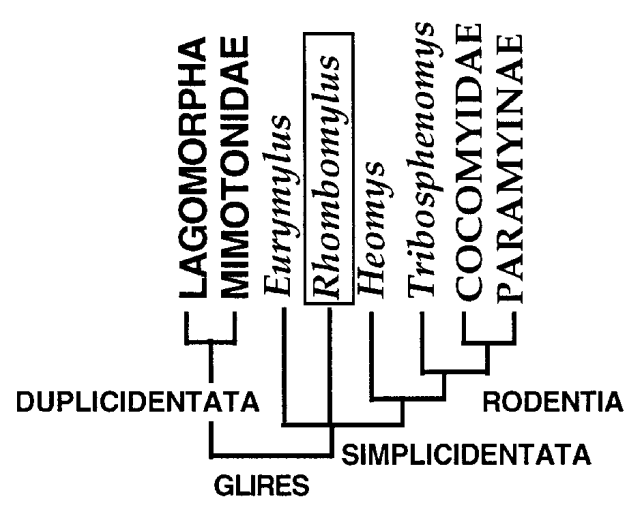

A
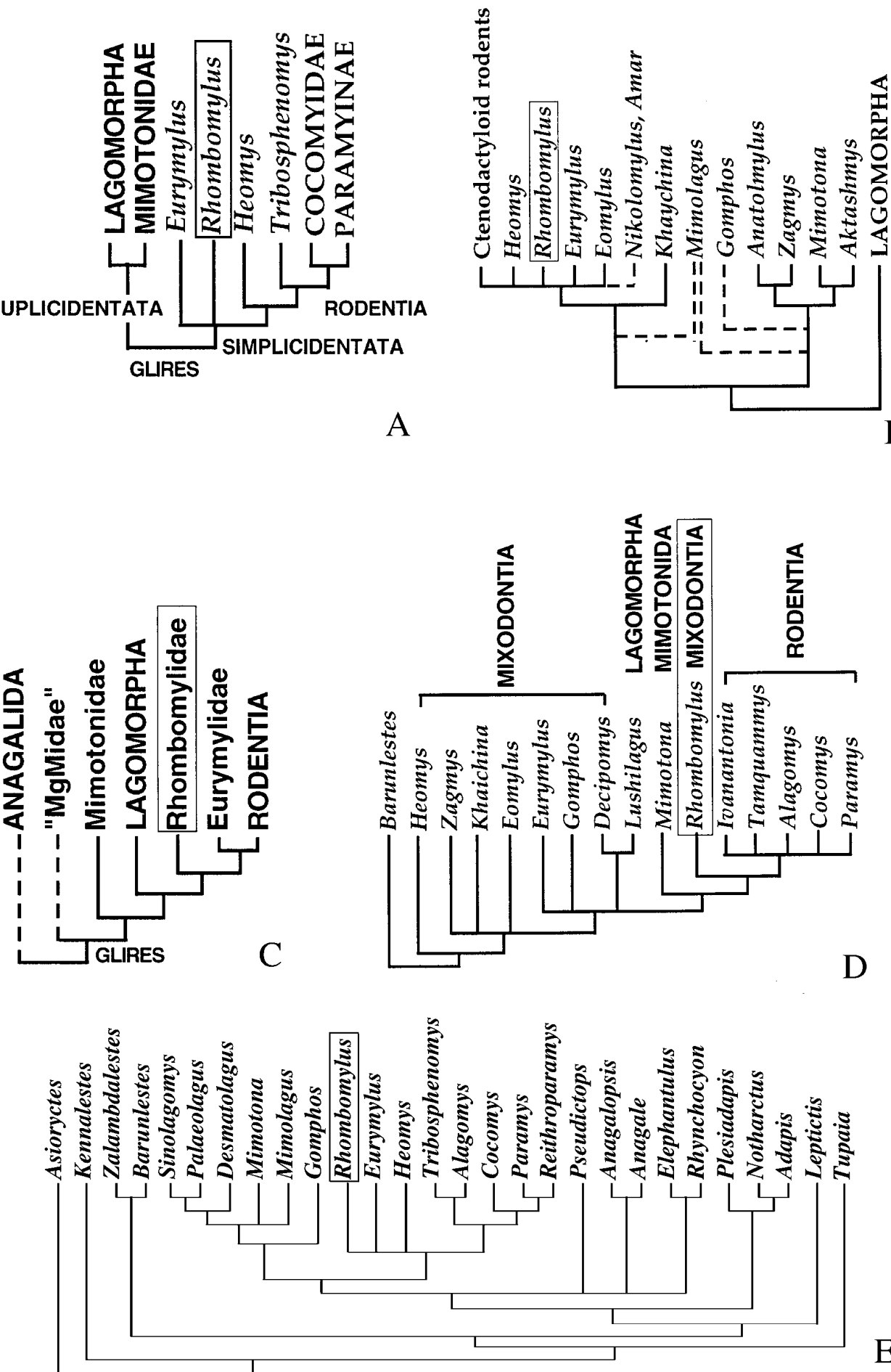

$\mathrm{E}$

Fig. 1. Phylogenetic hypotheses of basal Glires and the phylogenetic position of Rhombomylus, based on Meng et al. (1994), Averianov (1994), Li et al. (1987), Dashzeveg et al. (1998), and Meng and Wyss (2001). 
TABLE 1

Classification of Basal Gliroid Mammals

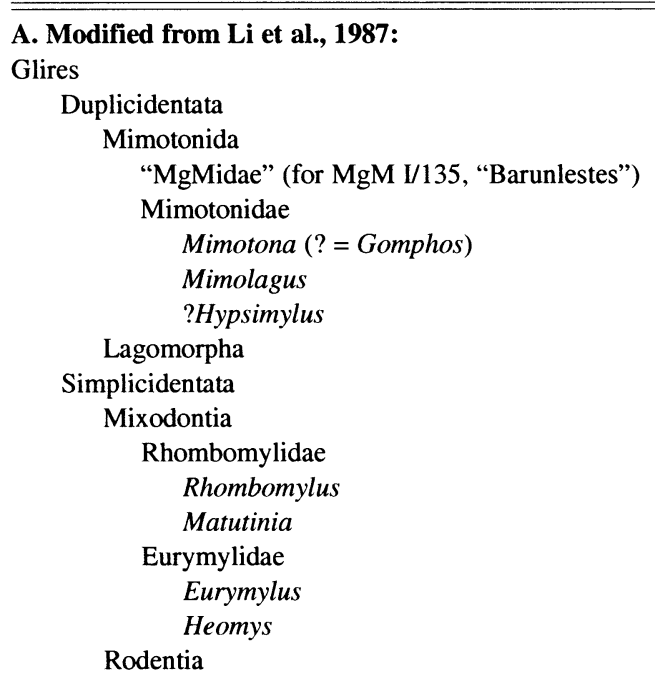

B. Modified from Dashzeveg and Russell, 1988:

Glires

Mixodontia
Eurymylidae
Eurymylinae
Eurymylus
Heomys
Rhombomylus (=Matutinia)
Eomylus
Amar
Khaychininae
Khaychina
Zagmyinae
Zagmys
Hypsimylinae
Hypsimylus
Mimotonidae
Mimotona
Gomphos

dae represent an ancestral stock from which the Leporidae and Ochotonidae have been derived, although he noticed that the absence of P2 and posteriorly extended lower incisor in Eurymylus differ from those of lagomorphs. Nonetheless, Wood (1942) regarded Eurymylus as a Paleocene lagomorph. Dawson (1967) and Van Valen (1964) placed a more lagomorph-like form, Mimolagus rodens Bohlin, 1951, in the family Eurymylidae.

Sych (1971) made an extensive study of

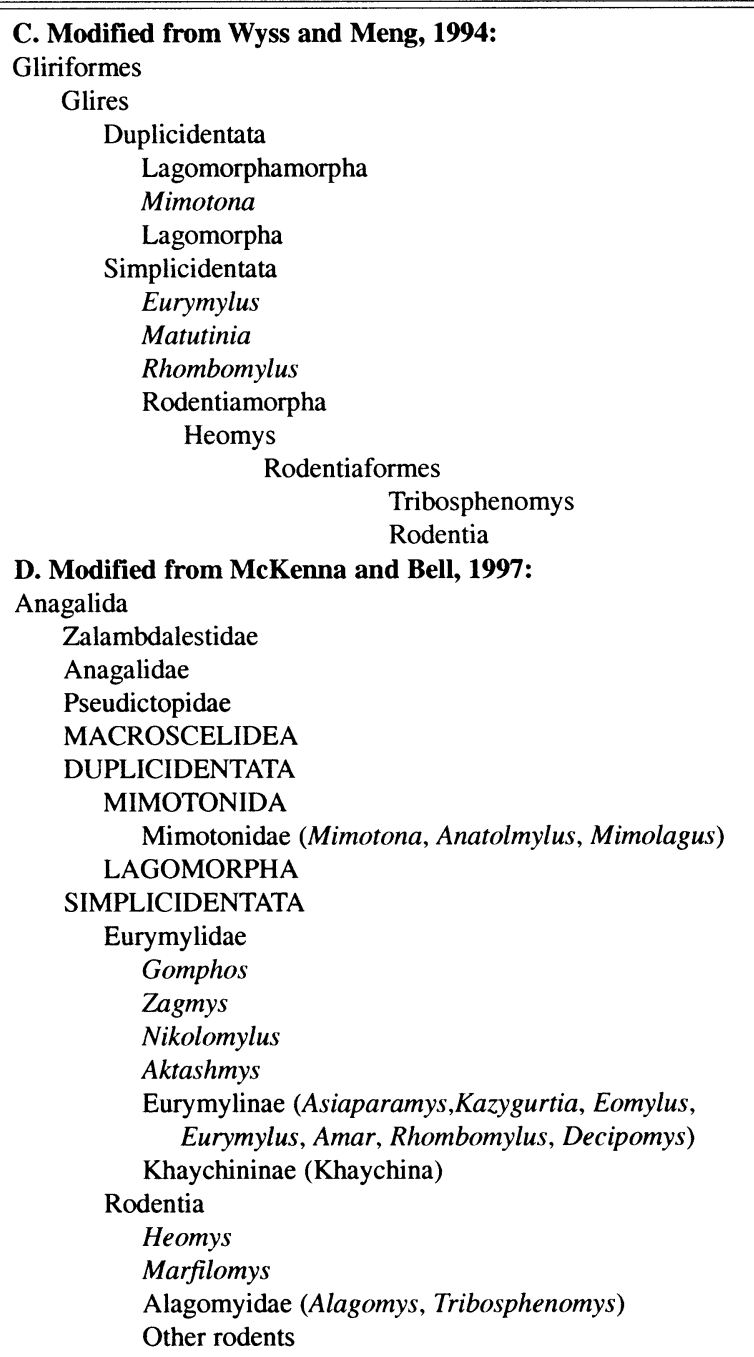

Eurymylus in comparison with lagomorphs, rodents and insectivores and concluded that Eurymylus does not belong to any of these groups. Sych (1971) instead invented a monotypic order Mixodontia for Eurymylus. In the same year, Szalay and McKenna (1971) proposed the order Anagalida, which comprises several Asian forms, including $\mathrm{Za}-$ lambdalestidae, Anagalidae, Pseudictopidae, and Eurymylidae. Szalay (1985) further included Mimotonidae in the Eurymylidae. Li and Ting (1985), following $\mathrm{Li}$ and Yan 
(1979), considered Mixodontia a suborder of Rodentia and expanded the Eurymylidae, the only family contained in the suborder Mixodontia, to include Heomys, Rhombomylus, Matutinia, and Eurymylus. Li et al. (1987) placed the four genera into two families: Rhombomylidae, for Rhombomylus and Matutinia, and Eurymylidae, for Eurymylus and Heomys. McKenna and Bell (1997), however, regarded Rhombomylidae as a junior synonym of Eurymylidae. The family Rhombomylidae has never been used in other studies since its proposal (Li et al., 1987). Dashzeveg and Russell (1988) further expanded Mixodontia in a study describing new taxa as well as reviewing early gliroid mammals from Asia. They lumped all nonrodent, nonlagomorph gliroid mammals into the order Mixodontia under the cohort Glires (table 1), a concept followed by Averianov (1994). With more material of basal gliroid mammals being discovered from Asia (Shevyreva et al., 1975; Shevyreva and Gabunia, 1986; Dashzeveg et al., 1987; Nessov, 1987; Dashzeveg and Russell, 1988; Dashzeveg et al., 1998; Averianov, 1994; McKenna and Meng, 2001), Eurymylidae now includes about a dozen genera (McKenna and Bell, 1997; table 1).

Another gliroid taxon that bears importantly on the taxonomy of Rhombomylus is Matutinia nitidulus from the early Eocene Lingcha Formation, Hengyang Basin, Hunan Province, China (Li et al., 1979; Ting et al., 2002). Dashzeveg and Russell (1988) argued that there is no sufficient generic distinction between Matutinia and Rhombomylus, and thus they referred $M$. nitidulus as a species of Rhombomylus. This assignment was followed by McKenna and Bell (1997). Based on additional, better preserved material of $M$. nitidulus, however, Ting et al. (2002) came to the conclusion that Matutinia is a valid genus (see below).

In classifications of Glires, paraphyletic taxa are often allowed (Li et al., 1987; Dashzeveg and Russell, 1988; Dashzeveg et al., 1998; Averianov, 1994). An effort to implant a phylogenetic taxonomy (de Queiroz and Gauthier, 1990, 1992) for Glires was proposed (Meng and Wyss, 1994, 2001; Wyss and Meng, 1996; table 1) in which taxonomic names are attached to a phylogenetically defined node to avoid paraphyletic taxa, and to reserve popular taxonomic names, such as Rodentia, for crown groups by means of phylogenetic definitions. The paraphyly of Mixodontia, Eurymylidae, and Mimotonidae, respectively, has been cautiously pointed out (Meng et al., 1994; Meng and Wyss, 1994; Wyss and Meng, 1996; Meng and Wyss, 2001).

Phylogeny of Rhombomylus: Taxonomic hierarchy of the Glires usually does not reflect the phylogeny (fig. 1). Given the similarity shared by Heomys and rodents, $\mathrm{Li}$ (1977) made a compelling case to relate Heomys with rodents, a view followed by many authors thereafter (Gingerich and Gunnell, 1979; Chaline and Mein, 1979; Hartenberger, 1980; Dawson et al., 1984; Li et al., 1987). Through the tie of Heomys, other eurymylids, including Rhombomylus, were also related to rodents ( $\mathrm{Li}$ et al., 1987; Wilson, 1989; Li and Ting, 1993). Although it is generally agreed that Rhombomylus is a stem taxon to the clade of rodents, its phylogenetic position with that clade is unstable. Figure 1 summarizes five phylogenetic hypotheses of basal gliroid mammals that highlight different placements of Rhombomylus among basal gliroid mammals.

Li et al. (1987), Meng et al. (1994), and Meng and Wyss (2001) proposed a similar phylogeny for Rhombomylus: it is a sister taxon, or one of the sister taxa, to the clade of Rodentia (fig. 1A, C, E). The relationship of Rhombomylus to Heomys was unresolved in the study by Averianov (1994; fig. 1B), although both are part of a polytomy that includes rodents. In a phylogenetic analysis by Dashzeveg et al. (1998), Rhombomylus was recognized as the sister group of Rodentia. The peculiar aspect of that phylogeny (fig. 1D) is the insertion of Mimotonida and Lagomorpha between Rhombomylus and rodents on the one hand and other "mixodonts" (Heomys, Zagmys, Khaichina, Eomylus, Eurymylus, Gomphos, and Decipomys) on the other. Heomys becomes the taxon most distantly related to rodents among eurymylids. Although this result indicates the similarity between Rhombomylus and rodents, it differs from all relationships of basal Glires proposed previously. The phylogeny of Dashzeveg et al. (1998), however, is based 

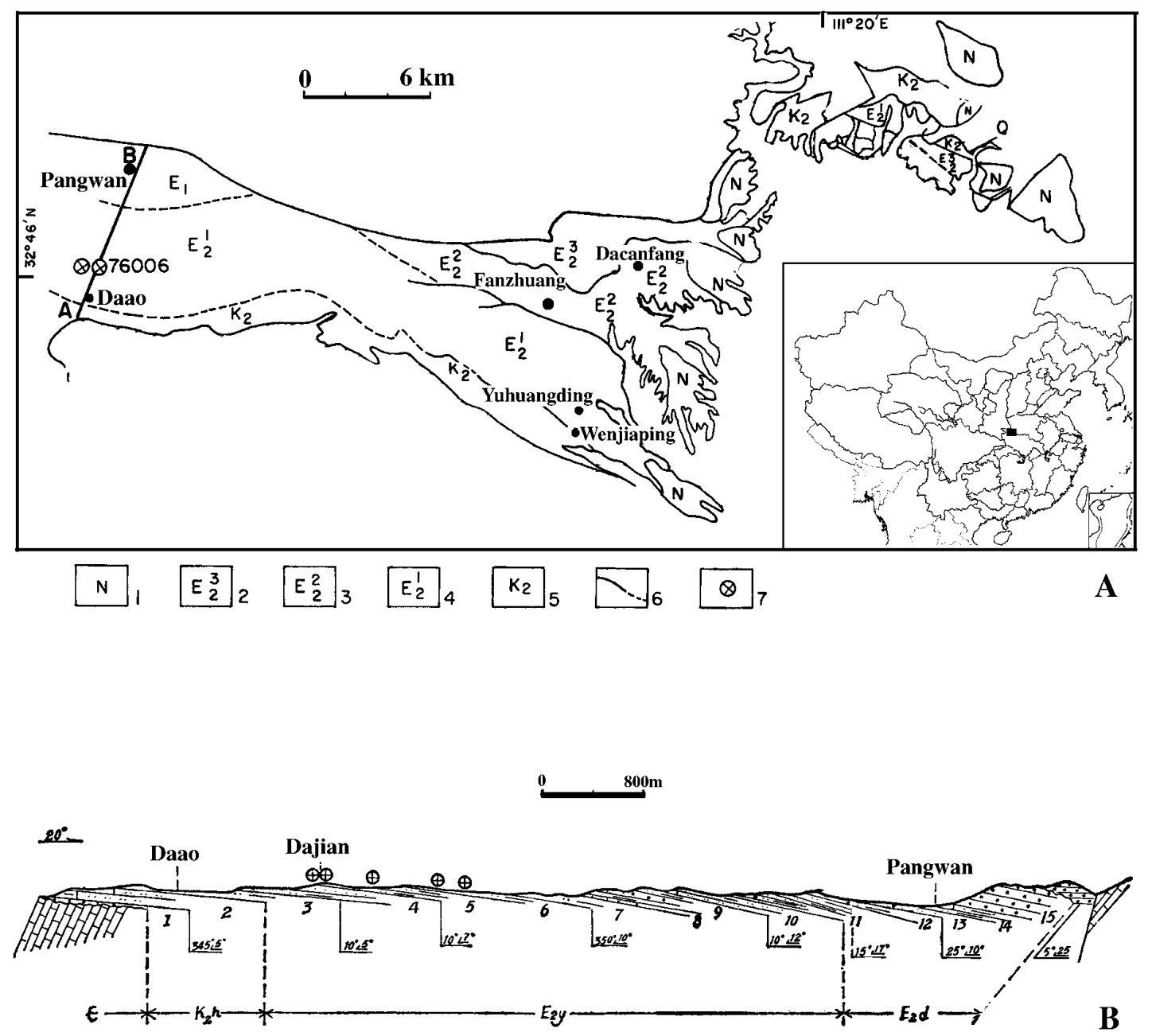

Fig. 2. A. Location and geological map of the Xichuan (Liquanqiao) Basin (1, Neogene; 2, Hetaoyuan Fm.; 3, Dacangfang Fm.; 4, Yuhuangding Fm.; 5, Hugang Fm.; 6, stratigraphical boundary; and 7, Dajian fossil localities). B. Section measured between Daao and Pangwan $\left(\mathrm{K}_{2} \mathrm{~h}\right.$, Hugang Fm; $\mathrm{E}_{2} \mathrm{y}$, Yuhuangding Fm.; $\mathrm{E}_{2} \mathrm{~d}$, Dacangfang Fm.). Modified from Xu et al. (1979) and D.E. Russell and Zhai (1987).

only on characters from the lower jaw and teeth; therefore, it may be highly biased by the restricted data. In a more recent phylogenetic analysis (Meng and Wyss, 2001), the relationships of Rhombomylus, Heomys, and Eurymylus remain unresolved, but they are closely related to the clade including rodents (fig. 1E).

\section{STRATIGRAPHY OF THE LOCALITY}

All specimens of Rhombomylus described in this work were collected from the early
Eocene Yuhuangding Formation at localities Dajian (IVPP site $76006,110^{\circ} 13^{\prime} \mathrm{E}, 32^{\circ} 47^{\prime} \mathrm{N}$, about $300 \mathrm{~m}$ northeast of the Wangjiayuan village) and Qingtanglin (400 m northwest of the Wangjiayuan village), Junxian County, Danjiang City, Hubei Province, China. The geological basin in which the Tertiary sediments were laid down was called Xichuan Basin or Liguanqiao Basin (fig. 2; see also Xu et al., 1979; Russell and Zhai, 1987). The study of the geology and biostratigraphy of this region started more than 70 years ago 
when two geologists (Li Ji and Zhu Sun) discovered fossil mammals at Fanzhuang in 1929. Teilhard de Chardin (1930) described these fossils. In 1959 regional geologists divided the terrestrial deposits in the basin into the Yuhuangding, Dacangfang, and Hetaoyuan formations, ranging from bottom up. The two formations at the bottom were then considered to be Cretaceous and the one on the top to be early Tertiary. Fieldwork by Li Chuankui and Tong Yongshen in 1960 recovered additional mammal fossils from the Yuhuangding Formation, which led them to think that all three formations were Eocene deposits. During the 1970s, several parties visited the Xichuan Basin and collected more mammal fossils, including numerous specimens of Rhombomylus, from the Yuhuangding Formation at the Dajian locality. Those field investigations further revealed the geology, stratigraphy, and faunas of the Liguanqiao Basin (Xu et al., 1979; $\mathrm{Ma}$ and Chen, 1991; Du et al., 1991). In addition to Rhombomylus, mammals from the $\mathrm{Yu}-$ huangding Formation now include Asiocoryphodon conicus Xu, 1976, Asiocoryphodon lophodontus Xu, 1976, "Coryphodon" flerowi, cf. Heptodon sp., Gobiatherium sp., ?Hunanictis sp., Advenimus hupeiensis Dawson et al., 1984, Hohomys lii Hu, 1995, "notoungulates", and Ctenodactyloidea gen. et sp. indet. The fossil assemblage suggests an early Eocene age of the Yuhuangding Formation A section through the Dajian locality was measured by $\mathrm{Xu}$ et al. (1979: 422-424, original in Chinese) and is briefly cited below:

\section{Dacangfang Formation}

(Early Middle Eocene)

15. Grayish, gray-black thick conglomerates intercalated with brown-reddish marl; graywhite, brown silt-mudstone at the top, $276.9 \mathrm{~m}$

14. Grownish silt-mudstone with marl and conglomerates, $80.3 \mathrm{~m}$

13. Light grayish sandstone, $30.5 \mathrm{~m}$

12. Light brownish sandy mudstone intercalated with gray sandstone and nodules, $72.4 \mathrm{~m}$

11. Brownish silt-mudstone intercalated with yellowish, gray sandstone. A 10-m-thick basal grayish pebbly sandstone and conglomerate mark the boundary between Da- cangfang Formation and Yuhuangding Formation, $184.1 \mathrm{~m}$

Yuhuangding Formation

(Late Early Eocene)

10. Brownish red mud-sandstone and silt-mudstone, containing calcic nodules, $72.3 \mathrm{~m}$

9. Brownish red silt-mudstone alternated with grayish brown sandstone and conglomerates, $121.1 \mathrm{~m}$

8. Dark yellowish, coarse sandstone intercalated with thin layers of silt-mudstone and conglomerates, $17.0 \mathrm{~m}$

7. Brownish, gray silt-mudstone intercalated with sandstone; 10-m-thick sandstone at the bottom, $88.9 \mathrm{~m}$

6. Brownish red silt-mudstone intercalated with yellow-brown sandstone; fragmentary bones of turtles and mammals, $51.8 \mathrm{~m}$

5. Brownish silt-mudstone intercalated with grayish, yellow-brown sandstone on a 6.7$\mathrm{m}$ layer of gritstone at the bottom. The middle silt-mudstone yields Heptodon sp., Rhombomylus turpanensis, Advenimus hubeiensis, Asiocoryphodon conicus, Hohomys lii and ?Hunanictis sp., collected from a basal dark reddish sandy mudstone, $66.9 \mathrm{~m}$

4. Brownish siltstone alternated with mudstone and sandstone; a 10-m grayish yellow, purple gritstone and conglomerates at the bottom, $112.3 \mathrm{~m}$

3. Brownish red mudstone alternated with siltstone, $61.1 \mathrm{~m}$

$$
\text { —Disconformity- }
$$

Hugang Formation (Late Cretaceous)

2. Brownish red siltstone intercalated with mudstone and sandstone, $84.4 \mathrm{~m}$

1. Brown conglomerates intercalated with siltstone, $6.5 \mathrm{~m}$

$$
\text { —Unconformity- }
$$

Cambrian limestone basement.

TAXONOMY

COHORT GLIRES LINNAEUS, 1758

MIRORDER SIMPLICIDENTATA WEBER, 1904

GENUS RHOMBOMYLUS ZHAI, 1978

TYPE SPECIES: Rhombomylus turpanensis Zhai, 1978 (= Rhombomylus laianensis Zhai et al., 1976; Rhombomylus cf. turpanensis Dashzeveg and Russell, 1988).

InCLUDED SPECIES: Type species only. 


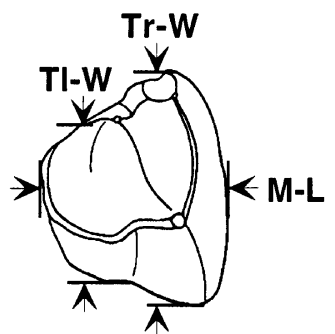

A

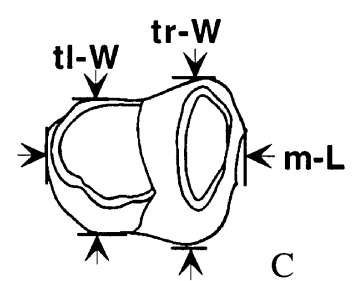

C

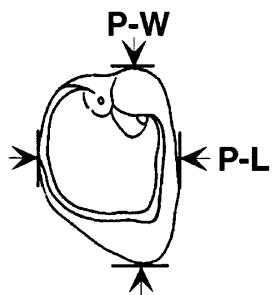

B

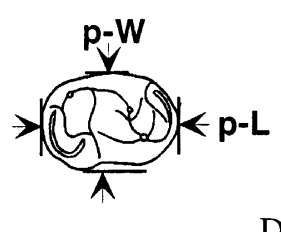

Fig. 3. Measured dimensions of the upper molar, upper premolar, lower molar, and lower premolar of Rhombomylus. M-L, upper molar length; m-L, lower molar length; P-L, upper premolar length; p-L, lower premolar length; P-W, upper premolar width; p-W, lower premolar width; Tl-W, upper molar talon width; tl-W, lower molar talonid width; Tr-W, upper molar trigon width; and tr-W, lower molar trigonid width.

Distributions AND AgE: Lower Eocene Shisanjianfang Formation at Shisanjianfang, Turpan Basin, Xinjiang Province, China (Zhai, 1978); lower Eocene Zhangshanji Formation, Laian, Anhui Province, China (Zhai et al., 1976); lower Eocene Youping Formation, Fangxian, Hubei Province of China (Lei, 1984; Huang, 1995); lower Eocene Yuhuangding Formation, Dajian, Junxian County, Hubei Province, China; and lower Eocene Bumban Member of the Naran Bulak Formation, Tsagan-Khushu, Mongolia (Dashzeveg et al., 1987; Dashzeveg and Russell, 1988).

Revised Diagnosis: A medium-sized gliroid mammal (see figs. 3-5, tables 2-5 for measurements) with a single pair of incisors in upper and lower jaws, respectively; dentition with tooth homology and formula being dI2, C0, P3-4, M1-3, di2, c0, p3-4, m13 (see figs. 6-9, 12-15); similar to Matutinia but differing from other simplicidentates in having a broad hypocone shelf on cheek teeth and cheek teeth being relatively anteroposteriorly long; further similar to Matutinia in having a specialized zygomatic arch formed by the maxilla and jugal, expansion of the mastoid of the petrosal and exposure of the mastoid on the skull roof, extensively interdigital sutures between premaxilla and frontal, contribution of the ectotympanic to the medial wall of the glenoid fossa, and the external auditory meatus contacting squamosal and blocking postglenoid fossa posteriorly. Differing from Matutinia in having higher tooth crown, stronger protocone and paracone, complete vertical groove on the lingual surface of cheek teeth, stronger crests on cheek teeth, and lacking a carotid foramen.

COMMENTs: Rhombomylus is most similar to Matutinia. Based on a rostrum of a skull (IVPP V5354) and referred specimens, Li et al. (1979: 77) briefly diagnosed Matutinia as "an intermediate form between Heomys and Rhombomylus, premolar nonmolarized, trigon with four cusps, metaconule rudimentary, posterior margin of the palate ending at the level of M2, paraconid reduced, trigonid higher than talonid, enamel of cheek teeth thick, M1 the largest of upper cheek teeth that decrease in size posteriorly" (original in Chinese). Dashzeveg and Russell (1988: 149) argued that the $\mathrm{p} 4$ of $M$. nitidulus shows "approximately intermediate characters" between Rhombomylus turpanensis and R. laianensis; that "no diagnostic distinguishing features of generic value are evident from the lower teeth"; and that "a difference in shape of M3 appears to be the major distinction separating this species from $R$. turpanensis; difference in crown height seems negligible." Thus, Dashzeveg and Russell considered $M$. nitidulus a species of Rhombomylus and regarded Matutinia as a junior synonym of Rhombomylus. Based on additional materials of $M$. nitidulus, Ting et al. (2002) concluded that the differences between Matutinia and Rhombomylus are sufficient to distinguish them as two valid genera. To facilitate comparison, we provide dental images of Matutinia in figure 10 and size comparison in figure 11. As shown by the images, the differences between Rhombomylus and Matutinia are not just in the shape of the M3, but are many, as outlined in the diagnosis. In general, features in Rhombomylus are more derived compared to those of Matutinia. 

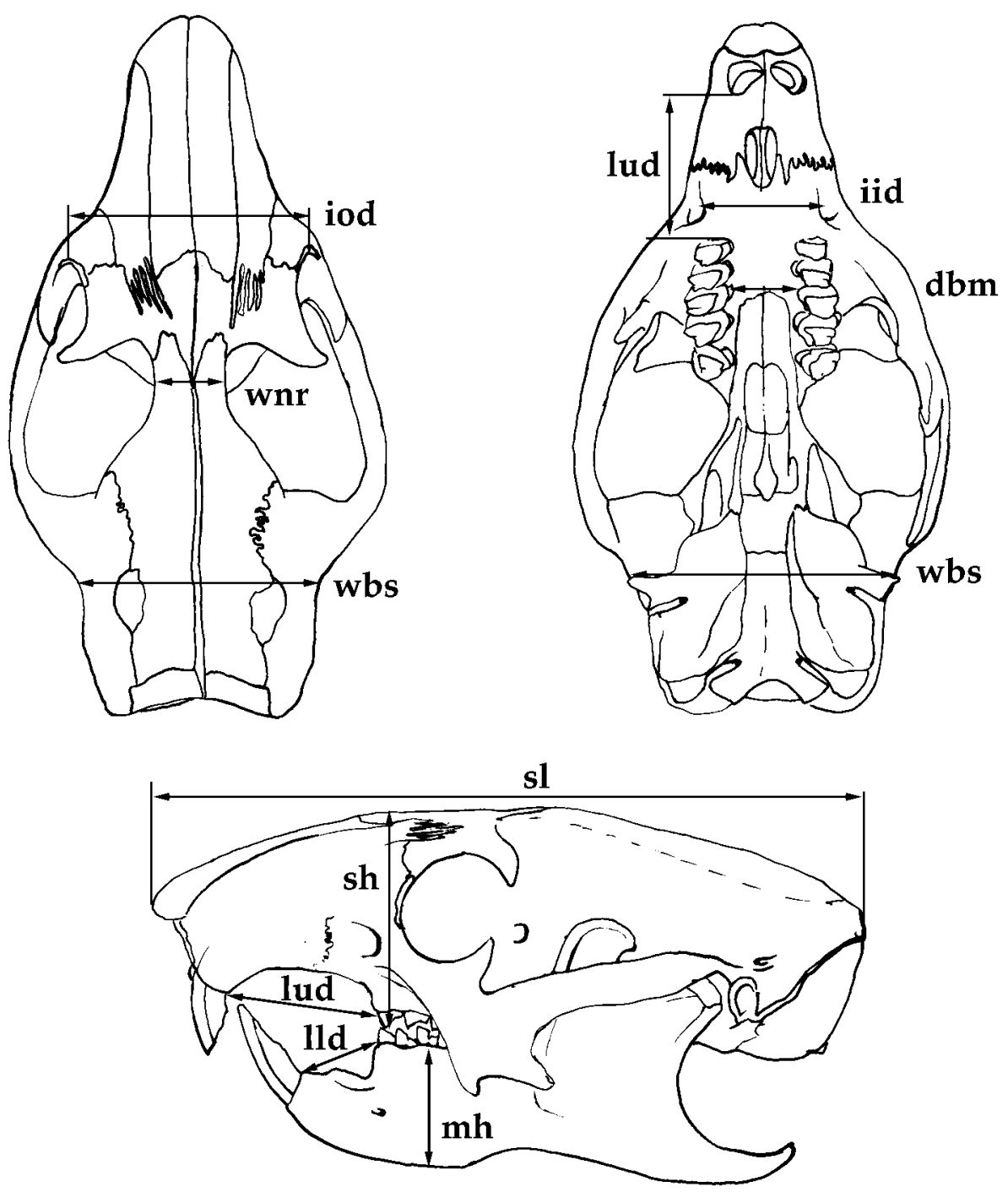

Fig. 4. Skull measurements of Rhombomylus. dbm, Distance between molar rows (between M1s); iid, interinfraorbital distance; iod, interorbital distance; lld, length of the lower diastema; lud, length of the upper diastema; mh, mandibular height (at m1); sh, skull height; sl, skull length; wbs, width between squamosal edges; and wnr, width of the narrowest region of the skull roof.

The fragmentary material of Eurymylus hampers a conclusive comparison with Rhombomylus. However, from the specimens described (Matthew and Granger, 1925; Matthew et al., 1929; Sych, 1971: fig. 1, Z. Pal. No. MgM-II/62-3), Eurymylus differs from Rhombomylus in having the following features: cheek teeth more transversely extended, a narrower hypocone shelf, double mental foramina, a relatively shorter but wider rostrum, a longer incisive foramina, and probably different enamel microstructure of the incisor (Sych, 1971; Martin, 1992, 1999).

As pointed out by McKenna and Meng (2001), the content of Eurymylus remains uncertain. Thus far only one species, E. laticeps, has been described (Matthew and Granger, 1925; Matthew et al., 1929; Sych, 1971; Dashzeveg and Russell, 1988), but specimens referred to Eurymylus may represent two taxa: one having a narrow hypocone shelf and more transversely widened cheek 

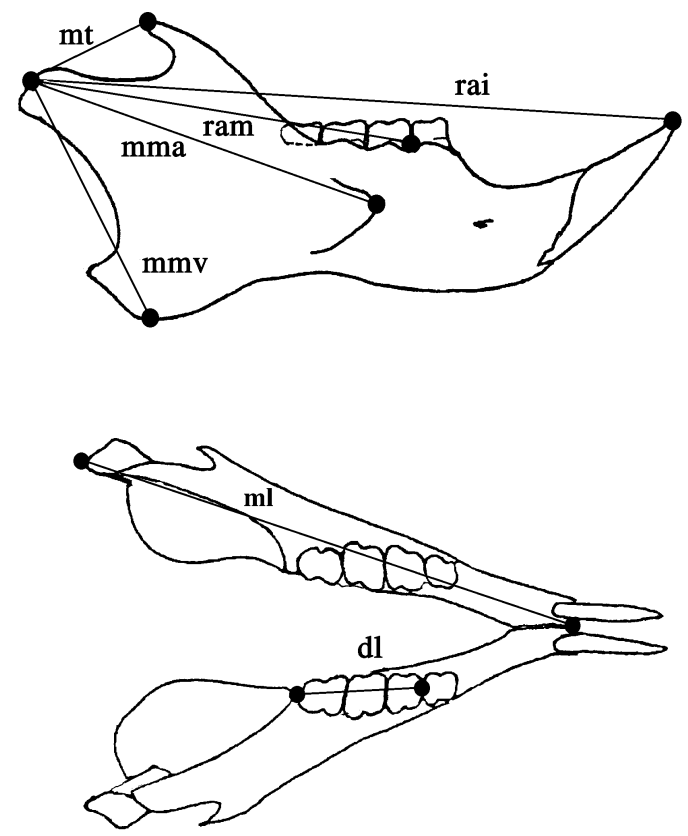

Fig. 5. Measurements of jaw muscle moment arms and resistance arms of the incisor and molar in Rhombomylus and other mammals. dl, Dental (molar) length; ml, mandibular length; mma, moment arm of the anteriormost fiber of the anterior deep masseter; mmv, moment arm of the ventralmost fibers of the superficial masseter; $\mathrm{mt}$, moment arm of the dorsalmost fibers of the temporalis rai, resistance arm of the incisor; and ram, resistance arm of the molar. Measurements were made between the landmarks using an Ultra-Cal Mark III caliper. The results are provided in appendix 4.

teeth (represented by AMNH 20422 [Matthew and Granger, 1925] and Z. Pal. No. MgM-II/62 [Sych, 1971: fig. 1]) and the other having longer cheek teeth with broader hypocone shelves (represented by PSS 20-162 [Dashzeveg and Russell, 1988: text-fig. 5] and Z. Pal. No. MgM-II/64 [Sych, 1971, fig. $3 a, b])$. The morphology of PSS 20-162 and MgM-II/64 appears more similar to that of Matutinia and Rhombomylus than are other specimens assigned to Eurymylus.

\section{Rhombomylus turpanensis Zhai, 1978}

R. laianensis Zhai et al., 1976.

Rhombomylus cf. turpanensis (Dashzeveg and Russell, 1988).
LECTOTYPE: V4362 (Dashzeveg and Russell, 1988), a left maxilla with P4-M3.

Distributions AND AgEs: As for the genus.

DiAGNOSIS: Same as that of the genus.

REFERRED SPECIMENS: See Materials and Methods.

COMMENTS: Rhombomylus turpanensis is based on the specimens from the lower Eocene Shisanjianfang Fm., Turpan Basin of Xinjiang. Because Zhai did not designate a type specimen for $R$. turpanensis, Dashzeveg and Russell (1988) chose an upper dentition (IVPP V4362) as the lectotype for the species. Mammals associated with $R$. turpanensis include Coryphodon sp., Hyopsodus sp., Heptodon tienshanensis, and Anatolostylops dubius (Zhai, 1978).

"Rhombomylus laianensis" was based on a right maxilla with P3-M3 (IVPP V5174), a right lower jaw with p4-m3 (IVPP V5175), and a left lower jaw with $\mathrm{m} 2-\mathrm{m} 3$ (IVPP V5176) from the lower Eocene Zhangshanji Fm. at a site south to the Wangjiagang village, Laian, Anhui Province (Zhai et al., 1976). No other mammal was known from this locality.

The specimens of Rhombomylus from Dajian display a range of size and shape variations owing to individual and ontogenetic variations. We cannot distinguish a systematic pattern among these variations, and therefore we regard all the specimens as belonging to one species. Because the variation displayed by the Dajian specimens convey morphologies that resulted in recognition of $R$. turpanensis and " $R$. laianensis", we lump all specimens of Rhombomylus reported previously (Zhai, 1978; Zhai et al., 1976; Dashzeveg and Russell, 1988) and those from Dajian into a single species, $R$. turpanensis.

The publication date of Rhombomylus presents a priority problem, as already realized by others (Dashzeveg and Russell, 1988; McKenna and Bell, 1997). Although "R. laianensis" was published first (Zhai et al., 1976), $R$. turpanensis was clearly designated as the type species of the genus (Zhai, 1978). It appears that the 1978 paper was expected to be published first, but was long in press. Therefore, we consider the species name $R$. turpanensis has priority over " $R$. laianensis". 


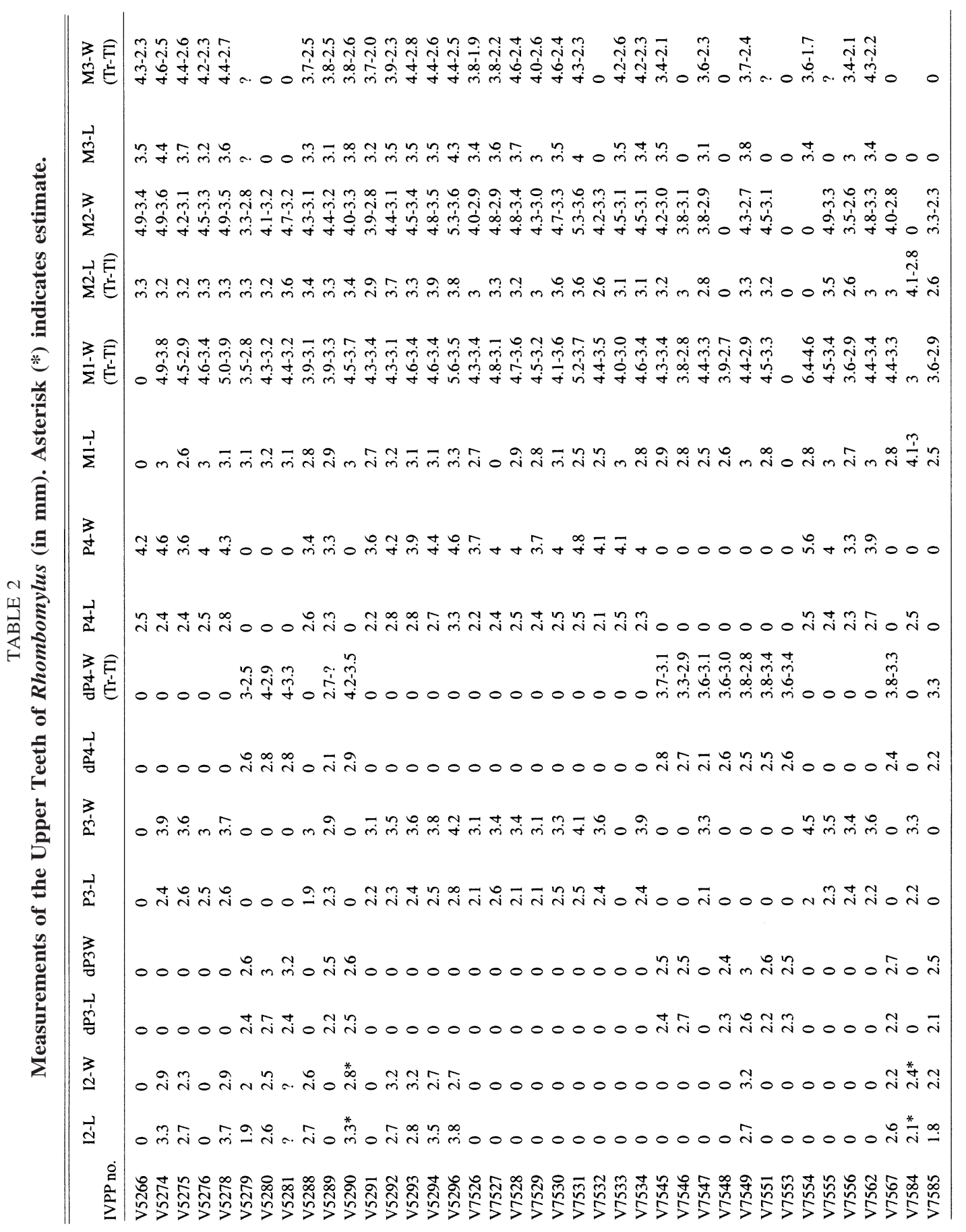




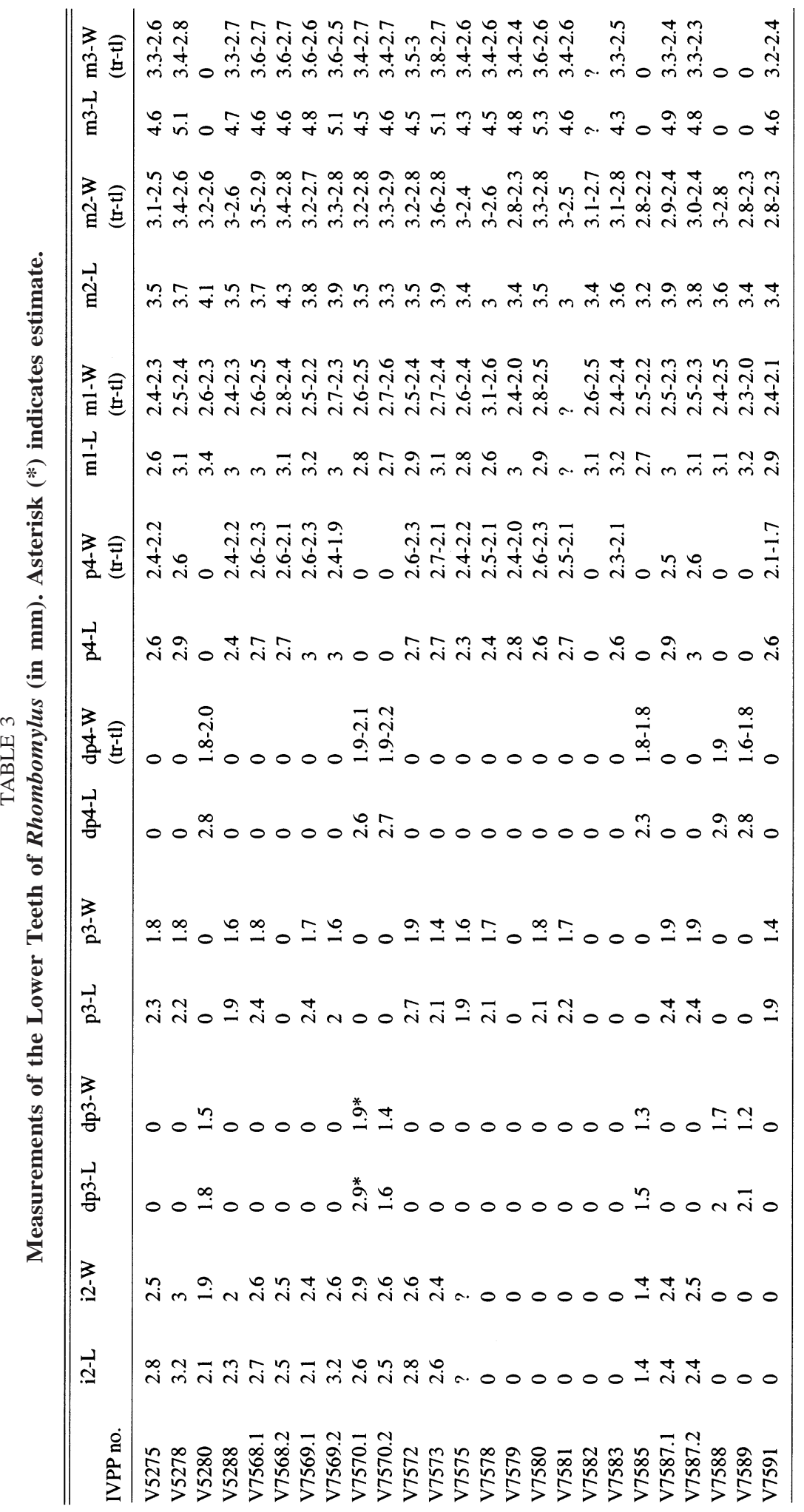


TABLE 4

\section{Skull Measurements of Rhombomylus (in mm)}

dbm, distance between molar rows; iid, inter-infraorbital distance; iod, interorbital distance; lud, length of the upper diastema; mh, mandibular height; sh, skull height; sl, skull length; wbs, width between squamosal edges; wnr, width of narrowest region of the skull roof. Asterisk (*) indicates estimate.

\begin{tabular}{llllllllll}
\hline \hline IVPP no. & sl & wbs & sh & lud & wnr & wnv & iid & iod & dbm \\
\hline V5274 & $?$ & $?$ & 21.3 & 15.2 & 7.7 & $?$ & 11.2 & 12.9 & $?$ \\
V5275 & 54.9 & $>18.8$ & 18.7 & 11.3 & 7 & $?$ & 9.8 & 16.8 & \\
V5276 & $54 *$ & 26.6 & 20.6 & $10.1 *$ & 8.9 & 8.4 & 11.5 & 16.5 & 5.0 \\
V5277 & 71.6 & 30.3 & 23.9 & 16.2 & 8.8 & $?$ & 13.5 & 21.7 & $?$ \\
V5278 & 84 & 30 & 26 & 18.1 & 7.6 & 8 & 14 & 28 & 7.0 \\
V5279 & 42 & 20.4 & 14 & 7.4 & $?$ & $?$ & 9.8 & $12.7 *$ & 4.5 \\
V5281 & $48.5 *$ & 24.6 & 17.9 & 8.9 & 8.66 & $?$ & 9.7 & 15 & 5.8 \\
V5283 & 41 & 18.3 & 13.6 & 7.7 & 8.1 & 7.9 & 8.3 & 12.2 & $?$ \\
V7585 & 34.3 & 16.6 & 13 & 6.2 & 8.7 & $?$ & 7.9 & 11.4 & 3.8 \\
V5284 & 46 & $16.1 *$ & $16.3 *$ & 7.9 & 7.5 & $?$ & $7.2 *$ & $11.5 *$ & $?$ \\
V5286 & $55 *$ & 26 & 20.8 & 9.8 & 8.4 & 8.7 & 11.4 & $?$ & 5.8 \\
V5289 & 55 & 23 & 17 & 9.1 & 7.8 & 7.5 & 9.6 & 16.4 & 5.4 \\
V5290 & 56.3 & 27.4 & 20.7 & 11.2 & 8 & 10 & 11.8 & 17.4 & 5.7 \\
V5291 & 50.5 & 22 & 18 & 9.1 & 8 & 7.6 & 9.3 & 14.4 & 4.3 \\
V5292 & 53.4 & 24.3 & 17.7 & 9.2 & 7.3 & 8 & 10.3 & 15.8 & 5.7 \\
V5293 & 62.3 & $24.8 *$ & 20.4 & 12.7 & 7.8 & 8 & 10.7 & 18.1 & 5.5 \\
V5294 & $58.7 *$ & 26.3 & 21 & 12.3 & 7.2 & 8.3 & 11 & 17.8 & 6.1 \\
V5295 & 62.2 & 30 & 21 & 12.5 & 9.3 & $?$ & 12.2 & 20.3 & $?$ \\
V5296 & 67.8 & $28.2 *$ & 22.2 & 13.6 & 7.3 & 0 & 12.8 & 21 & 5.7 \\
\hline
\end{tabular}

\section{OSTEOLOGICAL MORPHOLOGY}

\section{MATERIALS AND METHODS}

MATERIAL: Specimens from the lower Eocene Shisanjianfang Fm., Turpan Basin, Xinjiang Province (Zhai, 1978): IVPP V4361, a crushed skull, with right cheek teeth; IVPP V4363, a left lower jaw; IVPP V4364, a left lower jaw of a juvenile; IVPP V4365, an isolated $\mathrm{m} 3$; and IVPP V4366, a calcaneus (lost).

Specimens from the lower Eocene Zhangshanji Fm. at Laian, Anhui Province (Zhai et al., 1976): IVPP V5174 (type of " $R$. laianensis"), a right maxilla with P3-M3; IVPP V5175, a right lower jaw with p4-m3; and IVPP V5176, a left lower jaw with m2-m3.

Specimens from the lower Eocene Yuhuangding Fm. at Dajian: IVPP V5257-9, lower incisors; IVPP V5260, posterior half of skull; IVPP V5261, posterior half of skull; IVPP V5262, partial skull with internal side of the braincase and ear region exposed; IVPP V5263, left posterior quarter of a skull with the interior exposed; IVPP 5264, pos- terior portion of skull; IVPP V5265, middle part of a skull with partial left mandible and semicircular canals exposed; IVPP V5266, partial skull (sectioned into 33 slices to reveal internal cranial and auditory morphologies); IVPP V5267, partial skull (sectioned into 27 slices); IVPP V5273, skull; IVPP V5274, partial skull with associated left mandible; IVPP V5275, skull with associated mandibles and basicranium laterally compressed; IVPP V5276, partial skull with left P3-M3; IVPP V5277, skull with associated mandibles; IVPP V5278, skull with associated mandibles (largest individual); IVPP V5279, juvenile skull with dP3-4 and M3; IVPP V5280, skull of a young individual with associated mandible, dP3-M2, and erupting M3 (tip of left lower incisor cut for SEM study of enamel structures); IVPP V5281, partial skull with left dP3-M3 (M3 erupting); IVPP V5282, skull; IVPP V5283, juvenile skull with associated mandibles, dP3-4, erupting M3 and m3; IVPP V5284, juvenile skull with associated mandibles; IVPP V5286, skull with right M2-3, left P4, 
TABLE 5

\section{Mandible Measurements of Rhombomylus (in mm)}

lld, length of lower diastema; mh, mandibular height; mt, mandibular thickness. Asterisk (*) indicates estimate.

\begin{tabular}{llcl}
\hline \hline IVVP no. & Lld & $\mathrm{mh}$ & $\mathrm{mt}$ \\
\hline V5278 & 9.3 & 15.3 & 6.8 \\
V5280 & 3.9 & 6.3 & 3.9 \\
V5283 & 3.7 & 6.9 & 4.4 \\
V7585 & 2.7 & 5.5 & 4.0 \\
V5284 & 4.4 & 7 & 3.8 \\
V7568.1 & $5.4^{*}$ & 10.9 & 4.9 \\
V7568.2 & $?$ & 9 & 4.8 \\
V7569.1 & 5.4 & 8.5 & 4 \\
V7569.2 & 5.9 & 10 & 5 \\
V7570.1 & 5.3 & 8.5 & 5.2 \\
V7570.2 & 5.3 & 8.8 & 4.4 \\
V7572 & 6.3 & 10.2 & 5.2 \\
V7573 & 6.4 & 10.3 & 4.5 \\
V7575 & $?$ & 8.6 & 3.8 \\
V7578 & 6 & 10 & 5 \\
V7579 & $?$ & 11 & 6.2 \\
V7580 & $?$ & 9.2 & 5.6 \\
V7581 & $?$ & 10.5 & 5.9 \\
V7582 & $?$ & 6.5 & 3.5 \\
V7583 & $?$ & 7.9 & 3.7 \\
V7587.1 & $?$ & 10.8 & 4.5 \\
V7587.2 & 6.5 & 10.5 & 4.6 \\
\hline & & &
\end{tabular}

and M2-3 (peeled for brain endocast); IVPP V5287, partial skull; IVPP V5288, skull with associated mandibles; IVPP 5289, skull; IVPP V5290, skull with dP3-4, erupting P34 and molars; IVPP 5291, skull; IVPP V5292, skull with dP3-4 and molars; IVPP V5293, partial skull with P3-M3 all erupted; IVPP V5294, partial skull with P3-M3; IVPP V5295, skull with associated mandibles; IVPP V5296, partial skull with P3-M3; IVPP 5297, skull; IVPP V 7428, partial skull with associated postcranial elements (see below); IVPP V7486 and V7487, partial skulls (peeled for endocast); IVPP V7488, rostrum (ground to reveal cross-sectional structures of the nasal cavity); IVPP V7489, mandible with lower incisor (sectioned for SEM study of enamel structure); IVPP V7490, maxilla with P4-M3 (sectioned for SEM study of enamel structure); IVPP V7491, mandible with p4-m3 (sectioned for SEM study of enamel structure); IVPP V7492, partial skull (skull ground to show frontal and longitudi- nal sectional structures of the nasal cavity; left incisor cut for SEM study of enamel structure; and right cheek teeth used for SEM study of tooth wear); IVPP V7493, partial mandible with $\mathrm{p} 4-\mathrm{m} 2$ (used for SEM study of microwear); IVPP V7494, petrosal (peeled to show semicircular canals); IVPP V7495, partial mandible with $\mathrm{m} 1-2$ (sectioned to show pulp cavities of teeth); IVPP V7496, partial maxilla showing tooth roots; IVPP V7521 and V7522, skulls with associated mandibles; IVPP V7523-5, skulls; IVPP V7526, partial skull with all cheek teeth; IVPP V7527, partial skull with left P3-M3 and right P3-M2; IVPP V7528, partial skull with all cheek teeth; IVPP V7529, partial skull with all cheek teeth except last molars; IVPP V7530, partial skull with all cheek teeth; IVPP V7531, partial skull with all cheek teeth; IVPP V7532, partial skull with all cheek teeth except last molars; IVPP V7533, partial skull with teeth preserved except both P3s; IVPP V7534, partial skull with left cheek teeth; IVPP V7535, anterior part of skull with all cheek teeth; IVPP V7536, posterior half of skull with right M3; IVPP V7537, posterior half of skull with ear region exposed; IVPP V7538, posterior half of skull; IVPP V7539, posterior half of skull with right M3; IVPP V7540, posterior left quarter of skull; IVPP V7541, posterior right quarter of a skull; IVPP V7542, middle portion of skull; IVPP V7543, posterior skull with ear region exposed; IVPP V7544, posterior half of skull; IVPP V7545, partial skull with all cheek teeth; IVPP V7546, partial skull with both Dp3-M2; IVPP V7547, maxilla with all cheek teeth; IVPP V7548, partial skull with Dp3-m1; IVPP V7549, anterior half of skull with all cheek teeth; IVPP V7550; anterior half of skull with left dentition; IVPP V7551, right maxilla with dP3M3; IVPP V7552, left maxilla with dP4-M3; IVPP V7553, partial skull with dP3-4; IVPP V7554, left maxilla with P3-M3; IVPP V7555, anterior half of skull with cheek teeth; IVPP V7556, anterior left quarter of skull with dentition; IVPP V7557-62, anterior halves of skulls with cheek teeth; IVPP V7563, partial skull; IVPP V7564, partial skull with associated mandibles; IVPP V7565-7, anterior parts of skulls with associated mandibles; IVPP V7568.1, right man- 


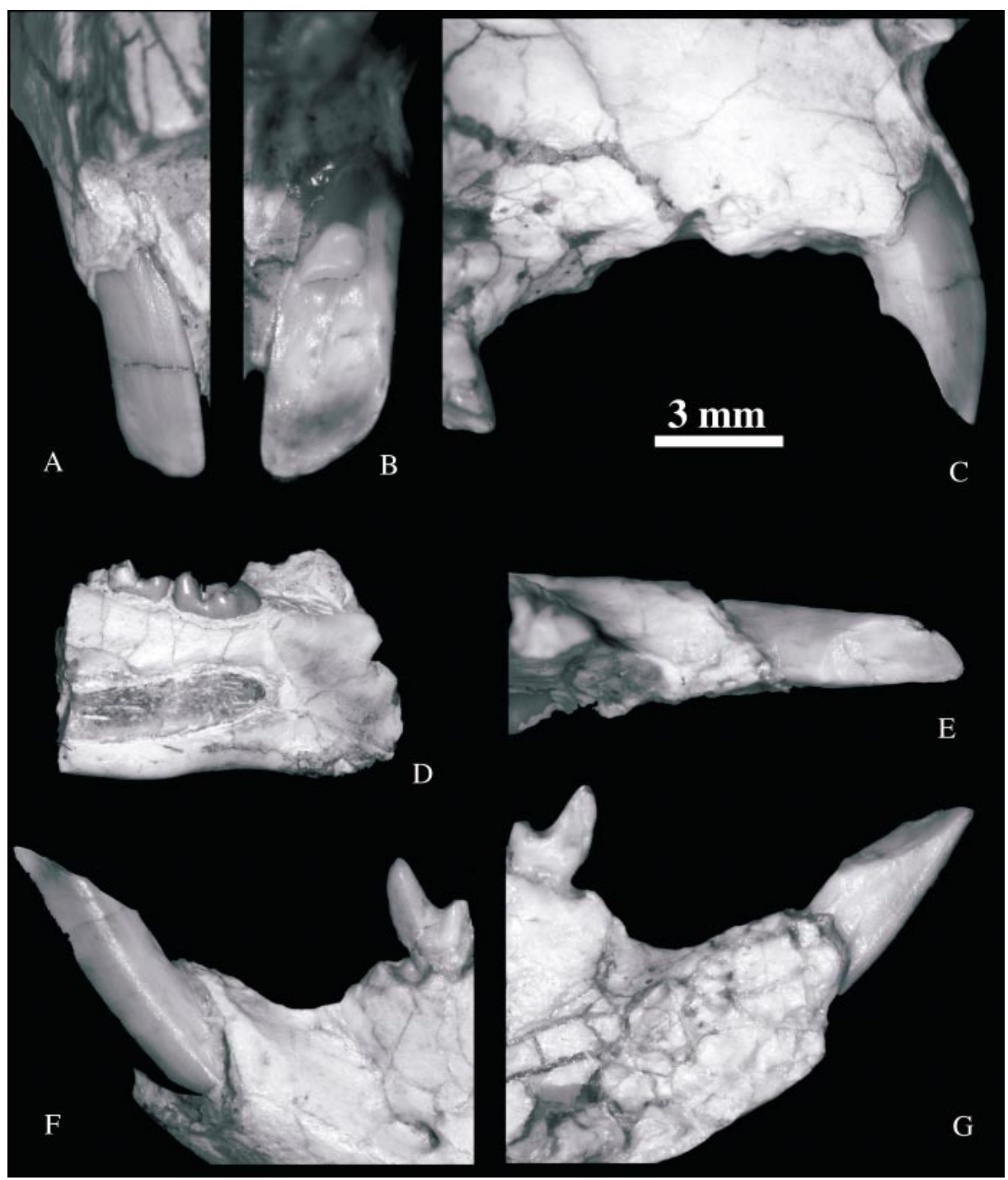

Fig. 6. Upper and lower incisors of Rhombomylus (IVPP V5288). A-C, Anterior, posterior, and lateral views of the right upper incisor (V5288); D, medial view of a fragmentary mandible showing the end of the lower incisor in the dentary; E-G, dorsal, lateral, and medial views of the left incisor (V5288). Scale bar for all except D.

dible with p3-m3; IVPP V7568.2, left mandible with p4-m3; IVPP V7569.1, right mandible with incisor and cheek teeth; IVPP V7569.2, left mandible with most of incisor and p3-m3; IVPP V7570.1, right mandible with most of incisor, broken dp3 and dp4m3; IVPP V7570.2, left mandible with most incisor and dp3-m3; IVPP V7571, left mandible with full dentition; IVPP V7572, left mandible with partial incisor and p3-m3; IVPP V7573, right mandible with full dentition; IVPP V7574, right mandible with full dentition; IVPP V7575, right mandible with partial incisor, broken p3 and complete p4m3; IVPP V7576, left mandible with cheek teeth; IVPP V7577, left mandible with cheek teeth; IVPP V7578, left mandible with p3m3; IVPP V7579, left mandible with p4-m3; 


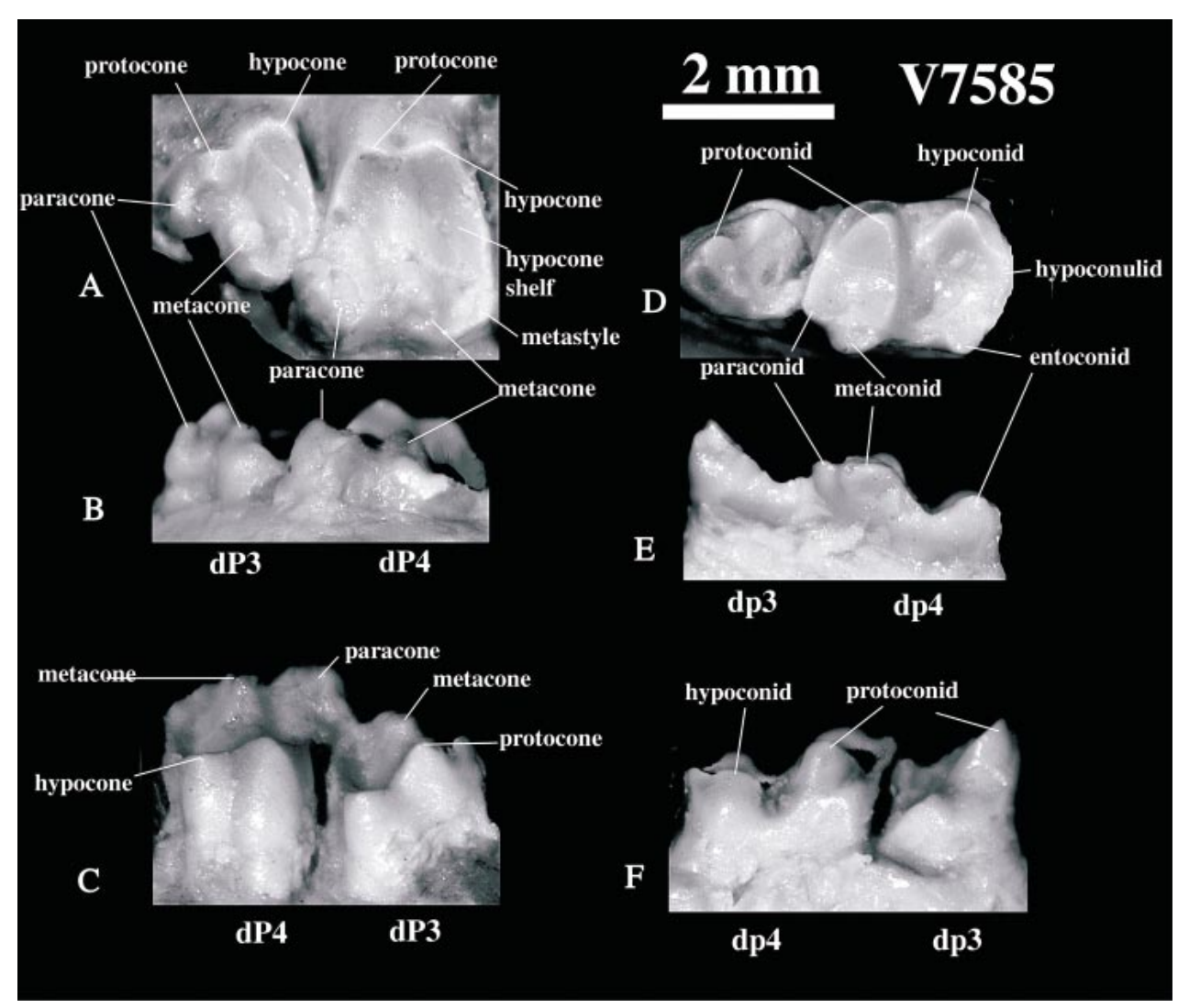

Fig. 7. Deciduous premolars of Rhombomylus (IVPP V7885). A-C, Occlusal, labial, and lingual views of the right $\mathrm{dP} 3$ and $\mathrm{dP} 4$; D-F, occlusal, lingual, and labial views of the right dp3 and dp4.

IVPP V7580, left mandible with broken p3 and complete p4-m3; IVPP V7581, left mandible with cheek teeth; IVPP V7582, left mandible with half dp4 and complete m1$\mathrm{m} 3$; IVPP V7583, right mandible with p4$\mathrm{m} 3$; and IVPP V7584, right maxilla with dP3-M2; IVPP V7585, juvenile skull with associated mandible (youngest and smallest individual); IVPP V7586, posterior part of skull; IVPP V7587, mandible; IVPP V758890, right dentaries; IVPP V7591, left mandible; IVPP V7592, right mandible; and IVPP V7593, left mandible.

PostCRANIAL SPECIMENS: IVPP V7428, articulated skeleton with both scapulae, both humeri with part of the shaft damaged, most parts of both ulnae and radii, part of the pelvis, a right femur, distal part of left femur, left patella, both tibiae (partially preserved), the distal portion of the left fibula, both calcanei, both astragali, left cuboid, left navic- ular, left ectocuneiform, left mesocuneiform, left metatarsals I-IV, some phalanges of the left foot, and some broken ribs; IVPP V5268, last cervical vertebra, first three thoracic vertebrae, first pair of ribs, and heads of second and third ribs; IVPP V5269.1-3, a segment of a vertebral column with six lumbar vertebrae, fused sacrum, the first two caudal vertebrae, and part of the pelvic girdle; IVPP V5270, the sacrum, first caudal vertebra, part of the pelvic girdle, and part of left femur; IVPP V5271, last two lumbar vertebrae, sacrum, and both ilia; IVPP V5272.1-3, four lumbar vertebrae; IVPP V5787, a middle segment of a possible clavicle; IVPP V7419, distal part of a right tibia; IVPP V7423, proximal parts of both the tibia and fibula; IVPP V7457 and V7458, middle parts of a left innominate; IVPP V7459, middle part of right innominate; IVPP V7460, dorsal portion of right ischium and part of a pubis; IVPP 
V7461, proximal portion of a humerus; IVPP V7462 and V7463, right humeri with both ends damaged; IVPP V7464, distal portion of a right humerus; IVPP V7465, left ulna and radius with the distal portion missing; IVPP V7480 and V7483, proximal parts of left femurs; IVPP V7481, distal portion of a left femur; IVPP V7482, distal part of a right femur; IVPP V7484, distal epiphysis of a femur with trochlea well preserved; IVPP V7485, proximal portion of a right femur with the third trochanter well preserved; IVPP V7498, proximal portions of right tibia and fibula in articulation; IVPP V7499, partial right pes; IVPP V7517, proximal half of a tibia; IVPP V7518, a partial left manus; IVPP V7519, distal half of a right ulna; and IVPP V7520, parts of right ischium and pubis. The assignment of the isolated specimens is mainly based on their similarities with corresponding elements in IVPP V7428.

IVPP V7418 (distal part of a left humerus), IVPP V7420 (left calcaneus), and IVPP V7421 (left astragalus) were assigned to Rhombomylus by $\mathrm{Li}$ and Ting (1993). Because of their distinct differences from corresponding elements of IVPP V7428, we exclude these specimens from Rhombomylus.

MEASUREMENTS: We made measurements of teeth, skulls, and mandibles using an Ultra-Cal Mark III calliper and a Microcode II digital measuring scope. The dimensions measured are illustrated in figures 3-5. The measurements are provided in tables 2-5. Additional measurements of some mandibular dimensions are presented in appendices 4 and 5. These data are used in the analysis of functional morphology.

DENTITION: Terminology for morphology and landmark structures of deciduous and permanent teeth is illustrated in figures 7-9 and 13. We follow Meng and Wyss (2001) for additional dental terms. Dentitions with different stages of eruption and wear are arranged in an ontogenetic sequence as illustrated in figures 12 and 14. All the photographs of cheek teeth are taken from casts that were photographically gray-toned using an RT SPOT digital camera mounted on a Nikon SMZ-U microscope.

EnAmel Microstructure: The upper left incisor of IVPP V7492, lower incisor of IVPP V7489, lower incisor of a juvenile in- dividual (IVPP V5280), lower cheek teeth of IVPP 7491, and upper cheek teeth of IVPP V7490 were sectioned for SEM study of enamel microstructures (figs. 16-20). All incisors were taken from mandibles and skulls with cheek teeth; thus, their identifications as Rhombomylus are secure.

In preparation of specimens for SEM study, a desired segment of incisor was cut using a saw or simply broken if the specimen was long enough. For cross-sectional view of the enamel, the posterior end of an incisor segment was slightly ground flat in a plane perpendicular to the long axis of the tooth. The flat end was then glued on a metal SEM stub using Krazy Glue. The rest of the specimen was coated with the glue for protection. The mounted specimen was then ground and polished by holding the stub parallel to the surface of the grinder. Therefore, all crosssectional views of the incisors were taken from the anterior ends of segments and are at a right angle to the longitudinal axis of the incisor. For longitudinal views, a segment of incisor was first ground on its lateral side to create a flat surface at a right angle to the enamel layer. The specimen was then glued on the stub by the ground, flat surface and was prepared in the same way for the cross section of the tooth. Each of the upper and lower incisors was cut at three regions: near the tip, at the middle, and close to the end of the tooth. In each case, the cross-sectional view of the enamel layer was examined first. After SEM cross-sectional views were taken, the specimen was removed from the stub using a sharp, straight-edged knife and was then prepared for longitudinal-sectional imaging. Therefore, a pair of cross-sectional and longitudinal views of incisor enamel microstructure was ensured for the same segment of each incisor (figs. 16-18).

For cheek tooth enamel structures, a maxilla and a mandible that bear teeth were cut into a desired size that fits to a large SEM metal stub. The flat surface that contacts the stub was prepared to be parallel to the occlusal surface of the tooth row, such that the cheek teeth in the same row can be ground simultaneously at the same level. The cheek teeth were cut at several levels through the tooth crowns from tips to bases. Images of enamel distributions at three levels were pro- 


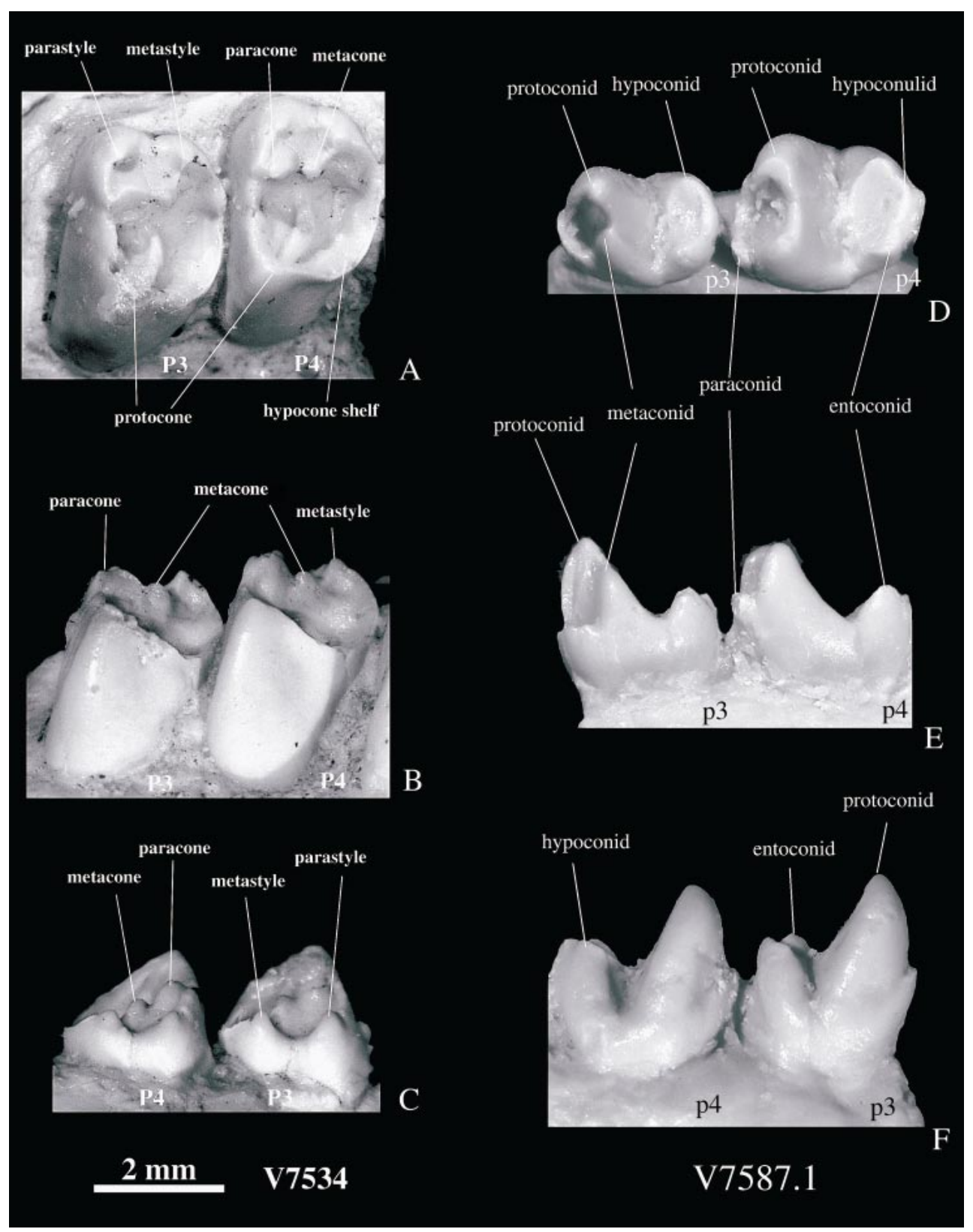

Fig. 8. Premolars of Rhombomylus. A-C, Occlusal, lingual, and labial views of the left upper P3 and P4 (IVPP V7534); D-F, occlusal, lingual, and labial views of the right lower p3 and p4 (IVPP V7587.1).

vided (fig. 19). SEM photographs of the enamel microstructures of the cheek were also taken at positions indicated in figure 19. Only those from the upper cheek teeth were illustrated (fig. 20). We follow Martin (1994,
1999) for terminology of enamel microstructure.

MicROWEAR: Wear facets from both upper and lower cheek teeth were examined (figs. $21,22)$. The first and second right upper mo- 


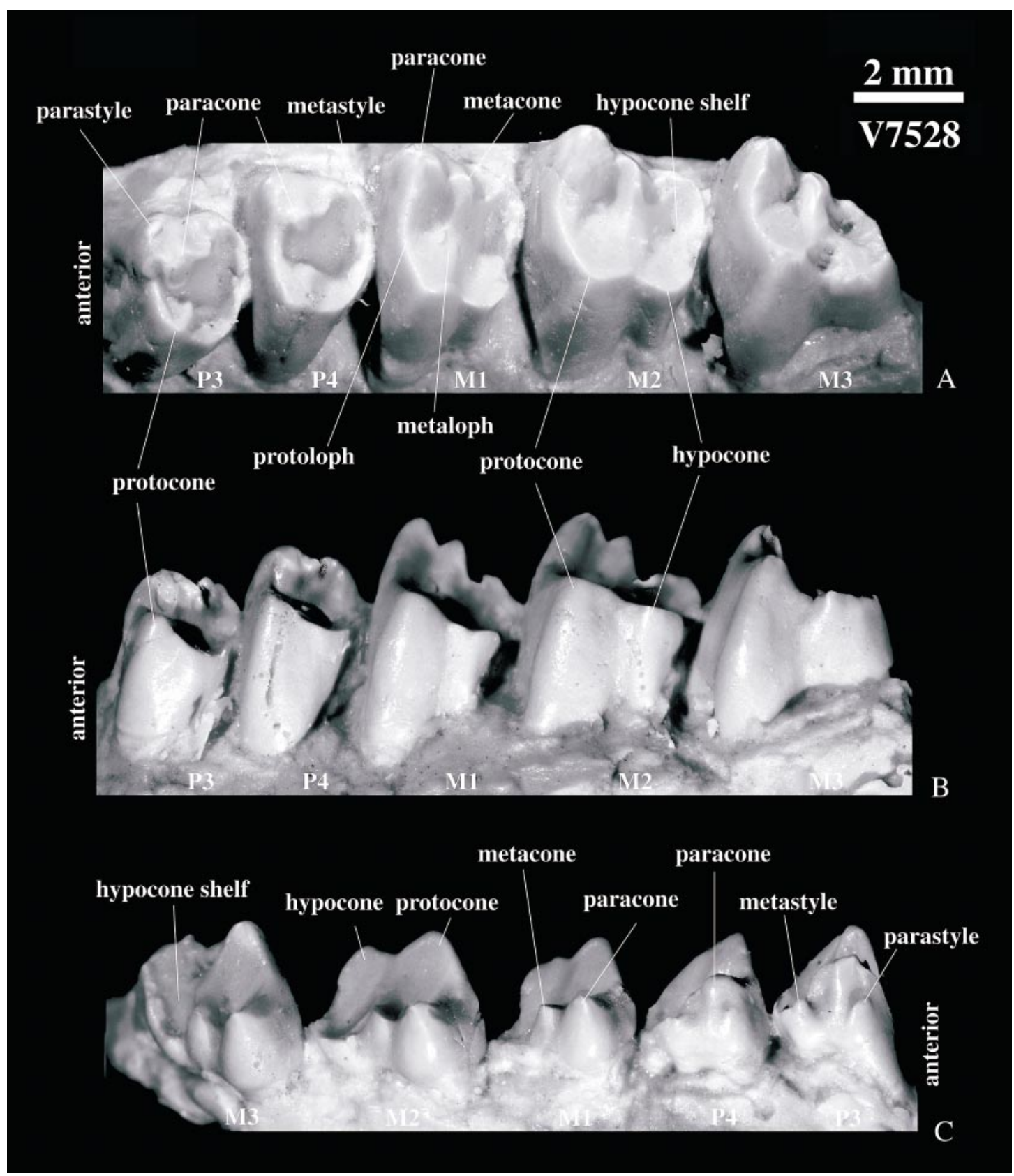

Fig. 9. Occlusal, lingual, and labial views of upper cheek teeth of Rhombomylus (IVPP V7528).

lars are taken from a broken skull (IVPP V7492) that has been cut to view the longitudinal and frontal sections of the nasal cavity. This individual has fully erupted P3-4 that were not yet worn. Wear on M1 and M2 is moderate so that only the enamel on the crown surface of each tooth was gone, but tooth cusps and ridges were not reshaped (fig. 21). The left lower teeth are from a fragmentary mandible that bears the $\mathrm{p} 4, \mathrm{~m} 1$, and the trigonid of the $\mathrm{m} 2$ (IVPP V7493). The p4 of the individual is fully erupted but 


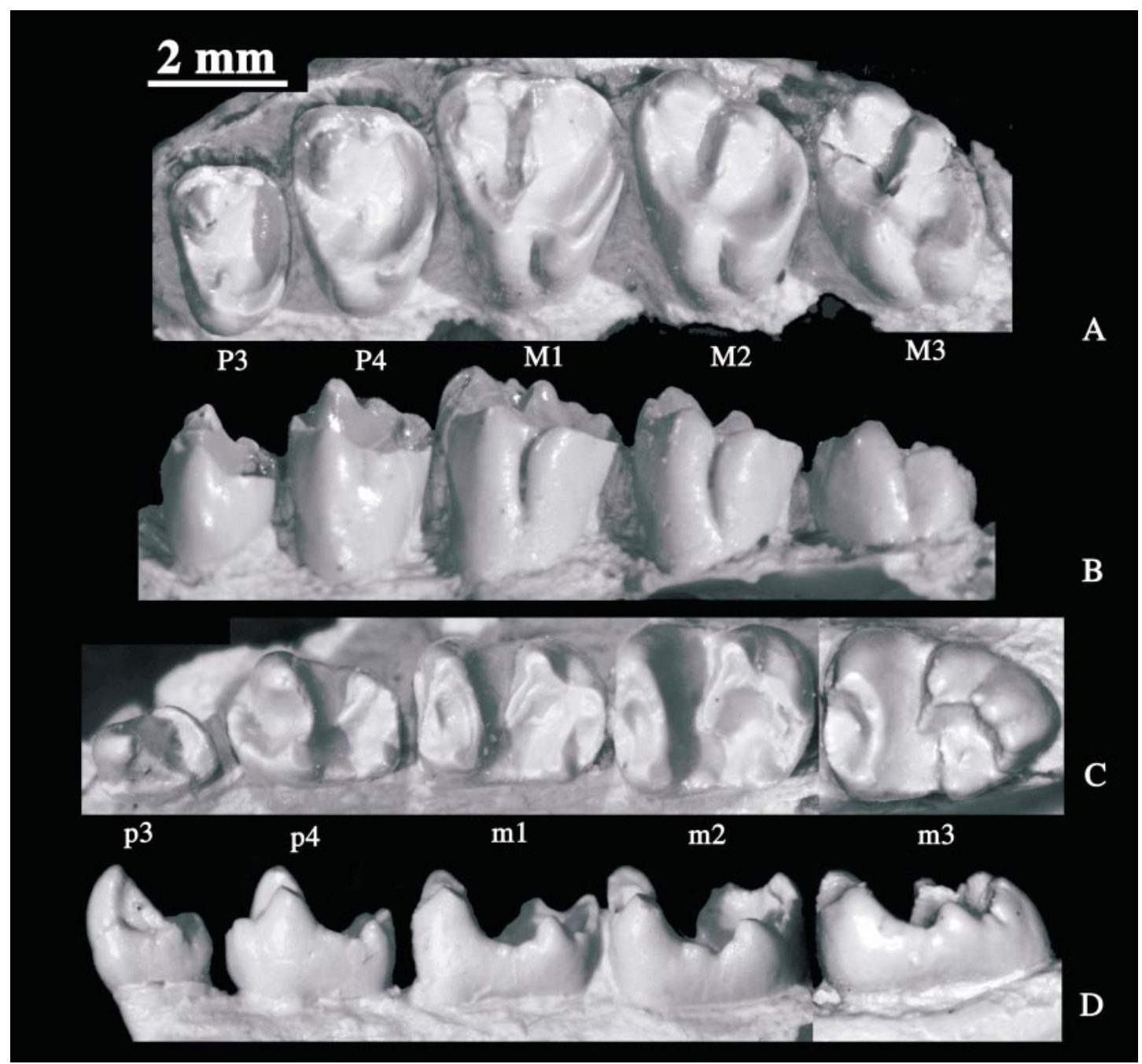

Fig. 10. Cheek teeth of Matutinia (IVPP V7442, V7443). A-B, Occlusal and lingual views of P3M3; C-D, occlusal and lingual views of p3-m3. Images photographically reversed to facilitate comparison with those of Rhombomylus. Modified from Ting et al. (2002).

shows no sign of wear; therefore, this specimen represents an individual of similar age to that of IVPP V7492.

The specimens were sputter-coated with gold as in the case for examining enamel microstructure, and observed using a scanning electron microscope. We provide only SEM images of the molars and their microwear (figs. 21, 22). The SEM photographs are oriented consistently to help understand directions of wear structures in relation to the entire tooth and the direction of jaw movement. We follow Butler (1985) and Rensberger
(1978) for terminology of wear facets and other features of wear.

SKULL: Reconstruction of the skull (figs. 23-25) is based on several specimens, primarily on IVPP V5278. Skull elements were described individually and were labeled either directly on the photographs or on accompanying line drawings (figs. 23-40). Anatomic terms were spelled out where space permits. Unless specified otherwise, we follow Novacek (1986b) for terminology of the skull elements.

Cross Sections: A total of four fragmen- 


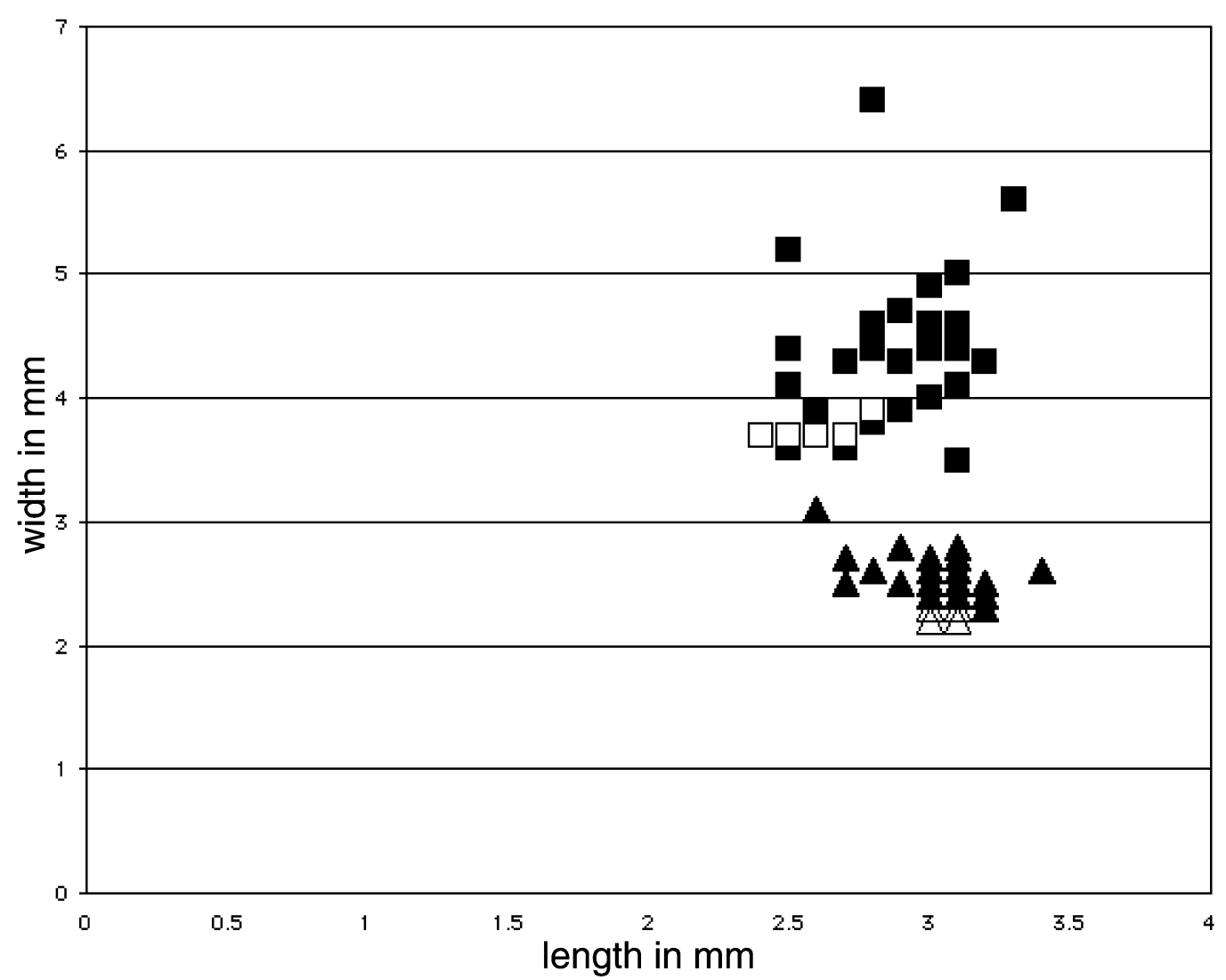

Fig. 11. Comparison of M1 (squares)/m1 (triangles) sizes of Matutinia and Rhombomylus. Solid symbols represent M1/m1 of Rhombomylus; empty ones are those of Matutinia. Measurements of Matutinia are from Ting et al. (2002).

tary skulls have been sectioned (figs. 41-49). The cross sections of the rostrum, primarily to show the nasal cavity, are cut from anterior to posterior from IVPP V7488 (figs. 41, 42). Longitudinal and frontal sections of the nasal cavity are from IVPP V7492 (figs. 43, 44). The left facial region and all left cheek teeth of V7492 were broken, with the exception of the incisor. The right P3-M2 are preserved. The left incisor was sectioned for investigation of its enamel microstructure, and the right cheek teeth were used for SEM study of microwear (see above). Longitudinal sections of the nasal cavity were obtained by grinding the left side of the specimen at an interval of $0.5 \mathrm{~mm}$ to the level just passing the medial sagittal plane of the skull (fig. 43). During grinding, the specimen was held with its sagittal plane parallel to the grinding wheel. The remaining right half of the spec- imen was then ground from skull roof to the hard palate at the same interval to reveal the frontal (horizontal) sections of the nasal cavity (fig. 44). At each interval during the grinding, the section of the ground specimen was directly scanned into the Photoshop program for Macintosh using a Microtek ScanMaker III scanner at its highest resolution. A total of 20 frontal and 15 longitudinal sections were obtained, and most of them were illustrated (figs. 43, 44). The original specimens are light-colored in bone and dark-colored in matrix. The images were inverted using the Photoshop program to provide the best results.

Only transverse cross sections of the braincase and basicranium were made from two posterior portions of skulls. IVPP V5266 was prepared before sectioning, whereas IVPP V5267 was sectioned without remov- 


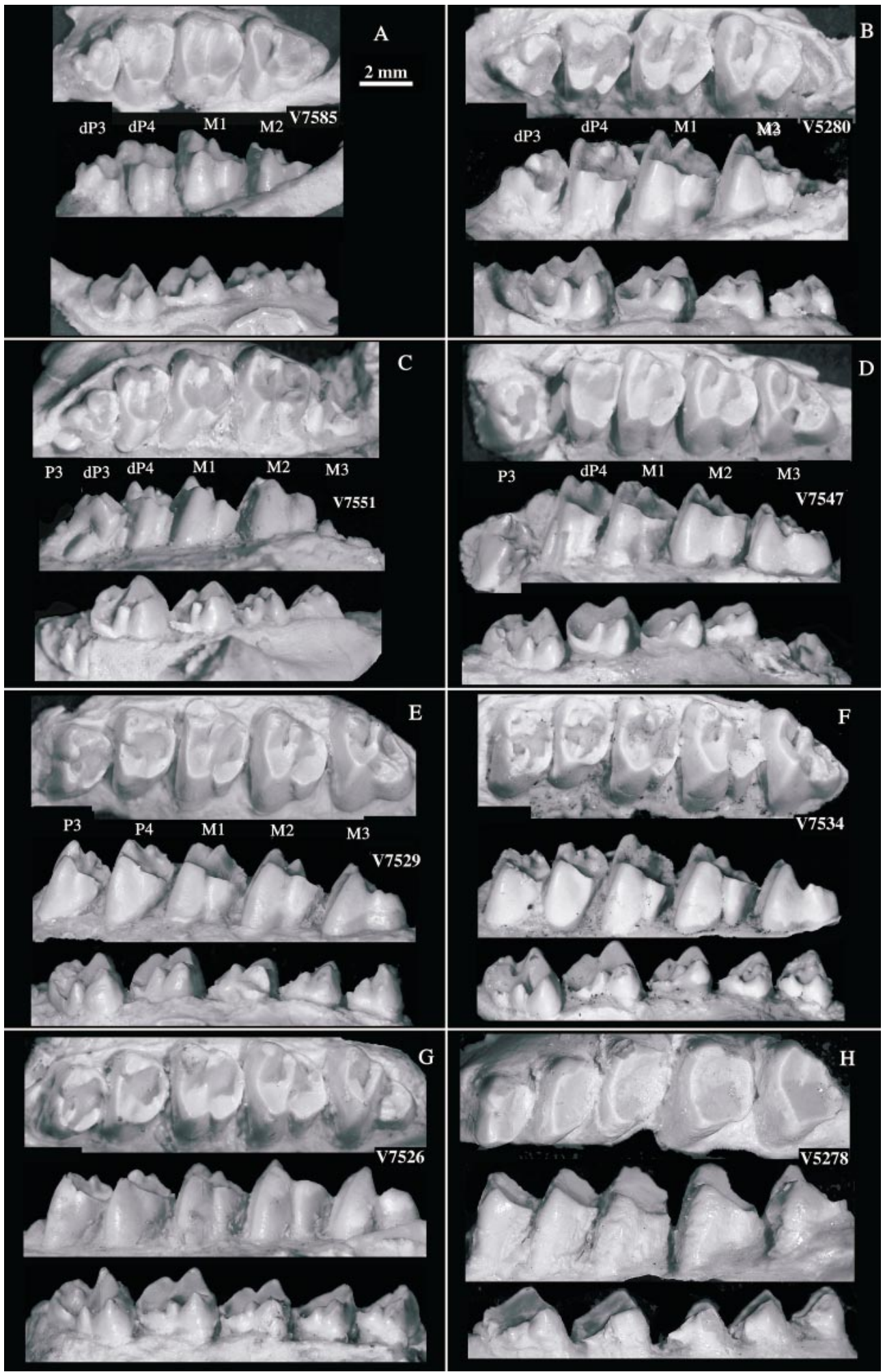


ing the original surrounding matrix. Both specimens were embedded in artificial resin and then cut into thin slides. A total of 33 slides were obtained from IVPP V5266 and 27 from IVPP V5267; each slide is $0.55 \mathrm{~mm}$ thick (except the remaining ends) at an interval of $0.3 \mathrm{~mm}$ that is caused by the cutting saw. The two sides of each slide for all were scanned using the Microtek ScanMaker III scanner at the highest input resolution. For IVPP V5266, 24 images of cross sections were illustrated (fig. 46). The original slides were numbered in a posteroanterior sequence. For convenience, however, our illustrations and description follow an anteroposterior order in keeping with sections of the nasal cavity. Because the skull has been slightly twisted during preservation, the cranial images in the slices are not symmetrical. Therefore, in the numbered order, the left side structures appear first. The left side of IVPP V5267 was seriously damaged during sectioning, but its right side is in better condition, especially in the ear region, than in IVPP V5266. We illustrated part of the ear and glenoid region of this specimen (figs. 47, 48). The 16 images of the ear region are from eight slides in a sequence (fig. 47). The image of the anterior side of each slide has been photographically reversed.

ENDOCAST: Endocasts were made by peeling off bones from three partial skulls (IVPP V5286, V7486, V7487) (figs. 50, 51). Terminology of the endocranial morphology follows Black (1920).

PostCRANial Skeleton: Comparisons of postcranial skeletons were based on published work (Wood, 1937, 1962; Kielan-Jaworowska, 1977, 1978; Szalay, 1977, 1984, 1985) and on our own observations of specimens (appendix 1). Selected measurements of bones are scattered in the text. The terminology of the skeletal elements primarily follows Cooper and Schiller (1975). Additional terms are from Wood $(1937,1962)$, Szalay (1977, 1985), Gebo and Rose (1993),
O'Leary and Rose (1995), and Muizon (1998). Abbreviations for foot structure mainly follow Szalay (1977, 1985). Attachments of muscles are based on comparisons with extant (Rinker, 1954; Klingener, 1964; Ryan, 1989) and fossil mammals (Kielan-Jaworowska and Gambaryan, 1994; Muizon, 1998). Positional terms refer to bone orientations in the articulated skeleton of normal quadrupedal mammals. Anatomical terms were directly labeled on the photographs of skeletal elements or explained in the figure captions (fig. 54-71).

\section{DENTITION}

INCISORS: There is only one pair of upper incisors (fig. 6). The incisor is contained within the premaxilla, with its posterior end intruding into the maxilla posterior to the premaxilla-maxilla suture. As in other gliroid mammals, the enamel covers the anterior (labial) surface of the incisor and wraps around on both medial and lateral surfaces of the tooth. The enamel coverage is a narrow stripe on the medial surface and extends nearly half the tooth depth on the lateral. In cross section, the anterior surface of the incisor is rounded and curves around to the lateral surface. Therefore, the cutting edge at the incisor tip is not straight; it is somewhat curved, deeper medially than laterally (fig. $6)$. In contrast, the medial and anterior surfaces of the tooth confine a nearly right angle in cross-sectional view. In posterior view the wear facet of the incisor tip is straight medially and rounded laterally (fig. 1A, B). The body curvature of the entire upper incisor is at a lower degree than are those in most rodents. The tip of the incisor points ventrally (fig. 3C), not posteroventrally as in many rodents. The crown length (height) of the incisor is shorter than those in most rodents.

The lower incisor within the mandible extends underneath the cheek teeth and ends at the level slightly posterior to m3 (fig. 6D).

$\leftarrow$

Fig. 12. Upper cheek teeth of Rhombomylus (IVPP V5278, V5280, V7526, V7529, V7534, V7547, V7585, V7551). Occlusal, lingual, and labial views of cheek teeth showing variations owing to ages and wear among individuals. See text for discussion. Some images were photographically flipped to facilitate comparison. 
The exposed crown is relatively shorter than those of rodents. Its tip is usually at the same level as the occlusal surface of the cheek teeth (figs. 6F, G, 52, 53). The only exception is IVPP V5278, the oldest individual in the collection, in which the incisor tip is slightly higher than the cheek tooth row (fig. 53D). The lower incisor is longer and less curved than its counterpart, but its enamel coverage and shape of cross sections are similar to those of the upper incisor. The wear facet on the tooth tip has a semicircular labial edge and a straight medial edge (fig. 6E). Presence of the wear facet on the lower incisor indicates that Rhombomylus was capable of moving the mandible anteriorly and honing the lower incisor against the edge of the upper one. This is characteristic of incisive gnawing of all gliroid mammals. The minimum distance the mandible could move anteroposteriorly is indicated by the distance between the tip of the upper incisor and the posterior edge of the wear facet on the lower incisor when the mandible is in its rest position (figs. 25, 79).

The specimens from Dajian represent a sequence of postnatal ontogeny of Rhombomylus (figs. 26-28). The skull of IVPP V7585 (figs. 26A, 27A, 28A) represents the youngest individual, in which $\mathrm{M} 2 / \mathrm{m} 2$ were not yet fully erupted (figs. 12A, 14A). The oldest one is IVPP V5278 (figs. 26F, 27F, $28 \mathrm{~F}$ ), in which all cheek teeth are heavily worn (figs. 12H, 14H). Many specimens of intermediate ages between IVPP V7585 and V5275 show various states of replacement of premolars and eruption of molars, but none of them shows any sign of incisor replacement. This suggests that the enlarged incisors of Rhombomylus are deciduous teeth. There is no evidence, however, for the loci of the enlarged incisors. We assume the incisors of Rhombomylus to be the second pairs in homology as those in living rodents and lagomorphs (Moss-Salentijn, 1978; Ooë, 1980; Luckett, 1985; Simoens et al., 1995).

Cheek Teeth: The upper tooth rows are parallel to each other (figs. 24, 27). Cheek teeth increase in size posteriorly (figs. 9, 12). In labial or lingual view, the upper cheek teeth incline posteriorly such that the anterior edge of each tooth stands out as the highest crest (fig. 9B). The lingual side of the upper cheek tooth is significantly higher than the labial one. For any fully erupted tooth the enamel does not enter the alveolus. The tooth morphology and size vary owing to different ages and degrees of wear. When a tooth crown was considerably worn, its lingual roots tend to grow out of the alveolus and the tooth crown becomes transversely wider. As in other gliroid mammals, the canine and the P1 are absent, and further differing from lagomorphs the P2 is also lost in Rhombomylus.

The $\mathrm{dP} 3$ and $\mathrm{dP} 4$ are preserved in IVPP V5279, V5280, V7551, V7567, V5281, V7584, and V7585. The dP3 is triangular with three spaciously separated roots. Each root supports an apex of the triangular tooth crown. On unworn specimens the dP3 consists of a small trigon and an expanded hypocone shelf (fig. 7A-C). The trigon bears three closely packed cusps, namely the protocone, paracone, and metacone. The protocone is the largest of the three and is positioned lingually. The paracone is the smallest and is at the anterior tip of the tooth. The metacone is posterolabial to the paracone and is separated from the other cusps by a shallow groove. A metastyle-like cuspule is lateral to the metacone. The hypocone is larger, lower, and more lingually positioned than the protocone. A broad hypocone shelf forms the posterior half of $\mathrm{dP} 3$ and is the widest region of the tooth. On slightly worn specimens (e.g., IVPP V5280, fig. 12B), the posterior surfaces of the protocone and metacone bear wear facets that slope into the broadly basined hypocone shelf. In older individuals (e.g., IVPP V5281, not illustrated) in which the dP3 is heavily worn, the crown becomes a flat surface inclined posteriorly and cusps are no longer distinguishable. Unlike dP4, the crown of dP3 is not significantly higher lingually (fig. 7C). The dP3 was replaced by the erupting P3 at the time when M3 is erupting (e.g., IVPP V7551, V7547; fig. 12C, D).

The dP4 is narrow lingually and broad labially (fig. 7A). It has one lingual and two labial roots. It is more molariform than is the $\mathrm{dP} 3$ in that the trigon is fully developed with a well-developed protocone, paracone, metacone, and hypocone. The paracone and metacone are separated by a transverse groove. Labial to the groove the edge of the tooth is 


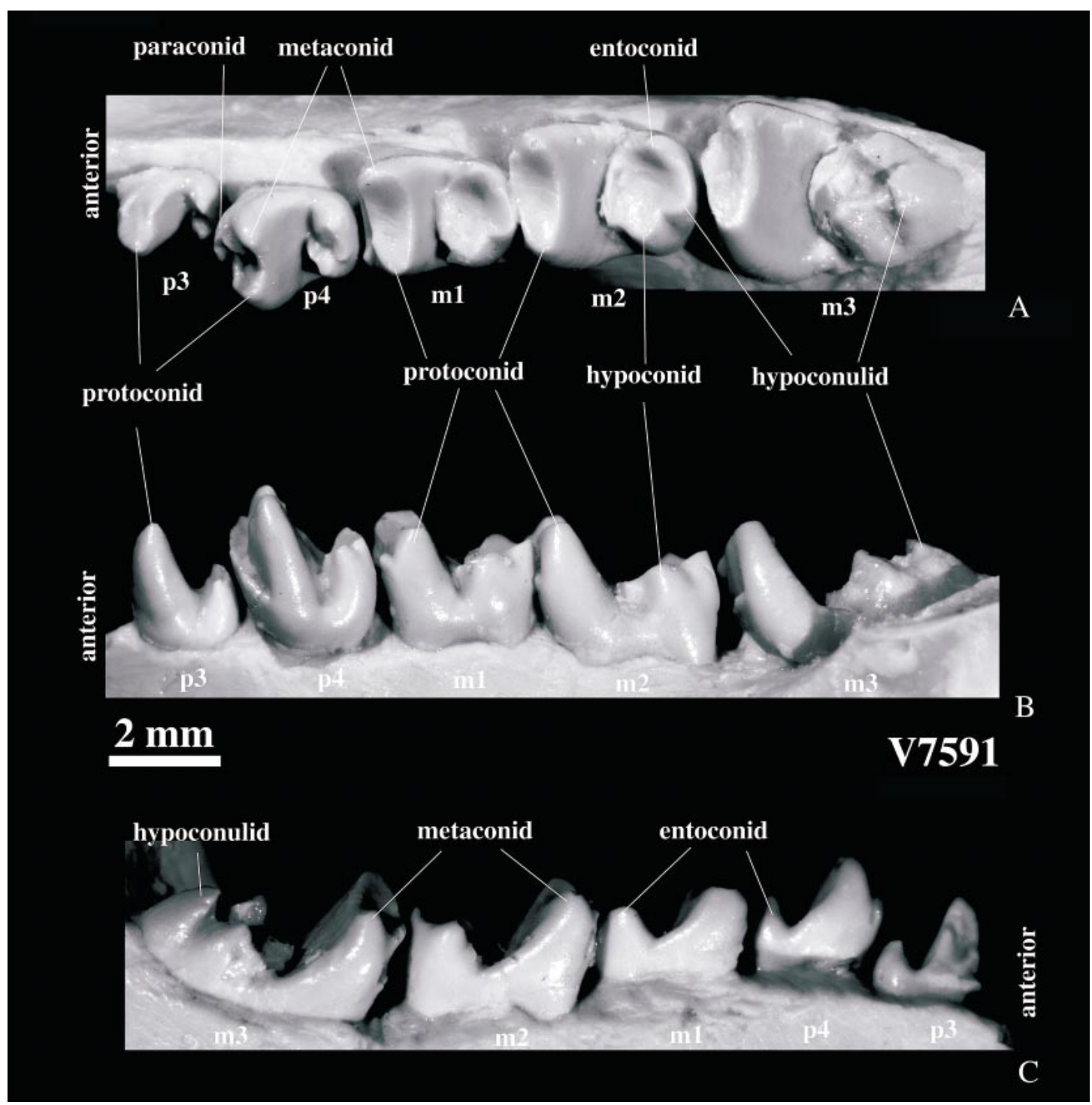

Fig. 13. Occlusal, labial, and lingual views of lower cheek teeth of Rhombomylus (IVPP V7591).

lingually indented, forming a shallow ectoflexus. The hypocone shelf is broader labially than lingually. The hypocone is nearly the same size as, but is lower than, the protocone; the two cusps are separated by a vertical groove on the lingual surface of the tooth (fig. 7C). The $\mathrm{dP} 4$ is replaced by $\mathrm{P} 4$ after M3 is erupted (IVPP V7528, V7547; figs. 9, 12D).

Differing from dP3, P3 is oval in outline and is transversely elongate (figs. 8, 9, 12). Its crown is higher lingually than labially.
The P3 lacks a hypocone and the vertical groove on the lingual surface. An unworn P3 has a sharp protocone and a labial cusp, presumably the paracone (figs. 9, 12E). The paracone is slightly higher than the protocone. An enamel ridge, the protoloph, which forms the anterior edge of the tooth, connects the two cusps. A weaker ridge, presumably the metaloph, extends from the protocone to the posterolingual base of the paracone. The two ridges confine a small basin between the protocone and paracone. The latter ridge dis- 


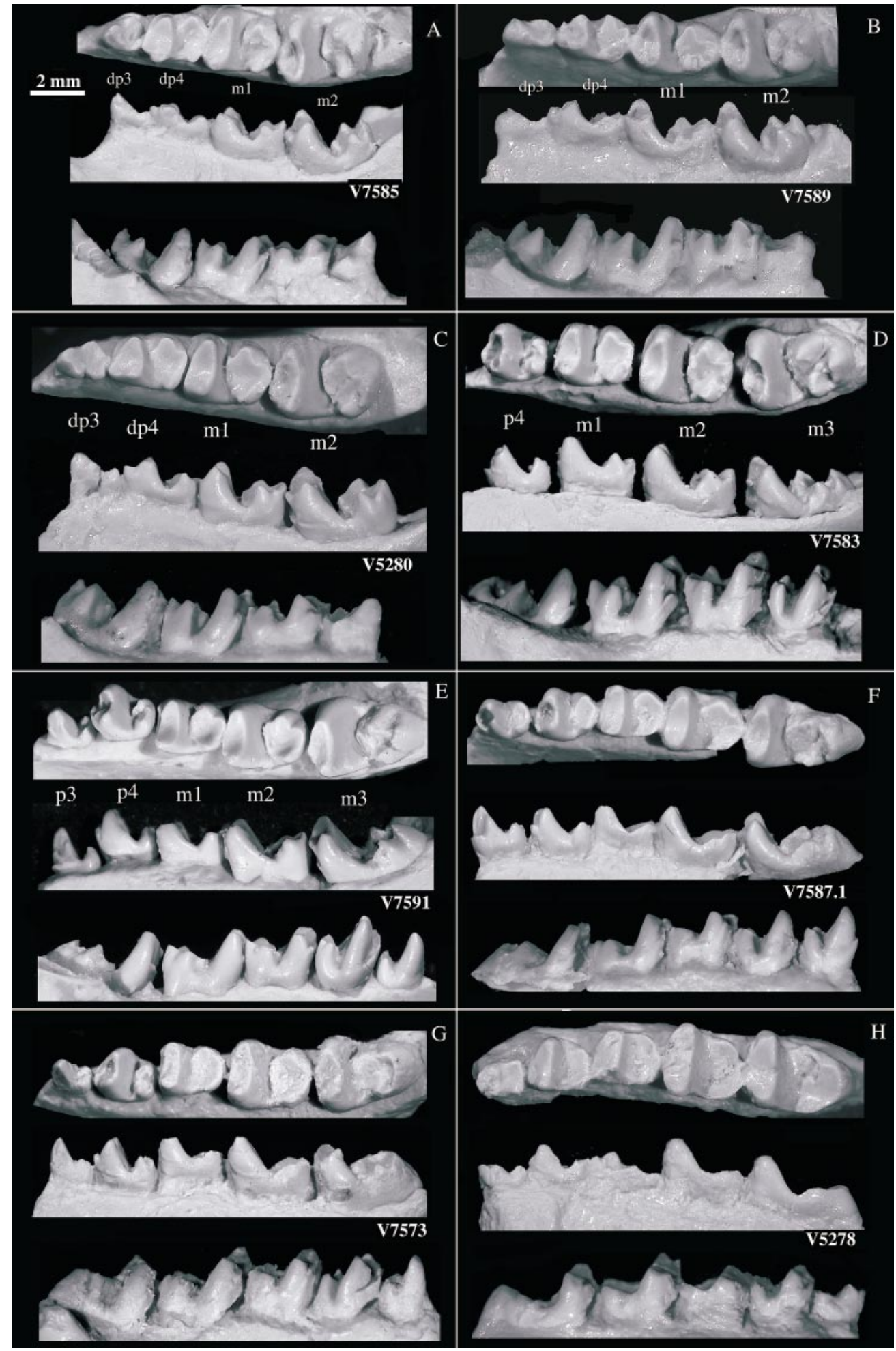


appears with wear and the confined basin becomes confluent with the hypocone shelf. Anterolateral to the paracone is a small parastyle-like cuspule. A metastyle-like cuspule is usually present posterolateral to the paracone. The metacone is not formed in most specimens. In some, however, an incipient metacone is developed immediately posterior to the paracone (fig. 8). In specimens that bear the metacone, the parastyle- and metastyle-like cuspules are usually more prominent, and a narrow external cingulum is commonly present. We consider these differences to be variations among individual organisms of the same species. In all cases, the posterior half of the P3 is a broad, deep, oval-shaped shelf.

Differing from the $\mathrm{dP} 4$, the $\mathrm{P} 4$ is less molariform in that all cusps, except the protocone, are not fully developed. The P4 is similar to but larger than the P3 (fig. 8). As in the $\mathrm{P} 3$, the $\mathrm{P} 4$ also shows the same variation of the metacone.

The M1 is significantly larger than the dP4 and P4 (figs. 9, 12). Its trigon is transversely elongate with the labial cusps being more marginally located. The paracone is higher and larger than the metacone and is more laterally extended; the two cusps are separated by a narrow, transverse groove. There is no external cingulum. The widest region of the tooth is between the paracone and the protocone. There is no anterior cingulum, nor is there any conule. The strong, curved protoloph connects the protocone and paracone and forms the anterior cutting edge of the tooth. A blunt metaloph extends from the protocone to the metacone. In contrast to the thick enamel layer of the protoloph, the metaloph is covered by a thin layer of enamel that is observed only in newly erupted teeth. The enamel is easily worn so that the metaloph in most specimens is made up of bare dentine. In specimens of older individuals, intensive wear has even deleted the metaloph. At that stage, the paracone and meta- cone are no longer distinguishable, the trigon becomes confluent with the hypocone shelf, and the enamel forms a loop surrounding the tooth (fig. 12H). Because the paracone and metacone are close to each other, the protocone and lophs from it form a compressed V shape. The protocone is the largest cusp of the tooth and is columnlike in lingual view (figs. 9, 12). The hypocone is lower, smaller, and more lingually positioned than the protocone. The two cusps are separated on the lingual surface by a shallow, vertical groove that extends into the alveolus. As in the premolars, the most conspicuous feature of the M1 is the expanded hypocone shelf, which is a broad basin forming the posterior half of the tooth. The basin is oval-shaped and posteriorly bordered by a complete enamel edge that extends from the hypocone and terminates as a metastyle at the posterolabial corner of the metacone.

M2 bears two lingual roots as shown on IVPP V7554, one supporting the protocone and the other the hypocone. M2 differs from M1 in being larger and having stronger cusps and lophs (figs. 9, 12). In some specimens, a dentine ridge is developed at the posterior side of the metaloph and extends posteriorly across the hypocone shelf, as shown in IVPP V7551 (fig. 12C). The hypocone shelf is further expanded on M2. In general morphology, however, M2 is similar to M1.

The M3 trigon is basically similar to those of M1 and M2 (figs. 9, 12). It differs from M1 and M2 in having a reduced metacone and a transversely narrower hypocone shelf. The tooth has a triangular outline in crown view. Although reduced, the M3 hypocone shelf is more complex than on M1 and M2 in bearing a strong dentine ridge that extends posterolingually from the posterior side of the metaloph to the hypocone. In addition, the posterolabial edge of the shelf is decorated with a series of small enamel conules. With wear, all these structures disappear.

Fig. 14. Lower cheek teeth of Rhombomylus (IVPP V5278, V5280, V7585, V7573, V7583, V7587.1, V7589, V7591). Occlusal, lingual, and labial views of cheek teeth showing variations owing to ages and wear among individuals. See text for discussion. Some images were photographically flipped to facilitate comparison. 


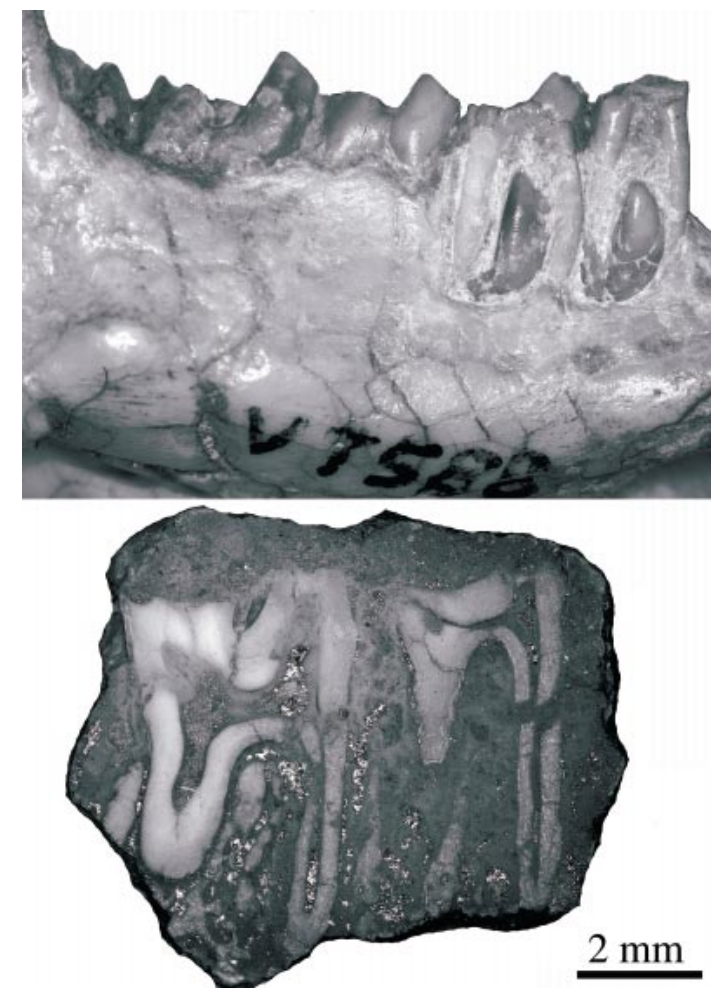

Fig. 15. Upper, A fragmentary mandible showing replacement of lower premolars of Rhombomylus (IVPP V7588); Lower, cross-section of $\mathrm{m} 1$ and $\mathrm{m} 2$ of Rhombomylus showing the roots and pulp cavity.

The lower cheek teeth increase in size posteriorly and lean forward. The dp3 displays some variation. In IVPP V7585 (fig. 7D-F), the dp3 is short, with an anterior cusp, presumably the protoconid, and a simple talonid that is wider than the anterior half of the tooth. In other specimens (IVPP V7589 and V5280 fig. 14B, C) the dp3 is long and shows differentiation of the trigonid and talonid. The trigonid bears a main anterior cusp, probably the protoconid, and a posterolingual cusp, possibly the metaconid. The shape of the talonid indicates presence of the hypoconid, entoconid and hypoconulid, although they are not definitely separated. The two roots of the deciduous premolars are more open than those of permanent premolars (fig. 15 , upper). Replacement of the dp3 appears to be slightly later than that of the dp4, after eruption of the $\mathrm{m} 3$.

The dp4 differs from the dp3 in being larg- er and more molariform (figs. 7, 14). Differing from the molars, the dp4 trigonid is relatively long and is narrower than the talonid. The trigonid bears the protoconid, paraconid and metaconid, with the paraconid projecting anteriorly. A weak anterior cingulum is at the anterior base of the tooth. The hypoconid, entoconid, and hypoconulid on the talonid are well developed, although in all specimens they were worn. The ectolophid extends from the hypoconid to the posterior wall of the trigonid.

The p3 also shows some variations. In IVPP V7591 and V7573 (figs. 13, 14G), the p3 has a high, conical anterior (trigonid) cusp. On the lingual base of this main cusp are two small swellings, of which the anterior one is probably the paraconid and the posterior the metaconid. A trigonid is not developed. The simple talonid bears two low cusps, presumably the hypoconid on the lateral side and the entoconid on the medial side; the two cusps are connected by a weak ridge. In IVPP V7587.1 (fig. 14F), however, the trigonid is more complex in having a significant metaconid and a small trigonid basin, so that the trigonid is wider than the talonid. The cusps on the talonid are stronger than those in IVPP V7591 and V7573, and a rudimentary hypoconulid appears to be present.

The $\mathrm{p} 4$ differs from the dp4 in being shorter and less molariform; its talonid is notably narrower than the trigonid (figs. 13, 14). Although the trigonid is wider than that of dp4, the paraconid of p4 is reduced and is much lower than the protoconid and metaconid. The trigonid is anteroposteriorly shortened. An inclined anterior cingulum extends dorsolingually from the base of the protoconid to the paraconid (fig. 13C). The posterior surface of the trigonid is high and steep. On the narrow talonid, the hypoconid, entoconid, and hypoconulid are distinct but closely packed. A short ectolophid extends from the hypoconid to the midline of the tooth, but it is separated from the posterior wall of the trigonid by a narrow gap, which differs from the condition in the dp4.

All lower molars have two long roots, with the posterior one being stronger. The pulp cavity of the tooth crown continues into the roots (fig. 15, lower). The tooth crown is 
broader at the base. The trigonid is significantly higher than the talonid and is anteroposteriorly shortened (figs. 13, 14). An anterior cingulum exists along the anterior base of the molar. The paraconid is reduced. On unworn specimens, the lingual side of the trigonid is slightly longer than the labial side, but with wear, the trigonid becomes nearly even in length labially and lingually and is transversely elongate. The entire trigonid is rimmed by an enamel loop. The metaconid is always higher than the protoconid in both juvenile and adult individuals.

The $\mathrm{m} 1$ trigonid is wider than that of the $\mathrm{dp} 4$. The posterior surface of the $\mathrm{m} 1$ trigonid is steep and bears an extensive shearing facet. The facet is broader lingually than labially. In posterior view, the cutting edge of the trigonid forms a curved line. The talonid is lower but longer than the trigonid. It bears the hypoconid, entoconid, and hypoconulid. In unworn teeth, the conical entoconid is the highest cusp of the talonid, separated from the transverse hypoconulid by a narrow gap, whereas the hypoconid is the broadest one. None of the cusps has an enamel ridge. The interior surfaces of all these cusps are covered with thin enamel, which is usually gone with slight wear. From the hypoconid a short ectolophid extends anteromedially. The enamel layer of the ectolophid forms a bulge, which may be interpreted as a mesoconid. A narrow, deep, and transverse groove fully separates the trigonid from the talonid. With wear, however, this groove and the talonid cusps are indistinct, and the talonid becomes broadly basined.

The $\mathrm{m} 2$ is larger than the $\mathrm{m} 1$. Its trigonid and talonid are more deeply separated, and the talonid cusps are further expanded. The mesoconid on the ectolophid is more prominent than that of the $\mathrm{m} 1$. In some cases, such as V7568.1, a small mesostylid occurs at the anterior base of the entoconid. Other structures are similar to those of the $\mathrm{m} 1$.

The $\mathrm{m} 3$ is the largest lower molar. Its trigonid is wider than, but morphologically similar to, that of the $\mathrm{m} 2$. The $\mathrm{m} 3$ talonid differs from that of $\mathrm{m} 2$ in being longer. All the talonid cusps are larger, especially so for the hypoconulid, than those of the $\mathrm{m} 2$. In young individuals the expanded hypoconulid is separated from the other two cusps by gaps. With wear, the cusps are no longer distinguishable and the talonid becomes a broad basin.

DisCussion: Although once regarded as synonyms (Dashzeveg and Russell, 1988), Rhombomylus is actually significantly different from Matutinia in dental morphology. Matutinia is generally smaller (figs. 10, 11). Its teeth have relatively lower crowns, more distinctive cusps, and lower ridges. The p3 is single-rooted in Matutinia, but doubled in Rhombomylus. The paracone and metacone on cheek teeth of Matutinia are more conical than those of Rhombomylus. These cusps, as well as the protoloph and metaloph extended from these cusps, are more distantly separated in Matutinia than in Rhombomylus. The hypocone shelf of the cheek teeth is more prominent in Rhombomylus than in Matutinia. In particular, the shelf on the M3 of Rhombomylus forms a lobe with complex structures on unworn specimens. The hypocone shelf on the M3 of Matutinia is simple and reduced. The ridges that confine the trigonid, the ectolophid, and talonid cusps are more distinctive in Matutinia than in Rhombomylus. On the upper teeth of Rhombomylus, the vertical groove on the lingual side of the cheek teeth extends into the alveolus, whereas the groove is short in Matutinia.

\section{EnAmel Microstructures}

The ever-growing incisors of rodents are very effective tools for food gathering and cutting, defense, and shaping the environment. These functions of incisors can be interpreted for early gliroid mammals on the basis of similar incisor morphology (Wahlert, 1989; Martin, 1993). Although the cheek teeth of gliroid mammals differ considerably, their incisors are similar in that they are rootless, ever growing, and deeply embedded in the premaxilla and mandible, and that the enamel is restricted primarily to the labial (external) surface of the incisor. In addition, the tooth enamel of gliroid mammals has complex microstructures. The fundamental microstructures typical of the incisor enamel of gliroid mammals are the various types of bands formed by decussating bundles (prisms) of hydroxyapatite crystallites, namely Hunter-Schreger bands (HSB) (Kor- 
venkontio, 1934; Wahlert, 1968, 1989; Boyde, 1978; Koenigswald, 1980, 1985; Koenigswald et al., 1987; Martin, 1992, 1993, 1994, 1999). The HSB are interpreted as strengthening architectures that prevent crack propagation of the enamel layer that forms the cutting edge of the tooth, which is under great pressure during gnawing (Lehner and Plenk 1936; Koenigswald, 1980; Pfretzschner, 1988; Koenigswald and Pfretzschner, 1991). For gliroid mammals that do not have HSB, such as Tribosphenomys (Meng et al., 1994), minute postmorton cracks occur in the enamel layer (Meng and Wyss, 1994). In addition to their functional significance, enamel microstructures have been considered phylogenetically informative for gliroid mammals at various levels (Korvenkontio, 1934; Wahlert, 1968, 1989; Boyde, 1978; Koenigswald, 1980, 1985; Koenigswald et al., 1987; Sahni, 1980; Martin, 1992, 1993, 1994; Meng and Wyss, 1994, 2001). As Boyde (1978: 57) stated, "The conclusion that genetic control of cell behavior determines the enamel structure explains the strong taxonomic basis found for the structures described here." However, alternative opinion on the phylogenetic value of the enamel microstructures in gliroid mammals exists (Dauphin et al., 1988).

Although extensive investigations of gliroid mammals have been made since the work of Korvenkontio (1934), the enamel microstructures in the early gliroid mammals, particularly those that are usually placed in "Mixodontia" or "Eurymylidae", are insufficiently described (Koenigswald, 1995; Martin, 1999). This is largely because of rareness of specimens. The enamel microstructures of Rhombomylus and cf. Rhombomylus have been illustrated and discussed elsewhere (Martin, 1992: pl. 5, figs. 4, 5; 1993: fig. 19; 1999). According to Martin, both single- and double-layered incisor enamel types exist in different species of Rhombomylus, although Martin's specimens were not assigned to any species of Rhombomylus. Here we describe additional incisor enamel from Rhombomylus turpanensis, the only species of the genus we recognize.

Most previous studies on enamel microstructure of gliroid mammals focused on the incisor enamel, whereas few have explored the cheek teeth. This is probably because of the concern, as stated by Martin (1993: 229), that "Rodent incisor enamel evolves independently from the other dental traits (Koenigswald, 1988; Koenigswald and Clemens, 1992) and is particularly appropriate for comparative studies, because the incisors have roughly the same shape in all rodents and therefore are not strongly influenced by differing biomechanical constraints, in contrast to hypsodont and brachydont molars." However, studies of living rodent teeth show that tooth development is regulated by molecular mechanisms common to most organs (Thesleff et al., 1995) and that in murines at least the molar enamel is similar to that of the incisor (Lyngstadaas et al., 1998). In addition, the molar enamel becomes fully matured in 14 to 17-day-old mice, indicating that enamel microstructures of cheek teeth are not so strongly subject to biomechanical constraints after birth (Lyngstadaas et al., 1998). Therefore, cheek teeth may bear phylogenetic information that has not been systematically documented.

To our knowledge, no study has been carried out on fossil gliroid mammals in which incisor and cheek teeth enamels from individuals of the same species at various ages are systematically examined. With our unique collection of Rhombomylus, we provide a thorough documentation of its incisor and cheek tooth enamels. We attempt to explore several issues: (1) whether the enamel microstructures vary at different segments of the same incisor; (2) whether the enamel structures change at different developmental stages of a species; (3) whether microstructures of the incisor enamel evolved independently from those of cheek teeth; (4) whether the molar enamel has any phylogenetic value for gliroid and higher level mammalian relationships; and (5) what are the relationships between structures of tooth enamel and the chewing function in Rhombomylus. As pointed out by Clemens (1997: 88), the studies of the enamel microstructure "underscore the need to analyze more than one section of a tooth in order to adequately describe the characteristic range of prismatic structure of a species." Our analysis is based on teeth from several individuals of different ages of Rhombomylus. 

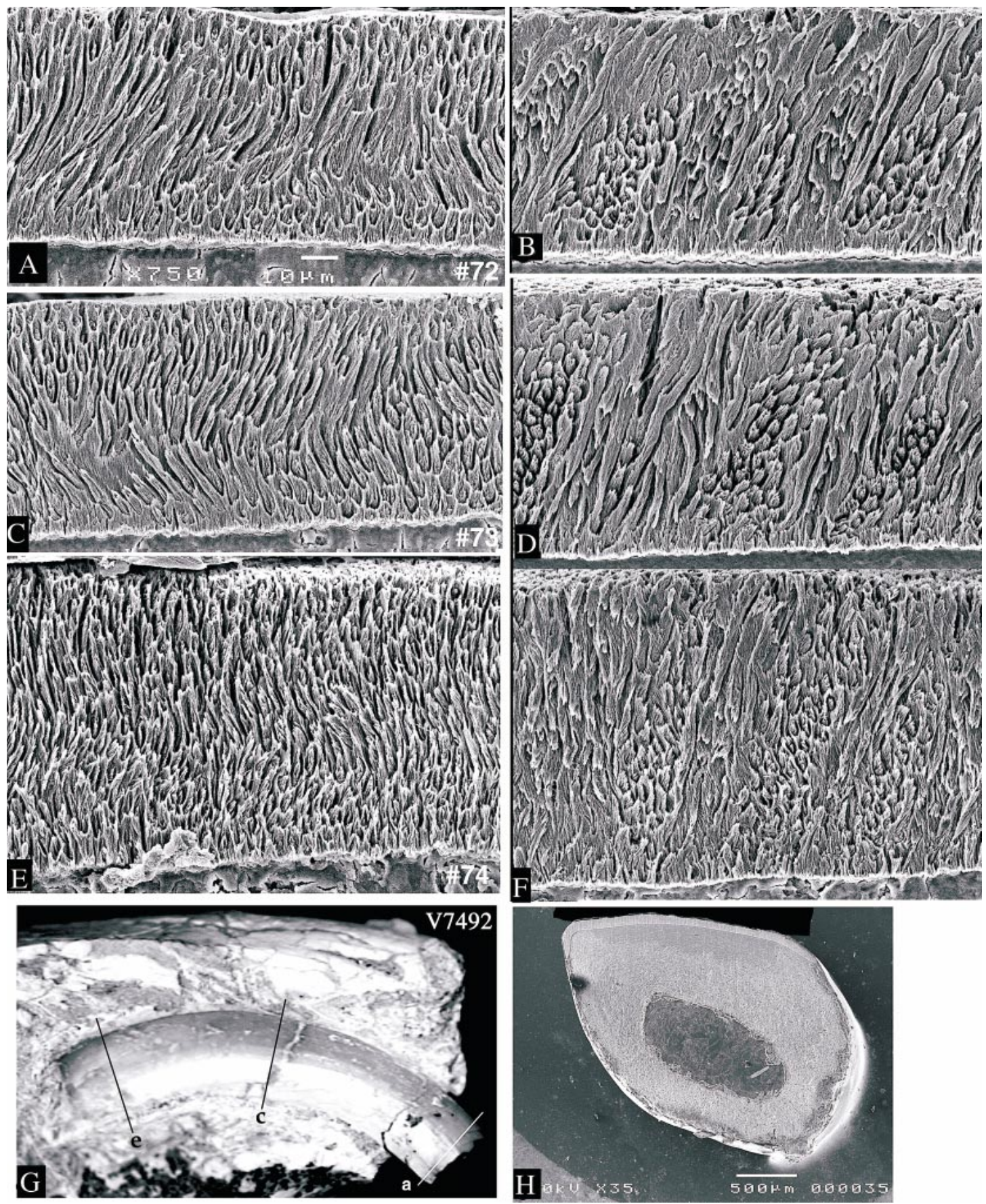

Fig. 16. Enamel structures of the left upper incisor of Rhombomylus (IVPP V7492; the rostrum of the same specimen was cut for the longitudinal and frontal sections of nasal cavity [figs. 43-44]) (the image in $\mathrm{G}$ was photographically reversed). A, C, E, Cross-sectional views of the incisor enamel microstructure at segments of the same tooth shown in $\mathbf{G} ; \mathbf{B}, \mathbf{D}, \mathbf{F}$, longitudinal-sectional views of the incisor enamel microstructure near a, c and e in $\mathbf{G} ; \mathbf{H}$, enamel distribution on the upper incisor. The scanning electronic microscopic images $(\mathrm{A}-\mathrm{F})$ are on the same scale. See text for description and discussion. 


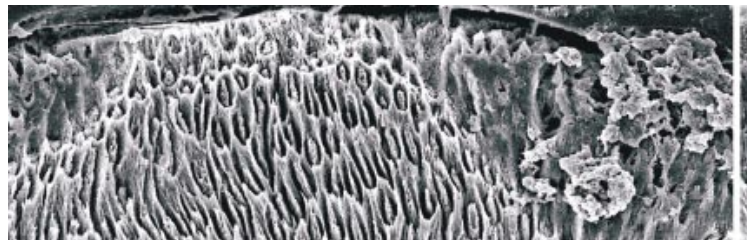

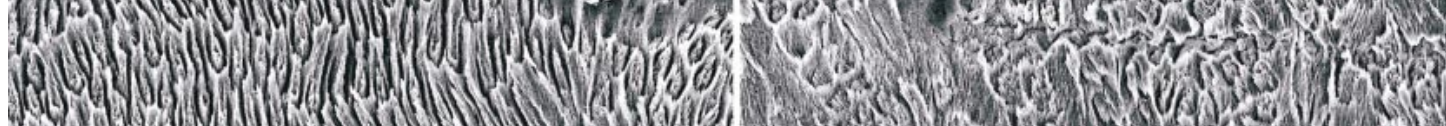
(1) 15.24 . 1.6.

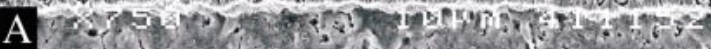

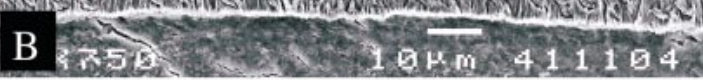

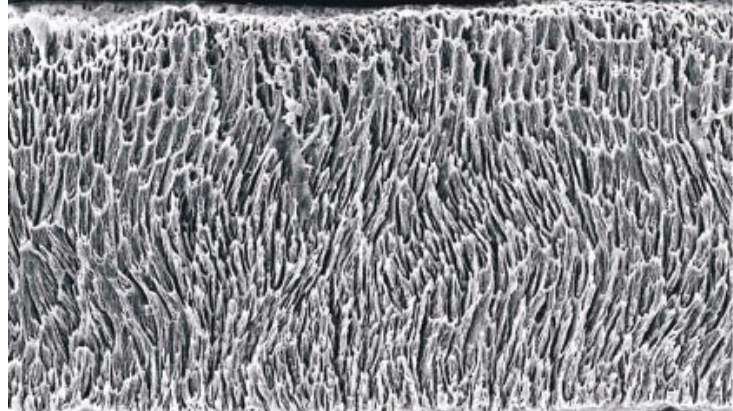

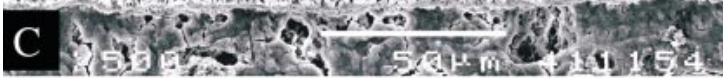

Fo6.6. 3.

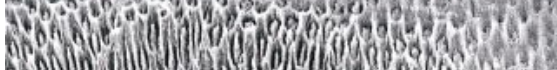

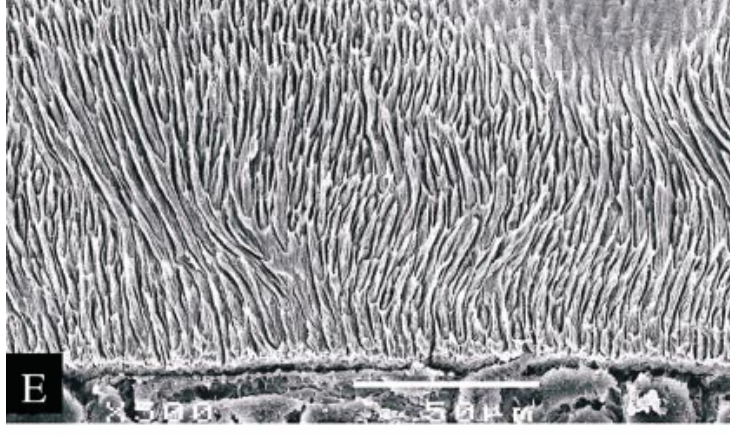

(1)

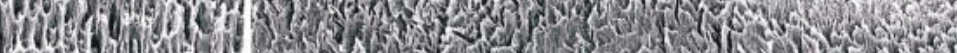

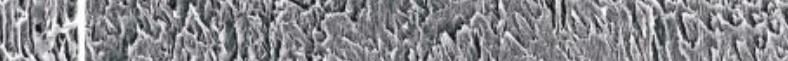

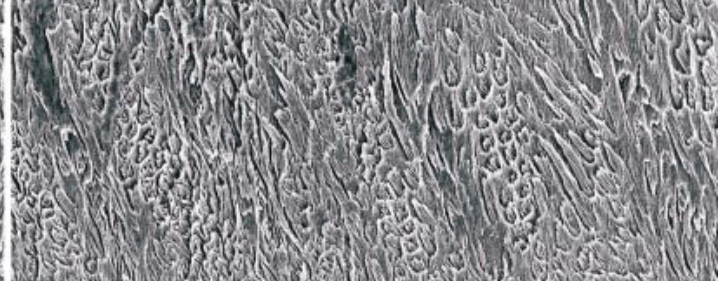

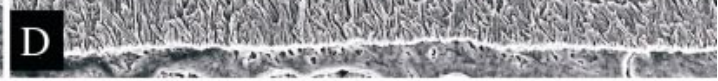

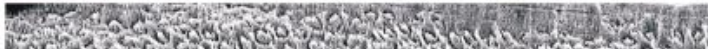

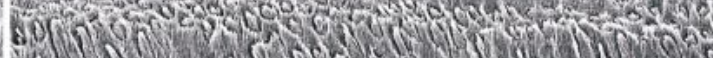

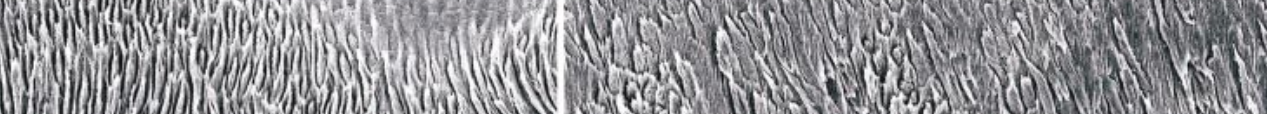
3.M. (y)

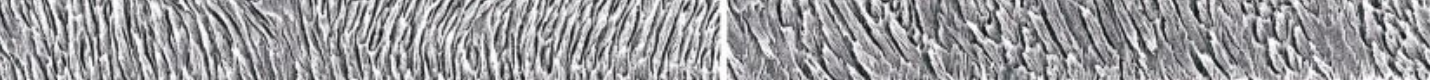

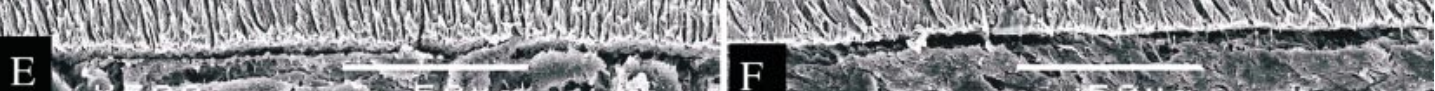
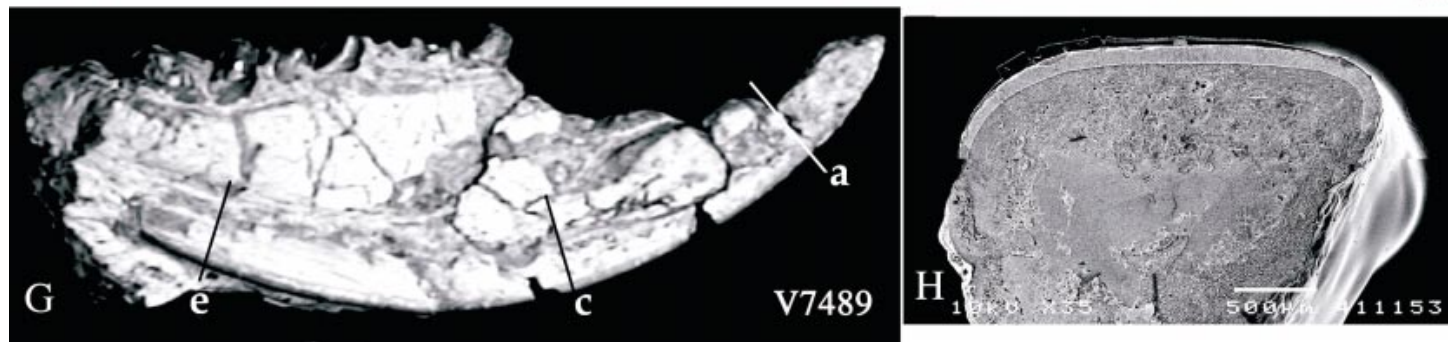
INCISOR ENAMEL: The enamel is distributed on the labial surface throughout the entirety of upper and lower incisors (figs. 16, 17). During preservation, the enamel may have undergone a certain degree of erosion or recrystallization, which alters the microstructure of the enamel. These changes take place more frequently near the tip of the incisor where the tooth is not protected by the jaw bone, as shown in IVPP V7489 (fig. 17A, B).

The enamel thickness is about $100 \mu \mathrm{m}$ in the lower incisor and $70 \mu \mathrm{m}$ in the upper for adult individuals that are approximately the same age (figs. 16, 17). The prismless external layer is very thin and shapes the smooth external surface of the enamel. After acid treatment, much of this layer was gone. In cross sections of lower and upper incisors, the prisms form an irregular wavy pattern. In contrast, HSB are distinctive in longitudinal sections of both upper and lower incisors. In both upper and lower incisors, the enamel is differentiated into an inner layer (portio interna, PI) with HSB and a thin, poorly defined outer layer (portio externa, PE) with radial enamel. The outer layer is more distinct in the lower incisor than in the upper incisor. The prisms in this layer are not well organized and do not show definitive apical bending in longitudinal section.

In longitudinal section, Hunter-Schreger bands of the inner layer in upper and lower incisors are inclined apically in relation to the enamel-dentine junction at an angle of $20-30^{\circ}$, according to the definition of Martin (1999). The HSB are not well defined and the number of prisms varies from band to band. By examining the HSB across entire sections of all examined specimens on the SEM monitor, we found that most bands consist of two to four rows of prisms; some bands may have up to five rows of prisms. There is no distinct transition zone between the HSB. Each prism is surrounded by the interprismatic matrix; both are separated by
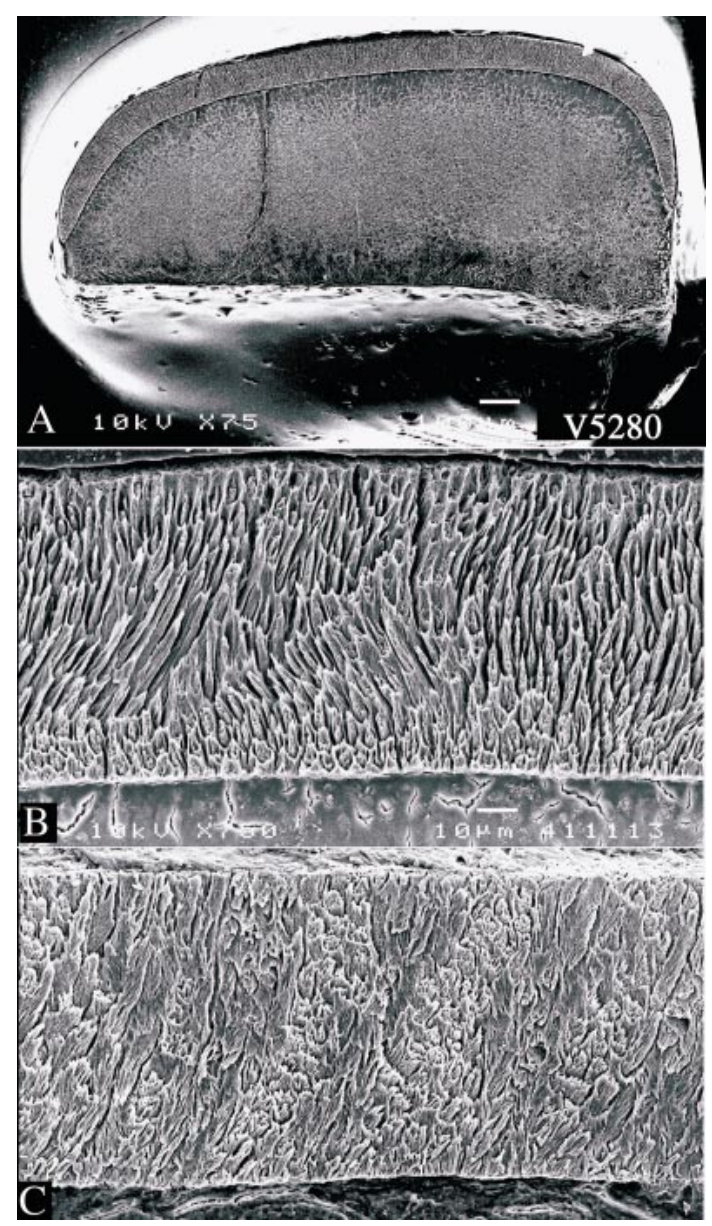

Fig. 18. Enamel distribution and microstructure of a lower incisor from a juvenile individual of Rhombomylus (IVPP V5280), in which deciduous premolars are present and germs of M3/m3 are completely embedded in bones. A, Distribution of the enamel on the labial surface of the incisor; B-C, cross and longitudinal sections showing microstructure of the enamel. See text for description and discussion.

Fig. 17. Enamel structures of the left lower incisor of Rhombomylus (IVPP V7489). A, C, E, Crosssectional views of the incisor enamel microstructure at segments of the same tooth shown in $\mathbf{G}$; $\mathbf{B}, \mathbf{D}$, $\mathbf{F}$, longitudinal-sectional views of the incisor enamel microstructure near a, c, and e in $\mathbf{G}$; $\mathbf{H}$, enamel distribution on the lower incisor. Note A and B are on the same scale, which differs from that of C-F. See text for description and discussion. 
a space, the prism sheath that was filled with proteoglycans in life. The inclination of prisms is roughly parallel to that of the crystallites of the interprismatic matrix. The enamel type is pauciserial.

Enamel microstructures taken from various segments of the same incisor do not show significant differences. This is true for both the upper and lower incisors. However, there exists a gradient of enamel thickness; that is, the enamel increases its thickness toward the posterior end of the tooth. This indicates that the enamel layer of the evergrowing incisor thickens with age.

In the young individual (IVPP V5280; fig. 18 ), in which deciduous teeth are present and germs of the M3 and m3 are not yet formed, the width of the lower incisor is only about half that in an adult. The enamel layer of this specimen is about $80 \mu \mathrm{m}$ thick, thinner than that of an adult individual. The enamel type, seen in both transverse and longitudinal sections, is similar to that of a mature individual. However, the outer layer in the juvenile lower incisor seems to be absent; therefore, the HSB seen in the longitudinal section extend from the enamel-dentine junction to the surface of the enamel.

Cheek Toотh EnAmel: Sectioned cheek teeth provide information on enamel distribution and its microstructures. Figure 19 shows the cross-sectional contours of enamel at three different levels of the P4-M3 from one individual and the $\mathrm{p} 4-\mathrm{m} 3$ from another. The expanding enamel contours from apical to basal sections indicate that the cheek teeth of Rhombomylus are narrower apically than basally. Similar to the incisor enamel, the cheek tooth enamel at a given position is slightly thinner apically than basally. The thickest enamel in upper cheek teeth is found around the protocone, measuring about 170 $\mu \mathrm{m}$, and the thinnest is between cusps on the occlusal surface, about $20 \mu \mathrm{m}$ (figs. 19A-C, 20). Among the upper cheek teeth, the enamel of the M1 is slightly thinner than it is on the P4 and M2-3. The enamel forming the anterior and medial edges of the upper cheek teeth is much thicker than that around the lateral and posterior borders of the teeth. This is in keeping with the fact that the anterior edge of the upper cheek teeth is the highest and widest region of the upper cheek teeth and functions as the main shearing edge.

For most of the lower cheek teeth, the enamel as seen in frontal crosssections forms two loops: one surrounds the trigonid and the other the talonid (fig. 19D-F). Similar to the upper cheek teeth, the enamel in the basined areas (trigonid and talonid) is very thin. The thickest enamel is around the posterior wall of the trigonid, whereas the thinnest is along the anterior edge of the trigonid and the posterior edge of the tooth (with the exception of the m3). The enamel may be completely absent at the central portion of the anterior edge of the trigonid, where cement fills the space between teeth. The posterior wall of the trigonid in a lower cheek tooth is the main cutting edge, which shears across the anterior edge of an upper molar during occlusion. When evenly grinding down the teeth of a dentition, the $\mathrm{m} 1$ is the first one of the cheek teeth to be worn off, suggesting the same sequence in life. Its thin enamel also indicates that the first molar was formed earlier than the last premolar and other molars. Unlike the upper cheek teeth, the enamel layers on the lateral and medial sides of the lower cheek teeth are almost equal in thickness.

SEM images (fig. 20) show that the cheek tooth enamel has more complex patterns of microstructure than does the incisor, and that the microstructure corresponds to the distribution of the enamel and probably to the functions of the tooth as well. Because the microstructure in the upper and lower cheek teeth is similar, we illustrate only that of the upper cheek teeth (fig. 20). The specific areas where the microstructures are examined are indicated in figure 19A, B. In general, the most complex microstructure is found in the thickest enamel along the primary shearing edges (fig. 20A, B). In this area the HunterSchreger bands are formed and the prisms with flat cross sections are densely compacted. In the occlusal section (fig. 20A), which is equivalent to the cross section of the incisor enamel, there is no distinctive pattern of prisms, but it can be seen that the prisms are surrounded by interprismatic matrix. In the longitudinal section (fig. 20B), the HSB are best developed in the middle portion of the enamel layer. Therefore, the enamel in 

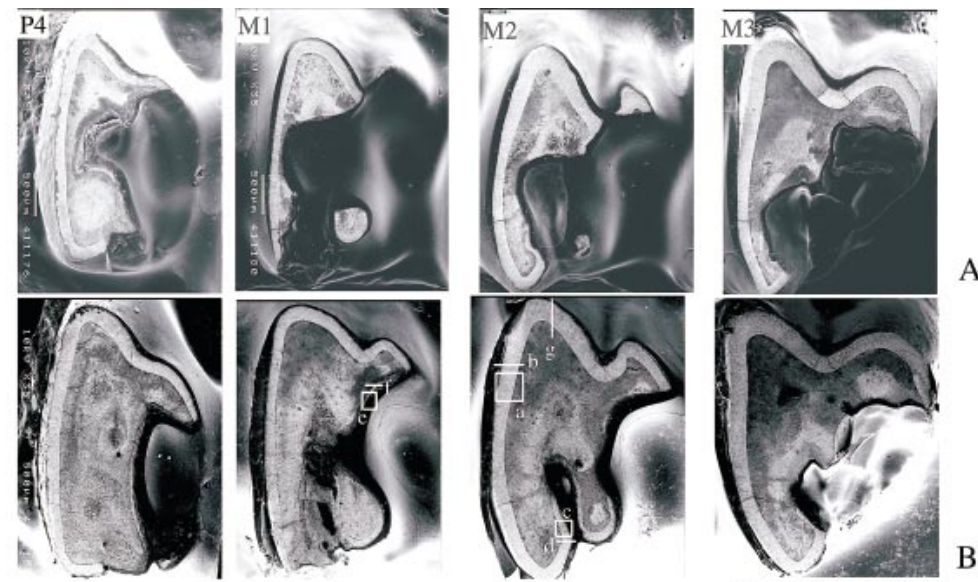

A
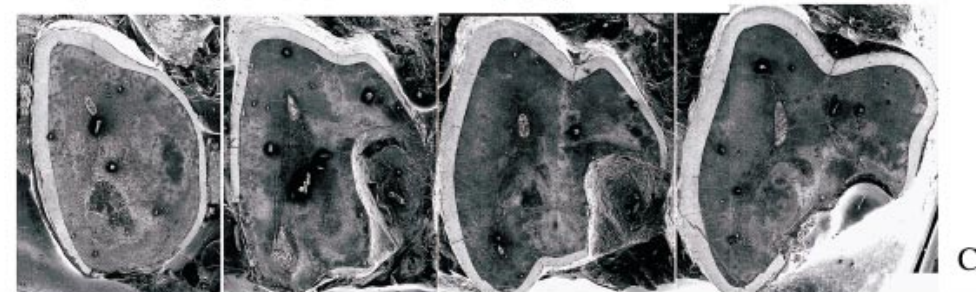

B
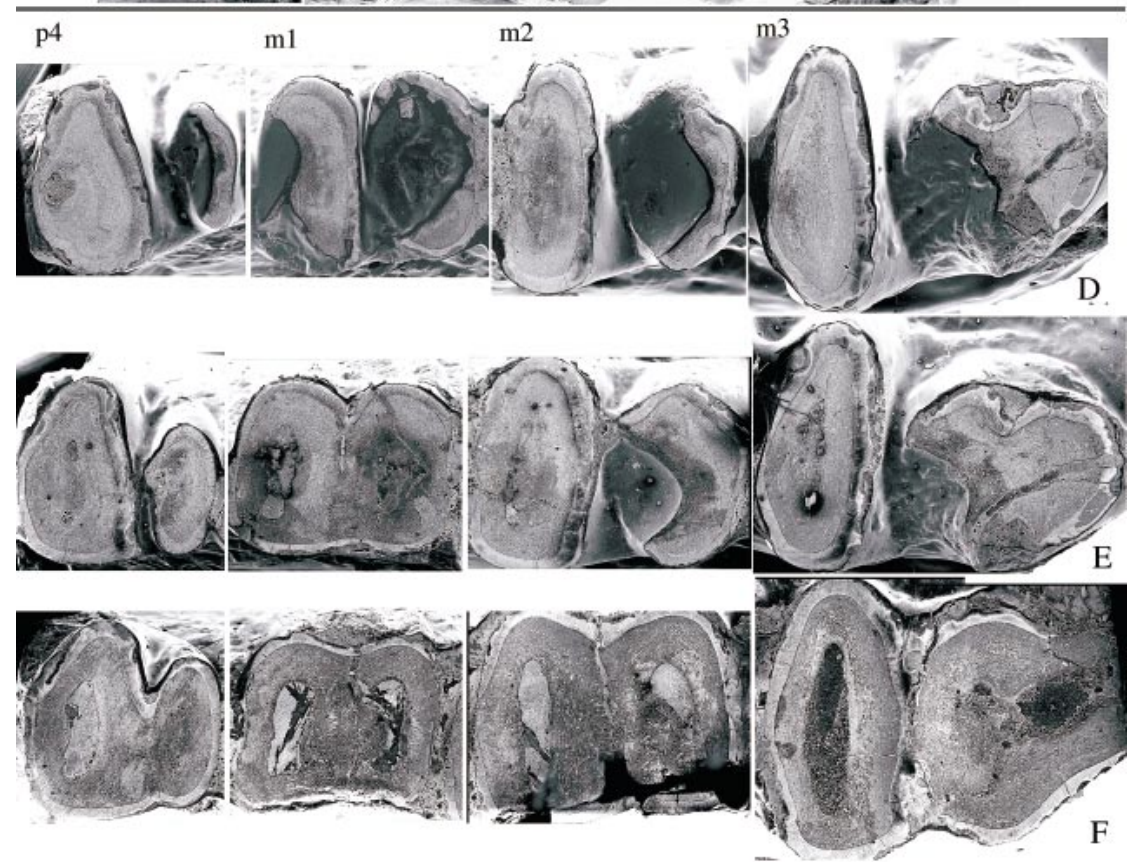

Fig. 19. Enamel distributions on cheek teeth of Rhombomylus. A-C, Enamel distributions in occlusal sections at three heights ranging from near the apex to the base of the left P4-M3 (IVPP V7490). DF, Enamel distributions in occlusal sections at three heights ranging from near the apex to the base of the right $\mathrm{p} 4-\mathrm{m} 3$ (IVPP V7491). The enamel microstructure of the areas labeled in B are illustrated in figure 20. All images are on the same scale. See text for description and discussion. 


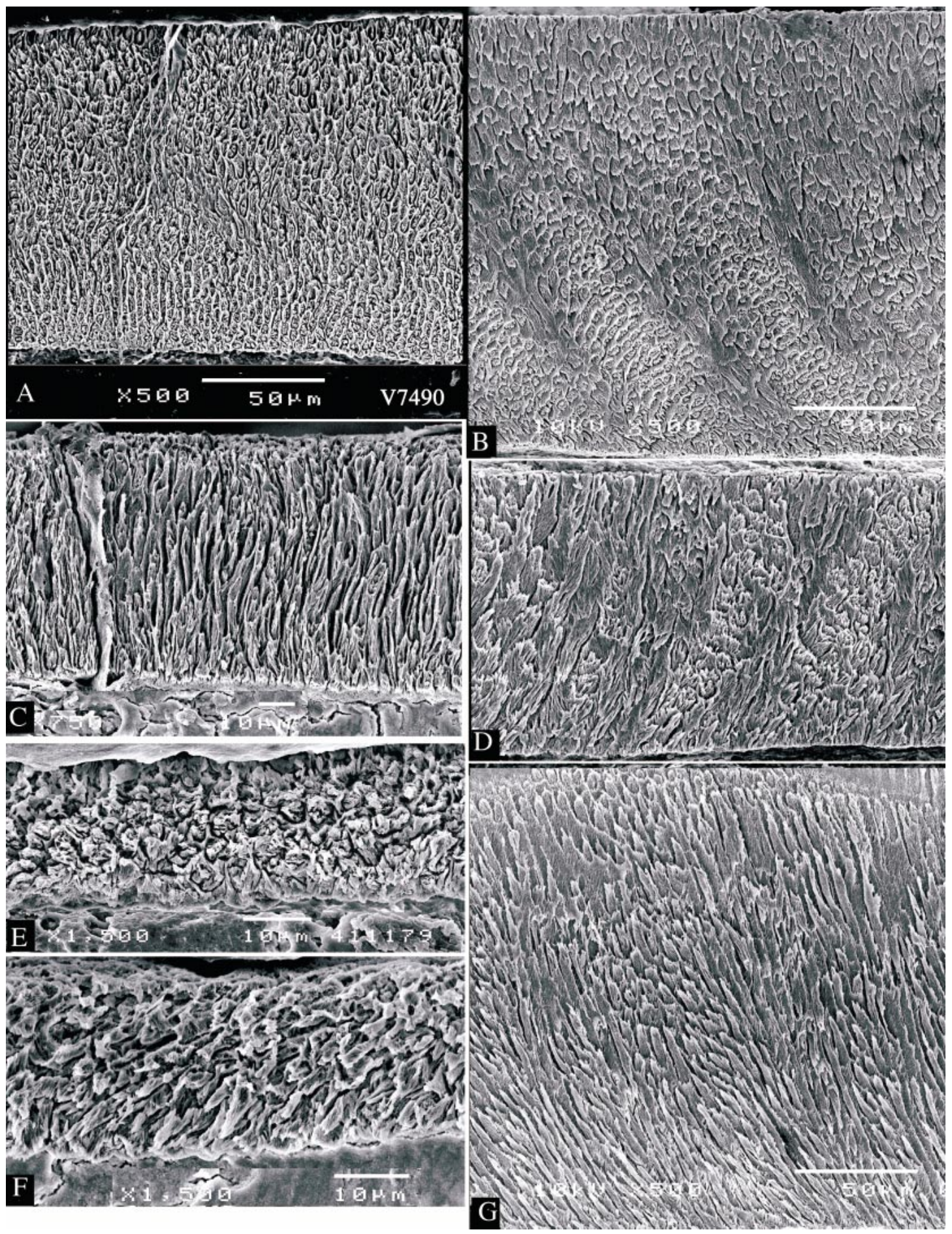

Fig. 20. Enamel microstructures of left upper molars of Rhombomylus (IVPP V7490), corresponding to sections labeled in figure 19. See text for description and discussion. 
this area is differentiated into three layers. With this multilayered structure, cracks parallel to the cutting edge are probably prevented when the cutting edge of the tooth is under pressure during food processing. In the transverse section (fig. 20G), the parallel prisms from HSB are revealed. The architecture of the enamel in this region appears to be weak, but it seems suitable for a region that receives compressional pressure during occlusion. The microstructure of the enamel posterior to the paracone is less complex than that of the anterior cutting edge. It lacks the outer layer and its HSB are similar to those of the incisor (fig. 20D). The enamel on the occlusal surface is the thinnest and weakest (fig. 20E, F) and lacks HSB.

DISCUSSION: In studying incisor enamel microstructure of gliroid mammals, samples are usually taken from segments at various positions along an incisor, depending on the preservation of the tooth. To make a meaningful comparison between species, one has to hypothesize that enamel samples taken at different segments of an incisor do not differ significantly in their microstructures. Our investigations of the upper and lower incisors show that the general enamel microstructure is the same wherever the enamel sample is taken from the same tooth. This observation may be generalized for other gliroid mammals. When incisor enamel microstructures of gliroid species are compared, the sampling location should not be a problem.

Previously it has been unclear whether the enamel microstructures change at different stages of ontogeny in a species. Koenigswald et al. (1993: 304) stated that "After mineralization in the depth of the jaw, enamel cannot be remodeled. This is a fundamental difference from bone. Therefore, biomechanical adaptations in enamel must first be established in the genome as a result of natural selection of previous generations and cannot be regarded as an immediate ontogenetic reaction to actual needs." By comparing the enamel from individuals of different ages of Rhombomylus, we may extend the conclusion of Koenigswald et al. to basal gliroid mammals. However, for the ever-growing incisor in gliroid species, such as Rhombomylus, the thickness of the enamel gradually increases during the life of an individual. Also, as shown in Rhombomylus, the outer layer is not developed in the incisor enamel of young individuals. The radial enamel of the outer layer helps to shape a sharp cutting edge, because it is slightly more resistant to wear than the inner layer and the dentine (Rensberger and Koenigswald, 1980; Fortelius, 1985). Thus, development or modification of the outer layer with radial enamel may be of some functional need that is necessary for adult individuals. It should be cautioned that age may be a factor affecting the morphology of the enamel microstructures in gliroid mammals.

It has been considered that the incisor of gliroid mammals is such a specialized structure and that rodent incisor enamel evolves independently of other dental traits (Koenigswald, 1988; Koenigswald and Clemens, 1992) so that the conservative form and universal function make the incisor enamel appropriate for phylogenetic study (Martin, 1993). For Rhombomylus and Tribosphenomys, in which both incisor and molar enamel microstructures are known, there is no clear evidence that the complex microstructures of the HSB is developed earlier in incisors than in molars and that incisor enamel has evolved independently of enamel of cheek teeth among gliroid mammals.

\section{Dental Wear}

Wear facets on teeth result primarily from contact between the lower and upper teeth during food processing and occlusion. Because of the uneven topology of the tooth crown, the contacts between the teeth develop at various places. For each contact area, a flat, smooth facet is generated on both upper and lower teeth (Butler, 1952). The facets are normally striated and pitted owing to ingestion of grit harder than the enamel, as part of, or as an accessory to, food (Rensberger, 1978). These corresponding facets between occlusal pairs of upper and lower teeth can be homologized over a variety of mammals (Butler, 1952; Crompton, 1971; Crompton and Hiiemae, 1970). Dental wear facets and microwear can be used to infer activities that caused them. These inferences fall mainly into two areas: the mechanisms related to chewing, such as chewing direction and type 
(e.g., compression vs. shearing) (Butler, 1952, 1972, 1985; Crompton and Hiiemae, 1970; Kay and Hiiemae, 1974; Hiiemae, 1978; Rensberger, 1978; Janis, 1990), and diet (what kind of food the teeth processed) (Lucas, 1979; Janis, 1995; Walker et al., 1978; Rensberger, 1978, 2000; Solounias et al., 1988; Solounias and Hayek, 1993; Hunter and Fortelius, 1994; Teaford and Walker, 1984; Grine, 1981; Grine and Kay, 1988). Most of these studies, however, are focused on primitive mammals, primates, and large ungulates. Although considerable studies of microwear for extant and fossil rodents have been conducted (Rensberger, 1975, 1978, 1982; Butler, 1980, 1985; Voss, 1988), little has been done on early eurymylids and mimotonids. This is probably because specimens of these species suitable for SEM study are limited. In this regard, the many specimens of Rhombomylus provide an opportunity to explore tooth wear in a basal gliroid.

DesCription: As described above, the cheek teeth of Rhombomylus differ most remarkably from those of other gliroid mammals in having an expanded hypocone shelf. In occlusion, the trigonid of a lower cheek tooth rests entirely in the hypocone shelf of the anterior upper cheek tooth, while the talonid of the lower tooth receives the trigon of its upper counterpart. Because the upper cheek teeth incline posteriorly and the lower ones lean anteriorly, the shearing surfaces of the teeth are slightly inclined. The primary shearing facets are formed between the anterior surface of an upper cheek tooth and the posterior surface of the trigonid of the corresponding lower cheek tooth. These surfaces are the widest area in both upper and lower teeth, and the wall bearing the shearing surface has the thickest enamel (see above).

On the upper cheek teeth (fig. 21), the primary shearing facet extends from the protocone to paracone, forming the anterior edge of the tooth. This shearing facet in an upper molar (e.g., M2) is caused by contact with the posterior wall of the trigonid of the corresponding lower molar (e.g., m2). It is transversely long and curved (in both occlusal and anterior views). Because of lack of the anterior cingulum and different shapes of crests and cusps, it is difficult to homologize the wear facets of Rhombomylus with those of rodents (Butler, 1980, 1985). However, by position and contact with the opposing lower molar, the primary shearing facet in Rhombomylus is probably a combination of facets 2 and 3 denoted to facets anterior to the paracone and protocone, respectively, in primitive rodents (Butler, 1980, 1985). The depth of the primary shearing facet varies from individual to individual. In general, an old individual tends to have a deeper facet than does a juvenile. In most cases, the facet is extensive, ranging from one-third to half the height of the anterior wall of the tooth.

The primary shearing facet is decorated with numerous fine pits and striations of different widths (fig. 21F). These structures most likely result from small inorganic particles, such as detrital grains in the soil (Rensberger, 1978), or by phytoliths (Walker et al., 1978), which have been drawn across the surface of the enamel under pressure sufficient to cause grooves. For grooves to be produced, the particles must be harder than the enamel (or dentinal) surface, and the pressure not so great as to cause them to be crushed. Occurrences of very short striae and pits may be the result of crushing of the particle. As shown in figure $21 \mathrm{~F}$, the striations can be as long as $200 \mu \mathrm{m}$. These striations are roughly parallel and are slightly curved. It appears that near the edge of the primary shearing facet, the striations are more vertically oriented, whereas along the basal area of the facet they become more horizontal.

Striations are common on the enamel at the lingual side and crown surface of the protocone (fig. 21E). On the occlusal surface, the harder enamel ridge is higher than the softer dentine. However, the enamel ridge is not a flat facet; instead, it is convex. The enamel near the dentine (on the right side of the line in fig. 21E) bears more pits than striations. The enamel on the outer side of the enamel ridge has numerous fine, parallel striae. The striae show a diagonal direction and are similar to the pattern that was referred to as furrows by Rensberger (1978). Furrows are parallel fabriclike grooves in which the diameters and spacing are uniform. The parallel grooves found on the enamel of Rhombomylus are not as distinct as those in rodents (e.g., Microtus in Rensberger, 1978).

Striations and pits also occur in elevated 

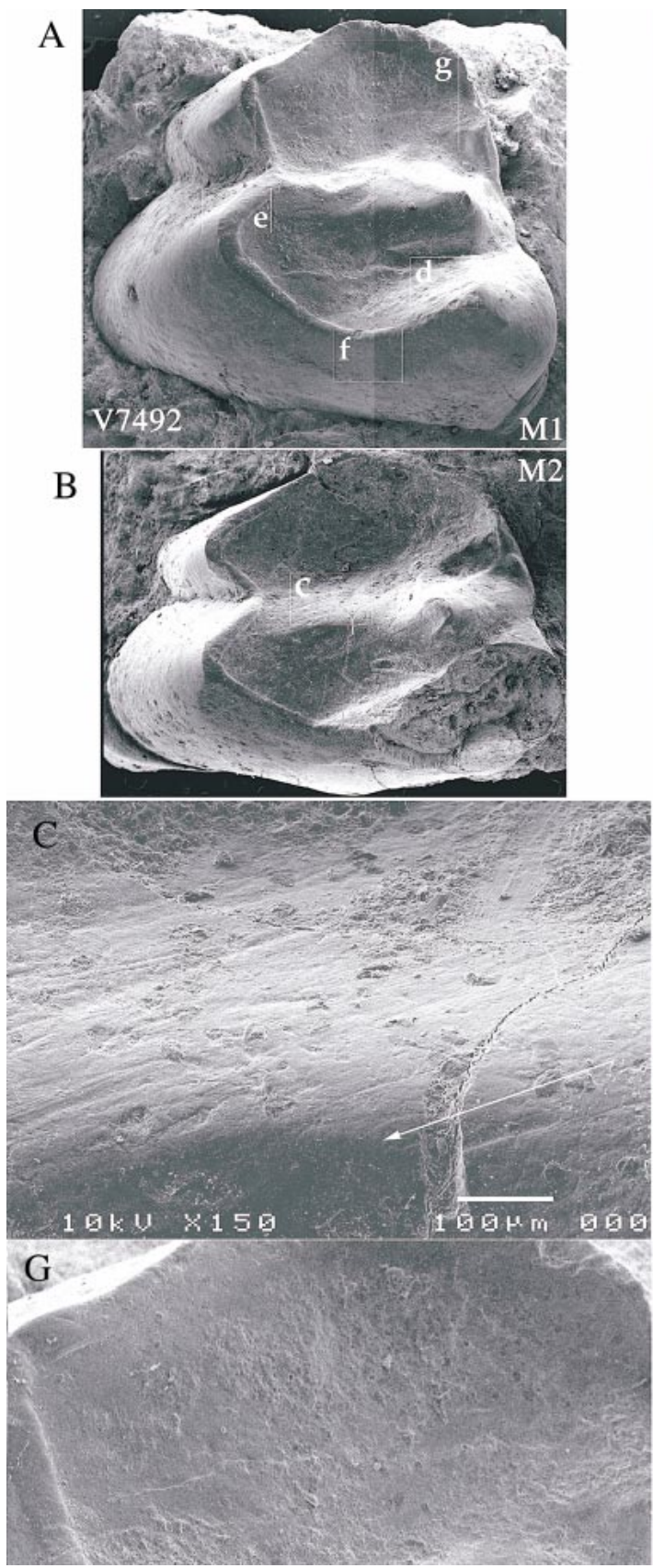
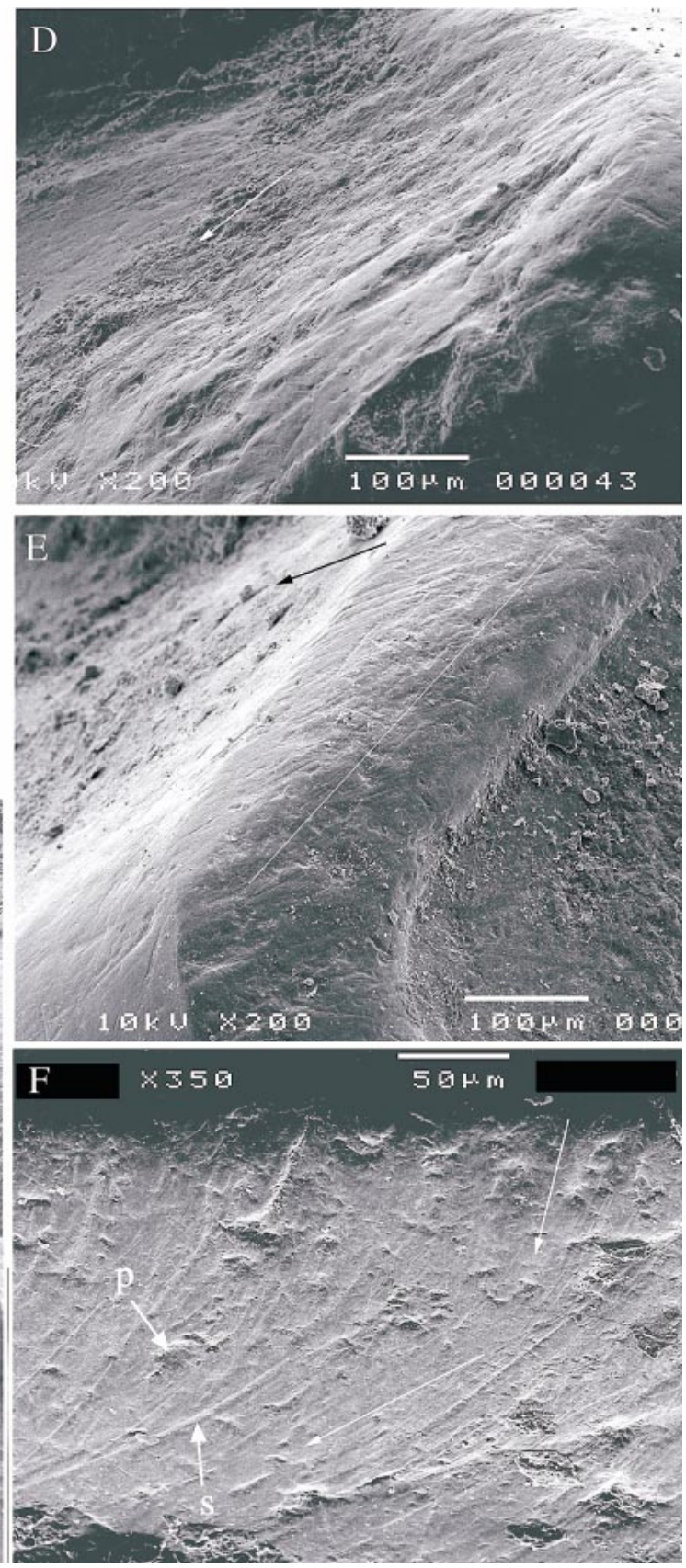

Fig. 21. A-B, Right M2 and M1 of Rhombomylus (IVPP V7492, from the same specimen that has been cut to view the longitudinal and frontal sections of the nasal cavity in figs. 43 and 44). The scanning electronic microscopic images $(\mathbf{C}-\mathbf{G})$ are close-up views of the areas labeled in A and B. All the figures are oriented in the same direction but are not on the same scale. Arrows indicate directions of strikes or movement of the lower teeth. p, pit; s, striation. See text for description and discussion. 

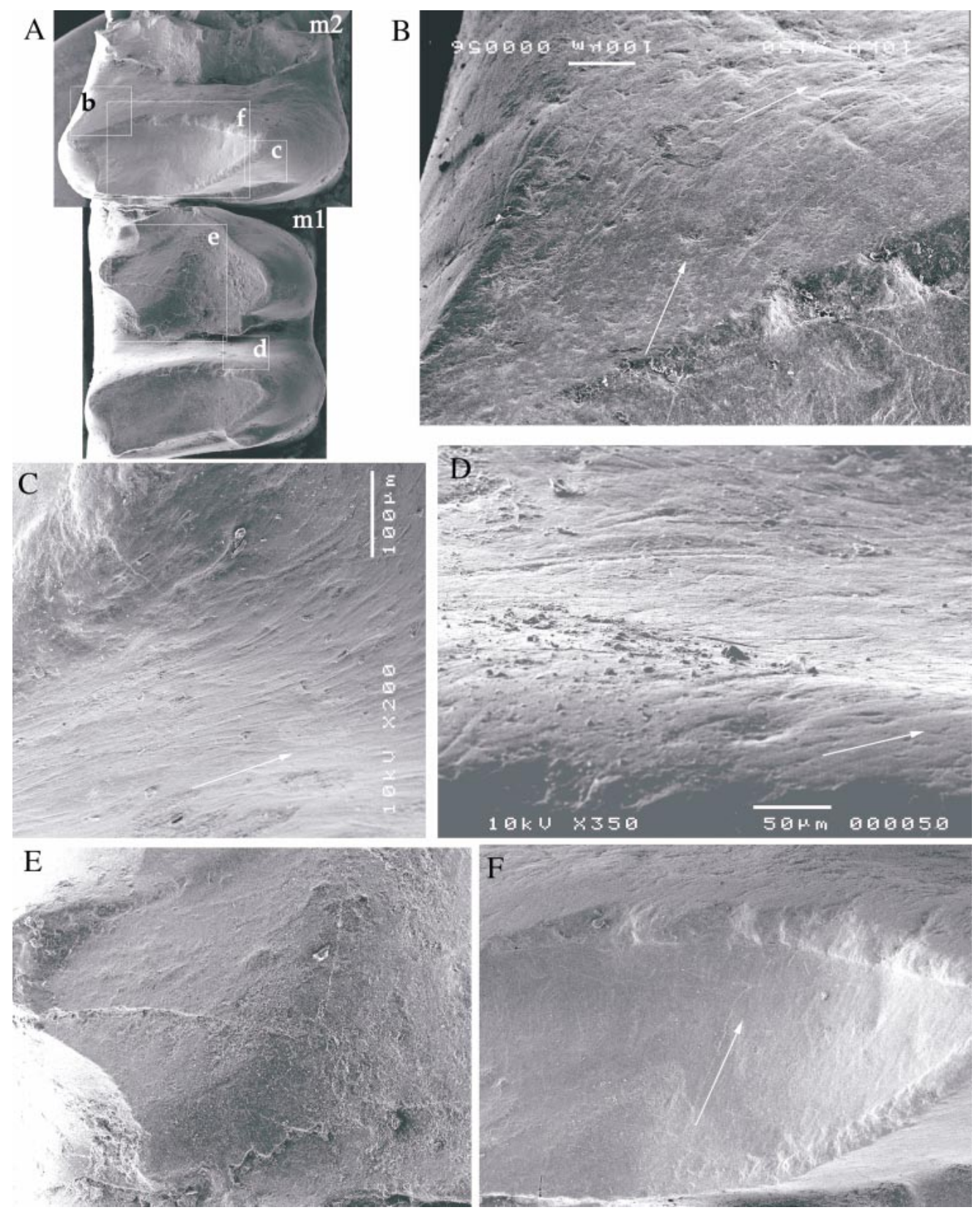

F

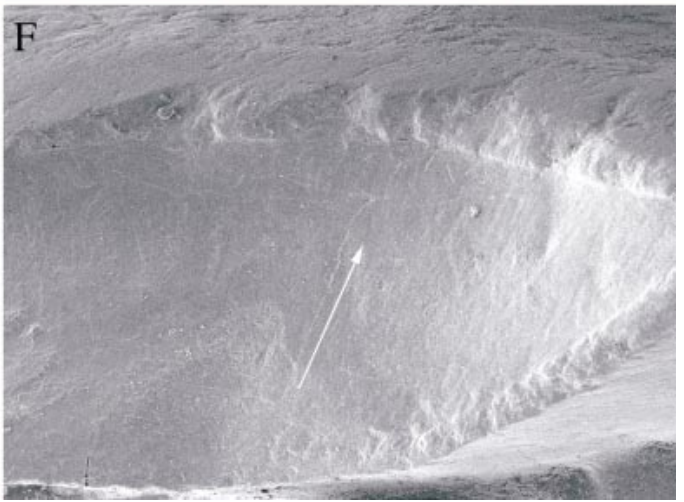

Fig. 22. A, Left $\mathrm{m} 1$ and the trigonid of $\mathrm{m} 2$ from a fragmentary mandible of Rhombomylus (IVPP V7493). The scanning electronic microscopic images (B-F) are close-up views of the areas labeled in A. All the figures are oriented in the same direction but are not on the same scale. Arrows indicate directions of strikes or movement of the teeth.

dentinal areas, such as the posterior side of the paracone (fig. 21D) and the metaloph (fig. 21C), where enamel is totally absent. On the posterior side of the paracone, the cusp is coarsely pitted and gouged. The coarse striae have the same direction of those seen in other areas of the tooth. The pits are denser and larger near the tip of the cusp than in 
other areas of the tooth. At the base of the paracone, or near the valley of the trigon basin (around the arrow in fig. 21D), the striations are finer. The surface of the metaloph is gently convex and bears fine pits and striae (fig. 21C). In addition to these structures, this area also shows the feature of polish, which probably results from abrasion by very fine and soft food material. Therefore, the striations on the dentine surface were probably not produced by tooth contact, differing from those on the primary shearing facet formed by enamel. The basined areas of the tooth are dentinal, such as the area labial to the enamel ridge (fig. 21E) and the hypocone shelf (fig. $21 \mathrm{G})$, where striations are lacking; nothing directional is observed. These regions have probably undergone polish and abrasion during food processing, but not punctuation from the opposing tooth during occlusion.

The primary shearing facet of the lower cheek teeth is on the posterior wall of the trigonid, extending from the protoconid and metaconid (fig. 22A, B). The facet bears pits and striations similar to those on upper cheek teeth in shape and density. Also similar to upper cheek teeth, the pits and striations on the cusp enamel are coarser apically than basally; striae are primarily transverse in direction; and the dentinal basins are somewhat polished and lack directional structures (fig. 22C-E).

See functional analysis of mastication for discussion.

\section{CRANIAL ELEMENTS}

General Shape of SkUll: The skull shape of Rhombomylus is similar to that of a nonspecialized rodent, such as Paramys (Wood, 1962) (figs. 23-28). Its outline is oval in dorsal and ventral views, with the rostrum tapering anteriorly. The narrowest part of the skull is behind the postorbital bar in dorsal view, posterior to M3 in ventral view. The widest part of the skull (counting the zygomatic arches) is across the middle level of zygomatic arches. On the skull roof there is usually an exposure of the mastoid between the squamosal and parietal. The surface of the mastoid exposure is rough and its area increases with age. The occipital region has a well-defined lambdoidal crest. In ventral view, the most prominent features of the Rhombomylus skull are a complete bulla that encloses the middle tympanic cavity and the expanded mastoid region between the basioccipital and squamosal. In lateral view, the skull of Rhombomylus is also rodentlike in having a distinct upper diastema that is significantly longer than its counterpart in the lower jaw. Another characteristic region of Rhombomylus is the strong and complex zygoma.

NASAL: The nasals form the roof of the nasal cavity; their anterior tips overhang the external nares. The two nasals are nearly rectangular, with anterior ends slightly flaring out; therefore, most of the nasal-premaxillary sutures are parallel (figs. 23, 26, 29). In dorsal view, the nasals extend posteriorly to the level of P3 and contact the frontals in a smooth, oblique suture. The posterolateral corner of each nasal extends more posteriorly than the median portion. The internal morphology of the nasal and nasal cavity can be assessed from cross sections (see Cross Sections). In lateral view, the nasals define a gentle arch along the dorsal margin of the rostrum (figs. 25, 28).

PREMAXILla: The premaxilla accommodates most of the upper incisor (the posterior end of the incisor extends into the maxilla) and constitutes the ventral and lateral walls of the nasal cavity (figs. 23-30). In anterior view, the external nares is roughly a triangular opening, with the premaxillae forming the two sides. In lateral view the deep premaxilla makes up most of the rostrum anterior to the infraorbital foramen in adult individuals (figs. 25, 28), as in other gliroid mammals. In juvenile individuals the rostrum and the premaxilla are proportionally shorter. This suggests that the elongation of the rostrum during postnatal ontogeny of Rhombomylus involves mostly lengthening of the premaxilla. The suture between the premaxilla and maxilla is simple and straight in young individuals (IVPP V7585; fig. 28, B) and gradually becomes complicated with aging. In the oldest individual (IVPP V5278; fig. 28F) it is interdigitated. As a typical gliroid feature, the posterior process of the premaxilla is well developed. It is as broad as the nasal and extends more posteriorly than the latter on the skull roof. The premaxilla- 


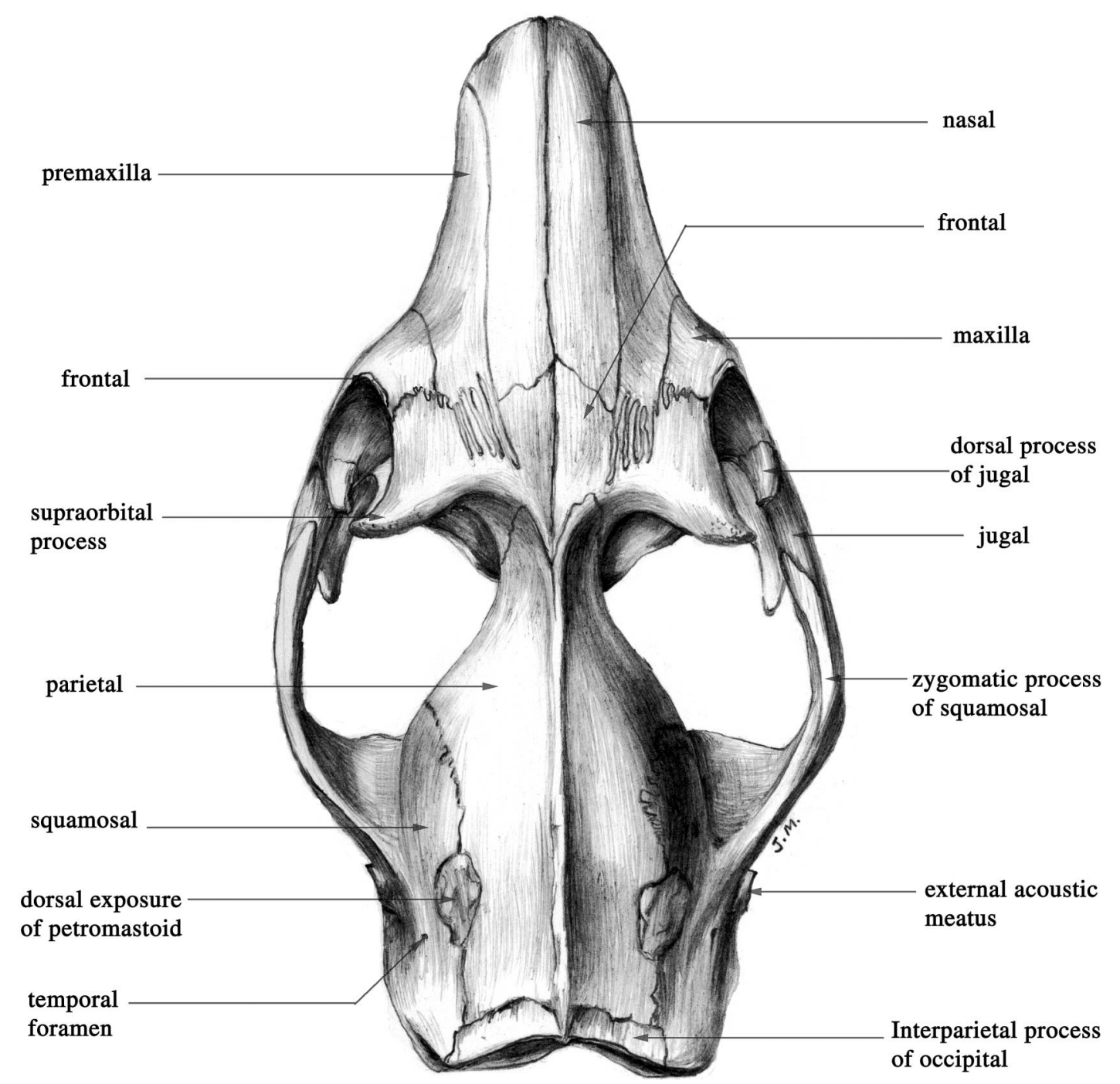

Fig. 23. Dorsal view of the skull of Rhombomylus. Reconstruction is based mainly on an adult skull (IVPP V5278).

frontal contact is complex, with small projections of the premaxilla extending posteriorly beyond the midpoint of the orbit, interdigtating with similarly narrow anterior projections of the frontal.

Ventrally the premaxilla constitutes the anterior half of the rostrum between the incisor and P3 (figs. 24, 27, 30) and contains the anterior portion of the incisive foramen. Lateral to the incisive foramen, the premaxilla commonly sends a small, pointed process to invade the palatal process of the maxilla that encloses the incisive foramen posteriorly (fig. 8). The incisive foramen is elongate and posteriorly positioned, a common condition seen in gliroid mammals. Its size is moderate compared to many rodents and all lagomorphs. The absolute size of the foramen and its distance from the incisor increase with age (fig. 30). The two incisive foramina are separated by the palatine processes (prevomerine processes) of the premaxillae, which form the entire medial division of the foramina and suture with the maxillae at the posterior edges of the incisive foramina. Within the nasal cavity, the palatine process 


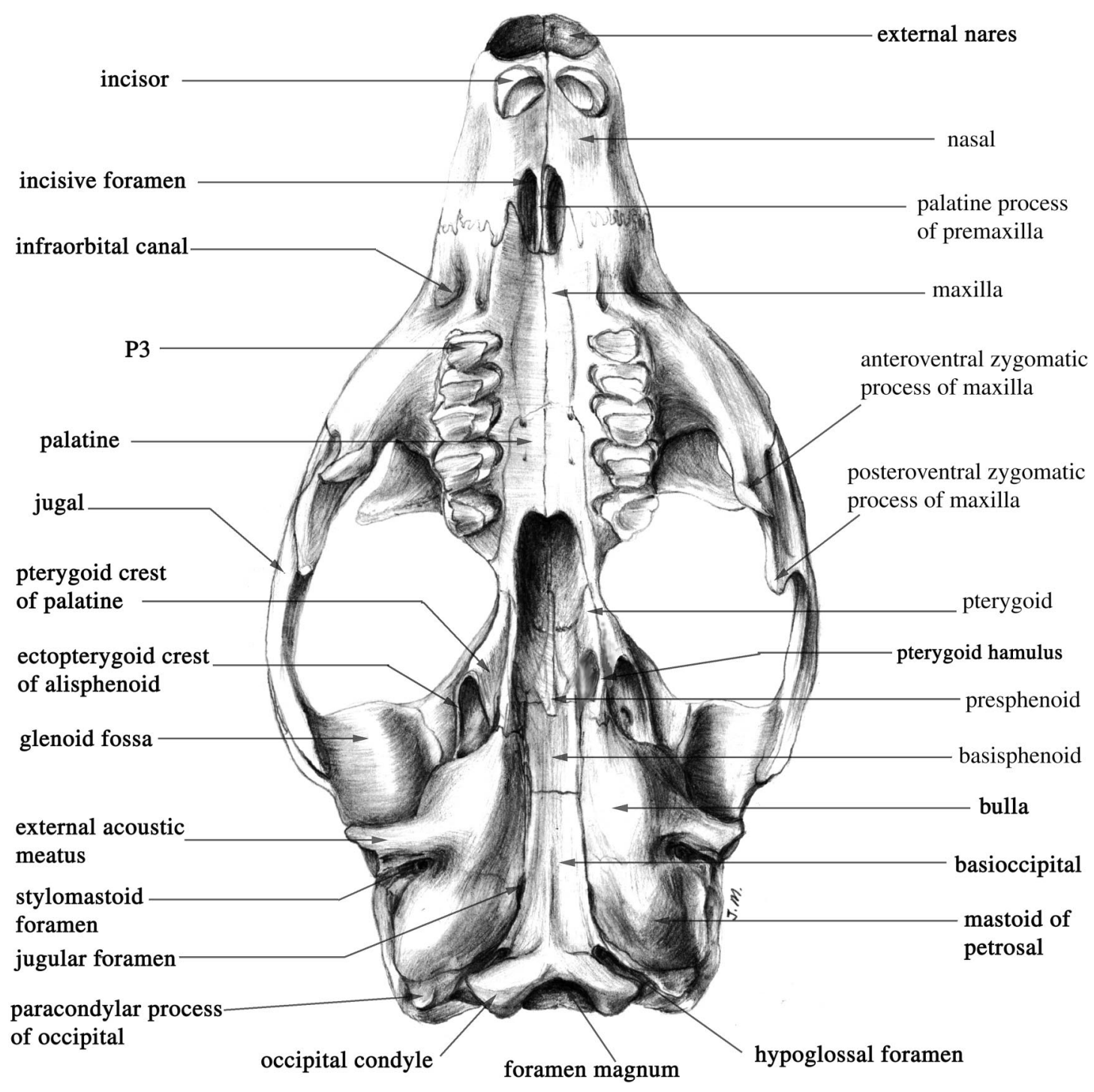

Fig. 24. Ventral view of the skull of Rhombomylus. Reconstruction is based mainly on an adult skull (IVPP V5278).

of the premaxilla forms an elongated fossa that in life supports the Jacobson's organ (see Cross Sections).

MAXILLA: The maxilla is a complex bone that contributes to the anterior and ventral edges of the orbit, forms the middle part of the palate, provides the posterior sidewall for the nasal cavity, accommodates the infraorbital canal and all cheek teeth, and bears a strong zygomatic process (figs. 23-32). On the facial region, the maxilla is solid, show- ing no fenestration. It contacts the premaxilla medially and the frontal and lacrimal posteriorly. The infraorbital canal is low in position and is wider than high (figs. 25, 28). The anterior opening of the canal, the infraorbital foramen, varies in size with age. In the oldest individual (IVPP V5278) it measures $3.8 \mathrm{~mm}$ in width and $2.4 \mathrm{~mm}$ in height, whereas in the youngest (IVPP V7585) it is $1.2 \times 1 \mathrm{~mm}$. With the exception of a faint groove leading anteriorly from the infraorbital foramen, 


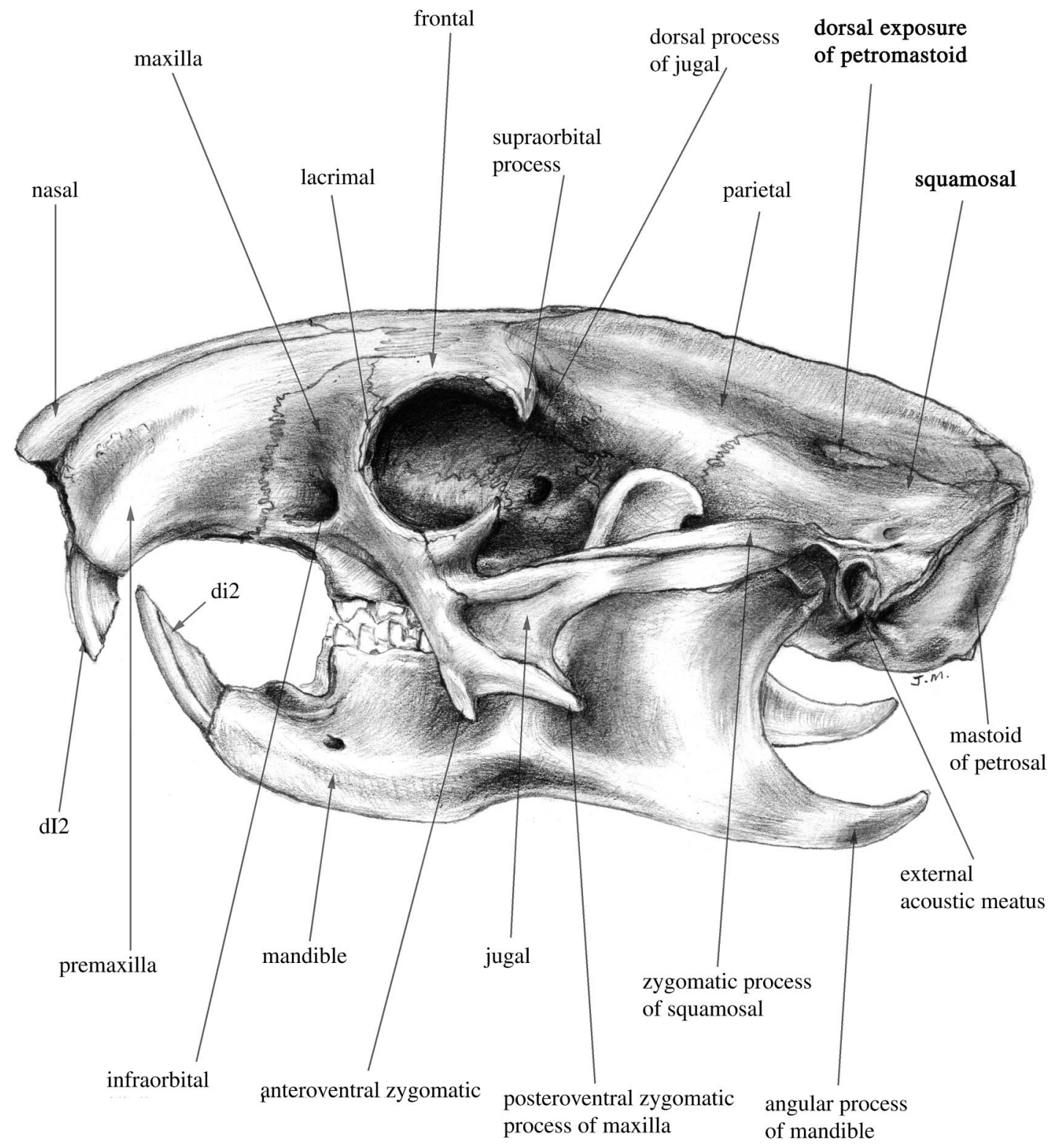

Fig. 25. Lateral view of the skull of Rhombomylus. Reconstruction is based mainly on an adult skull (IVPP V5278).

there is no scar or depression on the ventral and lateral sides of the foramen for attachment of jaw muscle. The maxilla forms the anteroventral edge of a well-defined orbit and separates the lacrimal and jugal. The zygomatic process of the maxilla is strong and complex (figs. 25, 28, 32). It descends posteroventrally to a level lower than the lower tooth row of the mandible in occlusal position and is posterodorsally overlapped by the jugal. Distally the maxillary process branches into anteroventral and posteroventral processes (fig. 32).

On the ventral side of the skull the palatal process of the maxilla is the largest component of the hard palate (figs. 24, 27). It en- 
closes the posterior half of the incisive foramen and ends immediately posterior to the M3. Anterior to the P4 there is a narrow anteroposteriorly oriented depression of unknown function. This depression appears to be too medially positioned to serve as an attachment site for jaw muscle. The anterior portion of the maxilla-palatine suture is opposite the M1, but in young individuals it is more anteriorly located at the level between the dp3 and dp4 as in IVPP V5280 (fig. $30 \mathrm{E})$. The lateral portion of the maxilla-palatine suture is straight and is medial to the molars. The palate is narrow. The tooth rows of the two sides are parallel. In ventral view the palatal process of the maxilla is gently concave. On each process, a narrow groove leads from a small anterior palatal foramen located near the maxilla-palatine suture and extends anteriorly in a curved course toward the incisive foramen. The posterior edge of the maxillary process of the zygoma is lateral to the M1.

The maxilla is a major element within the orbit, forming the floor and contributing to the medial wall (fig. 31). The orbital floor is large and flat, with roots of cheek teeth commonly exposed. In young individuals, the M3 germ is also visible in the maxillary floor of the orbit. The maxilla contributes to the anterolateral wall of the lacrimal foramen. The posterior opening of the infraorbital canal is roofed by a thin bony lamina of the maxilla, which separates the canal from the lacrimal. Because of this bony lamina, the infraorbital canal extends a little more posteriorly than estimated externally. The posterior opening of the infraorbital canal is low and its floor is confluent with that of the orbit. The orbital process of the maxilla is broad and contacts the lacrimal anteriorly, frontal dorsally, and orbitosphenoid and palatine posteriorly. It prevents the contact between the palatine and the lacrimal and between the palatine and the frontal. At the junction with the palatine, there is a slitlike space, which is identified as the sphenopalatine foramen.

PAlAtine: The palatine constitutes the posterior part of the hard palate, most of the roof and lateral wall of the choanal orifice, and part of the orbital wall (figs. 24, 27, 31). The palatal process is tongue-shaped, in which there are two pairs of small palatine foramina distributed asymmetrically. The larger, anterior palatine foramina are opposite M1, whereas the posterior ones are medial to the M2. The posterior emargination of the palate is between the last molars and forms a narrow arc with a weak postpalatine spine projecting posteriorly at its midline. The posterior margin does not thicken ventrally to form the postpalatine torus. Neither a postpalatine foramen nor a notch exists at this area. As the pars perpendicularis of the palatine extends posteriorly, the choanal orifice gradually widens, particularly so in skulls of young individuals. The pars perpendicularis of the palatine contributes posteriorly as the pterygoid crest that forms the anteroventral portion of the ectopterygoid fossa (figs. 24, 33). The ectopterygoid fossa is usually defined by the ectopterygoid crest of the alisphenoid laterally and the entopterygoid crest of the pterygoid medially in rodents and other mammals.

The delimitation of the orbital process of the palatine is best seen in IVPP V5289. The ventral portion of the process is between the maxilla anterolaterally and the alisphenoid posteromedially, whereas the dorsal portion is between the maxilla anteroventrally and the orbitosphenoid dorsally. Its dorsal tip does not reach the level of the ventral edge of the optic foramen. As already mentioned, between the anterior portion of the palatine and maxilla is the slitlike sphenopalatine foramen.

LACRIMAL: The lacrimal is best preserved in IVPP V5289 (figs. 26, 31). It is a small bone with only a narrow strip exposed on the facial region. The body of the lacrimal forms the anterior wall of the orbit dorsal to the posterior opening of the infraorbital canal. Within the orbit the lacrimal is surrounded by the frontal medially and by the maxilla ventrally and laterally. A large lacrimal foramen notches the lateral margin of the bone and is closed laterally by the maxilla. There is no lacrimal tubercle.

Jugal: The zygomatic arch of Rhombomylus is highly distinct in possessing a complex central plate that descends considerably below the ventral limit of the expanded mastoid of the petrosal of the skull (figs. 25, 28, 32). The large jugal bone is the main, central part of the specialized zygomatic arch. Its an- 


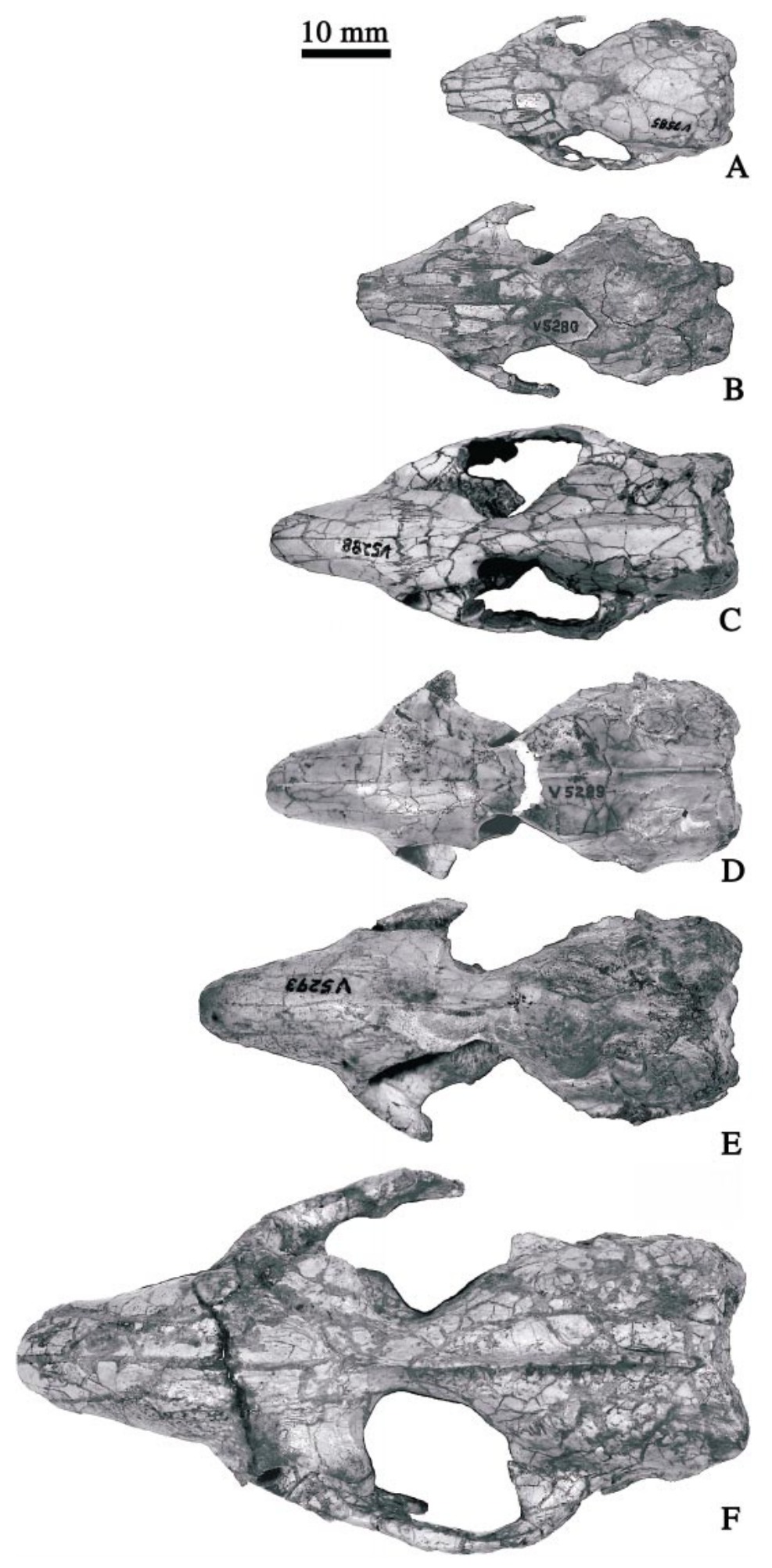


terior half overlaps the robust zygomatic process of the maxilla and its posterior half ventrally adjoins the zygomatic process of the squamosal. The jugal is transversely thinner than the zygomatic process of the maxilla and does not reach anteriorly to the lacrimal. On the anterior end of the jugal, a dorsal process projects posterodorsally to border the circular orbit, but does not meet the supraorbital process of the frontal; therefore, the orbit is open posteriorly. Posteroventral to the dorsal process of the jugal there is a smooth trough of unknown function, which is best seen in the skull of the oldest individual in our collection (IVPP V5278). The main body of the jugal contributes to the dorsal half of the central plate of the zygoma. The lateral surface of the plate is gently concave and is probably for attachment of the masseter muscle in life. The medial surface of the plate is smooth and gently convex. The central plate has two ventral processes, as has been mentioned in the description of the maxilla. The posterior one is longer than the anterior one and consists of two portions: the posteroventral process of the maxilla and the ventral process of the jugal. The posterior process of the jugal is the longest one and is dorsally overlapped by the zygomatic process of the squamosal; its posterior end forms the lateral wall of the glenoid fossa. The ventral edge of the posterior process is sharp, suggesting lack of attachment of muscle.

FRONTAL: Compared to the parietals and nasals that also form the roof of the skull, the frontals are short, but are more complex in shape (figs. 23, 26, 29). Anteriorly each frontal has a tricontact with the nasal, premaxilla, and maxilla, ranging from the medial to lateral, which is typical of gliroid mammals. The frontal-maxillary suture is restricted and is the narrowest of the three contacts. Medially the anterior end of the frontal forms a blunt wedge that intrudes between the nasals. The interdigtal frontal-premaxillary suture is more extensive in skulls of adult individuals. This condition differs from the long, narrow anterior process of the fron- tal that inserts between the premaxilla and maxilla in lagomorphs; it also differs from the simple suture in early rodents such as $\mathrm{Co}$ comys and Paramys. The frontal has a narrow connection with the lacrimal in the facial region. The supraorbital crest is a sharp, ridgelike crest forming the dorsal rim of the orbit. The supraorbital process is a strong posterolateral projection of the frontal that confines the orbit posterodorsally. Because of the projected crest and process, the orbital cavity is very deep. On the posterior side of the supraorbital process the frontal is a broad, concave surface that continues to the anterior portion of the parietal. Along the midline of the skull, the medial portions of the frontals converge posteriorly to form a spike inserting between the parietals; the tip of the spike marks the beginning of the sagittal crest and the narrowest section of the skull roof, which we call the postorbital constriction of the skull. The postorbital constriction delimits the region where the olfactory lobes are housed. Its width changes very little during postnatal ontogeny. As shown in figure 26, the skull representing the youngest individual (IVPP V7585) is about half the length of the oldest skull (IVPP V5278), but in both skulls, and those of intermediate size, the postorbital constriction is of similar width. Using this region as a reference point, it seems that during postnatal ontogeny of Rhombomylus, skull reshaping occurs primarily in the facial region and the braincase for the cerebral cortex.

The orbital process of the frontal is large and descends deeply to the level of the ventral rim of the optic foramen (fig. 31). It forms most of the dorsal midwall of the orbit. It meets with the lacrimal and maxilla anteriorly, the maxilla ventrally, and the orbitosphenoid and parietal posteriorly. The frontal-maxillary contact is the most extensive one. A small opening in the frontal anterodorsal to the optic foramen is probably the ethmoid foramen.

PARIETAL: The parietal is nearly half of the length of the skull, the longest bone in the

Fig. 26. Dorsal views of skulls of Rhombomylus. Skulls are in relative ages from the youngest to oldest (IVPP V7585, V5280, V5288, V5289, V5293, V5278). 

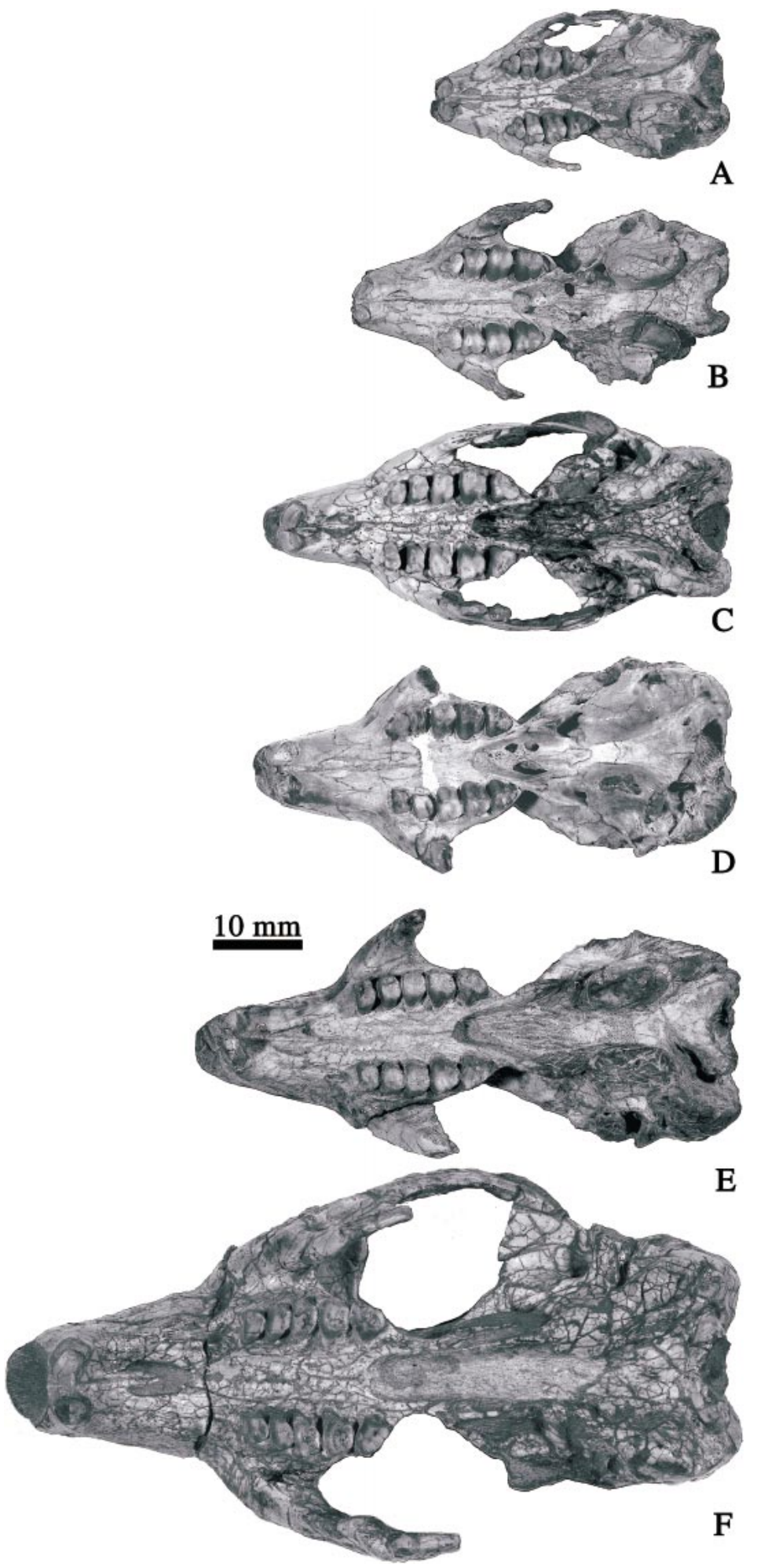
skull roof (figs. 23, 25, 26, 28), and roofs the entire cranial cavity. A sagittal crest extends along the full length of the parietals in adult skulls. As in several other features, the development of the sagittal crest is age dependent, ranging from none in very young (e.g., IVPP V7585; fig. 26A) to prominent in adult individuals (e.g., IVPP V5278; fig. 26F). The anterior borders of the parietals, at the narrowest region of the skull, are cleaved into two lobes by the posterior processes of the frontals. The broad, convex parietal spreads over the anterior and dorsal areas of the large temporal region. In the orbitotemporal region, the parietal sutures with the frontal, orbitosphenoid, alisphenoid, and squamosal. The ventralmost point of the parietal is at the triple junction with the alisphenoid and squamosal and marks the widest region of the parietal. From this point the parietal narrows posteriorly. The longest contact of the parietal is with the squamosal. An unusual feature on the roof of the skull is an exposure of the expanded mastoid of the petrosal between the parietal and the squamosal (figs. $23,25,26)$. The size of the exposure varies from specimen to specimen. The posterior edge of the parietal meets the interparietal process of the occipital, which forms the central lambdoidal crest. The parietal-squamosal suture does not reach the lambdoidal crest. Only a narrow process of the parietal, bearing the end of the sagittal crest, extends posteriorly to the edge of the lambdoidal crest; therefore, this projection bisects the interparietal process of the occipital.

SQuAmosal: The squamosal overlaps the petromastoid and parietal. It contributes to the sidewall of the cranial cavity, forms the glenoid fossa, and bears a long zygomatic process (figs. 23-28). In dorsal view, a temporal foramen is posterior to the root of the zygomatic arch. The base of the zygomatic process is a broad bony plate with a flat dorsal surface that is bounded with a low ridge laterally. The ventral side of the plate is the glenoid fossa. The temporal plate of the squamosal is convex and constitutes the ven- tral area of the temporal region and part of the lateral wall of the braincase. The zygomatic process of the squamosal is slender and stretches over the posterior half of the zygomatic arch. It overlaps the jugal in a beveling fashion so that the zygomatic process of the squamosal appears narrower laterally than medially; the reverse is true for the jugal.

In lateral view, the squamosal tightly surrounds the dorsal side of the auditory meatus of the ectotympanic; a distinct suture shows that it is not fused with the ectotympanic. The squamosal does not contribute to the lateral wall of the epitympanic recess in the auditory region. This condition is further confirmed by sectioned specimens (see below). Posterior to the auditory meatus, a triangular process of the squamosal wedges between the meatus and the expanded mastoid (figs. $31,34)$.

On the ventral side of the skull the squamosal contacts the alisphenoid and ectotympanic bulla, and posteroventrally with the mastoid of the petrosal (figs. 24, 33, 34). The glenoid fossa is broad, gently concave, and anteroposteriorly elongate. This architecture allows anteroposterior movement of the lower jaw as well as some degree of lateral shifting of the mandibular condyle during mastication. The jugal borders the fossa laterally. A triangular lip of the squamosal forms most of the medial wall of the glenoid fossa, whereas the ectotympanic contributes to the posterior part of the medial wall of the fossa. As in rodents, when the cheek teeth are in occlusion, the mandibular condyle is at the midpoint of the glenoid fossa. When the lower incisors are engaged with the uppers, the condyle is shifted to the anterior part of the fossa. When the condyle is shifted posteriorly against the external auditory meatus, the $\mathrm{m} 1$ trigonid will be against the M1 trigon so that full occlusion is not possible. The posterior end of the glenoid fossa is a socket, in which there is a foramen. The foramen is particularly large in IVPP V5263 and V5263. Although it appears to be unusual, the best

$\leftarrow$

Fig. 27. Ventral views of skulls of Rhombomylus. Skulls are in relative ages from the youngest to oldest (IVPP V7585, V5280, V5288, V5289, V5293, V5278). 

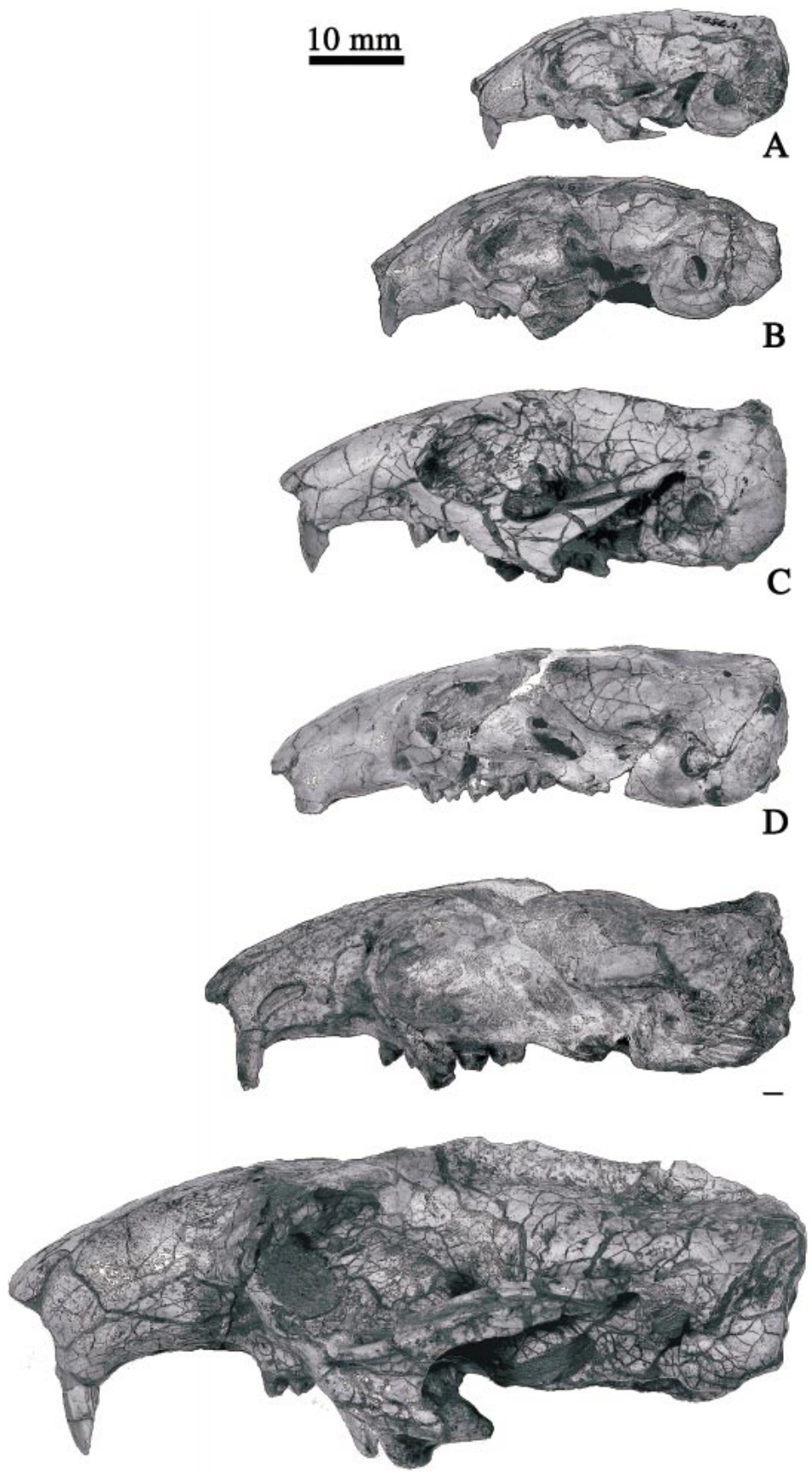

Fig. 28. Lateral views of skulls of Rhombomylus. Skulls are in relative ages from the youngest to oldest (IVPP V7585, V5280, V5288, V5289, V5293, V5278).

homolog of the foramen is the postglenoid foramen that transmits the external jugular vein in life. If our identification is correct, then one question is whether posterior move- ment of the mandibular condyle might affect the function of the blood vessel that goes through the foramen. By fitting the condyle to the glenoid foramen, we found the fora- 


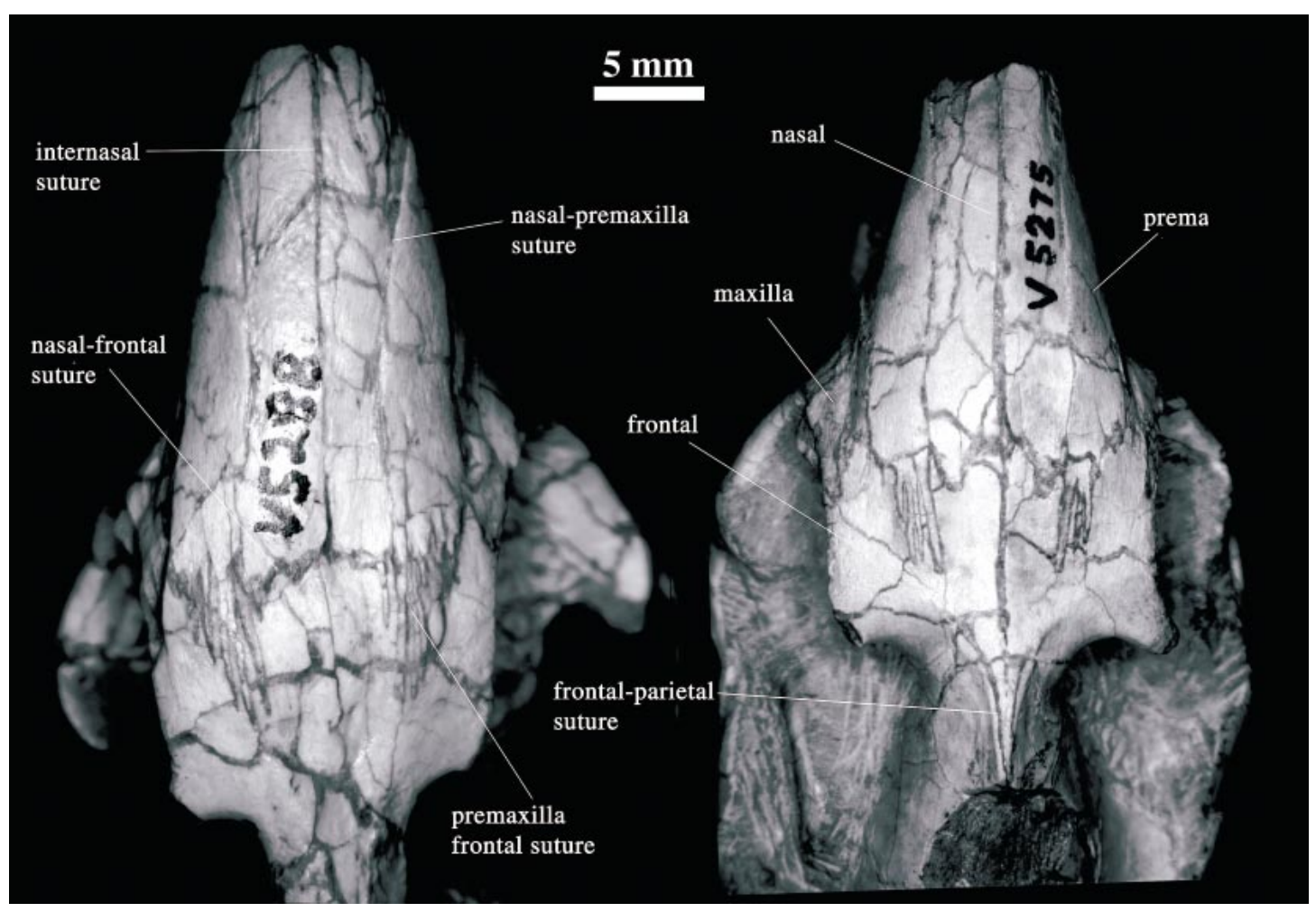

Fig. 29. Dorsal views of anterior skulls of Rhombomylus (IVPP V5288, V5275), showing the sutures between the nasal, premaxilla, and frontal.

men in question is not blocked by the condyle, suggesting that the foramen probably contained a functioning blood vessel. There is no postglenoid process. The external acoustic meatus is elongated and blocks the glenoid fossa posteriorly; it could function as a postglenoid process.

PRESPHENOID: The presphenoid is a spearhead-shaped bone, broad posteriorly and narrow anteriorly (figs. 24, 27, 33). With a faint ventral keel, it projects ventrally along the median line of the dorsal roof of the cavum defined laterally by the pterygoid. It sutures with the pterygoid laterally and overlaps the basisphenoid posteriorly. Its anterior extension cannot be surely estimated in all specimens, but it may reach the level of the posterior edge of the M3.

PTERYGOID: Lateral to the presphenoid the dorsal portion of the pterygoid is narrow. The main component of the pterygoid is the vertical process (figs. 24, 27, 33). The anterior part of the vertical process overlaps the medial side of the pars perpendicularis of the palatine, whereas the posterior part stands as the entopterygoid crest. The pterygoid hamulus extends posteriorly to meet an anterior projection of the bulla at its anteroventral corner. The hamulus is relatively thick compared to the slim process in most other mammals. Dorsal to the bridge is a large opening through which the anterior aperture of the Eustachian tube between the bulla and the alisphenoid is visible (figs. 24, 33).

BASISPHENOID: The basisphenoid is short and lies between the anterior halves of the two bullae. Anteriorly it contacts the pterygoid, and the suture between the two elements is ventrally overlapped by the posterior base of the presphenoid (figs. 24, 33A). Anterior to the opening of the Eustachian tube, the basisphenoid has a narrow contact with the alisphenoid as well as the petrosal process that roofs the opening of the Eustachian tube. The basisphenoid borders with the basioccipital posteriorly. The lateral edg- 

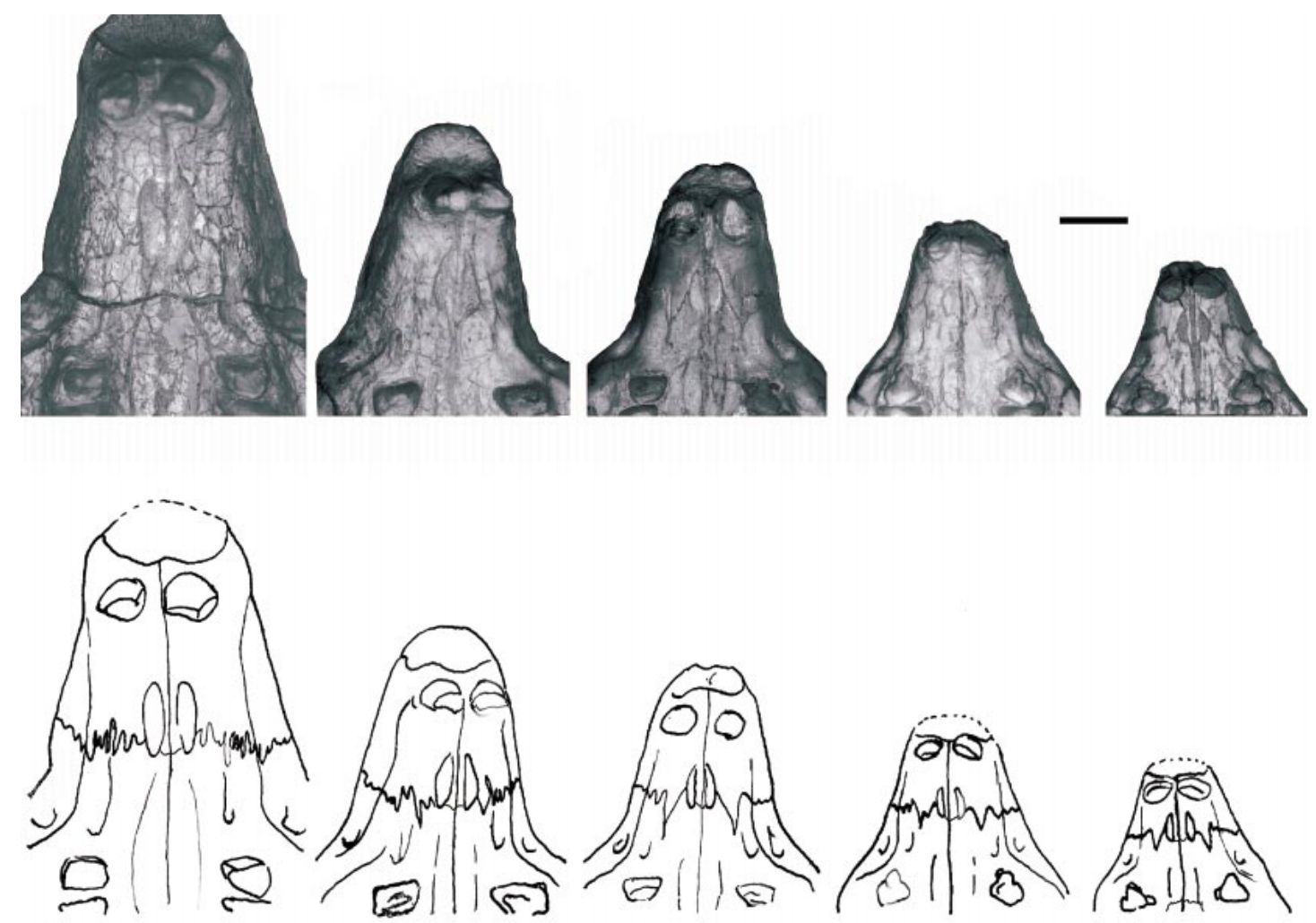

A

B

C

D

E

Fig. 30. Ventral views of the rostra of Rhombomylus (IVPP V5278, V5293, V5289, V5280, V7585), showing the conditions of the incisive foramen, premaxilla-maxillar suture, and shape of the diastema in individuals of different ages.

es of the basisphenoid and basioccipital are weakly ridged, confining a shallow, longitudinal valley along the central axis of these bones. Between the basisphenoid and the bullar wall there is a slit, which was previously identified as the foramen for the internal carotid canal (Li and Ting, 1985). Further preparations of several specimens show that the slit is blind.

ORBITOSPHENOID: The orbitosphenoid at the center of the orbital region (fig. 31) contacts the maxilla and frontal anteriorly, the frontal and parietal dorsally, the palatine ventrally, and the alisphenoid posteriorly. Its contact with the frontal is the most extensive. The optic foramen is large and lies in the center of the orbitosphenoid. The foramina of both sides are confluent with each other. Posteroventrally the orbitosphenoid forms the medial wall of the large sphenorbital fis- sure, which is laterally walled by the alisphenoid. In IVPP V5289 (fig. 31), an artificial opening exists posteroventral to the optic foramen in the orbitosphenoid.

AlisPHENOID: The alisphenoid is a complex bone bordering with several elements in the orbitotemporal region (figs. 24, 28, 31). In lateral view, the alisphenoid forms the lateral wall of a large sphenorbital fissure; the anterior margin of the alisphenoid is notched at the fissure. Dorsal to the notch the alisphenoid extends anterodorsally to contact the orbitosphenoid and parietal, but is separated from the frontal by the orbitosphenoidparietal connection. Ventral to the notch the alisphenoid joins the palatine lateral to the sphenorbital fissure. The alisphenoid contacts the squamosal posterodorsally and the bulla posteroventrally. The posterior border of the alisphenoid is a wedge, which inserts be- 

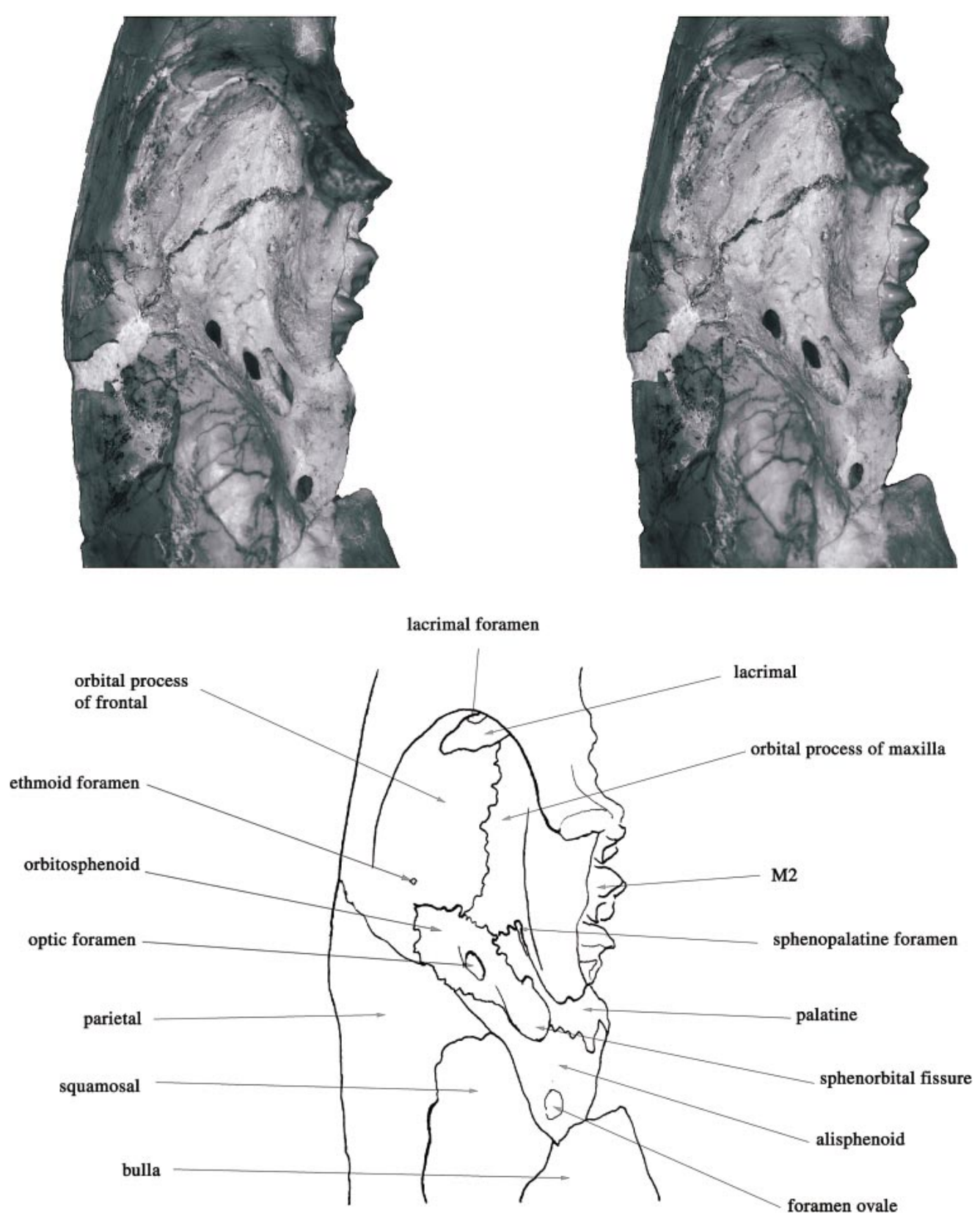

Fig. 31. Stereoscopic view of the lateral orbital and temporal regions of Rhombomylus (IVPP V5289).

tween the squamosal and the ectotympanic bulla. The contact with the squamosal is medial to the anterior half of the glenoid fossa formed by the squamosal; the alisphenoid does not contribute to the fossa. Within the triangular process of the alisphenoid is the elliptical foramen ovale that faces ventrolaterally. Immediately medial to the foramen ovale a foramen of the same size penetrates the posterior portion of the ectopterygoid crest of the alisphenoid. This foramen was called the foramen ovale accessorius ( $\mathrm{Li}$ and 

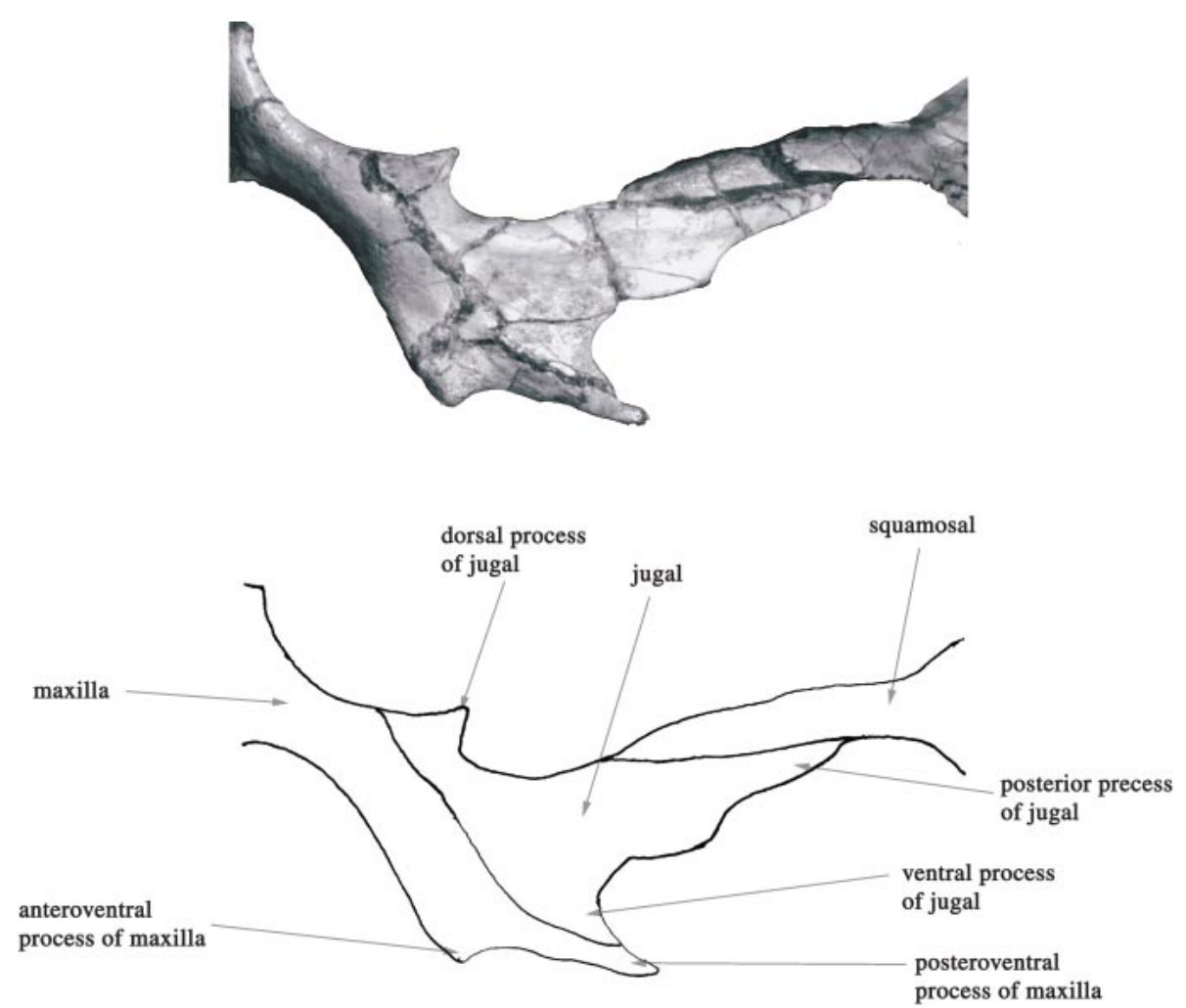

Fig. 32. Lateral view of the zygomatic arch of Rhombomylus (IVPP V5289).

Ting, 1985), a term coined by Wahlert (1974). However, Wahlert defined the foramen ovale accessorius as the opening lateral to the foramen ovale. The foramen in question is not the alisphenoid canal because the latter is usually situated anterior to the foramen ovale and runs for at least a short distance within the alisphenoid. We therefore tentatively called the foramen in question the medial foramen ovale. The ectopterygoid crest of the alisphenoid is a large lamina, gently concave on the medial surface and convex on the lateral surface. It constitutes the lateral wall of the ectopterygoid fossa. In ventral view, the alisphenoid has contacts posteriorly with the ectotympanic bulla and the petrosal process that forms the roof of the Eustachian tube. Medially, it has a narrow suture with the basisphenoid and a broad contact with the pterygoid. The ectopterygoid crest joins the palatine anteriorly.

OCCIPITAL: The occipital forms the most posterior portion of the skull (figs. 24, 27,
33-36). Ventrally, the trapezoidal basioccipital is quite long and adjoins the basisphenoid anteriorly at the level about a third of the bulla length from its anterior end. Laterally, the basioccipital borders the ectotympanic bulla anteriorly and the mastoid of the petrosal posteriorly. The slitlike jugular foramen lies between the basioccipital and the mastoid immediately posterior to the bulla-mastoid suture. The surface of the trapezoidal process bears a weak median ridge, on both sides of which are flat areas presumably for attachment of the rectus capti muscle. The paracondylar process of the occipital abuts the expanded mastoid posteriorly, forming the posterior extremity of this region and thickening to form a columnlike structure. The ventral surface of the paracondylar process is a semicircular, concave area. This concave area is probably for attachment of the diagastric muscle, or part of it. The rest of the muscle may also reach to the medial side of the expanded mastoid process, which, 
in contrast to the lateral convex part, is concave. The paracondylar process and the ventral lobe of the occipital condyle confine a deep trough, the ventral condyloid fossa. At the anteromedial end of the fossa lies the hypoglossal foramen. The ventral lobes of the occipital condyle are broad and gently convex. The intercondyloid incisure between the two condyles is gently concave.

In occipital view, the supraoccipital is fused to the rest of the occipital in all specimens, with the exception of IVPP V7585, which represents the youngest individual of Rhombomylus. In IVPP V7585 the supraoccipital is separated from the rest of the occipital by distinct sutures. The supraoccipital is a large, pentagonal, and concave bony lamina. It has an interparietal process that is exposed on the roof of the skull and constitutes the central part of the lambdoidal (nuchal) crest. The center area of the crest is concave anteriorly and intersects the posterior end of the sagittal crest; the parts lateral to the sagittal crest follow a gentle biconvex curvature. In posterior view the suture between the occipital and the eminence of the mastoid is definite. The trough between the ventral paracondyloid process and the occipital condyle continues to the posterior side of the occipital region between the condyle and the paroccipital process. The foramen magnum is oval shaped.

ECTOTYMPANIC: The auditory region is characterized by a fully developed bulla and an expanded mastoid of the petrosal (figs. 24, $25,27,28,33-36$ ), which is unique in early gliroid froms. The bulla is expanded and completely encloses the tympanic cavity ventrally. Its delimitation with other cranial elements can be determined by sutures. The bulla fuses with the expanded mastoid of the petrosal posteriorly in adults, but in young individuals (e.g., IVPP V5289, V5280, V7585) it is distinct. The external morphology of these specimens and the crosssections from others (see below) show that the bulla is formed by one element, the ectotympanic. In anterior view, the dorsal rim of the bulla is notched by the anterior opening of the Eustachian tube. The roof of this opening is formed by a small, flat process of the petrosal. The anteroventral point of the bulla develops a short process ("horn" of the bulla, fig. 33). In young individuals, the process is weak but the hamulus-bulla sutural connection is complete. In adults, the connection becomes robust.

The lateral aspect of the bulla is marked by the external auditory canal. The canal is short in juvenile skulls and is elongated and extended posterolaterally in adults. The canal blocks the glenoid fossa posteriorly and probably functions as the postglenoid process. At the posteromedial corner of the bulla, a small foramen, ventral to the jugular foramen and completely within the ectotympanic, is probably the stapedial foramen, but the small size suggests that it may not have contained any functional blood vessel in adult life. The carotid foramen is lost in Rhombomylus. The ectotympanic contributes to the posterior half of the medial wall of the glenoid fossa; a concave area is thus formed on the anterodorsal side of the basal external auditory meatus. The bony canal tightly joins the squamosal. Internally, the bulla does not have bony septa, in contrast to the partitioned mastoid space.

Petromastoid: The petromastoid is a complex bone that provides accommodations for the middle and inner ear, allows passages of several cranial nerves and vessels, and forms a large part of the sidewall of the braincase (figs. 24, 25, 27, 28, 33-39). Some internal structures of this complex are revealed in cross-sections (see below). At the basicranial region, the expanded mastoid is a prominence that connects with the ectotympanic bulla posteriorly. Between the mastoid and the external auditory meatus, there is a transverse depression in which the stylomastoid foramen that transmits the facial nerve is located. Externally, this portion of the mastoid appears to be a posterior extension of the bulla. However, the inflated mastoid is not considered to be part of the bulla because it does not enclose the middle tympanic cavity. Internally, the tympanic cavity enclosed by the bulla is well separated from the mastoid cavity by a septum, although the two spaces are confluent through an opening. The mastoid is abutted posteriorly by the paracondylar process of the occipital. In young individuals, the mastoid inflation is not significant in comparison with the bulla. It ap- 

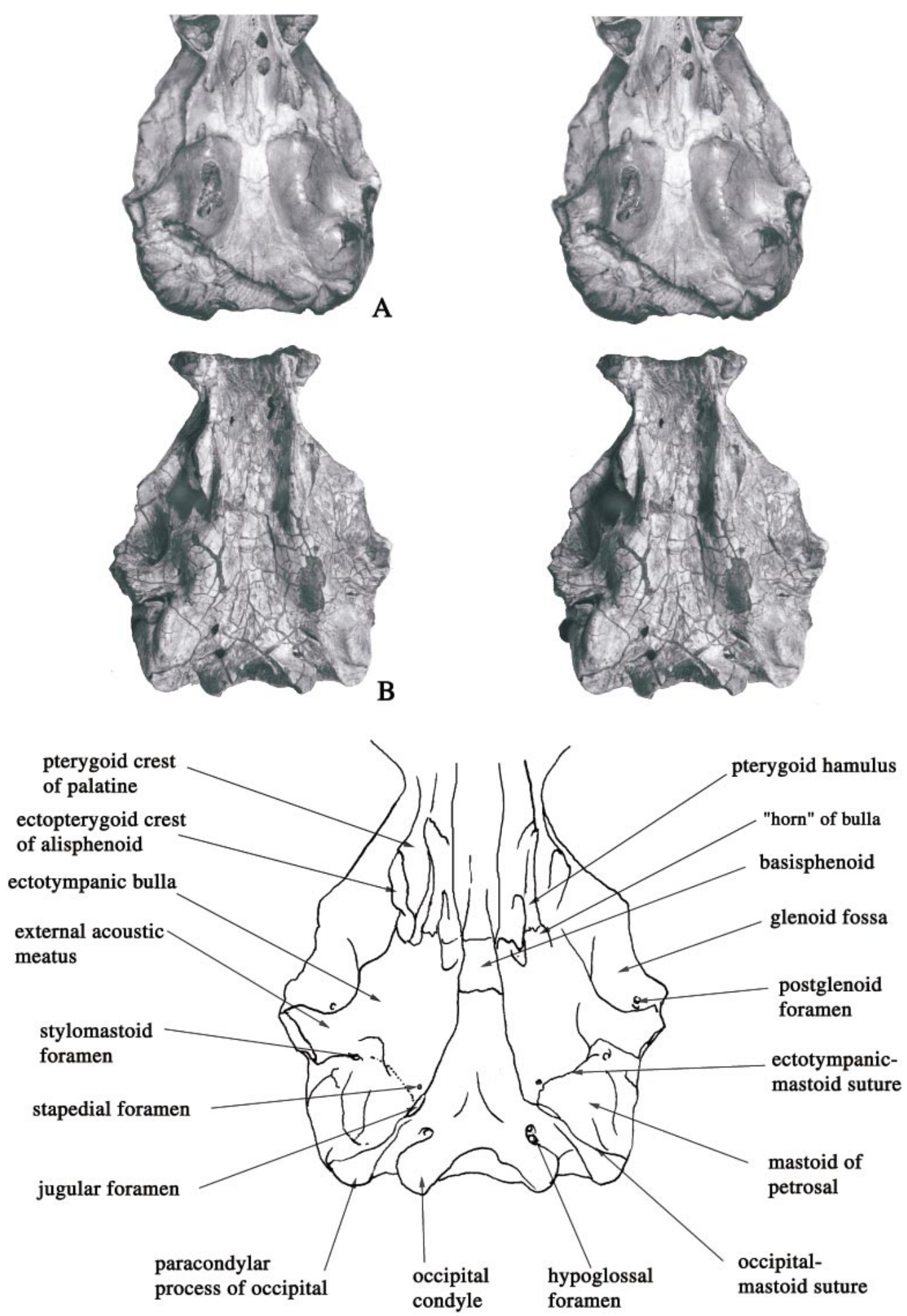

Fig. 33. Stereoscopic views of ventral basicranial regions of Rhombomylus (IVPP V5289, V5281). 

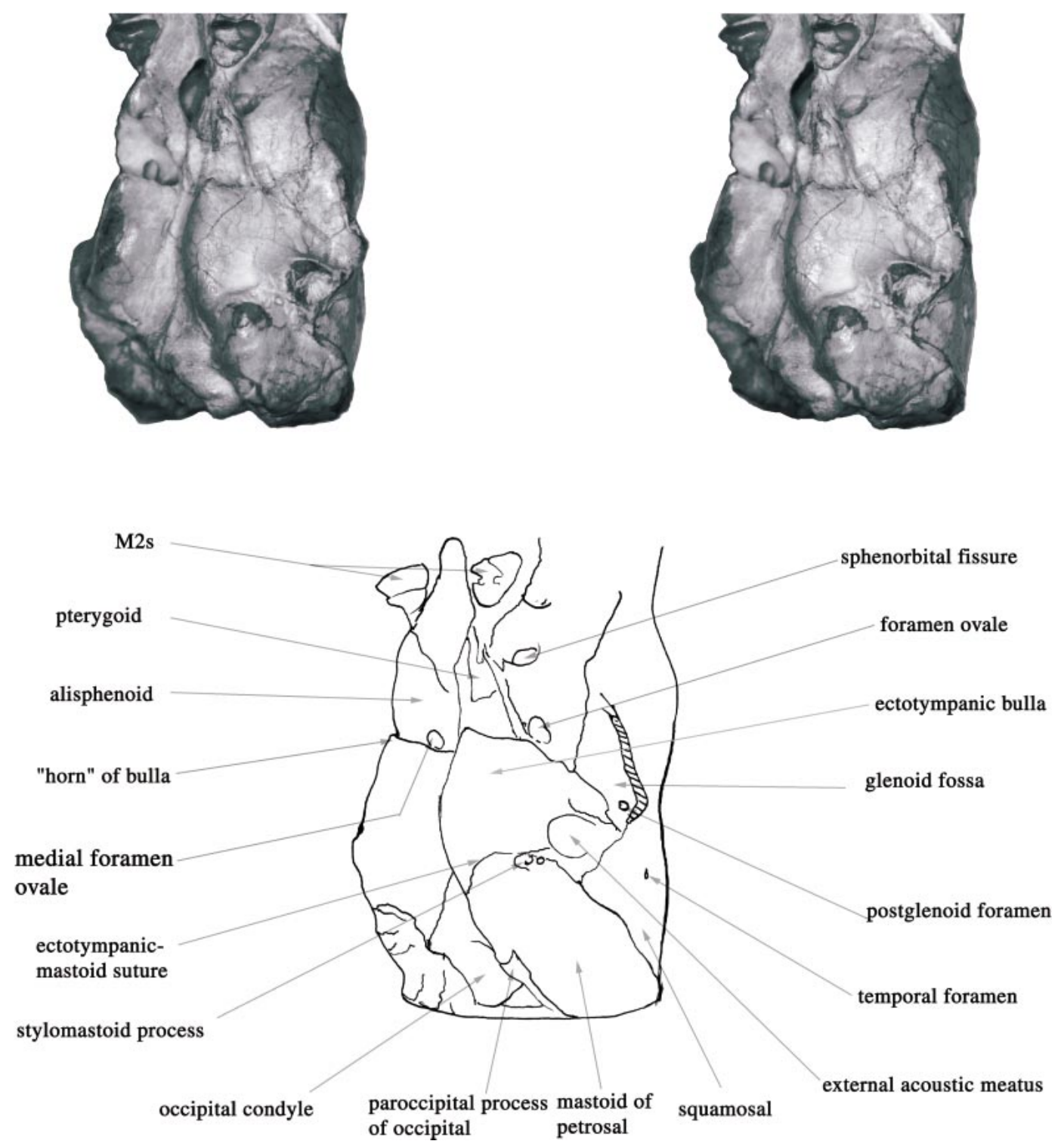

Fig. 34. Stereoscopic view of the ventrolateral basicranial region of Rhombomylus (IVPP V5289).

pears that the mastoid increases its size with age at a greater rate than does the bulla during postnatal ontogeny of Rhombomylus. Consequently, the inflated mastoid can be as long as, or even longer than, the ectotympanic bulla in adult individuals.

The expanded mastoid accounts for a large portion of the region on the lateral side of the skull, posterior to the squamosal (figs. 25, $28,34)$. The convex surface of the mastoid forms the posterior edge of the skull. The mastoid is also extensively exposed as a blunt triangular projection in the occipital region of the skull. The mastoid foramen is posterior to the squamosal-mastoid suture at the level of the temporal foramen. Because of its dorsal extension, a patch of the mastoid is exposed on the roof of the skull between the squamosal and the parietal. Internally, the massive mastoid consists of numerous sinuses partitioned by bony septa (figs. 36-38). The expansion of the petromastoid is not 

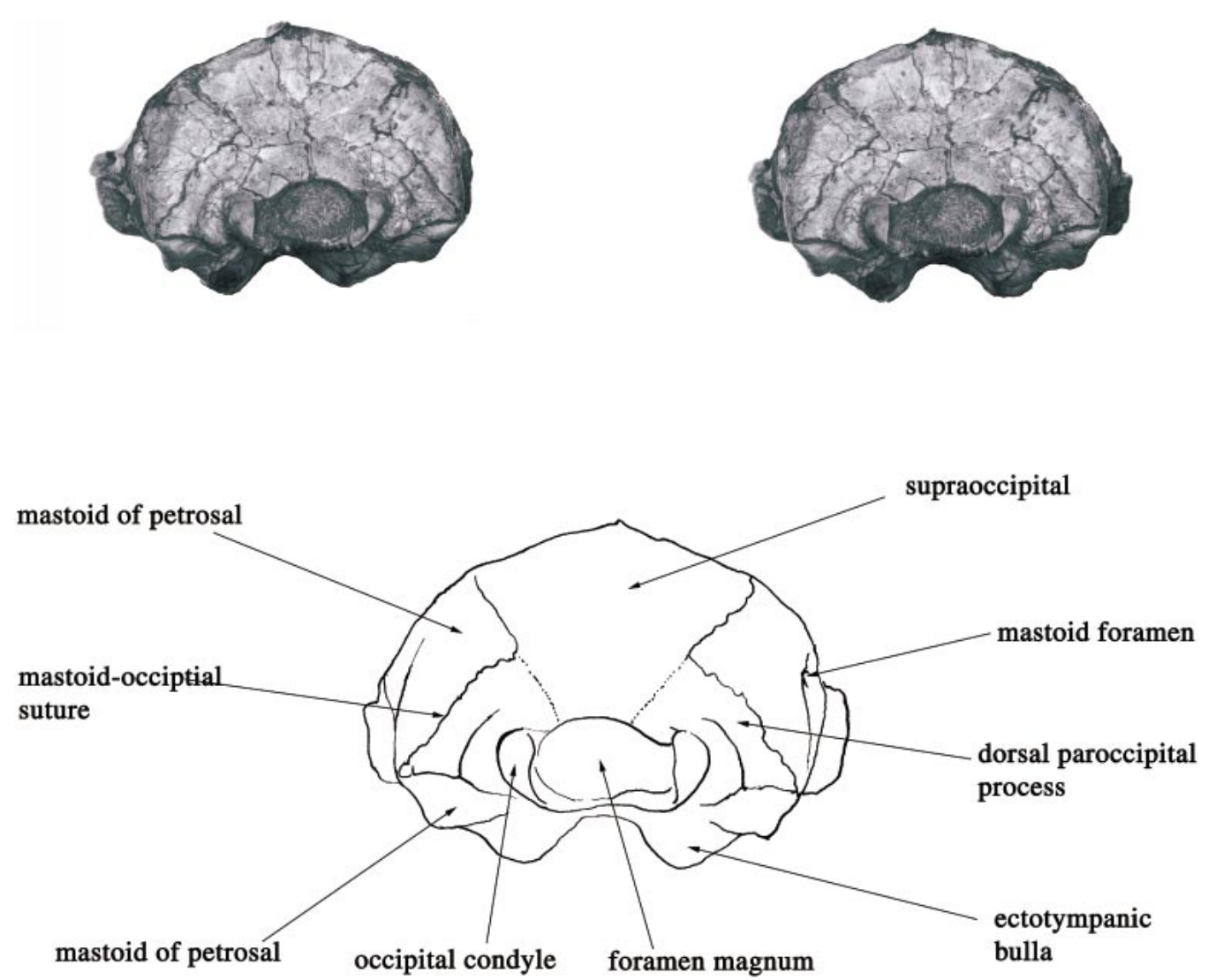

Fig. 35. Stereoscopic view of the occipital region of skull of Rhombomylus (IVPP V5281).

only external, but also occurs within the cranium (see below).

Morphologies of the tympanic cavity and middle ear are observed primarily from IVPP V5262, V5263, V7586 V7494, V5265 (figs. 36-40) and V5264 (not illustrated). These are basicranial specimens with broken bulla or isolated petrosals. Although the mastoid region shows a complex of septa and sinuses, the tympanic cavity, where the middle ear is located, is rather normal. The center structure within the cavity is the promontorium, which houses the cochlear canal. The promontorium is somewhat inflated as compared to some primitive forms such as leptictids (Novacek, 1986b) and other insectivores (MacPhee et al., 1988). The inflation may be attributed to two factors: increase in spiral turns of the cochlear canal and change in orientation of the cochlea. The inflation of the promontorium of Rhombomylus appears to be due to both factors, although the orientation probably plays a major role. The cochlear canal spiraling around a nearly horizontal axis pointing anterolaterally can be detected from IVPP V5262, in which a break at the surface of the promontorium reveals the cochlear canal (fig. 38). About two full turns of the cochlear canal are estimated for Rhombomylus. Because of the orientation, the ventral surface of the promontorium displays two swellings, separated by a saddleshaped trough (figs. 36, 37). The anterior one contains the main part of the cochlear canal, whereas the posterior part lodges the basal cochlear canal and part of the vestibule. The posterior part of the promontorium, as a general rule in mammals, bears two landmark openings: the fenestra vestibuli laterally and the fenestra cochleae posteriorly. The fenestra vestibuli (oval window or fenestra ovalis) is large, oval-shaped, and located in a pocket. 
The fenestra cochleae (round window or fenestra rotunda) has its long axis oriented nearly vertically. The ventral surface of the promontorium displays no sulcus or bony canal, suggesting absence of the carotid and stapedial artery. This is consistent with a minute stapedial foramen at the posteromedial corner of the bulla. Additional morphology of the inner ear is revealed in sectioned specimens (see below).

The anterolateral roof of the tympanic cavity is formed by the tegmen tympani of the petrosal, on which the tensor tympani fossa is broad and concave. The tensor tympani fossa is separated from a medial fossa by a bony ridge containing the canal for the greater petrosal nerve. In IVPP V7586 the same area is a large opening, which may have been created by preparation. An opening in this region is possibly the piriform fenestra. On the medial side of the promontorium, there is a broad, gently concave area formed by fusion of the petrosal and the folded ectotympanic. Immediately anterolateral to the promontorium is the tympanic opening of the canal for the facial nerve. There is no clear evidence that the facial nerve was contained in a bony canal. The exit of the facial nerve, the stylomastoid foramen, however, is located posterior to the base of the external auditory meatus at the junction of the bulla and the mastoid. This condition is similar to some rodents where the external auditory meatus is elongated, such as Tsaganomys (Bryant and McKenna, 1995), and suggests that the facial nerve is probably contained in a bony canal within the tympanic cavity. On the lateral side of the promontorium is the epitympanic recess. Unlike the normal mammalian condition, in which the recess is a shallow fossa, in Rhombomylus it is deeply pocketed in the expanded mastoid. This indicates that the malleus and incus were probably partly suspended in the expanded recess. The lateral side of the recess is floored by the lamina of the ectotympanic that forms the external auditory meatus and holds the dorsal part of the eardrum in life. The squamosal does not contribute to the lateral wall of the epitympanic recess. In IVPP V7586, a poorly preserved incus is in the epitympanic recess. Lateral to the epitympanic recess is the external auditory meatus, which is smoothly walled and has a narrow diameter compared to the inflated bulla. A small fossa for the stapedius muscle is defined by the gyrus of the lateral semicircular canal posterolateral to the fenestra cochleae. Further posteriorly the tympanic cavity is confluent with the expanded mastoid cavity.

The cranial side of the petromastoid forms a large part of the sidewall for the cranial cavity. The subarcuate fossa is moderate. Ventral to it is the internal auditory meatus. The two openings for the seventh and eighth cranial nerves are separated by a transverse crest. The semicircular canals are seen in crosssections and in IVPP V5265, V5263, and V7494. In the latter specimens the bone containing the canals and surrounding the subarcuate fossa was peeled off, revealing endocasts of the canals and the ampullae. The endocasts of the posterior and lateral canals are completely displayed, whereas that of the anterior is partially visible in IVPP V5265 and only the anterior and posterior canals are exposed in IVPP V5263. The three canals are completely exposed in IVPP 7494 (figs. 37, 39,40 ). Although the petromastoid is expanded, the inner ear, including the semicircular canals, remains normal. The three semicircular canals are oval in shape and are different in size. The anterior semicircular canal is the largest of the three; the maximum diameter of its loop is $3.2 \mathrm{~mm}$. The lateral canal is the smallest, with a loop diameter of $2.1 \mathrm{~mm}$, whereas that of the posterior semicircular canal measures $2.7 \mathrm{~mm}$. The diameter of each canal is about $0.3-0.4 \mathrm{~mm}$. The anterior and the posterior canals are nearly vertical and the lateral one is horizontal in orientation. The plane of each semicircular canal is nearly perpendicular to those of the other two. The plane of the anterior canal is oriented in a direction anterolaterally and that of the posterior one anteromedially. The three canals surround the space of the subarcuate fossa. As in other mammals, such as some early therians (Meng and Fox, 1995), the gyrus of the anterior canal forms the posterior rim of the subarcuate fossa and that of the lateral one surrounds the fossa for the stapedius muscle. Posteriorly, the anterior canal joins the anterior section of the posterior canal to form the crus commune. The anterior and lateral ampullae are at the lateral side of the vestibule, with that of the lateral one being more ventrally located and therefore closer to the fenestra vestibuli. The me- 

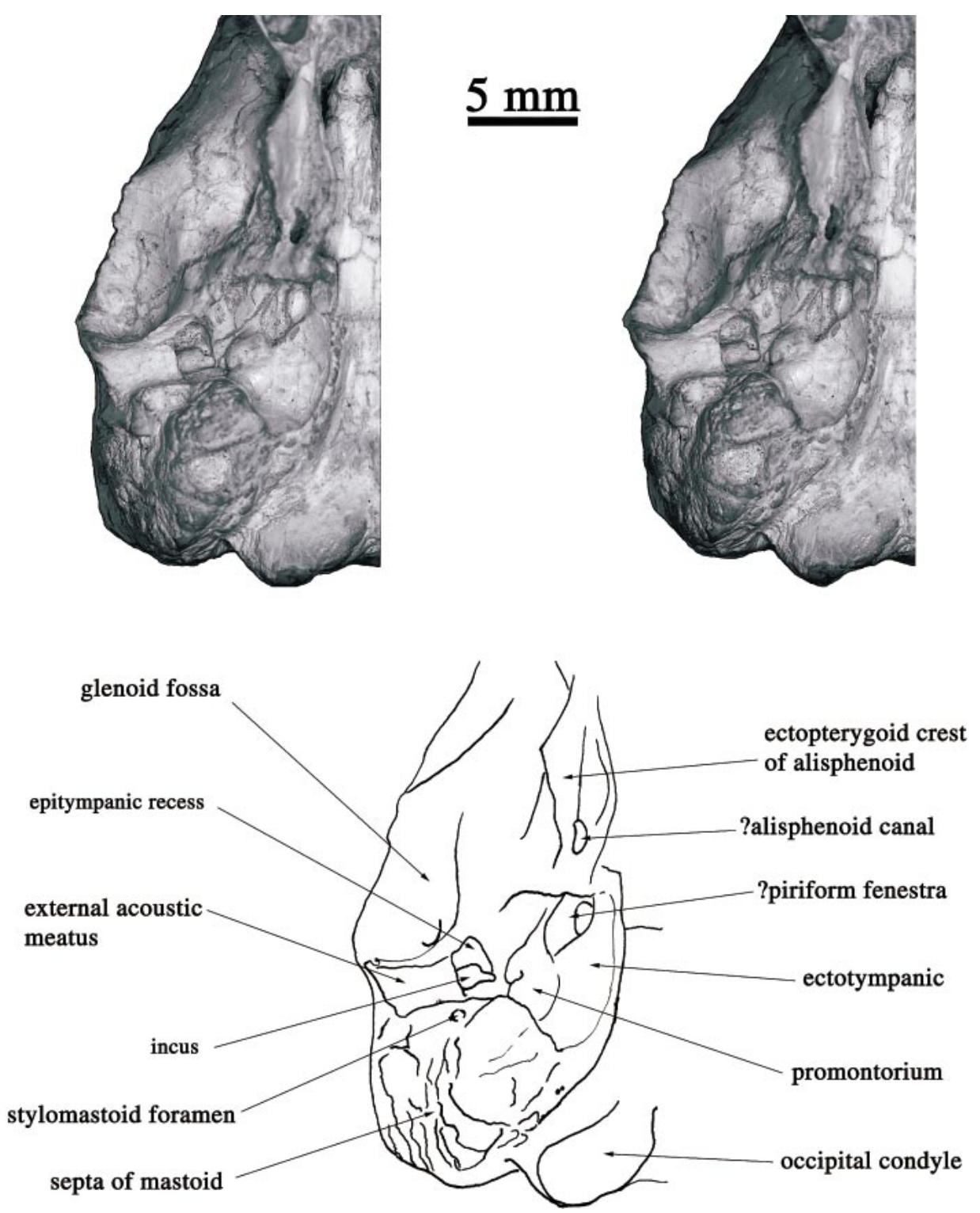

Fig. 36. Stereoscopic view of ventral basicranial and middle ear regions of Rhombomylus (IVPP V7586). The ectotympanic bulla was broken.

dial end of the lateral semicircular canal joins the vestibule immediately dorsal to the posterior ampulla, at the position posterior to the fenestra cochleae.

\section{Cross Sections of SkUll}

There are several methods of observing cranial morphology enclosed by bone. These include X-radiographs (Meng and Fox, 1995;
Fox and Meng, 1997), computer tomographic scan (Luo and Ketten, 1991), and serial sections (Kielan-Jaworowska et al., 1986; Allin, 1986; Novacek, 1986b; Graybeal et al., 1989; Hurum, 1994, 1998a, 1998b). Xradiographic pictures are two-dimensional, and computer tomographic scans are still unable to produce sharp images of detailed structures in complex systems such as the ear 

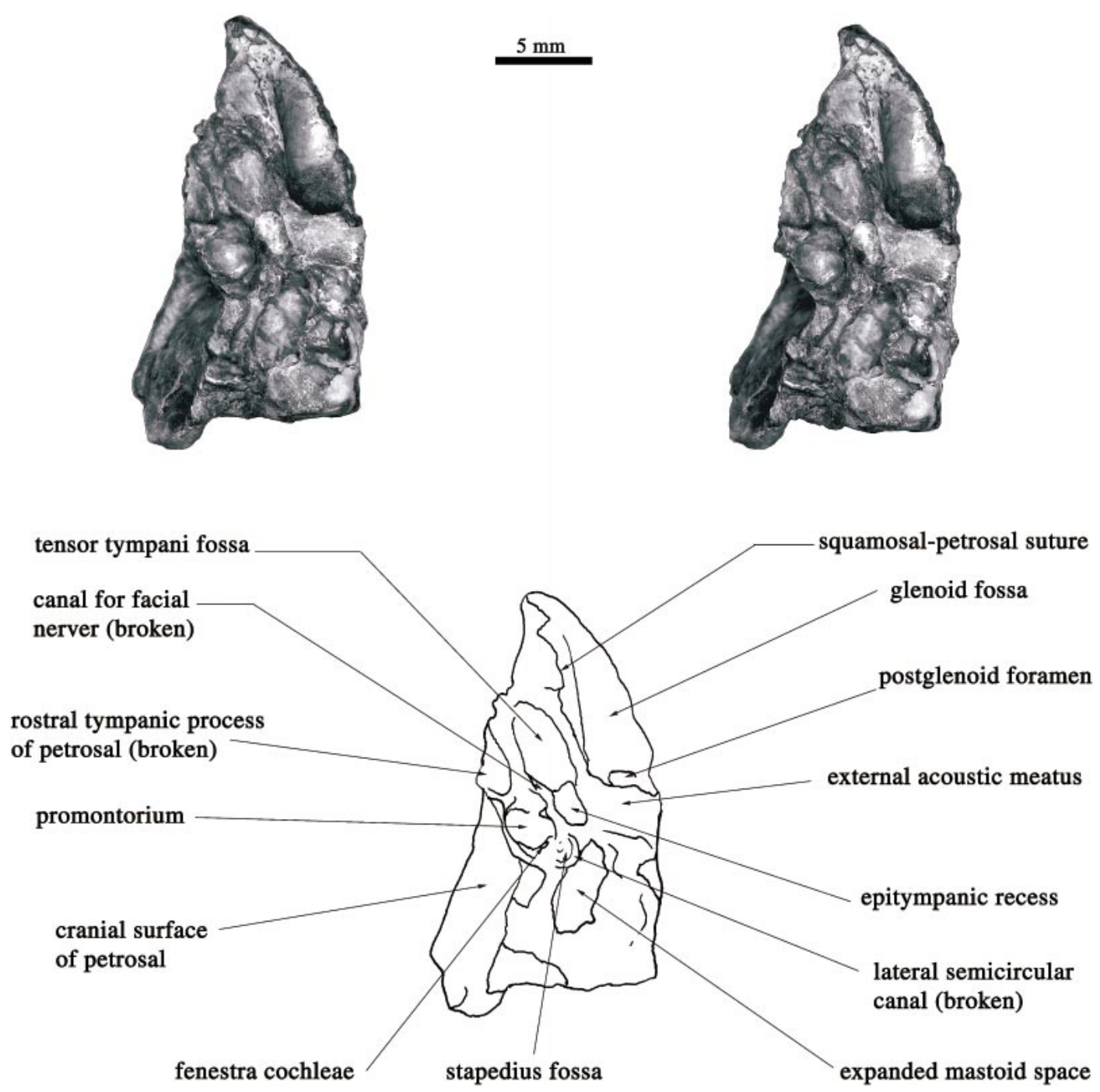

Fig. 37. Stereoscopic view of the lateral aspect of the ear region of Rhombomylus (IVPP V5263). The bulla was broken.

region of small fossil mammals. Serial sections can provide better images at the expense of the specimens. For taxa that are represented by many specimens, such as Rhombomylus, serial sections are a useful method to generate important information on fine cranial structure usually unobservable using conventional methods.

Previous studies using the above techniques focus primarily on early mammals or close relatives of mammals. Few such studies have been done in exploring early eutherian mammals and, consequently, we know very little about the internal anatomy, such as the nasal cavity, in early eutherians. Recognizing the importance of the internal morphology of the mammal skull as a source of phylogenetic data and the lack of information in this region, some workers (Moore, 1981; Novacek, 1993b) have encouraged effort for more serial sectional studies in comparative study of the internal structures of mammal skulls. Except for a preliminary study conducted by Ting and Li (1984), our study is the most detailed serial study of a basal gliroid mammal skull, which focuses on the nasal cavity 

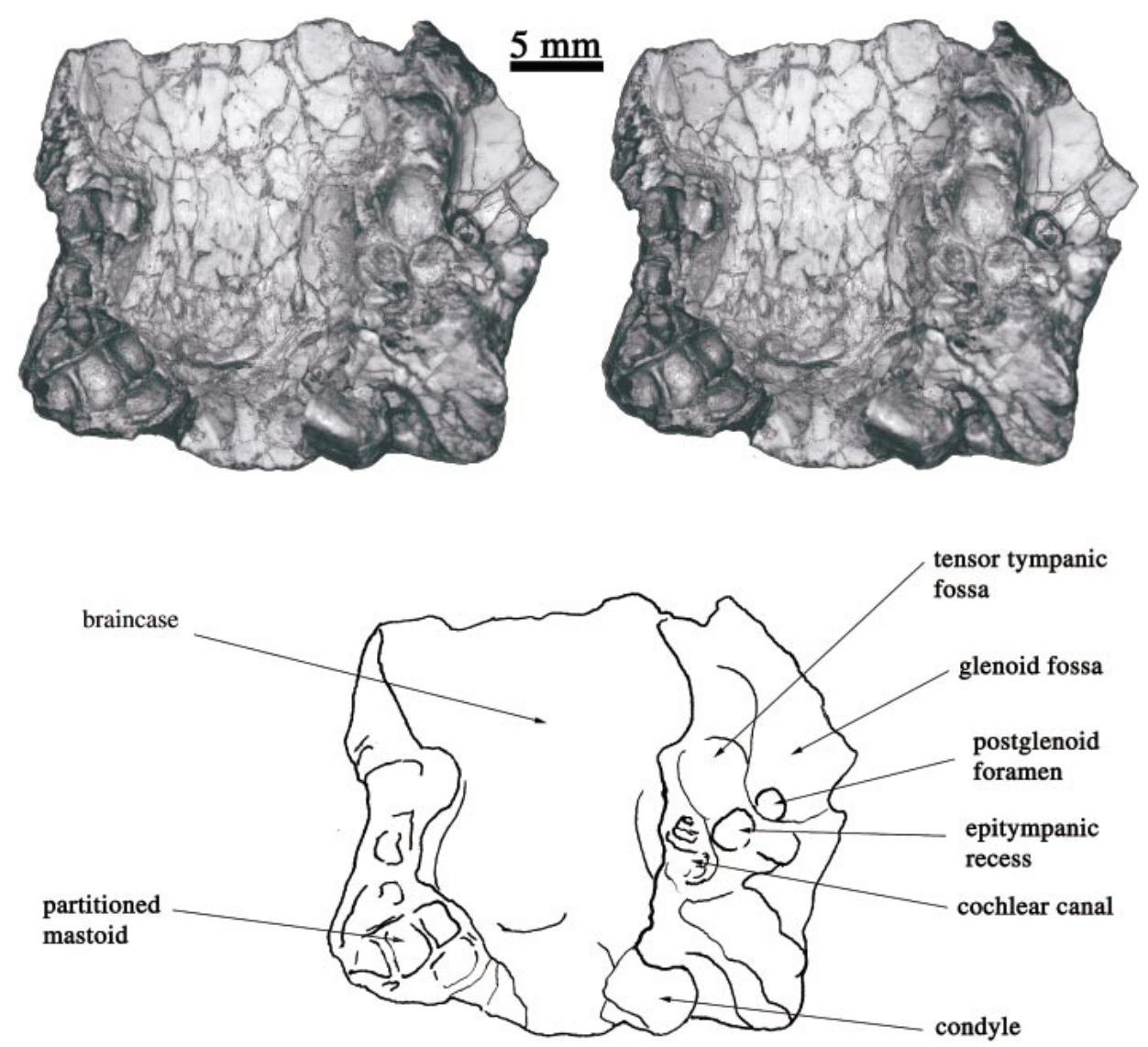

Fig. 38. Stereoscopic view of the internal braincase and ear region of Rhombomylus (IVPP V5262).

and braincase, including the auditory region. We hope that sectional morphologies of Rhombomylus will be useful not only in understanding the skull morphology of early gliroid mammals, but also in providing a basis for future comparative study with sectioned and CT-scanned specimens of other mammals.

NASAL CAVITY: The nasal cavity in mammals makes up the first part of the respiratory pathway and provides the distribution area for the peripheral olfactory apparatus (Moore, 1981). During its passage through the cavity, inspired air is moistened, cleaned of coarse particulate matter, subjected to olfactory analysis, and warmed. Except for some specialized groups, such as cetaceans in which highly specialized respiratory mechanisms prevail, the basic pattern of the nasal cavity is relatively conservative in most mammals. The structure of the nasal cavity in gliroid mammals is similar to those of other mammals, even though the rostrum of the skull in gliroid mammals has undergone considerable modification related to the specialized mastication. As shown in the guinea pig (Cooper and Schiller, 1975) and other rodents and lagomorphs, the floor of the nasal cavity is formed by the hard palate and the roof primarily by the nasal and frontal bones. Other skeletal elements in the lateral wall of the nasal cavity include the premaxilla, maxilla, palatine, and frontal, which are overlain on their medial surfaces by the lateral mass of the ethmoidal labyrinth, nasoturbinal, and maxilloturbinal. The cavity consists of the right and left fossae separated by a median septum that is bony in its posterior part but cartilaginous anteriorly. Posteriorly, the cavity ends at the paired posterior nasal aper- 

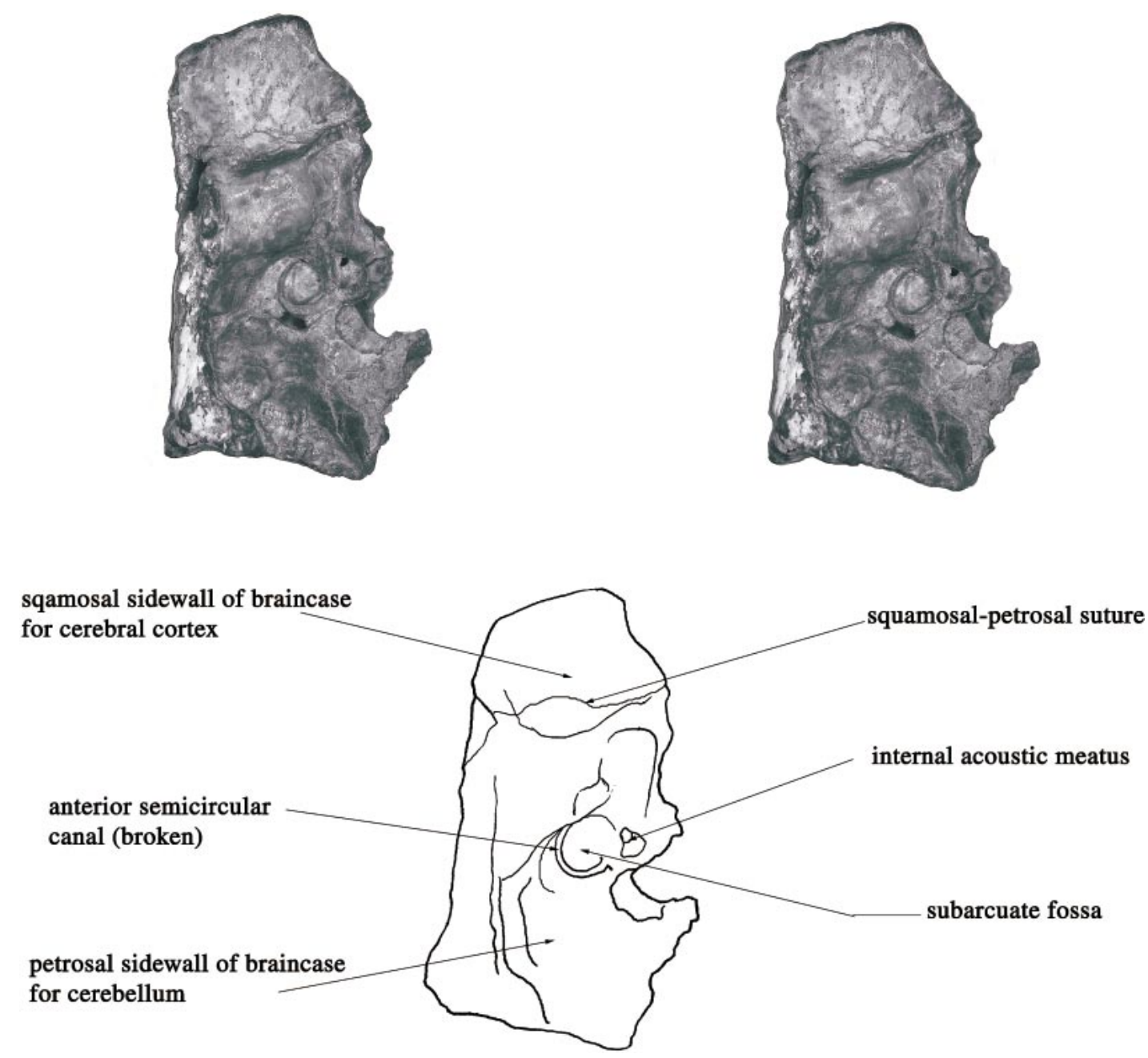

Fig. 39. Stereoscopic view of internal side of ear region and braincase of Rhombomylus (IVPP V5263).

tures. The area of mucous membrane is greatly increased in mammals by the formation of scroll-like bony plates, the turbinals, which project into the lumen of the nasal cavity from its lateral walls.

Cross sections of the nasal cavity of Rhombomylus are based on IVPP V7488, whereas frontal and longitudinal sections are based on IVPP V7492. The cross sectioning starts at the level near the anterior end of the incisive foramen (figs. 41, 42). At this level the contour of the rostrum is nearly circular, and the nasal cavity is roofed by the nasals, walled and floored by the premaxillae, and divided into two symmetrical halves by the vomer. The nasal is thick and has its lateral side bent ventromedially. The bent side is deep and contacts the dorsomedial portion of the maxillae in a flat surface-to-surface conjunction. This contact appears too weak to keep the nasal in anatomical place so that the nasals were pushed ventrally into the nasal cavity during preservation. A ventral ridge is formed along the medial line of the nasals and divides the dorsal region of the nasal cavity into two symmetrical, semicircular areas in crosssection. As this configuration continues posteriorly, the bent side of the nasal gradually straightens in keeping with the expansion of the nasal cavity. In section 12 


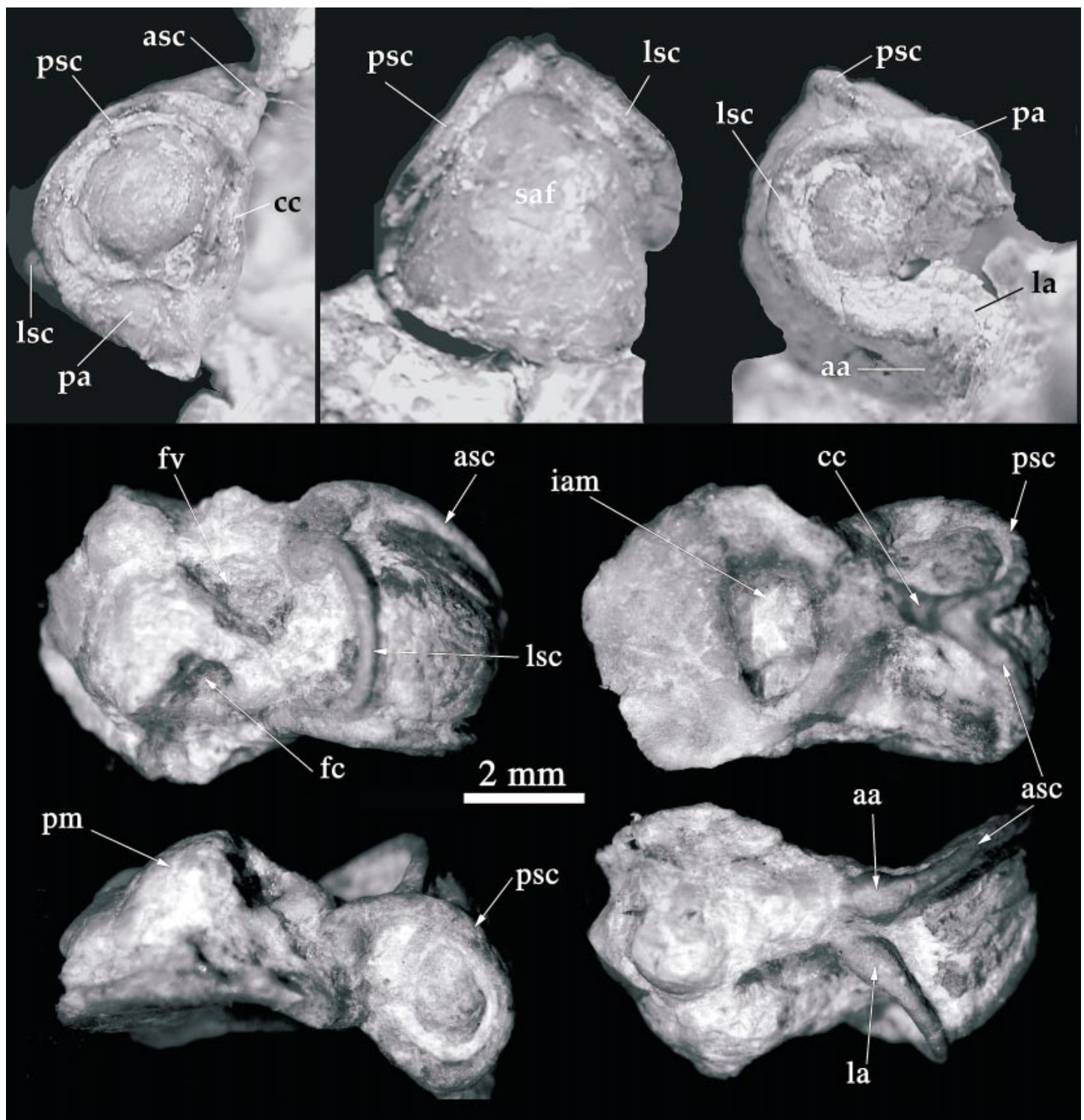

Fig. 40. Peeled petrosal showing casts of the inner ear of Rhombomylus (IVPP V5265, V7494). aa, anterior ampulla; asc, anterior semicircular canal; cc, crus commune; fc, fenestra cochleae; fv, fenestra vestibuli; iam, internal acoustic meatus; la, lateral ampulla; lsc, lateral semicircular canal; pa, posterior ampulla; pm, promontorium; psc, posterior semicircular canal; and saf, subarcuate fossa.

(fig. 42), the bent side of the nasal is sandwiched by a turbinal lamella ventrally and by the maxilla dorsally. The nasals are excluded from the nasal cavity by the frontal bone in section 14 and posterior ones (fig. 42). The entire inner surface of the nasal is smooth and there is no sign of pneumatization.

The sidewall of the nasal cavity is formed by the premaxilla that contains the incisor. The cross section of the alveolus of the incisor is oval. The incisor ends in the maxilla at sections 10 and 11 . On the anterior floor of the cavity, the passage of the incisive foramen is defined by the premaxilla anteriorly and is partly joined by the maxilla posteriorly. In forming the lateral wall of the inci- 

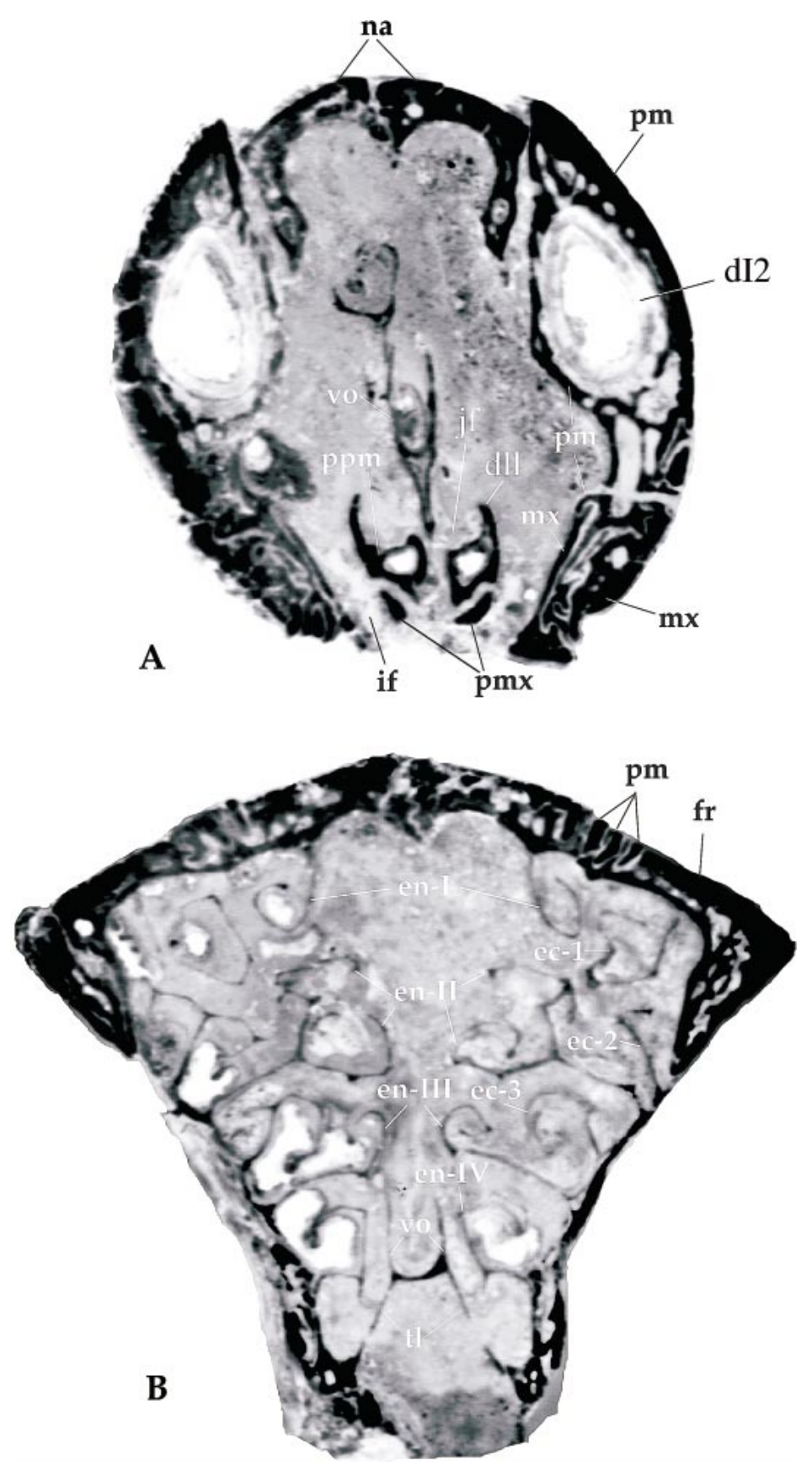

Fig. 41. Cross sections through anterior and posterior nasal cavity (IVPP V7488) of Rhombomylus, showing internal structures of the cavity. dll, dorsolateral lamina of the Jacobson's fossa; ec-1, ectoturbinal 1; ec-2, ectoturbinal 2; ec-3, ectoturbinal 3; en-I, endoturbinal I; en-II, endoturbinal II; en-III, endoturbinal III; en-IV, endoturbinal IV; fr, frontal; if, incisive foramen; jf, Jacobson's fossa. mx, maxilla; na, nasal; pm, premaxilla; pmx, palatine process of maxilla; ppm, palatine process of premaxilla; tl, transverse lamina; and vo, vomer. See text for discussion. 

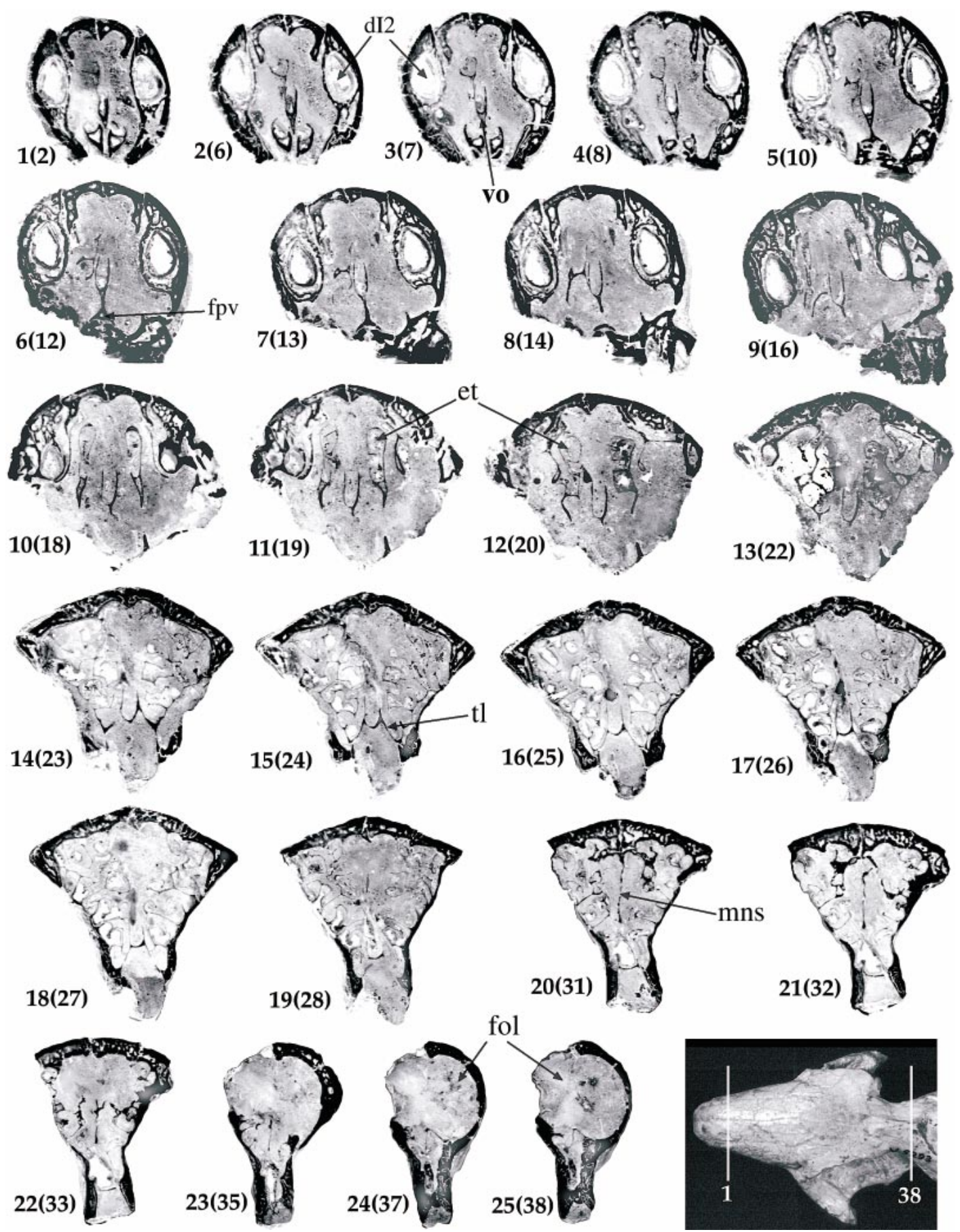

Fig. 42. Cross (coronal) sections through the rostrum of Rhombomylus (IVPP V7488), showing internal structures of the nasal cavity. The sequence of sections is indicated in the figure at the lower right corner (the specimen is not the one that was cut). Among a total of 38 sections, only 25 are illustrated. The number between brackets indicates the original position in the cutting sequence. et, ethmoturbinals; fol, fossa for olfactory lobe; fpv, footplate of vomer; mns, median nasal septum; and $\mathrm{t}$, transverse lamina. See text for discussion. 
sive foramen, the premaxilla has a complex suture with the maxilla (sections 2-4; fig. 42 ), contrasting sharply to the smooth contact between the nasal and maxilla in the roof of the nasal cavity. The palatine processes of the premaxillae (prevomerine process of the premaxilla of Novacek, 1985) form the medial separation of the incisive foramen ( $\mathrm{sec}$ tions 1-3; fig. 42). The processes from both sides are separated by a suture. The body of the process is a hollow structure with a triangular crosssection. Two thin lamellae are developed longitudinally along the dorsomedial and dorsolateral edges on the palatine process of the premaxilla. The dorsolateral lamella is significantly higher than the medial one (as seen particularly in sections 2 and 3; fig. 42). The space defined by the two lamellae, C-shaped in cross section, accommodates the Jacobson's capsule formed by the paraseptal cartilage in life; the capsule in turn houses the Jacobson's organ. We term the osseous space as the Jacobson's fossa and the projected laminae as the dorsomedial and dorsolateral processes of the Jacobson's fossa. These structures correspond to the shape of the Jacobson's capsule and organ (see discussion below) and therefore can be used to estimate the size and shape of the latter. The morphologies and relationships of the fossa to other elements, such as the maxilla, are similar to those of other mammals, such as marsupials, as illustrated by Goodrich (1930: fig. 404). From section 3, the medial wall of the incisive foramen is partially intruded by a slim palatine process of the maxilla, which abuts the ventral side of the posterior palatine process of the premaxilla. Posterior to section 3 , the premaxillary process reduces its size and finally fades away in section 5 . From section 6 on, the floor of the nasal cavity is primarily formed by the maxilla. A maxillary sinus is first developed ventral to the incisor (section 6 and posterior ones) and then expands into the maxilla (section 6; fig. 42). Only part of the palatine process of the maxilla is preserved, primarily on the left side, which forms the ventrolateral wall of the posterior nasal cavity. In sections 14-16, alveoli for the left M1 can be seen.

The lower part of the anterior nasal cavity is longitudinally divided into two halves by the vomer that is located along the median line dorsal to the suture between the palatine processes of the premaxillae. Anteriorly, the vomer has a pointed ventral tip in cross section and two narrowly separated wings of thin bone that extend dorsally and are almost parallel to each other to give the vomer the shape of a tuning fork. The vomer increases its height posteriorly and, in section 5 , a flat footplate is developed at its ventral end, and the profile of the vomer then takes the shape of a "champagne-glass" (fig. 42). The footplate of the vomer first stands on the very posterior end of the palatine processes of the premaxillae and then entirely on the maxilla posteriorly. Starting from section 8 , the shaft of the "champagne-glass"-shaped vomer disappears and the body of the vomer is separated from the foot and is suspended in the nasal cavity. This morphology is confirmed in the longitudinal sections in figure 43.

The bony turbinals in the two sectioned specimens (IVPP V7488, V7492) appear intact (figs. 41, 44). The configurations of the scroll-like structures, formed by extremely thin bony lamellae, suggest the bony turbinals are in their original shape and position. The maxilloturbinal is not surely detected in the cross-sectioned specimen but is probably revealed in the longitudinal and frontal sections (figs. 43, 44; see below). The anteroromost portion of the nasoturbinal is represented by a simple lamella at the position above the incisive foramen in the right side of the nasal cavity (fig. 42). The ethmoturbinals are well developed and are shown symmetrically in two sides of the nasal cavity. The anterior ends of the ethmoturbinals extend to the level near the end of the incisor. These parts of the ethmoturbinals are suspended in the nasal cavity, showing no connection with any surrounding structures. Although the anterior ethmoturbinals on each side of the cavity remain roughly as one unit, they already show a complex inrolling pattern of bony lamellae. The two masses of ethmoturbinals are also separated by the median vomer, which does not have any contact with the ethmoturbinals until the level of section 14. In section 14 and subsequent ones the ethmoturbinals project medially into the lumen of the nasal cavity from the lateral ethmoidal plate; each ethmoturbinal consists of a thin lamella that is plicated and under- 


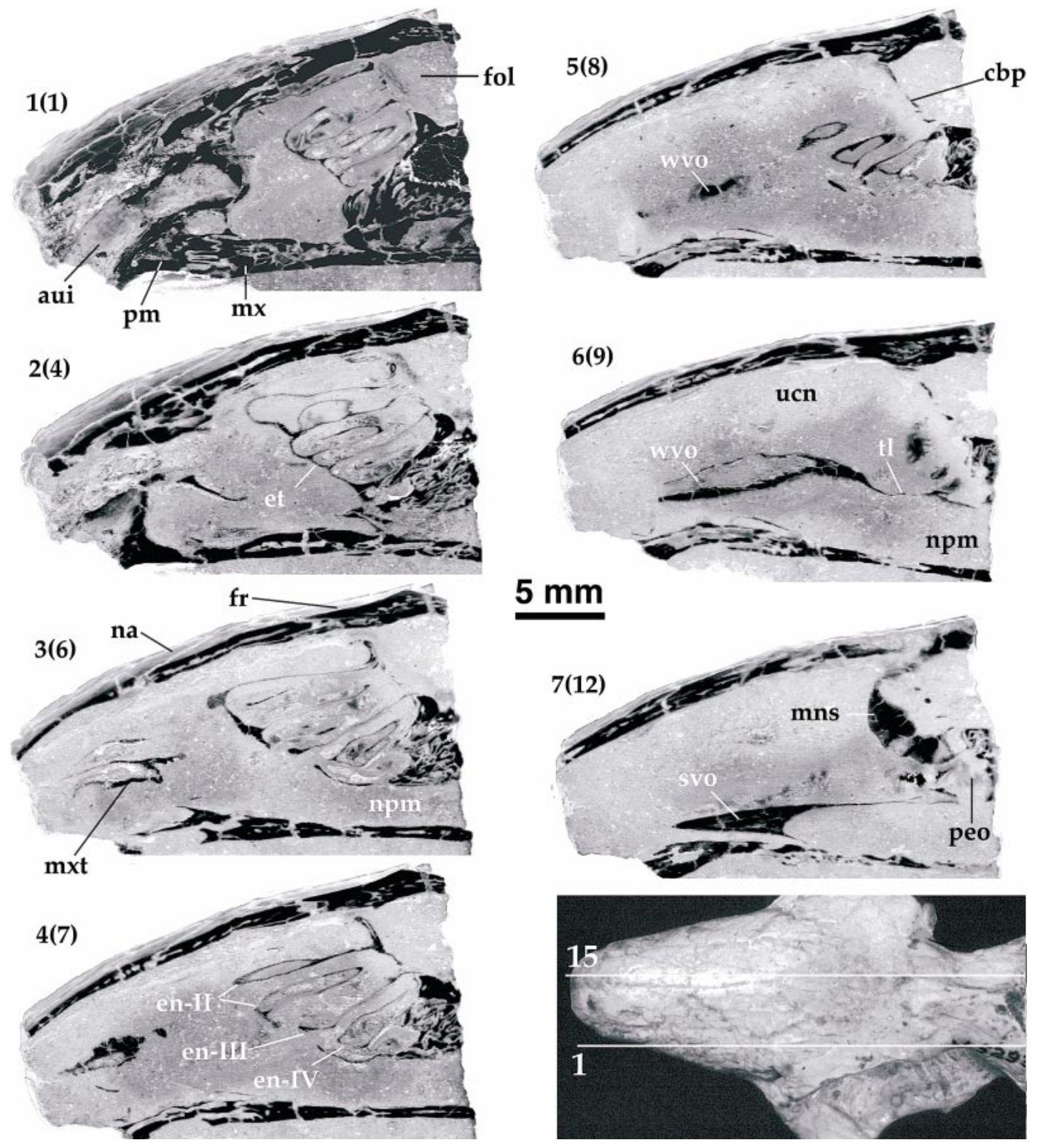

Fig. 43. Longitudinal (sagittal) sections through the rostrum of Rhombomylus (IVPP V7492), showing internal structures of the nasal cavity. The sequence of sections is shown in the figure at the lower right corner (the specimen therein is not the one that was cut). A total of 15 sections were made and 7 of them are illustrated. The number between brackets indicates the original position in the cutting sequence. aui, alveolus for upper incisor (dI2); cbp, cribriform plate; en-II, endoturbinal II; en-III, endoturbinal III; en-IV, endoturbinal IV; et, ethmoturbinals; fol, fossa for olfactory lobe; fr, frontal; mns, median nasal septum; mx, maxilla; mxt, maxilloturbinal; na, nasal; npm, nasophryngeal meatus (lower compartment of the nasal cavity); peo, posterior extension of olfactory region; pm, premaxilla; svo, stem of the vomer; svo, stem of vomer; tl, transverse lamina; ucn, upper compartment of the nasal cavity; and wvo, wing of the vomer. 

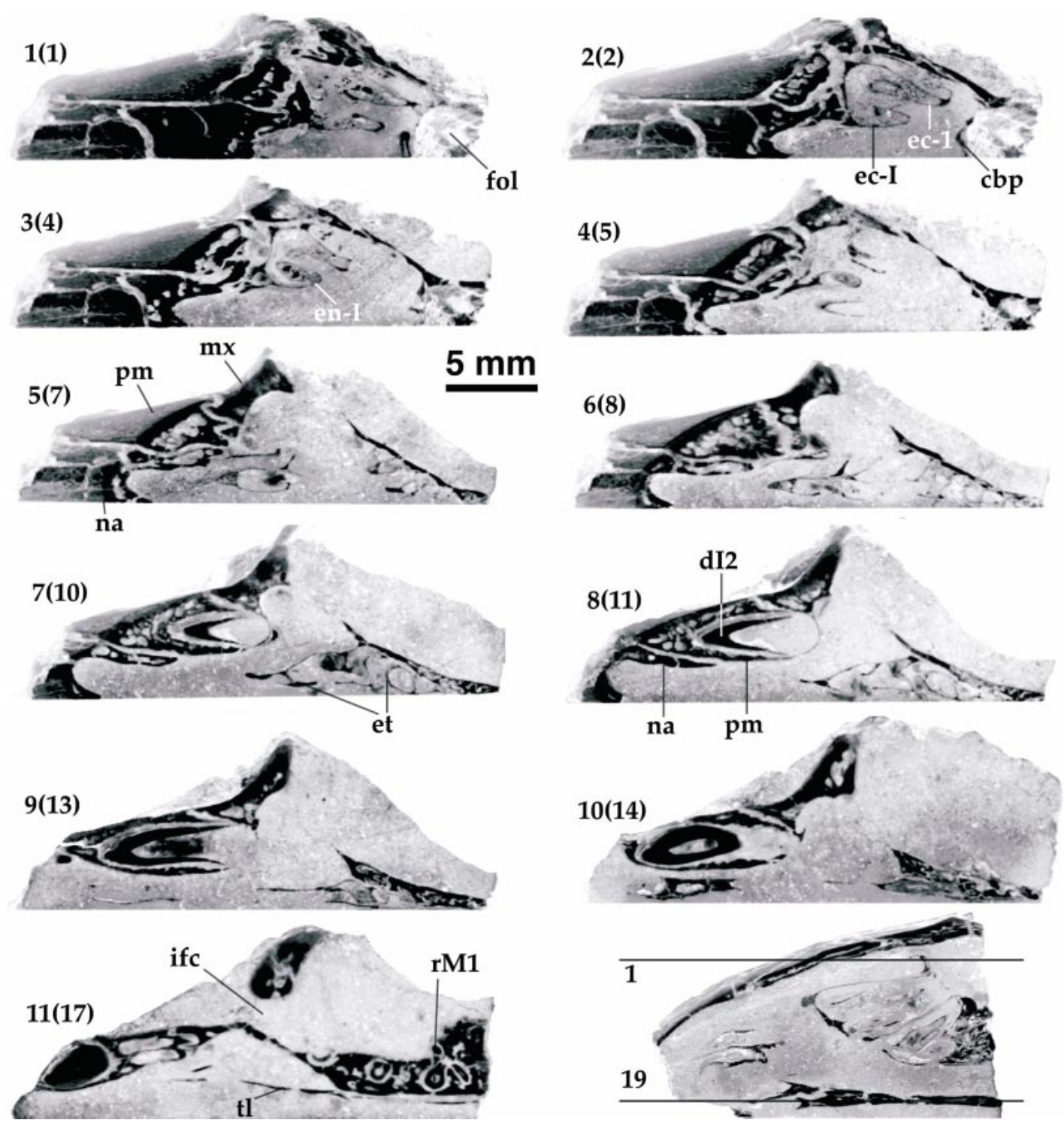

Fig. 44. Frontal (horizontal) sections through the rostrum of Rhombomylus (IVPP V7492), showing internal structures of the nasal cavity. The sequence of sections is shown in the figure at the lower right corner (the specimen is the left side of the same skull in fig. 43). A total of 15 sections were made and 11 of them are illustrated. The number between brackets indicates the original position in the cutting sequence. cbp, cribriform plate; dI2, second upper deciduous incisor; en-I, endoturbinal I; et, ethmoturbinals; fol, fossa for olfactory lobe; ifc, infraorbital canal; mx, maxilla; na, nasal; pm, premaxilla; rM1, roots of M1; tl, transverse lamina; and wvo, wing of the vomer.

goes branching and inrolling to form olfactory folds. Because of the complicated folds, sediment did not fill the spaces surrounded by the lamellae during fossilization, leaving some empty spaces in the ethmoturbinals. As in extant mammals (extensively described by
Paulli, 1900a, 1900b, 1900c; summarized by Moore, 1981, and more recently by Novacek, 1993b), the ethmoturbinals are arranged roughly into two rows. One row consists of the turbinals that extend medially close to the nasal septum in the center of the cavity and 
are called endoturbinals; the other row comprises ectoturbinals that end more laterally. Four endoturbinals are recognized, as shown in sections 17 and 18 (figs. 41B, 42). A distinct feature in Rhombomylus is division of the stem lamella of endoturbinal II into two branches that inroll in opposite directions: the ventral branch curving dorsally and the dorsal one ventrally. In contrast, endoturbinals III and IV inroll only dorsally. There are only a few ectoturbinals, and their number varies at different regions: two or three are found between endoturbinals I and II, one between II and III, and none between III and IV. The lamellae are distributed in such a pattern that their density in cross section appears to be even. In other words, the olfactory interspace between any two lamellae is not overly packed or spaced. This suggests an even distribution of the olfactory epithelium that may function most efficiently.

Another structure seen in section 14 and subsequent ones is the transverse lamina. The description of this structure and the interpretation of its function in mammals by Moore (1981: 244-245) precisely fit to the structure observed in Rhombomylus:

In many macrosmatic species the more posterior eth-
moturbinals are located within a recess formed by a
horizontal shelf of bone, the transverse lamina. This
is made up of two plates, ossifying in the posterior
lamina transversalis, which project medially from the
lateral ethmoidal plates of each side to articulate with
the wings projecting laterally from the vomer. It thus
divides the nasal cavity posteriorly into upper and
lower compartments. The upper compartment, hous-
ing the ethmoturbinals, is a capacious but blind ol-
factory recess lying below the cribriform plate and
usually prolonged posteriorly by a hollowing out of
the anterior surface of the body of the presphenoid
$\ldots$. The lower compartment, or nasophryngeal me-
atus, is respiratory in function, being continuous an-
teriorly with the inferior meatus and terminating pos-
teriorly at the posterior nasal aperture.

The transverse lamina is also visible in the longitudinal sections as a thin line of bone (fig. 43). In Rhombomylus, the lamina from the lateral ethmoidal plates appears to be fused to the vomer, instead of "articulating" with the latter as described by Moore. Although it is termed the transverse lamina, these plates in Rhombomylus are not horizontal, but are inclined. According to Moore (1981), the presence of the transverse lamina excludes the olfactory area from respiratory air currents; then, olfaction must depend on diffusion from the scent-laden inspired air or on the projection of puffs of air into the recess by sniffing. Therefore, the air within the olfactory recess will not be washed out during exhalation but is retained longer for olfactory analysis.

In addition to the transverse lamina, a thin, bony sagittal nasal septum divides the posterior upper compartment of the nasal cavity into two parts (fig. 42: 19-22). This vertical plate is also observed in longitudinal section (fig. 43). By its position, this septum is probably formed by the mesethmoid. A large contribution of the mesethmoid (also called the perpendicular plate) to the nasal septum is considered a derived mammalian condition and is found in several eutherian groups including rodents (Goodrich, 1930; de Beer, 1937; Novacek, 1993b). Anterior to section 19 , there is no such bony nasal septum in Rhombomylus. This is probably because the anterior part of the septum is cartilaginous, as in extant mammals. Posteroventral to the median nasal septum is a space that is probably the posterior extension of the olfactory cavity into the presphenoid region, as described in some rodents (Paulli, 1990c; Moore, 1981; Novacek, 1993b).

The roof of the posterior nasal cavity is mainly formed by the frontal. The frontal sinus is absent. Starting from section 21 or 22 , the cranial space for the olfactory lobes emerges dorsal to the posterior end of the nasal cavity. The two spaces are separated by the cribriform plate of the ethmoid. Crosssections through the olfactory lobe mark the narrowest region of the skull, which has a keyhole-shaped profile (fig. 42: 23-25).

In no section of the rostrum could we confirm the presence of a bony canal for the lacrimalnasal duct that presumably courses from the lacrimal foramen to the floor of the anterior nasal cavity. Because of breakage at the lacrimal region, the longitudinal sections of the left rostrum of IVPP V7492 do not include the lateralmost region of the nasal cavity. The lateralmost section starts at the level of the alveolus of the incisor (the tooth was removed for SEM study of microwear) (fig. 43). In longitudinal sections, the anteroposterior dimension of the ethmoturbinal can be better appreciated. The ethmoturbinals are 
located in the posterior nasal cavity and are oriented as oblique rows, with the dorsal plates being longer than the ventral ones. Laterally, the anteroventral edges of the ethmoturbinal are connected (fig. 43: sections 1-5), but medially, the edges of the endoturbinals are separated (fig. 43: sections 6 and 7). The ethmoidal origin of the turbinal can be confirmed by connection with the cribriform plate. The latter forms a roughly $50^{\circ}$ angle with the palatal plane of the skull and floors the olfactory fossa of the braincase. In sections 5 and 6 , the maxilloturbinals are observed. The vomer, the transverse lamina, and the bony nasal septum (mesethmoid) are all visible. The bony nasal septum is short and is ventral to the cribriform plate. The space under the median nasal septum is the posterior extension of the olfactory region. The highest point of the nasal cavity is immediately anterior to the anterior border of the cribriform plate.

The frontal sections again confirm the structures of the turbinals. They show that endoturbinal I and ectoturbinal 1 originate from the ethmoidal plate underneath the nasal and maxilla (fig. 44: sections 2 and 3 ) and are not from the cribriform plate. The rest of the ethmoturbinals are connected with the cribriform plate. In addition, the frontal sections reveal the open-ended incisor and its pulp cavity, the infraorbital canal, and roots of the cheek teeth.

BRAINCASE: Cross sections of the braincase and auditory region of Rhombomylus are based on IVPP V5266 and V5267 (figs. 4549). The anteromost section cuts through the braincase region corresponding to the cruciate fissure of the brain, which is the groove that separates the olfactory lobe anteriorly from the cerebral cortex posteriorly. It is the narrowest area of the skull, both internally and externally in Rhombomylus. The space for the olfactory lobe has a circular contour in cross section. The wall of the braincase immediately posterior to the olfactory fossa is formed primarily by the parietal. Because of breakage in both sectioned specimens, it is unclear which element forms the floor of the braincase in this region. The braincase expands posteriorly to reach its greatest dimension at the anterior end of the bulla or at the anterior portion of the glenoid fossa (sec- tions 5-8). The profile of the neocortex is roughly oval, wider than higher, but is not so transversely expanded. It indicates no significant expansion of the pyriform lobe of the cerebral cortex, being consistent with morphology of the endocranial cast (see below). The braincase for the anterior portion of the cerebrum is formed by the parietal, with partial contribution from the squamosal, particularly at the region shown in sections 5 and 6. Both the parietal and squamosal are thin, compared to those in leptictids (Novacek, 1986b). Posteriorly, starting from section 8, the squamosal is excluded from the braincase by the expanded mastoid of the petrosal. The basisphenoid is the main element to the floor of the braincase for the cerebrum. It is not clear from the sectioned specimens whether other bones contribute, or how much if any, to formation of the floor. The basisphenoid is flat and thin anteriorly at the level where the glenoid fossa begins (section 6). It gradually thickens posteriorly. Unlike the parietal and squamosal, the basisphenoid is not a solid bone; instead, it is quite spongy in its internal structure. In sections 8 and 9 , the basisphenoid bears a dorsal projection, but posterior to that projection, the dorsal surface of the basisphenoid is concave or flat.

The extensive petromastoid is the only bone forming the entire lateral wall of the posterior half of the braincase up to the foramen magnum. It extends anteriorly to the level of the midglenoid fossa and dorsally to meet the parietal. This expanded element can be roughly divided into an anterior and a posterior part. The anterior part is dorsal to the tympanic cavity; its medial side is vertical and confines a rectangular space in crosssectional view for the anterior cerebellum (sections 10-13). Still posteriorly, the internal auditory meatus notches the sidewall, so that the profile of the braincase becomes hexagonal (sections 14-17). The petromastoid is large but not solid; its internal space is divided by numerous septa into many small air sinuses. The posterior part of the petromastoid is the portion posterior to the bulla and the petrosal proper sections (18-24). This part is greatly inflated, kidney-shaped in cross section, and projected ventrally below the level of the basioccipital. The inflated mastoid region is hollowed and is braced in- 

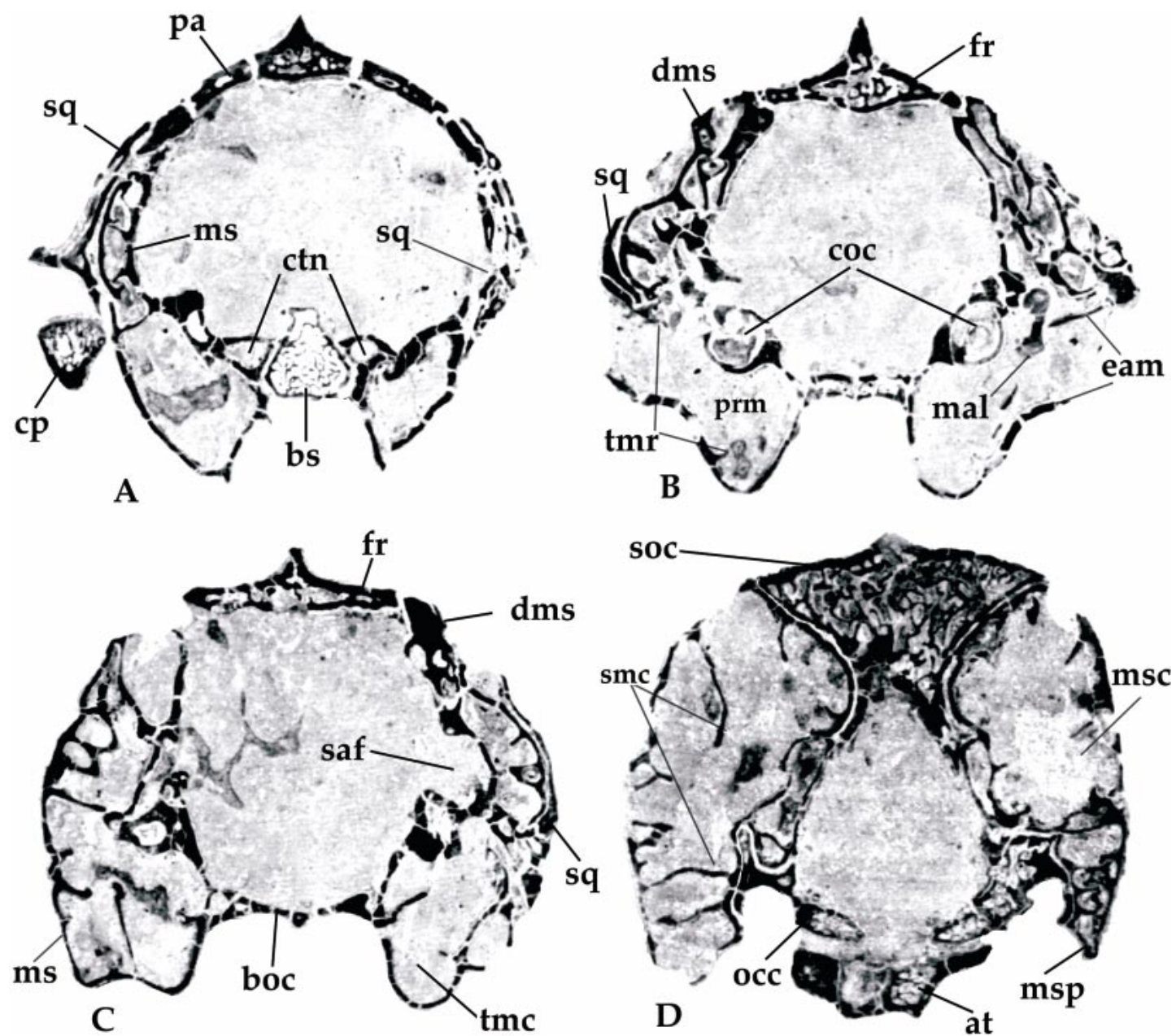

Fig. 45. Cross sections anteroposteriorly through the braincase and basicanium of Rhombomylus (IVPP V5266), showing some basic structures and terminology. at, atlas; boc, basioccipital; bs, basisphenoid; coc, cochlear canal; dms, dorsal exposure of mastoid of petrosal; eam, external acoustic meatus; fr, frontal; mal, malleus; ms, mastoid of petrosal; msc, mastoid cavity of petrosal; occ, occipital condyle; pa, parietal; prm, promontorium; saf, subarcuate fossa; smc, septum (septa) in mastoid cavity; soc, supraoccipital; sq, squamosal; tmc, tympanic cavity; and tmr, tympanic ridge (or ring) that holds the tympanium in life.

ternally by numerous bony septa that are oriented parallel either to the sagittal or to the frontal plane of the skull. Most of the septa in this region are not complete, with their free edges projected into the mastoid cavity. At the posterior end the bony septa are complete and bridge the lateral and medial walls of the cavity. The boundary between the petromastoid and the ectotympanic bulla is unclear in most sections through the bulla, but in sections 12 and 13 it appears to be lateral to the basisphenoid. A similar condition is also observed in IVPP V5267 (fig. 47). The mastoid cavity is confluent with the tympanic cavity. The squamosal does not contribute to the sidewall of the posterior braincase; instead, it overlaps the inflated mastoid laterally except the area where the petromastoid is exposed on the dorsal surface of the skull. The parietal is a thick bone bearing a sagittal crest and it roofs most of the braincase. It has an end-to-end contact with the petromas- 


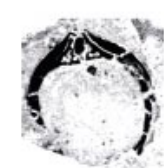

1
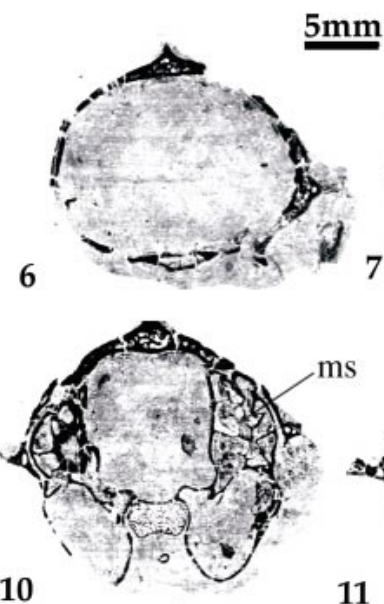

10

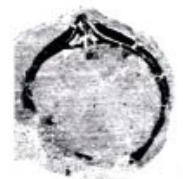

3

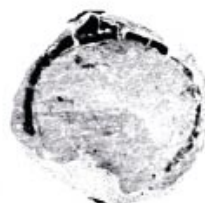

4

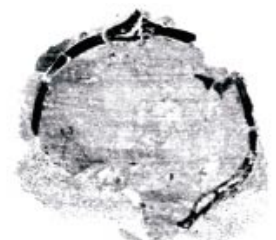

5
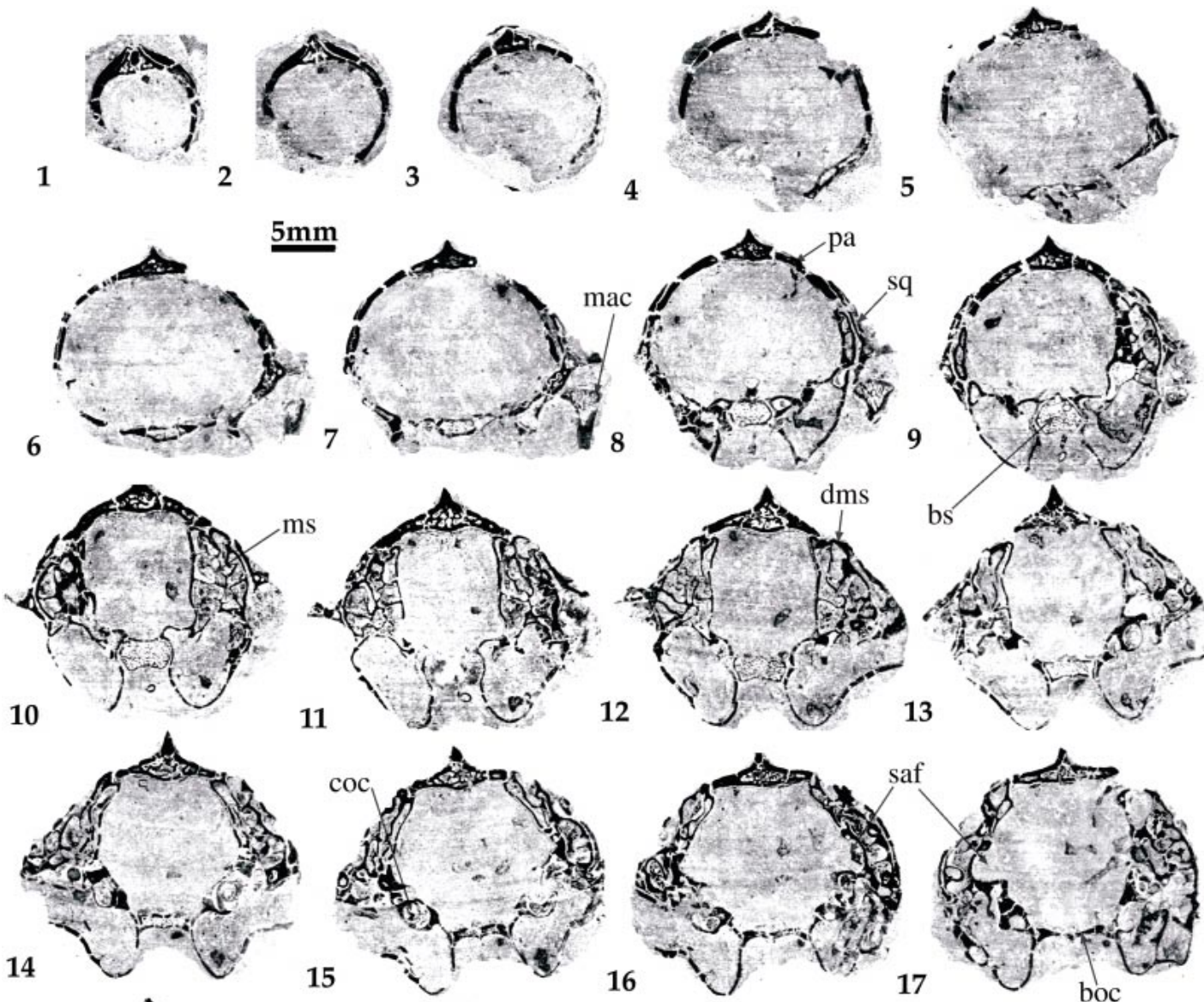

13
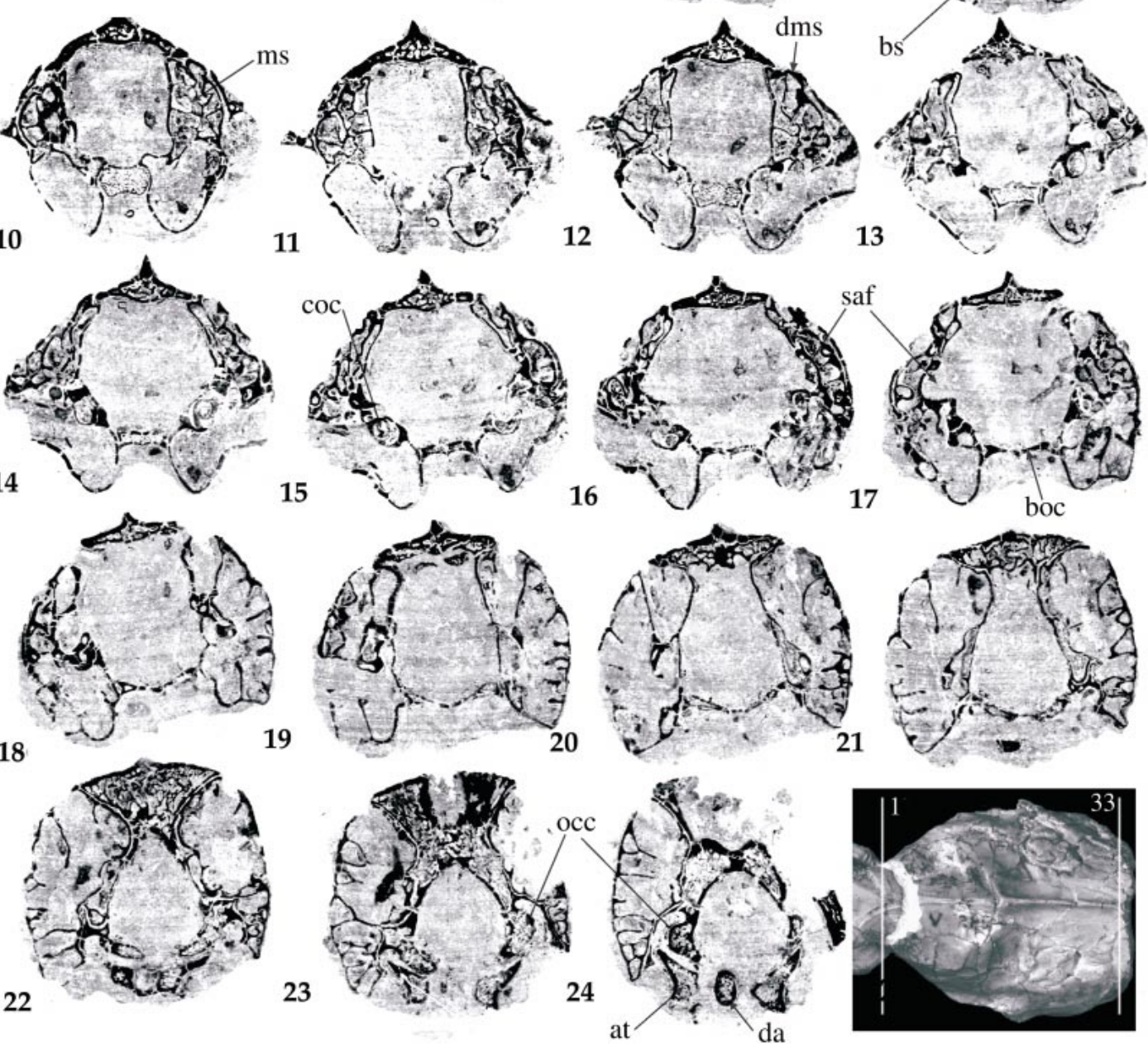

Fig. 46. Cross sections of the braincase and basicranium of Rhombomylus (IVPP V5266). The cutting sequence of the sections is shown in the figure at the lower right corner. Twenty-four sections out of 33 slides are illustrated. at, atlas; boc, basioccipital; bs, basisphenoid; coc, cochlear canal; da, dens of axis; ms, mastoid of petrosal; occ, occipital condyle; pa, parietal; saf, subarcuate fossa; and sq, squamosal. 


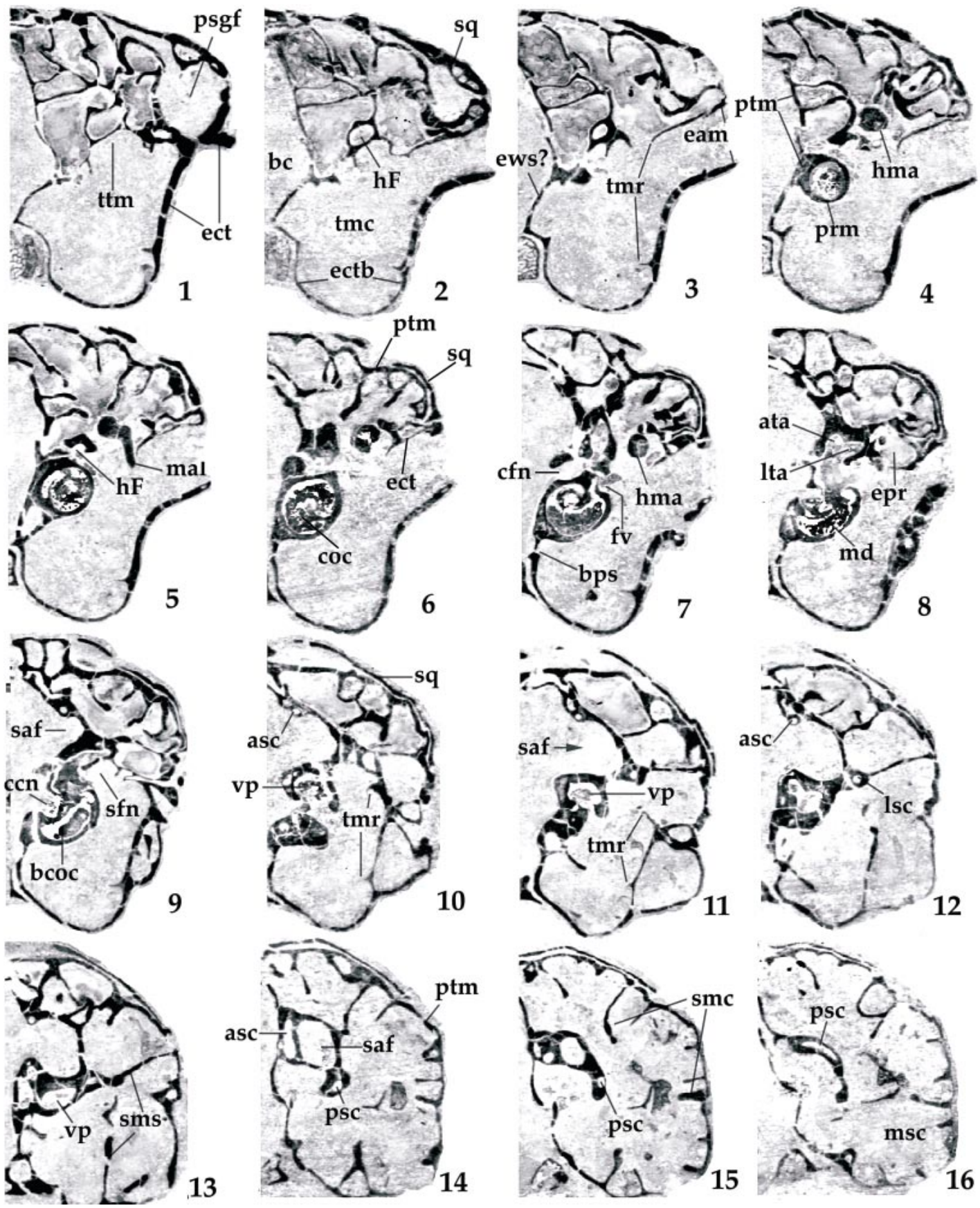

Fig. 47. Cross sections through the auditory region of Rhombomylus (IVPP V5267) (only the right side sections are illustrated), showing some inner and middle ear structures and related architectures; these sections are in a continuous sequence. asc, anterior semicircular canal; ata, anterior ampulla; bc, braincase; boc, basioccipital; bps, bulla (ectotympanic)-petrosal suture; bs, basisphenoid; ccn, canal for cochlear nerve cfn, canal for facial nerve; coc, cochlear canal; dms, dorsal exposure of mastoid of petrosal; eam, external acoustic meatus; ect, ectotympanic; ectb, ectotympanic bulla; epr, epitympanic recess; ews, epitympanic wing of the sphenoid; fc, fenestra cochleae (rotunda); fr, frontal; hF, hiatus Fallopii; hma, head of malleus; lsa, lateral semicircular canal; lta, lateral ampulla; mal, malleus; md, 
toid and overlaps the anterior part of the supraoccipital posteriorly. The braincase is closed posteriorly by the thick, internally spongy, supraoccipital as shown in sections 22 and 23 (fig. 46). The suture between the parietal and supraoccipital is clearly seen in several sections. The observation of the suture in the crosssections supports the identification of the supraoccipital, which differs from the previous idea that an interparietal is present in Rhombomylus (Li and Ting, 1985; fig. 43). The basioccipital starts roughly at section 15 and floors the central portion of the braincase. It borders the bulla anteriorly and the petromastoid posteriorly and becomes wider and more concave dorsally toward the foramen magnum; posteriorly, it ends at the occipital condyles. Near the foramen magnum, the suture between the petromastoid and the supraoccipital is complex. The occipital exposure of the petromastoid is extensive. The foramen magnum is completely surrounded by the occipital. From sections 22 to 24 , the atlas and dens of the axis were sectioned in IVPP V5266 (figs. 45, 46). The dens is about $2 \mathrm{~mm}$ long and oval in cross section, with its long axis vertically oriented.

Although the left side of the basicranium of IVPP V5267 is damaged, its right side is in better condition than that of IVPP V5267, and furnishes detailed morphologies of fine structures in the auditory region (fig. 47). Section 1 in figure 47 is approximately equivalent to section 11 in figure 46. Similarly, the air sinuses and septa are well developed in the petromastoid. Beginning from section 2 , the petrosal proper, by which we mean the structure consisting of the pars canalicularis and pars cochlearis, is in evidence. A canal, identified as the hiatus Fallopii that contains the greater petrosal branch of the facial nerve, is determined by its position at the anterior tip of the petrosal, lateral to the promontorium and anterior to the sulcus for the facial nerve. The canal has a greater diameter anteriorly than posteriorly. In slides 6 and 7 , the canal for the facial nerve, starting within the internal acoustic meatus, penetrates the petrosal at the level of the epitympanic recess as indicated by the head of the malleus (we assume the malleus is in its original anatomical position). In life, the epitympanic recess houses the articulation of the malleus and incus. Lateral to the promontorium, there is no clear evidence for a bony canal of the facial nerve.

The promontorium is completely cut through to reveal the cochlear canal. The apical section of the promontorium is nearly circular and shows that the promontorium is inflated and projects from the rest of the tympanic roof (section 4 in fig. 47). About two turns of the cochlear canal are estimated, which spirals clockwise around an axis that points anteroventrolaterally. This spiral is particularly visible in sections $4-10$. The modiolus and the primary osseous spiral lamina are exposed in section 8 . The secondary osseous spiral lamina is not very clear, but is probably present in the basal cochlear canal in slide 9. A narrow vestibular fissure and the development of the secondary osseous lamella are probably related to hearing of highfrequency air-conducted sounds (Meng and Fox, 1995 and references therein).

The medial border of the promontorium is not certain but is probably indicated at the bullar-petrosal suture as shown in section 7 . Given that suture, the medial margin of the promontorium is narrow. However, anteromedial to the promontorium there is an additional development of a bony structure that appears to be a canal on the ventromedial side of the promontorium in section 5. The ventral wall of the canal opens up in section 4 to form a broad, inclined plate that is continuous with the wall of the tympanic cavity. The plate extends anteriorly and contributes to the lateral floor of the midbraincase. By

\footnotetext{
$\leftarrow$

modiolus; ms, mastoid of petrosal; msc, mastoid cavity of petrosal; occ, occipital condyle; pa, parietal; prm, promontorium; psc, posterior semicircular canal; psgf, posterior socket of the glenoid fossa; ptm, petromastoid; saf, subarcuate fossa; sfn, sulcus for facial nerve; smc, septum (septa) in mastoid cavity; soc, supraoccipital; sq, squamosal; tmc, tympanic cavity; tmr, tympanic ridge (or ring) that holds the tympanium in life; and vp, vestibule proper.
} 

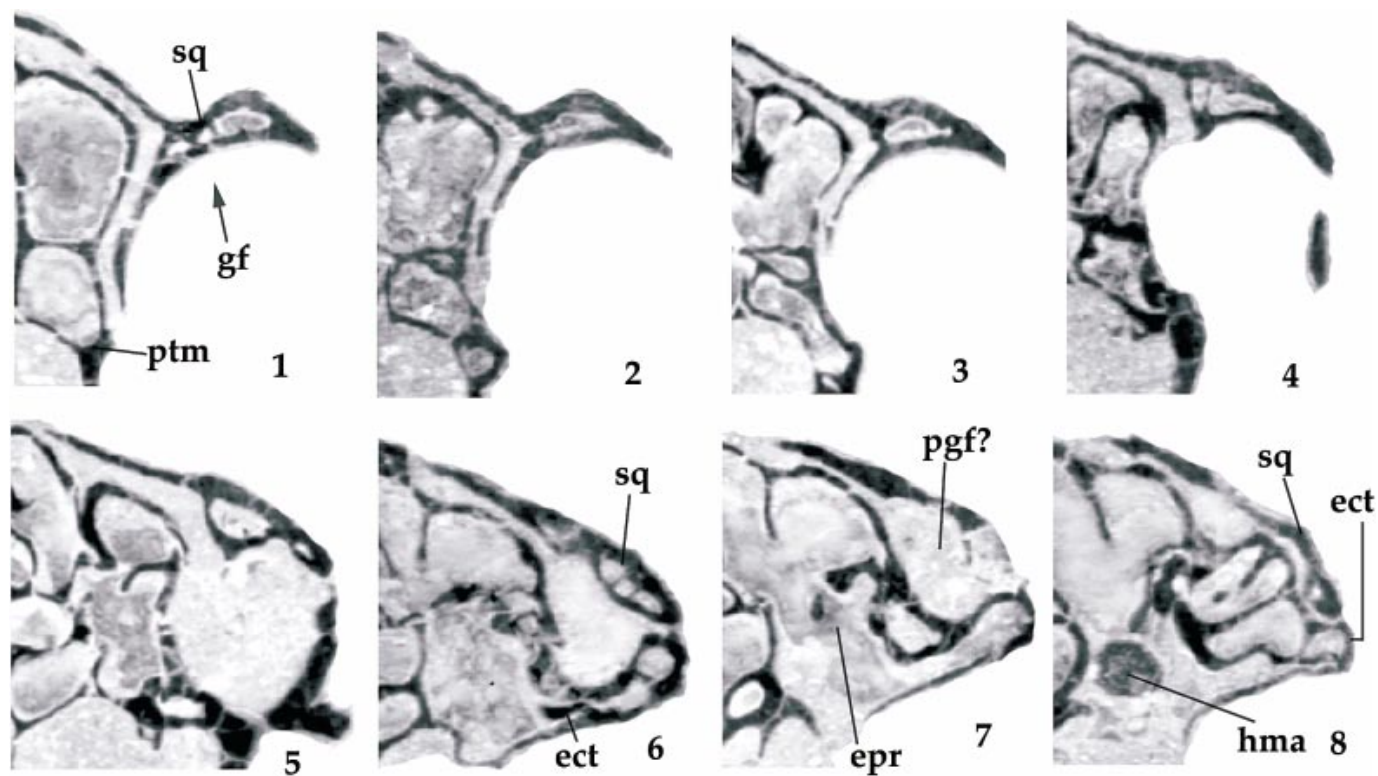

Fig. 48. Cross sections through the glenoid fossa and epitympanic recess of the ear region of Rhombomylus (IVPP V5267). ect, ectotympanic; epr, epitympanic recess; gf, glenoid fossa; hma, head of malleus; pgf, postglenoid foramen; ptm, petromastoid; and sq, squamosal.

position, the plate is similar to the epitympanic wing of the sphenoid as seen in erinaceomorphs (MacPhee et al., 1988). The cranial side of the promontorium is marked by the canal for the facial nerve and, ventral to it, the canal for the cochlear nerve. Although the mastoid region of the petrosal is inflated, the inner ear is normal. The vestibule proper is seen in a series of sections from 7 to 13 . The fenestra vestibuli opens into the vestibule and the rim of the fenestra is thicker than the rest of the promontorial

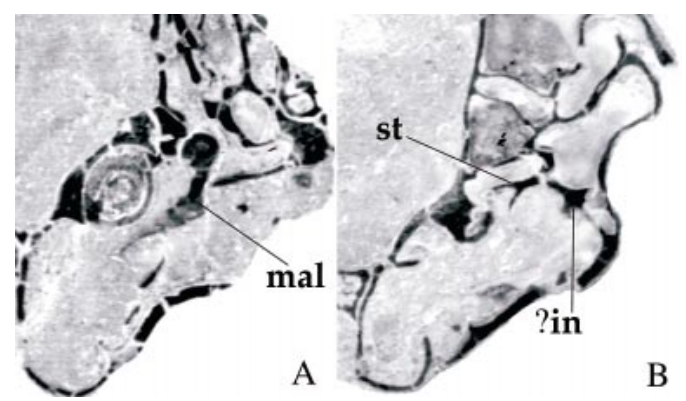

Fig. 49. Cross sections through the ear regions of Rhombomylus (IVPP V5266, V5267), showing the auditory region and ear ossicles. ?in, incus; mal, malleus; and st, stapes. wall. In general, the bony wall surrounding the vestibule is thicker than that of the promontorium containing the cochlea. The fenestra cochleae at the posterior end of the promontorium is transversely elongate (fig. 47: section 13). Confluent with, and dorsal to, the vestibule are the anterior and lateral ampullae, which continue distally as the semicircular canals. The gyrus of the anterior semicircular canal constitutes the edge of the subarcuate fossa as in other mammals (Meng and Fox, 1995; Fox and Meng, 1997). The posterior semicircular canal is exposed posteriorly within the mastoid region (fig. 47: sections 14-16).

The epitympanic recess is on the lateral side of the promontorium and occupied by the head of the malleus in sections 4-7 in IVPP V5267. The recess is floored by a lamina of the ectotympanic that forms the dorsal portion of the external auditory meatus. The partial roof of the recess is braced by many mastoid septa. The recess is confluent with the expanded mastoid space, so that the malleus-incus articulation must be partly suspended in life. Unlike many other mammals, in which the epitympanic recess is medially 
formed by the petrosal and laterally walled by the squamosal, the squamosal is excluded from the epitympanic recess by the expanded mastoid in Rhombomylus (fig. 47). The lateral wall of the epitympanic recess is instead completed by a combination of the ectotympanic and petrosal, as in rodents and lagomorphs (Novacek, 1986b).

The ectotympanic bulla is well developed and the tympanic cavity is spacious, within which there is no septum. The ectotympanic can be divided into two parts: one medial to the tympanic ridge and the other lateral to the ridge. The medial bullar part is thin and forms the floor of the tympanic cavity, whereas the lateral part is distinctively thicker and makes up the wall of the external auditory meatus. The tympanic ridge is a circular structure that supports the tympanic membrane. The maximum diameter of the circle formed by the ridge, at the position where the manubrium of the malleus is located (fig. 47: section 5), measures $5.8 \mathrm{~mm}$ in IVPP V5267. The dorsal part of the tympanic ridge is actually represented by the lip of the ectotympanic that makes up the medial end of the external auditory meatus. This part of the ectotympanic is a thin lamina with a sharp, free edge projecting ventromedially into the tympanic cavity. The projected part also floors the epitympanic recess. Judging from the position of the tympanic ridge, the tympanic membrane must have a high angle $\left(>70^{\circ}\right)$ to the horizontal plane of the skull.

Figure 48 shows some close-up images of the glenoid region and the epitympanic recess of the auditory region, which are of importance phylogenetically for gliroid mammals. Again, the squamosal overlaps the inflated petromastoid and constitutes the bulk of the glenoid fossa. The petromastoid also contributes to the medial and posterior walls of the glenoid fossa (sections 3-5). As seen externally, the fossa has a pocketed end confined by the squamosal dorsally and the ectotympanic ventrally. The depression continues backward as a canal between the squamosal and the mastoid. The canal is identified as the postglenoid foramen, which transmits the external jugular vein (Novacek, 1986b). The external auditory meatus closes the glenoid fossa posteriorly. Sections through the ear region (fig. 48) clearly show that the squamosal does not contribute to the formation of the epitympanic recess.

Discussion: The discussion is devoted to the following aspects that are related to the nasal cavity: the incisive foramen, Jacobson's fossa, vomer, ethmoid, and paranasal sinuses.

It is well known that mammals are characterized by development of the bony secondary palate. The palate forms the ventral floor of two nasopharyngeal passages that are separated by the vomer and open posteriorly as the choanae. The choanae are continued backwards by a fleshy false palate which ends opposite the epiglottis. During development, the secondary palate develops behind the original internal nostrils on the true palate by growth of the maxillae and palatines, which meet ventrally and join a vertical plate of the vomer. The original internal nostrils therefore open into the air passages enclosed by the palate, with only a pair of small incisive foramina (anterior palatine foramina or nasopalatine canals of Goodrich) remaining as parts of the original internal nostril communications (Goodrich, 1930). The vomeronasal organ (Jacobson's organ) (Jacobson, 1811) opens into the nasopalatine duct, which is a canal linking the oral and nasal cavities and is located caudal to the incisors in the anterior part of the hard palate within the incisive foramina (Broom, 1881, 1896a, 1896b, 1896c, 1897, 1902, 1915; Moore, 1981; Novacek, 1985; Poran, 1998; Giere et al., 1999; Keverne, 1999; SánchezVillagra, 2001). According to Goodrich (1930), therefore, the incisive foramina represent the anterior areas of the original nostrils, and small incisive foramina should be a derived mammalian feature. In lagomorphs and rodents these are more posteriorly located than in other mammals and are elongate in association with a marked expansion of Jacobson's organ (Novacek, 1993b). The large incisive foramina in gliroid mammals are probably secondarily achieved, as evidenced by the condition in Rhombomylus and other basal gliroid mammals such as Matutinia (Ting et al., 2002), Eurymylus (Sych, 1971), Heomys (Li, 1977), Mimotona (Li and Ting, 1985), and Sinomylus (McKenna and Meng, 2001).

The Jacobson's organ (= the vomeronasal organ, Jacobson, 1811; Bhatnagar and Reid, 
1996; Giere et al., 1999) occurs in the Amniota as a small sac, blind behind and opening in front toward the palatal surface at or near the internal nostril (Goodrich, 1930). In mammals, Jacobson's organ is a cigarshaped, oblong neuroepithelial and receptorfree epithelial tube situated bilaterally at the base of the anterior nasal septum (Wible and Bhatnagar, 1996). The organ appears to serve for smelling the liquid contents of the buccal cavity (Bhatnagar and Reid, 1996). In other studies (Coquelin et al., 1984; Halpern, 1987; Meredith and Fernández-Fewell, 1994) sensory input from the organ has also been implicated in the control of sexual behavior.

The Jacobson's organ and its related structures, particularly the Jacobson's capsule, have been used as data for mammalian phylogeny. This is particularly illustrated by de Beer's (1937: 398) review of the studies of the structures related to that organ by Broom (1881, 1896a, 1896b, 1896c, 1897, 1902, $1915,1926)$. Broom has examined the part of the paraseptal cartilage that encloses Jacobson's organ and forms Jacobson's capsule in various mammals. In monotremes, Jacobson's capsule forms anteriorly a complete cylinder with a "turbinal" projecting into its lumen. Broom found a simplification of the turbinal to an "outer bar" (the dorsolateral lamina we use here) in several mammalian groups, including marsupials, rodents, Xenarthra, Tubulidentata, Tupaia, Macroscelides, and Chrysochloris. These mammals are collectively called the "Archaeorhinata". Broom also distinguished another condition in which an outer bar is absent. This condition is found in the group "Caenorhinata", including Artiodactyla, Perissodactyla, Carnivora, Insectivora, Chiroptera, Hyracoidea, and Primates. (In regard to the taxonomy, note that rodents in de Beer's usage include what we call rodents and lagomorphs today. De Beer not only placed Lepus under the Rodentia (de Beer, 1937: 306) but also specifically stated (de Beer, 1937: 467) "Thus, in Rodentia, Lepus closely resembles Microtus." De Beer's Rodentia is apparently equivalent to our Glires and, therefore, Lagomorpha are included in the "Archaeorhinata".)

Because the Jacobson's capsule is formed by the paraseptal cartilage, it is unlikely to be preserved in fossils. However, the pre- maxilla that ventromedially supports the capsule can be preserved as the Jacobson's fossa, as we described in Rhombomylus. The shape of the fossa reflects at least to some extent the shape of the Jacobson's capsule and therefore the Jacobson's organ. As noted by Broom (1895: 478), "When the organ of Jacobson is well developed and much elongated, its bony support is correspondingly long, while when the organ is rudimentary the palatine process is short or absent. So that not only is there a close connection existing between the organ and the cartilage, but also an intimate association between the cartilage and the supporting bone." Novacek, based on several studies (Spatz, 1964; Kuhn, 1971; Zeller, 1987), also mentioned that the paraseptal cartilage forms an elongated trough posterior to the nasopalatine duct and that the trough is $\mathrm{C}$-shaped in cross section, housing the Jacobson's organ.

Judging from the distinct shape of the Jacobson's fossa, we may postulate that the Jacobson's capsule is well developed and the Jacobson's organ is elongated in Rhombomylus. A similar condition is probably present in other basal gliroid mammals that have the elongate incisive foramina. The condition of Rhombomylus is closer to that of rodents than of lagomorphs. In lagomorphs, the Jacobson's fossa is further elongated and its dorsolateral processes are more extensive. In other mammals (didelphids, primates, carnivores, perissodactyls, and artiodactyls), the processes are less developed and the fossa is relatively shallow and short. Therefore, a well-developed Jacobson's fossa and an elongated Jacobson's organ appear to be gliroid synapomorphies.

The ethmoid and the repositioned vomer are two neomorphic elements in the mammalian nasal region, as compared with the typical reptilian condition (Goodrich, 1930). The ethmoid consists of a perpendicular plate (with its superior projection, the crista galli), cribriform plate, and two masses of ethmoturbinals attached to the lateral plates. The ethmoid thus contributes to the roof and posterior parts of the lateral walls of the nasal cavity, to the nasal septum, and to most of the floor of the olfactory fossa of the cranial cavity. The lateral surface of the lateral plate is opposed to the posterosuperior part of the 
medial surface of the maxilla and the adjacent surface of the palatine bone, while from its medial surface are the ethmoturbinals (Allen, 1882; Moore, 1981). The area of the olfactory mucosa in mammals is increased by the development of the ethmoturbinals which form the main part of the labyrinths of the ethmoid. The mammalian turbinals serve to increase the efficiency of the warming, clearing, and moistening actions of the nasal mucosa, as well as to enhance the sense of smell. The terminology of the turbinals has been reviewed by Moore (1981) and Novacek (1993b). As shown in the detailed documentation of the ethmoturbinals in major mammalian groups by Paulli (1900a, 1900b, 1900c), the number, size and shape of these elements vary widely from species to species. Elaboration of these structures is associated with the degree of olfactory acuity (Dieulafe, 1906; Novacek, 1993b) and may be phylogenetically informative, although a correspondence between turbinal variation and phylogenetic pattern (Weber, 1904; Gregory, 1910) has not been compelling (Novacek, 1993b).

In summarizing Paulli's investigations, Moore (1981) pointed out that the typical ethmoid of rodents is similar to that of insectivores in that there are four endoturbinals, with the lamella of the second split to form two olfactory plates. This combination is seen in several other mammals, including chiropterans, hyracoids, some primates, and many carnivorans. But there are five endoturbinals in didelphid marsupials, seven in Tachyglossus, and three in Ornithorhyncus, which makes it difficult to polarize the states of the ethmoturbinal in mammals (Novacek, 1993b). The morphology of Rhombomylus suggests that the four-endoturbinal condition is probably primitive for gliroid mammals, and possibly for mammals as well. According to Moore (1981), the number of ectoturbinals in rodents is also low and they are arranged in a single row. Moreover, the olfactory region is prolonged posteriorly by a hollowing out of the body of the presphenoid. All these conditions are already present in Rhombomylus.

However, differences do exist. Unlike those mammals in which the two split olfactory plates of endoturbinal II inroll in the same direction (Paulli, 1900a, 1900b, 1900c; Moore, 1981; Novacek, 1993b), those of Rhombomylus inroll in opposite directions. In addition, all lamellae of endoturbinals in extant mammals as illustrated by Paulli (1900a, 1900b, 1900c; see also Moore, 1981, and Novacek, 1993b) inroll ventrally, whereas in Rhombomylus entoturbinals III and IV fold dorsally. In order to understand the phylogenetic significance of the turbinals, however, a more thorough investigation of the nasal cavity in early mammals is needed.

Another common feature of the skull in placental mammals is the presence of paranasal air sinuses. These pneumatisations are essentially extensions of the nasal cavity within the bones immediately adjacent to the nasal cavity. Commonly the paranasal sinus may be respectively called maxillary, frontal, or sphenoidal sinuses according to the bones the sinuses occupy. In some cases the sinuses can extend farther into other cranial bones such as the nasal and lacrimal. The studies of the nasal region of extant mammals by Paulli (1900b, 1900c) remains the most extensive accounts of the subject. According to Paulli, there is no evidence of pneumatisation in the skull of the monotremes and marsupials studied, which suggests that absence of such sinus is a primitive condition for mammals, although a maxillary sinus may have already occurred in some cynodonts (Kemp, 1979). The maxillary sinus is the only paranasal sinus present in insectivores and bats and occurs widely in most other placental mammals. It is therefore considered a primitive condition for placental mammals, although corroborative evidence for the primitive nature of the maxillary sinus would require study of the phylogenetic history of nasal pneumatisation, which has been unfortunately prevented by almost total lack of fragmented or sectioned skulls of fossil mammals (Moore, 1981). For those extant forms that have various paranasal sinuses, the number and extent of the sinuses vary greatly from one species to another, as well as within species or even between the two sides in the same individual. But the number of the sinuses is usually less than that of the intervals between the lamellae of the ethmoturbinals.

The maxillary sinus is the only chamber 
broadly present in rodents and lagomorphs. The sinus appears to be closely related to total body size, with the chamber being small or even completely lacking in smaller species, whereas in larger forms it may extend beyond the maxilla into the frontal bone (Moore, 1981; Novacek, 1993b). Additional sinuses are developed in some large forms such as the porcupine and capybara, which is probably a derived condition in gliroids. In Rhombomylus, there is no indication of any paranasal sinus except for the possible existence of the maxillary sinus, so that the nasal cavity remains relatively simple. This finding supports the hypothesis that the presence of the maxillary sinus is a primitive condition for placental mammals.

The function of paranasal sinuses has long been uncertain. It has been postulated that the sinus may (1) reduce the weight of the head; (2) be functionless, having arisen incidental to the evolutionary changes that have taken place in the shape of the mammalian cranium (Weidenreich, 1941); (3) allow the area of olfactory epithelium (turbinal) to be increased and the olfactory sense thus improved (Negus, 1958); (4) act as resonators; and (5) prevent heat loss from the nasal cavity (see Moore, 1981, for a more detailed discussion). Moore (1981) concluded that it is by no means certain that the sinuses serve the same functions in various mammals. The sinuses may have a combination of functions outlined above. For instance, they may serve simultaneously to reduce the weight of the head, to themoregulate the heat of the nasal cavity, and to increase the capacity of the nasal cavity for expanded turbinal.

\section{ENDOCRANIAL CASTS}

Several studies of endocranial casts of fossil mammals carried out around the turn of the last century (Marsh, 1874; Palmer, 1913; Moodie, 1916, 1922; Black, 1920) covered an array of taxa, including perissodactyls, artiodactyls, rodents, insectivores, carnivores, and primates. Since then, related studies have been continued on a variety of Mesozoic and Tertiary mammals (Simpson, 1927; Radinsky, 1973, 1974, 1975, 1976, 1977, 1981; Kielan-Jaworowska, 1984c; Novacek, 1982a;
Remy, 1999; Mödden and Wolsan, 2000; Saveliev and Lavrov, 2001). Brain evolution has been important in the study of mammals (Hofman, 1982; Deacon, 1990; Clark et al., 2001; de Winter and Oxnard, 2001), and brain morphologies of extant mammals have been used for phylogenetic reconstruction (Johnson et al., 1982a, 1982b, 1984, 1994; Kirsch, 1983; Kirsch and Johnson, 1983). In general, however, characters from the brain morphology for phylogenetic reconstruction of mammals are not very popular. This is partly attributable to sparse preservation of this important organ of the vertebrate body in fossils. Although many endocranial casts of various fossil mammals have been studied, the focusing issue was the evolution of the brain size and general shape. When detailed documentation in both fossil and extant mammals becomes available, endocranial morphology may prove to be a useful source for phylogenetic reconstruction of mammals.

DESCRIPTION: Three endocranial casts were prepared by peeling off the skull bones. IVPP V5286 and V7486 represent adult individuals in which all molars are fully erupted. They are significantly larger than IVPP V7487, which is probably a young individual. IVPP V5286 and V7487 were slightly distorted during preservation so that the cast is not symmetrical. The olfactory lobe is broken in both IVPP V7486 and V7487 but is preserved in IVPP V5286. The widest region of the endocranial cast between the extremities across the neocortex is $19.4 \mathrm{~mm}$ in IVPP V7486, $18.1 \mathrm{~mm}$ in V5286, and $15.5 \mathrm{~mm}$ in IVPP V7487. The length from the cruciate fissure to the posterior edge of the cerebellum is $27.2 \mathrm{~mm}$ in V7486, $26 \mathrm{~mm}$ in V5286, and $21.8 \mathrm{~mm}$ in V7487.

As shown in IVPP V5286, the olfactory lobes are sizable in comparison with the cerebral cortex (fig. 50) but are not transversely expanded as in Leptictis (Moodie, 1922; Novacek, 1982a, 1986b). The transverse cruciate fissure marks the boundary between the olfactory lobes and the cerebrum; it is broad, in contrast to a narrower, sharper fissure in Leptictis.

The cerebrum is heart-shaped in dorsal view (figs. 50, 51). In IVPP V5286 and V7486 the surfaces of the cerebrum casts are largely featureless, showing no convolutions 


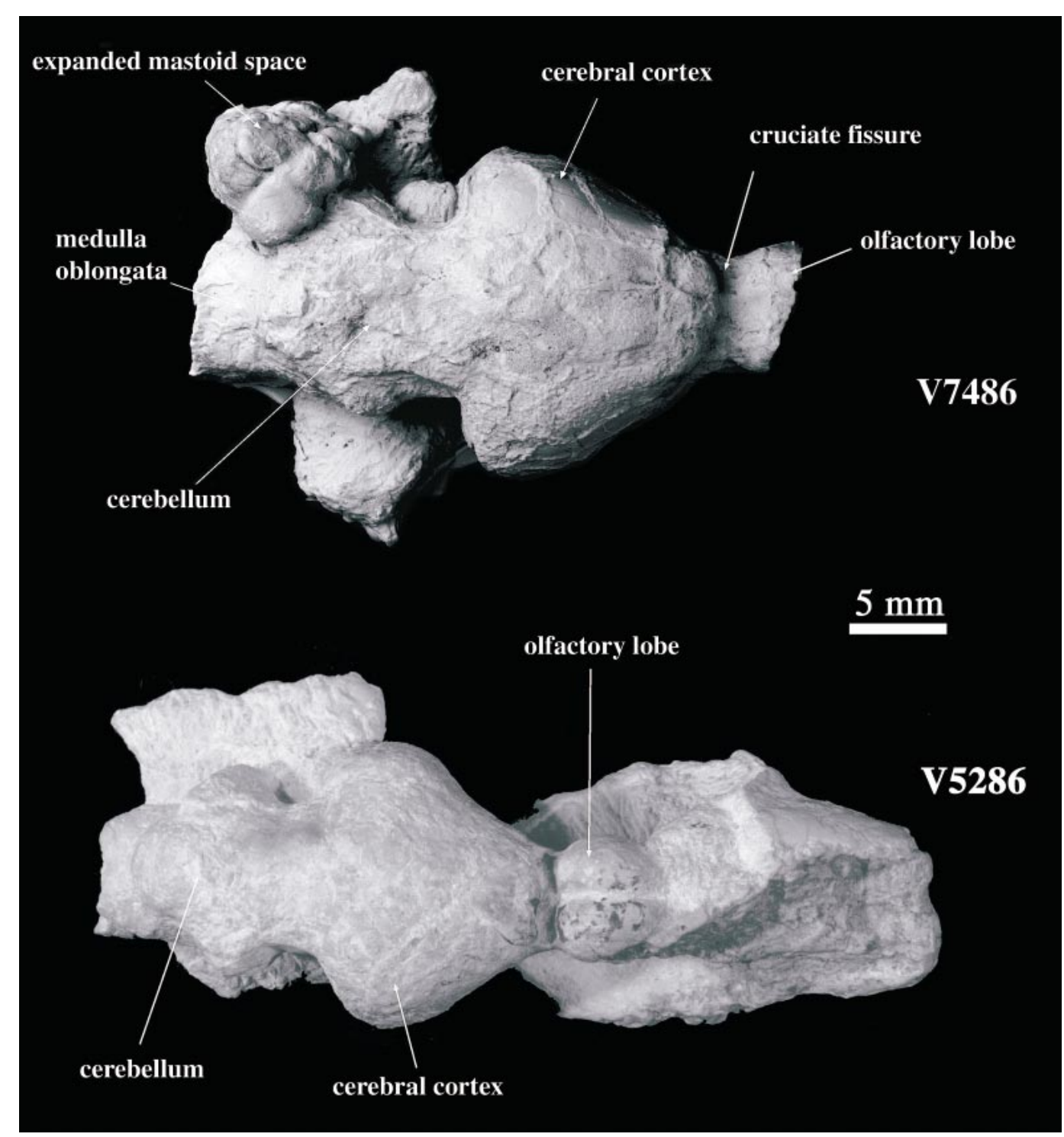

Fig. 50. Dorsal views of endocranial casts of Rhombomylus (IVPP V7486, V5286).

or grooves, such as the rhinal fissure. A similar condition is present in endocranial casts of some early rodents (Moodie, 1922; Wood, 1937). In IVPP V7487 a faint longitudinal fissure along the longitudinal axis of the cast indicates the course of the sagittal sinus. Also in IVPP V7487 two faint grooves diverge from the posterior end of the sagittal groove and extend posterolaterally (fig. 51). These two grooves probably indicate the transverse sinus, which marks the boundary between the cerebrum and cerebellum. Each groove ends laterally as a sedimentary cast of a small foramen, suggesting the exit for a branch of blood vessel to the skull roof. The cerebrum and cerebellum are relatively small compared to those of other mammals, such as Leptictis (Novacek, 1986b) and ischyromids (Wood, 1937). Because of the relatively unexpanded cerebrum, the brain segment posterior to the grooves of the transverse cerebral fissure is relatively narrow and long. 


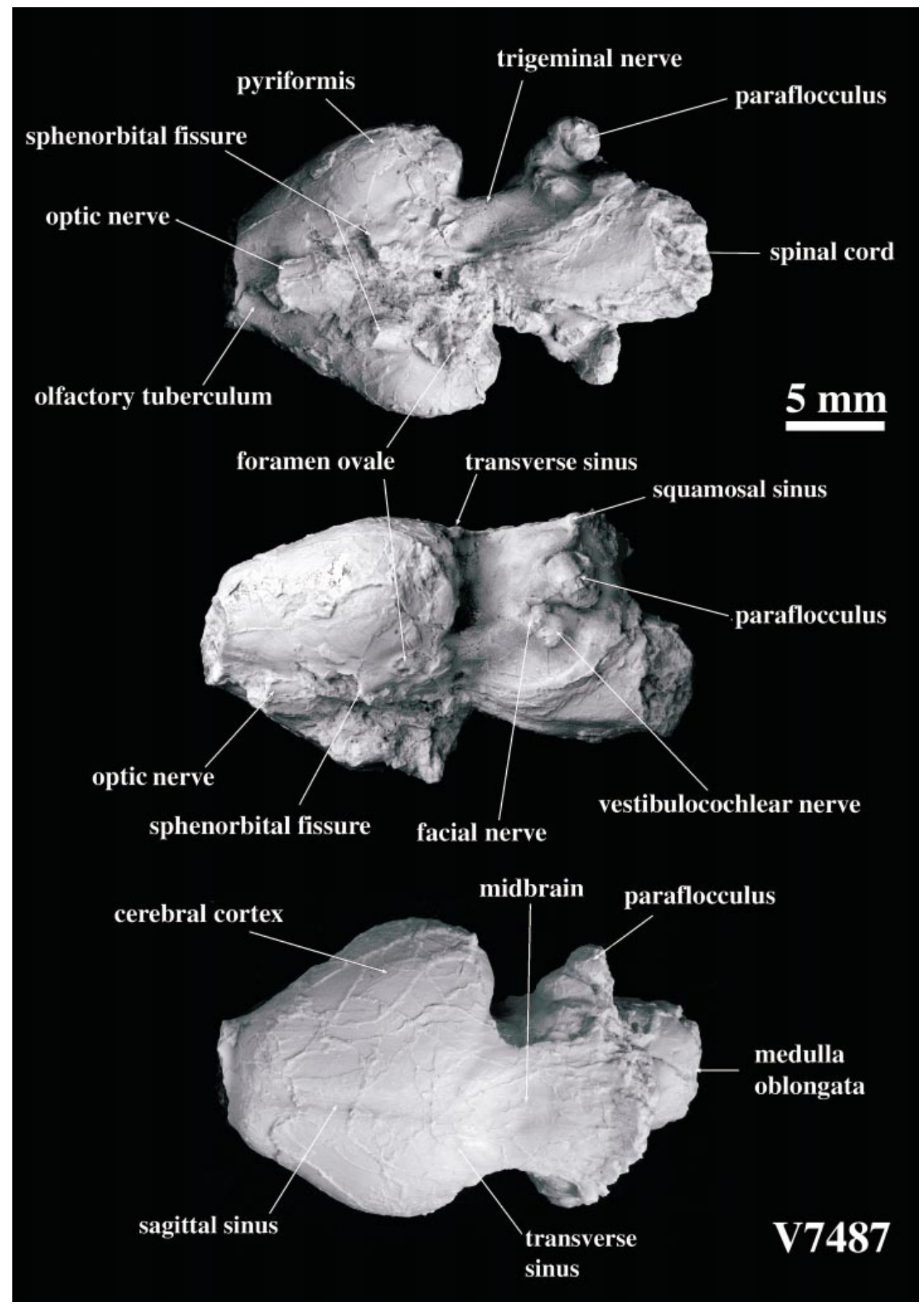

Fig. 51. Ventral, ventrolateral, and dorsal views of the endocranial cast of Rhombomylus (IVPP V7487). 
In all the three specimens, the dorsal surfaces of the midbrain and the cerebellum are flat and show no convolution. Pyriform lobes are not significantly inflated. Because of lack of the rhinal fissure, which separates the pyriform lobe from the cerebral cortex (neocortex) dorsally, the relative sizes of the two parts are uncertain. However, it is clear that both parts are not significantly expanded. As part of the paleocortex, the pyriform lobe is usually prominent in mammals having a keen sense of smell (Hildebrand, 1995).

On the ventral side, the area for the chiasma ridge was broken (fig. 51). The casts for the optic nerves are prominent in IVPP V7487. At the point of their emergence from the endocranium, the roots of the optic nerves appear unseparated from each other by a bony lamina, in contrast to the condition of Leptictis. This is consistent with the optic foramina seen in the skull. The cast of the sphenorbital fissure (= sphenorbital foramen of Butler, 1956; sphenoidal fissure of Wahlert, 1974) is preserved on each side in IVPP V7487. The sphenorbital fissure commonly conveys the oculomotor (III), trochlear (IV), and abducens (VI) nerves as well as the ophthalmic division and part of the maxillary division of the trigeminal (V) nerve. Posterolateral to the sphenorbital fissure is the cast for a large foramen ovale, which usually transmits the mandibular branch of the trigeminal nerve. Most of the eminence corresponding to the pituitary fossa was broken; some peripheral parts indicate an oval protuberance.

The brainstem posterior to the cerebrum is narrow. Immediately posterior to the foramen ovale is a low ridge that forms the lateral edge of the cast of the brainstem in ventral view. This ridge is interpreted as the cast of the trigeminal nerve, which originates anterior to the casts for the facial and cochlear nerves and extends anteriorly to the foramen ovale and foramen lacerum anterius. The ventral surface of the brainstem is floored by the basisphenoid and basioccipital and is smooth. Between this smooth area and the impression made by the petrosal bone is a blunt, curved ridge. This ridge is interpreted as a cast of a groove between the petrosal and the basioccipital. The lateral surfaces of the brainstem are molded in a characteristic fashion by the petrosal bones. In the middle of the area is the prominence corresponding to the internal acoustic meatus, which is divided into two parts: the anterodorsal one is the cast of the facial (VII) nerve, and the posteroventral one is that of the vestibulocochlear (VIII) nerve (fig. 51). At the dorsal area is a remarkable eminence corresponding to the subarcuate fossa of the petrosal (or the flocculus of the cerebellum). The distal portion of the eminence is more expanded than is its base, reflecting the shape of the subarcuate fossa. In addition to the endocranial casts, the cast of the inflated mastoid cavity is also revealed in IVPP V7486. The cast is divided into small sections by bony septa.

Discussion: The endocranial casts of Rhombomylus are compared to those of other Tertiary eutherians (Black, 1920; Moodie, 1922; Radinsky, 1973, 1974, 1975, 1976, 1977, 1981; Novacek, 1982a, 1986b; Remy, 1999; Mödden and Wolsan, 2000; Saveliev and Lavrov, 2001) and a variety of endocranial casts of living mammals made by Meng, including monotremes, marsupials, and representatives of most placental orders. The endocranial casts of Rhombomylus are more derived than those of didelphid marsupials in having a transversely expanded neocortex and relatively smaller olfactory lobes. Compared to other eutherian mammals, such as Leptictis (Novacek, 1986b) and Tupaia (Poonkhum et al., 2000), the endocranial casts of Rhombomylus display some primitive features. These include relatively large, but not transversely expanded, olfactory lobes, small and unconvoluted cerebrum and cerebellum, relatively narrow, long cerebrum (probably including the midbrain), and lack of the rhinal fissure. It is also more primitive than that of Ischyromys (Wood, 1937) in having a less inflated cerebral cortex. On the other hand, the Rhombomylus endocranial casts may have some derived eutherian conditions, such as the confluent roots of the optic nerves. However, because endocranial morphologies have not been systematically studied, it is difficult to incorporate them in a phylogenetic analysis. Therefore, we do not include brain characters in our phylogenetic analyses. 


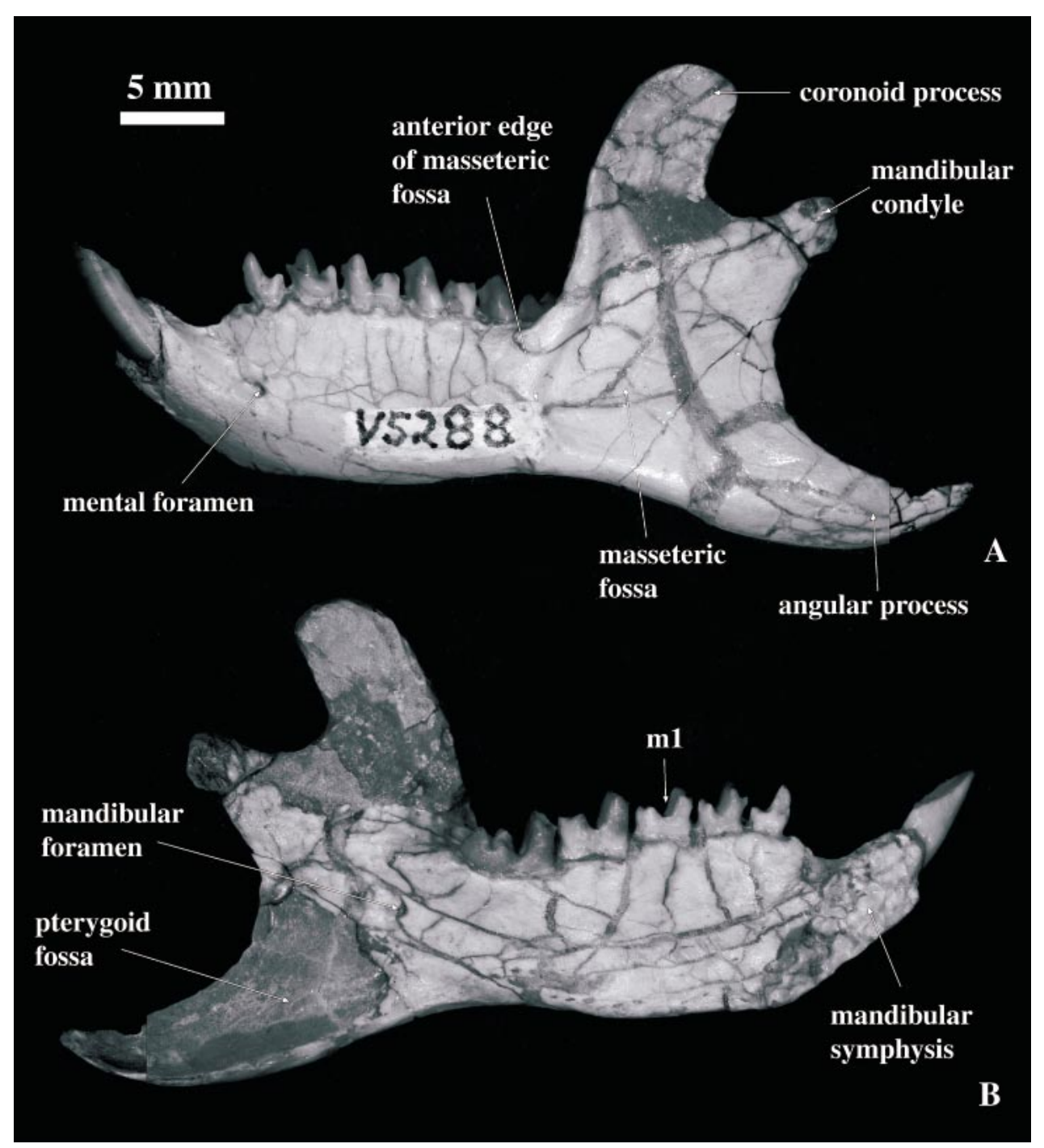

Fig. 52. Lateral and medial views of the mandible of Rhombomylus (IVPP V5288).

\section{MANDiBLE}

DESCRIPTION: Complete mandibles in association with skulls are preserved in several specimens (figs. 52, 53). These represent individuals of different ages. The symphysis of the mandible is extensive but is not fused in any case. This indicates certain relative movement of the two mandibles in life, which may be beneficial for unilateral transverse mastication. The bottom of the mandible is convex ventrally under the cheek teeth, with the deepest point below the $\mathrm{m} 2$, and is concave under the coronoid process.
The diastema is shorter than that of the upper jaw; its absolute and relative sizes increase with age. Usually, the diastema is smoothly curved, but in IVPP V5278, the oldest individual of the collection, the dorsal border of the jawbone anterior to the $\mathrm{p} 3$ forms a steep step (fig. 53D). On the lateral surface of the jaw, there are usually two small mental foramina: one below p3 and the other anteroventral to $\mathrm{p} 3$. In some individuals there may be only one foramen ventral to $\mathrm{p} 3$, or three, with the third one below p4 (IVPP V7570.2).

The coronoid process is relatively large 
compared to those of other gliroid mammals. In adults the ascending ramus starts lateral to the m3. Its anterior edge is nearly vertical and is slightly thickened. The coronoid process is smoothly rounded anterodorsally. Posteriorly, the process is hooklike. The masseteric fossa is broad and shallow, and does not have distinct dorsal and ventral masseteric crests. The anterior edge of the fossa is at the level of the $\mathrm{m} 3$ talonid (figs. 52, 53).

The angular process lies in the same plane of the cheek teeth and incisor; therefore, it is sciurognathous. The process is unusually long and is pointed at its end, in contrast to a short, blunt one in most gliroid mammals. While the mandibular condyle is in the resting position in the glenoid fossa and the cheek teeth are engaged, the posterior tip of the angular process reaches posteriorly to the level below the mastoid process (fig. 25). The lateral surface of the angular process is convex and the tip of the process bends medially. The posterior margin of the mandible between the process and the condyle has a broad semicircular profile.

The condyloid process is much higher than the tooth row. Its head is ovoid, slightly wider than long. The lateral portion is larger and less curved than the medial one. An interesting feature is that, as best shown in V5278, the medial tip of the condyle bends ventrally to form a hooklike structure. The ventral surface of the hooked area may serve as part of the area for origination of the lateral pterygoid muscle. The dorsal surface of this bent area is apparently the facet that contacts the medial wall of the glenoid fossa. On the medial side of the mandible, a blunt ridge runs from the condyle to the posterior end of the $\mathrm{m} 3$. A distinctive mandibular foramen is ventral to midpoint of the ridge. The medial surface of the angular process is the pterygoid fossa, a large concave area that is ventrally bounded by a prominent ridge. The size and depth of the fossa suggest a capacity for holding a large bulk of muscle.

It is clear that during postnatal ontogeny, the mandible increases its depth and length and becomes more robust with age. However, other mandibular structures, such as the angular process, coronoid process, mandibular condyle, diastema, length of the tooth raw, and position of the masseteric fossa, undergo very little change proportionally with age. This suggests that the function of the jaw in the individuals of different ages represented by our collection is similar. See the functional analysis of mastication for discussion of the mandible.

\section{PostCRANIAL ElEMENTS}

Vertebral Column: There are several segments of articulated vertebrae in our collection, but most of them preserve only parts of the vertebral column. IVPP V5268 has the last cervical vertebra and first three thoracic vertebrae (fig. 54A). The cervical vertebra has no diapophysis for ribs, and its body has no midventral keel; otherwise, it is not easy to distinguish it from anterior thoracic vertebrae. The body of each thoracic vertebra bears a distinct midventral keel, which is especially prominent on the second one. The dipophyses of the thoracic vertebrae are stout. The spinal processes in all four vertebrae were damaged. Their neural arches increase in length posteriorly, whereas the diameter of the vertebral canal slightly decreases posteriorly.

We do not know exactly how many lumbar vertebrae Rhombomylus has. IVPP V5269.13 has six successive lumbar vertebrae preserved and articulated posteriorly with the sacral series (figs. 54D, 55A). Some lumber vertebrae are also preserved in other specimens (figs. 54B-C, 55B). The thoracolumbar transition cannot be determined, as no posterior thoracic vertebra is identified in our collection. The anterior lumbar vertebrae of IVPP V5269.1 are damaged. The centra of the lumbar vertebrae are longer than wide and become more robust and thicker posteriorly. The ventral surfaces of the vertebral centra are concave and bear midventral keels that weaken posteriorly. The transverse processes of the lumbar vertebrae are wingshaped and are anterolaterally directed; they extend along the anterior border of the vertebral centrum. The base of the process is nearly as long as the centrum. The processes of the lumbar vertebrae become longer and broader toward the sacrum. The neural spines were damaged on all lumbar vertebrae. The spines are anterodorsally inclined and become nearly vertical and more slender pos- 


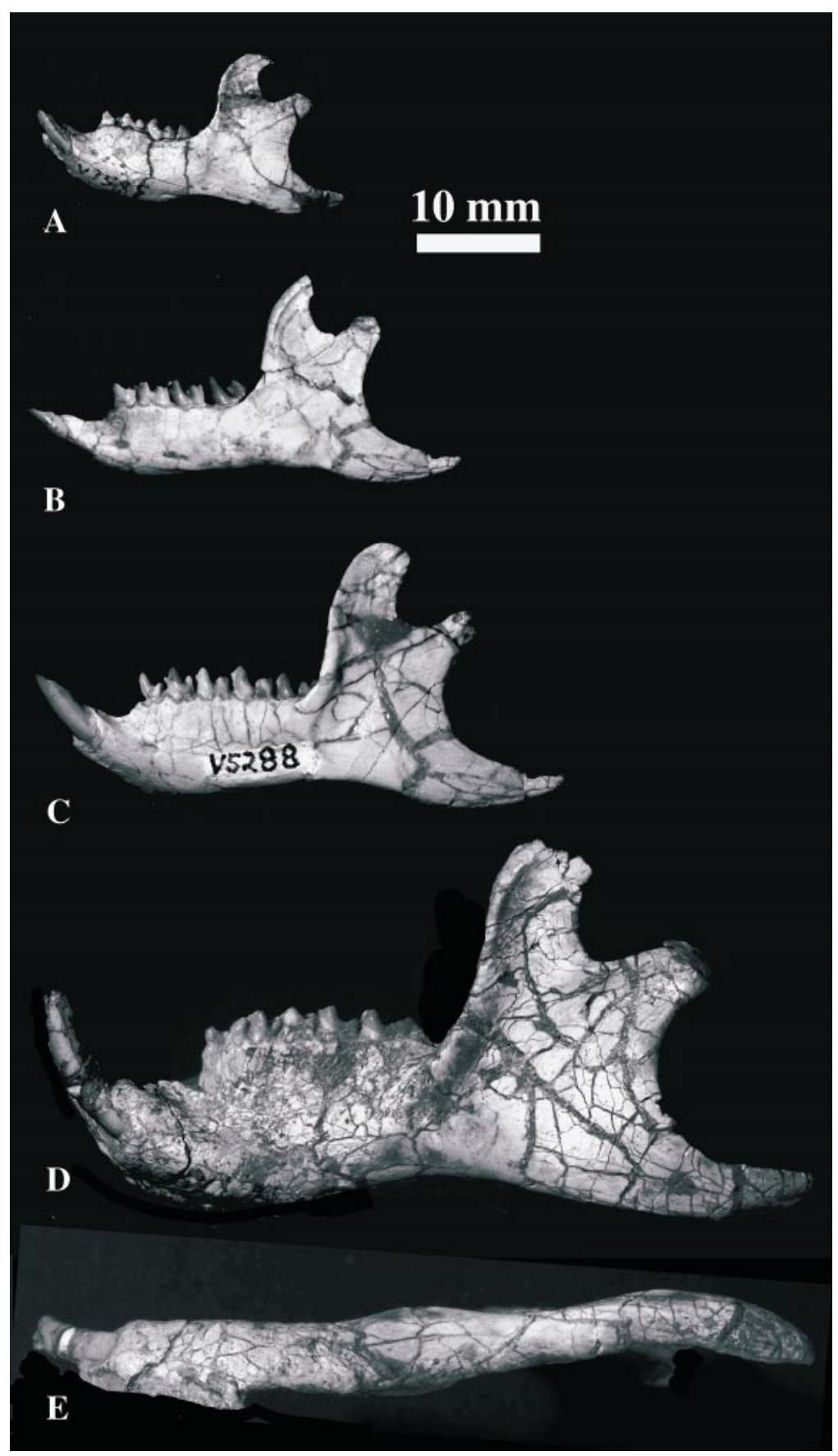

Fig. 53. A-D, Lateral views of mandibles from individuals of different ages of Rhombomylus (IVPP V7585, V5280, V5288, V5278); E, ventral view of the mandible (IVPP V5278). The angular process of V5280 (B) is reconstructed based on other specimens. 


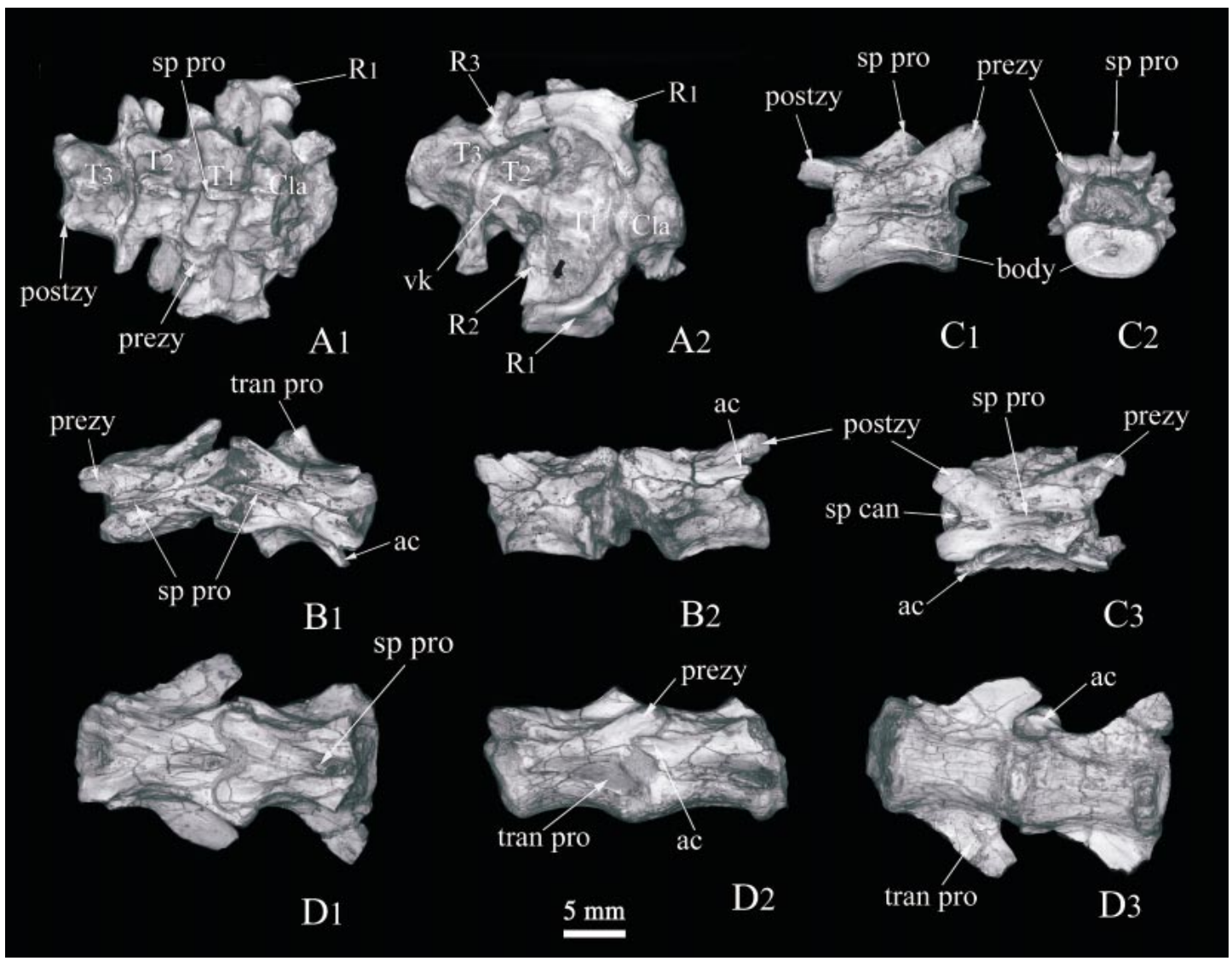

Fig. 54. Cervical, thoracic, and lumbar vertebrae of Rhombomylus (A, IVPP V5268; B, IVPP V5272.1; C, IVPP V5272.1; D, IVPP V5269.2). A, Last cervical and first three thoracic vertebrae and attached ribs: A1, dorsal view; A2, ventral view; B, two anterior lumbar vertebrae: B1, dorsal view; B2, lateral view; C, a middle lumbar vertebra: C1, lateral view; C2, anterior view; C3, dorsal view; D, two middle lumbar vertebrae: D1, dorsal view; D2, lateral view; D3, ventral view. ac, accessory process; body, vertebral body; Cla, last cervical vertebra; prezy, prezygapophysis; postzy, postzygapophysis; R1, first rib; R2, second rib; R3, third rib; sp can, spinal canal; sp pro, spinal process; T1, T2, T3, first three thoracic vertebrae; tran pro, transverse process; and vk, ventral keel of the vertebra.

teriorly. On the middle lumbar vertebrae (L3-5 in IVPP V5269, assuming six lumbar vertebrae in Rhombomylus) a triangular surface slopes and widens posteriorly from the back of the neural spines. The two lateral edges of the triangular surface converge to the medial margins of postzygapophyses. The prezygapophyses face mediodorsally and the postzygapophyses ventrolaterally. The facets on zygapophyses become nearly vertical and the prezygapophyses become longer and more slender posteriorly. The tips of the zygapophyses thicken to form small tubercles. On most lumbar vertebrae the ac- cessory processes are well developed and counterlocked with the zygapophyses. The accessory processes become successively weaker posteriorly and eventually are lost on the last lumbar vertebra.

The sacral series consists of three vertebrae that are fused to form an elongated and triangular sacrum (fig. 55). The sacral vertebrae are smaller than the lumbar vertebrae and gradually narrow posteriorly. The transverse process of $\mathrm{S} 1$ is robust, with its distal end being expanded to form the articular facet for the ilium. The facet is roughly triangular and slightly concave and faces postero- 


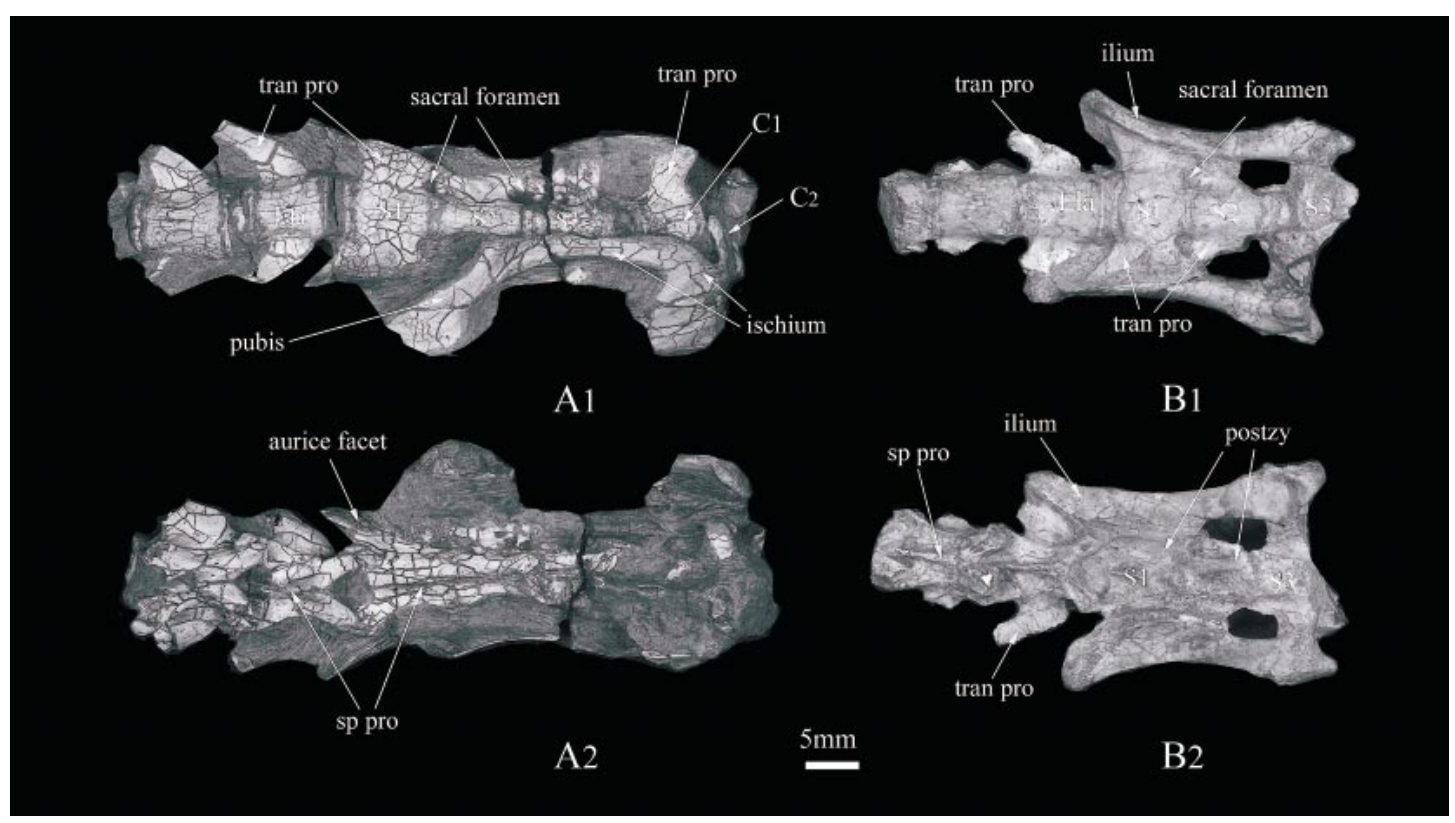

Fig. 55. Partial vertebral column and pelvic girdle of Rhombomylus (A, IVPP V5269.3; B, V5271). A, Last two lumbar vertebrae, sacral series, first two caudal vertebrae, and posteroventral part of pelvic girdle: A1 ventral view; A2, dorsal view; B, last two lumbar vertebrae, sacral series, first caudal vertebra, and ilium: B1, ventral view; B2, dorsal view. C1, first caudal vertebra; C2, second caudal vertebra; Lla, last lumbar vertebra; postzy, postzygaphosis; S1, S2, S3, first to third sacral vertebrae; sp pro, spinal process; and tran pro, transverse process.

laterally and slightly dorsally. The process is firmly synostosed with the ilium. The transverse process of $\mathrm{S} 2$ is anterolaterally oriented. It is synostosed with the posterior extremity of the transverse process of S1, and laterally encloses a sacral foramen. Laterally, the transverse process contributes to the posterior portion of the articular facet for the ilium. The transverse process of S2 joins that of S3 and encloses a smaller sacral foramen. The transverse process of S3 extends posterolaterally. The prezygapophysis of $\mathrm{S} 1$ is a thin, vertical, and high process with its articular facet facing medially and is larger than its counterpart on the postzygapophysis of the last lumbar vertebra. The postzygapophysis of S3 faces ventrolaterally. The neural spine of the sacral vertebrae is a thin, low ridge that extends along the entire length of the neural arch.

The first two caudal vertebrae are preserved in IVPP V5269 and V5270. Only the first one ( $\mathrm{C} 1$ in fig. 55A) is good enough to show some detailed morphology. It is shorter than S3. The ventral surface of its body is concave and bears no tubercle for the hemal arch. The winglike transverse process of $\mathrm{C} 1$ is slightly wider than that of $\mathrm{S} 3$ and tapers posterolaterally. The postzygapophysis of $\mathrm{C} 1$ is located more medially than that of S3 and is slightly vertically directed.

RIBS: The first pair of ribs are well preserved in IVPP V5268 (fig. 54A). They are anteroposteriorly flattened and more robust dorsally than ventrally. The neck is long. The tuberculum projects dorsally and is articulated with the stout diapophysis of T1. The head and neck of the second and third rib are preserved in situ. They are similar to those of the first rib but are more slender. Fragments of several posterior ribs are preserved in IVPP V7428 and in several uncataloged specimens. These ribs are long, curved, and gently rounded in crosssection.

SCAPULA: Both scapulae of IVPP V7428 (fig. 56) are partially preserved. The blade of the scapula is thin and roughly triangular in shape, with its anterior edge terminating at 


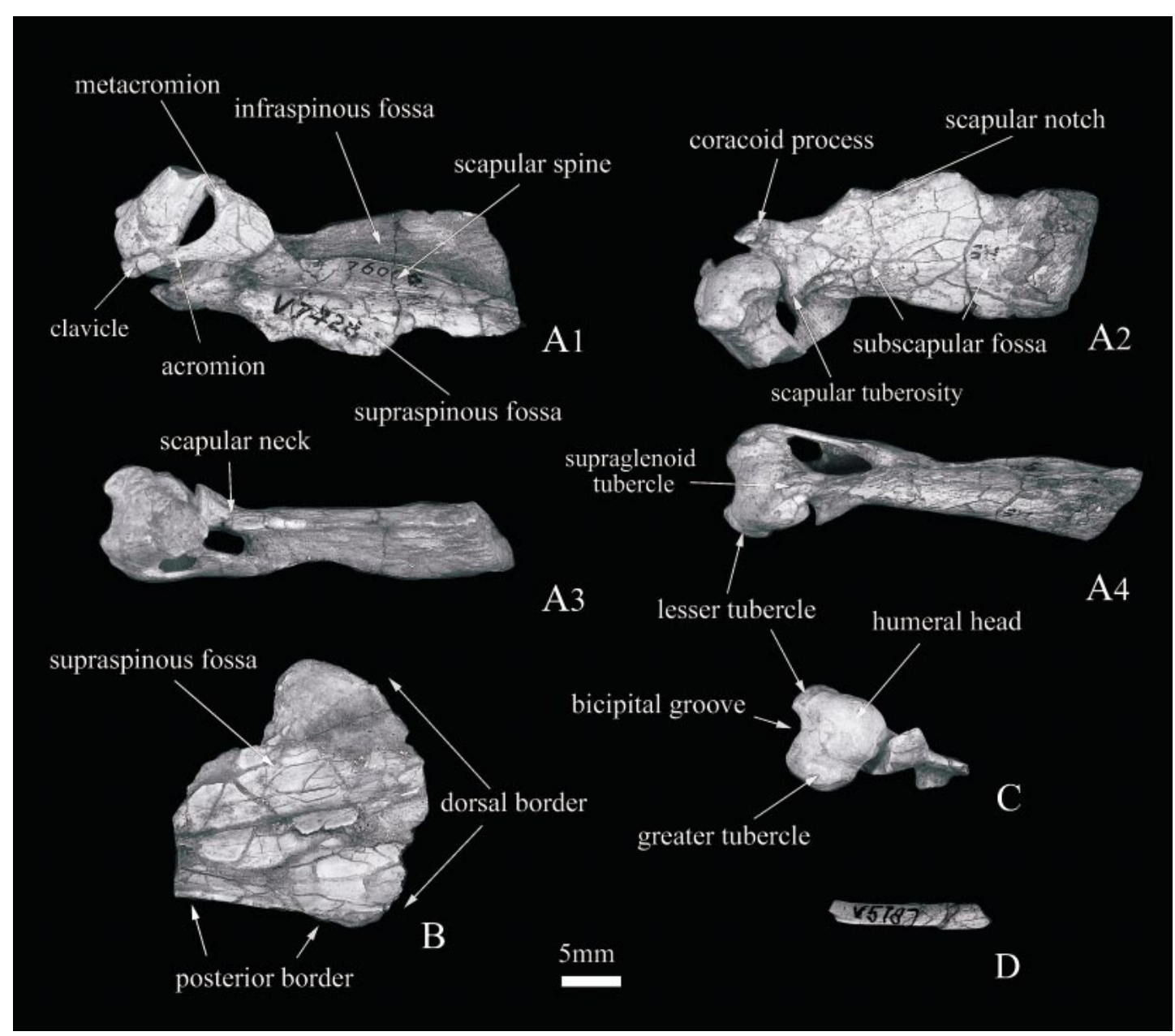

Fig. 56. Scapulae, clavicle, and proximal humerus of Rhombomylus (A, B, and C, IVPP V7428; D, IVPP V5287). A, Right scapula and articulated proximal humerus: A1, lateral view; A2, medial view; A3, posterior view; A4, anterior view; B, lateral view of dorsal part of left scapula; $\mathbf{C}$, anterior view of ventral part of left scapula and articulated proximal humerus; $\mathbf{D}$, middle segment of a possible clavicle.

the scapular notch. The caudal edge of the blade is straight and laterally reflected. Much of the dorsal edge of the scapular blade is damaged on both sides. The preserved portion indicates a convex dorsal border. The scapular spine starts just superior to the scapular neck and divides the lateral aspect of the scapular blade into supraspinous and infraspinous fossae. The spine is deflected posteriorly and makes the infraspinous fossa much more concave than the supraspinous fossa. The spine has a convex edge and is more elevated ventrally than dorsally. The ventral extremity of the scapular spine ex- pands to form a triangular plate. In lateral view, the plate bears a slender, long acromion process that points ventrally and slightly anteriorly and a stout metacromion that projects ventroposteriorly. In anterior view, the expanded plate of the scapular spine is very thin and the acromion process is slightly bent medially, with its apex pointing medioventrally. In medial view, both acromion and metacromion processes extend ventrally beyond the glenoid fossa. Both supraspinous and infraspinous fossae are narrow at their ventral portions and become wider dorsally, with the former being expanded at a greater 
degree. The short neck of the scapula is thin anteroposteriorly but thick lateromedially. The glenoid fossa is shallow and gently concave. It is pear-shaped and lateromedially narrow. Most of the fossa faces ventrally; its anterior portion faces posteroventrally and bears a supraglenoid tubercle (Muizon, 1998). The coracoid process is located just above the supraglenoid tubercle and is separated from it by a shallow notch. The process is strongly recurved medially with its tip pointing medioventrally. The posterior edge of the glenoid fossa is rounded, bearing a low projection, the scapular tuberosity (Muizon, 1998). The medial surface of the scapula reflects the relief of the structures on the lateral face of the bone to some degree. A small depression, probably for the origin of the caput longum of the triceps brachial muscle (Muizon, 1998), is dorsal to the scapular tuberosity on the medial surface of the scapula.

Clavicle: The lateral end of right clavicle is preserved in IVPP V7428 (fig. 56A). It is slender and slightly wider than the tip of the acromion. The articulation between the clavicle and acromion is not very strong. No other clavicle is certainly identified in our colection. A segment of slender bone looks similar to the clavicle of extant rodents and is considered to be the middle part of the clavicle of Rhombomylus (IVPP V5787, fig. 56D). Its size matches the clavicle of IVPP V7428.

HuMERUS: Both humeri of IVPP V7428 are preserved but the midshafts were damaged (figs. 56A, C, 57C). Supplemented by several humeral fragments (figs. 57A, B, D), most of the humeral morphology of Rhombomylus can be determined, although the precise length and torsion of the bone are uncertain. The estimated length of the humerus of IVPP V7428 is $40 \mathrm{~mm}$. The proximal and distal ends of the bone are twisted relative to each other. Because no humerus is complete, it is difficult to measure the exact degree of twisting (see Gambaryan and Kielan-Jaworowska, 1997). We estimate that the torsion of the humerus of IVPP V7428 is 10$20^{\circ}$. The proximal end is the most robust part of the bone. The epiphysis is unfused in all known specimens. The humeral head is nearly hemispherical and extends slightly beyond the greater tubercle. The head is also poste- riorly projected relative to the longitudinal axis of the shaft. The articular surface of the head has a curvature greater anteroposteriorly than mediolaterally and is longer anteroposteriorly than mediolaterally.

The greater tubercle is prominent but lower than the humeral head. It is separated from the humeral head by a shallow sulcus. Viewed proximally, the greater tubercle is obliquely oriented in an anteromedial-posterolateral direction and is about three times as long as wide. The anteromedial edge of the greater tubercle is more anteriorly positioned than is the lesser tubercle. The lesser tubercle is rounded and anterolaterally-posteromedially elongate (twice as long as wide) and is much smaller than the greater one. It is anteromedial to the humeral head and confluent with the latter proximally. A small groove on the proximal surface of the lesser tubercle is distinct from the smooth articular surface of the humeral head by its rough appearance. The groove is probably for insertion of the $\mathrm{m}$. subscapularis. The bicipital groove is shallow, broad, and well defined only proximally between the two tubercles.

The humeral shaft shows a sigmoid curvature in lateral view. Contributing to such a configuration is the deltopectoral crest, which runs distally on the anterior aspect of the shaft from the anteromedial corner of the greater tubercle for about half the length of the bone. The crest is low and rounded in its proximal portion and appears more elevated and sharper distally. On the lateral aspect of the humeral shaft there is a salient tricipital line (Muizon, 1998), which leads from the posterolateral corner of the greater tubercle, runs distally and anteriorly, and fuses with the deltopectoral crest. The tricipital line and the deltopectoral crest define the deltopectoral surface (Muizon, 1998) for insertion of several muscles. The medial surface of the distal part of the deltopectoral crest is also rough, suggesting insertion of the $\mathrm{m}$. pectoralis superficialis.

On the medial surface of the humeral shaft a low and small tuberosity for insertion of the $\mathrm{m}$. teres major extends out distally from the lesser tubercle. The posterior surface of the proximal humeral shaft bears a low, broad ridge that extends distally from the edge of the humeral head. Ventral to the hu- 
meral head, between the aforementioned tubercles and ridge, is a shallow fossa. The fossa and ridge probably provide attachment for the accessory head of the $\mathrm{m}$. triceps.

In cross section, the humeral shaft is nearly circular proximally, mediolaterally compressed in the middle portion, and transversely expanded distally. Two low ridges and a crest contribute to the flatness of the distal humerus. One ridge runs from the distal end of the deltopectoral crest to the entepicondyle; the other extends proximally from the entepicondyle as the medioposterior edge of the humeral shaft. The lateral epicondylar crest stretches along the lateral aspect of the humeral shaft. It is moderately developed, intermediate between those of Sciurus and Lepus. The curved ridges and crest give the distal portion of the humerus a twisted appearance.

The small ectepicondyle is on the lateral aspect of the distal end of the humerus and is the distal end of the lateral epicondylar crest. In contrast, the entepicondyle is a prominent tuberosity on the medial aspect of the distal humerus. Its apex, for the origin of the flexor muscles of the carpus and digits, is oval-shaped and projects distomedially. A shallow depression is present on the posterior aspect of the entepicondyle, probably for the attachment of helical ligament. The oval entepicondylar foramen is large. A shallow furrow extends distally from the foramen, between the entepicondyle and the medial rim of the humeral trochlea. The furrow extends onto the distal surface of the humerus and terminates at a tiny, presumably nutrient, foramen.

In anterior view, the capitulum is spindleshaped, with the articular surface continuing well onto its proximal surface. Dorsal to the capitulum is a well-defined radial fossa, which receives the head of the radius during full flexion of the forearm. The medial part of the capitulum is gradually constricted and confluent with the trochlea medially. The lateral surface of the capitulum is flattened and bears a pit for the radial collateral ligament (Gebo and Rose, 1993). In distal view the capitulum tapers posteromedially and merges with the lateral rim of the trochlea. The trochlea is shallow and broad. It wraps around the distal end of the humerus from the anterior to the posterior side. The trochlea is similar to a spiral, with its posterior portion being more lateral to the anterior. This configuration is also indicated by the oblique orientation of the lateral and medial trochlear rims relative to the axis of the humeral shaft. The medial trochlear rim is prominent and projects distally beyond both the capitulum and the entepicondyle. The lateral trochlear rim is sharp and confined to the posterior side of the humerus. The posterior surface of the distal humerus presents a deep, wide olecranon fossa dorsal to the trochlea. The fossa communicates with the radial fossa through several tiny foramina instead of a large supratrochlear fossa (Kielan-Jaworowska, 1978).

Li and Ting (1993) assigned a left, distal humerus (IVPP V7418) to Rhombomylus. As indicated by these authors, the bone is larger than all other specimens assigned to Rhombomylus. Its lateral epicondylar crest is much more prominent than those of other specimens, and its capitulum is more rounded and relatively shorter than those of other specimens assigned to Rhombomylus. These two differences allow us to exclude IVPP V7418 from Rhombomylus; the specimen probably represents a larger mammal with the ability to rotate the forearm more extensively.

ULNA: The ulna is shorter and much more slender than the humerus (fig. 58). It is moderately compressed mediolaterally. The proximal third of the bone is slightly recurved anteriorly, whereas its distal end is bent posteriorly. The olecranon process is the most robust region of the ulna. It expands laterally and bears a shallow groove for insertion of the $\mathrm{m}$. triceps and anconeus (O'Leary and Rose, 1995). Viewed anteriorly, the olecranon is slightly deflected medially. In posterior view, the olecranon process expands transversely and forms a convex surface facing posterolaterally. This surface bears a prominent, slightly curved medial edge and a weak, markedly curved lateral edge. The medial side of the olecranon bears a shallow, broad fossa, which extends distally as a furrow on the medial surface of the proximal ulna. The fossa and its distal extension provide the origin sites for the flexor carpi ulnaris and flexor digitorum profundus (Muizon, 1998). The posterior and proximal edges 


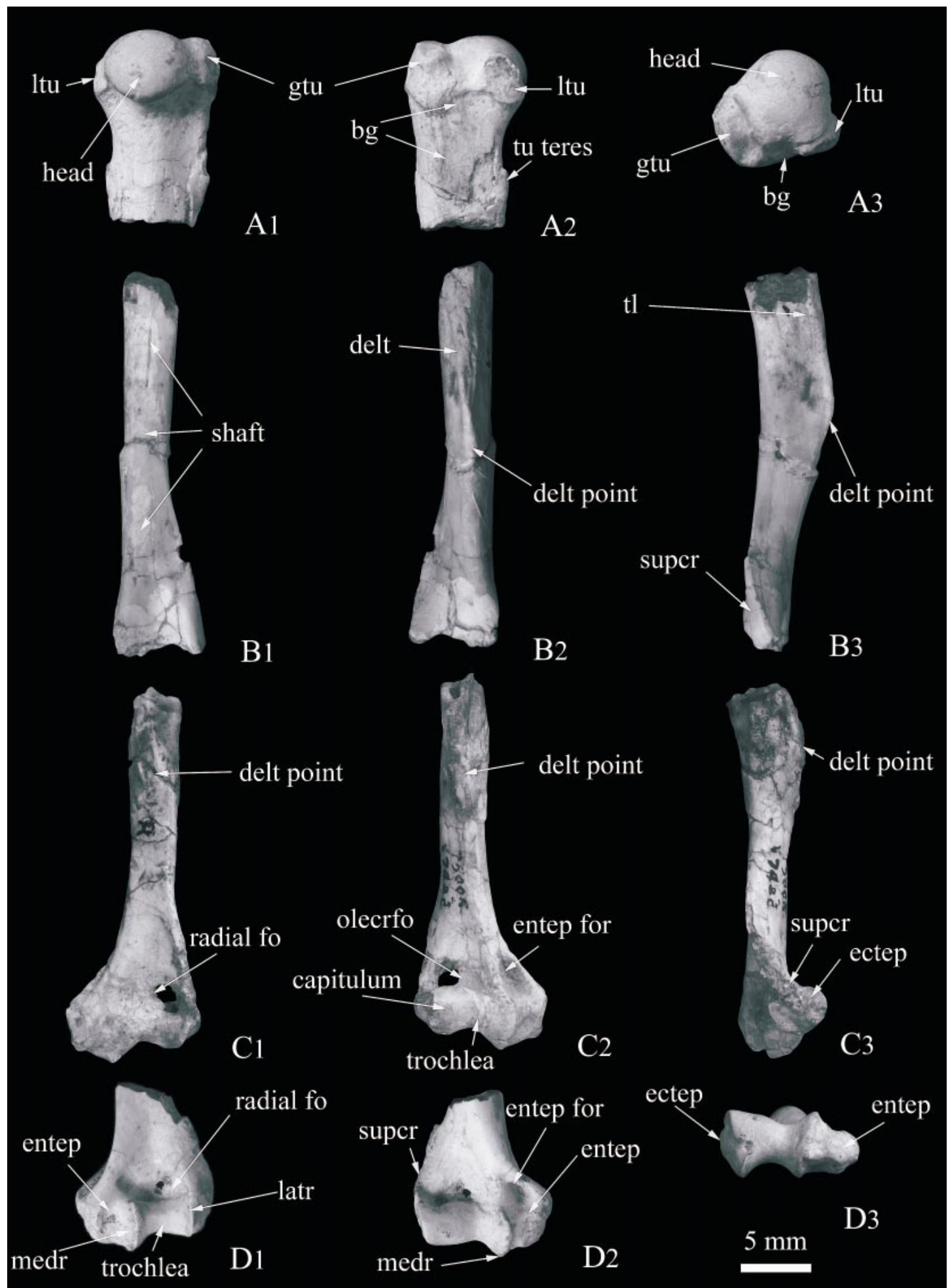

Fig. 57. Right humeri of Rhombomylus (A, IVPP V7461; B, IVPP V7463; C, IVPP V7428; D, IVPP V7464). A1-3, Posterior, anterior, and proximal views of a right proximal humerus. B1-3, Posterior, 
of the fossa form a continuous ridge for the attachment of the ulnar collateral ligament.

The trochlear notch is deep and concave. Both anconeal and coronoid processes are prominent. The anconeal process projects anterolaterally, with its medial limb being longer than the lateral one and reflected proximally. The articular facet of the trochlear notch extends onto the laterodistal surface of the coronoid process. The coronoid process bears a rounded ridge and projects mediolaterally. The low median ridge of the trochlear notch marks the proximodistal axis of the notch and is slightly oblique (about $15^{\circ}$ ) to the longitudinal axis of the ulnar shaft. The lateral margin of the trochlear notch is a semicircular incisure. The shape of the trochlear notch conforms to the spiral geometry of the humeral trochlea. The arc of the trochlear notch is less than a half circle, but, with the aid of radius, it can firmly hold the distal end of the humerus (fig. 58B4, B6). The radial notch of the ulna is distal to the lateral portion of the trochlear notch and is buttressed by the coronoid process. Its articular facet is slightly excavated and faces anterolaterally.

Viewed anteromedially, a small brachialis fossa is immediately distal to the coronoid process. Laterally, the supinator crest runs distally from the lateral end of the radial notch. The robust interosseus crest, continuing distally from the supinator crest, forms the large part of the anterior edge of the ulnar shaft. Most of the lateral surface of the ulnar shaft is occupied by a shallow, well-defined elongate fossa. The fossa is broad in its middle part and becomes narrower toward both ends. A prominent, oblique pronator ridge for attachment of the $\mathrm{m}$. pronator quadratus (Muizon, 1998) divides the medial surface of the distal ulna into a small, anterior area that faces anteromedially and a large, posterior area that faces posteromedially. As a result the distal ulnar shaft is triangular in cross section. The distal end is of the ulna is poorly preserved. It is mediolaterally compressed and bears a prominent styloid process.

RADIUS: The radius (fig. 58) of Rhombomylus is shorter than the humerus, with an estimated brachial index (radial length/humeral length $\times 100 \%$; see Gebo and Rose, 1993 ) of about 0.75 . The radius is more slender than the ulna. The radial head is ovalshaped and transversely wide. Most of the humeral articular surface is concave for articulation with the humeral capitulum, but a small medial portion is gently convex and articulates with the humeral trochlea. The anterior edge of the humeral articular surface bears a distinct capitular eminence at the point where the surface becomes convex. The ulnar facet of the radius accounts for most of the posterior surface of the radial head and extends slightly medially. The short radial neck is relatively straight and appears constricted distally. The bicipital tuberosity is a low ridge on the posterolateral side of the shaft distal to the neck. The radial shaft distal to the bicipital tuberosity is the thinnest part of the bone. From that point the radius becomes more robust distally; the distal end is the heaviest part of the bone.

MANUS: No manual element in our collection is directly associated with a skull or dentition of Rhombomylus. A fragmentary specimen (IVPP V7518) is tentatively identified as part of the left manus of Rhombomylus based on its size (fig. 59). Four metacarpals (Mc) and three proximal phalanges are preserved in this specimen. The metacarpals tightly contact each other proximally and spread out distally. Mc III, IV, and V are complete, of which Mc III is the longest and Mc V the shortest, about $60 \%$ as long as Mc III. Only the distal half of Mc II is preserved.

$\leftarrow$

anterior, and lateral views of the shaft of a right humerus. C1-3, Anterior, posterior, and lateral views of the distal part of a right humerus. D1-3, Posterior, anterior, and distal views the distal part of a right humerus. bg, bicipital groove; delt point, distal terminal point of deltopectoral crest; ectep, ectepicondyle; entep, entepicondyle; entep for, entepicondylar foramen; gtu, greater tubercle; latr, lateral ridge of humeral trochlea; ltu, lesser tubercle; medr, medial ridge of humeral trochlea; olecrfo, olecranon fossa; radial fo, radial fossa; supcr, supinator crest (lateral epicondylar crest); tl, tricipital line; and tu teres, tuberosity for attachment of M. teres major. 


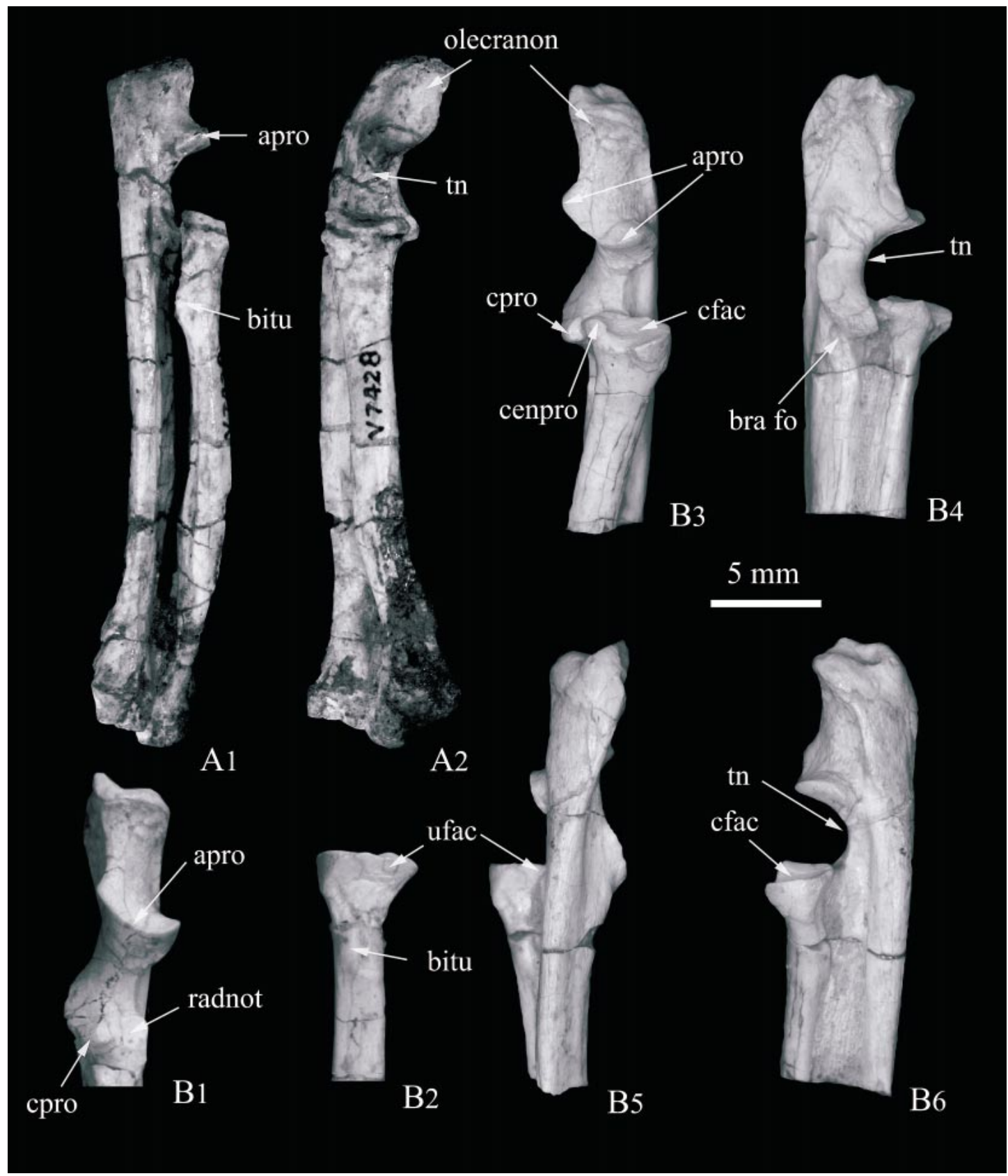

Fig. 58. Ulna and radius of Rhombomylus (A, IVPP V7428; B, IVPP V7465). A1-2, Medial and lateral views of the right ulna and radius. B, Proximal part of left ulna and radius. B1, anterior view of ulna; B2, posterior view of radius; B3-6, anterior, anteromedial, posterior, and lateral views of the ulna. apro, anoconeal process; bitu, bicipital tuberosity; bra fo, brachialis fossa; cenpro, central process; cfac, facet for humeral capitulum; cpro, coronoid process; radnot, radial notch; and tn, trochlear notch. 


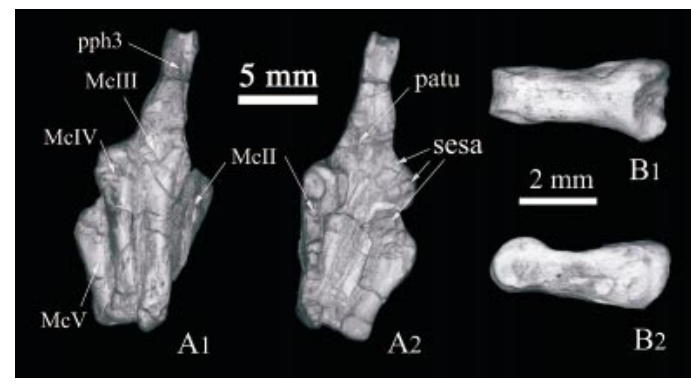

Fig. 59. Manus of Rhombomylus (IVPP V7518). A1-2, dorsal and ventral views of a partial left manus; B1-2, dorsal and ventral views of a proximal phalange. McII, McIII, McIV, and $\mathrm{McV}$, second to fifth metacarpals; patu, palmar tubercle; pph3, proximal phalange of third finger; and sesa, sesamoid bones.

Judging by its diameter, the bone seems to be shorter than Mc IV. Mc V is dorsoventrally flat and slightly concave ventrally. The proximal epiphysis is so expanded that it looks like a tuberosity projecting ventrolaterally. The proximal articular facet of $\mathrm{Mc} \mathrm{V}$ is slightly convex. The distal epiphysis expands transversely. The ventral surface of the distal shaft has a low, slim longitudinal middle keel. The distinct margins of the distal shaft and the medial keel define two small pits presumably for a pair of sesamoid bones. A displaced sesamoid ossicle is preserved nearby. The distal articular facet is convex and extends proximally onto the dorsal side and to a lesser degree to the ventral side of the distal epiphysis. The convexity of the facet is greater dorsally than ventrally. The distal epiphysis also has a sagittal ridge and a lateral and medial pit for attachment of collateral ligaments. The shaft of Mc IV is slightly flattened dorsoventrally. Its proximal epiphysis is dorsoventrally deep and is wedge-shaped with a narrow ventral side. The proximal articular facet and distal epiphysis are similar to those of Mc V. A pair of elongated sesamoid bones are on the ventral side of Mc IV.

The proximal epiphysis of Mc III is transversely compressed and bears a prominent ventral tubercle. The proximal end of Mc III projects proximally beyond those of Mc IV and V. The distal part of Mc III is similar to those of Mc IV and $\mathrm{V}$ but is more robust.
Mc II is similar to Mc III and IV but is slimmer.

The proximal phalanx of digit III is about half as long as Mc III. Its proximal end is expanded transversely and the distal end is about the width of the shaft. The proximal articular facet forms a shallow hemispheric fossa, facing proximodorsally. Its ventral lip bears a middle notch to accommodate the sagittal ridge of Mc III. The distal end of the bone bears a well-developed trochlea, the articular facet of which extends proximally onto the ventral side of the bone. The trochlea is slightly asymmetrical, indicating a slight degree of medial deviation during flexion (Jenkins and Parrington, 1976). The proximal half of the proximal phalanx of digit IV is similar to that of digit III. A displaced proximal phalanx is possibly from digit II of the same hand.

PelviC GiRdLE: Several fragments of the pelvic girdle reveal morphologies of its components (figs. 55A, B, 60). We estimate that the ilium extends about $60 \%$ of the total length of the pelvis and that the ilium and the ischium are oriented parallel to the axis of the vertebral column. The ilium has a large, elongate wing with lateral, medial, and ventral surfaces. The lateral surface of the iliac wing is expanded dorsally. Because of the lateral reflection of the anterior edge of the ilium, this surface is gently concave, forming a shallow superior gluteal fossa (Wood, 1962). The fossa is nearly vertical and faces laterally. The anterodorsal corner of the iliac wing is rounded. The posterior iliac spine is prominent and forms the anterior edge of a deep greater sciatic notch. The notch is on the dorsal margin of the iliac body and anterior part of the ischial body. The ventral surface of the iliac wing is also gently concave and is much narrower than the lateral surface. A slender crest extends anteriorly from the femoral process (Wood, 1962, see below for details) on the ventral surface of the iliac wing and delimits a narrow medial fossa and a narrower lateral fossa. The medial surface of the iliac wing is convex longitudinally and concave dorsoventrally. The articular facet for the sacrum is large and rogose.

A prominent femoral process is located at the border between the lateral and ventral 


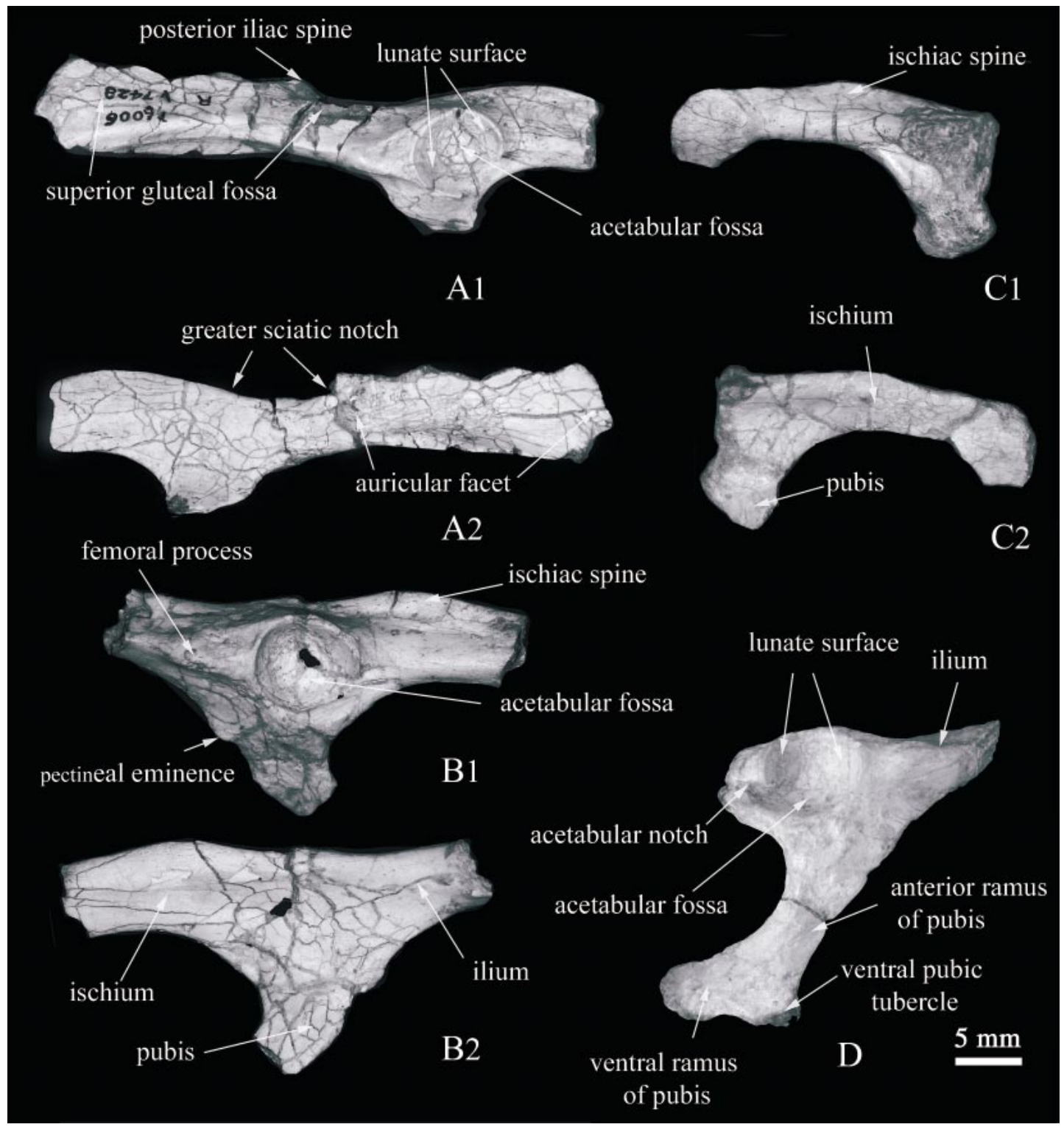

Fig. 60. Pelvic girdle of Rhombomylus (A, IVPP V7428; B, IVPP V7457; C, IVPP V7460; D, IVPP V7459). A1-2, lateral and medial views of a partial left innominate; B1-2, lateral and medial views of the middle part of a left innominate; C1-2, lateral and medial views of the partial right ischium and pubis; D, lateral view of the middle part of a right innominate.

surfaces of the iliac body, just anterior to the anterior margin of the acetabulum. The process bears a shallow fossa on its dorsal aspect. The sutures between innominate elements are indiscernible in any specimen. At the point corresponding to the junction of the ilium and pubis exists a small pectineal eminence (Wood, 1962).
The acetabulum is an open socket, nearly hemispherical in shape, and faces laterally. The margin of the acetabulum is well defined and laterally salient except for the posteroventral area, which is indented by the acetabular notch. The lunate surface, which is the articular facet for the femoral head, forms a circumferential band. The posterodorsal lobe 
of the lunate surface is very narrow and bears a prominence that bounds the deep acetabular notch laterally. The nonarticular surface in the center of the acetabulum is surrounded by the lunate surface except at the acetabular notch. The body of the ischium extends posteriorly along the same axis with the ilium, and is deeper but slightly thinner than the body of the latter. The dorsal margin of the anterior ischial body bears a low ischial spine. Posterior to the spine is a shallow lesser sciatic notch, which is much smaller than the greater sciatic notch. The posterior half of the ischial body is dorsoventrally shallower than the anterior part. The posterodorsal end of the ischium is thickened, but an ischial tuberosity is absent in all specimens. The ramus and the body of the ischium form a right angle. The symphyseal portion of the ischium is quite straight and is more slender than the ischial ramus.

The anterior ramus of the pubis (the acetabular process, Kielan-Jaworowska, 1978) tapers posteroventrally. It becomes extremely narrow at the point near its junction with the ventral ramus of the pubis (the symphyseal process of Kielan-Jaworowska, 1978). The two rami form an angle of about $135^{\circ}$. The ventral ramus bears a small ventral pubic tubercle. The obturator foramen appears oval and is much large than the acetabulum.

FEMUR: The right femur of IVPP V7428 is nearly complete (fig. 61), although it is crushed and twisted to some degree (fig. 61). It is much longer than the humerus. The bone gradually thickens distally to form a robust distal end. The right femur in IVPP V7428 was deformed so that it is curved and the shaft is convex laterally. The bone would be straighter in life. The estimated length of the right femur of IVPP V7428 is $66 \mathrm{~mm}$. The femoral head is well defined, with its long axis having a $120^{\circ}$ angle to the shaft. It is slightly shifted onto the proximal aspect of the neck; thus the anterior and posterior edges of the head are oblique to the axis of the neck. The articular surface of the head is quite globular and shows no extension onto the neck. The anterior border of the articular surface $(=$ the anterior edge of the femoral head) forms an acute angle (about $60^{\circ}$ ) with the femoral shaft. The greater trochanter is a massive process and extends slightly higher than the femoral head. It is anteroposteriorly expanded in proximal view. The lateral surface of the greater trochanter bears a low, rounded ridge, which extends distally as a sharp third trochanter (Wood, 1962). Posteriorly, the greater trochanter is marked by a prominent trochanteric crest that projects posteriorly and overhangs the trochanteric fossa. The fossa is very deep and extends distally to the level of the apex of the lesser trochanter. The third trochanter is triangular in anterior view and bears a knob on its tip. The lesser trochanter forms a low triangular flange, protruding posteromedially from the shaft. A rounded ridge runs laterally from the rough apex of the lesser trochanter and meets with the trochanteric crest of the greater trochanter. For most the part, the femoral shaft appears gently rounded in cross section. The distal femur is much more expanded anteroposteriorly than mediolaterally. The patellar trochlea (Gebo and Rose, 1993) is narrow and well defined by sharp, elevated trochlear ridges. The lateral trochlear ridge is slightly higher than the medial one. The femoral condyles project posteriorly with articular facets well recurved posteroproximally. The lateral condyle is slightly larger than the medial and protrudes slightly beyond the medial one in both distal and posterior directions. The intercondylar fossa is deep and separated from the patellar trochlea by a low ridge. The lateral surface of the lateral condyle and the medial surface of the medial condyle are flat. Small pits on the surfaces probably suggest the origin site for the popliteus muscle.

PATELLA: The left patella of IVPP V7428 (fig. 62) is well preserved. The bone is longer than wide. It is oval in general shape with a rounded proximal and tapered distal end. Its anterior surface is gently convex and rugose. The posterior surface is convex mediolaterally and slightly concave proximodistally and forms a smooth articular surface readily fitting the patellar trochlea of the femur.

TIBIA: No tibia is complete (fig. 63), so it is impossible to know the precise length of the bone. Both tibiae of IVPP V7428 lack the middle part of the shaft. Several other fragments preserve either the proximal or the distal portion of the bone. It is estimated that the length of the tibia is between those of the femur and the humerus. Viewed anteriorly, 


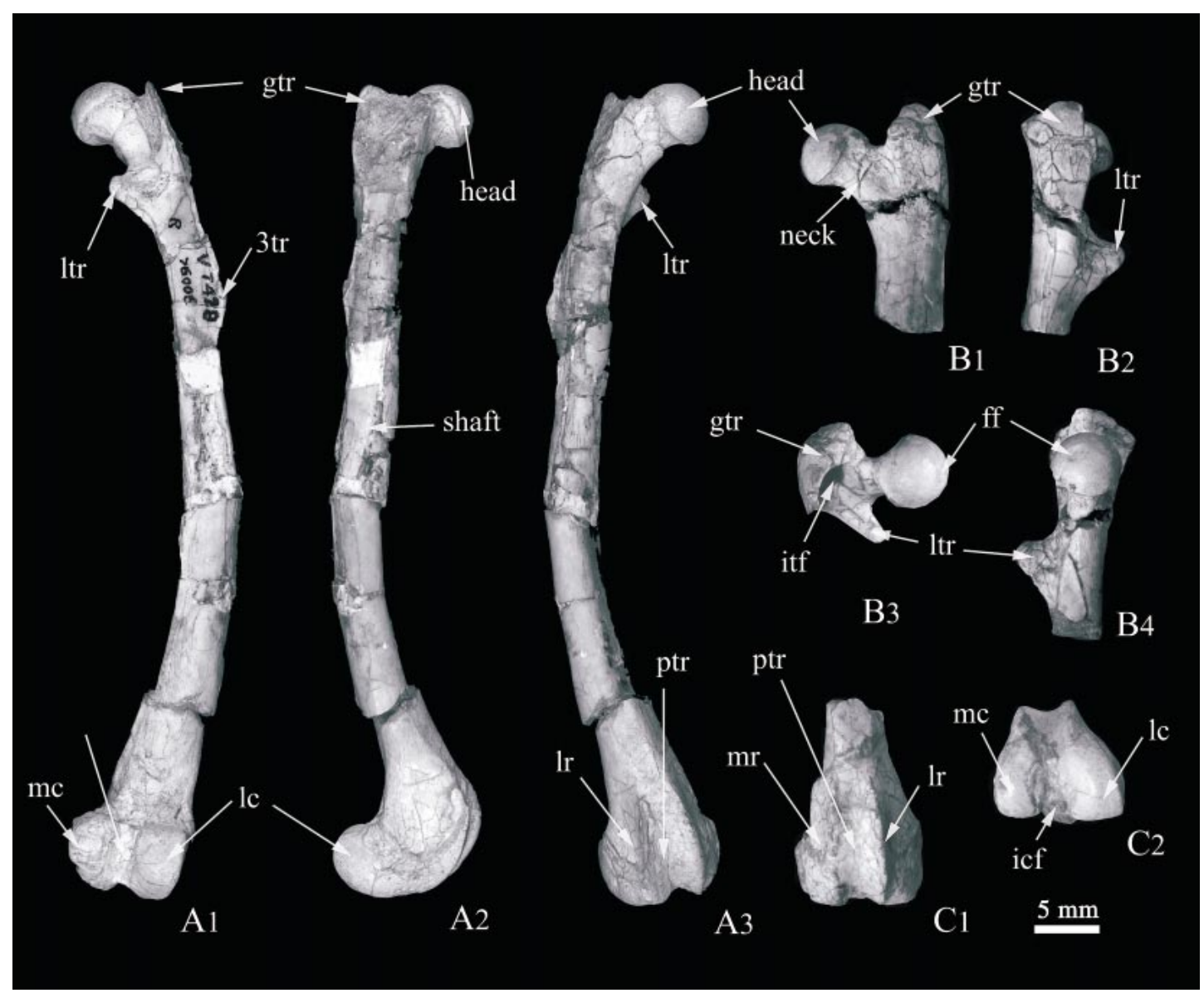

Fig. 61. Femurs of Rhombomylus (A and C, IVPP V7428; B, IVPP V7480). A1-3, Posterior, lateral, and anterior views of a right femur. B1-4, Anterior, lateral, proximal, and medial views of the proximal part of a left femur. C1-2, Anterior and distal views of the distal part of a left femur. 3tr, third trochanter; ff, favea for femoral head ligament; gtr, greater trochanter; itf, intertrochantic fossa (or trochantic fossa); lc, lateral condyle; lr, lateral rim of femoral trochlea; ltr, lesser trochanter; mc, medial condyle; mr, medial rim of femoral trochlea; and ptr, patella trochlea.

the bone has a low degree of sigmoid curvature, with the proximal part being slightly concave laterally and the distal part slightly convex laterally; the distal end of the bone expands medially.

The tibial plateau is subtriangular in proximal view. The tibial condyles are subequal in size; both are anteroposteriorly longer than transversely wide and are separated from each other by the intercondylar eminence. The medial condyle is oval in outline and gently concave, whereas the lateral one is subrectangular with its long axis oblique to the anteroposterior axis of the tibial plateau.
It is gently flat mediolaterally but distinctly convex anteroposteriorly. This is especially so in its posterior portion, which actually extends onto the posterior side of the tibial plateau. The condyles are confluent in front of the intercondylar eminence, forming a rugose surface called the anterior intercondyloid area (Muizon, 1998). This area is very short anteroposteriorly and separated from the tibial tuberosity by a short furrow. The tibial tuberosity has a rugose, convex surface that slopes down from the tibial plateau to the anterior surface of the tibia. The lateral surface is vertical and extends posteriorly from 


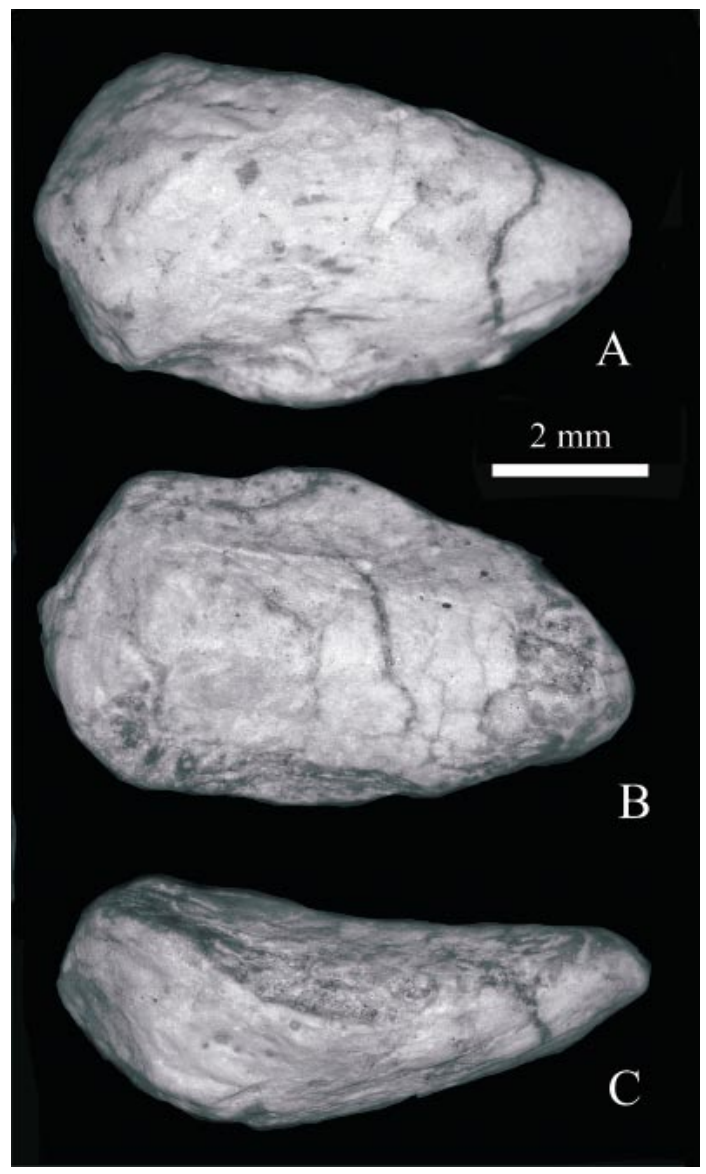

Fig. 62. Anterior, posterior, and medial views of the left patella of Rhombomylus (IVPP V7428).

the tibial tuberosity to the lateral side of the lateral condyle. The tibial crest is prominent on the anterior tibial shaft. The crest fades out abruptly in the middle portion of the shaft. On the posterior surface of the shaft a sharp crest buttresses the lateral condyle and forms the posterolateral edge of the proximal shaft. Distally it gradually migrates to the lateral surface of the shaft and becomes blunt; it eventually reaches the anterolateral corner of the distal epiphysis of the tibia. This crest is probably for the attachment of the interosseous membrane between the tibia and fibula. A short ridge that forms the posteromedial edge of the proximal tibia supports the medial condyle. Owing to the development of the crests and ridge, the proximal portion of the tibial shaft is roughly triangular in cross section. A large, deep lateral tibial fossa (Muizon, 1998) is on the lateral surface of the proximal shaft. The fossa becomes shallower distally and fades out at the level where the posterolateral crest begins to shift to the lateral side of the shaft. Posterior to the proximal end of the lateral tibial fossa and just ventral to the posterolateral overhang of the tibial plateau is a small facet for the proximal end of the fibula (FTp in fig. 63). A small medial tibial fossa (Muizon, 1998), probably for insertion of the m. semimembransous (Wood, 1962), is on the medial surface of the proximal shaft. The posterior surface of the proximal shaft has a narrow, deep fossa, which becomes shallower distally and fades out at the level of the popliteal notch (Muizon, 1998). The notch is an indentation on the posteromedial edge of the tibial shaft where the posteromedial popliteal line (Kielan-Jaworowska, 1978) ends. The popliteal line is oblique and joins the posteromedial edge of the tibia distal to the popliteal notch. The middle portion of the tibial shaft is much more slender than the proximal portion. Distally the shaft flares transversely and is compressed anteroposteriorly. The surfaces of the distal shaft are gently flat. Proximal to the distal epiphysis is the distal fibular facet, which faces laterally and contacts the distal end of the fibula.

The distal epiphysis of the tibia is about 1.5 times as wide transversely as anteroposteriorly. It bears a medial malleolus and a complex articular surface for the astragalus. The articular surface can be divided into medial and lateral astragalotibial facets (ATim and ATil). The ATim is on the lateral aspect of the tibial malleolus, facing laterally and almost vertical in orientation. The ATil faces distally. The facet is saddle-shaped with a low middle ridge and two side cavities. The ridge is rounded and oriented anteroposteriorly. The medial cavity of the ATil appears smaller than the lateral one. The latter is adjacent to the distal fibular facet. ATil, including the middle ridge and both cavities, extends posteriorly to the anterior surface of the posterior process of the tibia (tpp). This portion of the ATil is also called the distal astragalotibial facet (ATid, Szalay, 1985). The medial malleolus is a massive process occupying the whole anteroposterior length of the medial distal epiphysis. There is a shallow 


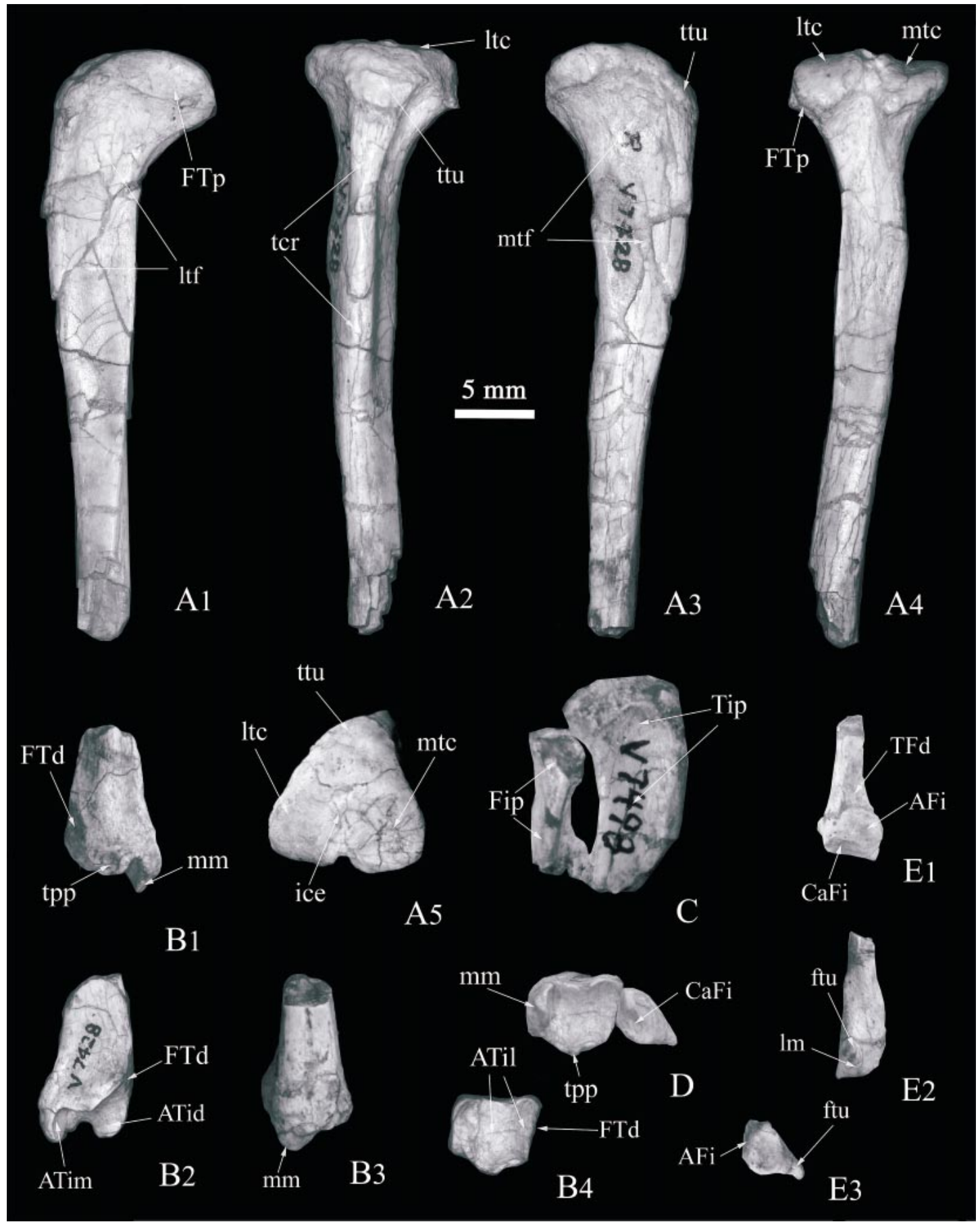

Fig. 63. Tibia and fibula of Rhombomylus (A, B, D, E, IVPP V7428; C, IVPP V7498). A1-5, Lateral, anterior, medial, posterior, and proximal views of the proximal part of a left tibia. B1-4, Posterior, anterior, medial, and distal views of the distal part of a left tibia. C, Proximal parts of right tibia and fibula in anterolateral view. D, Left tibia and fibular in distal view. E1-3, Medial, lateral, and distal views of a distal left fibula. AFi, astragalofibular facet; ATid, distal astragalotibial facet; ATil, lateral astragalotibial facet; ATim, medial astragalotibial facet; CaFi, calcaneofibular facet; Fip, proximal part 
but well-marked sulcus on the posterior surface of the medial malleolus, which is probably left by the tendon of the $\mathrm{m}$. flexor tibialis (Szalay, 1985). The posterior process of the tibia is thinner and much lower than the medial malleolus and is separated from the latter by a deep notch. The posterior side of the process has a shallow, vertical sulcus, probably to guide the tendon of the m. flexor fibularis (tff, Szalay, 1985; = m. flexor digitorum fibularis; m. flexor digitorum longus). The anterior edge of the lateral astragalotibial facet forms a low but sharp lip and is separated from the medial malleolus by a shallow notch. This notch and the deep notch between the medial malleolus and the posterior process of the tibia mark the end of a groove between the medial and lateral astragalotibial facets. The groove accommodates the medial rim of the astragalar trochlea.

FIBULA: Only proximal and distal fibular segments are known for Rhombomylus (fig. 63). The fibula is much more slender than the tibia and is probably as long as the latter, judging from the fibular facets on the tibia. Little morphology is available about the proximal portion of the bone. The distal end of the fibula is anteroposteriorly compressed and tightly contacts the distal tibia by the tibial facet. The lateral malleolus is well developed, with its apex being located at the anteromedial moiety. The distal epiphysis is much wider than the shaft; it is compressed and slightly twisted from the shaft. Two articular facets on the medial surface of the epiphysis are the astragalofibular (AFi) and the calcaneofibular $(\mathrm{CaFi})$. The AFi is nearly vertical and articulates with the lateral facet of the astragalar body. The $\mathrm{CaFi}$ is slightly concave and oblique and faces medially and distally. It is distally separated from the AFi by a weak crest. A prominent tubercle is on the posterolateral corner of the distal epiphysis.

ASTRAGALUS: Both astragali of IVPP V7428 are well preserved (figs. 64, 65). The body of the astragalus is broad and nearly square in dorsal view. Its medial surface is flat and vertical and is in articulation with the medial astragalotibial facet of the tibia. The helical lateral surface of the astragalar body articulates with the astragalofibular facet of the fibula. A ventrolaterally pointed process at the anteroventral corner of the lateral surface exaggerates the helix of the lateral surface. The process fits a notch on the anterior edge of the lateral malleolus of the tibia when the foot is fully dorsiflexed at the upper ankle joint. The astragalar trochlea is well grooved. It is slightly asymmetrical, with the bottom of the trochlear groove being slightly shifted medially. The medial edge of the trochlea is rounded and is lower than the sharp, lateral one. Both edges are semicircular, with the lateral one having a greater radius. The trochlea is narrower posteriorly. It almost extends to the ventral surface of the astragalar body and terminates anteriorly at a shallow fossa that may have accommodated the anterior lip of the lateral astragalotibial facet of the tibia. The calcaneoastragalar and the sustentacular facets account for most of the ventral side of the astragalus. The facets are subparallel and separated by the sulcus astragali. The concave calcaneoastragalar facet faces lateroventrally and extends slightly oblique to the midline of the astragalar body. The sustentacular facet is gently convex in its anterior half and concave in the posterior half. It extends anteriorly to the ventral base of the astragalar neck and ends posteriorly at the sustentacular hinge. The sulcus astragali is closed posteriorly.

The astragalar head is anteromedially offset from the astragalar body through a short and well-constricted neck. In anterior view, the head is compressed and twisted in relation to the body. Its width is about twice the depth. The astragalonavicular facet is extensive, convex, and spans the entire width of the head. The articular surface extends proximally onto the medial side of the astragalar

\footnotetext{
$\leftarrow$

of fibula; FTd, facet on tibia for distal fibula; FTp, facet on tibia for proximal fibula; ftu, fibular tubercle; ice, intercondylar eminence of tibia; lm, lateral malleolus of fibula; 1tc, lateral tibial condyle; ltf, lateral tibial fossa; mm, medial malleolus of tibia; mtc, medial tibial condyle; mtf, medial tibial fossa; tcr, tibial crest; TFd, facet for distal tibia on fibula; tpp, tibial posterior process; and ttu, tibial tuberosity.
} 


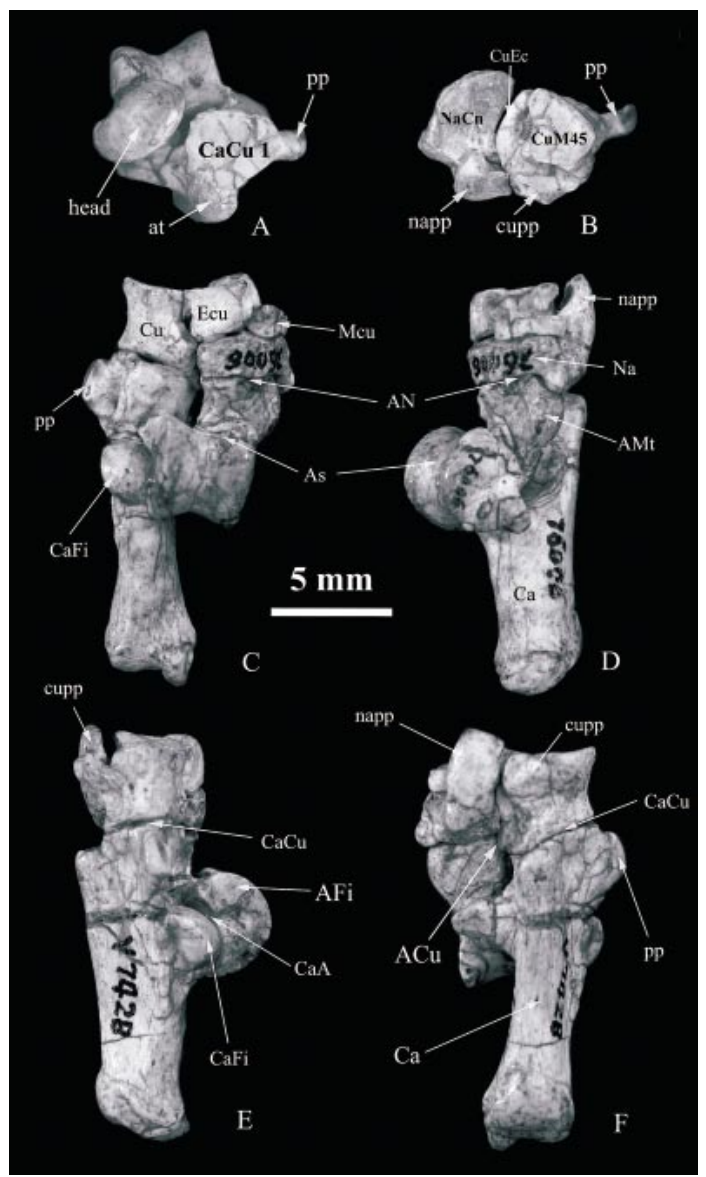

Fig. 64. Left tarsal bones of Rhombomylus (IVPP V7428), entocuneiform missed. A, Distal view of astragalus and calcaneus; B, distal view of cuboid and navicular; C-F, dorsal, medial, lateral, and ventral views of tarsal bones. $\mathrm{ACu}$, astragalocuboid joint; Afi, astragalofibular facet on astragalus; AMt, astragalomediotarsal facet on the head of astragalus; AN, astragalonavicular joint; As, astragalus; at, anterior planter process of calcaneus; Ca, calcaneus; $\mathrm{CaA}$, calcaneoastragalur joint; $\mathrm{CaCu}$, calcaneocuboid joint; $\mathrm{CaCu}$, calcaneocuboid facet of calcaneus; $\mathrm{CaFi}$, calcaneofibular facet on calcaneus; $\mathrm{Cu}$, cuboid; $\mathrm{CuEc}$, ectocuneiform facet on cuboid; cupp, plantar process of cuboid; Ecu, ectocuneiform; Mcu, mesocuneiform; Na, navicular; napp, plantar process of navicular; and pp, peroneal process of calcaneus.

neck, but this portion of the articular surface (AmT in fig. 65) is probably not for articulation with the navicular, because its dorsoventral depth does not match that of the articular surface on the navicular. Therefore, the navicular cannot slide onto the astragalar neck. A similar extension of the articular surface on the astragalar neck of Paramys was considered the articulation with the entocuneiform (Wood, 1962). Szalay (1985), however, found that in Paramys, the entocuneiform does not contact the astragalus, and he suggested that the extended part of articular surface on the astragalar head was for the medial tarsal bone. Given the general similarity of the foot morphology between Rhombomylus and paramyids, we follow Szalay's (1985) suggestion in identifying the facet, although we cannot completely exclude the possibility that this facet is actually for the entocuneiform. The astragalocuboid facet is the ventrolateral one-third of the surface of the astragalar head. There is no border between the astragalonavicular and astragalocuboid facets. Posterior to the astragalocuboid facet there is a small smooth surface on the astragalar neck, which contacts the anteromedial corner of the calcaneus.

CALCANEUS: Both calcanei of IVPP V7428 are well preserved (figs. 64, 66). The calcaneus is robust compared to the astragalus. The tuber calcanei (Szalay, 1984) is compressed transversely and expands posteriorly. It composes the posterior half of the bone, and has a sharp, high medial process and a rounded, low lateral one. The two processes define a broad, vertical groove. Ventral to this groove on the plantar side of the tuber calcanei there is a prominent, rounded tuberosity. The calcaneal protuberance (Muizon, 1998) is distinct on the dorsal side of the calcaneus. A lower ridge along the midline of the protuberance divides the protuberance into two parts. The medial, longer part articulates with the astragalus, whereas the lateral one articulates with the fibula. The calcaneoastragalar facet is gently convex and faces dorsomedially, whereas the calcaneofibular facet is strongly convex and faces dorsolaterally. Both facets are slightly oblique to the longitudinal axis of the calcaneus. The calcaneofibular facet is delimited laterally by a low ridge that extends posteriorly.

The small peroneal process is high on the lateral surface of the calcaneus. It is compressed dorsoventrally, projects laterally, and ends as a tubercle. A fine groove on its lateroventral surface is the passage for the ten- 


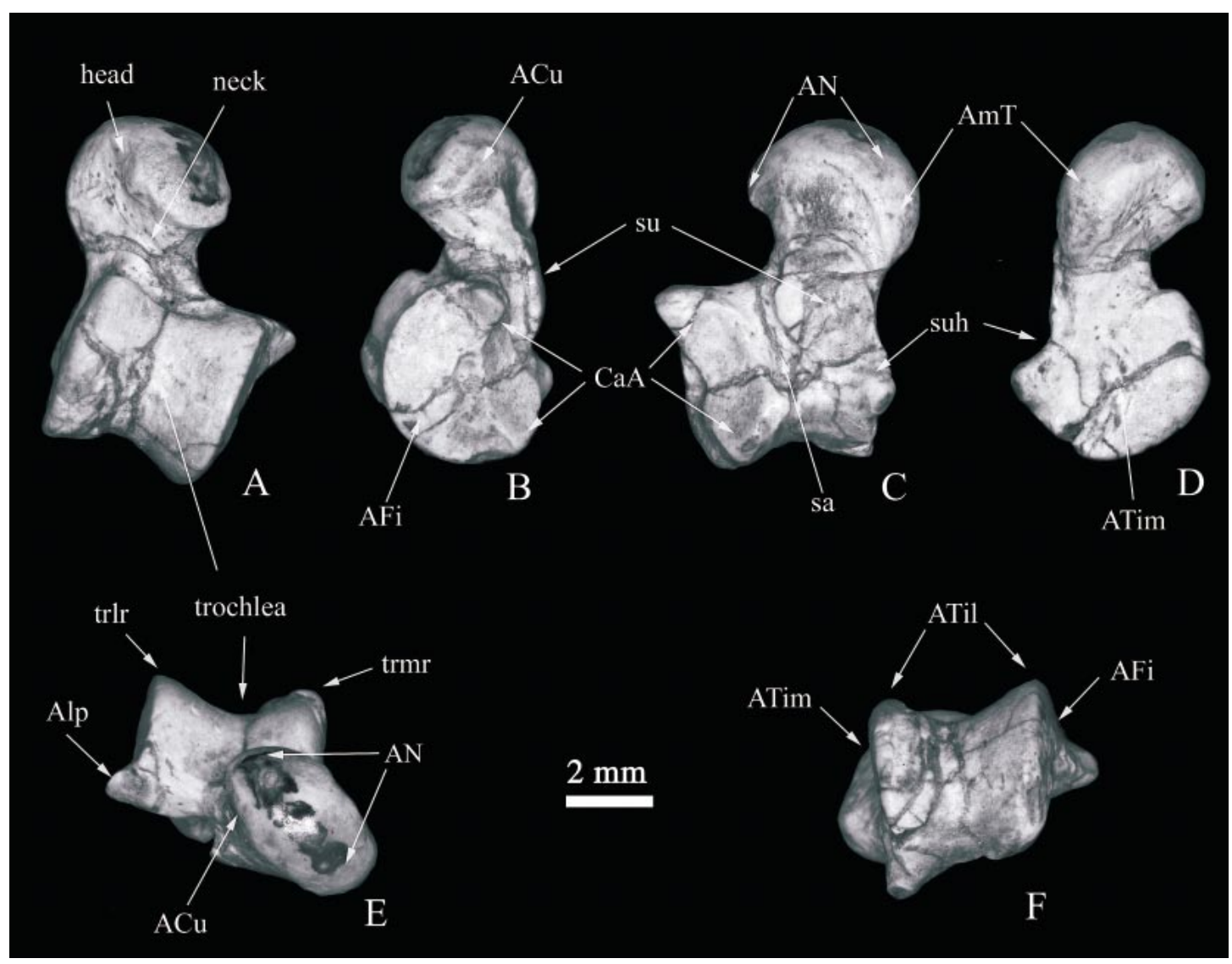

Fig. 65. Dorsal, lateral, ventral, medial, distal, and posterior views of the right astragalus of Rhombomylus (IVPP V7428). ACu, astagalocuboid facet; AFi, astragalofibular facet; Alp, lateral process of astragalus; AmT, astragalomediotarsal facet; AN, astragalonavicular facet; ATil, lateral astragalotibial facet; ATim, medial astragalotibial facet; CaA, calcaneoastragalar facet; sa, sulcus astragali; su, sustentacular facet; suh, sustentacular hinge; trlr, lateral rim of astragalar trochlea; trmr, medial rim of astragalar trochlea.

don of the $\mathrm{m}$. peroneus longus. A small notch on the anterior edge of the peroneal process is comparable to the groove for the tendon of the $\mathrm{m}$. peroneus brevis in paramyids.

The sustentaculum talus is located anteriorly on the medial side of the calcaneus. It is triangular in dorsal view and much broader transversely than anteroposteriorly. Its posterior edge is at the same level of the posterior border of the calcaneofibular facet, and its anterior edge is oblique and is anterior to the anterior border of the calcaneal protuberance. Fine crests and furrows on the anterior edge of the sustentaculum talus indicate attachment of the calcaneonavicular ligament.
The sustentacular facet is flat and directed dorsally and slightly anteriorly. The plantar surface of the sustentaculum talus is smooth and faces posteroplantarly. A groove at the junction of this surface and the medial surface of the calcaneal body marks the pathway for the tendon of the $\mathrm{m}$. flexor fibularis. The groove extends anteriorly on the medial surface of the calcaneus and separates the anterior plantar tubercle from the calcaneal body. The calcaneonavicular facet is at the anterior aspect of the calcaneal body. It is concave and oblique to the longitudinal axis of the calcaneus. In anterior view, the anterior plantar tubercle is ventral and slightly 


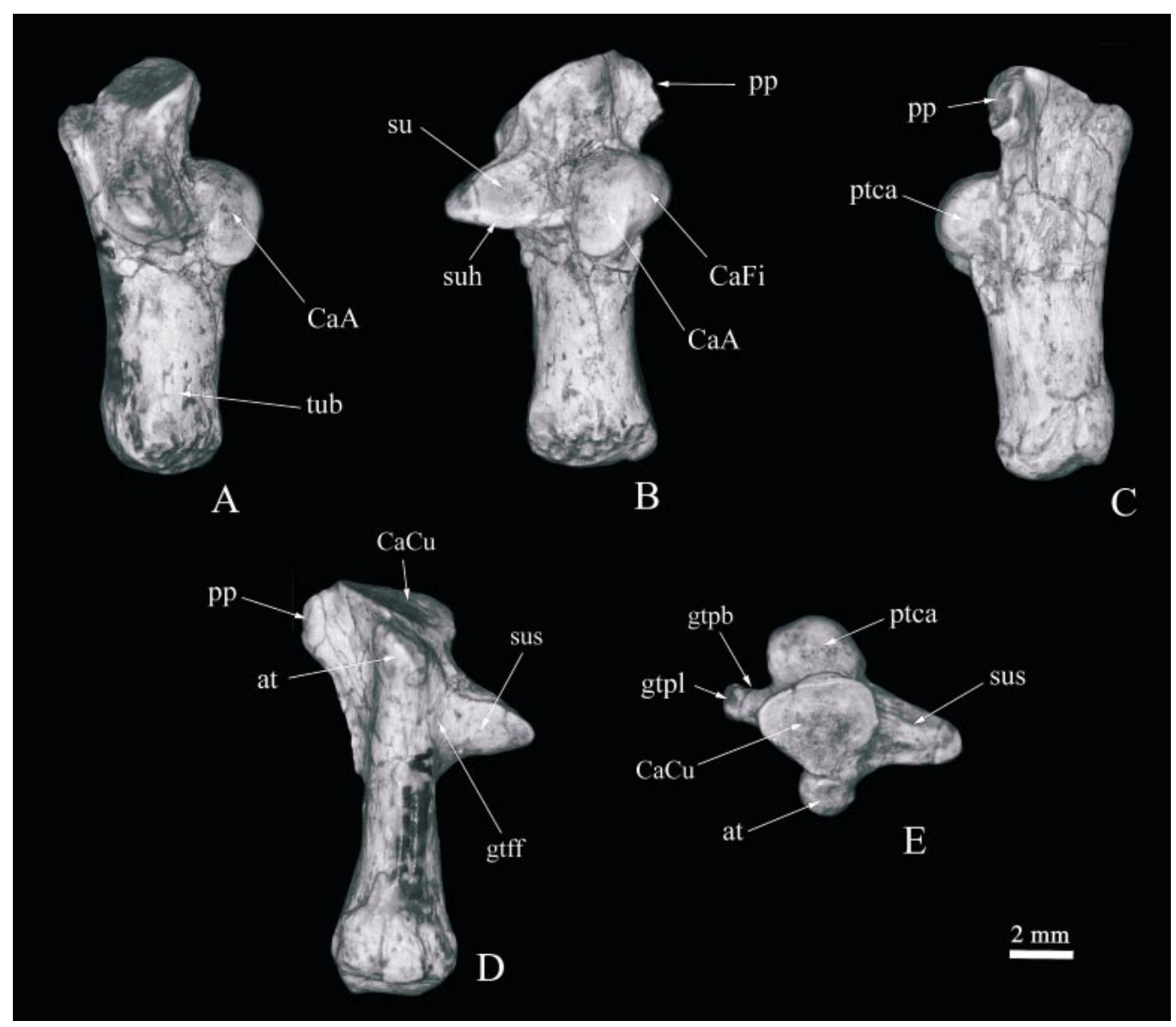

Fig. 66. Medial, dorsal, lateral, ventral, and distal views of the right calcaneus of Rhombomylus (IVPP V7428). at, anterior plantar tubercle; CaA, calcaneoastragalar facet; $\mathrm{CaCu}$, calcaneocuboid facet; $\mathrm{CaFi}$, calcaneofibular facet; gtff, groove for the tendon of M. flexor fibularis; gtpb, groove for the tendon of M. peroneus brevis; gtpl, groove for the tendon of M. peroneus longus; pp, peroneal process; ptca, calcaneal protuberance; su, sustentacular facet; suh, facet for the sustentacular hinge of astragalus; and sus, sustentaculum talus.

medial to the calcaneonavicular facet. Its anterior surface is flat and inclined in the same direction as the calcaneonavicular facet.

Li and Ting (1993) referred a left astragalus (IVPP V7421) and a left calcaneus (IVPP V7420) from a single individual to Rhombomylus. The astragalus differs from that of IVPP V7428 in having a larger size and a more laterally positioned head. The calcaneus is also larger than that of IVPP V7428 and lacks the calcaneofibular facet, which is distinctive from that of IVPP V7428. We exclude these two specimens from Rhombomylus. Like the distal humerus mentioned above (IVPP V7418), they prob- ably belong to a larger mammal yet to be identified.

NAVICULAR: The navicular has a complex shape (figs. 64, 67). It can be roughly divided into two parts, the body and the plantar process. The body is approximately triangular and is proximodistally short. Its concave proximal surface articulates with the astragalar head, whereas the distal surface contacts the ectocuneiform and the mesocuneiform. The medial surface of the body is moderately concave and contacts the articular process of the cuboid. The dorsal and the lateral surfaces are confluent and convex. The plantar process of the navicular is massive and com- 


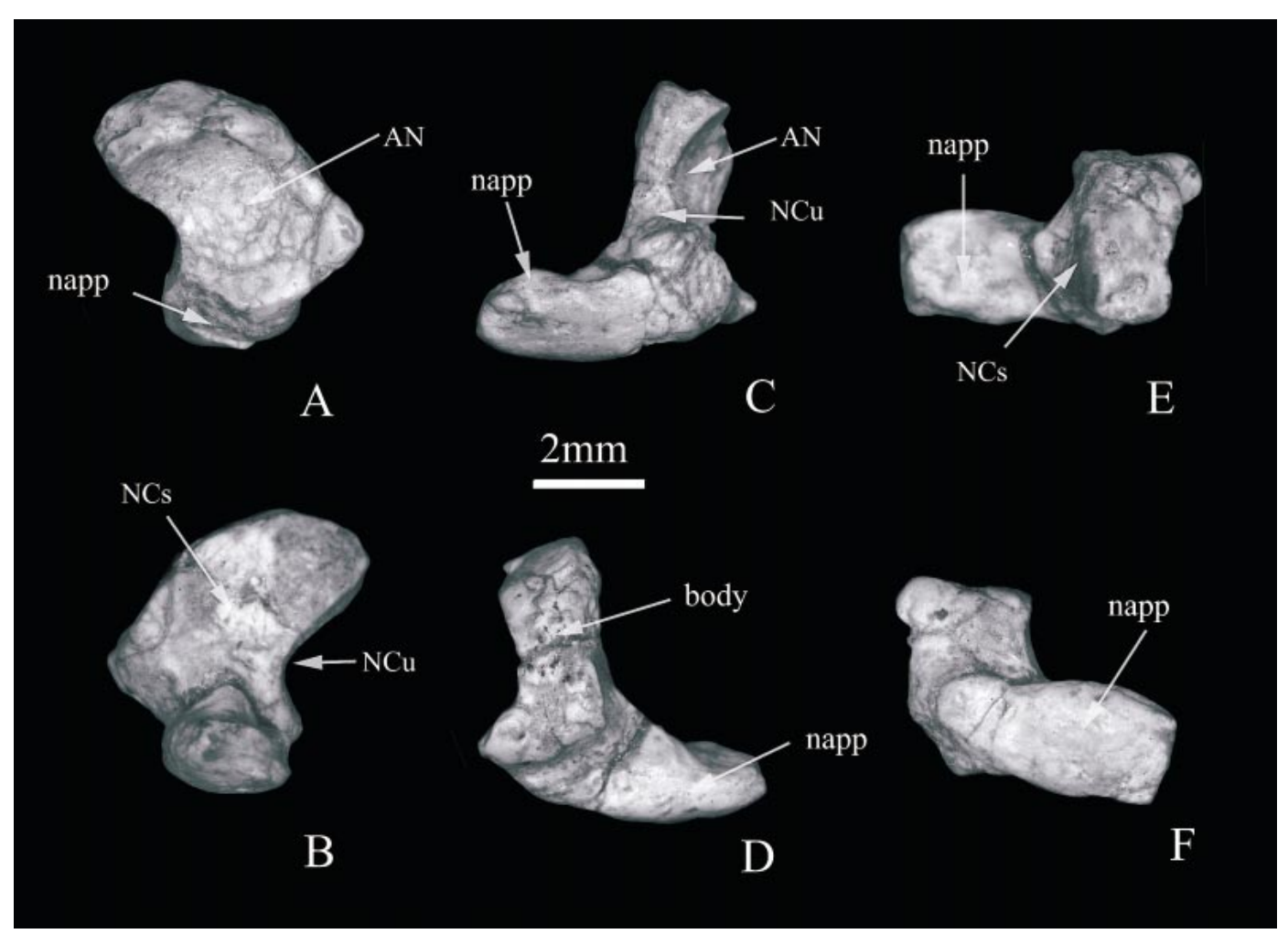

Fig. 67. Proximal, distal, lateral, medial, dorsal, and ventral views of the left navicular of Rhombomylus (IVPP V 7428). AN, astragalonavicular facet on navicular; napp, plantar process of navicular; NCs, articular facet for cuneiforms on the distal surface of navicular body; and $\mathrm{NCu}$, naviculocuboid facet on the lateral surface of navicular body.

pressed dorsoventrally; it extends distally from and at a right angle to the body. Its plantar surface is convex, bearing a rough tuberosity probably for attachment of the $\mathrm{m}$. abductor hallucis brevis. The dorsal surface of the process is concave, but has a low, rounded ridge posteriorly.

CuBoID: In dorsal view, the cuboid is nearly rectangular and is slightly wider than long (figs. 64, 68). The dorsal surface of the bone is gently flat. The posterior surface of the cuboid articulates with the calcaneus. A large articular process (Bohlin, 1951) accounts for most of the medial surface of the cuboid and is visible dorsally as a medial extension of the medioposterior corner of the bone. The posterior surface of the process forms the medial most portion of the calcaneal facet. A small, concave astragalar facet and a flat navicular facet are on the medial surface of the process. The navicular facet extends ventral- ly to the plantar side of the process. The anterior facet of the process articulates with the ectocuneiform. In anterior view, a large oval foramen lies between the articular process and the body of the cuboid. The medial surface of the cuboid anterior to the articular process is gently concave. The distal surface of the cuboid is triangular and slightly concave; it articulates with metatarsals IV and V. A massive plantar process is present on the ventral side of the cuboid. This process projects anteroventrally from the cuboid body and then turns anteriorly. The process also extends laterally as a small hook pointing anterolaterally. In anterior view, the plantar process surrounds the sulcus for the tendon of the $\mathrm{m}$. peroneus longus.

ECTOCUNEIFORM: Viewed anteriorly, the ectocuneiform is roughly wedge-shaped (figs. 64, 69). Its proximal surface bears two articular facets, the navicular and the cuboid. 


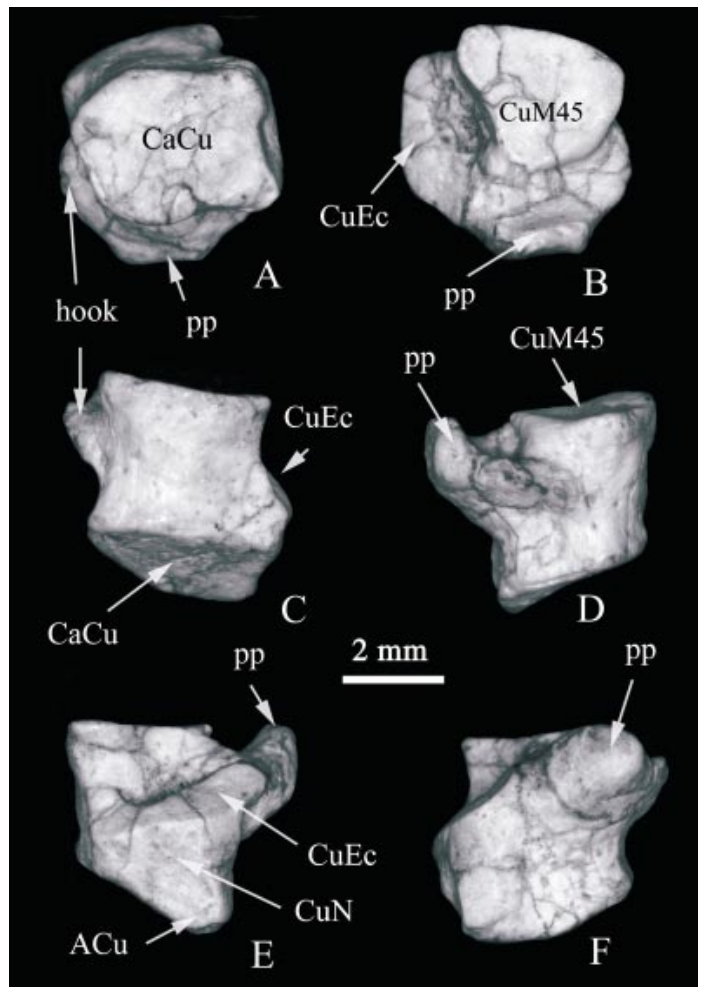

Fig. 68. Proximal, distal, dorsal, lateral, medial, and ventral views of the left cuboid of Rhombomylus (IVPP V 7428). Acu, astragalocuboid facet; $\mathrm{CaCu}$, calcaneocuboid facet; $\mathrm{CuEc}$, facet for ectocuneiform; CuM45, facet for metatarsals IV and V; hook, hook extending laterally from plantar process; and pp, plantar process.

The navicular facet is flat and triangular and faces proximally. The cuboid facet is transversely narrow and dorsoventraly elongate and faces proximolaterally. The lateral surface of the ectocuneiform is gently concave and contacts the anterior part of the medial surface of the cuboid at several points. However, no distinct articular facet is present. The distal surface of the ectocuneiform is smooth and articulates only with metatarsal III. The medial surface of the bone is gently concave and uneven, bearing pits and ridges. It contacts the lateral aspects of the mesocuneiform and the metatarsal II. The dorsal surface of the ectocuneiform is convex and roughly rectangular in outline. Viewed ventrally, the ectocuneiform bears two small tuberosities. One is located mediodistally and ventral to the facet for metatarsal III; the other is situ-

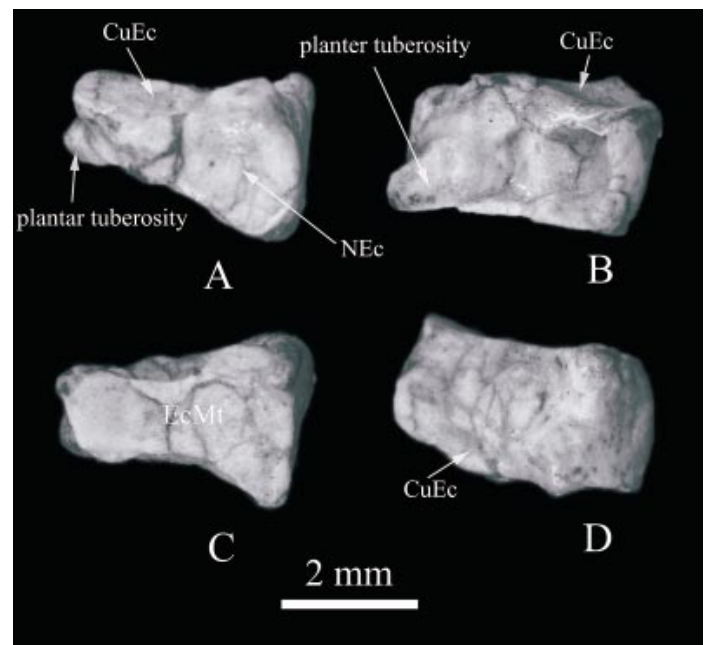

Fig. 69. Left ectocuneiform of Rhombomylus (IVPP V 7428). A, Proximal view (right up); B, medial view; C, distal view; D, lateral view. CuEc, facet for cuboid; EcMt, articular facet for metatarsal III; NEc, facet for navicular.

ated proximolaterally and ventral to the cuboid facet.

MEsocunEIFORM: The mesocuneiform is transversely narrow and much smaller than the ectocuneiform (figs. 64, 70). It articulates with the navicular proximally and with metatarsal II distally. Its notched laterodorsal corner accepts the process on the dorsolateral corner of the proximal end of metatarsal II.

ENTOCUNEIFORM: The entocuneiform is not preserved, but is inferred. A shallow groove

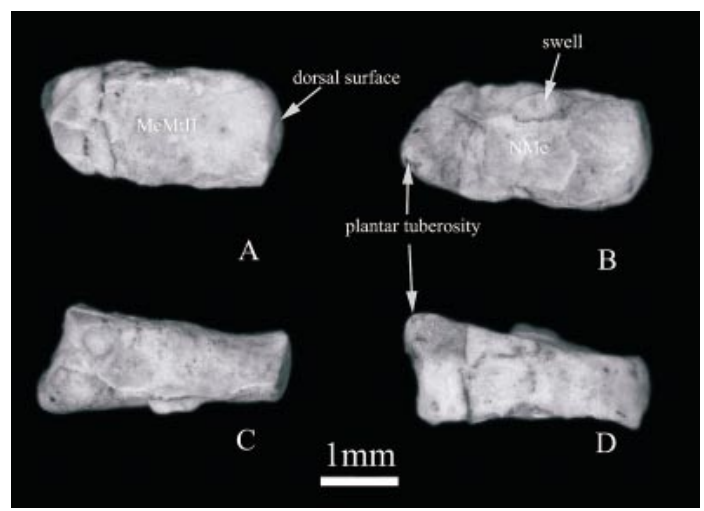

Fig. 70. Distal, proximal, medial, and lateral views of the left mesocuneiform of Rhombomylus (IVPP V 7428). McMtII, facet for the metatarsal II; and NMe, facet for navicular. 


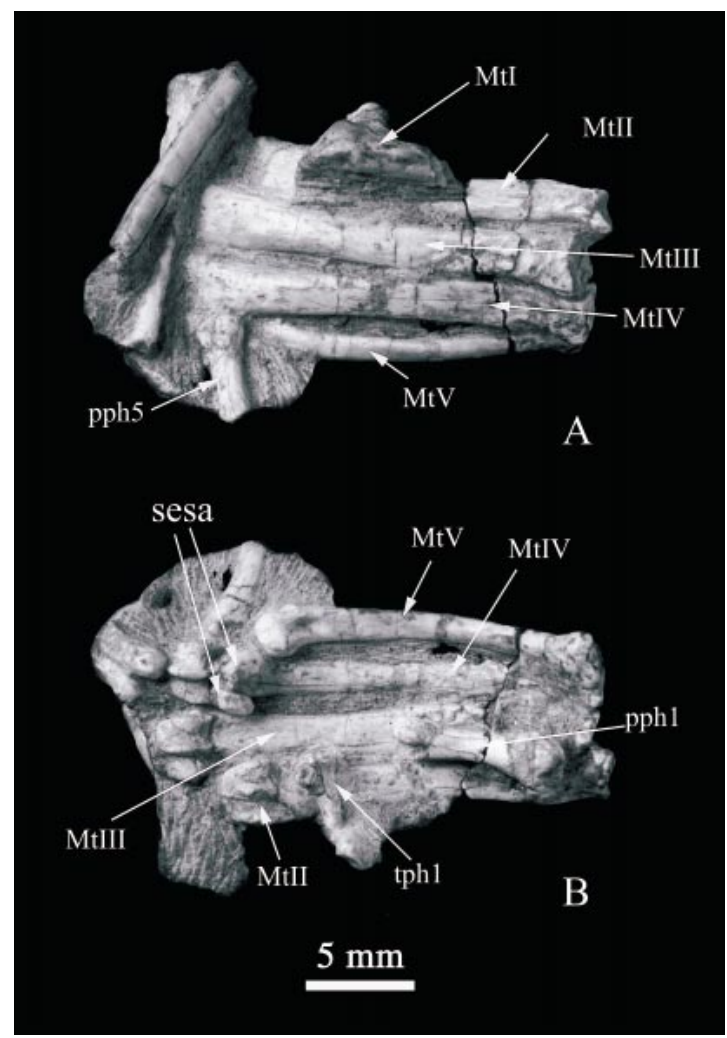

Fig. 71. Dorsal and ventral views of metatarsals and phalanges of the left pes of Rhombomylus (IVPP V 7428). MtI-MtIV, first to fifth metatarsals; pph1, proximal phalange of hallux; pph5, proximal phalange of fifth digit; sesa, sesamoid bones; and tph1, terminal phalange of hallux.

at the junction of the plantar process and the medial side of the navicular body possibly contacts the entocuneiform. In addition, the medial surface of the proximal metatarsal II bears a rough surface probably for contact with the entocuneiform. The indirect evidence shows that a large entocuneiform similar to that of paramyids (Wood, 1962; Szalay, 1985) is present in Rhombomylus.

Metatarsals: All metatarsals of the left foot in IVPP V7428 are well preserved, with the exception of Metatarsal I, of which the proximal half is broken (fig. 71). Metatarsals II-V tightly contact each other proximally and diverge slightly from one another distally. Metatarsal I was turned over. It is the shortest metatarsal, whereas metatarsal III is the longest and most robust. Metatarsal V is the most gracile and is much longer than metatarsal I. Metatarsals II-V are arched dorsally and expanded distally. Metatarsal I is less curved and expanded. Each of the metatarsals bears a small plantar tuberosity on its proximal end. The proximal surface of the metatarsal is wedge-shaped, with the ventral side being narrower. The proximal end of metatarsal II is dorsoventrally deep and mediolaterally compressed. Its medial surface bears a rough facet, which is slightly oblique to the longitudinal axis of metatarsal II. The facet suggests a slightly divergent hallux. The distal end of each metatarsal, bearing a salient ridge, is very similar to those of the metacarpals. The distal end of metatarsal V is slightly asymmetrical. A pair of sesamoid bones is associated with metatarsal III; others are lost.

The proximal and terminal phalanges of the hallux of the left pes in IVPP V7428 are well preserved. The proximal phalanx is a delicate bone. In lateral view the bone is moderately concave ventrally. Both ends are asymmetrical. The proximal articular facet forms a shallow, concave surface delimited by the lateral and medial ridges. The facet extends slightly to the dorsal side of the bone. Medial to the medial ridge is a small sulcus, probably for attachment of the collateral ligament. The distal end of the proximal phalanx bears a small sagittal ridge on its plantar surface. The distal articular facet is restricted to the medial half of the distal epiphysis and extends to the dorsal side of the distal epiphysis. The portion of the distal epiphysis lateral to the sagittal ridge is reduced to a small tubercle. The asymmetry of the proximal phalanx indicates divergence of the hallux. The distal phalanx of the hallux is somewhat transversely compressed. Its proximal articular facet is gently concave. Adjacent to the plantar edge of the proximal facet a prominent flexor tubercle (Jenkins and Parrington, 1976) occupies the plantar surface of the element. The phalanx tapers distally and curves slightly ventrally. The proximal phalanges of other digits of the left pes of IVPP V7428 are partially preserved. They are slender and elongate. The proximal ends of these phalanges are similar to those of the proximal phalanges of the manus but are much larger. 


\section{CHARACTER ANALYSES}

\section{Materials ANd Methods}

The sources of characters are from the literature and from our observations of specimens. The main references and specimens examined are listed in appendix 1. Characters are tabulated using MacClade in a data matrix (appendix 2). The numbering of each character in the following discussion corresponds to that in the data matrix of appendix 2. A number with an asterisk following a character refers to a feature discussed in Meng and Wyss (2001); additional discussion about those characters can be found therein. Characters already discussed in Meng and Wyss (2001) may be modified because the ingroup in the present study is more inclusive. Nonetheless, analyses focus on features that are not covered by Meng and Wyss (2001). Because the study is mainly based on fossil specimens, only dental and osteological characters are considered in our analyses. Selection criteria for characters included (1) use in previous studies, (2) amenability to description as discrete states, and (3) a non-autapomorphic distribution. A character is selected commonly from diagnosis of a taxon or from a list of characters in a phylogenetic analysis of a relevant mammalian group. All these characters will be compared with descriptions and illustrations of specimens, and most frequently with specimens or casts to confirm their accuracy. Polarity determination is based on outgroup comparison (Maddison et al., 1984).

We do not use autapomorphic characters. The derived condition of a character occurring in only one terminal taxon may reflect morphological variation among the selected ingroup, but it does not provide information about relationships. However, for multistate characters an autapomorphic state may be scored. For instance, the dI2 is scored as (0) small with root, (1) ever-growing but restricted to premaxilla, (2) large and extended into maxilla, or (3) the end of incisor extends into orbital region. State 3 occurs only in one taxon, Tsaganomys, among the selected taxa.

In describing the characters, we avoid phrasing a character and states in expressions that imply evolution a priori. For instance, in discussing the length of the infraorbital ca- nal, a relatively long canal is usually considered a primitive state, in contrast to a short, derived one, in eutherians. However, in advanced rodents, such as geomyoids, the infraorbital canal is relatively long because it is extended anteriorly, in relation to the modification of the rostrum for attachment of the masseter muscle. In this case, instead of terming the state as "secondarily elongated", we phrase it as "the canal extends anteriorly".

We also avoid using terms that imply a taxon in describing a character and its states. For instance, when phrasing the states of the zygomatic arch for rodents, we do not use the terms protrogomorphous, hystricomorphous, sciurogmorphous, and myomorphous. Each of these terms usually implies a particular rodent group. The goal of our analyses is to identify groups of the terminal taxa; therefore, using those taxonomically sensitive terms in character analyses appears to be logically circular. Therefore, we use neutral, descriptive, yet committal terms in describing the character and states. In addition, as Landry (1999) pointed out, some logical distortion is inserted when the term "Myomorpha" had to be extended to some hystricomorph forms, such as dipodoids.

\section{DENTITION}

Character 1: Incisor enamel-(O) completely surrounds the tooth, or (1) is restricted primarily to the labial surface (*1). In reporting the earliest zalambdalestid Kulbeckia, Archibald et al. (2001) considered the enamel distribution on the enlarged lower medial incisor in this taxon to be the same as in Glires. However, the actual cross section of the enlarged incisor in Kulbeckia shows that the enamel covers the entire tooth except the side in contact with the other incisor, which is different from the incisor enamel in Glires in which the enamel is absent on the lingual or medial surface (dorsal surface of Archibald et al., 2001). If two teeth are in contact, the enamel in the contacting area may be reduced, as shown by the enamel distribution in the cheek teeth of Rhombomylus. Whether this is the case for the enlarged incisor of Kulbeckia is unclear. Nonetheless, the enamel condition in Kul- 
beckia is different from the primitive condition for eutherians, in which the enamel surrounds the crown of the incisor. Pending studies of new zalambdalestid material from Mongolia and China may reveal the enamel distribution in zalambdalestids, for which we tentatively score a missing entry for this character. The enamel distribution on the enlarged incisor of Barunlestes is also unclear; a missing entry is used for this taxon as well.

Character 2: Incisor enamel-(O) one layer with radial enamel, (1) double-layered with $H S B$, or (2) single-layered with HSB $(* 2)$. Archibald et al. (2001: 62) stated that "scanning electron micrographs (SEMs) of the surface of the lower incisor did not reveal Hunter-Schreger bands [in Kulbeckia]." In our understanding, Hunter-Schreger bands are usually detected in the cross and longitudinal sections of the tooth enamel after the enamel is polished and treated with acid. It is not clear to us that the method of observation used by Archibald et al. (2001) would show whether the incisor enamel of $\mathrm{Kul}$ beckia and other zalambdalestids have the bands. Consequently, we code zalambdalestids with a missing entry. Traditionally, it was thought that single-layered incisor enamel characterizes lagomorphs, whereas doublelayered enamel is typical of rodents (Korvenkontio, 1934). Recent studies by Koenigswald $(1995,1996)$, however, show that single-layered enamel does not typify lagomorphs ancestrally, but rather that it is restricted to leporids. Ochotonids, in contrast, have multilayered enamel, a condition considered primitive relative to the leporid condition by Koenigswald. Martin (1999) postulated that single-layered, radial incisor enamel similar to that in Tribosphenomys and cf. Eomylus is primitive for Theria and that Hunter-Schreger bands are derived; an HSB inner zone developed from the primitive condition, producing double-layered enamel. Reduction of the outer layer of radial enamel (from a double-layered precursor) could have yielded the single-layered condition typical of leporids (but see Koenigswald, 1985: 409).

Character 3: Incisor enamel type-(0) radial, (1) pauciserial, (2) multiserial, or (3) uniserial. Korvenkontio (1934) distinguished three basic enamel types in rodent incisors, based mainly on width of the HSB: pauciserial, multiserial, and uniserial enamel. The distinction of HSB by their width proved not to be practical because a wide overlap in width between pauciserial and multiserial HSB makes it difficult to distinguish them (Sahni, 1980; Flynn et al., 1987; Dauphin et al., 1988). Martin's (1992, 1999) studies, however, redefined the pauciserial and multiserial enamels and regarded the pauciserial type the primitive condition for those that have the HSB in Glires. We follow Martin (1992) in polarizing this character. The enamel type in Tribosphenomys (Meng et al., 1994; Meng and Wyss, 1994; Meng and Wyss, 2001) and Sinomylus (Martin, 1999) is radial, showing no HSB. The enamel type of lagomorphs has been occasionally discussed in detail compared to those of rodents. Koenigswald (1985) considered lagomorph enamel to be multiserial. Based on our observations of fossil and living specimens, and following the criteria of Martin (1994), we think the enamel type in lagomorphs is best regarded as pauciserial.

Character 4: Upper diastema-(O) insignificant, (1) significant, (2) large owing to loss of canine and premolars, or (3) significantly larger than lower diastema (*54). As already discussed by others (Hartenberger, 1985; Li and Ting, 1985; Meng and Wyss, 2001), the long diastema between incisors and premolars of both jaws in gliroid mammals is due to loss of canines and anterior premolars. Zalambdalestes and Barunlestes retain the primitive dental formula of eutherian mammals; therefore, the diastema on their upper jaws stems not from the loss of teeth, but from increased spacing between them (Kielan-Jaworowska, 1975, 1984a, 1984b; Kielan-Jaworowska and Trofimov, 1980). The space is furnished by elongation of the rostrum in Zalambdalestes and Barunlestes. Archibald et al. (2001: 62) stated that "All [Glires and zalambdalestids] have narrow, elongate snouts. Zalambdalestes and Barunlestes fall between the ancestral condition in which most of the elongation is within the maxilla and the derived condition in Glires in which half or more of the elongation is in the premaxilla." Comparing the rostrum of gliroid mammals such as Cocomys (Li et al., 1989) and Rhombomylus with 
that of Zalambdalestes, Barunlestes, Kennalestes, Asioryctes (Kielan-Jaworowska, 1975, 1984a-b) or Leptictis (Novacek, 1986b) we think the rostrum of Glires is neither narrow nor elongated. In fact, the rostrum of Glires is actually wider and shorter than that of the nongliroid forms. This again indicates that the diastema in Zalambdalestes and Barunlestes probably arose independently of the condition in gliroid mammals (Meng and Wyss, 2001). Li and Ting (1985: table 3, ch. 14) considered "lower diastema shorter than upper diastema" to be a synapomorphy for eurymylids and rodents, and "long diastema between incisors and premolars" to be typical of gliroid mammals. We here combine these two characters into one.

Character 5: Lower incisors-(0) more than three pairs, or (1) three or fewer pairs (*3). Asioryctes and Kulbelkia have four pairs of lower incisors, whereas Kennalestes has three (Kielan-Jaworowska, 1981; Archibald et al., 2001). Other ingroup taxa have three or fewer pairs of lower incisors.

Character 6: Upper incisors-(0) more than three pairs, or (1) three or fewer pairs $(* 4)$. Asioryctes has five pairs of upper incisors and Kennalestes has four (Kielan-Jaworowska, 1981). The number of upper incisors in Kulbelkia is uncertain (Archibald et al., 2001). For Asioryctes there seems to be no problem with the tooth counting. The homology of each incisor in eutherian mammals that have four or fewer incisors is always problematic. However, the fact that the fourth and fifth incisors are discrete characters should not be ignored. In scoring these teeth, their loci can be specific or nonspecific; the latter was chosen for this study. Scoring these teeth this way does not affect the calculation of the ingroup relationships for this study, with the exception of the fourth lower incisor for Kulbelkia. Other ingroup taxa have three or fewer pairs of upper incisors.

Character 7: First lower incisor-(O) present, or (1) absent (*5).

Character 8: First lower incisor-(0) small, or (1) enlarged (*6).

Character 9: First upper incisor-(0) present, or (1) absent (*7). Meng and Wyss (2001) scored presence of the first upper incisor in both Barunlestes and Zalambdales- tes. In fact, I1 is not preserved in Barunlestes, although it was mentioned that the snout of Barunlestes extends "between I1 and P2" (Kielan-Jaworowska and Trofimov, 1980: 169) and was reconstructed as being present (Kielan-Jaworowska, 1975; Kielan-Jaworowska et al., 1979). Rougier et al. (1998) scored absence of the first upper incisor in Zalambdalestes, contra to Simpson (1926) and Kielan-Jaworowska (1984a, 1984b). The specimens described by Simpson (1926) and Kielan-Jaworowska (1984a, 1984b) appear to have some space anterior to the enlarged I2; therefore, presence of I1 seems probable. Until additional information on these taxa becomes available, we score "?" for Barunlestes and " 0 " for Zalambdalestes.

Character 10: First upper incisor-(O) small, or (1) enlarged (*8).

Character 11: Second deciduous lower incisor-(0) small and rooted, (1) ever-growing and extended beneath $p 3$ or $m 1$, or (2) beneath $m 3$ and beyond (*9). Primitively, the $\mathrm{di} 2$ and $\mathrm{i} 2$ are rooted and their roots are embedded in the anterior portion of the mandible. Li and Ting (1985) regarded i2 limited to the anterior end of mandible a primitive condition, in contrast to the derived condition in which di2 is below $\mathrm{m} 2$ or $\mathrm{m} 3$ or more posterior. Li and Ting (1985: fig. 6) coded an intermediately derived state for Lagomorpha, whereas Dashzeveg et al. (1998: table 3, fig. 12) considered di 2 below $m 3$ or farther back a derived condition for gliroid mammals including Lagomorph, which is represented by Lushilagus in their analysis. This coding appears contradictory to the observations that the di2 shaft extends only to the level of p3 or $\mathrm{p} 4$ in ochotonids and of $\mathrm{m} 2$ in leporids (Bohlin, 1942; Dawson, 1958; McKenna, 1982). In fact, a short incisor in Lagomorpha has long been recognized as one of the features distinguishing it from Rodentia (Gidley, 1912; Wood, 1957). We consider Dashzeveg et al.'s (1998) coding for Lagomorpha to be an error. In mimotonids, however, the di2 extends to m3 (Li and Ting, 1993; McKenna, 1994). However, a secondarily short di2 occurs in rodents, such as Hydrochoerus.

The homology of the enlarged lower incisor is one of the controversial issues in the study of gliroid and other eutherian mammals, as discussed in Meng and Wyss (2001). 
The enlarged lower incisor of Barunlestes and Zalambdalestes was identified as i1 (Kielan-Jaworowska, 1975, 1984a, 1984b; Kielan-Jaworowska and Trofimov, 1980) and was reported to reach at least to the level of p3 (Kielan-Jaworowska, 1975; Luckett, 1985). Archibald et al. (2001) considered this tooth to be homologous with the second deciduous lower incisor of living Glires on the basis of overall anatomy of the dentitions. If three upper and lower incisors represent the primitive eutherian incisor dental formula (Novacek, 1986a), then the homologies of these teeth with those in primitive eutherians, such as Kennalestes or Asioryctes, which have more than three incisors in both upper and lower jaws, will remain a matter of debate. In a recent study, the enlarged lower incisors of zalambdalestids were still considered to be the i1 instead of di2 (FostowiczFrelik and Kielan-Jaworowska, 2002).

Character 12: Second lower incisor replacement-(0) present, or (1) absent (*12). The incisors are primitively diphyodont in eutherian mammals. The loss of the permanent second upper and lower incisors has been regarded as a synapomorphy for Glires (Li et al., 1987) or as part of a derived condition for gliroid mammals (Luckett and Hartenberger, 1993: table II, "enlarged dI2 persists, ever-growing; I2 absent"). We follow $\mathrm{Li}$ et al. (1987) in considering the replacement of the second incisors as two separate characters, for i2 and I2, respectively.

Character 13: Deciduous I2-(O) small with root, (1) ever-growing but restricted to premaxilla, (2) large and extended into maxilla, or (3) the end of incisor extending into orbital region $(* 10)$. Incisors are primitively contained in the premaxilla in mammals. Li and Ting (1985: table 4) considered [d]I2 extends distally into maxilla a derived condition for gliroid mammals except lagomorphs. Mimotona shared with Lagomorpha the condition in having the dI 2 within the premaxilla (Li and Ting, 1993). In rodents, the incisor may extend farther into the maxilla to the level of M2, as in Tsaganomys (Bryant and McKenna, 1995). The end of the tooth creates a bulge at the orbital region.

Character 14: Anterior surface of dI2(0) smooth, or (1) grooved (*11).

Character 15: Second upper incisor re- placement-(0) present, or (1) absent. See above.

Character 16: Third lower incisor-(0) present, or (1) absent (*13).

Character 17: Third upper incisor-(O) present, or (1) absent (*15).

Character 18: Third upper incisor-(O) lateral to I2, (1) posterior to dI2, or (2) reduced and immediately behind dI2 (*16).

Character 19: Fourth upper incisor-(O) present, or (1) absent. The fourth upper incisor is present in Asioryctes and Kennalestes and absent in all other eutherains selected in the study.

Character 20: Fourth lower incisor-(0) present, or (1) absent. The fourth lower incisor is present in Asioryctes but absent in Kennalestes and other eutherians.

Character 21: Fifth upper incisor-(O) present, or (1) absent. The fifth upper incisor is present in Asioryctes. Its condition in Kennalestes is uncertain.

Character 22: Incisors-(0) conical or convex anteriorly and concave posteriorly, (1) anteroposteriorly compressed, (2) transversely compressed with rounded anterior surface, or (3) with flat, square-shaped anterior surface. The incisor is conical or has a convex labial surface and concave lingual surface in most conservative mammals. In gliroid mammals, the incisor displays variation in shape in addition to enlargement. In extant lagomorphs, both the upper and lower incisors are transversely wide and anteroposteriorly short. This condition, however, is not present in mimotonids and early lagomorphs, such as Palaeolagus. In Palaeolagus the incisors, particularly the lower ones, are anteroposteriorly longer than wide. In simplicidentates, the enlarged incisors are transversely narrow but anteroposteriorly deep. In crosssection, the incisor usually is rounded except the medial surface, which is usually flat. In some rodents, the incisors are very strong, with the anterior surface becoming squared. In crosssection, the tooth may be triangular; the enamel cap is thick, forms a straight line, and is the widest region of the tooth.

Character 23: Upper canine-(0) present, (1) reduced, or (2) absent (*17).

Character 24: Upper canine-(0) tworooted, or (1) single-rooted $(* 18)$. 
Character 25: Lower canine-(0) present, (1) reduced, or (2) absent (*19).

Character 26: Lower canine-(0) tworooted, or (1) single-rooted $(* 20)$.

Character 27: First lower premolar-(O) present, or (1) absent (*21).

Character 28: First upper premolar-(0) present, or (1) absent (*22).

Character 29: Second lower premolar(0) present, or (1) absent (*23). Frahnert (1999) assumed occurrence of at least three premolars in each jaw to be the Glires morphotype with further reduction toward the rodent morphotype (2/1) and lagomorph morphotype (3/2). Although absence of $\mathrm{p} 2$ has been considered a derived feature for gliroid mammals (Meng and Wyss, 2001), Archibald et al. (2001) thought that among some Glires three upper and lower premolars are retained, although they scored absence of p2 for Tribosphenomys and Mimotona, the representative taxa of Glires in their analysis. We are unclear about the evidence of Archibald et al. (2001) for presence of p2 in Glires and thus follow Meng and Wyss (2001) to score " 1 " for the group.

Character 30: Second upper premolar(0) present, or (1) absent (*24).

Character 31: Second upper premolar(0) bladelike, (1) conical, (2) triangular, or (3) hypsodont with enamel fold (*25). Differing from Meng and Wyss (2001), we scored an additional derived condition, P2 triangular for Hyopsodus, Phenacodus, and Anagale.

Character 32: Third lower premolars(0) present, or (1) absent (*26).

Character 33: Third lower premolar-(O) with longitudinal main cusp, (1) with metaconid, (2) with transverse rudiment trigonid and talonid, or (3) enlarged with enamel folds (*27). Differing from Meng and Wyss (2001), we score an additional "1" for Phenacodus.

Character 34: Third upper premolar-(0) present, or (1) absent. This character was not used in Meng and Wyss (2001) because all taxa included in that study possess the P3. P3 is lost in some advanced rodents selected for this study.

Character 35: Third upper premolar- $(0)$ large, triangular in crown view, (1) oval in crown view and multicuspate, (2) small and conical, (3) high-crowned and molariform, or (4) bilobed and elongated (*28).

Character 36: Fourth lower premolar(0) present, or (1) absent. The p4 is absent in muroid rodents. The p4 is also absent in Ivanantonia efremovi (Shevyreva, 1989), an early Eocene rodent with uncertain taxonomic position within Rodentia. Other than the absence of $\mathrm{p} 4$, the lower teeth morphology and enamel microstructures of this species are primitive, similar to those of Alagomys and Tribosphenomys (Hartenberger et al., 1997). This peculiar case either indicates an early divergent gliroid dental morphology or represents an anomalous case of dental development.

Character 37: Trigonid of $p 4-(0)$ singlecusped, (1) tricuspate, (2) bicuspate, or (3) with strong ridges $(* 29)$.

Character 38: Protostylid of $p 4-(0) a b-$ sent, or (1) present. The protostylid is a cingular cusp labial to the protoconid (Wood and Wilson, 1936). It occurs in Florentiamys and Gregorymys.

Character 39: Talonid of $p 4-(0)$ simple, (1) tricuspate, or (2) fully crested (*30).

Character 40: Upper fourth premolar(0) present, or (1) absent. The derived condition occurs in muroids only.

Character 41: Last upper premolar-(O) smaller or subequal to $M 1$, or (1) significantly larger than M1. State 1 occurs in Florentiamys and Gregorymys.

Character 42: Labial cusp(s) of P4-(O) single, (1) double, including a smaller metacone, (2) paracone and metacone equal in size, or (3) crested $(* 31)$.

Character 43: Styles of $\mathrm{P} 4-(0)$ absent, or (1) present. On the lingual side of the tooth, there are protostyle, entostyle and hypostyle in Florentiamys and Gregorymys.

Character 44: Anterior and posterior ridges of $\mathrm{P} 4-(0)$ weak or incomplete, (1) strong, continuous ridges surrounding the labial cusp, or (2) strong with cusp lost (*32).

Character 45: P4 hypocone-(O) absent, (1) small, or (2) very large and anteroposteriorly extended (*33).

Character 46: Labial cingulum on upper molars-(0) present, or (1) absent (*35).

Character 47: Third lower molar-(O) present, or (1) absent (*36). 
Character 48: Third upper molar-(O) present, or (1) absent (*37).

Character 49: Upper cheek teeth-(0) decrease in length and width both anteriorly and posteriorly, (1) increase in width posteriorly, (2) M3 greatly reduced in size, or (3) tooth row increases in width anteriorly (*38). State 3 was not recognized in Meng and Wyss (2001), but it occurs in muroids, in which the molars have the tendency to increase the size anteriorly.

Character 50: Cheek teeth-(0) brachydont, (1) some unilateral hypsodonty, (2) some hypsodont, or (3) hypsodont (*34). The degree of hypsodonty varies among gliroid mammals. If comparing those of lagomorphs and hystricognath rodents selected for this study, the former has a higher degree of hypsodonty in that the tooth crown enters deeply into the alveolus and the roots of the teeth are weak or absent.

Character 51: Upper tooth rows-(0) parallel, or (1) convergent anteriorly. The upper tooth rows are primitively parallel in most eutherian and gliroid mammals. In $\mathrm{Ca}$ via, Myocastor, and Neoreomys, they strongly converge anteriorly and $\mathrm{P} 4 \mathrm{~s}$ are separated by only a narrow gap. This must be related to the modification of mastication and movement of the tongue in those forms. Because of the anterior convergence of the tooth rows, there is no significant space between them in the mouth; therefore, the tongue has to be lodged primarily in the space between the mandibles.

Character 52: Lower cheek teeth-(O) subequal in size, (1) increase in size posteriorly, (2) m3 significantly reduced, or (3) increase size anteriorly (*39). Differing from Meng and Wyss (2001), we add state 3 for muroids.

Character 53: Upper molar crown-(O) subtriangular, (1) rectangular, wider than long, (2) quadrate, or (3) longer than wide $(* 40)$.

Character 54: Precingulum-(0) weak or absent, (1) distinctive along the anterior edge of the tooth, (2) confluent with the protocone to form a strong loph, or (3) forming the strongest loph of upper molars (*41). The precingulum is primitively weak or absent at the anterior protocone base. A distinctive precingulum runs from the anterior base of the protocone to the parastyle in anagalids, pseudictopids, and rodents (Wood, 1962; Dawson et al., 1984; Li et al., 1989; Dashzeveg, 1990b; Korth, 1984; Hu, 1993), but is lacking in Alagomys, Tribosphenomys, and most basal gliroid mammals (Dashzeveg, 1990a; Tong and Dawson, 1995; Meng et al., 1994; Dawson and Beard, 1996; Meng and Wyss, 2001). In later rodents, the cingulum may join the protoloph (Dashzeveg and Meng, 1998), in which case it is usually called the anteroloph (Flynn et al., 1986). In hystricognath rodents that have five lophs on their molars, the anteroloph is independent of the protoloph and is the strongest ridge of the tooth. Although in these forms the homologies of the third and fourth lophs have long been debated (Hoffstetter and Lavocat, 1970; Lavocat, 1973, 1976; Wood and Patterson, 1959; Patterson and Wood, 1982; Candela, 1999), there seems no disagreement about the homology of the anteroloph.

Character 55: Upper cheek teeth lingual cingulum-(0) absent, or (1) present (*42).

Character 56: Upper molar protoloph(0) weak, (1) complete, (2) not recognizable owing to expansion of cusps, (3) strong and forming the anterior shearing edge of the tooth, or (4) forming transversely elongate edge (*43). Meng and Wyss (2001) scored three states for this character. Here we recognize five. In early rodents such as Cocomys and Paramys, the protoloph, the ridge connecting the protocone and paracone, is weak and is often interrupted by a distinct protoconule (paraconule). This is similar to the condition in primitive eutherians such as Asioryctes and Kennalestes. In most advanced rodents, the protoloph becomes well developed and the protoconule is no longer recognizable. A protoloph may be overwhelmed by inflated cusps as in Florentiamys and Gregorymys, in which the expanded cusps become confluent and after wear result in a transversely oriented enamel loop. In nonrodent gliroid mammals, the protoloph forms the anterior shearing edge and usually the highest ridge of the tooth. Because the precingulum is absent, the protoloph contacts the posteroloph of the preceding tooth. In pseudictopids and anagalids, the protoloph is fully developed, but the precingulum is also present (Sulimski, 1968; Hu, 1993), and con- 
tacts an equally developed postcingulum of the preceding tooth and therefore separates the protoloph from the posteroloph of the preceding tooth. The homology of the protoloph in lagomorphs depends on the recognition of homologous cusps, an issue of debate for years (McKenna, 1982; Lopez Martinez, 1985). We accept Averianov's (1998) interpretation of the lagomorph molar cusps and lophs, and consider the protoloph condition in lagomorphs to be comparable to that of nonrodent gliroid mammals. In lagomorphs, however, the protoloph is transversely long and is as high as the remainder of the tooth.

Character 57: Posteroloph-(0) weak or absent, (1) distinct, (2) lost, (3) a continuous ridge connecting the hypocone and metacone, or (4) a transversely elongated ridge. The posteroloph is the posterior cingulum when elevated to form a crest equal in importance to the others (Wood and Wilson, 1936). It is primitively weak or absent. In most gliroid mammals it is distinct. The posteroloph (or cingulum) is absent in Mus, Rattus, Florentiamys, and Gregorymys. In macroscelideans, the loph connects the hypocone and metacone and surrounds a basined talon posteriorly. In lagomorphs, the posteroloph is transversely extended, being as wide as, or even wider than, the protoloph.

Character 58: Trigon basin-(O) narrow, (1) basined, (2) broad, (3) divided by mesoloph, (4) anteroposteriorly compressed $(* 44)$. Meng and Wyss (2001) scored two states for this character; here we recognize five. The trigon basin is narrow in Asioryctes and Kennalestes. The trigon is basined in Adapis, Notharctus, Hyopsodus, and Phenacodus. State 2 is scored for Tribosphenomys and Cynocephalus in which the paracone and metacone are well separated by a broad trigon basin. Accepting the interpretation of the lophs on the pentalophodont molar of hystricognath rodents (Hoffstetter and Lavocat, 1970; Lavocat, 1973, 1976; Candela, 1999), the trigon basin is divided by a well-developed mesoloph. The trigon is greatly compressed anteroposteriorly in lagomorphs.

Character 59: Centrocrista on upper molars-(0) present, (1) converged labially to form a mesostyle, (2) absent, or (3) the para- cone and metacone coalesce (*45). We recognize state 1 for Phenacodus.

Character 60: Molar mesostyle-(0) $a b$ sent, or (1) present. The mesostyle is a small cusp at the labial side of the tooth, between the paracone and metacone (Wood and Wilson, 1936). It is absent in Asioryctes and Kennalestes and present in various taxa selected for the analysis.

Character 61: Upper molar protocone(O) conical, (1) ridged and V-shaped, (2) ridged and anteroposteriorly compressed, or (3) U-shaped (*46). Accepting Averianov's (1998) interpretation of the lagomorph cusps and lophs, we recognize state 2 for lagomorphs.

Character 62: Conule-(0) weak, (1) distinctive, (2) absent, or (3) ridged (*47). The conule condition in Pseudictops, Anagale, and Anagalopsis is unclear. It appears that there is no indication of conules on the molar in specimens of the three taxa known to us. We tentatively scored absence (state 2) for these genera.

Character 63: Paracone and metacone(O) conical, (1) transverse, (2) V-shaped, (3) inflated, (4) anteroposteriorly long, (5) fused with lophs as strong transverse enamel ridges, or (6) confluent to form the anterior lobe of tooth $(* 48)$. Differing from Meng and Wyss (2001), we recognize three additional states for the character. In some hystricognath rodents the paracone and metacone become parts of strong transverse lophs of the lophodont teeth. The paracone and metacone are inflated in Plesiadapis, Nocharctus, and Adapis. They are also inflated in Mus and Rattus. Given Averianov's (1998) interpretation of the lagomorph molar cusps and lophs, we found that the paracone and metacone in lagomorphs are anteroposteriorly compressed in the anterior lobe of the upper molar, whereas the hypocone and its shelf constitutes the posterior half of the upper molar. An intermediate condition is present in some Eocene-Oligocene forms such as Desmatolagus, in which the trigon is the main part of the upper molar, with the paracone and metacone marginally located and still distinguishable. The hypocone and the posteroloph form the posterior, minor portion of the molar. In Palaeolagus and other lagomorphs, such as Ochotona, Prolagus, and 
Lepus, the paracone and metacone are no longer separable and the hypocone and posteroloph are expanded to form the posterior lobe of the tooth.

Character 64: Upper molar hypocone(0) weak or absent, or (1) strong. The hypocone is primitively absent or weak. The hypocone is not present in anagalids ( $\mathrm{Hu}$, 1993), nor is it present in Pseudictops (Sulimski, 1968). The hypocone condition in early rodents is a matter of debate. Flynn et al. (1986: 47) stated: "If the primitive rodent condition includes presence of hypocones on molars, then paramyine Ischyromyoidea, a protrogomorphous group, would be derived by their loss of the hypocone, an idea advanced by Korth (1984)." However, Korth (1984: 8) wrote: "hypocone on upper molars usually small" in paramyines. Butler (1985: 390) also made a similar observation, although he thought the hypocone is absent on $\mathrm{P} 4$ of paramyines. In the early Clarkforkian Paramys adamus the hypocone does exist on P4 and molars (Dawson and Beard, 1996). The hypocone is small in early ctenodactyloids (Dawson et al., 1984; Li et al., 1989; Dashzeveg, 1990a). A small hypocone may be present in Tribosphenomys (Meng et al., 1994; Meng and Wyss, 2001), but the cusp is absent in Alagomys (Dashzeveg, 1990b; Tong and Dawson, 1995; Dawson and Beard, 1996). The hypocone is usually large and continues labially as a strong posteroloph in some early gliroid mammals, such as Heomys and Mimotona. It is expanded to form a broad shelf in Rhombomylus and Matutinia (Ting et al., 2002).

Character 65: Cusps lingual to protocone on M1-(0) absent, (1) present as anterostyle and enterostyle, or (2) as protostyle and hypostyle. In murids, two small cusps occur on the lingual side of the protocone. These cusps are termed anterostyle and enterostyle (Jacobs, 1978; Jacobs et al., 1989). Jacobs et al. (1989) considered this feature a synapomorphy for the family Muridae. In geomyoids, cusps developed on the lingual side of the protocone and hypocone are termed, respectively, the protostyle and entostyle (Wahlert, 1983). Because the homologies of these cusps are uncertain (Jacobs et al., 1989), we tentatively treat these lingual cusps as different states in muroids and geomyoids.

Character 66: Cusps labial to protoconid on $\mathrm{ml-(0)}$ absent, (1) present, or (2) as protostylid and hypostylid (2). For the same reason stated above, we code the character separately.

Character 67: Mesoloph (mesolophule)— (0) absent, or (1) present. The mesoloph is a crest from the mesocone toward the buccal or lingual margins of the tooth (Wood and Wilson, 1936). This crest occurs as early as the middle Eocene in the muroid Pappocricetodon (Wang and Dawson, 1994). The homology of the mesoloph is a matter of long debate for hystricognath rodents, as summarized in several studies (e.g., Bryant and McKenna, 1995; Candela, 1999). In short, Lavocat $(1973,1974 a, 1974 b, 1976,1980$; Hoffstetter and Lavocat, 1970) considered that in the pentalophodont molar of the African hystricognathi, the third and fourth lophs are homologous to the mesoloph and metaloph. This tooth pattern is primitive for the "caviomorphs", which were considered to be paraphyletic by Bryant and McKenna (1995). In contrast, Wood (1974, 1980, 1984, 1985; Wood and Patterson, 1959, 1970; Patterson and Wood, 1982) regarded the third loph the metaloph, and the fourth one a neoloph. Bryant and McKenna (1995) thought that the third loph is the mesolophule, a crest extending labially from the metaconule (Flynn et al., 1986). This view is largely accepted by Candela (1999). For hystricognaths in which the third crest is present, we follow Bryant and McKenna in scoring it as the mesolophule. We note that in Bathyergoides (Lavocat, 1973; personal obse. of a cast, AMNH 114279), there are only four crests on the upper molar. We also observe that in Paraphiomys (Lavocat, 1973; personal obse. of casts, AMNH 114281-3, 11487) the mesolophule is incomplete; the mesolophule is a crest extending from the mure toward the metaloph that is connected to the posteroloph on P4-M2. Given the interpretation of the mesolophule for hystricognath rodents, the "mesoloph" used by some authors for the group may not be homologous with that in other rodents.

Character 68: Hypoflexus-(0) absent or shallow, (1) deep and anterolabially extend- 
ed, (2) deep and posterolabially extended, or (3) deep and anteroposteriorly compressed. The hypoflexus is the valley between the protocone and hypocone on the lingual side of the upper molar. The hypoflexus is absent or shallow in primitive forms. In advanced conditions, the hypoflexus may extend anterolabially as in Paraphiomys, or posterolabially as in Tataromys. In lagomorphs, the hypoflexus is deep and anteroposteriorly compressed.

Character 69: Mure-(0) absent, or (1) present. The mure (or endoloph) is the longitudinal crest that connects the protocone and hypocone (Wood and Wilson, 1936). In primitive forms, such as Asioryctes and Kennalestes, the hypocone is not developed or is very small and a mure is usually absent. In forms that have the hypocone, the protocone and hypocone are commonly connected by the mure. This connection is absent in lagomorphs. Bryant and McKenna (1995) considered that hystricognaths do not have the mure, which is not accurate, because some early hystricognaths, such as Paraphiomys, have the mure (Lavocat, 1973; personal obse. of casts). Owing to deepening of the hypoflexus, the mure is more labial or absent in hystricognath rodents.

Character 70: Hypoflexid-(O) shallow or absent, or (1) deep. The hypoflexid is the valley between the protoconid and hypoconid on the labial side of the lower cheek teeth. It becomes deep when the protoconid and hypoconid are large and the ectolophid is lingually positioned.

Character 71: Ectolophid-(0) present, or (1) absent. The ectolophid is the ridge from the protoconid to hypoconid, near the labial side of the tooth (Wood and Wilson, 1936). It is considered homologous with the cristid obliqua of nongliroid mammals (Meng and Wyss, 2001). This ridge may be lost in various forms such as lagomorphs, murids, and geomyoids.

Character 72: Metaloph-(O) extending to protocone, (1) to hypocone (parallel to the protoloph), or (2) posteriorly to join posteroloph. The metaloph is the ridge extended from the metacone. It usually runs lingually either to the protocone as a primitive condition or to hypocone as a derived condition. In some hystricognath rodents it turns back- wards to join the posteroloph. In Tataromys, the metaloph is usually connected to the hypocone but occasionally it turns to the posteroloph (Wang, 1997).

Character 73: Lower molar trigonid-(O) notably higher than talonid, (1) slightly higher or equal in height, or (2) flat occlusal surface (*49). In advanced rodents, the occlusal surface of a cheek tooth is flat so that the trigonid and talonid are at the same level.

Character 74: The posterior wall of trigonid-(0) present, (1) strongly crested, or (2) incomplete or absent $(* 50)$.

Character 75: Anteroconid of $m l-(0)$ absent, (1) single, or (2) bilobed. The anteroconid is a cuspid anterior to the protoconid and metaconid usually at the longitudinal axis of $\mathrm{m} 1$. The anteroconid is single on $\mathrm{m} 1$ of Cricetops (Carrasco and Wahlert, 1999) and bilobed in Mus and Rattus.

Character 76: Anterocone on $\mathrm{MI}-(0)$ absent, (1) present. The anterocone is a cusp anterior to the protocone and paracone, usually more labial to the longitudinal axis of M1. A single anterocone is present on M1 of some rodents such as Eumys and Eucricetodon, and a bilobed anterocone occurs in Cricetops, Mus, and Rattus. However, we did not include any taxon bearing a single anterocone in our analysis.

Character 77: Lower molar paraconid(0) distinct, (1) reduced, or (2) absent (*51).

Character 78: Lower molar metaconid(O) aligning with the protoconid, (1) more anterior than protoconid, or (2) more posterior (*52). In Neoreomys and Myocastor, the protoconid is extended more anteriorly while the metaconid is relatively posteriorly positioned, so that the ridges between the labial and lingual cusps are diagonal with the labial end directed anterolaterally.

Character 79: Mesoconid of lower molar-(0) absent, or (1) present. The mesoconid is a cusp on the ectolophid in the median valley (Wood and Wilson, 1936).

Character 80: Talonid basin of lower molar-(0) restricted, (1) broad, (2) filled with a large entoconid, (3) filled with hypolophid, or (4) separated as an independent lobe (*53). In addition to those used by Meng and Wyss (2001), we add two additional states: the talonid basin is filled with a large entoconid and with a hypolophid in rodents. 
Character 81: Entoconid of lower molar-(0) posteriorly positioned, or (1) anteriorly shifted. The entoconid is usually opposite the hypoconid. In many gliroid mammals, this cusp is shifted anteriorly.

Character 82: Hypolophid of lower molar-(0) absent, (1) present, or (2) very strong. The hypolophid (also called hypolophulid) is primitively absent. When present, the ridge usually extends from the entoconid to the hypoconid or to the ectolophid anterior to the hypoconid. The hypolophid is usually strong in hystricognath rodents. In some Tsaganomys, which was considered a sister group of hystricognath rodents (Bryant and McKenna, 1995), the hypolophid is usually not preserved in adult specimens (Bryant and McKenna, 1995; Wang, 2001), but is evident in juvenile specimens.

Character 83: Hypoconulid of $m 3-(0)$ present, (1) forming posteriorly and transversely substantial third lobe, (2) third lobe distinct but narrow, (3) hypoconulid incorporated into posterior cingulum, (4) third lobe anteroposteriorly elongated, or (5) hypoconulid and posteroloph absent (*56). Differing from Meng and Wyss (2001), we add state 4 for Tataromys and 5 for Florentiamys and Gregorymys.

\section{MANDiBLE}

Character 84: Lower diastema-(0) insignificant, or (1) significant (*55).

Character 85: Mandibular condyle height relative to tooth row-(O) even with tooth row, (1) somewhat higher and posterodorsally extended, or (2) significantly higher (*68).

Character 86: Horizontal ramus of mandible-(0) slender and long, (1) deep and short, with symphysis tapered anteriorly, or (2) deep and short, with a deep symphysis $(* 69)$.

Character 87: Mental foramina-(0) two or more, or (1) single. Dashzeveg et al. (1998) recognized this character. They also considered a single, enlarged mental foramen as an autapomorphic state for Decipomys, which is not included in this study. Two mental foramina are present in Barunlestes, Zalambdalestes (Dashzeveg et al., 1998; Rougier et al., 1998; Archibald et al., 2001) and some other nongliroid mammals. Archibald et al. (2001) considered the anterior and posterior mental foramina as separate characters that vary in position.

Character 88: Coronoid process-(O) large, (1) somewhat reduced, (2) greatly reduced but still as high as mandibular condyle, (3) reduced and located lateral to $\mathrm{m} 3$, or (4) nearly absent, forming a rudimentary process much lower than the condyle (*70). The coronoid process in caviomorph rodents is low and anteriorly positioned (lateral to m3). Differing from Meng and Wyss (2001), we add an additional state for those rodents.

Character 89: Angular process-(O) small, or (1) expanded (*71).

Character 90: Angular process-(0) origin in the same plane of incisor alveolus, (1) process bending inward, or (2) process origin lateral to incisor alveolus. The angular process of the mandible is usually within the same plane as the mandibular body in eutherian mammals or in the same plane as the incisor alveolus in some rodents. This condition is conventionally considered sciurognathy. In contrast, state 2 is called hystricognathy in the study of rodents. The sciurognath and hystricognath conditions have been used as main characteristics for two principal rodent subgroups, Sciurognathi and Hystricognathi (see Landry, 1999, for review). However, Landry (1999) recognized that in a typical sciurognath condition the angular process not only takes origin in the same plane as the incisor alveolus, but the middle of the process is pushed inward and downward and the posterior end is turned upward. In paramyids, the angular process takes origin in the same plane of the incisor alveolus, but the middle of the process is not pushed inward. Therefore Landry (1999) coined a new term "protrocognath", to describe the paramyid condition, but not to denote a taxon. We found the protrocognath condition also present in Rhombomylus and Matutinia but unknown in other basal gliroid taxa because of breakage in this region. Landry (1999) noted that a jaw of Mimolagus has the sciurognath condition. Landry did not compare the protrocognath condition with those of nongliroid eutherians. Although we avoid using terms such as sciurognath, hystricognath and protrocognath, which imply 
taxa a priori, we follow Landry (1999) in recognizing the difference between angular processes in Glires.

Character 91: Anterior edge of masseteric fossa-(0) confined posterior to cheek teeth, (1) extends below m3, (2) extends below $m 2$, or (3) below anterior half of $m 1$ or p4 (*72). The masseteric fossa is positioned farther anteriorly below $\mathrm{m} 1$ or even $\mathrm{p} 4$ in advanced rodents.

Character 92: Protuberance surrounding the base of incisor root-(0) absent, or (1) present. In geomyoid rodents, the lower incisor extends posteriorly beyond $\mathrm{m} 3$; its end projects lateral to the plane of the mandible and creates a capsule on the lateral surface of the coronoid process. In some rodents, such as Tsaganomys and Pedetes, the long lower incisor extends posteriorly even to the mandibular condyle, but it does not project laterally as a capsule. Presence of the capsule may increase the area for the attachment of the masseter muscle.

Character 93: Mandibular condyle-(0) ovoid, (1) cylindrical transversely, or (2) anteroposteriorly oriented. The mandibular condyle is ovoid in Asioryctes (Rougier et al., 1998), macroscelideans, Rhombomylus, and Matutinia. In other nongliroid eutherians the condyle is usually transversely cylindrical. The transverse length of the cylindrical condyle varies from species to species. For instance, the condyle of Tupaia is relatively narrower than that of Cynocephalus. In most gliroid mammals, the condyle is oriented anteroposteriorly, which appears to be an adaptation for anteroposterior movement of the lower jaw.

Character 94: Coronoid canal-(O) $a b-$ sent, (1) present. A short canal posterior to $\mathrm{m} 3$ pierces the anterior base of the coronoid process and confluent with the mandibular foramen is uniquely present in lagomorphs.

Character 95: Mandibular foramen-( 0 ) lower than tooth row, or (1) at level of tooth row or higher. The mandibular foramen is lower than the tooth row in many non-gliroid eutherians, such as Asioryctes, Kennalestes, Zalambdalestes, and Leptictis (Rougier et al., 1998). In other nongliroid and all gliroid mammals, the foramen is positioned at the level of, or higher than, the tooth row.

\section{CRANIAL ForAminA}

Character 96: Incisive foramina-(0) small and anteriorly positioned, (1) small and slightly posterior, (2) moderately elongated and at middle level of rostrum, (3) large and at middle level of rostrum, (4) greatly elongated and expanded posteriorly, (5) reduced but at middle, or (6) posteriorly positioned and significantly reduced (*57). The incisive foramen is a canal linking the oral and nasal cavities and transmits the nasopalatine duct (Broom, 1897; WöhrmannRepenning, 1984, 1991, 1992, 1993; Giere et al., 1999). The condition of the incisive foramen is unclear in Asioryctes and Kennalestes, because the anterior part of the rostrum in these forms is not well preserved (Kielan-Jaworowska, 1969). Nonetheless, the incisive foramen has been reconstructed as absent in Asioryctes, Kennalestes, Zalambdalestes, and Deltatheridium (Kielan-Jaworowska, 1984a, 1984b). Reduction of the canal occurs in some extant taxa, but the is regarded as secondary (Giere et al., 1999) because the canal is found in the main clades of mammals, including monotremes (Broom, 1896a; Zeller, 1989), marsupials (Broom, 1896b), and eutherians (Wöhrmann-Repenning, 1984, 1991, 1992, 1993). Nonetheless, some specimens show that the incisive foramen is present at least in Deltatheridium, and probably in Zalambdalestes (Gregory and Simpson, 1926; Rougier et al., 1998, fig. $1)$. It is likely that a small incisive foramen, positioned close to the incisor, is present in Asioryctes and Kennalestes. Nonetheless, we score a missing entry for each of the latter taxa. Enlarged and posteriorly shifted incisive foramina are a common feature in gliroid mammals (Wahlert, 1974; Novacek, 1985; Novacek and Wyss, 1986; Li et al, 1987; Li and Ting, 1993; Luckett and Hartenberger, 1993; Meng and Wyss, 2001). However, the sizes, shape, and positions of the foramina display considerable variation. It appears that the sizes of the foramina are not related to the size of the rostrum. For instance, Tsaganomys has a very extensive rostrum, but the incisive foramina are greatly reduced and posteriorly positioned (Bryant and McKenna, 1995; Wang, 2001).

Character 97: Infraorbital canal-(0) 
long, (1) short, or (2) anteriorly extended. Luckett and Hartenberger (1993) considered a short infraorbital canal a derived feature for gliroid mammals. Li and Ting (1985) scored a short infraorbital canal for leptictids and gliroid mammals. However, Novacek (1986b) considered the canal in leptictids to be moderately short, and recognized this as a derived condition. Li et al. (1987) employed a short infraorbital canal to group lagomorphs, eurymylids, and rodents, but not mimotonids. Recent studies demonstrated that the infraorbital canal in mimotonids is also short (Li and Ting, 1993; McKenna, 1994). In geomyoid rodents, the infraorbital canal is anteriorly extended so that the anterior opening (infraorbital foramen) of the canal is at the middle of the rostrum, near the premaxilla-maxillary suture (Wahlert, $1983,1985)$. This is probably related to the elongation of the rostrum and anterior shift of the attachment site for the masseteric muscle, as well as the modification of the canal itself. It has been postulated that the geomyoid condition is a secondary closing over of the dorsal portion of the myomorphic infraorbital foramen by a hypertrophid zygomatic plate; therefore, geomyoids do not have a true infraorbital canal (Landry, 1999). An elongate rostrum does not always correspond to a long infraorbital canal. For instance, the rostrum of the Tsaganomys is significantly extended, but its infraorbital canal remains short.

Character 98: Infraorbital foramen-(O) small and circular, (1) small and compressed, (2) small and sunk into side of rostrum, (3) extremely large, or (4) keyholeshaped. The infraorbital foramen is the anterior opening of the infraorbital canal and is primitively small and circular. In rodents, the foramen has been greatly modified in relation to diversification and anterior shift of the jaw muscles and to reconfiguration of the skull. In protrogomorphous rodents, the infraorbital foramen is small and circular, as in most other eutherians. In hystricomorphous rodents, the infraorbital foramen is extremely large, which allows the medial masseter to pass through the foramen and attach to the lateral surface of the rostrum. In sciuromorphous rodents, the lateral masseter muscle extends to the side of the rostrum over the zygomatic plate formed by the maxilla, not passing through the infraorbital foramen; the infraorbital foramen is small and more anterior. Because of the muscle attachment, the foramen is transversely compressed. In geomyoid rodents the infraorbital foramen is not visible anteriorly because its lateral margin is flush with the wall of the snout (Wahlert, 1985). Again, this condition may be derived from the myomorphous condition (Landry, 1999). In myomorphous rodents the foramen is keyhole-shaped. The medial masseter muscle passes through the foramen as in hystricomorphous rodents, whereas the lateral masseter is attached in front of the eye on the zygomatic plate of the maxilla as in sciuromorphous rodents. Although not included in our analysis, we note that Bathyergidae is protrogomorphous but hystricognathous. The protrogomorphy was derived from the hystricomorphous condition (Bryant and McKenna, 1995), so that Bryant and McKenna (1995) scored the hystricomorphous condition for Bathyergidae. Landry (1999) considered secondarily reduced infraorbital foramen a typical character of Bathyergidae. In addition, the high round infraorbital foramen of Rhizomys (not included in the study) is transformed from the myomorphous condition (Flynn et al., 1985).

Character 99: Postglenoid foramen-(0) medial to glenoid process, (1) on ventral side of basicranium and posterior to postglenoid process, (2) in glenoid fossa within the squamosal, (3) on lateral side of skull and between squamosal and ectotympanic or petrosal, (4) on lateral side of skull within squamosal, or (5) concealed by bulla. The postglenoid foramen opens ventral and medial to the glenoid process in Asioryctes and Kennalestes (Kielan-Jaworowska, 1981; Rougier et al., 1998). Rougier et al. (1998) also scored the same state for Zalambdalestes. However, from the illustration of that taxon (Kielan-Jaworowska, 1984a: fig. 1), the postglenoid foramen in Zalambdalestes is posterior to the glenoid process, as in Barunlestes (Kielan-Jaworowska and Trofimov, 1980). Rougier et al. (1998) scored the medially placed postglenoid foramen as state " 2 " for the selected group in their analysis, whereas we score this state as " 0 " because of the different ingroup. Wahlert (1974) con- 
sidered that the postglenoid foramen in rodents pierces the squamosal bone ventral to the zygomatic root and posteromedial to the glenoid fossa. However, Wahlert's observation is based mainly on skulls of Marmota monax and, as noted by Wahlert (1974), many Marmota skulls do not have this foramen. Related to the postglenoid foramen is the temporal foramen. Wahlert (1974: 374) wrote: "The temporal foramen is absent in Marmota. When present it is within the squamosal bone or in the squamoso-parietal suture, usually posterodorsal to the root of the zygomatic arch. It serves the same function as the postglenoid foramen and can take over the entire function of that opening." The temporal foramen was illustrated on the skull of Marmota (Wahlert, 1974: fig. 1). A foramen in a similar position in fossil taxa (Paramys, Reithroparamys, Sciuravus, Ischyromys, Ardynomys, and Prosciurus) was identified as the postglenoid foramen (Wahlert, 1974). In these forms, at least one temporal foramen coexists. In muroids, such as sigmodontines (Voss, 1988), the postglenoid foramen is dorsal to the external auditory meatus, between the bulla and the squamosal. Novacek (1985) considered absence of the postglenoid foramen to be a shared derived feature for Rodentia and Lagomorpha. In a later study, Novacek (1986b) called the foramen on the lateral side of the squamosal the pseudopostglenoid foramen and regarded it as a shared derived condition for rodents and lagomorphs. In all hystricognath and ctenodactylid (entodactyans of Landry, 1999) rodents, the foramen is between the squamosal and ectotympanic (Landry, 1999). In Erethizon and Dasyprocta a small gap is found between the squamosal and ectotympanic, but whether it represents a functional postglenoid foramen is unclear. We follow Landry (1999) in scoring the character for these two taxa. In fossil species, the postglenoid foramen is usually unclear because of poor preservation. In Tsaganomys, however, it is within the squamosal (AMNH 19019, 21675). The postglenoid foramen concealed ventrally by a trabeculated, flattened auditory bulla is a unique state in $C y$ nocephalus (Novacek, 1986b). In Anagale, the foramen is medial to the glenoid process.

Character 100: Left and right optic fo- ramina-(0) separate, or (1) confluent anteriorly along the skull midline (*73).

Character 101: Nasolacrimal foramen(0) in orbit, or (1) anterior to orbit. The nasolacrimal (lacrimal) foramen transmits the lacrimal duct (Wahlert, 1974). The duct runs from the foramen near the eye to the base of the nasal cavity and opens into the cavity. The foramen primitively lies inside the bony orbit in eutherians and Glires, except murids, myoxids, and dipodids, in which the foramen is anterior to the orbit (Frahnert, 1999).

Character 102: Nasolacrimal foramen(0) within lacrimal, (1) between lacrimal and maxilla, or (2) within maxilla. Commonly, the nasolacrimal foramen is situated in the lacrimal bone in eutherians except hyracoids, pholidotans, and some carnivores (Frahnert, 1999). In most rodents and lagomorphs (Wahlert, 1974; Frahnert, 1999), including early forms such as Cocomys ( $\mathrm{Li}$ et al., 1989), the foramen is between the lacrimal and the maxilla. Only in some hystricognaths is the foramen completely within the lacrimal, which Frahnert (1999) considered to be secondary because in early ontogeny of all sampled rodents, including hystricognaths, the nasolacrimal foramen is between the lacrimal and maxilla (Dierbach, 1985; Frahnert, 1999). However, because we score characters based on adult specimens, we code " 0 " for those hystricognaths that have the foramen in the lacrimal. In other hystricognathous rodents examined, including Myocastor and Erethizon, the foramen is in the maxilla. In Myocastor, a vestige of the lacrimal bone appears to frame the posterior corner of the foramen. The nasolacrimal foramen is situated in the maxilla as a pocketed-like structure in murids, myoxids, and dipodids in association with the specialized masseter muscle (Frahnert, 1999). Although not included in our analyses, we mention that in Rhizomys the nasolacrimal foramen is primarily within the orbit and is completely in the maxilla immediately ventral to the infraorbital foramen. In Bathyergus the foramen is in the maxilla but differs from that of Rhizomys in that it is distantly separated from and dorsal to the infraorbital foramen.

Medial to the nasolacrimal foramen and canal, there is a large, circular opening in the orbit of Cavia. Cooper and Schiller (1975: 
19) described: “The 'orbital hiatus' (Hiatus orbitalis), a large channel communicating between the orbit and the nasal cavity, is at the dorsorostral border of the orbit and is bounded medially by the ethmoid, ventrally by the maxilla, laterally by the lacrimal, and dorsally by the frontal and lacrimal. It is covered by a fibrous membrane which is not pierced by any vessels or nerves." This structure is unique to Cavia and is not found in any other rodents or eutherian mammals we examined; therefore, we did not include it as a character.

Character 103: Nasolacrimal duct-(O) having no relation with incisor, (1) lateral to posterior part of incisor, or (2) medial to incisor. The course of the nasolacrimal duct has been recognized as a critical feature for rodent relationships (Frahnert, 1999; Landry, 1999). The duct running medial to the posterior part of the incisor is present in ctenodactylids, hystricognaths, and heteromyids (Frahnert, 1999), whereas in other rodents, the duct is lateral to the posterior portion of the incisor. Landry (1999) coined two terms for these conditions: "entodacry" and "ectodacry", and bracketed the Hystricognathi and ctenodactylids in a new group "Entodacrya". Landry did not include heteromyids within the new group, although they have the entodacry condition, according to Frahnert (1999). Landry did not create a counterpart Ectodacrya for the other rodents. As Frahnert (1999) pointed out, because the enlarged incisor is not present in most eutherians, a meaningful comparison of the course of the nasolacrimal duct is difficult. For that reason, we consider the duct having no relation with the incisor as the state for nongliroid mammals. For gliroid taxa, Frahnert (1999) considered that the lateral course of the nasolacrimal duct is probably primitive for Glires for two reason. First, the duct runs lateral to the incisor in Daubentonia and lies lateral in the skull in Tupaia. Second, taxa displaying plesiomorphic characters in rodents, such as aplodontids and sciurids, have the lateral course of the nasolacrimal duct. The course of the nasolacrimal duct can be estimated by the positions of the lacrimal foramen and the incisor, but this cannot be decisive. Therefore, the scores of this character in fossils are tentative. In some cases, such as Hydrocho- erus and Agouti (not included in our analyses), the incisor is relatively short and the nasolacrimal duct is more posteriorly situated, so that it is unclear whether the duct would be lateral or medial to the incisor.

Character 104: Lateral vacuity of nasolacrimal duct-(0) absent, or (1) present. The lateral wall of the nasolacrimal duct is partially lost in Cavia, Dasyprocta, and Neoreomys, which creates a large opening on the medial wall of the infraorbital canal.

Character 105: Foramen ovale-(0) between alisphenoid and squamosal, (1) within alisphenoid, or (2) between alisphenoid and petrosal or bulla. The foramen ovale is posterolateral in the pterygoid region and transmits the mandibular branch of the trigeminal nerve and a minute meningeal artery (Wahlert, 1974). This foramen occurs in all mammals selected for the study, but its position varies. Judging from illustrations (Kielan-Jaworowska, 1981: figs. 2, 4), the foramen in Asioryctes is largely within the alisphenoid but is narrowly closed off by the squamosal posteriorly. Rougier et al. (1998) considered the foramen "in alisphenoid or between alisphenoid and squamosal" as one state. We consider it as two.

Character 106: Dorsal palatine foramen-(0) in palatine, (1) situated between palatine and maxilla, (2) in maxilla, or (3) absent. The dorsal palatine foramen leads into the palatine canal through the palatine bone and transmits the descending palatine artery and nerve (Wahlert, 1974). In many gliroid mammals, this foramen is situated between the palatine and maxilla in the floor of the orbit. In Rhombomylus and Matutinia, the palatine foramen is very small; its dorsal opening is not identified but is possibly within the slitlike depression we identified as the sphenopalatine foramen between the palatine and maxilla. We tentatively score state 1 for these two taxa. The condition in Barunlestes and Zalambdalestes varies. A large foramen is present lateral to M3 (Kielan-Jaworowska, 1984a, 1984b; Kielan-Jaworowska and Trofimov, 1980), similar to the condition of Leptictis (Novacek, 1986b). In Zalambdalestes, the foramen is between the palatine and maxilla, whereas in Barunlestes it is within the maxilla. In hystricognath rodents, the orifices of the palatine canals on the palate are either 
absent, as in Dasyprocta, or reduced and asymmetrically positioned, as in Myocastor and Cavia. Owing to the posterior shift of the orbit, the dorsal postpalatine foramen appears absent in the orbit.

Character 107: Supraorbital foramen(0) absent, or (1) present. The supraorbital foramen pierces the supraorbital process of the frontal that forms the anterior portion of the orbital roof. This foramen was identified in Anagalopsis (Bohlin, 1951) and is present in Tupaia and Cynocephalus.

Character 108: Sphenopalatine foramen-(0) in palatine, (1) between palatine and maxilla, (2) within maxilla, or (3) between maxilla and orbitalsphenoid. The sphenopalatine foramen transmits the sphenopalatine nerve, artery, and vein. Its position varies among rodents and other mammals (Wahlert, 1974). In Barunlestes the foramen is within the maxilla (Kielan-Jaworowska and Trofimov, 1980), a condition similar to that of Paradjidaumo (Wahlert, 1978). With the exclusion of the palatine from the orbital region in ctenodactylids and hystricognaths, the sphenopalatine foramen is situated between the maxilla anteriorly and orbitosphenoid posteriorly (Lavocat, 1974a; Landry, 1999). However, because of the posterior shift of the orbit in ctenodactylids and hystricognaths, the structures in the orbit are remodeled. We have not been able to verify the condition of the sphenopalatine foramen in those rodents and thus tentatively follow Lavocat (1974a) and Landry (1999) in scoring this character. However, we note a slitlike opening in the maxilla ventromedial to the lacrimal foramen in Myocastor, Dasyprocta, and Cavia (in which the opening is medial to the "orbital hiatus"). The position of this opening is similar to the sphenopalatine foramen in Erethizon and other eutherians. In Castor (not included in the analysis) the palatine is also excluded from the orbital region, and the sphenopalatine foramen is within the large orbital wing of the maxilla, similar to that of Erethizon.

Character 109: Posterior maxillary notch-(0) weak, (1) closed to form a foramen, or (2) forming a deep socket. The posterior maxillary notch is usually between the posterior end of the maxilla and the pterygoid extension of the palatine; it transmits the descending palatine vein (Wahlert, 1974). When the notch is closed, a foramen is formed. In macroscelideans, the notch is deep and large and is situated in the palatine.

Character 110: Alisphenoid canal-(O) absent, or (1) present. In rodents, the alisphenoid canal passes through the alisphenoid bone and transmits the internal maxillary artery and vein (Wahlert, 1974). The alisphenoid canal is present in Zalambdalestes (Rougier et al., 1998), but is unknown in $\mathrm{Ba}$ runlestes. In rodents, the shape and size of the canal vary considerably; it may be open, as in Cavia (Cooper and Schiller, 1975).

Landry (1999) considered a lateral alisphenoid canal to be a synapomorphy for Glires. This is a channel for the masticatory branch of the mandibular nerve that runs parallel to the alisphenoid canal and ends in the masticatory foramen (Wahlert, 1974). As already noted by Landry, we found the condition of this canal to vary drastically, and in some cases difficult to identify; therefore, we do not include this character in this analysis. Future work may clarify its significance.

Character 111: Sphenopterygoid canal(0) absent, or present (1). According to Wahlert (1974), the sphenopterygoid canal leads from the pterygoid fossa to the sphenoid fissure and transmits the internal maxillary artery; it is found only in geomyoids. The pterygoid fossa is the area for the origin of the internal pterygoid muscle. In primitive condition, this fossa is floored. Bryant and McKenna (1995) used the character "pterygoid fossa solid (0) and perforate (1)" in their analysis that primarily concerns hystricognathous rodents. Their character appears to be equivalent to the sphenopterygoid canal. According to these authors, the derived condition occurs independently in Hystricognathi (with the exception of Hydrochoerus, in which the end of the fossa is blocked by M3), geomyoids, Spalax, and Aplodontia (see also Tullberg, 1899; Patterson and Wood, 1982). However, the condition in Aplodontia appears different. The canal is better interpreted as the alisphenoid canal, which opens posteriorly at the posterolateral side of a small, but solid, pterygoid fossa. Landry (1999) considered that the pterygoid fossa "breaks through to the orbit, so that the internal pterygoid muscle elongates" a syn- 
apomorphy for Hystricognathi, and noticed that this condition also occurs in geomyids. We follow Landry in scoring this character for rodents. The sphenopterygoid canal is also found in lagomorphs.

Character 112: Sphenopalatine vacuity(0) absent, or (1) present. This foramen exists in the roof of the choana, between the palatine and pterygoid. Cooper and Schiller (1975: figs. 2-4, 2-5) referred to the opening in Cavia as the "sphenopalatine foramen", which is different from the usage of the term in this and other studies (e.g., Wahlert, 1974; Novacek, 1986b). This opening is named the sphenopalatine vacuity (Wahlert et al., 1993; Voss, 1988), which we follow here.

Character 113: Supraorbital notch-(O) absent, (1) present, or (2) closed to form a foramen. The supraorbital notch is an indentation in the supraorbital crest of the frontal bone. It permits passage of the frontal branch of the ophthalmic nerve and the superior ophthalmic artery to the top of the head and is present only in sciurids among rodents (Wahlert, 1974). A similar notch is present in Rhynchocyon. In Cynocephalus and Tupaia, a foramen is formed. A similar foramen is also present in Dasyprocta.

Character 114: Mastoid foramen-(0) two foramina in mastoid, (1) single within mastoid, (2) between mastoid and supraoccipital, or (3) absent. There are two mastoid foramina in the mastoid in Asioryctes, Kennalestes, and Zalambdalestes (Kielan-Jaworowska, 1981, 1984a). The mastoid foramen is usually single and situated on the occipital region between the mastoid of the petrosal and the supraoccipital (Wahlert, 1974) in many eutherians where the foramen is present. In Rhombomylus and Matutinia, the mastoid is inflated, and the single mastoid foramen is situated near the lambdoidal crest.

\section{Cranial Elements}

Character 115: Skull-(O) flat dorsally, or (1) arched. In lateral view, the profile of the skull roof in leporids shows a distinctive bending. McKenna (1982) considered an arched skull to be present only in leporids. In ochotonids, however, the condition varies. For instance, the skull roof is relatively flat in Ochotona princeps, but shows the bending in Ochotona curzoniae. Therefore, we score a " $0 / 1$ " for Ochotona.

Character 116: Nasal-(O) narrow anteriorly but expanded posteriorly, (1) nearly parallel, (2) broad anteriorly but narrow posteriorly, (3) broad throughout with rounded posterior margin, or (4) broad throughout with $V$-shaped posterior margin. Novacek (1985) considered a flared anterior nasal a synapomorphy for rodents and lagomorphs. Frahnert (1999) assumed the anteriorly flared nasal to be the morphotype for Glires. The nasal shows a variety of morphologies among the taxa selected for this study. For most gliroid forms, the anterior nasal flares to various degrees. However, the nasal in ochotonids is relatively narrow and parallel. In leporids the nasal is broad and gradually expanded posteriorly, and it ends with a V-shaped posterior margin. In most hystricognath rodents, the nasals are broad and end with rounded posterior margins. In lagomorphs and most hystricognath rodents, the anterior nasal is not flared. The nasal of Tsaganomys is flared anteriorly.

Character 117: Premaxilla-frontal suture-(0) simple, or (1) intensively interdigtized. The posterior end of the premaxilla primitively has a simple contact with the frontal. In Mus, Rattus, Rhombomylus, and Matutinia (Ting et al., 2002), the suture is extensively interdigtized.

Character 118: Premaxilla-(0) small without a posterior process, (1) with a long posterior process, (2) long posterior process contacting frontal broadly (wider than the nasal-frontal contact), (3) contacting frontal narrowly, or (4) process long and needleshaped inserting in frontal (*63). Differing from Meng and Wyss (2001), we recognize a different state for lagomorphs, in which the posterior process of the premaxilla is needlelike, with its tip inserting in the frontal. This condition is considered an apomorphy of lagomorphs (Frahnert, 1999). In Hydrochoerus, the condition is somewhat similar to that of lagomorphs. In rodents with large body size and broad rostrum, the premaxillafrontal contact may be wide but usually does not surpass the nasal-frontal contact. Exceptions are present in Bathyergoides, Paraphiomys (Lavocat, 1973), Tsaganomys (Bryant and McKenna, 1995), and sciurids and 
aplodontids (Frahnert, 1999), in which the premaxilla-frontal contact is much more extensive than that of the nasal-frontal.

Character 119: Premaxilla on rostrum(0) small, (1) substantial but tapering anteriorly, or (2) substantial and deep anteriorly. The premaxilla primitively has a small exposure on either the ventral or lateral surface of the rostrum in most eutherian mammals, but is large in Glires, corresponding to the enlarged upper incisor. In Zalambdalestes and Barunlestes, the premaxilla shows some degree of expansion, and it was reconstructed as large, although the upper incisors are not enlarged in these forms (Kielan-Jaworowska, 1975). The premaxilla of Kulbeckia is less expanded in comparison with the reconstructed condition of Zalambdalestes and $\mathrm{Ba}$ runlestes. Because the rostral region of known Zalambdalestes and Barunlestes specimens is generally poorly preserved, the reconstructed premaxilla of these taxa needs to be confirmed with better material in the future. We tentatively score state 1 for these two taxa. In lagomorphs and some rodents, the premaxilla is substantial and tapers anteriorly. A similar condition is present in Plesiadapis, which has an enlarged upper incisor, but not in Notharctus and Adapis, which have small upper incisors. In most hystricognath and many geomyoid rodents, the premaxilla is very deep on the rostrum so that it has a rectangular shape in lateral view.

Character 120: Transverse constriction of the palate at the rostrum-(0) absent, or (1) present. The derived condition appears in geomyoid rodents, in which the palate between the incisor and the cheek teeth is very narrow.

Character 121: Fenestration of maxilla(0) absent, or (1) present (*66).

Character 122: Antorbital fossa-(0) $a b$ sent, (1) shallow, associated with lateral extension of the zygomatic arch, or (2) distinctive (*67).

Character 123: Posterior edge of anterior zygomatic root-(0) posteriorly situated, (1) aligning with M2 or M1, or (2) anterior to M1 (*58). Korth (1984: 64) pointed out that "posterior margin of the anterior root of the zygoma even with the posterior margin of P4" is a primitive feature for subgroups of rodents; advanced rodents may have the zy- goma extended even more anteriorly. The extremity is in Pedetes (not included in our analysis), in which the zygomatic root reaches the middle of the rostrum.

Character 124: Ventral projection of maxilla at anterior zygomatic arch-(O) $a b$ sent, (1) present, or (2) strong and double (*59). State 2 was modified from Meng and Wyss (2001), in which it was considered an autapomorphy for Rhombomylus. New specimens show that Matutinia shares with Rhombomylus the same condition (Ting et al., 2002).

Character 125: Zygomatic fossa for masseter lateralis-(0) absent, (1) present, or (2) broad (*60). Differing from Meng and Wyss (2001), we add state 2 for Rhombomylus and Matutinia. The condition in Rhombomylus and Matutinia differs from that of lagomorphs in being broad.

Character 126: Zygomatic plate-(O) $a b$ sent, (1) partial (myomorph type), (2) complete (sciuromorph type), or (3) extensive (geomorph type). Traditionally, four types of cranial morphologies are recognized for rodents: protrogomorph, sciuromorph, hystricomorph, and myomorph. Each type consists of several features, most notably the infraorbital foramen and the structure of the zygomatic arch. In the protrogomorph and hystricomorph skulls, the origins of the lateral and superficial masseters are similarly on the ventral surface of the anterior zygomatic arch, but the origin of the medial masseter in the latter is shifted anteriorly through the large infraorbital canal. In both, a zygomatic plate of the maxilla is not present. In protrogomorph and sciuromorph skulls, the origin of the medial masseter is similarly on the inner surface of the anterior zygomatic arch, but the origin of the lateral masseter in the latter is shifted anteriorly. A large, inclined zygomatic plate of the maxilla is formed for the attachment of that muscle. In myomorph skulls, both origins of the lateral and medial masseters are shifted anteriorly. A partial zygomatic plate is formed in conjunction with a large, keyhole-shaped infraorbital foramen. Therefore, modification of the infraorbital canal is primarily related to the anterior shifting of the medial masseter, whereas the formation of the zygomatic plate is related to that of the lateral masseter. An incipient zy- 
gomatic plate appears in Ischyromys and Titanotheriomys (not included in the analysis). A special case is the geomyoid skull. As Landry (1999) proposed, the sciuromorph type of zygomatic arch in geomyoids may have evolved by closing over the dorsal part of the myomorphic infraorbital foramen by a hypertrophied zygomatic plate.

Character 127: Orbits-(0) posteriorly positioned, (1) shifted anteriorly, or (2) farther posteriorly shifted (*74). In gliroid mammals, the anterior edge of the orbit aligns with the $\mathrm{P} 4$ or farther anterior, which is more anteriorly situated than that in other mammals (Li et al., 1987; Meng et al., 1994). In addition to the conditions discussed by Meng and Wyss (2001), we also observe that the orbit of some hystricognath rodents is significantly more posterior. This change of relative position of the orbit is not owing to anterior shift of the cheek teeth; the teeth are posteriorly positioned in those forms as well. We consider the condition to be different from the state " 0 ", and coded it as a separate condition. In Cynochephalus, the orbit is enlarged and somewhat anteriorly shifted as in gliroid mammals.

Character 128: Orbit-(O) facing laterally, or (1) more anteriorly (*76).

Character 129: Anteroventral rim of orbit-(0) rounded, or (1) squared. The anteroventral rim of the orbit is usually smoothly rounded. In leporids this region is squared up.

Character 130: Alveolus of cheek teeth intruding into orbit floor-(0) absent, or (1) present. The orbital floor is formed by the maxilla and is usually flat. In leporids, the hypsodont cheek teeth extend in their alveoli and form a platform projecting dorsally in the orbit floor.

Character 131: Hard palate-(0) long, or (1) short $(* 64)$.

Character 132: Orbital wing of the palatine-(0) present, or (1) absent. In hystricognath and ctenodactylid rodents, the orbital wing of the palate is generally reduced and restricted in the back of the orbit (Lavocat, 1974a, 1994b; Landry, 1999).

Character 133: Palatine-lacrimal contact-(0) present, or (1) absent. The outgroup condition is unknown. A narrow palatine-lacrimal contact is present in Barunles- tes (Kielan-Jaworowska and Trofimov, 1980). Li et al. (1987) considered absence of lacrimal-palatine contact a synapomorphy for rodents, lagomorphs, and eurymylids. A similar condition, however, exists in other taxa, such as Plesiadapis (Gingerich, 1976).

Character 134: Lacrimal projection-(O) absent, or (1) present. A distinct projection from the lacrimal is present in some geomyoid rodents such as Florentiamys and Gregorymys.

Character 135: Lacrimal-(0) present, (1) greatly reduced or absent, or (2) replaced by membrane. In Myocastor the lacrimal is greatly reduced to a small piece sitting in the lacrimal foramen. A similar condition is present in Erethizon (Bathyergus, as well). Although the nasolacrimal foramen is in the maxilla in Rhizomys, the lacrimal is still present and medial to the foramen. In muroid rodents, the inner wall of the infraorbital canal is extensively eroded and the bony wall is replaced by a membrane, so that the bony lacrimal canal is lost (Landry, 1999). However, the lacrimal is present in Cricetops (Carrasco and Wahlert, 1999).

Character 136: Lacrimal facial process(0) present, or (1) absent. A distinct facial process of the lacrimal has been considered a primitive condition in eutherians (Novacek, 1986a). In Glires, the lacrimal usually has a narrow or no facial process. In some hysticognaths, such as Dasyprocta and Cavia, the process is distinct.

Character 137: Jugal-lacrimal contact(0) present, or (1) absent. The jugal was reconstructed to contact the lacrimal in Asioryctes, Kennalestes, and probably in Zalambdalestes, but the relationship is unclear in Barunlestes (Kielan-Jaworowska, 1975). This contact is lost in various taxa of the selected group. The anterior suture of the jugal with maxilla is fused in early ontogeny in lagomorphs. Whether it contacts the lacrimal is uncertain, although it appears unlikely. We score "?" for lagomorphs in this and the following character.

Character 138: Jugal-(O) dorsally overlapping maxilla, or (1) end-to-end contact with maxilla. The jugal commonly overlaps the maxilla along the zygomatic arch. The contact is end-to-end in Notharctus, Adapis, and hystricognath rodents. 
Character 139: Posterior projection of jugal-(0) absent, (1) present, or (2) elongate. The jugal forms the central part of the zygomatic arch. In lagomorphs, the jugal extends posteriorly behind the glenoid fossa and forms a projection of varied size (Landry, 1999). Novacek (1985) considered a posterior ventral zygomatic process, which is equivalent to our posterior projection of the jugal, to be a synapomorphy for Lagomorpha. The posterior projection is greatly elongated in ochotonids.

Character 140: Jugal-(0) large, or (1) reduced. A large jugal is present in Kennalestes and Asioryctes (Kielan-Jaworowska, 1975; Novacek et al., 1997). Landry (1999: 308) considered that "A reduced jugal bone reaching neither the lacrimal in front nor the glenoid fossa in the rear" to be a common feature for geomyoids and murids.

Character 141: Jugal contribution to glenoid fossa-(0) absent, (1) forming lateral wall of the fossa, or (2) forming anterolateral corner of the fossa. In some hystricognath rodents, the jugal extends posteriorly to the lateral side of the glenoid fossa and forms the lateral wall of a well-defined, trenchlike glenoid fossa. In Tsaganomys, the jugal is not preserved, but from the contact between the jugal and squamosal (Bryant and McKenna, 1995), the jugal contributes to the lateral wall of the glenoid fossa. In Cynocephalus the jugal forms the anterolateral corner of the transverse glenoid fossa.

Character 142: Dorsal process of jugal(0) absent or weak, (1) strong, or (2) connecting the postorbital process of frontal. The jugal has a distinctive dorsal process that defines the posteroventral edge of the orbit in Rhombomylus and Matutinia. In Tupaia and primates, this process dorsally contacts the postorbital process of the frontal to enclose the orbit.

Character 143: Jugal ventral process in zygomatic arch-(0) absent, or (1) present. A ventral process of the jugal in the zygomatic arch is present only in Rhombomylus and Matutinia.

Character 144: Anterior projection of frontal between premaxilla and maxilla-(O) absent, or (1) present (*65).

Character 145: Postorbital process of frontal-(0) absent, (1) present, or (2) con- tacting jugal process to form a complete ring $(* 75)$.

Character 146: Supraorbital crest-(O) absent, or (1) present. The dorsal rim of the orbit is formed by the frontal and is usually blunt. In many eutherians, a shelflike crest is developed, often associated with an enlarged orbit.

Character 147: Glenoid fossa-(0) transverse and concave, (1) flat, (2) longitudinally elongate and concave, (3) longitudinal in a well-defined trough, or (4) longitudinal but greatly shortened (61*). Differing from Meng and Wyss (2001), we recognize an additional state for some hystricognathous rodents. In most rodents, the fossa is longitudinal and rounded. In some hystricognathous rodents, the glenoid fossa becomes deep and well defined by the squamosal and jugal.

Character 148: Postglenoid process-(0) present, (1) rudimentary, or (2) absent (*62). A rudimentary postglenoid process occurs in Rhynchocyon and Elephantulus. A distinct process is present in various other nongliroid mammals. The process is absent in gliroid mammals.

Character 149: Glenoid fossa-(0) near the auditory region, or (1) anterodorsal. In most eutherians the glenoid fossa is lateral to the middle-ear region. In Glires the glenoid fossa, and therefore the jaw articulation and the posterior root of the zygomatic arch, has moved a considerable distance anterodorsally (Van Kampen, 1905; Landry, 1999).

Character 150: Orbital process of alisphenoid-(0) significant, or (1) greatly reduced. The aplisphenoid usually has a significant orbital wing that forms part of the lateral wall of the braincase in eutherians. In hystricognath rodents, this process is greatly reduced.

Character 151: Alisphenoid-frontal contact-(0) absent, or (1) present. When the alisphenoid contacts the frontal, the parietalorbitosphenoid contact is lost. Landry (1999: 293) considered "very large orbitosphenoid, separating the frontal from the alisphenoid" as a typical lagomorph character. $\mathrm{Li}$ et al. (1987) considered a large orbitosphenoid that is high in the orbit a synapomorphy of rodents. In hystricognath rodents, the alisphenoid-frontal contact is absent. Lack of the alisphenoid-frontal contact is not necessarily 
associated with reduction of the orbital process of the alisphenoid. For instance, the orbital process of the alisphenoid is large in lagomorphs, but it is separated from the frontal by a narrow orbitosphenoid-squamosal connection. In Cynocephalus the squamosal also contacts the frontal dorsal to the alisphenoid-frontal contact. This condition is not observed in other taxa under study, and is not used as a character.

Character 152: Alisphenoid-parietal contact-(0) present, (1) absent owing to a narrow squamosal-frontal contact, or (2) absent owing to a broad squamosal-frontal contact. In most lagomorphs and rodents, the alisphenoid is separated from the parietal by a broad squamosal-frontal contact. In Paramys (Wood, 1962; Wahlert, 1974) the alisphenoid has a narrow contact with the parietal. In $\mathrm{Co}$ comys (Li et al., 1989) and Cricetops, the four elements are in conjunction, so that we score " $0 / 1$ " for these taxa.

Character 153: Hamulus-bulla contact(0) absent, or (1) present. Landry (1999) pointed out that in rodents the hamulus reaches back to the ear region, arching over the opening of the pharyngeotympanic tube. It often touches or fuses with the bulla. This condition is present in Rhombomylus and Matutinia, but unknown in many early gliroid forms. This connection is also not observed in specimens of many extant rodents. It is likely that this connection is absent in hystricognathous rodents, based on the specimens observed.

Character 154: Interparietal-(O) absent, or (1) present. The interparietal is absent in Asioryctes, Kennalestes, and Zalambdalestes (Rougier et al., 1998). This bone is present in several groups of rodents. It is also present in lagomorphs except Prolagus (Dawson, 1969).

Character 155: Mastoid of petrosal-(O) small, or (1) inflated. Inflation of the mastoid of the petrosal occurs in Rhombomylus, Matutinia, Incamys (Patterson and Wood, 1982), and Tataromys (Wang, 1997).

Character 156: Exposure of mastoid of petrosal on skull roof-(0) absent, or (1) present. Character state 1 occurs in Rhombomylus and Matutinia (Ting et al., in press).

Character 157: Mastoid exposure on occiput-(0) present, or (1) absent. The mas- toid of the petrosal is usually exposed on the occiput of the skull in eutherians. In hystricognathous rodents the mastoid is covered posteriorly by the occipital. However, the mastoid is inflated in Incamys and is exposed in the occiput (Patterson and Wood, 1982). In Bathyergus, the mastoid tubercle of the paraoccipital is weak and the mastoid is exposed on the occiput, which differ from those of other hystricognathous rodents.

\section{Auditory Region}

Character 158: Bulla-(O) incomplete or ring-shaped, or (1) complete. The bulla is ring-shaped or incomplete in primitive forms, such as Asioryctes and Kennalestes. It becomes complete and fully encloses the tympanic cavity in many eutherian mammals. The composition of the bulla, however, differs from species to species (see below).

Character 159: External auditory canal(0) absent, or (1) present. The external auditory meatus is not necessarily associated with a complete bulla. For instance, in Reithroparamys (Meng, 1990) and Cocomys (Li et al., 1989), the bulla is complete but the external auditory meatus is not developed.

Character 160: Opening of external auditory meatus-(0) circular, or (1) notched. State 1 is present in some hystricognathous rodents, such as Incamys, Neoreomys, Dasyprocta, and Cavia. In Cavia, the notched region forms the opening of the infratympanic canal (Cooper and Schiller, 1975).

Character 161: Internal bullar septa-(0) absent, (1) few, (2) numerous, or (3) bullar wall spongy. In Prolagus the bulla has a few internal septa, in contrast to Ochotona, in which the wall of the bulla is a mass of spongy bone, and to leporids, in which there is no bony septum (Dawson, 1969).

Character 162: Alisphenoid contribution to bullar wall-(0) absent, or (1) present (*77). The compositions of the bulla in eutherian mammals display a complicated pattern (Novacek, 1977, 1985, 1986b; MacPhee, 1981). In an early study (Meng and Wyss, 2001: 45), the bulla was coded as a multistate character: "Auditory bulla-(0) unossified, (1) formed primarily of entotympanic, (2) entotympanic and ectotympanic, (3) petrosal, (4) ectotympanic, or (5) several elements." 
Here we code each of the bullar elements as an independent character. Alisphenoid contribution to the anterior bullar wall is found only in macroscelideans among selected taxa.

Character 163: Ectotympanic-(0) ringshaped or slightly expanded, (1) forming part of a compound bulla, or (2) forming the entire bulla. The ectotympanic is primitively horseshoe-shaped or slightly expanded as in Asioryctes and Kennalestes. It may participate in the formation of a complete bulla that consists of several elements, as in macroscelideans. In gliroid mammals and ungulates, the ectotympanic is expanded to form the entire bulla (Novacek, 1985; 1986b; Luckett and Hartenberger, 1993). The bulla in Anagalopsis was considered to be exclusively ectotympanic (Bohlin, 1951). The bullar composition of Anagale has been controversial. Simpson (1931) observed a segment of a slender curved bone within the bulla of $A n$ agale and interpreted it as the tympanic ring. Consequently, he thought the bulla of Anagale was purely of entotympanic origin. However, Bohlin (1951) pointed out that the bone fragment may be part of the malleus. McKenna (1963) confirmed that the bone fragment is merely part of the bullar wall and thought that the bulla of Anagale was a compound ossification composed of both the entotympanic and ectotympanic, because an ankylosed suture was observed between the two. McKenna (1963) also commented that because Anagalopsis is an old individual, the ankylosis may be difficult or impossible to see. Before convincing evidence becomes available on the bullar composition of Anagale and Anagalopsis, we tentatively score state 1 for the former and 2 for the latter.

Character 164: Entotympanic to bullar wall-(0) absent, (1) partial, or (2) substantial. State 2 exists in Leptictis (Novacek, 1985, 1986b) and Tupaia (Novacek, 1980: fig. 6).

Character 165: Petrosal contribution to bulla-(0) absent, (1) partial, (2) or entire. The petrosal expands to form the bulk of the bulla in "Euprimates" (Novacek, 1985, 1986b). The bullar composition is unclear in Plesiadapis (see Meng and Wyss, 2001).

Character 166: Course of distal internal carotid artery on promontorium-(O) absent,
(1) present as sulcus, (2) in bony canal within tympanic cavity, (3) medial to tympanic cavity, or (4) absent owing to loss of the artery. The internal carotid artery and its phylogenetic significance in mammals have been discussed extensively in several studies (MacPhee, 1981; Wible, 1986, 1987). Those of gliroid mammals were further discussed in others (Bugge, 1985; Lavocat and Parent, 1985; Novacek, 1985; Meng, 1990; Bryant and McKenna, 1995; Wahlert, 2000). Whether the arterial system can be reliably inferred from osteological features, such as sulci and canals, remains problematic (Conroy and Wible, 1978; Cartmill and MacPhee, 1980; MacPhee and Cartmill, 1986; MacPhee et al., 1988). Nonetheless, inferred artery systems, or simply the osteological structures, have been generally used as features in phylogenetic studies. It is widely accepted that both an internal carotid artery and a stapedial artery on the ventral surface of the promontorium, which may or may not leave impressions on the bone, is the primitive eutherian condition (MacPhee, 1981; Wible, 1983, $1986,1987)$. The condition in which the internal carotid and stapedial arteries bifurcate within the tympanic cavity is present in sciuravids (Wahlert, 1974; Meng, 1990), theridomorphs (Lavocat and Parent, 1985), Cocomys (Li et al., 1989), and Paramys (Lavocat and Parent, 1985; Wahlert, 2000). Three additional conditions of the internal carotid system exist in rodents. First, the promontory branch is lost, while the stapedial artery is still present, as in Reithroparamys (Meng, 1990). Second, both arteries are present, but their bifurcation is medial to the medial bullar wall, as in Rattus (Wible, 1987) and Gregorymys (Wahlert, 1988) in which the distal portion of the internal carotid artery courses medial to the bullar wall. Finally, the entire internal carotid system is lost, as in Tsaganomys (Bryant and McKenna, 1995) and other hystricognathous rodents. In extant leporids and Prolagus, the internal carotid artery pierces the medial bullar wall and does not enter the tympanic cavity. In Palaeolagus the internal carotid artery takes a more lateral course (Meng, 1991) and leaves a short impression on the anterior portion of the promontorium. Previously, an extratympanic bifurcation of the internal carotid and stapedial 
arteries in Rhombomylus was implied by Ting and Li (1984), although the identification of the foramen for the internal carotid artery in Rhombomylus was questioned (Meng, 1990). With observations of more and better preserved skulls of Rhombomylus, it is clear that the carotid foramen is absent in this genus. In this regard, Matutinia presents an interesting case that appears to be similar to the condition of Rattus (Ting et al., 2002). In Matutinia three foramina coexist and are interpreted as the jugular, carotid, and stapedial (Ting et al., 2002). These foramina were considered as evidence for an extratympanic bifurcation of the internal carotid artery in Matutinia (Ting et al., 2002). The Rhombomylus condition is probably more derived than that of Matutinia in lacking the internal carotid artery and the foramen transmitting the artery. Moreover, the foramen for the stapedial artery in Rhombomylus is further reduced than in Matutinia and is too small to transmit a functional artery. In some studies (e.g., Rougier et al., 1998), absence or presence of the groove for the internal carotid artery is used as two character states. In that case, presence of the internal carotid artery is implied even if a groove is absent on the promontorium. Because absence of the groove on the promontorium may be attributed to several factors as shown above, we here recognize these character states. Contra Rougier et al. (1998), we follow Novacek (1986b) to score presence of the groove for Leptictis.

Character 167: Course of stapedial artery-(0) groove on promontorium, (1) in bony canal, or (2) absent. In muroids, the stapedial artery courses transversely across the promontorium ventral to the fenestra rotunda. See Meng (1990) for discussion of this and the following characters.

Character 168: Stapedial artery-(0) ventral to fenestra rotunda, or (1) partially shielding the fenestra.

Character 169: Facial nerve-(0) exposed in the tympanic cavity, (1) partially enclosed by bone, or (2) completely enclosed in a bony tube.

Character 170: Squamosal contribution to epitympanic recess-(0) present, or (1) absent. In the nongliroid mammals such as Zalambdalestes and Leptictis the squamosal forms the lateral wall of the epitympanic recess in which the articulation of the malleus and incus is located (Novacek, 1986b; Rougier et al., 1998). In gliroid mammals, the ectotympanic excludes the squamosal from the middle ear region. A similar condition is present in macroscelideans, although the ectotympanic forms only the lateral portion of the bulla and meatal tube in the latter (Novacek, 1986b).

Character 171: Tensor tympani muscle(0) uncovered, (1) partially covered, or (2) in a deep fossa. The tensor tympani muscle is usually attached to a depression at the anterolateral side of the promontorium. In some rodents, development of bony projection may deepen the depression so that the muscle is partially or substantially covered by bone.

Character 172: Meato-cochlear connection-(0) absent, or (1) present. See Meng (1990) for discussion of this character.

Character 173: Fenestra rotunda-(0) facing posteroventrally, or (1) turning laterally. The fenestra rotunda usually faces posteriorly. In hystricognathous rodents it is large and twisted to open posterolaterally.

\section{POSTCRANIUM}

Character 174: Intercentrum and arches of atlas-(0) separated or synostosed with suture in adults, or (1) fused without suture in adults. In known specimens of Kennalestes and Asioryctes, the atlas arches are preserved with no intercentrum attached; the atlas intercentrum and arches in Barunlestes are synostosed and have a suture between them (Kielan-Jaworowska, 1977, 1978). In most extant eutherians, these elements are completely fused in adults.

Character 175: Joint between atlantal and axial parts of the axial body-(0) present in adult, or (1) absent. The atlantal and axial portions of the axial body are not completely fused in Asioryctes, Zalambdalestes, and $\mathrm{Ba}$ runlestes. In all extant eutherians and other fossil groups where the structure is known the two parts are fused in adults.

Character 176: Number of sacral vertebrae-(0) two, (1) three, or (2) four or more (modified from Geisler, 2001). Barunlestes has two sacral vertebra; this is the primitive condition for eutherians. Rhombomylus, sim- 
ilar to rodents and many other taxa, has three. Lagomorphs, Hyopsodus, Phenacodus, and Cynocephalus have four or more sacral vertebra.

Character 177: Neck of the scapula-(O) short, or (1) long. The length of the scapular neck is the distance between the glenoid and the base of the scapular spine. Most eutherians, including Rhombomylus, have a short scapular neck. A long neck is characteristic of most lagomorphs, although Palaeolagus has a relatively short scapular neck (Dawson, 1958). In rodents, Neoreomys, Myocastor, Cavia, Rattus, and Mus also have a long scapular neck.

Character 178: Acromion-(O) overhanging glenoid ventrally, or (1) not encroaching upon glenoid ventrally. Rhombomylus and most rodents retain the primitive eutherian condition in having a large acromion plate that bears the acromion and metacromion processes, although its acromion process is relatively slender. In lagomorphs, the acromion is dorsal to the glenoid. In Palaeolagus, the acromion reaches the level of the glenoid (Dawson, 1958), while in other lagomorphs the acromion process is reduced. $\mathrm{Ca}$ via, Dasyprocta, and Mus also have a relatively short acromion.

Character 179: Greater tuberosity of humerus-(0) small and lower than humeral head, or (1) prominent and even with or higher than the head (modified from Rose and Lucas, 2000). The greater tuberosity is low in Rhombomylus, as in most rodents. In lagomorphs, the greater tuberosity is high in Ochotona, Prolagus, and Lepus, but low in Palaeolagus (Wood, 1937).

Character 180: Entepicondylar foramen-(0) present, or (1) absent. The function of the entepicondylar foramen is for the passage of the median nerve and brachial artery (Geisler, 2001). Landry (1958) argued that the structure is a primitive condition for mammals, related to abduction of the forearm. Rhombomylus, Palaeolagus, Paramys, and Sciurus retain an entepicondylar foramen, which is lost in most extant rodents and lagomorphs.

Character 181: Radial fossa-(O) small and shallow, or (1) large and deep. The radial fossa holds the head of the radius when the forearm fully extends. The development of the fossa indicates the degree of forearm extension. Rhombomylus, Barunlestes, and lagomorphs have a large and relatively deep radial fossa. Leptictis, Tupaia, Plesiadapis, Northarctus, Paramys, Sciuravus, Sciurus, and Rattus retain a shallow radial fossa.

Character 182: Supratrochlear foramen-(0) absent, or (1) present. The development of the supratrochlear foramen facilitates forearm flexion and extension. Terrestrial mammals, especially cursorial ones, often develop this foramen. This foramen is absent in Rhombomylus, Palaeolagus, Paramys, Sciuravus, Sciurus, Rattus, and Marmota, but is present in Barunlestes, Prolagus, Lepus, Myocastor, Cavia, and Mus.

Character 183: Ulna and radius-(O) completely separate, or (1) fused distally. Primitively, the radius and ulna are separate and are sizable. The distal end of the ulna is greatly reduced and fused with the shaft of the radius in Petrodromus; therefore, only the radius articulates with the carpals. In $C y$ nocephalus, the distal parts of the two bones are fused but both are articulated with carpals. State 0 is present in Rhombomylus and other taxa in which the bones are known.

Character 184: Posterior edge of proximal ulna-(0) convex posteriorly, or (1) straight or concave posteriorly (modified from Geisler, 2001). In mammals that are capable of climbing, the proximal ulna often has a convex posterior edge. Terrestrial mammals usually have a ulna with relatively straight or concave posterior edge (Argot, 2001; Szalay and Sargis, 2001). In Rhombomylus, the posterior edge of the proximal ulna is slightly convex, much less than those of Zalambdalestes, Barunlestes, Tupaia, Sciurus, Rattus, and Mus. In lagomorphs, the posterior edge of the proximal ulna is straight in Prolagus and Lepus, but is slightly convex in Palaeolagus.

Character 185: Central process of the radial head-(O) poorly developed or absent, or (1) well developed. The central process on the head of the radius is often well developed in terrestrial mammals and poorly developed in arboreal groups (Szalay and Sargis, 2001). The process is seen in Hyopsodus, Phenacodus, Rhombomylus, lagomorphs (except Palaeolagus), Cavia, and Dasyprocta. Most rodents do not have this process. 
Character 186: Ulnar facet on the radial head-(0) convex and ovoid, or (1) flat. A convex and ovoid ulnar facet on the radial head facilitates the rotation of the forearm at the proximal radioulnar joint, while a flat facet limits the movement (Rose and Lucas, 2000; Szalay and Sargis, 2001). Rhombomylus, Barunlestes, Hyopsodus, Phenacodus, Leptictis, Tupaia, Petrodromus, Rhynchocyon, lagomorphs, and Cavia have a relatively flat ulnar facet, whereas most rodents have a convex, ovoid facet.

Character 187: Distal articulator surface of radius-(O) with a single concave fossa, or (1) splitting into scaphoid and lunate fossae (Geisler, 2001: ch. 142). In Hyopsodus, Phenacodus, and Rhynchocyon the scaphoid and lunate fossae are well separated on the distal articulator surface of the radius. In lagomorphs, such as Prolagus and Lepus, the fossae are separated by a fine ridge. Rodents selected for this study, where the structure is known, have one articular fossa.

Character 188: Scaphoid and lunate-(O) separated in adult, (1) fused in adult. The scaphoid and lunate are separate in Asioryctes, Matutinia, and Paramys and are inferred to be separate in those taxa that have separate scaphoid and lunate fossae on the distal articulator surface of the radius. The two elements are fused in Barunlestes, Cynocephalus, Tupaia, Reithroparamys, and all extant rodents compared here.

Character 189: Centrale-(0) present in adult, or (1) absent in adult (Geisler, 2001). The centrale is absent in Hyopsodus and Phenacodus. In Glires in which carpals are known, the centrale is present. In Matutinia, however, the bone was fused with an other carpal element (Ting et al., 2002). The condition in unknown in Rhombomylus.

Character 190: Epipubic bone-(O) present, or (1) absent. The epipubic bone is present in Zalambdalestes (Novacek et al., 1997). Kielan-Jaworowska (1977) predicted the presence of the bone in Kennalestes and Asioryctes, which was confirmed by the presence of the bone in another asioryctid eutherian, Ukhaatherium (Novacek et al., 1997). In other taxa where the relevant part of the pelvic girdle is known, this bone is absent.

Character 191: Articular surface on the femoral head-(O) with pronounced lateral and posterior extension, or (1) with little extension (modified from Szalay and Sargis, 2001). The lateral extension of the articular surface on the femoral head indicates the abduction of the femur. In ambulatory mammals the femur has a greater degree of lateral extension than in cursorial ones (Jenkins and Camazine, 1977). Lagomorphs show the lateral extension of the articular surface. All extant rodents, including arboreal Sciurus, do not have the extension. The condition in $\mathrm{Ba}$ runlestes, Hyopsodus, Rhombomylus, and Paramys is similar to that of rodents.

Character 192: Greater trochanter-(0) lower than or even with the femoral head, or (1) higher than the head (Rose and Lucas, 2000). In most gliroid mammals where the structure is known, the greater trochanter is higher than the femoral head; only in Matutinia, Reithroparamys, Paraphiomys, and Neoreomys is the greater trochanter low. In Hyopsodus, Cynocephalus, and Notharctus the greater trochanter is lower than the femoral head.

Character 193: Femoral neck-(O) short and broad, or (1) long and narrow (Ross et al., 1998). A short and broad femoral neck is characteristic of lagomorphs and some other gliroid mammals including Rhombomylus, Matutinia, Paramys, Neoreomys, Sciurus, and Marmota. The femoral neck is short and broad in Barunlestes, Phenacodus, Cynocephalus, Tupaia, Rhynchocyon, Plesiadapis, and Notharctus.

Character 194: Lesser trochanter-(O) medially directed, or (1) posteromedially directed (Rose and Lucas, 2000). Except for Paradjidaumo and Sciurus, in which the lesser trochanter is somewhat medially directed, all rodents selected here have a posteromedially directed lesser trochanter. A similar condition is present in Rhombomylus, Barunlestes, Hyopsodus, Phenacodus, Leptictis, Cynocephalus, Petrodromus, Rhynchocyon, and Prolagus. Lepus, Palaeolagus, and primates have a medially directed lesser trochanter.

Character 195: Third trochanter-(O) prominent, (1) small, or (2) absent (modified from Rose and Lucas, 2000). The loss of the third trochanter in didelphids was thought a significant phylogenetic attribute (Szalay and Sargis, 2001). Absence of this structure is 
seen only in Myocastor among the gliroid mammals we examined. A prominent third trochanter is present in other rodents, Hyopsodus, Phenacodus, Anagalopsis, and primates. The third trochanter is small in Rhombomylus, Lepus, Palaeolagus, and Cavia.

Character 196: Patellar trochlea-(0) broad and shallow, or (1) narrower and elevated. The femoral trochlea is the place where the patella slides over the femur (see below).

Character 197: Distal femur-(0) as anteroposteriorly deep as mediolaterally wide, or (1) deeper than wide. An elevated and relatively narrow trochlea is often associated with an anteroposteriorly deep distal femur. It commonly indicates cursorial or saltatorial locomotion (Rose, 1999). A shallow patellar trochlea and distal femur are present in Hyopsodus, Tupaia, Plesiadapis, Mimolagus, Paramys, Neoreomys, Sciurus, and Marmota. These conditions are probably associated with a relatively generalized locomotor pattern, if not arboreal habit.

Character 198: Proximal tibia and fibula-(0) separate, or (1) fused. The fusion of the proximal tibia and fibula occurs in $\mathrm{Pe}$ trodromus and Rhynchocyon.

Character 199: Distal tibia and fibula(0) separate, (1) fused at distal ends, or (2) fused at midshaft. The tibia and fibula are separate in Asioryctes (Kielan-Jaworowska, 1977). In Tribosphenomys, Rhombomylus (Li and Ting, 1985, 1993), and early rodents such as Paramys (Wood, 1962), the two elements are separate. Among rodents, many muroids display fusion of the tibia and fibula (Luckett and Hartenberger, 1993). However, the muroid condition appears different in that a suture along the fusion area is recognizable. In describing the postcranial specimens of Mimolagus, Bohlin (1951) considered them to be skeletal bones of a rodent incertae sedis, although these postcranials were found together with the skull (the holotype) of Mimolagus. Among those postcranials, the tibia and fibula are separate, which perhaps was the reason that Bohlin hesitated to assign the postcranial specimens to Mimolagus, as Bohlin (1951) considered Mimolagus to be a lagomorph, whereas the postcranial elements show a mixture of rodent and lagomorph characters. However, the similarities to ro- dents, such as the separate tibia and fibula and lack of the calcaneus-fibula contact (both were recognized by Bohlin [1951]), are primitive features; it is not surprising that such features are present in a primitive lagomorph. The tibia and fibula of Pseudictops are preserved (Sulimski, 1968). Judging from the illustration (Sulimski, 1968: pl. III), these bones are separate. In Zalambdalestes (Novacek et al., 1997), macroscelids, and lagomorphs the distal tibia and fibula are fused up to the midpoint of the shaft. The fusion of the tibia and fibula was regarded as a synapomorphy for Lagomorpha (Li et al., 1987).

Character 200: Fibula-femur articulation-(0) absent, or (1) present. In lagomorphs and rodents, the head of the fibula articulates with the ventral side of the lateral condyle of the proximal head of the tibia; therefore, the head of the fibula does not contact the femur. In some lagomorphs and rodents, a fibular articular projection is developed so that the head of the fibula articulates with the tibia at a position more distal to the tibia-femur articulation surface. In Petrodromus and Rhynchocyon, the head of the fibula articulates with the femur.

Character 201: Fibula-(O) robust relative to the tibia, or (1) slim. A slim fibula is present in lagomorphs and many extant rodents, such as Myocastor, Cavia, Dasyprocta, Sciurus, Marmota, Rattus, and Mus. A similar condition is also found in Zalambdalestes, Leptictis, Cynocephalus, Tupaia, and macroscelideans. A relatively robust fibula is in Asioryctes, Hyopsodus, Phenacodus, Anagale, Pseudictops, Notharctus, Mimolagus, Rhombomylus, Matutinia, Tribosphenomys, Paramys, Reithroparamys, and Neoreomys.

Character 202: Tibial malleolus-(0) prominent, (1) reduced, or (2) bearing a convex articulation facet for the astragalus. A tibial malleolus is characteristic of macroscelideans. A reduced tibial malleolus is widely distributed in lagomorphs and many rodents, including Florentiamys, Gregorymys, Paraphiomys, Myocastor, Cavia, Dasyprocta, Sciurus, Marmota, Rattus, and Mus. Rhombomylus, Tribosphenomys, and Paramys bear a prominent tibial malleolus, similar to those of Asioryctes, Hyopsodus, Phen- 
acodus, Cynocephalus, Tupaia, and Notharctus.

Character 203: Posterior process on distal end of tibia-(0) absent, or (1) present. A distinctive posterior process or flange on the distal end of the tibia is typical in rodents (Szalay, 1985; Luckett and Hartenberger, 1993). A similar condition, although less distinctive than in Paramys, was present in Rhombomylus (Li and Ting, 1993). A modest posterior process of the tibia is present in Leptictis (Szalay, 1985). A similar process appears present in Mimolagus (Bohlin, 1951: figs. 26-34). Because it is difficult to judge the degree of the height of the process, we code " $0 / 1$ " for Leptictis and Mimolagus.

Character 204: Astragalar trochlea-(O) absent, or (1) present. Absence of the astragalar trochlea is present in Ukhaatherium and Asioryctes (Horovitz, 2000). The astragalar trochlea evolved independently in placentals and some marsupials (Szalay, 1984, 1994). The trochlea is present in selected placentals, in which the bone is known, except in Cynocephalus where it is absent.

Character 205: Lateral astragalar trochlear border-(0) higher than medial border, or (1) as high as the medial border (modified from Rose and Lucas, 2000; Hooker, 2001). The asymmetrical astragalar trochlea is the primitive condition for eutherians and is widely distributed in various groups, including Zalambdalestes, Hyopsodus, Phenacodus, Anagale, Leptictis, Pseudictops, lagomorphs, Mimolagus, Rhombomylus, Matutinia, Paramys, Reithroparamys, Florentiamys, Sciuravus, Paraphiomys, Sciurus, Marmota, Rattus, and Mus. A relatively symmetrical trochlea is found in primates, $T u$ paia, Petrodromus, Tribosphenomys, Neoreomys, Myocastor, Cavia, and Dasyprocta.

Character 206: Posterior trochlear shelf of astragalus-(O) absent or weakly developed, or (1) well developed. The posterior trochlear shelf of the astragalus limits the posterior extension of the bone and therefore plantar flexion of the foot. It is present in Plesiadapis, Notharctus, and Adapis.

Character 207: Ventral limit of astragalar trochlea-(O) not exceeding midpoint on posterior surface of body, or (1) more ventral (Hooker, 2001). The development of the posterior trochlear shelf in primates limits the astragalar trochlea to a relatively high point so that the ventral limit of the astragalar trochlea does not exceed the midpoint on the posterior surface of the astragalar body. Although Cynocephalus and Tupaia lack the posterior trochlear shelf, the ventral limit of the trochlea is similar to that of primates.

Character 208: Lateral process of astragalus-(0) present, or (1) absent (Geisler, 2001). Presence of the lateral process of the astragalus is probably a primitive eutherian condition and is widely distributed in fossil taxa, such as Zalambdalestes, Hyopsodus, Phenacodus, Anagale, Leptictis, Plesiadapis, Notharctus, Adapis, Rhombomylus, and Matutinia. It is absent in lagomorphs and most rodents.

Character 209: Astragalofibular facet(O) sloping obliquely and laterally, or (1) flat (vertical) (modified from Ross et al., 1998). An oblique astragalofibular facet probably facilitates the transverse movement of the upper ankle joint. It is present in Asioryctes, Plesiadapis, Notharctus, and Adapis. It probably represents the primitive condition of eutherians as it is also present in Ukhaatherium (Horovits, 2000: fig 5) and various marsupials (Szalay, 1994). Most eutherians have a vertical and somewhat flat astragalofibular facet, which, together with the trochlea, limits the transverse mobility of the upper ankle joint (Szalay, 1984).

Character 210: Dorsal astragalar foramen-(0) present, or (1) absent. Presence of the dorsal astragalar foramen, indicating the development of the astragalar canal, is the primitive condition of therians. The foramen and canal are lost in many placentals (Horovitz, 2000). In taxa compared here, the foramen is present only in Petrodromus, Anagale, Pseudictops, Plesiadapis, Notharctus, Adapis, and Mimolagus.

Character 211: Sustentacular facet on astragalus-(O) on ventral side of the bone, or (1) shifted to ventromedial side of the bone. In Petrodromus, Elephantulus, and Rhynchocyon (unknown in Macroscelides), as well as in Tupaia, the sustentacular facet of the astragalus is shifted to the ventromedial side of the bone. In other taxa in which the astragalus is known, the sustentacular facet is on the ventral side of the bone.

Character 212: Sustentacular facet on as- 
tragalus-(O) separate from the astragalonavicular facet, or (1) confluent. In hystricognathous rodents examined, the sustentacular and astragalonavicular facets are confluent. A similar condition exists in Tupaia and $C y$ nocephalus. In macroscelideans (unknown in Macroscelides) the two facets are separated by an angled border, which is different from the smoothly confluent surfaces in Tupaia and Cynocephalus.

Character 213: Long axes of calcaneoastragalar and sustentacular facets of astragalus-(0) forming an angle, or (1) roughly parallel (modified from Geisler, 2001). The parallel condition occurs in lagomorphs, Mimolagus, Rhombomylus, Paramys, Neoreomys, Dasyprocta, Marmota, Rattus, and Mus.

Character 214: Astragalar neck-(O) short (neck ratio: length divided by body width $<1$ ), or (1) moderate (neck ratio subequal to 1) or long (neck ratio $>1$ ) (modified from Rose and Lucas, 2000; Ross et al., 1998). A short astragalar neck is present in various eutherians, including Asioryctes, Uhkaatherium, Zalambdalestes, Phenacodus, Anagale, Pseudictops, Plesiadapis, Mimolagus, Rhombomylus, Matutinia, and various rodents examined here. Hyopsodus, Cynocephalus, Tupaia, macroscelideans, primates, lagomorphs (unknown in Ochotona), and Sciuravus have a relatively long astragalar neck.

Character 215: Astragalonavicular facet on astragalus-(0) transverse, or (1) dorsoventrally extended. The derived condition occurs in lagomorphs (Bleefeld and McKenna, 1985). Although the gross morphology of the astragalus in Pedetes is similar to that of lagomorphs, the astragalonavicular facet on the astragalus is transversely oriented.

Character 216: Cuboid facet on astragalus-(0) absent, or (1) present. The head of the astragalus articulates with the navicular, but in Asioryctes (Kielan-Jaworowska, 1977), anagalids (Simpson, 1931; Bohlin, 1951), and macroscelideans (Szalay, 1985) it also has a small contact with the cuboid. The contact is absent in lagomorphs (Szalay, 1985: p. 125, fig. 15). Although Mimolagus was considered a lagomorph (Bleefeld and McKenna, 1985; Szalay, 1985), an astragalar cuboid facet is present in the taxon (Bohlin,
1951). An articular facet for the astragalus exists on the cuboid of Mimolagus (Bohlin, 1951). The astragalus does not articulate with the cuboid in other taxa examined.

Character 217: Calcaneus-(O) relatively broad, or (1) narrow and proportionally long. In dorsal or plantar view, the sustentacular and peroneal processes are transversely extended in primitive forms (Szalay, $1985,1994)$. This condition exists in most eutherians in which the calcaneal fibular facet is commonly absent. In mimotonids, Mimolagus, and lagomorphs, these processes are compressed and the body of the calcaneus appears relatively long and slim. A similar condition appears to be in Zalambdalestes (Kielan-Jaworowska, 1978), Pseudictops (Sulimski, 1968), macroscelideans, and primates (Szalay and Drawhorn, 1980).

Character 218: Calcaneofibular facet (CaFi) on calcaneus-(O) present as an oblique surface, (1) absent, (2) modified as facing dorsally and broad, or (3) restricted and convex. The upper ankle joint has three contacts: the astragalus-tibia, astragalus-fibula, and calcaneus-fibula in metatherians (Szalay, 1985, 1994). This condition is present in Asioryctes (Kielan-Jaworowska, 1977). The condition in Rhombomylus and Heomys looks similar to that of Asioryctes (contra to $\mathrm{Li}$ and Ting, 1993). The derived condition for eutherian mammals is that the calcaneus is shifted more ventral to the astragalus and the calcaneus-fibula contact is lost (bi-contact of Szalay, 1985). The bi-contact state is present in Mimolagus (Bohlin, 1951; Szalay, 1985) and Tribosphenomys (Meng and Wyss, 2001). Li and Ting (1985) also indicated lack of $\mathrm{CaFi}$ in mimotonids, Anagalidae, Leptictidae, and Zalambdalestidae. The CaFi is present in lagomorphs, macroscelideans, and Pseudictops (Szalay, 1985; Li and Ting, 1985). The question then is whether the CaFi in these forms is a primitive retention or secondarily acquired. The lagomorph condition is considered to be primitive by several authors ( $\mathrm{Li}$ and Ting, 1985; Luckett and Hartenberger, 1993), but Szalay (1985: 120) thought it a secondary acquisition, which is followed by $\mathrm{Li}$ et al. (1987). Szalay (1985: p. 120, fig. 14) also pointed out that Pseudictops has the calcaneus-fibula contact and stated that "Pseudic- 
tops shares this feature with the Leporoidea, almost certainly convergently, or possibly parallel with them." We found that the CaFi in lagomorphs is different from the condition of Asioryctes and Rhombomylus in facing more dorsally and is broad. Averianov (1991) described some isolated calcanea, probably belonging to the mimotonid Anatolmylus and Aktashmys, which are similar to those of Mimolagus in general morphology and in lacking the calcaneum-fibula facet. The $\mathrm{CaFi}$ is more restricted and somewhat convex in macroscelideans.

Character 219: Calcaneoastragalar facet (CaA) on calcaneus-(O) diagonal to the long axis of the bone, (1) narrow facet roughly parallel to the long axis, or (2) facet short showing no obvious orientation. In most eutherians, the calcaneoastragalar facet either shows no apparent directions in its dimensions or it is elongate but oriented diagonally to the long axis of the calcaneus body. In mimotonids (Averianov, 1991), Mimolagus (Bohlin, 1951; Szalay, 1985), and lagomorphs the $\mathrm{CaA}$ is oriented such that the major axis is nearly parallel to the long axis of the calcanear body. This condition can be inferred from the articular facet on the astragalus, in which the $\mathrm{CaA}$ has a similar orientation. In macroscelideans, the $\mathrm{CaA}$ on the calcaneus is short and its orientation is either unrecognizable or perpendicular to the long axis of the bone.

Character 220: Calcanealoastragalar facet - (O) near the distal end of the bone (the tuber being relatively long), or (1) at the midpoint or near the proximal end (tuber short) (modified from Hooker, 2001). The long tuber is the condition for primitive eutherians, such as Uhkaatherium and Zalambdalestes, Hyopsodus, Phenacodus, macroscelideans, and basal gliroid mammals including Mimolagus, Rhombomylus, Heomys, and Tribosphenomys. Lagomorphs and many rodents exhibit state 1. Marmota, Rattus and Mus, however, display state 0 .

Character 221: Sustentaculum on the calcaneus-(0) anteromedial to and separated by $a$ distinctive sulcus calcanei from the CaA, or (1) immediately medial to CaA. The sustentaculum on the calcaneus is anteromedial to the calcaneus-astragalus $(\mathrm{CaA})$ facet and both are separated by a distinctive sul- cus calcanei in most eutherian taxa we selected here. In contrast, the sustentaculum in lagomorphs is immediately medial to $\mathrm{CaA}$ facet, while in macroscelids, the sustentaculum is anterior to the $\mathrm{CaA}$ facet (Szalay, 1985: p. 125, fig. 14). The condition in Mimolagus is similar to that of lagomorphs (see Szalay, 1985: fig. 18).

Character 222: Calcanear-navicular contact (CaN)-(O) absent, or (1) present. Primitively, the calcaneus does not extend more distally than the astragalus; therefore, the astragalar-navicular articulation (ANa) and the calcaneum-cuboid articulation $(\mathrm{CaCu})$ are at the same level and the ankle is more flexible in rotation. In some lagomorphs, the calcaneus is anteriorly extended to contact the navicular. The foot bones are more firmly interlocked so that sideward movement of the foot is limited but fore-and-aft movement of the foot is emphasized (Dawson, 1958). Dawson (1958: 15, 69) pointed out that in Palaeolagus haydeni and Megalagus turgi$d u s$, "the astragalus reaches approximately the same distance distally as does the calcaneum, but in Hypolagus and the recent leporids the calcaneum extends more distally than the astragalus, making possible a calcaneonavicualr contact." The calcaneonavicualr contact is not present in ochotonids (McKenna, 1982). The calcaneonavicualr facet also occurs on the calcaneus of Mimolagus (Bohlin, 1951; Szalay, 1985), suggesting that the calcaneus extends more distally than does the astragalus.

Character 223: Cuboid-(O) short, or (1) elongate. A relatively long and narrow cuboid is present in Asioryctes, macroscelideans, and Plesiadapis.

Character 224: Medial tarsal sesamoid bone-(0) absent, or (1) present. Szalay (1985) considered presence of a medial tarsal sesamoid bone as unique for rodents, including early forms such as Paramys. His view was followed by Luckett and Hartenberger (1993). A medial tarsal sesamoid bone was probably present in Matutinia (Ting et al., 2002). Whether this minute bone occurs in other early gliroid mammals is unknown. In Rhombomylus the articular surface of the astragalar head extends proximally to the medial side of the astragalar neck, which may 
have articulated with a medial tarsal sesamoid bone.

Character 225: Plantar process of navicular-(0) short, or (1) elongate. The derived condition is seen in leporids, Mimolagus, Rhombomylus, Matutinia (Ting et al., in press) and in hystricognathous rodents.

Character 226: Metatarsal IV-(O) present, or (1) absent. The metatarsals are reduced to three in Cavioidea (Landry, 1999: 303). According to Cooper and Schiller (1975), digits IV and V are lost in Cavia.

Character 227: Metatarsal V-(O) present, or (1) absent. The fifth digit including the metatarsal is lost in Myocastor, Cavia, and Dasyprocta.

\section{PHYLOGENETIC ANALYSES}

\section{Materials ANd Methods}

TAXA SELECTED: The present study focuses on testing the monophyly of Glires, as well as that of Rodentia; it is not attempted for a thorough phylogeny of placental mammals. The goal is to explore whether any of the selected gliroid taxa will, or will not, be clustered with any of the selected nongliroid taxa in a parsimonious analysis based on osteological data. To accomplish this goal, we selected a total of 50 terminal taxa, traditionally accorded the rank "genus", for analyses. In so doing, we assume generic monophyly for the selected taxa. For fossil taxa, this assumption is probably valid because most of the fossil genera, if not monotypic, consist of a small number of species. For extant genera, this assumption may hold at a lower level of confidence. Among the selected taxa, 33 are gliroid mammals. For nongliroid mammals we follow Meng and Wyss (2001) in focusing on those that are previously implicated in the origin of rodents and lagomorphs both by morphological and molecular studies.

Because the analyses are at the superordinal level of placental mammals, we chose two Cretaceous eutherians, Asioryctes and Kennalestes, as outgroups for the analyses. These two taxa are collectively referred to as "the outgroup taxa". All other 48 taxa constitute the ingroup. The selected taxa and sources of data are listed in appendix 1.

Differing from Meng and Wyss (2001), we include two additional fossil gliroid taxa in the analysis: Sinomylus and Matutinia. Sinomylus was described recently by McKenna and Meng (2001). Matutinia was previously considered a junior synonym of Rhombomylus (Dashzeveg and Russell, 1988; McKenna and Bell, 1997), but is now regarded as a valid taxon (Ting et al., 2002). Several gliroid taxa that are represented by very fragmentary material are not included in this study. These include Asiaparamys, Kazygurtia, Nikolomylus, Anatolimys, Khaychina, Aktashmys, Eomylus, Amar, Zagmys, Decipomys, and Ivanantonia. Discussion of these taxa can be found in Meng and Wyss (2001) and references therein.

Significantly differing from Meng and Wyss (2001) is the inclusion of more fossil and extant rodents in this study. Given the limited capacity of current computer programs for phylogenetic analyses and the diversity of rodents, we chose only a few extant taxa that represent the conventional sciuromorphous, hystricomorphous, and myomorphous groups, noting that these are not necessarily monophyletic. These extant taxa are commonly used in molecular systematic analyses of mammals. Therefore, the morphological data we amassed here may be used in combination with molecular data in future studies. Because of the limited taxa of rodents used in this study, the results of the phylogenetic analyses should not be accepted as a conclusive intraordinal phylogenetic relationship for Rodentia.

DATA: We tabulated 227 characters and 608 states across 50 terminal taxa using MacClade (Maddison and Maddison, 1992) based on literature and our observations of specimens and casts. The data matrix is presented in appendix 2. The number of scored character states (nonmissing data) and the completeness of each taxon (the number of nonmissing data divided by the total number of characters [227 in this case]; see Simmons, 1993) are also listed in appendix 2.

Missing data stem from several sources. First, character states may be unknown due to lack of preservation. This is particularly common for some early gliroid mammals known from fragmentary material. For instance, Sinomylus (McKenna and Meng, 2001) is represented only by a rostrum. This taxon has the lowest completeness (35\%) 
among all selected for this study. Second, certain states are not applicable to a given taxon. For instance, the shape and size of i3 cannot be coded for taxa that lack this tooth, such as rodents. Logically, we allow hierarchical coding of multistate characters that are related to this kind of missing data (Simmons, 1993; Simmons and Geisler, 1998). For example, i3 present or absent is coded as one character, whereas shape of i3 is regarded as a separate, dependent character for those that have i3. Third, in some cases, the morphology of a taxon may be unobservable. For instance, owing to fusing of bones, the alisphenoid-frontal suture cannot be determined in Tupaia. Despite their differing sources, these missing data are treated equally by the PAUP* program; therefore, we denote all these missing data by question marks in our data matrix.

To compare how dental and skeletal characters affect reconstruction of Glires phylogenies, we partitioned the data into two subsets. Three datasets, including a dental, a skeletal, and a combined matrix, were formed to serve as the basis for phylogenetic analyses. The dental matrix includes the first 95 characters, whereas the skeletal matrix contains the remaining 132 characters listed in appendix 2.

ANALYSES: Phylogenetic Analysis Using Parsimony, PAUP* (Swofford, 2000), was employed to reconstruct cladograms of the selected taxa. For all analyses we used the heuristic search option, with the rooting option imposing a monophyletic ingroup and a paraphyletic outgroup (Asioryctes and Kennalestes). All characters are weighted equally. Characters were optimized using both the delayed transformation (DELTRAN) and accelerated transformation (ACCTRAN) options. Each data matrix was analyzed in three runs in which character types are different. These include (1) all characters unordered, (2) all ordered, and (3) partially ordered as irreversible-up (or normal). Those that were irreversibly ordered include characters 11 , $16,17,19,20,21,23,25,27,28,29,30,32$, $34,36,47,48,226$, and 227. These are occurrences of teeth and digits (0, present; 1 , absent). When a character of binary states is ordered as irreversible-up, the character states can be transformed in only one direc- tion from state 0 to 1 in the calculation, which operationally prohibits reacquisition of a lost tooth or a digit.

Clade robustness was assessed using branch lengths, branch supports, and bootstrap values. Branch lengths are from the tree descriptions given by PAUP*. Branch support indices were calculated in the following steps (Bremer, 1988, 1994). First, most parsimonious trees (MPT) of $s$ steps were obtained by heuristic searches. Then, five additional searches were conducted sequentially. In each search trees with steps $s+1, s$ $+2, s+3, s+4$, and $s+5$ were kept respectively. Five strict consensus trees resulted from the five sets of trees. By comparing topologies of the five consensus trees and that of the MPT, the branch support indices can be determined. For a given branch (node) in the consensus tree of the most parsimonious trees, the branch support index is the total number of that branch in the five consensus trees of nearly parsimonious trees. In this case, the maximum branch support index is 5 . If a branch has an index of 5, it means that the branch remains in at least all the trees that have the length of $s+5$. If the index is 0 , the branch occurs only in the most parsimonious trees. That only trees with $s+$ 5 steps were obtained in generating the supporting indices is decided by the computer capacity to calculate the data. For our data, heuristic searches for trees with $s+5$ steps reached the limit of the computer capacity. The bootstrap value (Felsenstein, 1985; Sanderson, 1995) was obtained from 1000 bootstrap replicates using the full heuristic option. As pointed out elsewhere (Meng and Wyss, 2001), although including the bootstrap values, we are aware of the limitations of this method (Kluge and Wolf, 1993; Carpenter, 1996).

\section{RESUlts}

Cladograms: Five cladograms are illustrated out of numerous trees produced during our analyses. The statistics of each cladogram is presented below. Cladogram 1 is the strict consensus tree of 768 equally most parsimonious trees (MPT) based on the dental matrix with all characters unordered (fig. 72). Each MPT has the following parameters: tree 


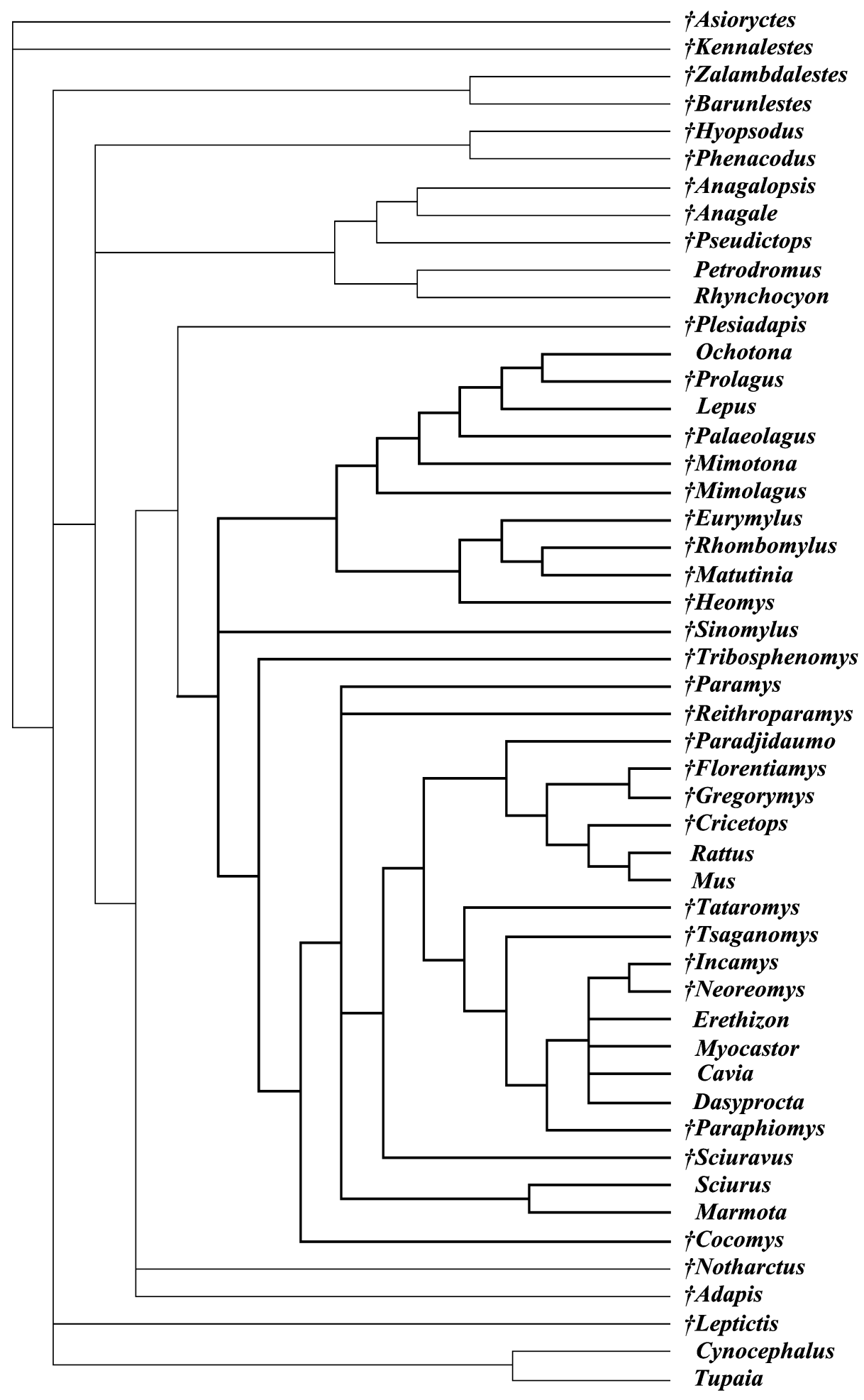


length $(\mathrm{TL})=352$; consistency index $(\mathrm{CI})=$ 0.5114 ; homoplasy index $(\mathrm{HI})=0.4886$; retention index $(\mathrm{RI})=0.8499(\mathrm{CI}$ excluding uninformative characters, HI excluding uninformative characters and rescaled consistency [RC] index are not cited). Statistics of the consensus tree of the 768 MPT include $\mathrm{TL}=361, \mathrm{CI}=0.4986, \mathrm{HI}=0.5014, \mathrm{RI}=$ 0.8421 , and $\mathrm{RC}=0.4199$.

Cladogram 2 is the strict consensus of 440 MPT $($ MPT TL $=501, \mathrm{CI}=0.3952, \mathrm{HI}=$ 0.6048 , and $\mathrm{RI}=0.7200$ ) derived from the skeletal matrix with all characters unordered (fig. 73). The strict consensus of the 440 MPT has $\mathrm{TL}=558, \mathrm{CI}=0.3548, \mathrm{HI}=$ 0.6452 , and $\mathrm{RI}=0.6673$.

Cladogram 3 is the strict consensus of 10 MPT (MPT TL $=876, \mathrm{CI}=0.4315, \mathrm{HI}=$ 0.5685 , and $\mathrm{RI}=0.7765$ ) derived from the combined data matrix with all characters unordered (fig. 74). The strict consensus of the $876 \mathrm{MPT}$ has $\mathrm{TL}=887, \mathrm{CI}=0.4262, \mathrm{HI}=$ 0.5738 , and RI $=0.7715$.

Cladogram 4 is the strict consensus of two MPT (MPT TL $=1027, \mathrm{CI}=0.3681, \mathrm{HI}=$ 0.6319 , and $\mathrm{RI}=0.7902$ ) derived from the combined data matrix with all characters ordered (fig. 75). The only difference of the two MPT is the positions of Mimotona and Mimolagus. Figure 75 illustrates one of the two. In the other tree, the positions of the two taxa are switched.

Cladogram 5 is the strict consensus of six MPT $($ MPT TL $=882 ; \mathrm{CI}=0.4286$; $\mathrm{HI}=$ 0.5714 ; $\mathrm{RI}=0.8072$ ) derived from the combined data matrix with some characters ordered irreversibly $(11,16,17,19,20,21,23$, $25,27,28,29,30,32,34,36,47,48,226$, and 227) (fig. 76). The consensus tree of the six $\mathrm{MPT}$ has $\mathrm{TL}=882, \mathrm{CI}=0.4286, \mathrm{HI}=$ 0.5714 , and $\mathrm{RI}=0.8072$.

Cladogram 3 (fig. 74) is our preferred relationship of the selected taxa. Bremer index, assigned branch length, and bootstrapping value for each node are attached to the clad- ogram. The trees that are used for calculating the Bremer supporting indices are as follows: 877 steps (249 trees), 878 (2491), 879 $(17,850), 880(87,932)$, and $881(362,600)$. The calculation was truncated in searching for trees of 881 steps because of computer memory outage. Therefore, the number of trees with 881 steps is incomplete. Nonetheless, the results provide an estimate of robustness for nodes. If a node has a Bremer supporting index of 5 , that node is presented in all the 363600 trees with tree length of 881 steps or fewer. That node is therefore considered to be robust. If a node has a zero value for the index, the node occurs only in the 10 MPT. Bootstrapping value was obtained from three runs of full heuristic searches, each with 1000 replicates. The bootstrapping value for a node resulted from the three calculations may be different. The difference is no more than 3\%. Apomorphic states from both ACCTRAN and DELTRAN options that diagnose nodes and terminal taxa of cladogram 3 are presented in appendix 3. Note that character states supporting the same node can be quite different in the two lists. Those that diagnose the main nodes of interest for this study are listed below.

GLIRES (NODE 81), ACCTRAN: Incisor enamel restricted primarily to the labial surface (1-1-0.5; the numbers stand for character-character state-consistency index); upper diastema large owing to loss of canine and premolars (4-2-0.75); first lower incisor absent (7-1-1.0); first upper incisor absent (9-1-0.25); second deciduous lower incisor extending to beneath $\mathrm{m} 3$ and beyond (11-2$1.0)$; second lower incisor replacement absent (12-1-1.0); deciduous I2 large and extended into maxilla (13-2-1.0); second upper incisor replacement absent (15-1-1); third lower incisor absent (16-1-0.333); third upper incisor posterior to dI2 (18-1-0.667); incisors transversely compressed with rounded surface (22-2-0.75); upper canine absent

$\leftarrow$

Fig. 72. The strict consensus of 768 equally most parsimonious trees (MPT) based on the dental matrix with all characters unordered. Each MPT tree has the following parameters: tree length (TL) = 352 ; consistency index $(\mathrm{CI})=0.5114$; homoplasy index $(\mathrm{HI})=0.4886$; retention index $(\mathrm{RI})=0.8499$. Statistics of the consensus tree are: $\mathrm{TL}=361, \mathrm{CI}=0.4986, \mathrm{HI}=0.5014, \mathrm{RI}=0.8421$, and $\mathrm{RC}=$ 0.4199 . 


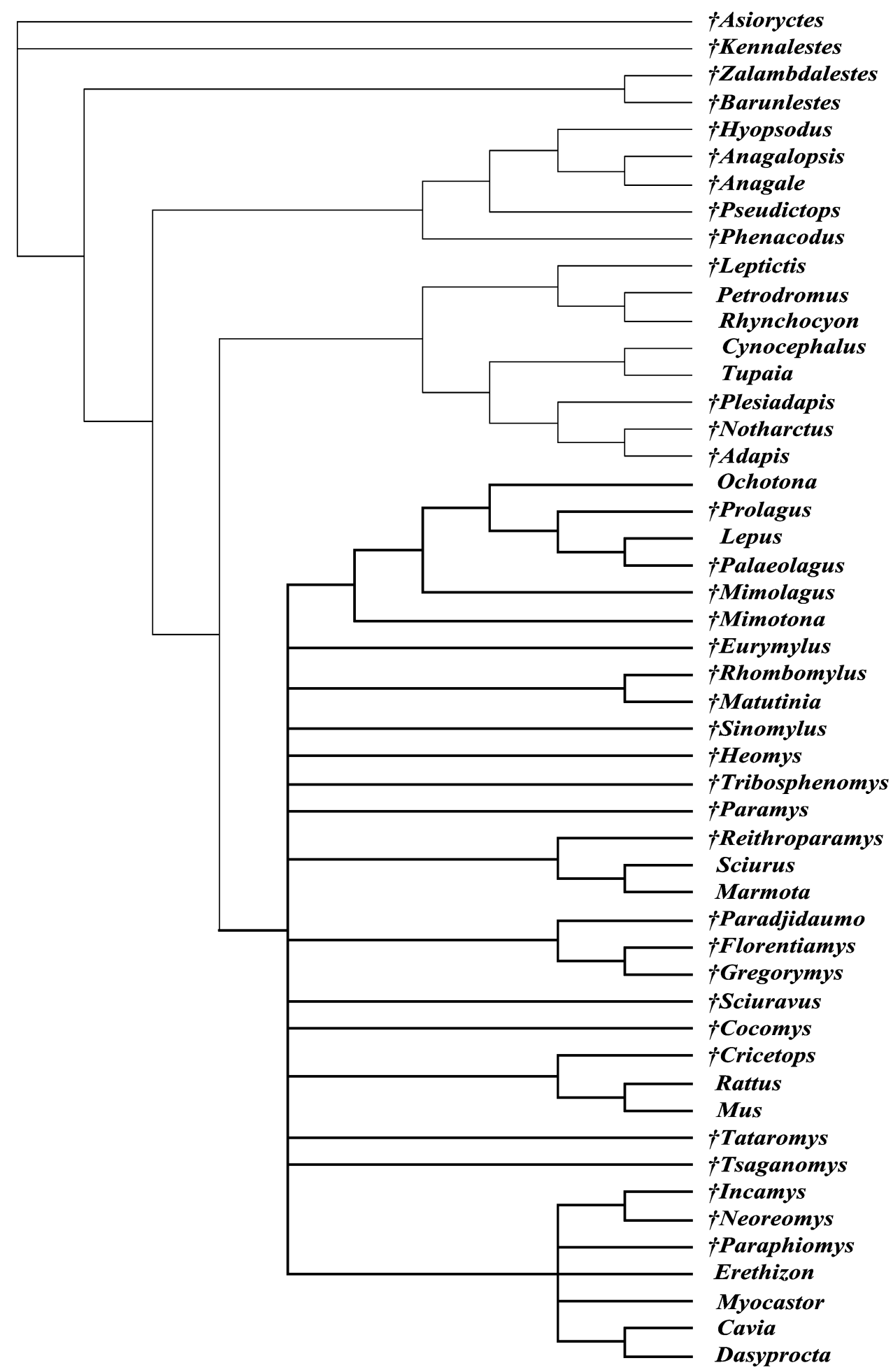

Fig. 73. The strict consensus of 440 MPT $(\mathrm{MPT} \mathrm{TL}=501, \mathrm{CI}=0.3952, \mathrm{HI}=0.6048$, and $\mathrm{RI}=$ 0.7200 ) derived from the skeletal matrix with all characters unordered. The strict consensus has $\mathrm{TL}=$ $558, \mathrm{CI}=0.3548, \mathrm{HI}=0.6452$, and $\mathrm{RI}=0.6673$. 
(23-2-0.5); lower canine absent (25-20.333 ); first lower premolar absent (27-1$0.333)$; first upper premolar absent (28-1$0.25)$; second lower premolar absent (29-10.333 ); second upper premolar conical (31$1-0.6)$; third upper premolar oval in crown view and multicuspate (35-1-0.667); trigonid of p4 bicuspate (37-2-0.5); talonid of p4 simple (39-0-0.182); centrocrista on upper molars absent (59-2-0.75); lower molar paraconid absent (77-2-0.4); lower diastema significant (84-2-1.0); horizontal ramus of mandible deep and short (86-1-1.0); coronoid process somewhat reduced (88-10.667); angular process expanded (89-1$0.5)$; mandibular condyle anteroposteriorly oriented (93-2-0.5); incisive foramina moderately elongate (96-2-0.545); infraorbital canal short (97-1-0.5); postglenoid foramen in glenoid fossa within the squamosal (992-0.5); nasolacrimal foramen between lacrimal and maxilla (102-1-0.4); nasolacrimal lateral to posterior part of incisor (103-1$1.0)$; sphenopalatine vacuity present (112-1$0.5)$; nasal broad anteriorl and narrow posteriorly (116-2-0.444); premaxilla contacting frontal narrowly (118-3-0.571); premaxilla on rostrum substantial and tapering anteriorly (119-1-0.667); posterior edge of anterior zygomatic root aligning with M2 or M1 (123-1-667); orbits shifted anteriorly(127-1-0.5); jugal-lacrimal contact absent (137-1-0.25); glenoid fossa longitudinally elongate and concave (147-2-0.8); postglenoid process absent (148-2-0.667); glenoid fossa shifted anterodorsally (149-1-1.0); third trochanter small (195-1-0.182); dorsal astragalar foramen absent (210-1-0.25); and long axes of calcaneoastragalar and sustentacular facets of astragalus roughly parallel (213-1-0.143). DELTRAN: Incisor enamel restricted primarily to the labial surface (1$1-0.5)$; first lower incisor absent (7-1-1.0); first upper incisor absent (9-1-0.25); second deciduous lower incisor extending to beneath $\mathrm{m} 3$ and beyond (11-2-1.0); second lower incisor replacement absent (12-1-1.0); deciduous I2 large and extended into maxilla (13$2-1.0$ ); second upper incisor replacement absent (15-1-1.0); incisors transversely compressed with rounded surface (22-2-0.75); upper canine absent (23-2-0.5); lower canine absent (25-2-0.333); first lower pre- molar absent (27-1-0.333); first upper premolar absent (28-1-0.25); second lower premolar absent (29-1-0.333); second upper premolar conical (31-1-0.6); third upper premolar oval in crown view and multicuspate (35-1-0.667); centrocrista on upper molars absent (59-2-0.75); conule distinctive (621-0.3); paracone and metacone transverse (63-1-0.667); lower molar paraconid absent (77-2-0.4); lower diastema significant (842-1.0); horizontal ramus of mandible deep and short (86-1-1.0); angular process expanded (89-1-0.5); mandibular condyle anteroposteriorly oriented (93-2-0.5); infraorbital canal short (97-1-0.5); nasolacrimal foramen between lacrimal and maxilla (102-1$0.4)$; nasolacrimal lateral to posterior part of incisor (103-1-1.0); premaxilla on rostrum substantial and tapering anteriorly (119-10.667); posterior edge of anterior zygomatic root aligning with M2 or M1 (123-1-667); orbits shifted anteriorly (127-1-0.5); palatine-lacrimal contact absent (133-1-0.5); postglenoid process absent (148-2-0.667); glenoid fossa shifted anterodorsally (149-11.0 ); and squamosal contribution to epitympanic recess absent (170-1-0.5).

DUPLICENDENTATA (Node 58), ACCTRAN: incisor enamel single-layered with HSB (2$2-0.667)$; anterior surface of dI2 grooved (14-1-0.5); anterior and posterior ridges of P4 strong surrounding the labial cusp (441-0.5); mandibular condyle height relative to tooth row significantly higher (85-2-0.5); coronoid process nearly absent (88-4$0.667)$; anterior edge of masseteric fossa below m3 (91-1-0.3); incisive foramina greatly elongate and expanded posteriorly (96-40.545 ); postglenoid foramen on lateral side of skull and between squamosal and ectotympanic or petrosal (99-3-0.5); optic foramina confluent anteriorly along the skull midline (100-1-0.333); sphenopterygoid present (111-1-0.333); mastoid foramen absent (114-3-0.429); skull arched (115-11.0); nasal broad throughout with rounded posterior margin (116-3-0.444); premaxilla posterior process long and needle-shaped (118-4-0.571); ventral projection of maxilla at anterior zygomatic arch present (124-11.0); zygomatic fossa for masseter lateralis present (125-1-1.0); hard palate short (131$1-0.5)$; posterior projection of jugal present 


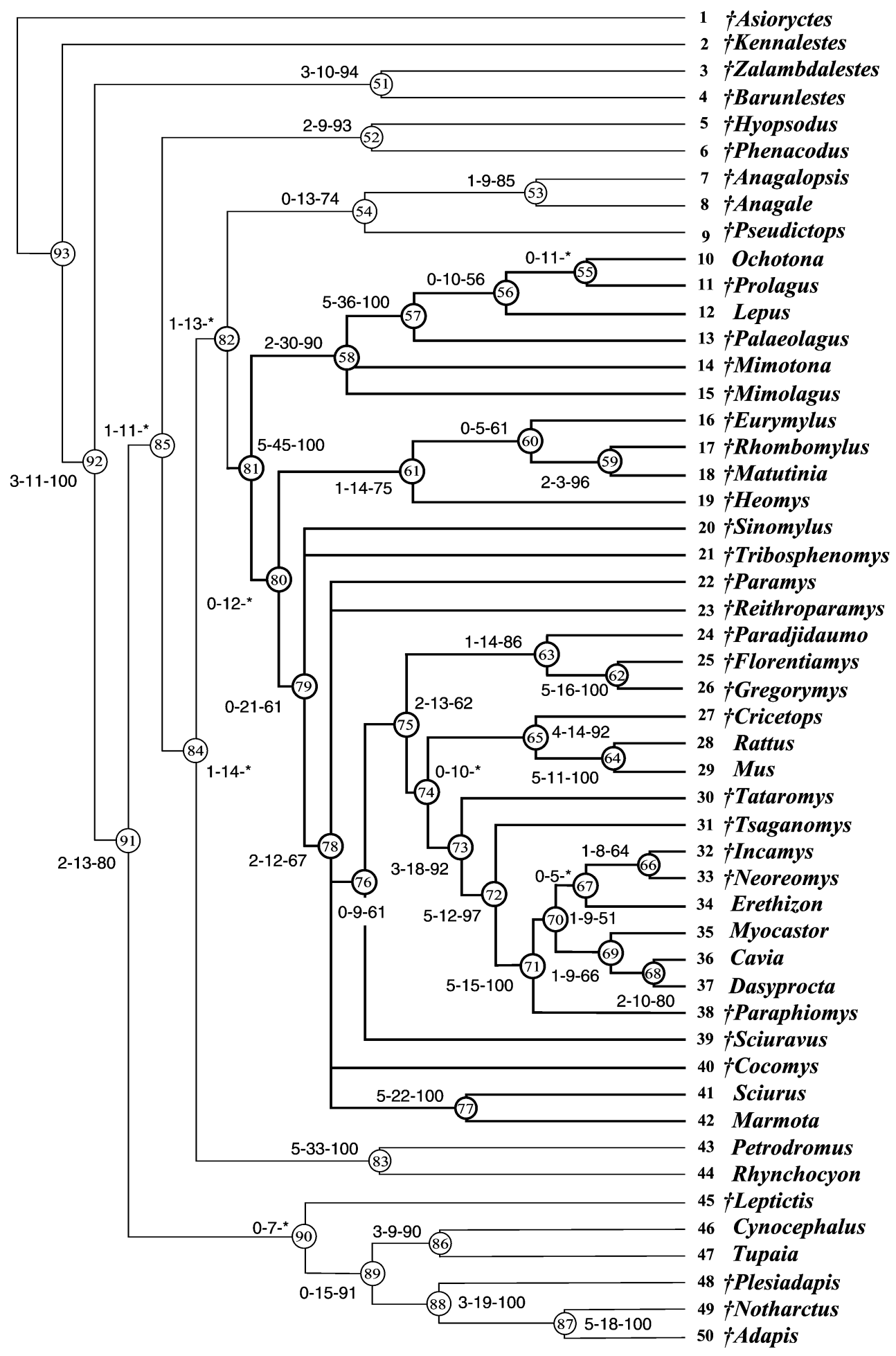


(139-1-1.0); anterior projection of frontal between premaxilla and maxilla present (144-1-1.0); postorbital process of frontal present (145-1-0.222); glenoid fossa longitudinal but greatly shortened (147-4-0.8); alisphenoid-parietal contact absent owing to a broad squamosal-frontal contact (152-2$0.286)$; interparietal present (154-1-0.143); number of sacral vertebrae four or more (176-2-0.5); acromion not encroaching upon glenoid ventrally (178-1-0.2); articular surface on the femoral head with pronounced lateral and posterior extension (191-0$0.167)$; lesser trochanter medially directed (194-0-0.167); astragalonavicular facet on astragalus dorsoventrally extended (215-1$1.0)$; calcaneoastragalar facet on calcaneus narrow and roughly parallel to the long axis (219-1-0.5); and sustentaculum on the calcaneus immediately medial to calcaneoastragalar facet (221-1-0.5). DELTRAN: Incisor enamel single-layered with HSB (2-20.667); upper diastema large owing to loss of canine and premolars (4-2-0.75); third upper incisor posterior to dI2 (18-1-0.667); anterior and posterior ridges of $\mathrm{P} 4$ strong surrounding the labial cusp (44-1-0.5); anterior edge of masseteric fossa below m3 (91-1$0.3)$; incisive foramina greatly elongate and expanded posteriorly (96-4-0.545); premaxilla posterior process long and needle-shaped (118-4-0.571); ventral projection of maxilla at anterior zygomatic arch present (124-11.0); zygomatic fossa for masseter lateralis present (125-1-1.0); anterior projection of frontal between premaxilla and maxilla present (144-1-1.0); long axes of calcaneoastragalar and sustentacular facets of astragalus roughly parallel (213-1-0.143); astragalonavicular facet on astragalus dorsoventrally extended (215-1-1.0); calcaneoastragalar facet on calcaneus narrow and roughly parallel to the long axis (219-1-0.5); sustentaculum on the calcaneus immediately medial to calcaneoastragalar facet (221-1-0.5); and plantar process of navicular elongate (225$1-0.333)$.

LAGOMORPHA (Node 57), ACTRAN: Second deciduous lower incisor ever-growing and extended beneath $\mathrm{p} 3$ or $\mathrm{m} 1$ (11-1-1.0); deciduous $\mathrm{I} 2$ ever-growing but restricted to premaxilla (13-1-1.0); third upper incisor reduced and immediately behind dI2 (18-2$0.667)$; incisors anteroposteriorly compressed (22-1-0.75); second upper premolar hypsodont with enamel fold (31-3-0.6); third lower premolar enlarged with enamel folds (33-3-1.0); third upper premolar highcrowned and molariform (35-3-0.667); trigonid of $\mathrm{p} 4$ with strong ridges (37-3-0.5); talonid of p4 fully crested (39-2-0.182); anterior and posterior ridges of $\mathrm{P} 4$ strong with cusp lost (44-2-0.5); P4 hypocone very large and anteroposteriorly extended (45-2$0.286)$; M3 greatly reduced in size (49-2$0.375)$; cheek teeth hypsodont (50-3-0.5); m3 significantly reduced (52-2-0.375); upper molar protoloph forming transversely elongate edge (56-4-0.571); posteroloph of molar as a transversely elongate ridge (574-0.571); trigon basin anteroposteriorly compressed (58-4-0.5); paracone and metacone coalesced (59-3-0.75); upper molar protocone ridged and anteroposteriorly compressed (61-2-0.429); conule ridged (62-3$0.3)$; paracone and metacone confluent to form the anterior lobe of tooth (63-6-0.667); hypoflexus deep and anteroposteriorly compressed (68-3-0.6); hypoflexid deep (70-10.2 ); ectolophid absent (71-1-0.167); talonid basin of lower molar separated as an independent lobe (80-4-0.8); hypoconulid of $\mathrm{m} 3$ incorporated into posterior cingulum (83-30.556); nasals broad throughout with $\mathrm{V}$ shaped posterior margin (116-4-0.444); fenestration of maxilla present (121-1-1.0); anteroventral rim of orbit squared (129-1-0.5); alveolus of cheek teeth intrusion of orbit floor present (130-1-0.5); supraorbital crest present (146-1-0.143); distal tibia and fibula

$\leftarrow$

Fig. 74. The strict consensus of $10 \mathrm{MPT}$ (MPT $\mathrm{TL}=876, \mathrm{CI}=0.4315, \mathrm{HI}=0.5685$, and $\mathrm{RI}=$ 0.7765 ) derived from the combined data matrix with all characters unordered. The strict consensus has $\mathrm{TL}=887, \mathrm{CI}=0.4262, \mathrm{HI}=0.5738$, and $\mathrm{RI}=0.7715$. Numbers attached to nodes are Bremer indexassigned branch length-bootstrapping value. Supporting synapomorphs for some nodes are discussed in the text and those for all nodes are listed in appendix 3. 


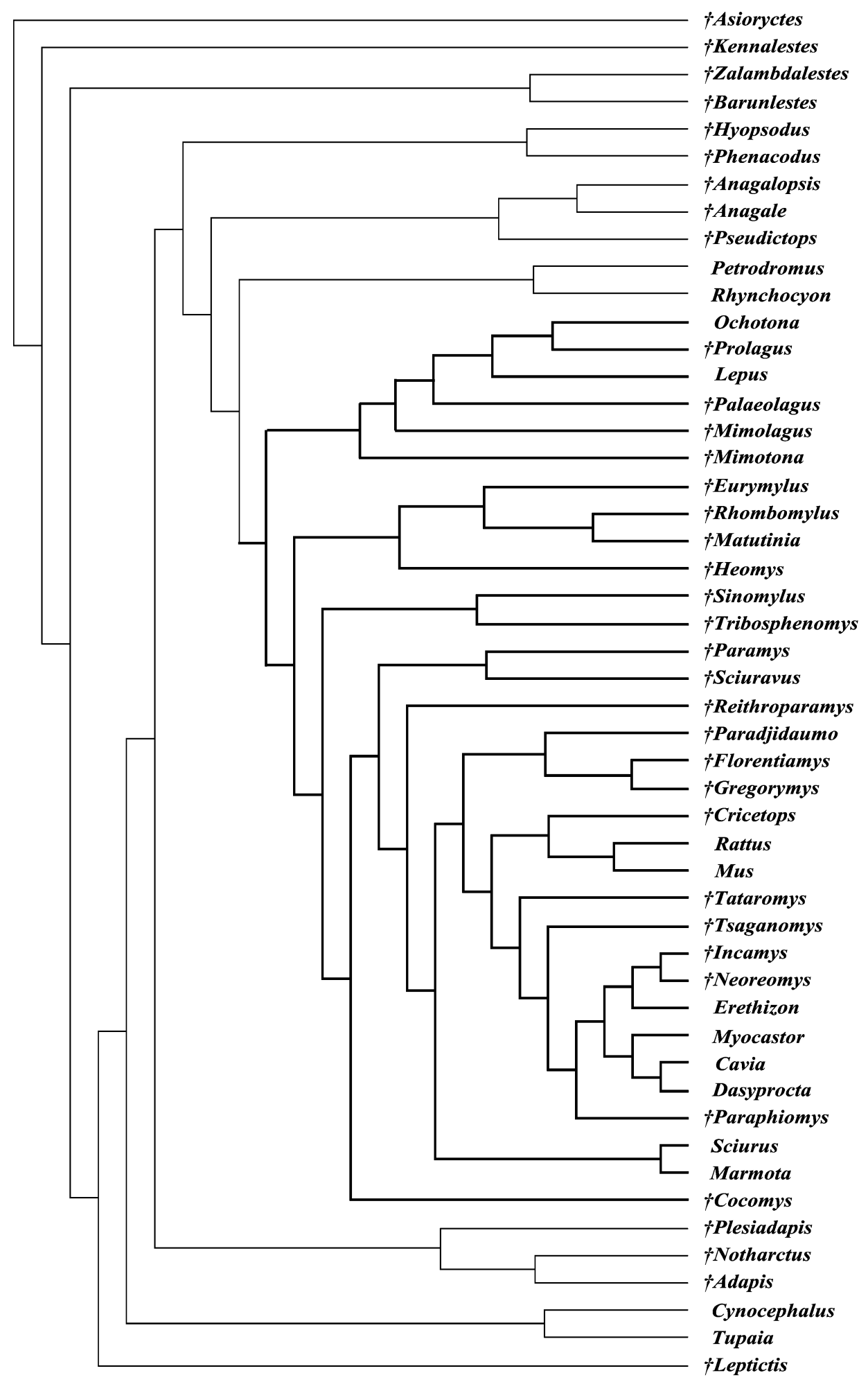


fused at midshaft (199-2-0.333); fibula slim (201-1-0.125); astragalar neck moderate or long (214-1-0.143); calcaneofibular facet on calcaneus facing dorsally and broad (218-20.6 ); and calcaneoastragalar facet at about midpoint along length or nearer to proximal than distal end (220-1-0.167). DELTRAN: Second deciduous lower incisor ever-growing and extended beneath $\mathrm{p} 3$ or $\mathrm{m} 1$ (11-11.0); deciduous I2 ever-growing but restricted to premaxilla (13-1-1.0); anterior surface of dI2 grooved (14-1-0.5); third lower incisor absent (16-1-0.333); third upper incisor reduced and immediately behind dI2 (182-0.667); incisors anteroposteriorly compressed (22-1-0.75); second upper premolar hypsodont with enamel fold (31-3-0.6); third lower premolar enlarged with enamel folds (33-3-1.0); third upper premolar highcrowned and molariform (35-3-0.667); talonid of p4 fully crested (39-2-0.182); anterior and posterior ridges of $\mathrm{p} 4$ strong with cusp lost (44-2-0.5); P4 hypocone very large and anteroposteriorly extended (45-2$0.286)$; M3 greatly reduced in size (49-2$0.375)$; cheek teeth hypsodont (50-3-0.5); m3 significantly reduced (52-2-0.375); upper molar protoloph forming transversely elongate edge (56-4-0.571); posteroloph as a transversely elongate ridge (57-4-0.571); trigon basin anteroposteriorly compressed (58-4-0.5); paracone and metacone coalesced (59-3-0.75); upper molar protocone ridged and anteroposteriorly compressed (61-2-0.429); conule ridged (62-3-0.3); paracone and metacone confluent to form the anterior lobe of tooth (63-6-0.667); hypoflexus deep and anteroposteriorly compressed (68-3-0.6); hypoflexid deep (70-10.2 ); ectolophid absent (71-1-0.167); talonid basin of lower molar separated as an independent lobe (80-4-0.8); hypoconulid of $\mathrm{m} 3$ incorporated into posterior cingulum (83-3$0.556)$; mandibular condyle significantly higher than tooth row (85-2-0.5); coronoid process nearly absent, forming a rudimentary process much lower than the condyle (88-20.5 ); postglenoid foramen on lateral side of skull and between squamosal and ectotympanic or petrosal (99-3-0.5); left and right optic foramina confluent anteriorly along the skull midline (100-1-0.333); sphenopalatine vacuity present (112-1-0.5); skull arched (115-1-1.0); fenestration of maxilla present (121-1-1.0); hard palate short (131-1-0.5); posterior projection of jugal present (139-11.0); glenoid fossa longitudinal but greatly shortened (147-4-0.8); alisphenoid-parietal contact absent owing to a broad squamosalfrontal contact (152-2-0.286); interparietal present (154-1-0.143); external auditory canal present (159-1-0.167); number of sacral vertebrae four or more (176-2-0.5); acromion not encroaching upon glenoid ventrally (178-1-0.2); articular surface on the femoral head with pronounced lateral and posterior extension (191-0-0.167); distal femur deeper than wide (197-1-0.1); distal tibia and fibula fused at midshaft (199-2-0.333); fibula slim (201-1-0.125); astragalar neck moderate or long (214-1-0.143); calcaneofibular facet on calcaneus facing dorsally and broad (218-2-0.6); and calcaneoastragalar facet at midpoint or near proximal end (220-10.167).

Simplicendentata (Node 80), ACCTRAN: Upper diastema significantly larger than lower diastema (4-3-0.75); third upper incisor absent (17-1-0.333); second upper premolar absent (30-1-0.333); dorsal palatine foramen situated between palatine and maxilla (106-1-0.5); sphenopalatine foramen between palatine and maxilla (108-10.429 ); hamulus-bulla contact present (153$1-0.5)$; course of distal internal carotid artery on promontorium absent owing to loss of the artery (166-4-0.267); course of stapedial artery as groove on promontorium (167-0$0.2)$; distal articulation surface of radius with single concave fossa (187-0-0.333); posterior process on distal end of tibia present (203-1-1.0); calcaneus relatively broad

$\leftarrow$

Fig. 75. The strict consensus of $2 \mathrm{MPT}(\mathrm{MPT} \mathrm{TL}=1027, \mathrm{CI}=0.3681, \mathrm{HI}=0.6319$, and $\mathrm{RI}=$ $0.7902)$ derived from the combined data matrix with all characters ordered. The only difference of the two MPT is the positions of Mimotona and Mimolagus. In the other tree the positions of the two taxa are switched. 


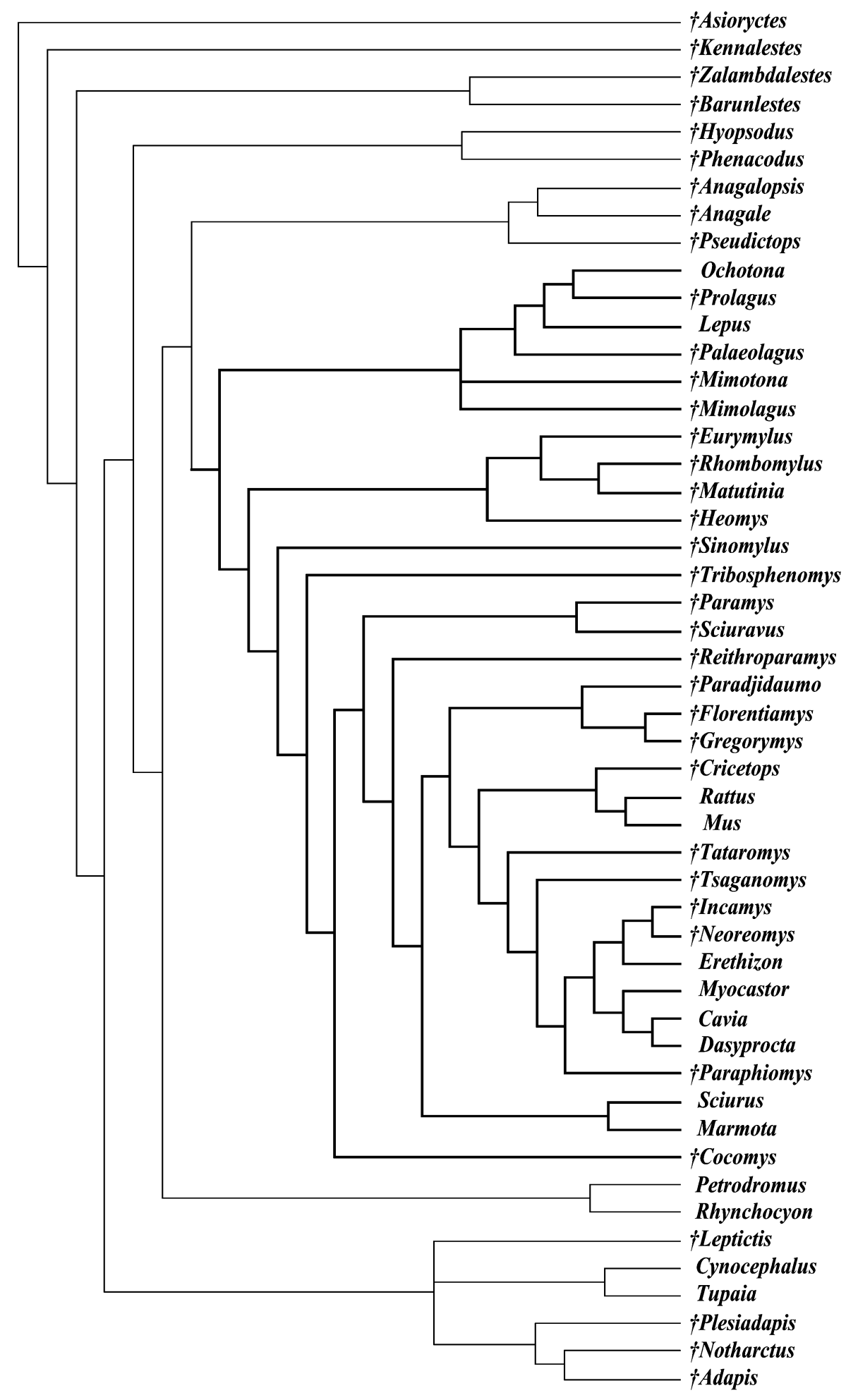


(217-0-0.25); and medial tarsal sesamoid bone present (224-1-1.0). DELTRAN: Upper diastema significantly larger than lower diastema (4-3-0.75); third lower incisor absent (16-1-0.333); third upper incisor absent (17-1-0.333); second upper premolar absent (30-1-0.333); trigonid of p4 bicuspate (372-0.5); mandibular condyle height relative to tooth row somewhat higher and posterodorsally extended (85-1-0.5); coronoid process somewhat reduced (88-1-0.667); incisive foramina moderately elongate (96-2-0.545); dorsal palatine foramen situated between palatine and maxilla (106-1-0.5); sphenopalatine foramen between palatine and maxilla (108-1-0.429); premaxilla contacting frontal narrowly (118-3-0.571); jugal-lacrimal contact absent (137-1-0.25); glenoid fossa longitudinally elongate and concave (147-20.8 ) hamulus-bulla contact present (153-1$0.5)$; posterior process on distal end of tibia present (203-1-1.0); calcaneus relatively broad (217-0-0.25); and medial tarsal sesamoid bone present (224-1-1.0).

EURYMYLIDAE (Node 61), ACCTRAN: Incisor enamel double-layered with HSB (2-10.667); labial cusp of P4 double, including a smaller metacone (42-1-0.231); mure present (69-1-0.2); mandibular condyle ovoid (93-0-0.5); sphenopalatine vacuity absent (112-0-0.5); mastoid foramen single within mastoid (114-1-0.429); jugal ventral process in zygomatic arch present (143-1-1.0); mastoid of petrosal inflated (155-1-0.333); exposure of mastoid of petrosal on skull roof present (156-1-1.0); facial nerve partially enclosed by bone (169-1-0.222); central process of the radial head well developed (185-1-0.2); centrale absent in adult (189$1-0.5)$; lateral process of astragalus absent (208-1-0.2); and calcaneofibular facet on calcaneus present as an oblique surface (218-0-0.6). DELTRAN: Incisor enamel double-layered with HSB (2-1-0.667); labial cusp of $\mathrm{P} 4$ double, including a smaller metacone (42-1-0.231); mure present (69-1-
$0.2)$; and calcaneofibular facet on calcaneus present as an oblique surface (218-0-0.6).

RodENTIA (Node 78), ACCTRAN: Incisor enamel double-layered with HSB (2-1$0.667)$; incisor enamel type pauciserial (3-1$0.6)$; talonid of p4 tricuspate (39-1-0.182); upper cheek teeth increase in width posteriorly (49-1-0.375); upper molar crown quadrate (53-2-0.429); precingulum distinctive along the anterior edge of the tooth (54-1$0.6)$; molar mesostyle present (60-1-0.143); mure present (69-1-0.2); entoconid of lower molar anteriorly shifted (81-1-0.333); hypoconulid of $\mathrm{m} 3$ incorporated into posterior cingulum (83-3-0.556); angular process bending inward (90-1-0.667); and posterior edge of anterior zygomatic root anterior to M1 (123-2-0.667). DELTRAN: Incisor enamel double-layered with HSB (2-1$0.667)$; third upper premolar small and conical (35-2-0.667); talonid of p4 tricuspate (39-1-0.182); upper cheek teeth increase in width posteriorly (49-1-0.375); upper molar crown quadrate (53-2-0.429); precingulum distinctive along the anterior edge of the tooth (54-1-0.6); molar mesostyle present (60-1-0.143); mure present (69-1-0.2); hypoconulid of $\mathrm{m} 3$ incorporated into posterior cingulum (83-3-0.556); single mental foramen (87-1-0.167); angular process bending inward (90-1-0.667); postglenoid foramen on lateral side of skull within squamosal (99-4-0.5); sphenopalatine vacuity present (112-1-0.5); nasal broad anteriorly but narrow posteriorly (116-2-0.444); posterior edge of anterior zygomatic root anterior to M1 (123-2-0.667); alisphenoid-frontal contact present (151-1-0.25); ulnar facet of the radial head convex and ovoid (186-0-0.2); distal articulation surface of radius with single concave fossa (187-0-0.333); scaphoid and lunate fused in adult (188-1-0.25); and long axes of calcaneoastragalar and sustentacular facets of astragalus roughly parallel (213-1-0.143).

$\leftarrow$

Fig. 76. The strict consensus of six MPT $($ MPT TL $=882, \mathrm{CI}=0.4286, \mathrm{HI}=0.5714, \mathrm{RI}=0.8072)$ derived from the combined data matrix with some characters ordered as irreversible-up $(11,16,17,19$, $20,21,23,25,27,28,29,30,32,34,36,47,48,226$, and 227). The consensus tree has TL $=882$, CI $=0.4286, \mathrm{HI}=0.5714$, and $\mathrm{RI}=0.8072$. 


\section{DISCUSSION}

Comparison of Cladograms: Calculations based on both dental and skeletal data identified the clade of Glires, but the dental results differ from the skeletal results in several aspects. The dental tree has a better resolution for gliroid mammals, although dental characters are fewer (95) than the skeletal characters (132). The lower resolution of the skeletal tree is probably the result of missing cranial and postcranial data in many fossil taxa. If a clade is present in both the dental and skeletal trees, it is retained in the tree derived from the combined data, such as the duplicendentates and the hystricognaths excluding Tsaganomys. However, if a clade is present only in either the dental or the skeletal tree, it may not be identified in the combined tree. For instance, the clade consisting of Paradjidaumo, Florentiamys, and Gregorymys is identified in the skeletal tree but not in the dental tree; however, it is present in the combined tree. The clade consisting of Reithroparamys, Sciurus, and Marmota is present in the skeletal tree but not in the dental tree; it is not identified in the combined tree.

In the dental tree, Plesiadapis is the sister group of the Glires. This indicates the dental similarity between early plesiadapids and gliroid mammals, as recognized by McKenna (1961). In the skeletal tree, the sister group of the Glires is a clade that comprises the following groups: (((Leptictis, (Petrodromus, Rhynchocyon)) ((Tupaia, Cynocephalus) ((Plesiadapis (Notharctus, Adapis $)))$. The grouping of Hyopsodus, Phenacodus, Anagale, Anagalopsis and Pseudictops is unconventional.

When characters are ordered or partially ordered (figs. 75, 76), the number of MPTs is reduced to two and six, respectively. Consequently, the two consensus trees of the ordered MPTs have higher resolutions, particularly in the clade of Glires, than do the unordered MPTs. Nonetheless, the differences between these consensus trees are not enormous. The unordered and partially ordered consensus trees are more similar in that the clade consisting of Anagalopsis, Anagale, and Pseudictops forms the sister group of the Glires. They are also similar in having a clade comprising Leptictis, Cynocephalus, Tupaia, and primates (Plesiadapis, Notharctus, and Adapis).

Nongliroid Mammals: The phylogenetic placement of various nongliroid mammals in this analysis may be relevant to higherlevel mammalian phylogeny. However, as stated by Meng and Wyss (2001), because this study is intended solely to test relationships among basal gliroid mammals (not a fullscale analysis of interordinal relationships among placentals), we ascribe little significance to the relationships suggested outside the clade Glires. Nonetheless, we would like to comment on a recent proposal that specifically addressed the Glires relationship. In a recent study of Cretaceous and Tertiary eutherians, Archibald et al. (2001: 64) concluded that the results of their analysis "support a superordinal clade including zalambdalestids and Glires". Based on that phylogeny, the authors reasoned that presence of zalambdalestids in the 85-90-Myr-old faunas at Dzharakuduk "argues that the superordinal clade including Glires had separated from other superordinal placental clades by this time". Therefore, they called zalambdalestids late Cretaceous relatives of rabbits and rodents and considered their results to be concordant with molecularly based estimates for the superordinal diversification of placentals.

Although Archibald et al.'s (2001) study identified zalambdalestids among the Cretaceous eutherians selected for their analysis as the sister taxon of the Glires, it did not sufficiently test whether the Glires, represented by Tribosphenomys and Mimotona in their analysis, is the Tertiary group that is most closely related to zalambdalestids. In addition to Tribosphenomys and Mimotona only two Tertiary ungulates, Protungulatum and Oxyprimus, were included in Archibald et al.'s (2001) study. These ungulates are distantly related to the Glires, and their separation from Tribosphenomys and Mimotona is not a surprise. As shown in the Introduction, however, a Glires relationship has been proposed for many other Tertiary eutherians, particularly primates, leptictids, tree shrews, pseudictopids, anagalids, and macroscelideans. None of these taxa was included in the study of Archibald et al. (2001). An ap- 
propriate phylogenetic analysis to test Glires relationships should involve these taxa. When relevant Tertiary taxa were included in a previous study (Meng and Wyss, 2001) and in the present study, zalambdalestids are not the sister group of the Glires. In the tree based on the dental characters (fig. 72), a polytomy is formed among zalambdalestids, Leptictis, (Cynocephalus + Tupaia), and a clade consisting of the remaining taxa selected for this study. In all other trees (figs. 73-76), zalambdalestids are the sister group to all other Tertiary taxa and are distantly related to the Glires. This relationship is relatively strongly supported by Bremer index, branch length, and bootstrap values at nodes 91 and 92 (fig. 74). Our study thus shows that a superordinal clade including zalambdalestids and Glires is unsupported.

GLIRES: Glires monophyly has been one of the most controversial issues in higherlevel mammalian systematics (Wilson, 1949; Wood, 1957; McKenna, 1975; Luckett and Hartenberger, 1985, 1993; Li and Ting, 1985; Novacek, 1992; Meng and Wyss, 2001). As mentioned above, Rodentia and Lagomorpha have each been related to various mammalian groups. The most received relationships include groupings of rodent-primate (McKenna, 1961), rodent-leptictid (Szalay, 1977, 1985), and lagomorph-zalambdalestid to the exclusion of rodents (Szalay and McKenna, 1971; McKenna, 1975). All of these hypotheses deem any similarity between rodents and lagomorphs as convergence. As pointed out elsewhere (Meng and Wyss, 2001), however, dismissing the derived resemblance of rodents and lagomorphs to convergence requires identification of some third taxon sharing a unique common ancestry with one of the two groups, but lacking the derived similarities common to both. To our knowledge, no such third taxon has seriously threatened the sister-group relationship between lagomorphs and rodents. Instead, the conception that rodents and lagomorphs share a common ancestor with other basal gliroid mammals such as eurymylids and mimotonids, an assumption amounting to an expanded concept of Glires, was favored by many morphological studies (Li, 1977; Li and Yan, 1979; Gingerich and Gunnell, 1979; Gingerich and McKenna, 1980; Har- tenberger, 1980, 1985; Korth, 1984; Dawson et al., 1984; Flynn et al., 1986; Li et al., 1987; Wilson, 1989; Novacek, 1992; Luckett and Hartenberger, 1993; Meng et al., 1994; Shoshani and McKenna, 1998; Meng and Wyss, 2001).

During the last decade the challenge to the monophyly of Glires and Rodentia from some molecular studies has been severe. Based on analysis of amino acid sequence data, Graur et al. (1991) first questioned whether the guinea pig (Cavia porcellus) is a rodent. Their result suggested a closer grouping of the guinea pig with primates, not with other rodents, which would make the conventional Rodentia, and therefore Glires, nonmonophyletic. This hypothesis was supported by several other molecular studies ( $\mathrm{Li}$ et al., 1992a; Graur et al., 1996; D'Erchia et al., 1996). In other molecular studies the relationships of Rodentia and Glires were less certain (Ma et al., 1993; Martignetti and Brosius, 1993; Honeycutt and Adkins, 1993; Porter et al., 1996; Stanhope et al., 1996; Huchon et al., 1999). In addition, a primatelagomorph clade (Easteal, 1990; Penny et al., 1991), a primate-rodent-lagomorph relationship that is further associated with tree shrews (Miyamoto and Goodman, 1986; Czelusniak et al., 1990), and a primate supraordinal clade comprised of Primates, Dermoptera, Scandentia, Lagomorpha, and Rodentia (Stanhope et al., 1993) have been proposed. More recent molecular studies with larger datasets (Madsen et al., 2001; Murphy et al., 2001a, 2001b) support rodent monophyly and the Rodentia-Lagomorpha sistergroup relationship. Conclusions of these more recent molecular studies are consistent with those of recent morphological studies (e.g., Meng and Wyss, 2001; this study). In all the five cladograms obtained in this study (figs. 72-76) the Glires is identified. The clade is robust and supported by high values of Bremer index, branch length and bootstrapping value (5, 45, and 100) (fig. 74). Based on morphological data, the monophyly of Glires containing Rodentia, Lagomorpha and their stem taxa holds firmly.

It has been suggested that some derived characters shared by rodents and lagomorphs, such as loss of canines, could be attributed to evolution of gnawing function; 
therefore, they are convergences (Graur et al., 1996). Absence of canines occurs in other mammalian groups, such as Plesiadapis, in which the incisors are enlarged as well, but Plesiadapis did not gnaw. Whether character states converged should be judged by character congruence in a hierarchy of phylogeny. Our analyses show that the enlarged incisors and other derived similarities shared by rodents and lagomorphs cannot be simply explained as independent evolution adapted to gnawing function.

In discussing Glires relationships, Graur et al. (1996: 335) also suggested that "One simple and intriguing possible resolution of the conflict between morphological and molecular data is to assume that many morphological 'synapomorphies' used in support of Glires are actually ancestral character states that have been retained in some mammalian orders but were lost in others. If this reversal of character-state polarity proves valid, then the ancestral eutherian morphotype may have resembled a rodent species much more closely than is currently recognized in the morphopalaeontological literature." Viewed within the phylogeny obtained in this study, a rodentlike ancestral eutherian morphotype appears unlikely. Features common for rodents and lagomorphs, such as losses of the incisors, canines, and premolars, modification of the glenoid fossa, and enlarged upper and lower incisors, are apparently derived conditions that evolved in the lineage toward rodents and lagomorphs; they are not retentions of a rodentlike eutherian morphotype that was present in the Cretaceous.

Duplicendentata: Duplicidentata was once considered a subgroup within Lagomorpha (McKenna, 1975; Szalay, 1977). In addition to Duplicidentata, McKenna (1975) included Pseudictopidae and Zalambdalestidae within the Lagomorpha. Szalay (1977) considered Anagalida, Mixodontia, and Macroscelididae to be lagomorphs as well. In later studies (Li et al., 1987; McKenna and Bell, 1997), Duplicidentata was generally applied to "Mimotonida" plus Lagomorpha that have two pairs of upper incisors. Although the Duplicidentata was previously considered to be paraphyletic in some studies (Li et al., 1987; Averianov, 1994), the clade was recognized in other studies (Meng et al.,
1994; Wyss and Meng, 1996; Meng and Wyss, 2001) and is here again corroborated in all five cladograms (figs. 72-76). The Duplicidentata is recognized and is relatively robust in having a Bremer index of 2, a branch length of 30 , and a bootstrap value of 90. Within the Duplicidentata, those that are stem taxa to the Lagomorpha (node 57), represented by Mimotona and Mimolagus, do not form a clade. This implies that the family "Mimotonidae" is paraphyletic.

SimPliCENDENTATA: Rodents and eurymylids usually form the group Simplicidentata, characterized mainly by a single pair of incisors in both lower and upper jaws. This group is recognized in the analyses based on the combined data. However, similar to an early study (Meng and Wyss, 2001), this clade is weakly supported (fig. 74).

EURYMYLIDAE: As pointed out by Meng and Wyss (2001), the names "Eurymylidae", "Eurymyloidea", and "Mixodontia" have been variably used for nonrodent simplicidentates (e.g., Sych, 1971; Li and Yan, 1979; Li and Ting, 1985, 1993; Dashzeveg et al., 1987; Li et al., 1987; Dashzeveg and Russell, 1988; Averianov, 1994; Dashzeveg et al., 1998). Although Dashzeveg and Russell (1988: 155) used "presence of a single incisor, little or no unilateral hypsodonty and widely separated protoconid and hypoconid" to group the Eurymylidae, a term roughly equivalent to the "Mixodontia" of $\mathrm{Li}$ et al. (1987), previous phylogenetic analyses usually recognized eurymylids as a paraphyletic assemblage (Li et al., 1987; Averianov, 1994; Meng et al., 1994; Meng and Wyss, 2001). However, this study identifies a clade consisting of Eurymylus, Heomys, Rhombomylus and Matutinia, which are the core members of the Eurymylidae. The clade is supported by a Bremer index of 1 , a branch length of 14, and a bootstrap value of 75 . In the skeletal tree eurymylids were not clustered as a clade, probably because cranial and postcranial material are sparse in Heomys and Eurymylus. In the dental tree, interestingly, the clade of Eurymylidae is clustered with that of duplicidentates. It indicates that although eurymylids are similar to rodents in lacking the I3 and P2, other dental features, such as the bipartite structure of the lower cheek teeth, show similarities to dupliciden- 
tates. This reflects the early view that Eurymylus was a relative of lagomorphs (Wood, 1942; see also Sych, 1971).

The phylogenetic position of Sinomylus is unstable. It was predicted that Sinomylus should occupy a sister-group position to the clade consisting of other eurymylids and Rodentia because Sinomylus primitively retained the P2. That relationship is only identified in the cladogram in which tooth occurrence was ordered as normally irreversible, which in this particular character means that regaining the lost P2 is impossible. Given that this genus is represented by the least complete material among the selected taxa, its phylogenetic position cannot be confidently recognized until better material becomes available.

LAGOMORPHA: The monophyly of Lagomorpha has never been questioned. In this study this clade is strongly supported by a Bremer index of 5, a branch length of 36 , and a bootstrap value of 100 . The robustness of the clade reflects the fact that lagomorphs were morphologically specialized in many aspects of the dentition, cranium, and postcranium, as indicated above by the large number of character states diagnosing the node. As the sister group of rodents, lagomorphs have a much lower species diversity. It is probably attributable to their specialized features in mastication and locomotion (see below).

Rodentia: Compared to Lagomorpha, Rodentia is less strongly supported, with a Bremer index of 2, a branch length of 30 , and a bootstrap value of 90 . In an earlier study in which only fossil taxa were involved (Meng and Wyss, 2001), whether fossil taxa such as Cocomys and Paramys are members of the crown clade Rodentia could not be adequately addressed. The present study shows a polytomic relationship for these fossil taxa and extant rodents. Therefore, their membership in the crown group Rodentia remains uncertain. Within Rodentia, the conventional sciurognath rodents are apparently paraphyletic. In contrast, hystricognath rodents form a robust clade. Three relevant nodes (node 71, 72 and 73) are strongly supported by Bremer indices of 5,5 and 3 , branch lengths of 15 , 12 and 18, and bootstrap values of 100, 97 and 92, respectively (fig. 74). Node 71 cor- responds to the Hystricognathi (Bryant and McKenna, 1995; McKenna and Bell, 1997), and node 72 to the Hystricognathiformes (Bryant and McKenna, 1995) or Hystricognatha (McKenna and Bell, 1997). That Tataromys forms the sister group of the Hystricognathiformes is also consistent with the phylogenetic placement of the Ctenodactylidae in the study by Bryant and McKenna (1995). Tataromys as a basal taxon of the Ctenodactylidae was also recognized in other studies (Wang, 1997; Dashzeveg and Meng, 1998).

Cavia is deeply nested in the clade of Rodentia. When the strict consensus tree (fig. 74) was manipulated in MacClade, it was shown that removing Cavia from rodents and clustering it with the clade of primates increased the tree length by 100 steps. If Cavia were not a rodent and were more closely related to primates as suggested by some molecular studies (Graur et al., 1991; Li et al., 1992 [Nature]; D'Erchia et al., 1996), all these extra steps in morphological transformation involved in the selected taxa must be considered as convergence and require explanation. Based on morphological data, we do not see any mechanism that would explain such convergence, and thus think that the guinea pig is a rodent.

\section{DIVERGENCE TIME OF GLIRES}

According to the molecular time-scale (Kumar and Hedges, 1998), rodents are the earliest placental group, next only to Edentata, that made their first appearance about 110 million years ago (Ma) in the Cretaceous, and Lagomorpha occurred about 90.8 $\pm 2.0 \mathrm{Ma}$. Other molecular studies (Janke et al., 1994; Huchon et al., 2000) also suggested that the common ancestor of rodents was separated from other eutherian orders in the Cretaceous. The fossil record tells a different story. For instance, the earliest known rodent, Acritoparamys, occurred in the late $\mathrm{Pa}$ leocene Clarkforkian of North America, about $57 \mathrm{Ma}$ (Dawson and Beard, 1996; Woodburne and Swisher, 1995), only half the estimated time for the branching off of rodents estimated by the molecular clock hypothesis (Kumar and Hedges, 1998). The statement (Kumar and Hedges, 1998: 918) 
that "molecular divergence times among sciurognath rodents are roughly four times older than their fossil-based estimates" may have over estimated the differences, because conventional sciurognath rodents (see Landry, 1999, for an alternative view of the terminology), such as Acritoparamys, were found in early Eocene. Still, the $110 \mathrm{Ma}$ divergence time for rodent is twice as old as what is indicated by the earliest fossil record.

Apparently, the molecular clock hypothesis tends to push rodent divergences at various levels of the group deeper into history than what the fossil record indicates. The discrepancy is enormous. These molecular data contradict the conventional view of a postCretaceous-Tertiary boundary radiation of placental orders as shown by the fossil record (Gingerich, 1977; Novacek, 1992; Archibald and Deutschman, 2001). Below we consider five hypotheses relevant to the discrepancy between fossil and molecular dates for eutherian mammals in general and Glires in particular. These hypotheses include (1) the conventional post-K-T boundary radiation of placental orders, (2) lower preservation rates of Cretaceous mammals, (3) lack of morphological diagnostic features in Cretaceous taxa, (4) hidden fossil records in Cretaceous, and (5) deep superordinal branching. Problems of the molecular clock hypothesis itself is another relevant issue that has been discussed in other molecular studies (e.g., Ayala, 1997; Bromham et al., 1999; RodríguezTrelles et al., 2001) and is beyond the scope of this study.

We will focus on the phylogeny and geological distributions of gliroid mammals in addressing the divergence issues, because gliroid mammals represent a special case for several reasons. Taken as a whole, they display the longest missing duration among all mammals between the fossil record and the divergence time estimated by the molecular clock (Bromham et al., 1999). As the most diverse placental mammals, rodents have the densest fossil record from the early Paleocene to Recent. Most of them have been always small and many are smaller than most known Cretaceous mammals so that the preservation rate is a less influential factor than in other mammals when the fossil record is concerned.
Hypothesis 1 exemplifies the conventional view of eutherian radiation ("Explosive Model" of Archibald and Deutschman, 2001) that emphasizes a post-K-T boundary burst radiation of modern placental orders, presumably taking place in the empty niches left by the extinction of dinosaurs (Gingerich, 1977; Novacek, 1992). The monophyly and geological distributions of basal Glires support this view. As shown in figures 74 and 77 , the stem taxa, including eurymylids, mimotonids, and alagomyids, to both clades of rodents and lagomorphs were found within the Paleogene and were bracketed in the monophyly of the Glires. Moreover, the earliest occurrence of immediate outgroups to the Glires, such as anagalids, pseudictopids, macroscelideans, and primates, are all distributed in early Paleogene as well. These indicate that the cladogeneses between the Glires and other placental mammals and between rodents and lagomorphs took place no earlier than the K-T boundary. This contradicts the earlier divergence times for rodents and lagomorphs estimated by some molecular studies.

Hypothesis 2 was called the "Short Fuse Model" in Archibald and Deutschman (2001). It indicates that the splits of modern placental orders are deep in the Cretaceous, probably resulting from the break-up of continents (Hedges et al., 1996), but the early placental forms did not show up in the fossil record because Cretaceous mammals are small and fragile, and their preservation as fossils is less likely (Cooper and Fortey, 1998; Foote et al., 1999a). However, Foote et al. (1999a, 1999b) showed that even if very low preservation rates for Cretaceous mammals were assumed, the gap between the molecularly estimated occurrence and fossil record is unacceptably too large. Cretaceous mammals, including "triconodonts", symmetrodonts, multituberculates, therians, and eutherians, flourished on the earth (Lillegraven et al., 1979; McKenna and Bell, 1997; Novacek et al., 1998). These mammals are generally small. It seems plainly unreasonable that these Cretaceous mammals left remains in the fossil record, whereas traces of supposed Cretaceous placental orders remain totally undetected. Although Cenozoic mammals are in general larger than Cretaceous 


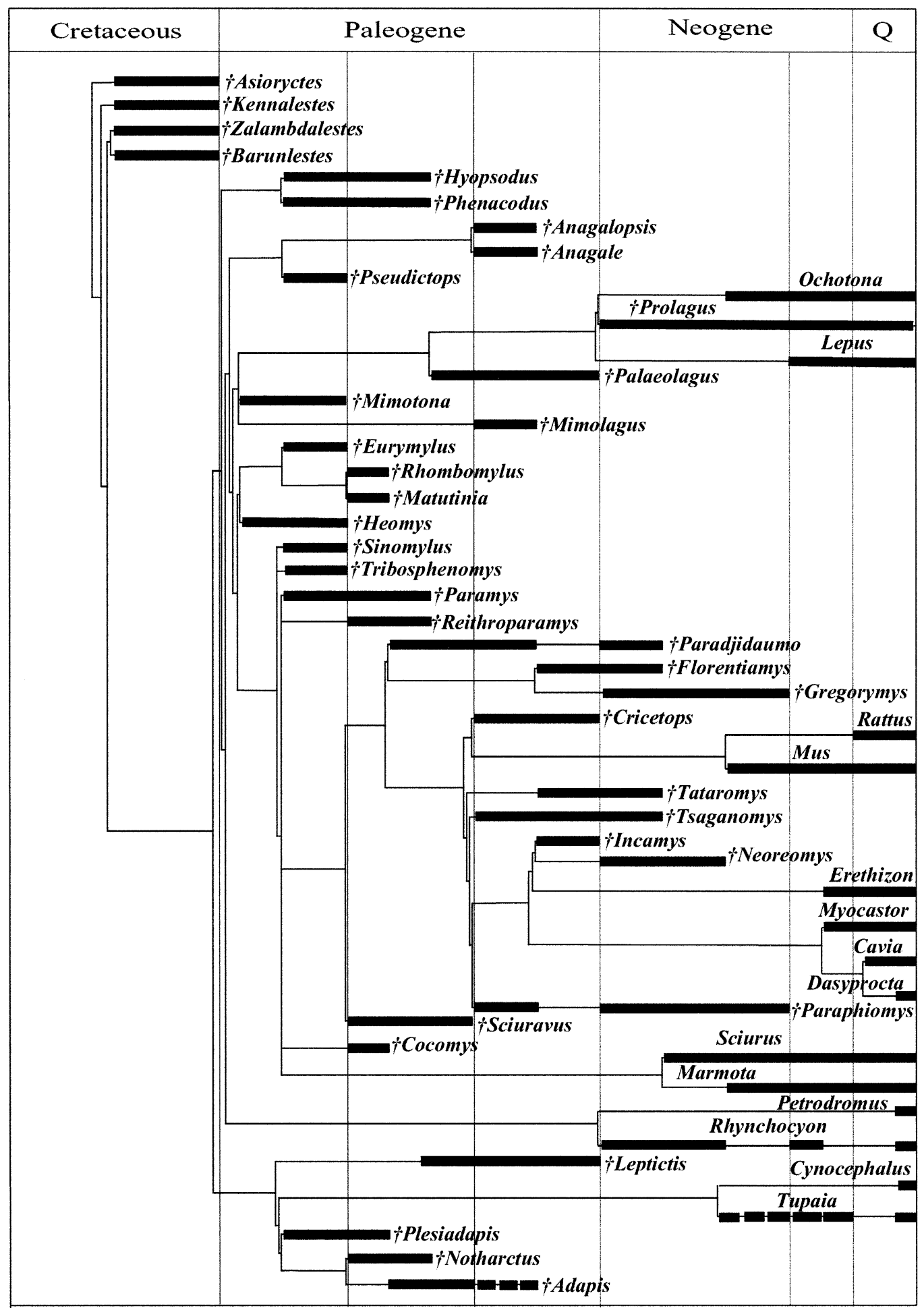

Fig. 77. Phylogenetically constrained distributions of selected taxa. Fine lines represent cladistic relationships of figure 74. Solid lines are geological distributions of taxa (based on McKenna and Bell, 1997). 
mammals so that Cenozoic preservation rates of fossils may be greater than those of the Cretaceous (Cooper and Fortey, 1998; Foote et al., 1999a), this assumption does not seem adequately applicable to the Glires. This is because early gliroid mammals are always small and many are smaller than most Cretaceous mammals.

Moreover, the molecular clock hypothesis extends not only the divergences of the ordinal lineages of Rodentia and Lagomorpha into the Cretaceous, but also those of subgroups into much earlier periods of the Tertiary than their earliest fossil records. According to the molecular time-scale, sciurognath rodents made their first appearance about $112 \pm 3.5 \mathrm{Ma}$, followed by divergences of hystricognaths $(109 \pm 3.2 \mathrm{Ma})$, Gerbillidae/Muridae $(66.2 \pm 7.6 \mathrm{Ma})$, Muridae/ Cricetidae (65.8 $\pm 2.2 \mathrm{Ma})$, and Mus/Rattus (40.7 \pm 0.9 Ma) (Kumar and Hedges, 1998; see also Janke et al., 1994). The fossil record shows that hystricognath rodents first appeared in late Eocene or early Oligocene in Africa, Asia, and South America (Wood, 1968; Gagnon, 1992; Gingerich, 1993; Wyss et al., 1993, 1994; Bryant and McKenna, 1995; McKenna and Bell, 1997) and that Mus and Rattus diverged about 8-14 Ma (Jacobs and Pilbeam, 1980; Jaeger et al., 1986). The first record of the extant genus Mus may only date back to 5.7 Ma (Jacobs and Downs, 1994). Poor preservation owing to small size of organisms does not seem to explain the lack of hystricognath rodents through the early Eocene, the entire Paleocene, and part of the Cretaceous, nor does it explain the absence of Mus and Rattus in the entire early Tertiary.

Hypothesis 3 suggests that cladogeneses of rodents took place about $110 \mathrm{Ma}$ but the lineage remained cryptic morphologically until it evolved diagnostic characters across the K$\mathrm{T}$ boundary (phylogenetic fuse of Cooper and Fortey, 1998; Foote et al., 1999a). Both hypotheses 1 and 3 imply fast morphological modifications during a short period across the K-T boundary. The differences are that in hypothesis 1 the morphological changes are coeval with cladogeneses, whereas in hypothesis 3 the cladogeneses are scattered during the Cretaceous and are decoupled from punctuated morphological changes within mammalian lineages. Hypothesis 3 also requires that placental lineages, e.g., Glires, have low rates of morphological changes during the Cretaceous but high rates during the Tertiary and that morphological evolution is decoupled with molecular evolution during a long period. The decoupling of molecular and morphological evolution gains no support. Instead, molecular and morphological evolutions appear to be related (Omland, 1997). Lacking diagnostic characters is not a good argument for the absence of subgroups of rodents, such as Mus, in the early Tertiary record; this is particularly so when the cladogenesis of Mus and its sister taxon is recognized in the fossil record (Jacobs and Downs, 1994).

Hypothesis 4 is called the "Garden of Eden" (Foote et al., 1999a). It implies that deep splitting of modern placental mammals took place in areas where fossil records of Cretaceous placentals are unknown or sediments of relevant ages are not preserved. Potential areas of this kind include Africa, Australia and Antarctica. The hypothesis further assumes that early Tertiary, about 60-65 Ma, early members of eutherian orders dispersed to northern continents from these, or one of these, southern continents, and were recognized as the post-K-T radiation (Bromham et al., 1999). The hypothesis intimates that known Cretaceous eutherians from northern continents have nothing to do with modern placental orders, which conflicts with studies that higherlevel taxa such as ungulates may have evolved from these northern eutherians (Archibald, 1996). Nonetheless, the Garden of Eden hypothesis can be tested by finding fossils in the southern continents (Foote et al., 1999a). Fossils from the early Cretaceous (Rich et al., 1997, 1999) were considered to be hedgehogs, which was therefore thought to corroborate the hypothesis of Garden of Eden (Rich et al., 2001). However, whether these fossils were actually hedgehogs remains controversial (Kielan-Jaworowska et al., 1998; Archer et al., 1999). Additional material from cranial and postcranial skeleton of these fossils would clarify their taxonomic identification.

The phylogeny and geological distributions of basal gliroid mammals show that the early divergence area, if not the origin center, 
of the Glires is in Asia (fig. 77). Among all basal gliroid taxa, such as Mimotona, Mimolagus, Rhombomylus, Matutinia, Eurymylus, Heomys, Tribosphenomys, and Sinomylus, only one species of Alagomys was found outside of Asia (Dawson and Beard, 1996). If gliroid mammals took their origin in the southern continents during the Cretaceous, the large bodies of water present between Asia on the one hand and the southern continents on the other in late Cretaceous (Smith et al., 1994) were geographic barriers for the migration of these gliroid mammals. Some mechanisms have to be assumed for their geographic dispersal. Discovery of gliroid mammals in areas that are potential dispersal route for these mammals also tests for the Garden of Eden hypothesis. However, absence of basal Glires, such as mimotonids and eurymylids, outside central Asia neither shows the origin of the Glires in southern continents, nor does it support dispersal of Glires from those continents.

Again, the Garden of Eden hypothesis hardly explains the absence of subgroups of rodents, such as Mus and Rattus, in the early Tertiary as predicted by molecular clock hypothesis. Given that rodents and lagomorphs have a relatively dense fossil record since early Eocene, hidden origin centers for various groups of rodents, such as Mus and Rattus, are untenable.

Hypothesis 5 is called the "Long Fuse Model" by Archibald and Deutschman (2001). This hypothesis argues that although members of modern placental orders are not present in the Cretaceous, stem taxa to them can be found well back in the Cretaceous. This hypothesis was supported by a study of ungulates (Archibald, 1996), but was challenged by others (Novacek et al., 1998, 2000). Archibald and Deutschman (2001: 114) stated: "it suggests that the Long Fuse Model may be correct for some clades (ungulates and their relatives), but beyond this, the fossil record cannot distinguish between the Explosive and Long Fuse models. Further, this means that, for the most part, the fossil record cannot be used to support or argue against molecular studies that extend superordinal clades of placentals back into the Cretaceous." It must be noted that molecular studies not only extend superordinal clades of placentals into the Cretaceous, they do too for intraordinal groups, such as Hystricongathi and Sciurognathi of Rodentia. Contrary to the above statement, however, Archibald et al. (2001) presented a case study in which not only a superordinal clade including zalambdalestids and Glires was recognized, but also one thought to be concordant with molecularly based estimates for the superordinal diversification of placentals. It is true that the fossil record itself cannot distinguish between the Explosive and Long Fuse models, but with phylogenetic constraints, these patterns can be recognized from the fossil record, as demonstrated by Archibald et al. (2001).

Archibald et al. (2001: 64) concluded that the results of their analysis "support a superordinal clade including zalambdalestids and Glires". Based on that phylogeny, the authors reasoned that presence of zalambdalestids in the 85-90-Myr-old faunas at Dzharakuduk "argues that the superordinal clade including Glires had separated from other superordinal placental clades by this time". These authors therefore called zalambdalestids Late Cretaceous relatives of rabbits and rodents, a scenario once favored by McKenna $(1982,1994)$. Although Archibald et al.'s (2001) study identified zalambdalestids to be more closely related to the Glires than to other Cretaceous eutherians selected for their analysis, it did not sufficiently address whether the Glires, represented by Tribosphenomys and Mimotona in their analysis, is the Tertiary group that is most closely related to zalambdalestids. As introduced above, Glires have been related to primates, leptictids, tree shrews, pseudictopids, anagalids, and macroscelideans. All these are Tertiary groups. An appropriate phylogenetic analysis to test Glires relationships should involve these taxa. In Archibald et al.'s (2001) study, however, only two Tertiary basal ungulates, Protungulatum and Oxyprimus, were included. Because these ungulates are distantly related to the Glires, the proposed zalambdalestids-Glires link (Archibald et al., 2001) was weakly supported. When relevant taxa are included in a previous study (Meng and Wyss, 2001), and particularly in the present study (figs. 74, 77), zalambdalestids are not the closest group to the Glires. There- 
fore, the conclusions are unsupported that the superordinal clade including Glires had separated from other superordinal placental clades some 85-90 Ma and that rabbit and rodent relatives were found in the Cretaceous.

In a previous study that involved fewer taxa and characters (e.g., Meng and Wyss, 2001), zalambdalestids were nested within crown placentals. It is therefore feasible to postulate that at a broad level of the mammalian phylogeny, such as the clade of "Euarchontoglires" of Murphy et al. (2001b), placentals may have occurred within the Cretaceous. Murphy et al. (2001b), based on their molecular data and the study of Archibald et al. (2001), suggested that zalambdalestids may be early representatives of Euarchontoglires. However, in the present study zalambdalestids form the outgroup to all selected Tertiary taxa in all cladograms (e.g., fig. 77) except in the dental tree in which a polytomy was formed. In other words, zalambdalestids are not within the crown group placentals. As shown in figure 74 , this relationship (nodes 91 and 92) is quite stable. Both nodes are supported by Bremer indices 2 and 3, branch lengths of 13 and 11 , and bootstrap values of 80 and 100 . Thus, it remains an open question whether modern placental mammals have occurred in the Cretaceous, either at an ordinal level, such as Rodentia and Lagomorpha, or at a higher level, such as Glires.

In summary, phylogenies and geological distributions of gliroid and other eutherian mammals selected for this study indicate that divergences of the Glires from other placental clades, between Lagomorpha and Rodentia, and between sciurognath and hystricognath rodents do not predate the CretaceousTertiary boundary. The results of our analyses support the traditional view that modern eutherian orders evolved after the K-T boundary in an explosive pattern. They disagree with the hypotheses that discrepancies between the molecular clock dating and fossil record for modern placental divergences are owing to low preservation rates of Cretaceous mammals, lack of morphological diagnostic features in Cretaceous species, unknown Cretaceous placental fossil records in southern continents, or superordinal splitting of eutherian mammals in the Cretaceous.

\section{FUNCTIONAL ANALYSES OF MASTICATION}

Mammals are characterized by a set of structures used for mastication or intraoral processing of food, such as the secondary palate, heterodont teeth with precise occlusion, and modification of jaw muscles. Within mammals these structures are further diversified (Tullberg, 1899; Turnbull, 1970). A specialized mammalian masticatory apparatus in gliroid mammals is characterized by separate incisor biting and cheek tooth chewing (Vinogradov, 1926; Becht, 1953; Landry, 1957, 1970; Hiiemae, 1971a; Hiiemae and Houston, 1971; Wood, 1965; Druzinsky, 1995). Gnawing is accomplished by enlarged upper and lower incisors that are labially covered with enamel and are kept honed to a chisel-like edge by contact of the teeth. Gnawing is also associated with several structural modifications in other regions of the dentition, in the skull and mandible, and in the jaw musculature. Because of their distinctive patterns, the masticatory apparatuses have been used as the most important features to distinguish gliroids from other mammals as well as to characterize principal subgroups within rodents.

Studies on the masticatory apparatus of gliroid mammals were carried out long ago. For instance, Cope (1888) analyzed the mechanical causes for the origin of the rodent dentition. He thought that increase in length of the incisor was the first factor in the order of time and importance during rodent evolution, which resulted in several structural changes, such as loss of the postglenoid process and specialized muscle arrangements. However, because of sparse specimens of basal gliroid mammals, early evolution of the most important apparatus characterizing the group has not been systematically investigated. Our knowledge about this apparatus is only from fragmentary teeth and jaws. Complete skulls and lower jaws are unknown from early relatives of rodents and lagomorphs, except Rhombomylus. In this section, we analyze the functional aspects of the masticatory apparatus of Rhombomylus and 
discuss their implication for evolution of the complex in early gliroid mammals.

\section{SKULl SHAPE}

The skull shape, such as the relative breadth, was thought to relate to the direction of chewing in pocket gophers (Merriam, 1895) and in some capromyid rodents (Woods and Howland, 1979). Those rodents with a propalinal power stroke have a relatively narrow skull (dolichocephalic), whereas others with an oblique power stroke have a broad skull (platycephalic). The relative breadth is the ratio of the zygomatic breadth to the least interorbital constriction (Wilkins and Woods, 1983; Wilkins, 1988; Wilkins and Cunningham, 1993). The zygomatic breadth measures at the widest points on the outer surface of the arches, and the least interorbital constriction is between the orbits at the dorsal surface of the skull. Other studies show that mastication and skull morphologies are not significantly related (Wilkins, 1988; Wilkins and Cunningham, 1993). A platycephalic condition may be related to fossorial life (Wilkins and Woods, 1983) or to hearing of substrate-borne vibrations (Wilkins and Cunninham, 1993). In Rhombomylus this ratio is about $2(47 / 24 \mathrm{~mm})$ in V5278; it is considerably smaller than those of some living rodents, which range from 2.4 to about 5. However, the ratio may be biased by the relatively small orbit in Rhombomylus, that is, relatively greater interorbital constriction, which does not adequately reflect the skull shape in general. Comparing the ratio of the zygomatic breadth to the skull length of Rhombomylus with those derived from measurements in living rodents (Wilkins and Cunningham, 1993: table 1), the skull of Rhombomylus is narrow, although Rhombomylus chewed transversely (see below). This demonstrates that skull breath does not correlate to the direction of mastication in early gliroid mammals.

In rodents there are traditionally four types of zygomasseteric morphology, the protrogomorph, sciuromorph, hystricomorph, and myomorph. If a small infraorbital foramen results from a secondary closing over of the dorsal portion of the myomorphic infraorbital foramen by a hypertrophid zygomatic plate
(Landry, 1999), then a geomorph skull condition may be distinguished. The geomorph condition, however, is superficially similar to that of sciuromorphs, although its zygomatic plate is more extensive. The protrogomorph type is common in early rodents (Wood, 1962; Li et al., 1989; Korth, 1994) and is retained only in Aplodontia among living rodents. In other types of skull morphology the infraorbital canal, the zygomatic plate, and the rostrum are specialized so that the origin of at least one bundle of jaw muscles is significantly shifted anteriorly. The anterior placement of the muscle emphasizes anteroposterior movement of the lower jaws for gnawing. The lagomorph condition differs from those of rodents in lacking enlargement of the infraorbital foramen and zygomatic plate. In addition, the jaw muscles do not shift anteriorly (see below). The skull of Rhombomylus is most nearly comparable to the protrogomorph condition. However, because of the ventral process of the zygoma, the origin of the superficial masseter does not originate on the ventral surface of the zygomatic arch ventral to the infraorbital foramen, as in the protrogomorph skull. The skull morphology of Matutinia (Ting et al., 2002) is similar to that of Rhombomylus. Whether this condition is present in other eurymylids is unknown.

\section{ReCOnstruction of JAw Musculature}

Functionally, the masticatory apparatus is a complex adapted to optimal transmission of force from muscles to teeth. The functional activity of the jaws in rodents comprises three parts, chewing, gnawing, and shifting between them, which are powered by different combinations of muscle activities (Becht, 1953; Wood, 1965). Chewing by the cheek teeth is characterized by a vertical or transverse movement, with the condyle located toward the posterior end of the glenoid cavity and the incisors separated. This would have involved the use of the main part of the temporal, the deep masseter, and the internal pterygoid. Gnawing is a type of incisive movement carried out by means of upper and lower pairs of enlarged incisors and is capable of reducing hard fibrous substances in which the separated material is not always 


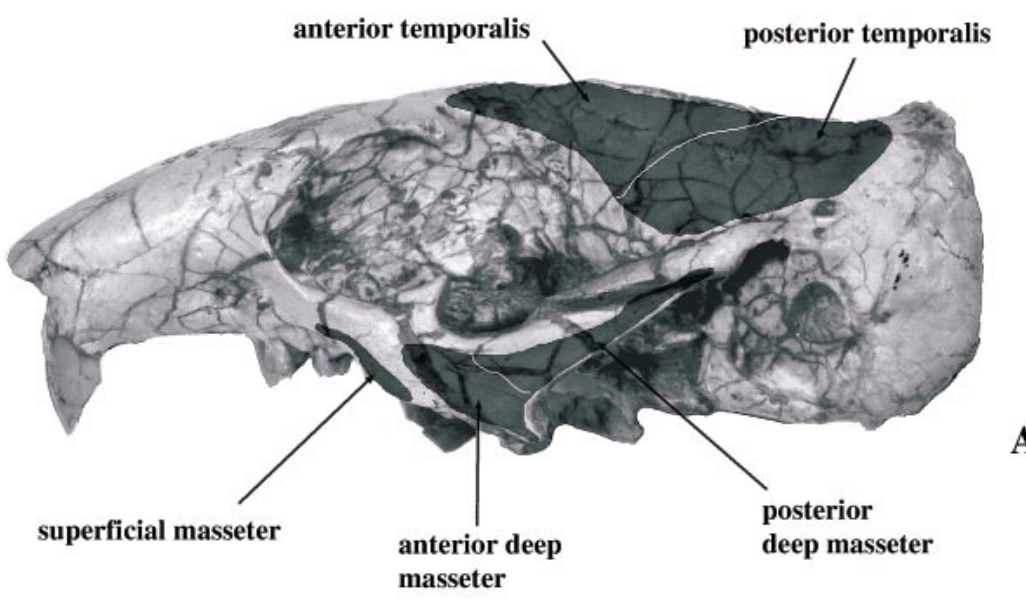

A
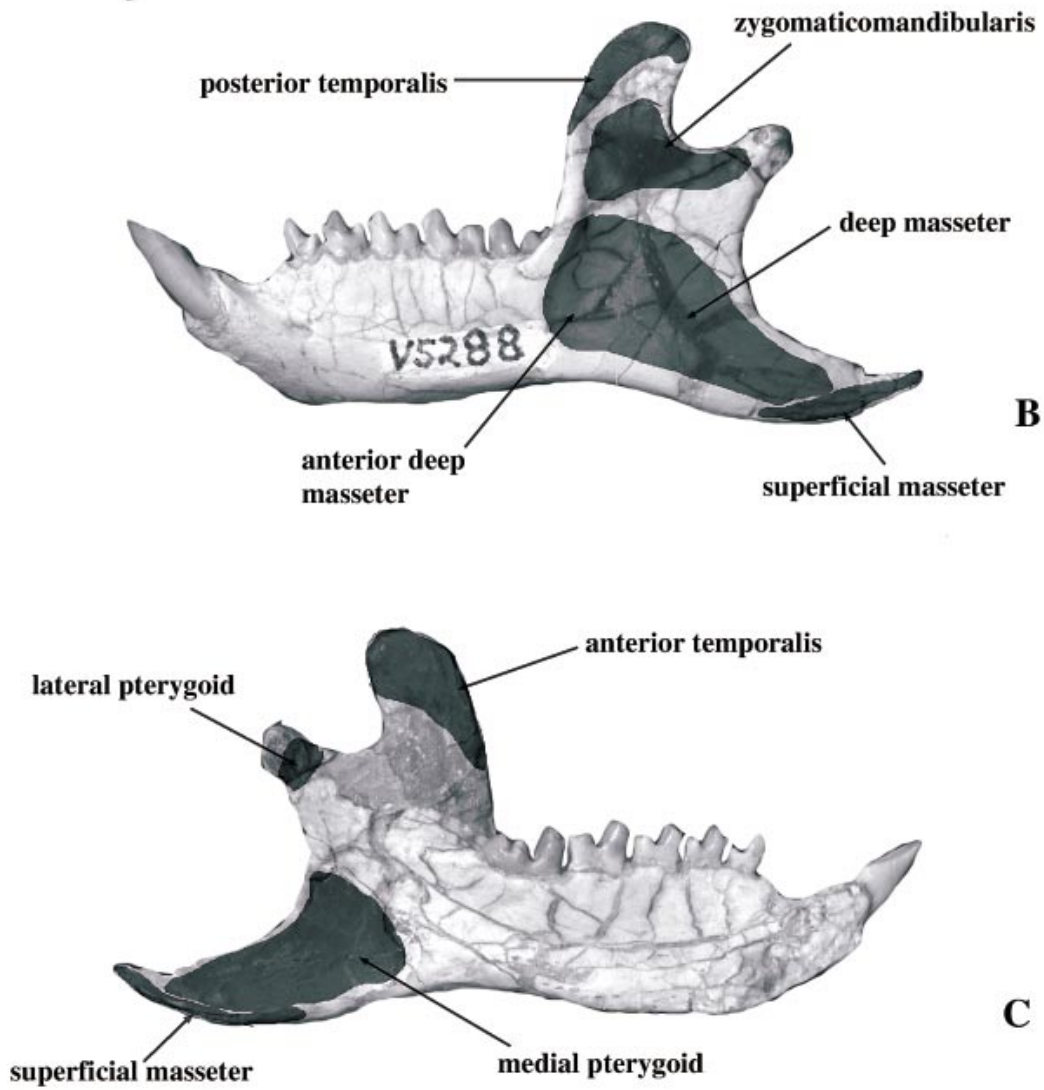

C

Fig. 78. Estimated areas of origin (A) and insertion (B, C) of jaw muscles in Rhombomylus. A, Lateral view of the skull (the skull being slightly transversely compressed). B, Lateral view of the mandible. $\mathbf{C}$, Medial view of the mandible. 
ingested (Moore, 1981). During gnawing the condyle moves forward to the anterior end of the glenoid fossa and the cheek teeth are disengaged. The same combination of muscles plus the masseter superficialis would provide the motion of the lower incisor against the upper incisor. The shifting component from chewing to gnawing would be brought about by the anterior portion of the temporal, the lateral pterygoid, and the masseter superficialis. The posterior portion of the temporal and the digastric are responsible to move the jaw from gnawing back to chewing.

To understand the mastication of Rhombomylus, we attempted to reconstruct its jaw muscles. Although myological features, particularly those associated with mastication, are important in rodent relationships and evolution (Woods and Hermanson, 1985), this array of characters can only be inferred from osteological structures for fossils. The jaw muscle reconstruction of Rhombomylus is based on comparison with rodent skeletal features in the following studies: Turnbull (1970), Hiiemae and Houston (1971), Cooper and Schiller (1975), Gorniak (1977), Woods and Howland (1979), Byrd (1981), Druzinsky (1995), Ball and Roth (1995), and Thorington and Darrow (1996). These studies provided detailed anatomies of jaw muscles, as well as their origin and insertion sites in protrogomorph, sciuromorph, hystricomorph, and myomorph rodents. Because the subdivisions of muscle groups are not always clear and because boundaries of origins and insertions of these muscle bundles may not be recognizable on the skeleton (Thorington and Darrow, 1996), reconstruction of the masticatory muscles of Rhombomylus can only be made with certain conjecture for major muscle bundles. We reconstruct the following portions of masticatory musculature for Rhombomylus: the superficial masseter, deep masseter, zygomaticomandibularis, temporalis, and pterygoid (medial and lateral) muscles. Smaller muscles with relatively minor effect on mastication are untreated. These include the digastric, transverse mandibular, mylohyoid, geniohyoid, stylohyoid, genioglossus, hyoglossus, and styloglossus.

SUPERFICIAL MASSETER: For many rodents, the origin of the superficial masseter is a ten- don that attaches to a masseteric tuberosity on the side of the rostrum. For Aplodontia, the only protrogomorphic taxon among extant rodents, it originates on the ventral side of the zygomatic arch immediately ventral to the infraorbital foramen (Thorington and Darrow, 1996). The area for the origin of the superficial masseter is slightly concave. The muscle inserts on the angular process of the mandible. The superficial masseter is a relatively conservative muscle and changes very little in its origin and insertion during evolution (Wood, 1965) and among groups of rodents (Thorington and Darrow, 1996).

There is no masseteric tuberosity or concave area to indicate the origination of the superficial masseter in Rhombomylus. Because of the special architecture of the zygomatic arch, the superficial masseter of Rhombomylus cannot originate from the ventral surface of the arch as in Aplodontia or in primitive rodents such as paramyines (Wood, 1962, 1965). It is most likely, however, that the superficial masseter originates on the flat area posteroventral to the infraorbital foramen and immediately ventral to the anterior edge of the orbit (figs. 78, 79). This area is also the anterior surface of the zygomatic root of the maxilla. It is possible that the origin may extend ventrally at a certain degree on the anterior surface of the zygomatic process of the maxilla. Such an extended origin is seen in the Old World squirrel Ratufa (Thorington and Darrow, 1996), although at a more anterior position than in Rhombomylus.

The superficial masseter of Rhombomylus must insert on the ventral edge of the angular process and may wrap over under the medial side of the process, as in rodents (figs. 78, 79). Because of the long angular process, the superficial masseter is elongate posteriorly. While the mandible is at its rest position, the longest extension of the muscle, measuring from the anteromost point for the origin to the posterior tip of the angular process for the insertion, is about $50 \mathrm{~mm}$ in IVPP V5278. The superficial masseter is therefore the longest jaw muscle in Rhombomylus. As the major muscle to move the mandible forward, the superficial masseter must be long enough so that it can work to bring the jaw forward at least the minimum working dis- 
tance (fig. 79). The minimum working distance is between the tip of the upper incisor and the posterior edge of the wear facet on the lower incisor while the mandible is at rest. In IPVV V5278, the minimum working distance is $9.3 \mathrm{~mm}$. Because of the relatively low position of the origin for the superficial masseter in Rhombomylus, the muscle fibers must be oriented quite horizontally, and the anterior pull provided by the superficial masseter can be significant (fig. 79A, B). Differing from those of rodents, the origin and insertion of the superficial masseter in Rhombomylus are more posterior. Although the elongate angular process gives the muscle enough length to work, it is less efficient mechanically than those of rodents in which both the origin and insertion of the superficial masseter are more anteriorly located.

DeEP MAsSeTER: The deep masseter has been differentiated into two portions, the anterior and posterior, at least at its origin in rodents. This differentiation is especially advanced in sciuromorphs in which the anterior deep masseter is larger and extends to a broad zygomatic plate anteroventral to the infraorbital foramen, whereas the posterior deep masseter generally originates from the ventral and lateral sides of the zygomatic arch (Ball and Roth, 1995; Thorington and Darrow, 1996). The anterior fibers of the anterior deep masster insert on the anterior area of the masseteric fossa, whereas the rest of the deep masseter insert on the ventral portion of the masseteric fossa. In general, the action line of the anterior deep masseter is nearly perpendicular to the moment arm of the mandible, while the posterior one has an acute angle to the moment arm and, therefore, less mechanical advantage (Thorington and Darrow, 1996). In the protrogomorph Aplodontia, this differentiation is not so distinctive (Thorington and Darrow, 1996). In some rodents that have a similar deep zygomatic arch, such as Capromys, the posterior deep masseter originates from the lateral jugal fossa and is correspondingly broad (Woods and Howland, 1979). In comparison, the deep masseter muscle of Rhombomylus must have originated from the lateral jugal fossa and probably extended posteriorly on the lateral surface of the zygomatic arch (figs. 78, 79). An anterior deep masseter may have differentiated and attached to the area anterior to the fossa. Its anterior extremity does not seem to extend anteriorly over the level of the midpoint of the orbit, which differs from the condition in all rodents. The deep masseter inserts on the ventral surface of the masseteric fossa of the mandible, dorsal to the insertion of the superficial masseter but ventral to that of the zygomaticomandibularis (figs. 78, 79). Given the size and depth of the lateral jugal fossa, the deep masseter must have been sizable and supplies the main force for mastication as in rodents.

ZYGOMATICOMANDIBULARIS: The zygomaticomandibularis had been considered either as part of the masseter (masseter medialis of Tullberg, 1899: 61-62; masseter profundus of Howell, 1932: 410-411) or as a separate muscle (Müller, 1933; Turnbull, 1970). The latter is commonly accepted in recent rodent anatomy (e.g., Ball and Roth, 1995; Thorington and Darrow, 1996) and is followed herein. The zygomaticomandibularis originates on the medial surface of the zygomatic arch in Aplodontia and sciuromorphous rodents. In the latter, the anterior portion of the zygomaticomandibularis may spread onto the orbital surface of the maxilla, lateral to the posterior opening of the infraorbital canal, as well as on the dorsal surface of the zygomatic process of the squamosal. In hystricomorph rodents the anterior portion of the zygomaticomandibularis is large and spreads anteriorly through the enlarged infraorbital foramen on to the snout. In Rhombomylus, the zygomaticomandibularis probably originates on the medial surface of the jugal and on the dorsomedial side of the zygomatic process of the squamosal (figs. 78, 79). There may be a muscle bundle, via a tendon, inserting on the lateral side of the zygomatic arch with the tendon passing through the trough between the ventral postorbital process and the arch. It seems unlikely that any portion of the muscle attaches to the lateral orbital surface. The large zygomatic plate provides a surface for origination of a potentially large anterior portion of the zygomaticomandibularis. However, if the zygomaticomandibularis did attach to the entire medial surface of the zygomatic plate, the ventral portion may have functioned primarily for anterior pull rather than for occlusion. 
This is because the ventral zygomatic plate is so low that it is at the same level of the insertion of the zygomaticomandibularis on the mandible. In living rodents, the insertion of the zygomaticomandibularis is on the dorsal masseteric fossa and the ventral areas of the coronoid and condyloid processes. Because the masseteric fossa is relatively posterior to the zygomatic arch in Rhombomylus, the zygomaticomandibularis probably provides an anterior component of pull for the mandible. In sciuromorphous rodents the origin and insertion of the zygomaticomandibularis is more dorsoventral, with little contribution to anterior pull of the mandible. In hystricomorphous and myomorphous rodents, the anterior portion of the zygomaticomandibularis originates much more anteriorly, providing a major force for the anterior pull of the mandible.

TEMPORALIS: Smith and Savage (1956) recognized the basic differences in the mechanics of the masticatory system in herbivores and carnivores, due to their different diets. In addition to other features, such as the transversely oriented glenoid fossa, carnivores have a massive temporalis that provides force for the jaws to close fast, and carnassials that shear precisely, with little transverse movement. The mandibular condyle is low and the coronoid process is high, generating a large moment arm for the temporalis. Herbivores, in contrast, have large masseter and pterygoid muscles but a small temporalis, favoring primarily transverse mastication (Schumacher, 1961; Turnbull, 1970). Their mandibular condyle is high and the moment arm for the masseter is increased. The lagomorph masticatory apparatus is basically herbivorous, and rodents remain as a distinctive group (Turnbull, 1970). In addition to a large masseter, the temporalis is sizable in many rodents. The retracting temporalis counteracts the protracting masseter and makes possible the anteroposterior jaw movements (Weijs and Dantuma, 1975; Weijs, 1980). In the case of Cavia in which the temporalis is relatively small, the temporalis plays a "braking" or compensatory role during closing and power stroke (Byrd, 1981). During incisal biting, the posterior portion of the temporalis may have a role in stabilizing the mandibular condyle in the glenoid fossa (Satoh, 1998).

A well-developed temporalis appears to be present in Rhombomylus, judging from the large temporal area of the skull and a considerable coronoid process of the mandible for the origin and insertion of the temporalis. The temporalis probably spread from the posterior side of the supraorbital process to the lambdoidal crest, to the dorsal side of the zygomatic process of the squamosal, and to the posterior orbital wall (figs. 78, 79). It extends medially to the sagittal crest. The temporalis inserts on the dorsal and anterior edges as well as on the lateral and medial surfaces of the coronoid process (figs. 78, 79). The coronoid process of Rhombomylus is relatively large and has a vertical anterior edge; it is most similar to that of Aplodontia among living rodents. In other rodents the process is greatly reduced and the anterior edge is inclined posteriorly, partly owing to the anterior shifting of the masseteric fossa. The coronoid process of Rhombomylus is more posteriorly positioned than those of rodents. In this configuration, the anterior portion of the temporalis must provide primarily vertical force for the power stroke of chewing.

PTERYGOID: It is generally accepted that the pterygoid muscles of mammals, particularly herbivores, have a major role in the production of the lateral mandible movement (Smith and Savage, 1956; Schumacher, 1961; Turnbull, 1970; Greaves, 1978). The medial (internal) pterygoid originates from the low portion of the pterygoid fossa, probably anterior to the oval foramen and its ventral surface, as well as the anterior projection of the auditory bulla. It inserts on the broad pterygoid fossa and angular process on the medial surface of the mandible. The lateral (external) pterygoid originates on the concave area of the lateral pterygoid crest and inserts on the medial surface of the condylar neck of the mandible. Because of the orientation of the fibers, the medial pterygoid has a small contribution to the anterior pull of the mandible and is therefore less important in the incisor bite (Hiiemae, 1971; Hiiemae and Houston, 1971; Thorington and Barrow, 1996). The main function of this muscle is to control or balance the lateral component of the deep masseter in the chewing stroke 

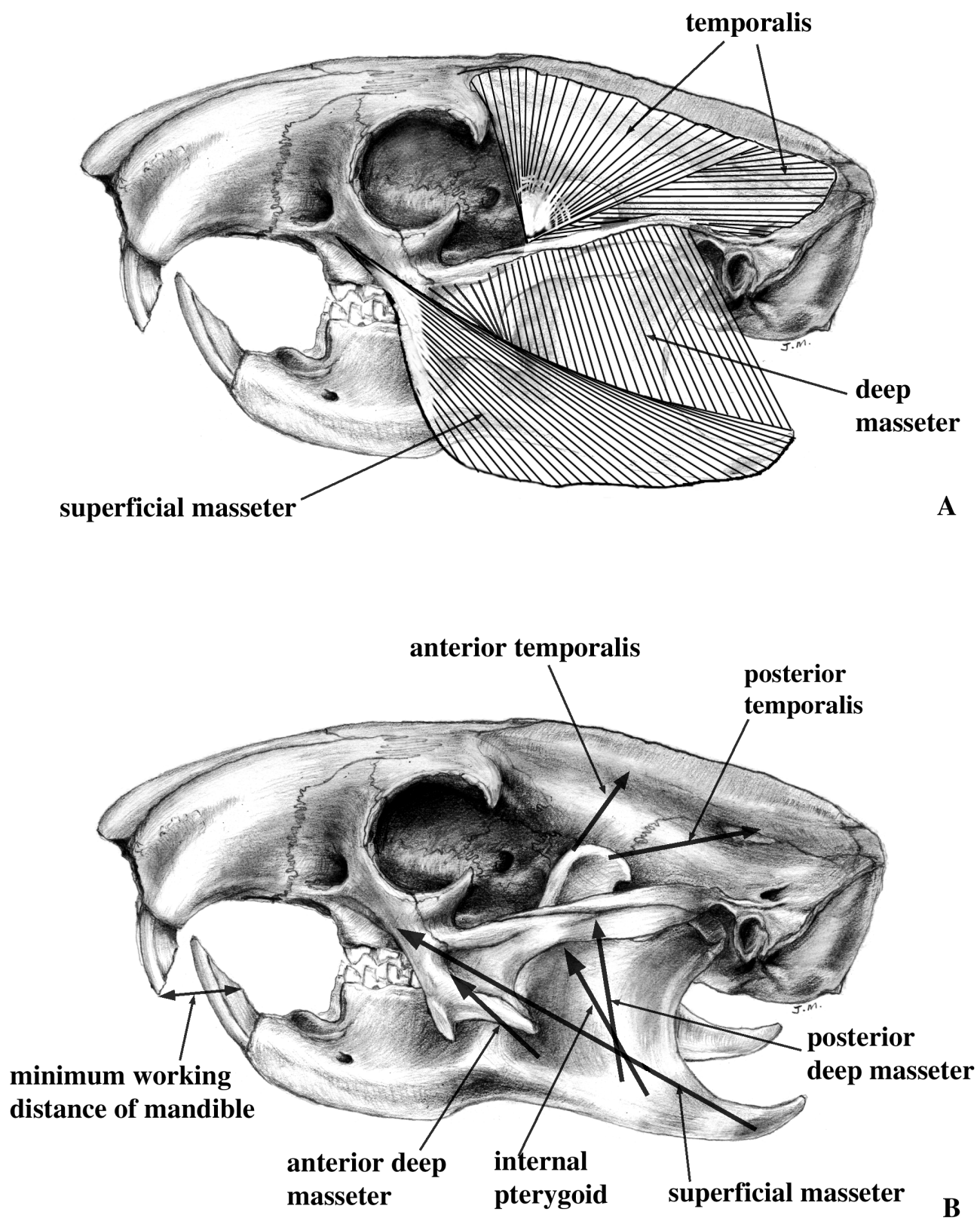

Fig. 79. A, Reconstructed major jaw muscles of Rhombomylus. B, Estimated working lines of masticatory muscles.

(Hiiemae, 1971). A role of the medial pterygoid in balancing the deep masseter is also present around an axis of rotation connecting the biting tips of incisors and the jaw joint (Satoh, 1998). The small lateral pterygoid has a different function, probably serving to protract the mandible in preparation for the incisor bite (Thorington and Darrow, 1996) or having an action in controlling and regulating the positions of the condylar neck during gross movements of the jaw (Hiiemae and Houston, 1971). 
Because of the relatively deep pterygoid fossa in Rhombomylus, it is probable that its pterygoid muscle was bulky (fig. 78C). Given its orientation inferred from the fossa, the internal pterygoid in Rhombomylus probably functioned to balance the power applied by the masseter as in extant rodents. Because the angular process curves medially around a vertical sagittal plane of the skull, the medial pterygoid possibly provides an anterior component of force for mandible movement.

\section{MANDIBLE}

It has been realized that large herbivores have a large angular process and a high mandibular condyle, but a reduced coronoid process, whereas carnivores have just the opposite (Wolff-Exalto, 1951; Becht, 1953; Smith and Savage, 1956; Moss, 1968; Turnbull, 1970; Scapino, 1972; Radinsky, 1985). Lagomorphs were considered to be structurally intermediate between rodents and ungulates (Weijs and Dantuma, 1981; Lopez Martinez, 1985). These features bear importantly on the masticatory function because they directly reflect the moment arms of the acting muscles that provide power for mastication and gnawing. Although the anterior placement of jaw muscles in early gliroid mammals can be recognized from the position of the anterior root of the zygomatic arch and the anterior edge of the masseteric fossa in early gliroid mammals, the relationship of these structures to the jaw muscles has not been considered in detail because of the fragmentary nature of fossil specimens. With the reconstruction of the Rhombomylus masticatory musculature above, it is clear that jaw muscles of early gliroid mammals differ from those of extant rodents in their size and orientation. In comparison with other gliroid and nongliroid mammals, we here provide a quantitative analysis on the moment arms of jaw muscles for Rhombomylus.

The specimens (both fossil and living species) and measurements for the analysis are listed in appendices 4 and 5. Mandibular landmarks and related measurements are provided in figure 5. Most of these landmarks follow Thorington and Darrow (1996) and Velhagen and Roth (1997). Because of the phylogenetic level of this study, in which in- traspecific variation is insignificant, measurement of a single, carefully selected specimen for each taxon suffices for the analysis (Radinsky 1985; Marcus, 1990; Velhagen and Roth, 1997). In addition, complete mandibles in fossils are rare, leaving us with only the single specimen approach.

In viewing the plotted results it should be borne in mind that although the resultant power for biting and chewing is directly related to the moment arms of muscles, it is also affected by the direction (or the angle with the moment arm) of muscle fibers, type and size of muscles, gape angle, and structures of the skull (Turnbull, 1970; Hiiemae, 1971a, 1971b; Hiiemae and Houston, 1971; Woods and Howland, 1979; Weijs, 1975, 1980; Weijs and Dantuma, 1975, 1981; Weijs et al., 1987; Wilkins and Cunningham, 1993; Ball and Roth, 1995; Thorington and Darrow, 1996; Satoh, 1997, 1998, 1999). In addition, the measurement is not the actual effort arm for the muscle, which is the perpendicular distance between the jaw joint and the line of action of each muscle. The estimated working lines of masticatory muscles are illustrated in figure 79B.

RESULTS: Figure 80A plots the ratios of the moment arm of the anteriormost fibers for the deep masseter (mma) to the resistance arms of the molar (ram) and incisor (rai). The moment arm of the anteriormost fibers of the deep masseter is measured between the mandibular condyle and the anterior edge of the masseteric fossa. The resistance arms of the molar and the incisor are between the condyle and, respectively, the anterior edge of the $\mathrm{m} 1$ and the tip of the incisor. The plot shows that the moment arm of the anterior deep masseter in gliroid mammals is generally greater than are those in other groups of mammals. Although the mma/ram and mma/ rai ratios differentiate Glires from other mammals, they cannot distinguish ungulates, carnivores, and other mammals.

Figure $80 \mathrm{~B}$ illustrates the ratios of the moment arm of the ventralmost fibers for the superficial masseter ( $\mathrm{mmv}$ ) to the resistance arms of the molar (ram) and incisor (rai). The moment arm of the ventralmost fibers of the superficial masseter is between the mandibular condyle and the lowest point of the angular process. The ratios of the moment arm 

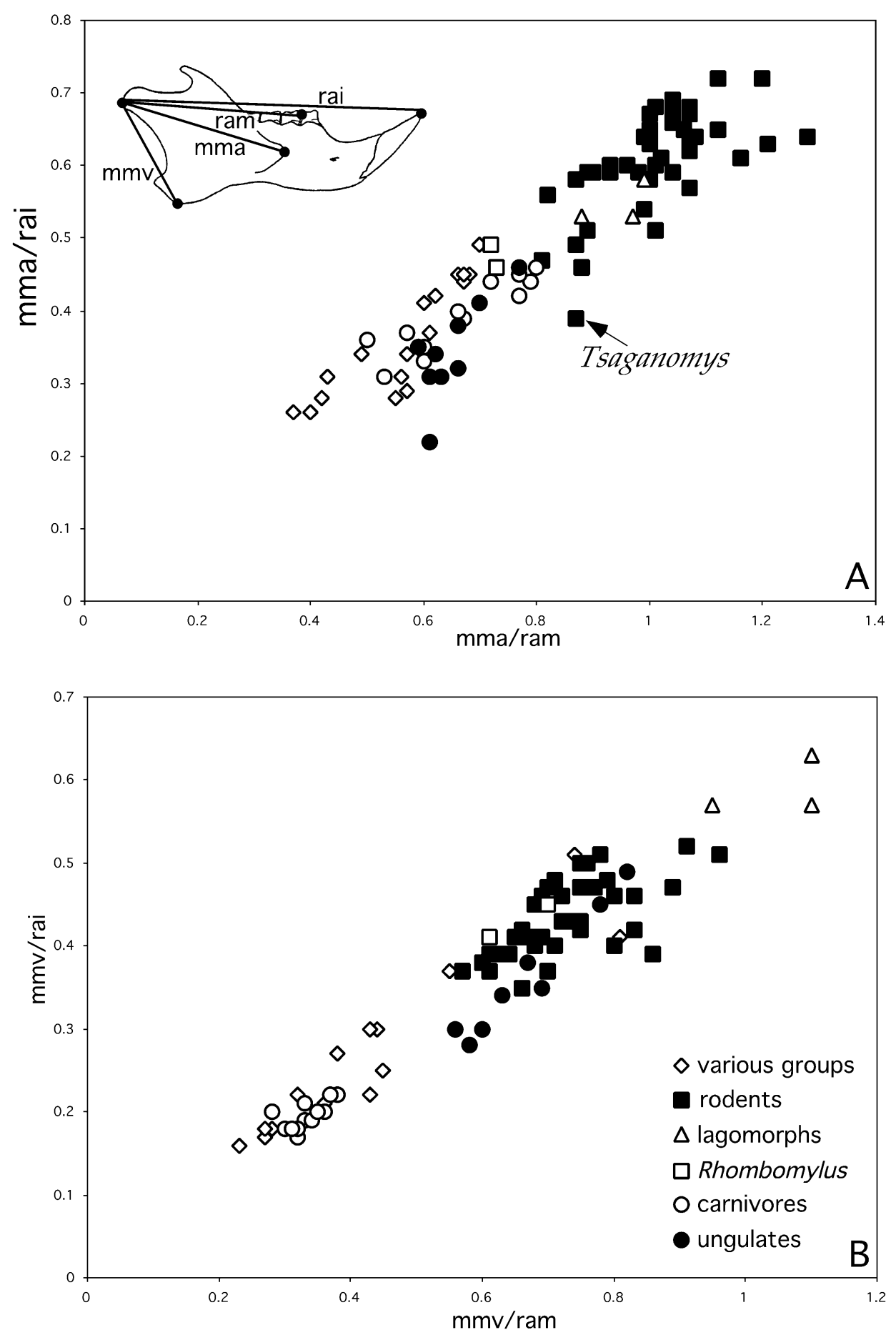

Fig. 80. A, Plotted ratios of the moment arm for the anterior deep masseter (mma) to resistance arms of the molar (ram) and incisor (rai). B, Ploted ratios of the moment arm for the ventralmost fiber of the superficial masseter (mmv) to the resistance arms of the molar and incisor. See text for discussion. 
of the superficial masseter to resistance arms of the molar and incisor have a different pattern compared to those of the anterior deep masseter. Rodents, Rhombomylus, and ungulates are similar in the mmv/ram ratio. Lagomorphs are outstanding in having the greatest $\mathrm{mmv} / \mathrm{ram}$ and $\mathrm{mmv} / \mathrm{rai}$ ratios, which result from deepening of the angular process and elevation of the mandibular condyle. $C y$ nocephalus and Plesiadapis (empty diamonds clustered with the solid squares and dots in fig. 80B) are comparable to those of Rhombomylus, rodents, and ungulates in the $\mathrm{mmv} / \mathrm{ram}$ and $\mathrm{mmv} / \mathrm{rai}$ ratios. Carnivores and insectivores are characterized by small $\mathrm{mmv} / \mathrm{ram}$ and $\mathrm{mmv} / \mathrm{rai}$ ratios, corresponding to a small angular process in those forms. A small angular process, like those of carnivores and insectivores, is probably a primitive condition in eutherian mammals.

Figure $81 \mathrm{~A}$ plots ratios of the moment arm of the dorsalmost fibers for the temporalis to the resistance arms of the molar and incisor. These ratios in carnivores are generally great, while those of ungulates are small. They vary among other mammals. Gliroid mammals and other primitive forms overlap with both ungulates and carnivores in these plots.

Figure $81 \mathrm{~B}$ illustrates the ratios of the total length of the molars to the resistance arms of the molar and the length of the mandible. It shows that the relative molar length, which is one of the parameters for chewing area of teeth, is not significantly different among mammals. The low value in feliform carnivores results from the reduction of teeth (i.e., only the $\mathrm{m} 1$ among molars is retained). For the caniform the relative length of the molars remains in the range of other mammals. This is because although the $\mathrm{m} 2$ and $\mathrm{m} 3$ are reduced in size, the $\mathrm{m} 1$ is enlarged.

DISCUSSION: The extraordinary ecological and evolutionary success of the rodents has been attributed in part to their adaptations for biting and chewing (Thorington and Darrow, 1996). Development of separate biting and chewing phases entails an emphasis on the masseter muscle, in contrast to the emphasis on the temporalis of many other mammals (Turnbull, 1970). The size and orientation of the rodent masseters represent a masticatory pattern in which a protrusive component of the lower jaw is substantial. Our plots show that the deepening of the angular process for attachment of the superficial masseter and the anterior shifting of the anterior deep masseter are the most important features in the evolution of jaw musculature that characterize the gliroid mammals. For rodents, the long moment arm of the anterior deep masseter is achieved by anterior extension of the anterior deep masseter, which can be inferred from the position of the anterior edge of the masseteric fossa in fossils. In many rodents the moment arm of the anterior deep masseter is significantly longer than the resistance arm of the molar so that the mma/ram ratio is greater than 1 . The increase of the moment arm of the anterior deep masseter provides a great mechanical advantage for rodents in both cheek teeth chewing and incisor biting.

Schumacher (1961) concluded that the protracting component of rodent jaw muscle is about $50 \%$ of the total masticatory force, whereas in herbivores, which share a strongly developed masseter and pterygoids, it amounts to only about $25 \%$. Many observations (Stark and Wehrli, 1935; Becht, 1953; Turnbull, 1970; Hiiemae, 1971a; Satoh, 1999) indicated that the masseter is the largest of the masticatory muscles in rodents, although the most developed subdivision of the muscle varies among different groups. In the evolutionary history of rodents the areas of origin of some parts of the masseter have extended anteriorly (Wood, 1965). Because the masseter exerts the largest force among all the masticatory muscles, the force to gnaw hard food material can be increased most efficiently by increasing the value of the effort arm for the masseter. Given a fixed area of insertion the moment arm for the masseter becomes larger when the area of its origin extends more anteriorly (Satoh, 1999). In addition to anterior shift, the origin of the masseter muscle is also dorsally extended. It is equally important that the insertion of the anterior deep masseter migrates anteriorly during evolution of rodents, which increases the effort moment arm of the masseter as well. Dorsal extension of the origin and anterior shift of insertion for the deep masseter avoid horizontal orientation of the muscle resulting from anterior extension of the origin area, therefore compensating for the loss of verti- 

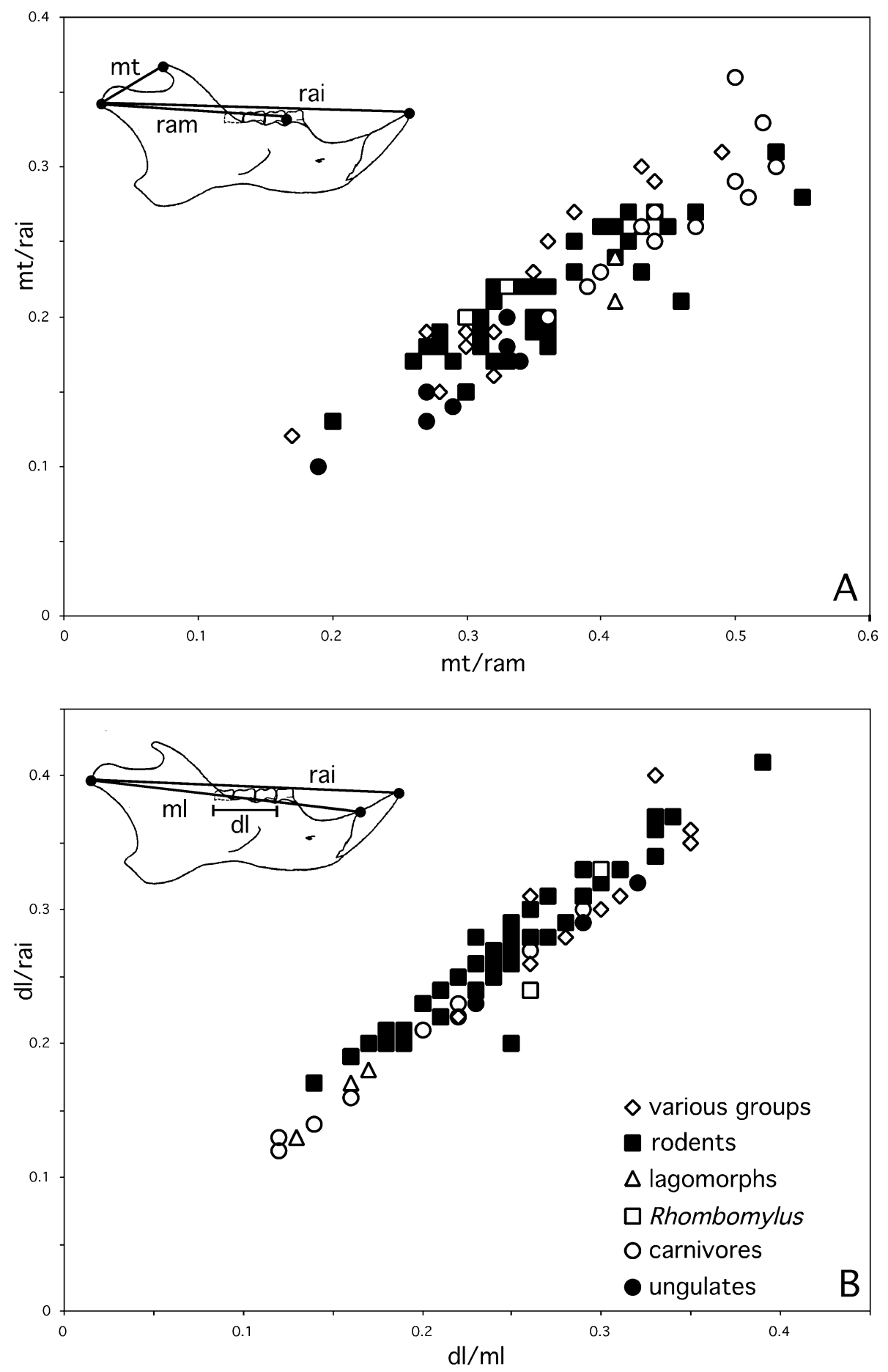

Fig. 81. A, Plotted ratios of the moment arm for the dorsal fibers of the temporalis (mtd) to resistance arms of the molar (ram) and incisor (rai). B, Ploted ratios of the total molar length (dl) to resistance arms of the molar (ram) and incisor (rai). See text for discussion. 
cal bite force caused by horizontal orientation of the masster (Weijs and Dantuma, 1981).

The mma/rai ratio of Tsaganomys is the lowest among the plotted samples of rodents and is even lower than those of Rhombomylus and some other mammals (fig. 80A). This is owing to a relatively posteriorly located masseteric fossa and, more importantly, to unusually long incisors that are probably used for digging. Given the phylogenetic position, in conjunction with other cranial and dental specializations of Tsaganomys (Bryant and McKenna, 1995), the short moment arm of the anterior deep masseter for incisor biting in Tsaganomys is probably secondarily developed for fossorial life. The long, procumbent incisors and thick enamel of Tsaganomys recall those in molerats, which use their incisors for digging (Merwe and Botha, 1998). Interestingly, lengthening of the incisor is at the expense of the moment arm of the anterior deep masseter. The loss of advantage of the moment arm may be compensated by a stronger masseter muscle, similar to that of Bathyergidae, which has developed perhaps the most massive masseter muscles among rodents, although anterior shifting of the masseter in the family is not significant (Tullberg, 1899; Wood, 1965).

The moment arm of the anterior deep masster in lagomorphs is comparable to those of many rodents. However, differing from rodents, the relatively long moment arm for the anterior deep masseter in lagomorphs results from the dorsal elevation of the mandibular condyle, not from anterior shifting of the masseteric fossa. Compared with rodents, the position of the anterior edge of the masseteric fossa is relatively posterior in lagomorphs. This indicates that anterior movement of the lower jaw in lagomorphs is not so significant as in rodents, which is concordant with a short glenoid fossa and a short distance between the lower and upper incisors in lagomorphs. These observations suggest that incisive biting in lagomorphs is less efficient than in rodents. The most distinguishable feature of lagomorphs is their long moment arms for the superficial and posterior deep masseters, as shown in figure 81B, which result from elevation of the mandibular condyle and deepening of the angular process. In this regard, the lagomorph jaw apparatus was considered to be intermediate between those of ungulates and rodents (Weijs and Dantuma, 1981).

The moment arm of the anterior deep masseter in Rhombomylus is intermediate between other gliroids and other mammals, indicating that a posteriorly positioned anterior deep masseter is a primitive condition for gliroid mammals. Because of the relatively posterior placement of the deep masster, the incisor bite of Rhombomylus was probably less powerful than in more advanced gliroid mammals, in keeping with the relatively slender incisors of Rhombomylus. In contrast, the ratios of the moment arm of the ventralmost fibers of the superficial masseter to the resistance arms of the molar and incisor are like those of rodents, suggesting that the superficial masseter is a relatively conservative muscle that changed little during the gliroid evolution.

As discussed in describing the mandible, although the masseteric fossa in Rhombomylus is less anterior, the angular process is longer than in most rodents. The posterior elongation of the angular process extends the insertion of the superficial masseter more posteriorly than in rodents, which gives the superficial masseter sufficient length to do the work in moving the lower jaw anteriorly. Even though the origin of the masseters is not so anteriorly placed in Rhombomylus, the gnawing function has already achieved. This mechanism, however, is less efficient than that of rodents, because more posteriorly and horizontally positioned masseter muscles have relatively shorter moment arms.

The plot shows that the moment arm of the dorsalmost fibers of the temporalis in carnivores is generally greater, while that of ungulates is smaller, than in other mammals (fig. 81). The differences among groups are not so remarkable as those of the masseters. Gliroid mammals and the primitive forms (mainly insectivores) overlap with both ungulates and carnivores in this plot. However, a parameter that is not shown by our plot is the size and shape of the coronoid process, which are functionally related to the activity and presence of the temporalis muscle (Moss and Meehan, 1970). For carnivores, the coronoid process is much larger than those of 
gliroid mammals and ungulates (Turnbull, 1970). Even though some Glires may have a relatively long moment arm for the dorsal temporalis, the actually power produced by the temporalis may not be comparable to that of carnivores, because of the small size of the muscle in gliroid mammals. Radinsky (1985) considered that the carnivore condition, with a small angular process and large temporal fossa and coronoid process, represents the primitive condition of the mammalian skull and jaw proportions. In most ungulates, the temporal fossa is reduced, indicating reduction of the temporalis, and the angular process is expanded, corresponding to expansion of the masseter and pterygoid musculature. The reduced coronoid process in gliroid mammals is apparently a derived condition. In this regard, the lagomorph condition is extreme in that the coronoid process is almost lost and the temporalis is a small bundle of muscle.

The amount of food that can be broken down in one chewing stoke depends on the occlusal area and thus the length of the row of cheek teeth (Weijs and Dantuma, 1981). Interestingly, the total length of the molars in lagomorphs is short (fig. 81B). However, lagomorphs differ from rodents in having two molariform premolars, with the $\mathrm{p} 3$ being enlarged. This gives lagomorphs an occlusal area of cheek teeth that matches those of rodents. However, in comparison with ungulates the entire cheek tooth row is short in rodents and lagomorphs (Weijs and Dantuma, 1981). In large ungulates the premolars are usually molarized so that the chewing surfaces are increased. The quantitative relationship of the area between the chewing surface and the body weight of a herbivorous mammal is unknown to us. It is apparent, however, that the chewing surface at any given time is two-dimensional, whereas the body size is three-dimensional. Although rodents have fewer cheek teeth compared to ungulates, the proportional chewing area in relation to the body mass may not be smaller than those of large sized ungulates. In addition, the repeated fracture of food particles by mastication is a mechanical process, and the rate at which it takes place depends on the chewing rate of any given mammal (Lucas and Peters, 2000).

\section{DENTITION}

INCISORS: For functional activities of the rodent incisors, three postures of the lower incisors have been recognized (Krumbach, 1904; Druzinsky, 1995): (1) rest position, in which the lower incisors are roughly parallel to one another; (2) catching or spearing position, in which the tips of the lower incisors are spread apart; and (3) gnawing and wedging position, in which the tips of the lower incisors are brought together. These postures are possible because the mandibular symphysis is movable. In Rhombomylus, the symphysis is not fused and is probably movable, suggesting that the lower incisors could display more than one posture.

Many studies also show that there are three basic types of incisal function in gliroid mammals: (1) cropping, in which the tips of the upper and lower incisors are occluded; (2) gnawing, in which the tips of the lower incisors move dorsally, passing posterior to the tips of the upper incisors; and (3) toothsharpening, in which the lower incisors are protruded anterior to the tip of the upper ones and their lingual sides are ground against the upper, producing thin enamel edges (Krumbach, 1904; Vinogradov, 1926; Taylor and Butcher, 1951; Ardran et al., 1958; Ardran and Kemp, 1960; Landry, 1957, 1970; Becht, 1953; Hiiemae and Houston, 1971; Hiiemae and Ardran, 1968; Every, 1975; Weijs and Dantuma, 1975; Gorniak, 1977; Byrd, 1981; Offermans and de Vree, 1990; Druzinsky, 1995). Cropping (tip-to-tip occlusion) of the incisors has been found in lagomorphs (Ardran et al., 1958), but has never been reported in any investigation of rodents (Druzinsky, 1995).

Among the three functions, the tooth sharpening ability is important functionally and phylogenetically (Druzinsky, 1995). Length regulation of the ever-growing incisors is not by wear but by purposeful sharpening (Taylor and Butcher, 1951). Landry (1999) considered that the incisor chisel is deliberately shaped by the animal. Although many mammals, such as multituberculates, plesiadapids, and tillodonts, possess enlarged incisors, none of them has developed the elliptical incisor chisels of Glires (with the exception of Daubentonia). Therefore, Landry 
(1999) regarded this feature as a useful synapomorphy for the Glires. Sharpening is critical for biting executed by an ever-growing incisor. It hones the edges of the incisors, improves the cutting performance of the teeth, and reduces the chance of breaking the tips of the incisors by avoiding tip-to-tip occlusion of the upper and lower incisors (Druzinsky, 1995). During the upper incisor sharpening, the mandible is brought anteriorly and the labial edge of the lower incisor contacts the posterior tip of the upper incisor and is dragged across the lingual surface to create the wear facet. The lower incisor chisel is probably the most characteristic aspect of gliroid incisor shaping. During the sharpening of the lower incisors, the mandible is protruded farther forward and the lingual surface of the lower incisor is dragged across the tip and labial surface of the upper incisor in an arc that corresponds to the concave wear facet of the lower incisor (Druzinsky, 1995). Although the enlarged lower incisor of zalambdalestids has been cited as evidence for a zalambdalestid-Glires relationship (Archibald et al., 2001), a lower incisor chisel is not present in zalambdalestids. This may be attributed to several factors: a procumbent incisor that curves very little dorsally, a small upper incisor that cannot shape the lower one, or the incapability of anterior movement of the lower jaw in zalambdalestids. In contrast, early gliroid mammals, such as Rhombomylus, are capable of self-sharpening of the incisors.

As Vinogradov (1926; see also Druzinsky, 1995) recognized, the ventral end of the wear facet on the lingual surface of the lower incisor represents the limit of anterior movement of the mandible, and the dorsal end of the lingual facet of the upper incisor represents the superior limit of movement of the mandible. The distance between the tip of the upper incisor and the ventral edge of the wear facet on the lingual surface of the lower incisor while the mandible is in its rest position is the minimum working distance of the mandible (fig. 79B). This distance indicates how far the mandible can protrude anteriorly from its rest position to the incisorsharpening position. It is possible that the mandible can protrude even farther anteriorly. Comparing various species of gliroid mammals, we found that rodents generally have a longer minimum working distance of the mandible than do lagomorphs. This can be told from three features. First, in rest position the upper and lower incisors are more distantly separated from each other in rodents. Second, the wear facet on the lower incisor in rodents is much longer than that in lagomorphs, which means the mandible must protrude farther anteriorly to create such a long wear facet. Finally, the glenoid fossa is long in rodents, in contrast to a very short fossa in lagomorphs. Rhombomylus is similar to rodents in all of these features. The minimum working distance of the mandible has a certain relationship with the length of the glenoid fossa. In IVPP V5278, the minimum working distance is $9.3 \mathrm{~mm}$ and the maximum length of the glenoid fossa is $10.4 \mathrm{~mm}$. When the ventral edge of the wear facet on the lower incisor meets the tip of the upper incisor, the mandibular condyle is completely moved out of the glenoid fossa. This suggests two possibilities. First, during incisor sharpening the position of the mandible is maintained by jaw muscles and the mandibular condyle does not function as a fulcrum. Second, the glenoid fossa may have an anterior extension of fibrocartilage that supports the mandibular condyle, as in some living murids (Weijs, 1975). In this latter case the condyle functions as the fulcrum against the extended glenoid fossa while the incisors work. Although Rhombomylus is similar to rodents in several masticatory features, it differs in that the jaw muscles were not shifted anteriorly. Thus, it may be postulated that gnawing and self-sharpening of the incisors evolved earlier than anterior shift of the masticatory muscles in gliroid mammals.

The robustness of the incisors appears to have a certain correlation to that of the anterior masseter in sciuromorphous rodents at least. Squirrels with the most massive anterior deep massters generally have the most robust incisors and presumably have the strongest incisor bites (Thorington and Darrow, 1996). The incisors of Rhombomylus are relatively slender, possibly corresponding to the relatively smaller masseter and to a diet on soft food.

Cheek Teeth: In spite of their diversity, mammalian teeth basically function by trans- 
mitting forces from the jaw muscles to food items. Several forms of tooth activities can be recognized (Rensberger, 1973). Simple cutting is the process in which a relatively sharp edge is pressed against another surface. Crushing occurs when blunt surfaces are brought together. Shearing is a motion of two relatively flat surfaces across one another in a plane oblique or perpendicular to the occlusal plane. Grinding is the motion of flat or irregular surfaces across one another in a plane more or less parallel to the occlusal plane. These processes do not differ in a clear-cut way; instead, they are variations of a fundamental relationship (Rensberger, 1973). In gliroid mammals the anterior placement of masseter muscles emphasizes anteroposterior movement of the lower jaws. Therefore, it has often been thought that anteroposteriorly, or propalinally, chewing is typical of rodents (Gidley, 1912; Wood, 1973; Li et al., 1987). Propalinal chewing is not common in mammals generally (Gans et al., 1978; Hiiemae, 1978; Weijs, 1980), nor does it appear to be common among the Glires (Offermans and de Vree, 1990). Even though all gliroid mammals are able to protrude their mandibles forward for incisor biting, they do not necessarily chew propalinally. Studies of mastication demonstrate that a variety of patterns of masticatory movements are present in rodents. In addition to propalinal chewing, the movements of rodent mandibles can be transverse, asymmetrically forward, symmetrically forward, or in twophased occlusion (buccal and lingual) (Ardran et al., 1958; Ardran and Kemp, 1960; Hiiemae and Ardran, 1968; Weijs and Dantuma, 1975, 1981; Gorniak, 1977; Butler, 1980, 1985; Byrd, 1981; Bekele, 1983; Offermans and De Vree, 1990; Druzinsky, 1995; Satoh, 1997). Even for rodents that are capable of bilateral propalinal chewing, such as murines, a transverse component in grinding is also produced by mandibles pulled toward the midsagittal plane around the center of rotation at the fibrocartilage area in the mandibular symphysis while the mandible is moving forward (Hiiemae and Ardran, 1968; Weijs, 1975; Weijs and Dantuma, 1975; Satoth, 1999). Primitive rodents have twophase masticatory movements inferred from wear facets, in which the lower jaw move- ment consists of a transverse (buccal phase) and an obliquely forward (lingual phase) component (Butler, 1985). This primitive condition was thought to be retained in aplodontoids (Rensberger, 1982) and sciurids (Butler, 1980, 1985), but based on striae, a primary transverse mode of chewing is said to be present in living Aplodontia (Koenigswald et al., 1994).

The movement of the lower jaw is determined primarily by dental features, not by the muscles that provide the force for the movement or by cranial morphologies in other mammals (Kallen and Gans, 1972; Gans et al., 1978) and in rodents (Offermans and De Vree, 1990; Wilkins, 1988; Wilkins and Cunningham, 1993). Offermans and de Vree (1990, 1993) suggested that rodent masticatory patterns can be predicted with reasonable reliability from the dental structures, such as the occlusal relations between the opposing molar cusps and ridges, the inclination of the occlusal surfaces, the transverse width between upper and lower tooth rows, and the orientation of the tooth rows relative to the sagittal plane of the skull. In particular, propalinal mastication is possible only when the teeth meet the following conditions. First, the molars of both sides can occlude simultaneously, that is, they are isognathic. In most mammals, including primitive forms such as didelphid marsupials (Crompton and Hiiemae, 1970; Hiiemae and Crompton, 1971), the dentitions are anisognathic, meaning unequal width of jaws, or more specifically the upper dental arch is wider than the lower one (Moore, 1981). The anisognathic condition is probably also more common in rodents (Ryder, 1978; Becht, 1953; Offermans and de Vree, 1990; Druzinsky, 1995). Second, the teeth must stay in occlusion during forward movement, and tooth rows must be parallel to the sagittal plane. However, tooth rows that are nearly parallel are a primitive condition for gliroid mammals because it occurs in most other mammals and in early gliroids such as Heomys (Li, 1977), Cocomys (Li et al., 1989), Paramys (Wood, 1962), and Rhombomylus. In these forms, mastication is either transverse or has a considerable component of transverse movement (Butler, 1980, 1985). This implies that parallel tooth rows are necessary, but not decisive, for bi- 
lateral propalinal mastication in rodents and that those with parallel tooth rows may not possess propalinal mastication. Third, propalinal movements are possible only in those that have a flat occlusal surface of cheek teeth. High cusps and transverse, uneven ridges on the tooth crown prevent anteroposterior protrusion but guide or force a lateral movement of the mandible during the grinding phase. In gliroid mammals with these crown structures, such as Rhombomylus, chewing is necessarily unilateral and is buccolingually directed. This corresponds to the primitive condition seen in other basal mammals such as didelphid marsupials and early mammals (Hiiemae and Jenkins, 1969; Crompton and Hiiemae, 1970; Hiiemae and Crompton, 1971; Hiiemae, 1976; Crompton et al., 1977). Finally, the incisors should not restrict propalinal movement. When the mandible is in rest position, the tip of the lower incisor may be only slightly posterior to the upper incisor. Overhang of the upper incisor restricts propalinal movement of the mandible, as in Mesocricetus auratus (Gorniak, 1977; Offermans and de Vree, 1990). However, for most gliroid mammals in which there is no postglenoid process, it is unclear whether at the beginning of grinding the mandible can be retracted from the rest position to increase the phase of grinding. For Rhombomylus, in which the external auditory meatus functions as a stop for the mandibular condyle, the possible distance for anteroposterior movement of the mandible can be measured. Although a gap is present between the upper and lower incisors in their rest positions, propalinal movement is still unlikely in Rhombomylus because the high, transverse ridges on the cheek teeth prevent an anteroposterior power stroke.

Thus, it is a more parsimonious argument that ectental, or nearly transverse, jaw movements are primitive for gliroid mammals, and that propalinal chewing has evolved, probably more than once, within rodents (Offermans and de Vree, 1990; Druzinsky, 1995). This is supported by the anisognathic condition of tooth rows and by the unilateral mastication with a considerable transverse component in Rhombomylus.

Other dental features that bear significantly on the function of mastication include in- clinations of the cheek teeth and, therefore, inclination of the occlusal surfaces. There are two types of tooth inclination in rodents. The first type is mediolateral inclination in which the crown surfaces of the upper cheek teeth tilt outward from the midline and the lower ones slant inward, facing dorsomedially. In many mammals, including Rhombomylus, this condition is weakly present. A strong inclination is seen both in living species, such as Cavia (Byrd, 1981; Offermans and de Vree, 1990), and in fossils, such as Tsaganomys (Bryant and McKenna, 1995). According to Offermans and de Vree (1990), in rodents that chew unilaterally this inclination affects the degree of lateral excursion required to bring the lower cheek teeth into alignment with the upper ones in preparation for the grinding phase. This condition appears to be beneficial for mastication that has at least a lateromedial component of jaw movement.

In the other type of inclination the lower cheek teeth slant anteriorly whereas the upper ones slant posteriorly, as in Rhombomylus and lagomorphs (Weijs and Dantuma, 1975; Lopez Martinez, 1985). Some rodents, such as Tsaganomys, have the components of the two types of inclinations. The second type of inclination can also be shown by the enamel bands of teeth in a longitudinal section (Lyngstadaas et al., 1998; Koenigswald et al., 1994). Although the inclinations of the lower and upper teeth are at opposite directions, the angle of inclination is the same. The angle of inclination approaches the direction of the muscle resultant force so that the teeth, particularly the enamel ridges, will be subjected only to compressive forces (Weijs and Dantuma, 1975). Therefore, the direction of resultant force of the jaw muscles for mastication may be estimated by measuring the inclination of the cheek teeth and the enamel bands seen in longitudinal section. The inclination angle of the cheek teeth in Rhombomylus is age and size dependent. Older, and thus larger, individuals have a greater angle. In IVPP V5278, the oldest and largest individual in the collection, the angle (between the anterior surface of the upper tooth and the frontal plane of the skull) is about $20-25^{\circ}$. This angle indicates that shearing between upper and lower teeth is in 
an inclined, not a vertical, plane. The orientation of the plane is roughly parallel to the estimated working line of the jaw muscles (fig. 79B).

\section{ENAMEL STRUCTURES}

INCISOR ENAMEL: The incisor enamel in gliroids is limited primarily to the labial surface of the tooth and wraps to various degrees on the medial and lateral surfaces of the incisor, presumably functioning to brace the enamel better against the stresses of gnawing (Wood, 1965). Owing to its high mineral content ( $93 \%$ by volume), compared with that of dentine $(69 \%)$, enamel is the dental material most resistant to wear (Waters, 1980). Although enamel is more brittle than dentine, its property to resist fracture is improved by decussation of hydroxyapatite crystal prisms (Rensberger, 1995, 2000). The prism decussation is first seen in early Paleocene condylarths (Koenigswald et al., 1987) and evolved in many mammals. It was thought that in rodents the prism decussation, or Hunter-Schreger bands (HSB), strengthens the incisors and prevents crack-propagation of the enamel layer (Lehner and Plenck, 1936; Koenigswald, 1980, 1985; Pfretzschner, 1988; Koenigswald and Pfretzschner, 1991). The radial enamel of the outer layer helps to shape a perpetual sharp cutting edge because of its slightly higher wear resistance than the inner layer of the enamel and dentin (Rensberger and Koenigswald, 1980; Fortelius, 1985). However, in leporid lagomorphs, cutting edges of the incisors are formed even though they have only one inner layer (Koenigswald, 1995). Although Rhombomylus has the gross morphology of incisor typical of rodents, the external layer of the incisor enamel is poorly developed. Nonetheless, a sharp edge is formed at the tip in both upper and lower incisors. In Tribosphenomys (Meng et al., 1994; Meng and Wyss, 2001), Sinomylus and cf. Eomylus (Martin, 1999; McKenna and Meng, 2001), the incisor enamel has only one radial layer, but the tooth forms a chisel-shaped cutting edge.

The single-layered radial enamel in Tribosphenomys, Sinomylus, and cf. Eomylus was considered primitive for Glires (Martin, 1999). The transformation of the enamel types during evolution of gliroid mammals is represented primarily by formation of the inner layer consisting of HSB and by diversification of the HSB into pauciserial, multiserial, and uniserial types within rodents (Martin, 1994, 1999). Functional and phylogenetic analyses on the incisor enamel microstructures focused mainly on the number of layers, thickness, and complexity of the decussation of hydroxyapatite crystal prisms (HSB) of the enamel. Another feature that we think is important for incisor function is the density of prisms (or abundance of prisms, Clemens, 1997), that is, the number of prisms per unit enamel volume. The prisms are rodlike elements consisting of bundled hydroxyapatite crystallites with a diameter of 3-5 $\mu \mathrm{m}$ and is the most important constructional element of enamel (Koenigswald and Clemens, 1992; Martin, 1999). The density of the prisms is related to the amount of the interprismatic matrix, size of the prism sheath, and the diameter and shape of each prism. The radial enamel in Tribosphenomys (Meng et al., 1994; Meng and Wyss, 1994, 2001), Sinomylus, and cf. Eomylus (Martin, 1999) has the lowest prism density but relatively higher percentage of interprismatic matrix and larger space of prism sheath than those of other gliroid mammals. The prism sheath in life contains a higher content of unmineralized organic material (proteoglycans) (Clemens, 1997), and the interprismatic matrix consists of unbundled hydroxyapatite crystallites. The enamel with a higher percentage of the prism sheath and interprismatic matrix may have a lower degree of mechanical strength. Multiserial and uniserial enamel are considered to be derived independently from pauciserial enamel (Martin, 1994, 1997, 1999), and have the highest density of prisms. Increase in prism density likely increases the hardness as well as toughness of the enamel, as an adaptation for processing tougher food. In this regard, the pauciserial incisor enamel of Rhombomylus appears stronger than the radial enamel of Tribosphenomys and Sinomylus, but it is weaker than the multiserial and uniserial enamel in more advanced rodents (Korvenkontio, 1934). Prisms are anisotropic in their resistance to abrasion (Rensberger, 2000). Because prisms are more evenly distributed 
in the uniserial enamel, the latter probably resists wear and fracture better than does the multiserial enamel. The evolutionary transformation of the enamel types was thought to be related to environmental changes during the geological history (Mess et al., 2001), but this correlation needs to be corroborated from additional evidence.

Cheek TоOтH EnAmel: The functional significance of tooth enamel has been widely studied for mammals in general (Rensberger, 1995,2000 ) and for rodents in particular (Koenigswald et al., 1994). The organization of the enamel microstructure is known to have an adaptive relationship to the stresses generated during mastication (Rensberger, 2000). The prism decussation in enamel (or the formation of the Hunter-Schreger bands) of mammals is usually associated with large tooth size. Mammals with molars more than $6 \mathrm{~mm}$ wide usually have HSB and smaller teeth often lack this structure. The main exceptions to this association occur in rodents and whales (Koenigswald et al., 1987; Rensberger, 2000). Lagomorphs are similar to rodents in having HSB in the cheek tooth enamel. Two aspects of enamel structures of cheek teeth in gliroid mammals may be considered: the distribution of enamel on the teeth and the enamel microstructure.

Distribution of enamel on cheek teeth can be observed directly from naturally worn teeth, or by grinding the teeth (Meng and Wyss, 1994; this study). Based on available data, two kinds of enamel distributions can be recognized: one represented by Rhombomylus and the other by Tribosphenomys (Meng and Wyss, 1994). The enamel distribution on the cheek teeth of Rhombomylus is similar to that of lagomorphs (Weijs and Dantuma, 1981; Lopez Martinez, 1985), in which the thickest enamel forms the anterior wall of the upper tooth and the posterior wall of the trigonid of the lower tooth. The enamel is also reinforced at the lingual sides of the upper teeth and buccal sides of the lower teeth. The two walls from the matching upper and lower teeth constitute the main shearing surfaces (comparable to wear facet 1 in lagomorphs; Lopez Martinez, 1985). In contrast, the enamel on the occlusal surface of the tooth is very thin and disappears completely with light wear after eruption of the tooth. It is probable that mastication in Rhombomylus has two components: shearing and crushing, as in lagomorphs, with the lower jaw moving primarily transversely during mastication (Weijs and Dantuma, 1981; Lopez Martinez, 1985). This is supported by the analysis of microwear of the cheek teeth of Rhombomylus (see below). The same enamel distribution and mastication are assumed for some other basal gliroid mammals, such as Eurymylus, that have cheek teeth with relatively high, transversely widened crowns, well-developed enamel crests, and relatively high trigonids.

Although the enamel along the anterior wall and the lingual side of the cheek tooth is the thickest in Tribosphenomys, it differs from Rhombomylus in having much thicker enamel on the occlusal surface of teeth (Meng and Wyss, 1994). After considerable wear, enamel loops are formed at cusps. In addition, the posterior wall of the trigonid is so weak that it does not furnish a shearing facet for the lower cheek teeth. The condition in Tribosphenomys is similar to that of early rodents, such as Cocomys and Paramys, and extant sciurids. This type of enamel distribution suggests an emphasis on crushing rather than shearing for mastication.

Similar to the incisor, the molars of Tribosphenomys have the same radial enamel that lacks HSB (Meng and Wyss, 1994). In contrast, the cheek tooth enamel of Rhombomylus has HSB, which in some areas of the tooth resembles that of the incisor. This shows that development of HSB occurs simultaneously in incisors and cheek teeth of gliroid mammals. As in the case of the incisor, the radial enamel in the molar of Tribosphenomys is mechanically less resistant to stress than enamel with HSB, such as that of Rhombomylus. However, the enamel microstructure varies within the same tooth. The most complex structure is found in the enamel crests that function as shearing facets, whereas the simplest and weakest is in the thin enamel on the occlusal surface of the tooth.

\section{MicROWEAR}

Studies of microwear are primarily focused on inference of diet and jaw move- 
ments. The relationship between dental wear and chewing patterns provides a reliable picture of the evolution and diversity of mammalian mastication (Teaford, 1985; Maas, 1989). Differences in occlusal mechanics that affect patterns of microwear are dependent on dietary specialization, and the two aspects of teeth are related in a complicated way (Rensberger, 1995, 2000). Most studies concerning diet interpretation from dental wear patterns are based on large mammals, particularly primates and ungulates (e.g., Walker et al., 1978; Lucas, 1979; Teaford, 1985; Teaford and Walker, 1984; Kay, 1987; Solounias et al., 1988; Janis, 1990; Hunter and Fortelius, 1994). Relatively few analyses have been done for rodents. In contrast, studies of microwear in rodents are usually related to chewing mechanics (Butler, 1980, 1985; Wilkins, 1988; Wilkins and Woods, 1983; Wilkins and Cunningham, 1993; Woods and Howland, 1979; Koenigswald, 1980; Koenigswald et al., 1994). In these studies the microwear facets and striations are regarded as the determining evidence for the direction of mandible movements. Again, dental wear pattern in early Glires is generally little known.

The striations on the primary shearing facet on the cheek teeth of Rhombomylus are formed by tooth contact during power strokes of the jaw movement. The orientations of these striations suggest that lower tooth occlusion follows a path as shown by the arrows in figure $21 \mathrm{~F}$. In the early stage of occlusion when the upper and lower teeth are engaged, the vertical component is the dominant lower jaw movement. Then the lower teeth shift more horizontally during the shear contact. When the trigonid (protoconid) of the lower tooth contacts the hypocone shelf of an upper tooth, the lateral shift of the lower jaw is completed; there is only compressive effect between the trigonid and the hypocone shelf, as reflected by absence of striations on the hypocone shelf.

The chewing cycle of living rodents can be divided into as many as 16 phases (Weijs and Dantuma, 1975). The precise cycle of mastication may never be known in fossils, but, based on morphologies of the jaws and teeth and the striations on the wear facets, the following phases of jaw movements of
Rhombomylus may be deduced. (1) Because of the anisognathic dentition, upper and lower teeth on both sides cannot work simultaneously. In the rest position, the labial half of a lower tooth must contact the lingual half of an upper tooth. (2) During occlusion the lower jaws first open and move labially to the working side to prepare for occlusion. (3) The early stage of occlusion has a dominant component of vertical movement, which brings the upper and lower teeth into contact. Shearing may have taken place in this stage. (4) Immediately after the teeth contact, the working lower jaw moves dorsomedially, with a predominant transverse component of movement. At this stage, shearing between the anterior surface of the trigon and the posterior surface of the trigonid is the main activity of the teeth and the most significant means of subdividing food. Again, the shearing movement is in an inclined plane, corresponding to the inclination of the cheek teeth. (5) The enlarged hypocone shelf receives the high trigonid, and the talonid basin receives the protocone. This stage is primarily compression in which food is crushed. (6) The lower jaws return to rest position.

The chewing style of Rhombomylus is more similar to that of lagomorphs than rodents. In lagomorphs, chewing is fully transverse, and an important element is the transverse shear between the posterior wall of the trigonid and a facet along the anterior margin of the upper molar (Weijs and Dantuma, 1981; Butler, 1985; Lopez Martinez, 1985). Moreover, the trigonid is worn against an expanded posterior cingulum on the upper molar. Similar characters are found in both mimotonids and eurymylids (Butler, 1985). However, as we show above, the jaw and skull morphologies, and therefore the jaw musculature, of Rhombomylus are different from those of lagomorphs. It implies that if the chewing patterns in Rhombomylus, other eurymylids, mimotonids, and lagomorphs, are similar, as suggested by tooth morphology and wear patterns, they may have evolved independently. Mastication in the simplicidentate Rhombomylus has evolved in a direction different from that of rodents.

Cheek teeth of primitive rodents, such as paramyids, are usually cuspidate (Wood, 1962). Chewing in paramyids involved a 
two-phase movement, resembling that of primates (Butler, 1980, 1985). The lower molar passes lingually across the upper in a relatively transverse direction during the buccal phase, and then obliquely forward during the lingual phase. In sciurids, there is a phase of anterior movement in addition to a lateral one (Butler, 1980, 1985). In Aplodontia, transverse jaw movement is dominant (Koenigswald et al., 1994), but there is no vertical shearing because of the flat occlusal surface of its dentition. Wear patterns in other early gliroid mammals, such as Eurymylus and Mimotona (Butler, 1985), are similar to those of Rhombomylus. Within the phylogenetic constraint, the evolutionary tendency of mastication in gliroid mammals appears to be reduction of vertical and transverse components of mandibular movement but increase of horizontal and propalinal components.

In the leporids studied by Weijs and Dantuma (1981), shearing is used primarily in hay mastication, whereas crushing is used in feeding on carrots. Although microwear rate is rapid and may reflect only short-term dietary variation (Walker et al., 1978; Skogland, 1988; Teaford and Oyen, 1989), dietary preference of soft vegetation for Rhombomylus is supported by the following factors: (1) lack of enamel ridges on the occlusal surface except the shearing edges on upper teeth; (2) predominance of fine striations and pits on wear facets of tooth enamel ridges; (3) a combination of fine striations and polished areas on dentinal areas, suggesting a significant component of compression during the occlusion; and (4) lack of distinct pits and striations in the hypocone shelf.

\section{Conclusions}

The masticatory apparatus and mastication of Rhombomylus are similar to those of lagomorphs in the following aspects: (1) The upper cheek teeth are considerably wider than the lower ones. (2) The cheek teeth have transverse shearing ridges. The main shearing facets are on the anterior surface of the upper cheek teeth and the posterior wall of the trigonid of the lower one. (3) The thickest enamel forms the anterior edge of the upper tooth and the posterior wall of the trigonid of the lower. (4) The enamel microstructure is more like that of lagomorphs than that of rodents. (5) Power strokes of chewing are unilaterally and buccolingually directed, starting with the buccal edges of the upper and lower molars aligned. Mastication involves both shearing and crushing power strokes. The mandible movement is primarily transverse during mastication.

The masticatory apparatus and mastication of Rhombomylus are similar to those of rodents in the following aspects: (1) I3 is absent. (2) P2 is absent. (3) The glenoid fossa is anteroposteriorly elongated. (4) The minimum working distance of the mandible is relatively long, compared to that of lagomorphs. (5) The upper diastema is longer than the lower one. (6) The wear facets on the incisors are long. (7) The general morphology of the mandible is rodentlike.

The masticatory apparatus of Rhombomylus shows several primitive conditions in comparison to more advanced gliroid mammals. (1) The origins of the masseter muscles are relatively posteriorly located; they do not pass the anterior edge of the orbit. (2) The insertions of the masseter muscles are also posteriorly located. The anterior extremity of any muscle insertion is behind the posterior edge of the orbit. The posterior extremity, represented by the posterior end of the angular process, is behind the level of the external auditory meatus. These sharply contrast with the conditions in rodents and lagomorphs. In the latter, the origin of some muscles is anterior to the orbit and the anterior limit of the muscle can reach to, or even pass, the anterior edge of the orbit. The insertions of the muscles in rodents and lagomorphs usually do not pass posterior to the ear region, or even the glenoid fossa. (3) The coronoid process of the mandible and the temporal region of the skull are relatively large. (4) The incisors are slender and the tooth crown is relatively short. (5) The enamel is pauciserial and has lower prism density. It is, however, more advanced than the radial enamel of Tribosphenomys and Sinomylus. (6) Although the mandible of Rhombomylus can be protruded anteriorly and the elongate glenoid fossa allows such a movement, the mastication is primarily transverse.

Several general conclusions can be made for evolution of the masticatory apparatus of 
gliroid mammals. First, the propalinal movement of the mandible that permits gnawing and incisor sharpening is a gliroid synapomorphy that is found in all Glires in which the lower incisors are known. In other mammals the lower incisors may be enlarged, but they are unable to protrude anteriorly to perform self-sharpening. Corresponding to this inability, their glenoid fossa is not anteroposteriorly elongate, contrasting with the condition in rodents.

Second, transverse and oblique jaw movements are probably primitive in gliroid mammals. Although all gliroid mammals are capable of anteroposterior movements of the mandible for incisal biting and sharpening, only some advanced forms are capable of propalinal mastication. The propalinal mastication is a derived condition and has been independently evolved several times in rodents.

Third, evolution of the gnawing function is prior to and independent of that of propalinal chewing. It is also prior to the anterior shift of the jaw muscles.

Fourth, anterior shift of the jaw muscles is a general trend for gliroid mammals, particularly rodents. It distinguishes gliroid mammals from other placentals. The modification of jaw musculature was originally and primarily to emphasize the strength of incisor biting by increasing the moment arms of the muscles.

Fifth, modifications of the jaw musculature already occurred in early gliroids, such as Rhombomylus, and diverged in several directions independently among various gliroid groups, particularly rodents.

Sixth, acquisition of some cranial features such as the anteroposterior elongate glenoid fossa is originally related to gnawing and incisor sharpening, not to mastication. For early gliroid mammals, such as Rhombomylus, an elongate glenoid fossa is only beneficial for gnawing; it does not seem to be critical for transverse chewing.

Seventh, reduction of teeth is a general evolutionary trend in rodents; however, enamel ridges and bands functioning as grinding edges are generally increased. Mastication evolved from primarily shearing to grinding, which is more efficient for processing vegetable food with tough fabrics.
Finally, and in general, the evolution of rodent mastication is a refining process that involves many aspects of the masticatory apparatus including the skull, mandible, jaw muscles, tooth morphologies, tooth size, enamel structures, and chewing rates. Refinements of these masticatory features often evolved independently in different directions and in mosaic combinations. Thus, the heterochronical acquisition of masticatory features and the mosaic pattern of these features convey phylogenetic information at various levels of Glires.

\section{FUNCTIONAL ANALYSIS OF POSTCRANIAL SKELETON}

Postcranial specimens are poorly known for some early gliroid mammals, including Mimolagus (Bohlin, 1951; Bleefeld and McKenna, 1985), Heomys (Li and Ting, 1985, 1993), Tribosphenomys (Meng and Wyss, 2001), and Matutinia (Ting, et al. 2002). Locomotion of Rhombomylus can only be inferred by comparison with extant mammals in which locomotion is adequately known (Szalay and Sargis, 2001). The model mammals used for comparison include Sciurus (habitual climbers, but also active on the ground), Marmota (mainly terrestrial walkers, good swimmers, and occasional climbers), Erethizon (terrestrial walkers, slow ascending climbers), Dasyprocta (terrestrial walkers, runners and jumpers), Rattus (good runners, climbers, and diggers), Cavia (terrestrial walkers and diggers), Lepus (terrestrial walkers, runners, and jumpers), Ochotona (terrestrial walkers, runners, and diggers), Tupaia (agile climbers, although some species are not habitual [Sargis, 2001] and active terrestrial walkers), and Rhynchocyon (terrestrial walkers and jumpers). It is unclear which locomotion is primitive for gliroid mammals. Although highly conjectural, it seems that walking on the ground is the easiest method of locomotion for early mammals, because it requires less morphological modification than climbing, fast running, jumping, and digging. Those behaviors are usually associated with distinctive morphological specialization. In the following comparison, we identify morphological modifications for locomotion of Rhombomylus. 
Muscle attachments in fossil taxa are based on their analogs in extant mammals (KielanJaworowska and Gambaryan, 1994; Rinker, 1954; Evans and Christensen, 1979).

\section{FORELIMB}

SCAPULA: The angular appearance of the scapula in Rhombomylus is similar to those of terrestrial mammals such as Lepus, Ochotona, Cavia, Dasyprocta, and Rhynchocyon, in which both anterior and posterior angles of the scapular blade are relatively distinct. In Sciurus and Tupaia, only the posterior angle is distinct. In contrast, the dorsal and anterior borders of the scapula are confluent to form a long convex border. The slow climber Erethizon and occasional climber Marmota also lack a distinct anterior scapular angle. The difference between the above strict terrestrial mammals and those capable of climbing is probably related to the different rotational modes of the scapula in the two forms (Argot, 2001). The dorsal border of the scapula is the place the $\mathrm{m}$. serratus inserts anteriorly. The angular scapula with somewhat straight dorsal border helps the posterior portion of $\mathrm{m}$. serratus anterior to resist the dorsal gliding of the scapula in movement on horizontal substrates (Jolly, 1967 in Argot, 2001), while the confluent and convex anterodorsal border provides mechanical advantage for this muscle to rotate the scapula extensively, which is crucial in climbing. In terms of the angulation of the scapular blade, Rhombomylus is more similar to terrestrial mammals than to climbing forms.

Argot (2001) found that in didelphimorphs, the posterodorsal angle (caudal angle in Argot's paper) of the scapula is much more posteriorly extended in arboreal forms than in terrestrial forms. A similar condition is present in fossorial eutherians (Smith and Savage, 1956). The extended posterodorsal angle presumably increases the lever arm of the $\mathrm{m}$. teres major (Smith and Savage, 1956). The posterodorsal angle of the scapula in Rattus, Rhynchocyon, Cavia, Lepus, and Ochotona is more extended than those in $T u$ paia, Sciurus, Marmota, and Erethizon. In Tupaia, and more distinctly in Sciurus, a small fossa is developed on the posterodorsal angle to accommodate the strong $\mathrm{m}$. teres major. This structure seems to correspond to ascending climbing in these agile animals (Thorington et al., 1997). With regard to the shape of the posterodorsal angle of the scapula, Rhombomylus is similar to Marmota and Erethizon.

The scapular spine provides the insertion for the $\mathrm{m}$. trapezius that is active in abduction, protraction, and retraction of the scapula. The spine and the acromion are also the places where deltoid muscles originate; the latter are the retractors and abductors of the humerus. In Tupaia, Sciurus, Marmota, and Erethizon the scapular spine and the acromion are strong, which is in accord with rotation and abduction of the scapula and humerus in climbing. The acromion plate usually extends ventrally and anteriorly beyond the glenoid fossa. The clavicle is thick and has a strong articulation with the acromion. It appears that the clavicle can act as a bracing structure in the rotation of scapula and abduction/adduction of the forelimb. These structures are relatively reduced or even lost in Lepus, Ochotona, Cavia, Dasyprocta, and Rhynchocyon, probably as an adaptation for terrestrial life, because walking and running do not require extensive rotation of the scapula or abduction/adduction of the limbs. The scapular spine and acromion of Rhombomylus are intermediate in size between those of climbers and terrestrial runners, suggesting a certain degree of rotation of the scapula and abduction of the arms. The acromion process of Rhombomylus is slender, and the clavicle is slim. The weak articulation between the clavicle and shoulder girdle does not support frequent abduction of the forelimb with a heavy load in climbing or digging.

Argot (2001) found that the m. omotransversarius is more developed in terrestrial didelphids than in more scansorial or arboreal forms, which appears to be also true for eutherians. The m. omotransversarius originates on the metacromion process of the scapula and helps to pull the glenoid cavity forward during locomotion. In Lepus, Ochotona, Cavia, and Dasyprocta the metacromion process is large and extends posteriorly to form the ventral extremity of the spine, in contrast to the reduced acromion process in these mammals. The metacromion process is usually small in climbers. The relative size 
of the metacromion process in Rhombomylus is smaller than that of terrestrial runners, but larger than that of climbers.

The coracoid process in Tupaia, Sciurus, Rattus, Marmota, and Erethizon is large, robust, and projects anteroventrally. It is reduced in Cavia, Dysaprocta, and Rhynchocyon. In Lepus and Ochotona, the process is large but projects medially and ends with a thin tip. The tip of the coracoid process is the origin of $\mathrm{m}$. coracobrachialis, which adducts and rotates the forelimb. The basal part of the coracoid process is for the origin of one head of the $\mathrm{m}$. biceps, the major flexor of the forearm. The robust and anteroventrally projected coracoid process in Tupaia, Sciurus, Rattus, and Marmota indicates that both muscles are well developed in these mammals and they play an important role in climbing. The $\mathrm{m}$. coracobrachialis is also developed in Lepus and probably in Ochotona; it helps to hold the shoulder joint while the animal is running. The muscle seems not well developed in Cavia, Rhynchocyon, and Dasyprocta because of a reduced coracoid process. The $\mathrm{m}$. biceps probably is more developed in terrestrial runners than in climbers. In this regard, the supraglenoid tuberosity from which another head of the biceps originates is usually more robust in terrestrial runners than in climbers. The tuberosity is smaller in Rattus and Marmota than in Sciurus and Tupaia. In the orientation of the coracoid process, Rhombomylus is similar to climbers, but the small size of the process may suggest limited capability of adducting the humerus. The supraglenoid tuberosity of Rhombomylus, on the other hand, is quite robust and similar to those of Lepus and Ochotona.

The pear-shaped articular facet of the glenoid fossa is common in many terrestrial and arboreal mammals (Roberts, 1974). The fossa is narrower than the surface of the humeral head so that abduction and adduction of the humerus at the joint are possible. The differences between the widths of the glenoid fossa and humeral head are greater in arboreal climbers than in terrestrial runners. $\mathrm{Le}$ pus may represent an extreme example in which the glenoid fossa expands posteriorly and the posterior part of the glenoid fossa is as broad as the humerus head. Thus, abduc- tion and adduction of the humerus is limited, if possible at all, in Lepus. In Rhombomylus, the glenoid fossa is pear-shaped and narrower than the humeral head, allowing abduction and adduction of the forearm. As in Tupaia, Sciurus, Rattus, Marmota, Rhynchocyon, Lepus, and Ochotona, the glenoid fossa of Rhombomylus has an anterior lip. It helps to prevent dislocation of the shoulder joint when the humerus is highly flexed.

Humerus: Szalay and Sargis (2001) found that arboreal marsupials differ from terrestrial ones in that their humeral heads are less beaked posteriorly. Similar differentiation is found in eutherians compared in this study. Owing to enlargement of the greater tubercle, the humeral head in Cavia, Dasyprocta, Lepus, Ochotona, and Rhynchocyon is posteriorly shifted relative to the humeral shaft. Such a shifting increases the lever arms of the muscles (see below). The structure in Rhombomylus is similar to that of climbing forms, which show little shifting in the humeral head. On the other hand, in all eutherians compared here, except Erethizon, the humeral head overhangs the shaft posteriorly to some degree and the articular surface of the head faces posteroproximally. Such an orientation allows the humerus to flex against the scapula sufficiently, although tensile and stressing forces loaded on the shoulder joint may be different in various locomotor patterns.

The greater tubercle is the insertion of the m. supraspinatus and infraspinatus. Both muscles play important roles in stabilizing the shoulder joint. The supraspinatus is also involved in protraction of the humerus and the infraspinatus is mainly an abductor and external rotator of the humerus (Jenkins and Weijs, 1979; Argot, 2001). The enlargement of the greater tubercle in Cavia, Dasyprocta, Lepus, Ochotona, and Rhynchocyon emphasizes the role of the supraspinatus in protracting the humerus during running, jumping, or digging. The infraspinatus in these mammals mainly acts as a joint stabilizer because abduction of the humerus at the joint is limited by the enlarged greater tubercle. The lesser tubercle is mainly for the insertion of the $\mathrm{m}$. subscapularis, which is an adductor of the humerus. In Sciurus and Tupaia, the lesser tubercle is even more conspicuous than the 
greater one. A large lesser tubercle increases the lever arm of the m. subscapularis (Argot, 2001). The enlargement of the greater tubercle in Rhombomylus indicates terrestrial locomotion. The relatively small lesser tubercle suggests that power adduction exerted by the subscapularis is limited. The greater and lesser tubercles of Rhombomylus are similar to those of Marmota and Erethizon in shape and size.

The lateral epicondylar crest is the place for origin of extensor muscles of the forearm and manus. This crest is well developed in Sciurus and Marmota, but reduced in Lepus, Ochotona, Dasyprocta, and Rhynchocyon. A large lateral epicondylar crest is usually associated with climbing and manipulative ability of the manus (Szalay and Sargis, 2001). A reduced epicondylar crest is usually associated with forms that are not good at climbing or manipulating objects with hands. Reduction of the lateral epicondylar crest may simplify the distal limb musculature, an adaptation for high-speed locomotion. The crest is moderately developed in Rhombomylus, Rattus, Tupaia, and Erethizon, which probably indicates some degree of manipulative ability of the manus.

The entepicondyle provides the site for attachment of major flexor muscles of the hand. Its size is related to rotation and flexion of the forelimb as well as pronation of the manus. The entepicondyle is reduced in $\mathrm{Le}$ pus, Ochotona, Cavia, and Dasyprocta, corresponding to simple movements of the manus in these terrestrial mammals. Although a well-developed entepicondyle is not always associated with arboreal lifestyle, such as in Rhynchocyon, climbing forms typically have a large entepicondyle. Rhombomylus has a moderate entepicondyle, comparable to those of Erethizon and Tupaia, but smaller than those of Sciurus, Rattus, and Marmota.

The development of the olecranon and radial fossae on the humerus indicates the range and stability of the forearm in extension and flexion. The large, deep fossae in terrestrial forms, such as Cavia, Dasyprocta, Lepus, Ochotona, and Rhynchocyon, indicate capacity of full extension and flexion of the forearm. In these mammals, the bony plate between the two fossae is perforated and a large supratrochlear foramen is formed.
These structures, as well as the anconeal process of the ulna and radial head, form a locking mechanism that helps stabilizing the elbow joint. Such a mechanism is probably an adaptation to high loading of the joint in terrestrial locomotion. The locking mechanism of the elbow joint is further strengthened by the lateral lip and ridges of the capitulum in Lepus. In Sciurus, Tupaia, Rattus, Marmota, and Erethizon, however, the olecranon and radial fossae are shallow, the supratrochlear foramen is absent, and the capitulum is spindlelike, lacking the lateral lip. The morphology of the distal humerus in these mammals indicates incomplete extension and flexion and high mobility of the elbow joint. The olecranon fossa in Rhombomylus is similar to that of typical terrestrial forms, although its humeral capitulum is somewhat spindlelike and lacks the lateral lip. Therefore, the forearm of Rhombomylus is probably capable of extending to a greater degree than those of climbing mammals, and at the same time it can reach a wider transverse range than those of typical terrestrial forms. Lack of the supratrochlear foramen in Rhombomylus suggests that extension and flexion of the forearm are less extensive than in Lepus and $\mathrm{Ca}$ via.

ULNA AND RADIUS: The olecranon process of the ulna is the lever arm of the triceps muscle, which is the major extensor of the forearm. In Sciurus, Rattus, and Tupaia, the posterior surface of the olecranon process is convex and oblique; it suggests habitual flexion of the forearm in these taxa (Szalay and Sargis, 2001). In Dasyprocta, Rhynchocyon, Lepus, Ochotona, Erethizon, and Marmota, the olecranon process is straight and the lever arm of the triceps is elongated as the result of elongation of the forearm. This gives the muscle advantage to work more efficiently in terrestrial locomotion. Rhombomylus resembles Sciurus, Rattus, and Tupaia in having an olecranon process with a convex posterior border.

The shape of the radial notch on the ulna and its counterpart on the radius are good indicators of the forearm rotation (Taylor, 1974; MacLeod and Rose, 1993; Argot, 2001). In Dasyprocta, Cavia, Rhynchocyon, Lepus, and Ochotona, the articular facets are relatively flat and the bones tightly contact 
each other to limit flexibility at the joint. This is commonly seen in terrestrial locomotors. In climbers, such as Sciurus and Tupaia, the ulna-radial joint is more flexible. The ulnaradial joint of Rhombomylus is similar to those of the climbing mammals.

In Dasyprocta, Rhynchocyon, Lepus, Ochotona, and Cavia, the long axis of the trochlear notch is nearly parallel to the direction of the ulnar shaft. In Sciurus, Rattus, and Tupaia, the axis is oblique to the ulnar shaft. The radial head is anterior to the ulna in those with the notch parallel to the shaft, but is anterolateral to the ulna in those with an oblique notch. The anteriorly located radius is probably suitable to weight loading in anteroposterior movements of the forelimb, which is inducted by the parallel trochlear notch. The orientation of the trochlear notch and the location of the radial notch of Rhombomylus are similar to those of Sciurus, Rattus, and Tupaia.

The radial articular surface for the capitulum is another elbow feature indicative of locomotion. In Sciurus, Rattus, Tupaia, Marmota, and Erethizon, the surface is gently concave and slightly convex medially, allowing rotation of the radius around the ulna as well as maintaining the articulation of the radius and capitulum of the humerus. In Dasyprocta, Rhynchocyon, Lepus, and Ochotona, the surface is concave in the central portion but convex medially and laterally. This configuration reinforces the humeroradial articulation in movement but limits the rotation of the radius. These structures in Rhombomylus are more similar to those of Sciurus than to those of Lepus.

\section{HINDLIMB}

PELVIC GiRdLE: The pelvic girdle transmits the body weight to the femur and provides places for attachment of hindlimb muscles. The femoral process is for the origin of the m. rectus femoris, a powerful extensors of the shank (Taylor, 1976). A distinct femoral process is present in all extant mammals compared here, except Erethizon, which has a rough pit for the origin of the rectus femoris. Rhombomylus, like most mammals studied here, has a distinct femoral process, which means that its rectus femoris is well developed.

The morphology of the acetabulum is related to the range of the femoral rotation or excursion (Elftman, 1929; Jenkins and Camazine, 1977). In Lepus, Ochotona, and Cavia the acetabulum is deep and has a narrow acetabular notch that restricts the range of movement of the femur. The acetabulum of Rhombomylus is similar to that of other extant mammals studied here in being shallow and open, with the acetabular notch relatively broad. This configuration probably suggests a wide range of femoral rotation.

Jenkins and Camazine (1977) found that in carnivores the orientation of the ischium is related to the posture of the hindlimb. A more vertical ischium indicates a more abducted femur. This holds true for other mammals. For instance, Sciurus, Rattus, and Tupaia have a more vertical ischium than do Lepus, Ochotona, Rhynchocyon, and Cavia, and the femur in the former group is more abducted than in the latter. The orientation of the ischium in Rhombomylus is more similar to that of Sciurus.

The configuration of the margins of the articular surface on the femoral head is related to the limb posture and excursion (Jenkins and Camazine, 1977). A larger angle between the anterior margin of the articular surface and the femoral shaft indicates more abducted posture of the femur, given that the margins of the head and the acetabulum are more or less aligned (Jenkins and Camazine, 1977). However, other factors also contribute to limb posture and excursion, such as the orientation of the acetabulum. For instance, the acetabulum faces lateroventrally in Lepus but laterally in Sciurus, the femur is less abducted in Lepus than in Sciurus, even though the angle between the anterior margin of the articular surface and the femoral shaft is larger in Lepus than in Sciurus. Ochotona and Rhynchocyon have the same condition as Lepus. In Rhombomylus, the angle between the anterior margin of the articular surface and the femoral shaft is similar to that of Lepus and is larger than those of Sciurus, Rattus, and Tupaia; however, the orientation of its acetabulum is similar to those of the latter. It is probable that the femur of Rhombomylus 
is more abducted than those of Sciurus, Tupaia, and Lepus.

The greater trochanter of the femur is for the insertion of the gluteals and the $\mathrm{m}$. gemelli, which extend or abduct the femur. Szalay and Sargis (2001) found that in marsupials the trochanter is somewhat larger in terrestrial forms than in arboreal forms. The relationship does not universally apply to eutherians. For instance, Dasyprocta has the highest trochanter, indicating the importance of the gluteals as the main extensor in cursorial locomotion. However, the trochanter of Sciurus, which needs both extension and abduction of the femur in locomotion, is prominent; it is higher than those of Lepus and Ochotona. Rhombomylus has a moderate greater trochanter with the size similar to that of Sciurus, but it is difficult to evaluate whether it is particularly related to extension or abduction.

The lesser trochanter of the femur is probably more indicative of the locomotion pattern than the greater one. Two muscles insert on the lesser trochanter, the quadratus femoris (originating from the ischium near the ischiac tuberosity) that extends and rotates the femur outward and the iliopsoas that flexes the femur and rotates it inward (Taylor, 1976). A large and medially directed lesser trochanter emphasizes the ability of these muscles in rotating, extending, and flexing the femur in arboreal forms, such as Sciurus (Taylor, 1976). In terrestrial forms, such as Lepus and Ochotona, the lesser trochanter is small and posteromedially directed. The extension and flexion of the femur and the rotational ability of the limb are limited. The lesser trochanter is large and posteromedially directed in Rhombomylus, which is similar to that of Rhynchocyon. As in Rhynchocyon and Lepus, the limb of Rhombomylus can extend and flex considerably, but its rotation is limited.

The third trochanter of the femur is for the insertion of the m. gluteus superficialis, an abductor of the femur. The third trochanter in Sciurus, Rattus, and Tupaia is large, but it is usually small in terrestrial mammals. This trochanter in Rhombomylus is similar to that of Tupaia in location and shape, but is much smaller.

The femoral shaft of Rhombomylus is most similar to that of Lepus. The elongated and relatively slim femoral shaft of Lepus and Rhombomylus differs from a relatively short, sturdy femoral shaft in other mammals. Elongation of the femoral shaft, relative to the humerus, increases the length of the stride and provides advantage for cursorial locomotion. Other modifications for cursorial locomotion are seen on the distal femoral epiphysis, such as a relatively deep patellar trochlea and the anteroposteriorly deep epiphysis. These conditions are collectively found in cursorial terrestrial mammals such as Lepus, Rhynchocyon, and Dasyprocta. Noncursorial mammals have a relatively wider distal epiphysis as well as shallow, wider patellar grooves. The distal femur of Rhombomylus is similar to that of Lepus.

TIBIA AND FiBULA: Elongation of the shank is present in Lepus, Ochotona, and Rhynchocyon, and to a lesser degree in Dasyprocta, Tupaia, Sciurus, and Cavia. The long shank also increases the length of the stride and is particularly advantageous for cursorial locomotion. Although the exact length of the tibia and fibula of Rhombomylus is unknown, it seems long based on preserved parts. Unlike in Lepus, Ochotona, and Rhynchocyon, the tibia and fibula are not fused distally in Rhombomylus. However, in Dasyprocta, a cursor and jumper, the tibia and fibula are separate as well.

ANKLE AND FoOT: The pes of Rhombomylus is more similar to that of rodents, such as Paramys (Wood, 1962; Szalay, 1985), than to those of lagomorphs. The upper ankle joint in all these groups is characterized by the deep trochlea of the astragalus and malleoli of the tibia and fibula, which limit the movement at the joint to a fore-and-aft rotation. However, the movements at lower and transverse ankle joints are quite different between Rhombomylus and Paramys on the one hand and lagomorphs on the other. Lagomorphs have flatter articular facets between the astragalus and calcaneus, which restricts gliding between the two bones. The curved and large articular facets between the astragalus and calcaneus in Rhombomylus and Paramys allow considerable inversion and eversion at this joint. In advanced lagomorphs, the astragalar head is laterally compressed and anterior to the astragalar body, and the 
calcaneocuboid articulation is distal to the astragalonavicular articulation, forming a locking mechanism that prevents the ankle joint from transverse moving. In Rhombomylus and Paramys, the astragalar head is offset medially and is compressed slightly obliquely relative to the horizontal plane. The calcaneocuboid and astragalonavicular articulations are aligned at the same level and allow transverse movement of the ankle. The ankle of Rhombomylus and Paramys appears less specialized and are more movable in multiple directions than those of lagomorphs.

SUMMARY: For many mammals, the forelimbs are not only involved in locomotion, but also function as the tool to explore and manipulate food items. The adaptation for these activities is often indicated by the morphology of the manus. The forelimb of Rhombomylus does not show much specialization toward arboreal, terrestrial cursorial, or fossorial behavior. It is probably capable of extensive movement in anteroposterior directions, but with limited abduction and adduction. It may also have the capacity of supination and pronation to some extent. This capability is, as seen in many extant rodents, most likely related to food manipulation, rather than to climbing or digging. The hindlimb of Rhombomylus shows many characteristics of terrestrial mammals; some features even suggest capability of running or jumping. The hindlimb is much longer than the forelimb, indicating asymmetrical gait in Rhombomylus. It seems that Rhombomylus was basically a terrestrial mammal that could walk or jump on the ground with an asymmetrical gait. It probably also used the manus for food manipulation, as in many rodents.

\section{ACKNOWLEDGMENTS}

Field collecting of Rhombomylus specimens from the early Eocene Yuhuangding Formation, Hubei Province, China, has lasted for more than two decades from 1975 to 1998, to which numerous colleagues have contributed their time. These include $\mathrm{Xu} \mathrm{Yu}$ Xuan, Qiu Zhang-Xiang, Yan De-Fa, and Chen Guan-Fang of the Institute of Vertebrate Paleontology and Paleoanthropology (IVPP), Beijing, and workers from the 12th provincial geological team (1975); Xu Yu-
Xuan, Qiu Zhan-Xiang, Yan De-Fa (IVPP) and the 12th provincial geological team (1976); Yan De-Fa and Han Shi-Jing (IVPP, 1977); Li Chuan-Kui and Qiu Zhu-Ding (IVPP, 1980); Li Chuan-Kui, Ting Su-Yin and Xie Shu-Hua (IVPP, 1984); Wang YuanQing and Zhang Li-Bo (IVPP, 1991); Wang Yuan-Qing, Guo Jian-Wei and Wang Yuan (IVPP, 1992); Wang Yuan and Zhou Wei (IVPP, 1993); and Wang Yuan and Meng Jin (1998). We are so grateful to the effort of our colleagues in accumulating the Rhombomylus specimens that make this research possible. We especially wish to thank $\mathrm{Xu} \mathrm{Yu}$ Xuan, Yan De-Fa, Qiu Zhan-Xiang, Qiu Zhu-Ding, Ting Su-Yin, Chen Guan-Fang, Wang Yuan-Qing, Guo Jian-Wei, Wang Yuan, and Zhang Li-Bo (IVPP) for allowing us to work on the material they collected during many years.

Most of the specimens were skillfully prepared by Xie Shu-Hua and Chang Shao-Wu of IVPP, and some by Edward Pedersen of the American Museum of Natural History (AMNH). We thank Katherine Doyle for allowing the use of the mammal collection at the Department of Biology, University of Massachusetts, Amherst. For scanning electronic microscopic images of enamel microstructures and microwear we thank Dale Callaham of the University of Massachusetts, Amherst, and Angela Klaus of the AMNH. For using their facility for thin sectioning of specimens we thank the Radiological Department of People's Hospital, Beijing Medical University.

We have benefited from collaborations on related projects and by discussions on various issues related to this project with numerous colleagues during the course of the study. We are grateful to Robert Asher (AMNH), Ann R. Bleefeld (AMNH), Percy M. Butler (Royal Holloway College, Surrey), Margery C. Coombs (University of Massachusetts, Amherst), Mary R. Dawson (Carnegie Museum, Pittsburgh), Robert J. Emry (National Museum of Natural History, Washington, D.C.), Lawrence J. Flynn (Harvard University, Cambridge), Jean-Louis Hartenberger (Université de Montpellier II, Montpellier, France), William W. Korth (Rochester Institute of Vertebrate Paleontology, Rochester), W. Patrick Luckett (University of 
Puerto Rico, San Juan), Malcolm C. McKenna (AMNH), Michael J. Novacek (AMNH), Frederick S. Szalay (Hunter College, CUNY, New York), Richard H. Tedford (AMNH), Su-Yin Ting (Louisiana State University, Baton Rouge), Robert S. Voss (AMNH), John H. Wahlert (AMNH), Robert W. Wilson (University of Kansas, Lawrence), Albert E. Wood (New Jersey), and André R. Wyss (University of California, Santa Barbara).

We are indebted to Robert J. Emry and Lawrence J. Flynn for their kindness and time to painstakingly review the manuscript. Their instructive comments on both scientific contents and writing have significantly improved the manuscript.

This work would have been impossible without generous financial support from many institutions and organizations. Most of the fieldwork was supported by the Institute of Vertebrate Paleontology and Paleoanthropology, Beijing, the Chinese Academy of Sciences, and the National Natural Science Foundation of China (special funds for major state basic research projects of China [G200007707] and an NNSFC grant [49928202] to Meng). Jin Meng has been supported by the University of Massachusetts, Amherst, the American Museum of Natural History, New York, and the National Science Foundation (DEB-9796038, EAR0120727 [SC\#S0123714]). Yao-Ming Hu has been supported by a fellowship from the American Museum of Natural History.

Lastly, J. Meng extends his very special thanks to Yu Liu for her support, both personally and professionally, during the course of the project over the past years.

\section{REFERENCES}

Adkins, R.M. and R.L. Honeycutt. 1993. A molecular examination of archontan and chiropteran monophyly. In Primates and their relatives in phylogenetic perspective, R.D.E. MacPhee (editor): 227-249. New York: Plenum Press.

Allard, M.W., M.M. Miyamoto, and R.L. Honeycutt. 1991. Tests for rodent polyphyly. Nature 353: 610-611.

Allen, H. 1882. On a revision of the ethmoid bone in the Mammalia, with special reference to the description of this bone and of the sense of smell in the Chiroptera. Bulletin of the Museum of Comparative Zoology 10: 135-64.
Allin, E.F. 1986. Auditory apparatus of advanced mammal-like reptiles and early mammals. In $\mathrm{N}$. Hotton, P.D. MacLean, J.J. Roth, and E.C. Roth (editors), The ecology and biology of mammallike reptiles: 283-294. Washington DC: Smithsonian Institution Press.

Archer, M., R. Arena, M. Bassarova, K. Black, J. Brammall, B. Cooke, P. Creaser, K. Crosby, A. Gillespie, H. Godthelp, M. Gott, S.J. Hand, B. Kear, A. Krikmann, B. Mackness, J. Muirhear, A. Musser, T., Myers, N. Pledge, Y. Wang, and S. Wroe. 1999. The evolutionary history and diversity of Australian mammals. Australian Mammalogy 21: 1-45.

Archibald, J.D. 1996. Fossil evidence for a Late Cretaceous origin of "hoofed" mammals. Science 272: 1150-1153.

Archibald, J.D, A.O. Averianov, and E.G. Ekdale. 2001. Late Cretaceous relatives of rabbits, rodents, and other extant eutherian mammals. Nature 414: 62-65.

Archibald, J.D., and D.H. Deutschman. 2001. Quantitative analysis of the timing of the origin and diversification of extant placental orders. Journal of Mammalian Evolution 8: 107-124.

Ardran, G.M., F.H. Kemp, and W.D.L. Ride. 1958. A radiographic analysis of mastication and swallowing in the domestic rabbit: Oryctolagus cuniculus (L.). Proceedings of the Zoological Society of London 130: 257-274.

Ardran, G.M., and F.H. Kemp. 1960. Biting and mastication. A cineradiographic study. Dental Practitioner (Bristol) 11: 23-34.

Argot, C. 2001. Functional-adaptive anatomy of the forelimb in the Didelphidae, and the paleobiology of the Paleocene marsupials Mayulestes ferox and Pucadelphys andinus. Journal of Morphology 247: 51-79.

Averianov, A.O. 1991. Tarsals of Glires (Mammalia) from the early Eocene of Kirgizia. Geobios 24: 215-220.

Averianov, A.O. 1994. Early Eocene mimotonids of Kyrgyzstan and the problem of Mixodontia. Acta Palaeontologica Polonica 39: 393-411.

Averianov, A.O. 1998. Homology of the cusps in the molars of the Lagomorpha (Mammalia) and certain general problems of homology in the morphological structures. Paleontological Journal 32: 76-81.

Avis, V. 1959. The relation of the temporal muscle to the form of the coronoid process. American Journal of Physical Anthropology 17: 99104.

Avis, V. 1961. The significance of the angle of the mandible: an experimental and comparative study. American Journal of Physical Anthropology 19: 55-61.

Ayala, F.J. 1997. Vagaries of the molecular clock. 
Proceedings of the National Academy of Science of the United States of America 94: 77767783.

Ball, S.S., and V.L. Roth. 1995. Jaw muscles of New World squirrels. Journal of Morphology 224: 265-291.

Beard, K.C. 1990. Gliding behaviour and palaeoecology of the alleged primate family Paromomyidae (Mammalia, Dermoptera). Nature 345: 340-341.

Beard, K.C. 1993a. Phylogenetic systematics of the Primatomorpha, with special reference to Dermoptera. In: F.S. Szalay, M.J. Novacek, and M.C. McKenna (editors), Mammal phylogenyplacentals: 129-150. New York: Springer.

Beard, K.C. 1993b. Origin and evolution of gliding in Cenozoic Dermoptera (Mammalia, Primatomorpha). In R.D.E. MacPhee (editor), Primates and their relatives in phylogenetic perspective: 63-90. New York: Plenum.

Becht, G. 1953. Comparative biologic-anatomical researches on mastication in some mammals. Proceedings of the Koninklijke Nederlandse Akademie van Wetenschappen Series C, Biological and Medical Sciences 56: 56: 508-527.

Bekele, A. 1983. The comparative functional morphology of some head muscles of the rodents Tachyoryctes splendens and Rattus rattus. Mammalia 47: 395-419.

Bhatnagar, K.P., and K.H. Reid. 1996. The human vomeronasal organ-I: historical perspectives. A study of Ruysch's (1703) and Jacobson's (1811) reports on the vomeronasal organ with comparative comments and English translations. Biomedical Research 7: 219-229.

Black, D. 1920. Studies on endocranial anatomy II- on the endocranial anatomy of Oreodon. Journal of Comparative Neurology 32: 271314.

Black, C.C. 1965. Fossil mammals from Montana. Rodents from the early Oligocene Pipestone Springs local fauna. Annals of Carnegie Museum 38: 1-48.

Bleefeld, A.R., and M.C. McKenna. 1985. Skeletal integrity of Mimolagus rodens (Lagomorpha, Mammalia). American Museum Novitates 2806: 1-5.

Bohlin, B. 1942. The fossil mammals from the Tertiary deposit of Taben-Buluk, western Kansu, part I: Insectivora and Lagomorpha. Palaeontologica Sinica Series C 8: 1-113.

Bohlin, B. 1946. The fossil mammals from the Tertiary deposit of Taben-Buluk, western Kansu, part II: Simplicidentata, Carnivora Artiodactyla, Perissodactyla, and Primates. Palaeontologica Sinica Series C, 8b: 1-259.

Bohlin, B. 1951. Some mammalian remains from Shi-her-ma-cheng, Hui-hui-pu area, western
Kansu. Report from the scientific expedition to the northwestern provinces of China under leadership of Dr. Sven Hedin. Vertebrate Palaeontology 5: 1-47.

Boyde, A. 1978. Development of the structure of the enamel of the incisor teeth in the three classical subordinal groups of the Rodentia. In P.M. Butler and K.A. Joysey (editors), Development, function and evolution of teeth: 43-58. New York: Academic Press.

Brandt, J.F. 1855. Beiträge zur nähern Kenntniss der Säugethiere Russlands. Mémoires de 1Ácadmie Impériales des Sciences de Saint-Pétersbourg 6: 1-375.

Bremer, K. 1988. The limits of amino acid sequence data in angiosperm phylogenetic reconstruction. Evolution 42: 785-803.

Bremer, K. 1994. Branch support and tree stability. Cladistics 10: 295-304.

Bromham, L., M.J. Phillips, and D. Penny. 1999. Growing up with dinosaurs: molecular dates and the mammalian radiation. Trends in Ecology and Evolution 14: 113-118.

Broom, R. 1881. The organ of Jacobson in the rabbit. Quarterly Journal of Microscopical Science 21: 549-570.

Broom, R. 1895. On the homology of the palatine process of the mammalian premaxillary. Proceedings of the Linnean Society of New South Wales 10: 477-485.

Broom, R. 1896a. Observations on the relations of the organ of Jacobson in the horse. Proceedings of the Linnean Society of New South Wales 21: 9-13.

Broom, R. 1896b. On the comparative anatomy of the organ of Jacobson in the marsupials. Proceedings of the Linnean Society of New South Wales 21: 591-623.

Broom, R. 1896c. On the organ of Jacobson in the Monotremata. Journal of Anatomy and physiology. 30: 70-80.

Broom, R. 1897. A contribution to the comparative anatomy of the mammalian organ of Jacobson. Transactions of the Royal Society of Edinburgh 39: 231-255.

Broom, R. 1902. On the organ of Jacobson in the elephant shrew. Proceedings of the Zoological Society of London 1902: 224-228.

Broom, R. 1915. On the organ of Jacobson and its relations in the "Insectivora" Part II. Talpa, Centetes, and Chrysochloris. Proceedings of the Zoological Society of London 25: 157-162.

Broom, R. 1926. On the organ of Jacobson and some other structures in the nose of Caenolestes. Proceedings of the Linnean Society of London 1926: 419-424.

Bryant, J.D., and M.C. McKenna. 1995. Cranial anatomy and phylogenetic position of Tsagan- 
omys altaicus (Mammalia: Rodentia) from the Hsanda Gol Formation (Oligocene), Mongolia. American Museum Novitates 3156: 1-42.

Bugge, J.A. 1985. Systematic value of the carotid arterial pattern in rodents, In W.P. Luckett and J.-L. Hartenberger (editors), Evolutionary relationships among rodents: a multidisciplinary analysis: 355-380. New York: Plenum.

Burchardt, B. 1978. Oxygen isotope paleotemperatures from the Tertiary period in the North Sea area. Nature 275: 121-23.

Butler, P.M. 1952. The milk-molars of Perissodactyla, with remarks on molar occlusion. Proceedings of the Zoological Society of London 121: 777-817.

Butler, P.M. 1956. The skull of Ictops and the classification of the Insectivora. Procedings of the Zoological Society of London 126: 453481.

Butler, P.M. 1972. Some functional aspects of molar evolution. Evolution 26: 474-483.

Butler, P.M. 1980. Functional aspects of the evolution of rodent molars. Palaeovertebrata, Mémoire Jubilaire en Hommage a René Lavocat: 249-262.

Butler, P.M. 1982. Directions of evolution in the mammalian dentition. In K.A. Joysey and A.E. Friday (editors), Problems of phylogenetic reconstruction: 235-244. London: Academic Press.

Butler, P.M. 1985. Homologies of molar cusps and crests, and their bearing on assessments of rodent phylogeny. In W.P. Luckett and J.-L. Hartenberger (editors), Evolutionary relationships among rodents - a multidisciplinary analysis: 381-401. New York: Plenum.

Byrd, K.E. 1981. Mandibular movement and muscle activity during mastication in the guinea pig (Cavia porcellus). Journal of Morphology 170: 147-169.

Candela, A.M. 1999. The evolution of the molar pattern of the Erethizontidae (Rodentia, Hystricognathi) and the validity of Parasteiromys Ameghino, 1904. Palaeovertebrata (Montpellier) 28: 53-73.

Carleton, M.D. 1984. Introduction to rodents. In S. Anderson, and J.K. Jones, Jr. (editors), Orders and families of Recent mammals: 155165. New York: Wiley.

Carpenter, J.M. 1996. Uninformative bootstrapping. Cladistics 12: 177-181.

Carrasco, M.A., and J.H. Wahlert. 1999. The cranial anatomy of Cricetops dormitor, and Oligocene fossil rodent from Mongolia. American Museum Novitates 3275: 1-14.

Cartmill, M., and R.D.E. MacPhee. 1980. Tupaiid affinties: the evidence of the carotid arteries and cranial skeleton. In W.P. Luckett (editor), Com- parative biology and evolutionary relationships of tree shrews: 95-132. New York: Plenum.

Chaline, J., and P. Mein. 1979. Les rongeurs et l'evolution. Paris: Doin.

Clark, D.A., P.P. Mitra, and S.S.-H. Wang. 2001. Scalable architecture in mammalian brains. Nature 411: 189-193.

Clemens, W.A. 1997. Characterization of enamel microstructure and application of the origins of prismatic structures in systematic analyses. In W.v. Koenigswald and P.M. Sander (editors), Tooth enamel microstructure: 85-112. Rotterdam: A. A. Balkema.

Conroy, G.C., and J.R. Wible. 1978. Middle ear morphology of Lemur variegata, some implications for primate paleontology. Folia Primatologica 29: 81-85.

Cooper, A., and R. Fortey. 1998. Evolutionary explosions and the phylogenetic fuse. Trends in Ecology and Evolution 13: 151-156.

Cooper, C., and A.L. Schiller. 1975. Anatomy of the guinea pig. Cambridge, MA: Harvard University Press.

Cope, E.D. 1883. The extinct rodentia of North America. The American Midland Naturalist 17: 43-57, 165-174, 370-381.

Cope, E.D. 1888. The mechanical causes of the origin of the dentition of the Rodentia. American Naturalist 22: 3-11.

Coquelin, A., A.N. Clancy, F. Macrides, E.P. Noble, and R.A. Gorski. 1984. Pheromonally induced release of luteinizing hormone in male mice: involvement of the vomeronasal system. Endocrinology 100: 1224-1229.

Corbet, G.B., and J. Hanks. 1968. A revision of the elephant-shrews, family Macroscelididae. Bulletin of the British Museum of Natural History 16: 4-111.

Crompton, A.W. 1971. The origin of the tribosphenic molar. In D.A. Kermack and K.A. Kermack (editors), Early mammals. Zoological Journal of the Linnean Society 50(Supplement 1): $65-87$.

Crompton, A.W., and K. Hiiemae. 1970. Functional occlusion and mandibular movements during occlusions in the American opossum $\mathrm{Di}$ delphis marsupialis. Zoological Journal of the Linnean Society 49: 21-47.

Crompton, A.W., A.J. Thexton, P. Parker, and K.M. Hiiemae. 1977. The activity of the jaw and hyoid musculature in the Virginian opossum, Didelphis virginiana. In B. Stonehouse and D. Gilmore (editors), The biology of marsupials: 287-305. London: Macmillan.

Cuvier, G. 1800-1805. Leçons d'anatomie comparée. Paris: Baudouin. 5 vol.

Czelusniak, J., M. Goodman, B.F. Koop, W.W. De Jong, and G. Matsuda. 1990. Perspective from 
amino acid and nucleotide sequences on cladistic relationships among higher taxa of Eutheria. In H.H. Genoways (editor), Current Mammalogy 2: 545-572. New York: Plenum.

Dashzeveg, D. 1988. Holarctic correlation of nonmarine Palaeocene-Eocene boundary strata using mammals. Journal of the Geological Society (London) 145: 473-478.

Dashzeveg, D. 1990a. The earliest rodents (Rodentia, Ctenodactyloidea) of Central Asia. Acta Zoologica Cracoviensia 33: 11-35.

Dashzeveg, D. 1990b. New trends in adaptive radiation of early Tertiary rodents (Rodentia, Mammalia). Acta Zoologica Cracoviensia 33: 37-44.

Dashzeveg, D., J.-L. Hartenberger, Martin, T., and S. Legendre. 1998. A peculiar minute Glires (Mammalia) from the early Eocene of Mongolia. Bulletin of Carnegie Museum of Natural History 34: 194-209.

Dashzeveg, D., and J. Meng. 1998. New Eocene ctenodactyloid rodents from the eastern Gobi Desert of Mongolia and a phylogenetic analysis of ctenodactyloids based on dental features. American Museum Novitates 3246: 1-20.

Dashzeveg, D., and D.E. Russell. 1988. Palaeocene and Eocene Mixodontia (Mammalia, Glires) of Mongolia and China. Palaeontology 31: 129-164.

Dashzeveg, D., D.E. Russell, and L.J. Flynn. 1987. New Glires (Mammalia) from the early Eocene of the People's Republic of Mongolia. 1. Systematics and description. Proceedings of the Koninklijke Nederlandse Akademie Van Wetenschappen Series B Physical Sciences 90: 133-154.

Dauphin, Y., Denis, A., and Denys, C. 1988. Les différents types d'émail chez les Rongeurs (Mammalia): conséquences d'un réexamen de leurs caractéristiques et de leurs définitions. Comptes Rendus de l'Académie des Sciences Series II 306: 93-98.

Dawson, M.R. 1958. Later Tertiary Leporidae of North America. University of Kansas Paleontological Contributions Article 6: 1-75.

Dawson, M.R. 1961. The skull of Sciuravus nitidus, a middle Eocene rodent. Postilla 53: 1-13.

Dawson, M.R. 1967. Lagomorph history and the stratigraphic record. C. Teichert and E.L. Yockelson (editors), Essays in paleontology and stratigraphy. University of Kansas Special Publication 2: 287-316.

Dawson, M.R. 1969. Osteology of Prolagus sar$d u s$, a Quaternary ochotonid (Mammalia: Lagomorpha). Palaeovertebrata 2: 157-190.

Dawson, M.R. 1977. Late Eocene rodent radiations: North America, Europe and Asia. Géobios Spécial 1: 195-209.
Dawson, M.R., and C.K. Beard. 1996. New Late Paleocene rodents (Mammalia) from Big Multi Quarry, Washakie Basin, Wyoming. Palaeovertebrata 25: 301-321.

Dawson, M.R., C.-K. Li, and T. Qi. 1984. Eocene ctenodactyloid rodents (Mammalia) of eastern central Asia. Carnegie Museum of Natural History Special Publication 9: 138-150.

Deacon, T.W. 1990. Rethinking mammalian brain evolution. American Zoologist 30: 629-705.

De Beer, G.R. 1937. The development of the vertebrate skull. Oxford: Clarendon Press.

De Beer, G.R., and W.A. Fell. 1936. The development of the Monotremata-part III. The development of the skull of Ornithorhynchus. Transactions of the Zoological Society of London 23: 1-42.

De Blainville, H.M.D. 1816. Prodrome d'une nouvelle distribution systématique du règne animal. Bulletin des Sciences par la Société Philomatique de Paris 3: 105-124.

De Queiroz, K., and J. Gauthier. 1990. Phylogeny as a central principle in taxonomy: Phylogenetic definitions of taxon names. Systematic Biology 39: 307-322.

De Queiroz, K., and J. Gauthier. 1992. Phylogenetic taxonomy. Annual Review of Ecology and Systematics 23: 449-480.

D’Erchia, A.M., C. Gissi, G. Pesole, C. Saccone, and U. Arnason. 1996. The guinea-pig is not a rodent. Nature 381: 597-600.

De Winter, W., and C.E. Oxnard. 2001. Evolutionary radiations and convergences in the structural organization of mammalian brains. Nature 409: 710-714.

Dice, L.R. 1933. Some characters of the skull and skeleton of the fossil hare, Palaeolagus haydeni. Papers of the Michigan Academy of Sciences and Art and Letters 18: 301-306.

Dierbach, A.R. 1985. Zur Morphogenese des Craniums von Cavia porcellus L., Teil 1: Einführung, Systematik und beschreibender Teil. Morphologisches Jahrbuch 131: 441-476.

Dieulafe, L. 1906. Morphology and embryology of the nasal fossae of vertebrates (translated by H. W. Loeb). Annals of Otology Rhinology \& Laryngology 15: 1-60, 267-349, 513-84.

Druzinsky, R.E. 1995. Incisal biting the mountain beaver (Aplodontia rufa) and woodchuck (Marmota monax). Journal of Morphology 226: 79101.

Du, H.-J., J. Cheng, W.-M. Wu, and A.-C. Ma. 1991. A discussion on Paleogene mammalian fossil horizons and concerned problems of Liguanqiao and Yuanqu Basins. Earth ScienceJournal of China University of Geosciences 16: 113-126.

Easteal, S. 1990. The pattern of mammalian evo- 
lution and the relative rate of molecular evolution. Genetics 124: 165-173.

Ehik, J. 1926. The right interpretation of the cheekteeth tubercles of Titanomys. Annales Historico-Naturales Musei Nationalis Hungarici 23: $178-186$.

Elftman, H.O. 1929. Functional adaptations of the pelvis in marsupials. Bulletin of the American Museum of Natural History 58: 189-232.

Emry, R.J., and R.W. Thorington. 1982. Descriptive and comparative osteology of the oldest fossil squirrel, Protosciurus (Rodentia: Sciuridae). Smithsonian Contributions to Paleobiology 47: $1-35$.

Emry, R.J., and W.W. Korth. 1996. The Chadronian squirrel "Sciurus" jeffersoni Douglass, 1901: a new generic name, new material, and its bearing on the early evolution of Sciuridae (Rodentia). Journal of Vertebrate Paleontology 16: 775-780.

Evans, F.G. 1942. The osteology and relationships of the elephant shrews (Macroscelididae). Bulletin of the American Museum of Natural History 80 : 85-125.

Evans, H.E., and G.C. Christensen. 1979. Miller's Anatomy of the dog. Philadelphia: W. B. Saunders.

Every, R.G. 1975. Significance of tooth sharpness for mammalian, especially primate, evolution. F. Szalay (editor), Approaches to primate paleobiology. Contributions to Primatology 5: 293-325.

Felsenstein, J. 1985. Confidence limits on phylogenies: an approach using the bootstrap. Evolution 39: 783-791.

Flynn, L.J. 1994. Roots of rodent radiation. Nature 370: 97-98.

Flynn, L.J., L.L. Jacobs, and I.U. Cheema. 1986. Baluchimyinae, a new ctenodactyloid rodent subfamily from the Miocene of Baluchistan. American Museum Novitates 2841: 1-58.

Flynn, L.J., D.E. Russell, and D. Dashzeveg. 1987. New Glires (Mammalia) from the early Eocene of the People's Republic of Mongolia, 2: incisor morphology and enamel microstructure. Proceedings of the Koninklijke Nederlandse Akademie Van Wetenschappen Series B Physical Sciences 90: 143-154.

Flynn, L.J., M. Sarwar, and J.-J. Jaeger. 1985. Evolution of the Rhizomyine zygoma. Palaeovertebrata, Montpellier, 15: 129-138.

Foote, M., J.P. Hunter, C.M. Janis, and J.J. Sepkoski, Jr. 1999a. Evolutionary and preservational constraints on origins of biological groups: divergence times of eutherian mammals. Science 283: 1310-1314.

Foote, M., J.P. Hunter, C.M. Janis, and J.J. Sep- koski, Jr. 1999b. Divergence times of eutherian mammals. Science on Line 285: 2031a.

Fortelius, M. 1985. Ungulate cheek teeth: Developmental, functional, and evolutionary interrelations. Acta Zoologica Fennica 180: 1-76.

Fostowicz-Frelik, L., and Z. Kielan-Jaworowska. 2002. Lower incisor in zalambdalestid mammals (Eutheria) and its phylogenetic implications. Acta Palaeontologica Polonica 47: 177180.

Fox, R.C., and J. Meng. 1997. An X-radiographic and SEM study of the osseous inner ear of multituberculates and monotremes (Mammalia): implications for mammalian phylogeny and evolution of hearing. Zoological Journal of the Linnean Society 121: 249-291.

Frahnert, S. 1999. Morphology and evolution of the Glires rostral cranium. Mitteilungen aus dem Museum für Naturkunde in Berlin Zoologische Reihe 75: 229-246.

Friant, M. 1932. Contributions à l'étude de la differentiation des dents jugales chez les mammifères. Publications Diverses du Muséum National d'Histoire Naturelle 1: 1-132.

Gagnon, M. 1992. Succession of mammalian communities in the Fayum of Egypt in relation to the age of the Jebel Qatrani Formation. Journal of Vertebrate Paleontology 12: 29A.

Galbreath, E.C. 1967. A skeleton of Geomyoid rodent Gregorymys curtus (Matthew) from the early Miocene of South Dakota. Transactions of the Illinois State Academy of Sciences 60: 272281 .

Gambaryan, P.P., and Z. Kielan-Jaworowska. 1997. Sprawling versus parasagittal stance in multituberculate mammals. Acta Palaeontologica Polonica 42: 13-44.

Gans, C., F. De Vree, and G.C. Gorniak. 1978. Analysis of mammalian masticatory mechanisms: process and problems. Zentralblatt für Veterinärmedizin Reihe C 7: 226-244.

Ganz, C.L. 1968. A study of the Eocene condylarthran mammal Hyopsodus. Smithsonian Miscellaneous Collections 153: 1-90.

Gazin, C.L. 1968. A study of the Eocene condylarthran mammal Hyopsodus. Smithsonian Miscellaneous Collections 153: 1-89.

Gebo D.L., and K.D. Rose. 1993. Skeletal morphology and locomotor adaptation in Prolimnocyon atavus, an Early Eocene hyaenodontid creodont. Journal of Vertebrate Paleontology 13: 125-144.

Geisler, J.H. 2001. New morphological evidence for the phylogeny of Artiodactyla, Cetacea, and Mesonychidae. American Museum Novitates 3344: 1-53.

Gervais, P. 1848. Zoologie et paléontologie (animaux vertébrés) ou nouvelles recherches sur les 
animaux vivants et fossiles de la France. Paris: Arthus Bertrand.

Gidley, J.W. 1906. Evidence bearing on toothcusp development. Proceedings of Washington Academy of Sciences 8: 91-110.

Gidley, J.W. 1912. The lagomorphs as an independent order. Science 36: 285-286.

Giere, P., C. Freyer, and U. Zeller. 1999. Opening of the mammalian vomeronasal organ with respect to the Glires hypothesis: a cladistic reconstruction of the therian morphotype. Mitteilungen aus dem Museum für Naturkunde in Berlin Zoologische Reihe 75: 247-255.

Gingerich, P.D. 1976. Cranial anatomy and evolution of early Tertiary Plesiadapidae (Mammalia, Primates). University of Michigan Papers on Paleontology 15: 1-140.

Gingerich, P.D. 1977. Patterns of evolution in the mammalian fossil record. In A. Hallam (editor), Patterns of evolution as illustrated by the fossil record: 469-500. Amsterdam: Elsevier.

Gingerich, P.D. 1993. Oligocene age of the Gebel Qatrani Formation, Fayum, Egypt. Journal of Human Evolution 24: 207-218.

Gingerich, P.D., and G.F. Gunnell. 1979. Systematics and evolution of the genus Esthonyx (Mammalia, Tillodontia) in the early Eocene of North America. University of Michigan Contributions to Paleontology 25: 125-153.

Gingerich, P.D., and R.D. Martin. 1981. Cranial morphology and adaptations in Eocene Adapidae - the Cambridge skull of Adapis uparisiensis. American Journal of Physical Anthropology $56: 235-257$.

Gingerich, P.D., and M.C. McKenna. 1980. Mammalian paleontology in China. Society of Vertebrate Paleontology News Bulletin 118: 4244.

Goodrich, E.S. 1930. Studies on the structure and development of vertebrates. New York: Dover.

Gorniak, G.C. 1977. Feeding in golden hamster, Mesocricetus auratus. Journal of Morphology 154: 427-458.

Graur, D. 1993. Molecular phylogeny and the higher classification of eutherian mammals. Trends in Ecology and Evolution 8: 141-147.

Graur, D., L. Duret, and M. Gouy. 1996. Phylogenetic position of the order Lagomorpha (rabbits, hares and allies). Nature 379: 333-335.

Graur, D., W.A. Hide, and W.-H. Li. 1991. Is the guinea-pig a rodent? Nature 351: 649-652.

Graur, D., W.A. Hide, A. Zharkikh, and W.-H. Li. 1992. The biochemical phylogeny of guinea pigs and gundis, and the paraphyly of the order Rodentia. Comparative Biochemistry and Physiology B, Comparative Biochemistry 101: 495498.

Graybeal, A, J. Rosowski, D.R. Ketten, A.W.
Crompton. 1989. Inner ear structure in Morganucodon, an early Jurassic mammal. Zoological Journal of the Linnean Society 96: 107117.

Greaves, W.S. 1978. The jaw lever system in ungulates: a new model. Journal of Zoology (London) 184: 271-285.

Greaves, W.S. 1980. The mammalian jaw mechanism - the high glenoid cavity. American Naturalist 116: 432-440.

Green, H.L.H.H. 1930. A description of the egg tooth of Ornithorhynchus, together with some notes on the development of the palatine processes of the premaxilla. Journal of Anatomy 74: 512-522.

Green, H.L.H.H., and R. Presley. 1978. The dumb-bell bone of Ornithorhynchus. Journal of Anatomy 127: 216.

Gregory, W.K. 1910. The orders of mammals. Bulletin of the American Museum of Natural History 27: 1-525.

Gregory, W.K. 1920. On the structure and relations of Notharctus, an American Eocene primate. Memoir of the American Museum of Natural History New Series 3: 49-243.

Gregory, W.K., and G.G. Simpson. 1926. Cretaceous mammal skulls from Mongolia. American Museum Novitates 225: 1-20.

Grine, F.E. 1981. Trophic differences between "gracile" and "robust" australopithecines: a scanning electron micro scope analysis of occlusal events. South African Journal of Science 77: 203-230.

Grine, F.E., and R.F. Kay. 1988. Early hominid diets from quantitative image analysis of dental microwear. Nature 333: 765-768.

Guo, J.-W., Y. Wang, and X.-A. Yang. 2000. A new Early Eocene ctenodactyloid rodent (Rodentia, Mammalia) from Liquanqiao Basin and the associated mammalian fossils. Vertebrata PalAsiatica 38: 303-313.

Halpern, M. 1987. The organization and function of the vomeronasal system. Annual Review of Neuroscience 10: 325-362.

Hartenberger, J.-L. 1977. A propos de l'origine des Rongeurs. Géobios, Spécial 1: 183-193.

Hartenberger, J.-L. 1980. Données et hypothèses sur la radiation initiale des rongeurs. Palaeovertebrata, Mémoire Jubilaire en hommage a René Lavocat: 285-301.

Hartenberger, J.-L. 1985. The order Rodentia: major question on their evolutionary origin, relationships and suprafamilial systematics. In W.P. Luckett and J.-L. Hartenberger (editors), Evolutionary relationships among rodents: a multidisciplinary analysis: 1-33. New York: Plenum.

Hartenberger, J.-L., D. Dashzeveg, and T. Martin. 
1997. What is Ivanantonia efremovi. Paläontologische Zeitschrift 71: 135-143.

Hasegawa, M., Y. Cao, J. Adachi, and T.-A. Yano. 1992. Rodent polyphyly? Nature 355: 595.

Hedges, S.B., P.H. Parker, C.G. Sibley, and S. Kumar. 1996. Continental breakup and the ordinal diversification of birds and mammals. Nature 381: 226-229.

Herring, S.W. 1972. The role of canine morphology in the evolutionary divergence of pigs and peccaries. Journal of Mammalogy 53: 500-512.

Herring. S.W, and R.P. Scapino. 1973. Physiology of feeding in miniature pigs. Journal of Morphology 141: 427-460.

Hiiemae, K. 1971a. The structure and function of the jaw muscles in the rat (Rattus norvegicus L.) - II. Their fibre type and composition. Zoological Journal of the Linnean Society 50: 101109.

Hiiemae, K. 1971b. The structure and function of the jaw muscles in the rat (Rattus norvegicus L.) - III. The mechanics of the muscles. Zoological Journal of the Linnean Society 50: 111132.

Hiiemae, K.M. 1976. Masticatory movements in primitive mammals. In D.J. Anderson, and B. Matthews (editors), Mastication: 105-118. Bristol: Wright.

Hiiemae, K.M. 1978. Mammalian mastication: a review of the activity of jaw muscles and the movements they produce in chewing. In P.M. Butler, and K.A. Joysey (editors), Development, function and evolution of teeth: 359-398. London: Academic Press.

Hiiemae, K.M., and G.M. Ardran. 1968. A cinefluorographic study of mandibular movement during feeding in the rat. Journal of Zoology (London) 154: 139-154.

Hiiemae, K.M., and A.W. Crompton. 1971. A cinefluorographic study of feeding in the American opossum, Didelphis marsupialis. In A.A. Dahlberg (editor), Dental morphology and evolution: 299-334. Chicago: University Chicago Press.

Hiiemae, K., and W.J.B. Houston. 1971. The structure and function of the jaw muscles in the rat (Rattus norvegicus L.). Their anatomy and internal architecture. Zoological Journal of the Linnean Society 50: 75-99.

Hiiemae, K.M., and F.A. Jenkins. 1969. The anatomy and internal architecture of the muscles of mastication in the American opossum, Didelphis marsupialis. Postilla 140: 1-49.

Hildebrand, M. 1995. Analysis of vertebrate structure, 4th ed. New York: Wiley.

Hinton, M.A.C. 1926. Monograph of the voles and lemmings (Microtinae) living and extinct, vol. 1. London: British Museum (Natural History).

Hofman, M.A. 1982. Encephalization in mammals in relation to the size of the cerebral cortex. Brain Behavior and Evolution 20: 84-96.

Hoffstetter, R., and R. Lavocat, 1970. Découverte dans le Déséadien de Bolivie de genres pentalophodontes appuyant les affinités africaines des Rongeurs caviomorphes. Comptes Rendus de l'Académie des Sciences de Paris 271: 172175.

Honeycutt, R.L., and R.M. Adkins. 1993. Higher level systematics of eutherian mammals: an assessment of molecular characters and phylogenetic hypotheses. Annual Review of Ecology and Systematics 24: 279-305.

Hooker, J.J. 2001. Tarsals of the extinct insectivoran family Nyctitheriidae (Mammalia): evidence for archontan relationships. Zoological Journal of the Linnean Society 132: 501-529.

Horovitz, I. 2000. The tarsus of Ukhaatherium nessovi (Eutheria, Mammalia) from the Late Cretaceous of Mongolia: an appraisal of the evolution of the ankle in basal therians. Journal of Vertebrate Paleontology 20: 547-560.

Howell, A.B. 1932. The saltatorial rodent Dipodomys: the functional and comparative anatomy of its muscular and osseous systems. Proceedings of American Academy of Arts and Sciences 67: 377-536.

Hu, Y.-M. 1993. Two new genera of Anagalidae (Anagalida, Mammalia) from the Paleocene of Qianshan, Anhui and the phylogeny of anagalids. Vertebrata PalAsiatica 31: 153-182.

Hu, Y.-M., J. Meng, and C.-K. Li. 2000. Postcranial skeleton of Rhombomylus from the Early Eocene of China: Implications to locomotion and phylogeny. Journal of Vertebrate Paleontology 20(supplement to number 3): 49A-50A.

Huang, X.-S. 1995. A new Hyopsodus from the early Eocene of Fangxian, Hubei. Vertebrata PalAsiatica 33: 39-46.

Huchon, D., F.M. Catzaflis, and E.J.P. Douzery. 1999. Molecular evolution of the nuclear von Willebrand Factor Gene in mammals and the phylogeny of rodents. Molecular Biology and Evolution 16: 577-589.

Huchon, D., F.M. Catzaflis, and E.J. Douzery. 2000. Variance of molecular datings, evolution of rodents and the phylogenetic affinities between Ctenodactylidae and Hystriconathi. Proceedings of the Royal Society of London B 267: 393-402.

Hunter, J.P., and M. Fortelius. 1994. Comparative dental occlusal morphology, facet development, and microwear in two sympatric species of Listriodon (Mammalia: Suidae) from the middle 
Miocene of western Anatolia (Turkey). Journal of Vertebrate Paleontology 14: 105-126.

Hurum, J.H. 1994. The snout and orbit of Mongolian multituberculates studied by serial sections. Acta Palaeontologica Polonica 39: 181224.

Hurum, J.H. 1998a. The braincase of two late Cretaceous Asian multituberculates studied by serial sections. Acta Palaeontologica Polonica 41: 253-275.

Hurum, J.H. 1998b. The inner ear of two late Cretaceous multituberculate mammals, and its implications for multituberculate hearing. Journal of Mammalian Evolution 5: 65-93.

Hürzeler, J. 1936. Osteologie und Odontologie der Caenotheriden. Abhandlungen der Schweizerischen Palaeontologischen Gesellschaft 58: 189.

Illiger, C. 1811. Prodromus systematis mammalium et avium additis terminis zoographicis utriudque classis. Berlin: C. Salfeld.

Jacobs, L.L. 1977. A new genus of murid rodent from the Miocene of Pakistan and comments on the origin of the Muridae. Paleobios 25: 111.

Jacobs, L.L. 1978. Fossil rodents (Rhizomyidae and Muridae) from Neogene Siwalik deposits, Pakistan. Bulletin of the Museum of North Arizona 52: 1-95.

Jacobs, L.L., and W.R. Downs. 1994. The evolution of murine rodents in Asia. National Science Museum Monographs (Tokyo) 8: 149156.

Jacobs, L.L., and D. Pilbeam. 1980. Of mice and men: fossil-based divergence dates and molecular "clock". Journal of Human Genetics 52: 152-166.

Jacobs, L.L., D.A. Winkler, and P.A. Murry. 1989. Modern mammal origins: evolutionary grades in the Early Cretaceous of North America. Proceedings of the National Academy of Sciences of the United States of America 86: 49924995.

Jacobson, L. 1811. Description anatomique d'un organe observé dans les mammifères. Annales du Muséum National d'Histoire Naturelle 18: 414-424.

Jaeger, J.-J. 1988. Rodent phylogeny: New data and old problems. In M. J. Benton (editor), The phylogeny and classification of the tetrapods, vol, 2. Mammals: 177-199. Oxford: Clarendon Press.

Jaeger, J.-J., H.-y. Tong, and C. Denys. 1986. Age de la divergence Mus-Rattus comparaison des données paléontologiques et moléculaires. Comptes Rendus de l'Académie des Sciences de Paris 302: 917-922.

Janis, C.M. 1990. The correlation between diet and dental wear in herbivorous mammals and its relationship to the determination of diets in extinct species. In A.J. Boucot (editor), Evolutionary paleobiology of behavior and coevolution: 241-259. New York: Elsevier.

Janis, C.M. 1995. Correlations between craniodental morphology and feeding behavior in ungulates: reciprocal illumination between living and fossil taxa. In J.J. Thomason (editor), Functional morphology in vertebrate paleontology: 76-98. New York: Cambridge University Press.

Janke, A., G. Feldmaier-Fuchs, W.K. Thomas, von A. Haeseler, and S. Pääbo. 1994. The marsupial mitochondrial genome and the evolution of placental mammals. Genetics 137: 243-256.

Jenkins, F.A., Jr., and S.M. Camazine. 1977. Hip structure and locomotion in ambulatory and cursorial carnivores. Journal of Zoology (London) 181: 351-370.

Jenkins, F.A., Jr., and F.R. Parrington. 1976. The postcranial skeletons of the Triassic mammals Eozostrodon, Megazostrodon and Erythrotherium. Philosophical transactions of the Royal Society of London B 273: 387-431.

Jenkins, F.A. Jr., and W.A. Weijs. 1979. The functional anatomy of the shoulder in the Virginia opossum (Didelphis virginiana). Journal of Zoology (London) 188: 379-410.

Johnson, J.I., J.A.W. Kirsch, R.L. Reep, and R.C. Switzer III. 1994. Phylogeny through brain traits: more characters for the analysis of mammalian evolution. Brain Behavior and Evolution 43: 319-347.

Johnson, J.I., J.A.W. Kirsch, and R.C. Switzer, III. 1982a. Phylogeny through brain traits: the distribution of categorizing characters in contemporary mammals. Brain Behavior and Evolution 20: 97-117.

Johnson, J.I., J.A.W. Kirsch, and R.C. Switzer, III. 1984. Brain traits through phylogeny: evolution of neural characters. Brain Behavior and Evolution 24: 169-176.

Johnson, J.I., R.C. Switzer, III, and J.A.W. Kirsch. 1982b. Phylogeny through brain traits: fifteen characters which adumbrate mammalian genealogy. Brain Behavior and Evolution 20: 7283.

Kallen F.C., and C. Gans. 1972. Mastication in the little brown bat, Myotis lucifugus. Journal of Morphology 136: 385-420.

Kay, R.F. 1977. The evolution of molar occlusion in the Cercopithecidae and early catarrhines. American Journal of Physical Anthropology 46: 327-352.

Kay, R.F. 1987. Analysis of primate dental microwear using image processing techniques. Scanning Microscopy 1: 657-662.

Kay, R.F., and K.M. Hiiemae. 1974. Jaw move- 
ment and tooth use in recent and fossil primates. American Journal of Physical Anthropology 40: 227-256.

Kay, R.F., R.W. Thorington Jr., and P. Houde. 1990. Eocene plesiadapiform shows affinities with flying lemurs not primates. Nature 345: 342-344.

Kemp, T.S. 1979. The primitive cynodont Procynosuchus: functional anatomy of the skull and relationships. Philosophical Transactions of the Royal Society of London B 285: 73-122.

Keverne, R.B. 1999. The vomeronasal organ. Science 286: 716-720.

Kielan-Jaworowska, Z. 1969. Preliminary data on the Upper Cretaceous eutherian mammals from Bayn Dzak, Gobi Desert. Results of the PolishMongolian Palaeontological Expeditions, Part I. Palaeontologia Polonica 19: 171-191.

Kielan-Jaworowska, Z. 1975. Preliminary description of two new eutherian genera from the Late Cretaceous of Mongolia. Results of the Polish-Mongolian Palaeontological Expeditions, Part VI. Palaeontologia Polonica 33: 513.

Kielan-Jaworowska, Z. 1977. Evolution of the therian mammals in the Late Cretaceous of Asia. Part II. Postcranial skeleton in Kennalestes and Asioryctes. Palaeontologia Polonica 37: 65-83.

Kielan-Jaworowska, Z. 1978. Evolution of the therian mammals in the Late Cretaceous of Asia. Part III. Postcranial skeleton in Zalambdalestidae. Palaeontologia Polonica 38: 3-41.

Kielan-Jaworowska, Z. 1981. Evolution of the therian mammals in the Late Cretaceous of Asia. Part VI. Skull structure in Kennalestes and Asioryctes. Results of the Polish-Mongolian Palaeontological Expeditions, Part IX, Palaeontologia Polonica 42: 25-71.

Kielan-Jaworowska, Z. 1984a. Evolution of the therian mammals in the Late Cretaceous of Asia. Part V. Skull structure in Zalambdalestidae. Results of the Polish-Mongolian Palaeontological Expeditions, Part X. Palaeontologia Polonica 46: 107-117.

Kielan-Jaworowska, Z. 1984b. Evolution of the therian mammals in the Late Cretaceous of Asia. Part VII. Synopsis. Results of the PolishMongolian Palaeontological Expeditions, Part X. Palaeontologia Polonica 46: 173-183.

Kielan-Jaworowska, Z. 1984c. Evolution of the therian mammals in the Late Cretaceous of Asia. Part VI. Endocranial casts of Eutherian mammals. Palaeontologia Polonica 46: 157171.

Kielan-Jaworowska, Z., Bown, T.M., andLillegraven, J.A. 1979. Eutheria. In J.A. Lillegraven, Z. Kielan-Jaworowska, and W.A.
Clemens (editors), Mesozoic mammals: the first two-thirds of mammalian history: 221-258. Berkeley: University of California Press.

Kielan-Jaworowska, Z., R.L. Cifelli, and Z.-X. Luo. 1998. Alleged Cretaceous placental from down under. Lethaia 31: 267-268.

Kielan-Jaworowska, Z., and P.P. Gambaryan. 1994. Postcranial anatomy and habits of Asian multituberculate mammals. Fossils and Strata 36: 1-92.

Kielan-Jaworowska, Z., R. Presley, and C. Poplin. 1986. The cranial vascular system in taeniolabidoid multituberculate mammals. Philosophical Transactions of the Royal Society of London B 313: 525-602.

Kielan-Jaworowska, Z., and B.A. Trofimov. 1980. Cranial morphology of the Cretaceous eutherian mammal Barunlestes. Acta Palaeontologica Polonica 25: 167-185.

Kirsch, J.A.W. 1983. Phylogeny through brain traits: objectives and method. Brain Behavior and Evolution 22: 53-59.

Kirsch, J.A.W., and J.I. Johnson. 1983. Phylogeny through brain traits: tree generated by neural characters. Brain Behavior and Evolution 22: $60-69$.

Klingener, D. 1964. The comparative myology of four dipodoid rodents (Genera Zapus, Napaeozapus, Sicista, and Jaculus). Miscellaneous Publications, Museum of Zoology University of Michigan 124: 1-100.

Kluge, A.G., and A.J. Wolf. 1993. Cladistics: what's in a word? Cladistics 9: 183-199.

Koenigswald, W.v. 1980. Schmelzmuster und Morphologie in den Molaren der Arvicolidae (Rodentia). Abhandlungen der Senckenbergischen Naturforschenden Gesellschaft 539: 1129.

Koenigswald, W.v. 1985. Evolutionary trends in the enamel of rodent incisors. In W.P. Luckett and J.-L. Hartenberger (editors), Evolutionary relationships among rodents: a multidisciplinary analysis: 404-422. New York: Plenum.

Koenigswald, W.v. 1988. Enamel modification in enlarged front teeth among mammals and the various possible reinforcements of the enamel. In D.E. Russell, J.-P. Santoro, and D. Sigogneau-Russell (editors), Teeth revisited: Proceedings of the VIIth International Symposium on Dental Morphology: 147-167. Memoires du Museum National d'Histoire Naturelle C 53.

Koenigswald, W.v. 1995. Lagomorpha versus Rodentia: the number of layers in incisor enamels. Neues Jahrbuch für Geologie und Paläontologie, Monatshefte 10: 605-613.

Koenigswald, W.v. 1996. Die Zahl der Schmelzschichten in den Inzisiven bei den La- 
gomorpha und ihre systematische Bedeutung. Bonner Zoologische Beiträge 46: 33-57.

Koenigswald, W.v., and W.A. Clemens. 1992. Levels of complexity in the microstructure of mammalian enamel and their application in studies of systematics. Scanning Microscopy 6: 195-218.

Koenigswald, W.v., T. Martin, and H.U. Pfretzschner. 1993. Phylogenetic interpretation of enamel structures in mammalian teeth: Possibilities and problems. In F.S. Szalay, M.J. Novacek, and M.C. McKenna (editors), Mammal phylogeny-Placentals: 303-314. New York: Springer.

Koenigswald, W.v., and H.U. Pfretzschner. 1991. Biomechanics in the enamel of mammalian teeth. In N. Schmidt-Kittler and K. Vogel (editors), Constructional morphology and biomechanics: 113-125. Berlin: Springer.

Koenigswald, W.v., J.M. Rensberger, and H.U. Pfretzschner. 1987. Changes in the tooth enamel of early Paleocene mammals allowing increased diet diversity. Nature 328: 150-152.

Koenigswald, W.v., P.M. Sander, M.B. Leite, T. Mörs, and W. Santel. 1994. Functional symmetries in the schmelzmuster and morphology of rootless rodent molars. Zoological Journal of the Linnean Society 110: 141-179.

Korth, W.W. 1984. Earliest Tertiary evolution and radiation of rodents in North America. Bulletin of Carnegie Museum of Natural History 24: 171.

Korth, W.W. 1994. The Tertiary record of rodents in North America. Topics in Geobiology 12: 1319.

Korth, W.W., and R.J. Emry. 1991. The skull of Cedromus and a review of the Cedromurinae (Rodentia, Sciuridae). Journal of Paleontology 65: 984-994.

Korvenkontio, V.A. 1934. Mikroskopische Untersuchungen an Nagerincisiven unter Hinweis auf die Schmelzstruktur der Backenzahne. Annales Zoologici Societatis Zoologicae-Botanicae Fennicae 'Vanamo' 2: 1-274.

Krumbach, T. 1904. Die unteren Schneidezahne der Nagetiere, nach Gestalt und Funktion betrachtet. Zoologischer Anzeiger 27: 273-290.

Kuhn, H.-J. 1971. Die Entwicklung und Morphologie des Schadels von Tachyglossus aculeatus. Abhandlungen der Senckenbergischen Naturforschenden Gesellschaft 528: 1-192.

Kumar, S., and S.B. Hedges. 1998. A molecular timescale for vertebrate evolution. Nature 392: 917-920.

Landry, S.O., Jr. 1957. The interrelationships of the New and Old World hystricomorph rodents. University of California Publications in Zoology 56: 1-118.
Landry, S.O. Jr. 1958. The function of the entepicondylar foramen in mammals. The American Midland Naturalist 60: 100-112.

Landry S.O., Jr. 1970. The Rodentia as omnivores. Quarterly Journal of Biology 45: 351372.

Landry S.O., Jr. 1974. The fundamental relationship of the Lagomorpha and Rodentia (abstract). In First International Theriological Congress Transactions Moscow 2:202-203.

Landry, S.O., Jr. 1999. A proposal for a new classification and nomenclature for the Glires (Lagomorpha and Rodentia). Mitteilungen aus dem Museum für Naturkunde in Berlin, Zoologische Reihe 75: 283-319.

Lavocat, R. 1973. Les rongeurs du Miocène d'Afrique Orientale I. Miocène inférieur. Mémoires et Travaux de l'E.P.H.E. Institut de Montpellier 1: 1-284.

Lavocat, R. 1974a. What is an hystricomorph? In I.W. Rowlands and B.S. Weir (editors), The biology of hystricomorph rodents: 7-20. London: Academic Press.

Lavocat, R. 1974b. The Interrelationships between the African and South American rodents and their bearing on the problem of the origin of South American monkeys. Journal of Human Evolution 3: 323-326.

Lavocat, R. 1976. Rongeurs du Bassin Déséadian de Salla-Luribay. In Rongeurs Caviomorphes de l'Oligocène de Bolivie. Paleovertebrata (Montpellier) 7: 15-90.

Lavocat, R. 1980. The implications of rodent paleontology and biogeography to the geographical source and origin of Platyrrhine Primates. In A.B. Chiarelli and R.C. Ciochon (editors), Evolutionary biology of New World monkeys and continental drift: 93-102. New York: Plenum.

Lavocat, R., and J.-P. Parent. 1985. Phylogenetic analysis of middle ear features in fossil and living rodents. In W.P. Luckett, and J.-L. Hartenberger (editors), Evolutionary relationships among rodents - a multidisciplinary analysis: 333-354. New York: Plenum.

Lehner, J., and H. Plenk. 1936. Die Zähne. W.v. Möllendorff (editor), Handbuch der mikroskopischen Anatomie des Menschen 5/3: 447-708.

Lei, Y.-Z. 1984. The analysis of the mammal fauna of the Paleogene of Central-southern China. Journal of the Yichang Institute of Geology and Minerals, Chinese Academy of Geology 8: 4150.

Li, C.-K. 1977. Paleocene eurymyloids (Anagalida, Mammalia) of Quianshan, Anhui. Vertebrata PalAsiatica 15: 103-118.

Li, C.-K., C.-s. Chiu, D.-K. Yan, and S.-H. Hsieh. 1979. Notes on some early Eocene mammalian 
fossils of Hengdung, Hunan. Vertebrata PalAsiatica 17: 71-80.

Li, C.-K., and S.-Y. Ting. 1985. Possible phylogenetic relationships of eurymylids and rodents, with comments on mimotonids. In W.P. Luckett and J.-L. Hartenberger (editors), Evolutionary relationships among rodents: a multidisciplinary analysis: $35-58$. New York: Plenum.

Li, C.-K., and S.-Y. Ting. 1993. New cranial and postcranial evidence for the affinities of the eurymylids (Rodentia) and mimotonids (Lagomorpha). In F.S. Szalay, M.J. Novacek, and M.C. McKenna (editors), Mammal phylogenyplacentals: 151-158. New York: Springer.

Li, C.-K., R.W. Wilson, M.R. Dawson, and L. Krishtalka. 1987. The origin of rodents and lagomorphs. In H.H. Genoways (editor), Current mammalogy 1: 97-108. New York: Plenum.

Li, C.-K., and D.-F. Yan, 1979. The systematic position of eurymylids (Mammalia) and the origin of Rodentia. 12th Annual Conference and 3rd National Congress Paleontological Society of China, Beijing. Abstracts of Papers: 155156.

Li, C.-K., J.-J. Zheng, and S.-Y. Ting. 1989. The skull of Cocomys lingchaensis, an Early Eocene Ctenodactyloid Rodent of Asia. In C.C. Black and M.R. Dawson (editors), Papers on fossil rodents in honour of Albert Elmer Wood. Natural History Museum of Los Angeles County Science Series 33: 179-192.

Li, W.-H., W.A. Hide, and D. Graur. 1992a. Origin of rodents and guinea-pigs. Nature 359: 277278.

Li, W.-H., W.A. Hide, A. Zharkikh, and D. Graur. 1992b. The molecular taxonomy and evolution of the guinea pig. The Journal of Heredity 83: 174-181.

Lillegraven, J.A. 1969. Latest Cretaceous mammals of upper part of Edmonton Formation of Alberta, Canada, and review of marsupial-placental dichotomy in mammalian evolution. University of Kansas Paleontological Contributions Article 50 (Vertebrata 12): 1-122.

Lillegraven, J.A., Z. Kielan-Jaworowska, and W.A. Clemens (editors). 1979. Mesozoic mammals: the first two-thirds of mammalian history. Berkeley: University of California Press.

Linnaeus, C. 1758. Systema naturae per regna tria naturae, secundum classes, ordines, genera, speciescum characteribus, differentiis, synomymis, locis, Editio decima reformata, Vol. 1. Stockholm: Laurentii Salvii.

Lopez Martinez, N. 1985. Reconstruction of ancestral cranioskeletal features in the order Lagomorpha. In W.P. Luckett, and J.-L. Harten- berger (editors), Evolutionary relationships among rodents: a multidisciplinary analysis: 151-189. New York: Plenum.

Lucas, P.W. 1979. The dental-dietary adaptations of mammals. Neues Jahrbuch fur Geologie und Palaontologie Monashefte 8: 486-512.

Lucas, P.W., and C.R. Peters. 2000. Function of postcanine tooth crown shape in mammals. In M.F. Teaford, M.M. Smith, and M.W.J. Ferguson (editors), Development, function and evolution of teeth: 282-289. Cambridge: Cambridge University Press.

Luckett, W.P. 1977. Ontogeny of amniote fetal membranes and their application to phylogeny. In M.K. Hecht, P.C. Goody, and B.M. Hecht (editors), Major pattern in vertebrate evolution: 439-516. New York: Plenum.

Luckett, W.P. 1980. Comparative biology and evolutionary relationships of tree shrews. New York: Plenum.

Luckett, W.P. 1985. Superordinal and intraordinal affinities of rodents: developmental evidence from dentition and placentation. In W.P. Luckett and J.-L. Hartenberger (editors), Evolutionary relationships among rodents: a multidisciplinary analysis: 227-276. New York: Plenum.

Luckett, W.P. 1993. Developmental evidence from the fetal membranes for assessing Archontan relationships. In R.D.E. MacPhee (editor), Primates and their relatives in phylogenetic perspective: 149-186. New York: Plenum.

Luckett, W.P., and J.-L. Hartenberger. 1985. Evolutionary relationships among rodents: comments and conclusions. In W.P. Luckett and J.L. Hartenberger (editors), Evolutionary relationships among rodents: a multidisciplinary analysis: 685-712. New York: Plenum.

Luckett, W.P., and J.-L. Hartenberger. 1993. Monophyly or polyphyly of the order Rodentia: possible conflict between morphological and molecular interpretations. Journal of Mammalian Evolution 1: 127-147.

Luo, Z.-X., and D.R. Ketten. 1991. CT scanning and computerized reconstructions of the inner ear of multituberculate mammals. Journal of Vertebrate Paleontology 11: 220-228.

Lyngstadaas, S.P., C.B. Møinichen, and S. Risnes. 1998. Crown morphology, enamel distribution, and enamel structure in mouse molars. Anatomical Record 250: 268-280.

Ma, A.-C., and Chen, J. 1991. On biostratigraphical subdivision of Yuhuangding Formation in Li Guanqiao Basin of eastern Qinling region. Scientia Geologica Sinica 1991: 21-29.

Ma, D.-P., A. Zharkikh, D. Graur, J.L. VandeBerg, and W.-H. Li. 1993. Structure and evolution of opposum, guinea pig, and porcupine cyto- 
chrome $b$ genes. Journal of Molecular Evolution 36: 327-334.

Maas, M.C. 1989. Enamel microwear: an experimental study of cause and effect. American Journal of Physical Anthropology 78: 264-265.

MacLeod, N., and K.D. Rose. 1993. Inferring locomotor behavior in Paleogene mammals via eigenshape analysis. American Journal of Science 293A: 300-355.

MacPhee, R.D.E. 1981. Auditory regions of primates and eutherian insectivores. morphology, ontogeny, and character analysis. Contributions to Primatology 18: 1-282.

MacPhee, R.D.E. 1993. Summary. In R.D.E. MacPhee (editor), Primates and their relatives in phylogenetic perspective: 363-373. New York: Plenum.

MacPhee, R.D.E., and M. Cartmill. 1986. Basicranial structures and primate systematics. In D.R. Swindler and J. Erwin (editors), Comparative primate biology 1: systematics, evolution, and anatomy: 219-275. New York: Alan R. Liss.

MacPhee, R.D.E., M.J. Novacek, and G. Storch. 1988. Basicranial morphology of early Tertiary erinaceomorphs and the origin of primates. American Museum Novitates 2921: 1-42.

Maddison, W.P., M.J. Donoghue, and D.R. Maddison. 1984. Outgroup analysis and parsimony. Systematic Zoology 33: 83-103.

Maddison, W.P., and D.R. Maddison. 1992. MacClade-analysis of phylogeny and character evolution. Sunderland, MA: Sinauer.

Madsen, O., M. Scally, C.J. Douady, D.J. Kao, R.W. DeBry, R. Adkins, H.M. Amrine, M.J. Stanhope, W.W. de Jong, and M.S. Springer. 2001. Parallel adaptive radiations in two major clades of placental mammals. Nature 409: 610614.

Major, C.J.F. 1893. On some Miocene squirrels. Proceedings of the Zoological Society of London 1893: 179-215.

Marcus, L. 1990. Traditional morphometrics. In F.J. Rohlf and F.L. Bookstein (editors), Proceedings of the Michigan Morphometrics Workshop: 77-122. Ann Arbor: University of Michigan Museum of Zoology.

Marsh, O.C. 1874. Small size of the brain in Tertiary mammals. American Journal of Sciences and Arts 8: 535.

Martignetti, J.A., and J. Brosius. 1993. Neural BC1 RNA as an evolutionary marker: guinea pig remains a rodent. Proceedings of the National Academy of Science of the United States of America 90: 9698-9702.

Martin, T. 1992. Schmelzmikrostructur in den incisiven alt-und neuweltlicher hystricognather
Nagetiere. Palaeovertebrata, Mémoire Extraordinaire 1-f 168.

Martin, T. 1993. Early rodent incisor enamel evolution: phylogenetic implications. Journal of Mammalian Evolution 1: 227-254.

Martin, T. 1994. On the systematic position of Chaetomys subspinosus (Rodentia, Caviomorpha) based on evidence from the incisor enamel microstructure. Journal of Mammalian Evolution 2: 117-131.

Martin, T. 1997. Incisor enamel microstructure and systematics in rodents. In W.v. Koenigswald and P.M. Sander (editors), Tooth enamel microstructure: 163-175. Rotterdam: Balkema.

Martin, T. 1999. Phylogenetic implications of Glires (Eurymylidae, Mimotonidae, Rodentia, Lagomorpha) incisor enamel microstructure. Mitteilungen aus dem Museum für Naturkunde in Berlin Zoologische Reihe 75: 257-273.

Matthew, W.D. 1910. On the osteology and relationships of Paramys and the affinities of the Ischyromyidae. Bulletin of the American Museum of Natural History 28: 43-72.

Matthew, W.D., and W. Granger. 1925. Fauna and correlation of the Gashato Formation of Mongolia. American Museum Novitates 189: 1-12.

Matthew, W.D., W. Granger, and G.G. Simpson. 1929. Additions to the fauna of the Gashato Formation of Mongolia. American Museum Novitates 376: 1-12.

McKenna, M.C. 1961. A note on the origin of rodents. American Museum Novitates 2037: 1-5.

McKenna, M.C. 1963. New evidence against tupaioid affinities of the mammalian Family Anagalidae. American Museum Novitates 2158: $1-16$.

McKenna, M.C. 1975. Toward a phylogenetic classification of the Mammalia. In W.P. Luckett, and F.S. Szalay (editors), Phylogeny of the primates: 21-46. New York: Plenum.

McKenna, M.C. 1982. Lagomorpha interrelationships. Géobios Mémoire Spécial 6: 213-224.

McKenna, M.C. 1994. Early relatives of flopsy, mopsy, and cottontail. Natural History 103: 5658.

McKenna, M.C., and S.K. Bell. 1997. Classification of mammals above the species level. New York: Columbia University Press.

McKenna, M.C., Z. Kielan-Jaworowska, and J. Meng. 2000. Earliest eutherian mammal skull, from the late Cretaceous (Coniacian) of Uzbekistan. Acta Palaeontologica Polonica 45: 1-54.

McKenna, M.C., and J. Meng. 2001. A primitive relative of rodents from the Chinese Paleocene. Journal of Vertebrate Paleontology 21: 565572.

Meng, J. 1990. The auditory region of Reithroparamys delicatissimus (Rodentia, Mammalia) 
and its systematic implications. American $\mathrm{Mu}-$ seum Novitates 2972: 1-35.

Meng, J. 1991. Auditory features of Palaeolagus (Lagomorpha, Mammalia) and its implications to some character polarities. Journal of Vertebrate Paleontology 11 suppl. 3: 47A.

Meng J. 2000. Phylogeny and divergence of Rodentia (Mammalia). Journal of Vertebrate $\mathrm{Pa}$ leontology 20, suppl.58A.

Meng, J. 2001 Glires (rodents and lagomorphs) from Asia: implications to phylogeny and divergence of mammals. In An International Symposium on Exploring the History of Life on the Earth: paleontology in China during the last 15 Years. Abstracts of Papers: 41-42. Beijing: National Natural Science Foundation of China.

Meng, J., and R.C. Fox. 1995. Osseous inner ear structures and hearing in early marsupials and placentals. Zoological Journal of the Linnean Society 115: 47-71.

Meng, J., and A.R. Wyss. 1994. The enamel microstructure of Tribosphenomys (Mammalia, Glires): functional and phylogenetic implications. Journal of Mammalian Evolution 2: 185203.

Meng, J., and A.R. Wyss. 2001. The morphology of Tribosphenomys (Rodentiaformes, Mammalia): phylogenetic implications for basal Glires. Journal of Mammalian Evolution 8: 1-71.

Meng, J., A.R. Wyss, M.R. Dawson, and R.-J. Zhai. 1994. Primitive fossil rodent from Inner Mongolia and its implications for mammalian phylogeny. Nature 370: 134-136.

Meng, J., R-J. Zhai, and A.R. Wyss. 1998. The late Paleocene Bayan Ulan fauna of Inner Mongolia, China. Special volume of the Symposium on Cretaceous and Early Tertiary Mammals of Asia. Bulletin of Carnegie Museum of Natural History 34: 148-185.

Meredith, M., and G. Fernández-Fewell. 1994. Vomeronasal system, LHRH, and sex behaviour. Psychoneuroendocrinology 19: 657-672.

Merriam, C.H. 1895. Monographic revision of the pocket gophers, family Geomyidae (exclusive of the species of Thomomys). North American Fauna 8: 1-258.

Merwe, M.v.d., and A.J. Botha. 1998. Incisors as digging tools in molerats (Bathyergidae). South African Journal of Zoology 33: 230-235.

Mess, A., B. Mohr, and T. Martin. 2001. Evolutionary transformations of hystricognath Rodentia and the climatic change in the Eocene to Late Oligocene time interval. Mitteilungen aus dem Museum für Naturkunde in Berlin Zoologische Reihe 77: 193-206.

Miller, M.E. 1964. Anatomy of the dog. London: W. B. Saunders.
Mills, J.R.E. 1966. The functional occlusion of the teeth of Insectivora. Zoological Journal of the Linnean Society 47: 1-25.

Mills, J.R.E. 1978. The relationship between tooth patterns and jaw movements in the Hominoidea. In P.M. Butler and K.A. Joysey (editors), Development, function, and evolution of teeth: 341-353. London: Academic Press.

Miyamoto, M.M., and Goodman, M. 1986. Biomolecular systematics of eutherian mammals: phylogenetic patterns and classification. Systematic Zoology 35: 230-240.

Mödden, C., and M. Wolsan, 2000. External brain morphology of the late Oligocene musteloid carnivoran Bavarictis gaimersheimensis. Acta Palaeontologica Polonica 45: 301-310.

Moodie, R.L. 1916. On the sinus paranasales of two early Tertiary mammals. Journal of Morphology 28: 141.

Moodie, R.L. 1922. On the endocranial anatomy of some Oligocene and Pleistocene mammals. Journal of Comparative Neurology 34: 348371.

Moody, P.A., V.A. Cochran, and H. Drugg. 1949. Serological evidence on lagomorph relationships. Evolution 3: 25-33.

Moore, W.J. 1981. The mammalian skull. Cambridge: Cambridge University Press.

Moss, M.L. 1968. Functional cranial analysis of mammalian mandibular ramal morphology. Acta Anatomica 71: 423-447.

Moss, M.L., and M.-A. Meehan. 1970. Functional cranial analysis of the coronoid process in the rat. Acta Anatomica 77: 11-24.

Moss-Salentijn, L. 1978. Vestigial teeth in the rabbit, rat and mouse. Their relationship to the problem of lacteal dentitions. In P.M. Butler and K.A. Joysey (editors), Development, function, and evolution of teeth: 13-29. London: Academic Press. W.P. Luckett (editor), Comparative biology and evolutionary relationships of tree shrews: 35-93. New York: Plenum.

Muizon, C.de. 1998. Mayulestes ferox, a borhyaenoid (Metatheria, Mammalia) from the early Palaeocene of Bolivia: phylogenetic and palaeobiologic implications. Geodiversitas 20: 19-142.

Müller, A. 1933. Die Kaumuskulatur des Hydrochoerus capybara und ihre Bedeutung für die Formgestaltung des Schädels. Morphologisches Jahrbuch 72: 1-59.

Murphy, W.J., E. Eizirik, W.E. Johnson, Y.-P. Zhang, O.A. Ryder, and S.J. O'Brien. 2001a. Molecular phylogenetics and the origins of placental mammals. Nature 409: 614-618.

Murphy, W.J., E. Eizirik, S.J. O’Brien, O. Madsen, M. Scally, C.J. Douady, E. Teeling, O.A. Ryder, M.J. Stanhope, W.W. de Jong, and M.S. 
Springer. 2001b. Resolution of the early placental mammal radiation using Bayesian phylogenetics. Science 294: 2348-2351.

Negus, V. 1958. The comparative anatomy and physiology of the nose and paranasal sinuses. London: Livingstone.

Nessov, L.A. 1987. Results of search and investigation of Cretaceous and Early Paleogene mammals on the territory of the USSR. Ezhegodnik Vsesoiuznogo Paleontologicheskogo Obshchestva 30: 199-218. [in Russian]

Novacek, M.J. 1977. Aspects of the problem of variation, origin, and evolution of the eutherian audirory bulla. Mammal Review 7: 131-149.

Novacek, M.J. 1980. Cranioskeletal features in tupaiids and selected Eutheria as phylogenetic evidence. In W.P. Luckett (editor), Comparative biology and evolutionary relationships of tree shrews: 35-93. New York: Plenum.

Novacek, M.J. 1982a. The brain of Leptictis dakotensis, an Oligocene leptictid (Eutheria: Mammalia) from North America. Journal of Paleontology 56: 1177-1186.

Novacek, M.J. 1982b. Information for molecular studies from anatomical and fossil evidence on higher eutherian phylogeny. In M. Goodman (editor), Macromolecular sequences in systematic and evolutionary biology: 3-41. New York: Plenum.

Novacek, M.J. 1985. Cranial evidence for rodent affinities. In W.P. Luckett and J.-L. Hartenberger (editors), Evolutionary relationships among rodents-a multidisciplinary analysis: $59-81$. New York: Plenum.

Novacek, M.J. 1986a. The primitive eutherian dental formula. Journal of Vertebrate Paleontology 6: 191-196.

Novacek, M.J. 1986b. The skull of leptictid insectivorans and the higher-level classification of eutherian mammals. Bulletin of the American Museum of Natural History 183: 1-111.

Novacek, M.J. 1990. Morphology, paleontology, and the higher clades of mammals. In H.H. Genoways (editor), Current mammalogy 2: 5981. New York: Plenum.

Novacek, M.J. 1992. Mammalian phylogeny: shaking the tree. Nature 356: 121-125.

Novacek, M.J. 1993a. Mammalian phylogeny: morphology and molecules. Trends in Ecology and Evolution 8: 339-340.

Novacek, M.J. 1993b. Patterns of diversity in the mammalian skull. In J. Hanken and B.K. Hall (editors), The skull: patterns of structural and systematic diversity: 438-545. Chicago: University of Chicago Press.

Novacek, M.J. 1996. Where do rabbits and kin fit in? Nature 379: 299-300.

Novacek, M.J., K.-Q. Gao, M.A. Norell, and G.W.
Rougier. 1998. Ghost lineages, phylogeny, and ranges of selected vertebrate lineages across the K/T boundary. Journal of Vertebrate Paleontology Supplement 18: 67A.

Novacek, M.J., G.W. Rougier, D. Dashzeveg, and M.C. McKenna. 2000. New eutherian mammal from the Late Cretaceous of Mongolia and its bearing on the origins of the modern placental radiation. Journal of Vertebrate Paleontology Supplement 20: 61A.

Novacek, M.J., G.W. Rougier, J.R. Wible, M.C. McKenna, D. Dashzeveg, and I. Horovitz. 1997. Epipubic bones in eutherian mammals from the Late Cretaceous of Mongolia. Nature 389: 483-486.

Novacek, M.J., and A.R. Wyss. 1986. Higher-level relationships of the recent eutherian orders: morphological evidence. Cladistics 2: 257-286.

Nowak, R.M. 1999. Walker's mammals of the world, 6th ed. Baltimore, MD: Johns Hopkins University Press.

Offermans, M., and F. de Vree. 1990. Mastication in springhare: a cineradiographic study. Journal of Morphology 205: 353-367.

Offermans, M., and F. de Vree. 1993. Electromyography and mechanics of mastication in the springhare, Pedetes capensis (Rodentia, Pedetidae). Belgian Journal of Zoology 123: 231261.

O'leary M.A., and K.D. Rose. 1995. Postcranial skeleton of the Early Eocene mesonychid Pachyaena (Mammalia: Mesonychia). Journal of Vertebrate Paleontology 15: 401-430.

Olson, E.C. 1971. Vertebrate paleozoology. New York: Wiley- Interscience.

Omland, K.E. 1997. Correlated rates of molecular and morphological evolution. Evolution 51: 1381-1393.

Ooë, T. 1980. Développment embryonnaire des incisives chez le lapin (Oryctolagus cuniculus L.) - Interprétation de la formule dentaire. Mammalia 44: 259-269.

Osborn, H.F. 1902. American Eocene primates, and the supposed rodent family Mixodectidae. Bulletin of the American Museum of Natural History 17: 169-214.

Palmer, R.W. 1913. The brain and brain case of a fossil ungulate of the genus Anoplotherium. Proceedings of the Zoological Society of London, part 4: 878-893.

Parker, W.K. 1885a. On the structure and development of the skull in the Mammalia-Part II. Edentata. Philosophical Transactions of the Royal Society of London 176: 1-120.

Parker, W.K. 1885b. On the structure and development of the skull in the Mammalia-Part III Insectivores. Philosophical transactions of the Royal Society of London 176: 121-276. 
Parrington, F.R., and T.S. Westoll. 1940. On the evolution of the mammalian palate. Philosophical transactions of the Royal Society of London 230: 305-355.

Patterson, B., and A.E. Wood. 1982. Rodents from the Deseadan Oligocene of Bolivia and the relationships of the Caviomorpha. Bulletin of the Museum of Comparative Zoology 149: 371-543.

Paulli, S. 1900a. Ueber die Pneumaticität des Schädels bei den Säugethieren. I. Ueber den Bau des Siebbeins. Ueber die Morphologie des Siebbeins der Pneumaticität bei den Monotremen und den Marsupialiern. Morphologisches Jahrbuch 28: 147-178.

Paulli, S. 1900b. Ueber die Pneumaticität des Schädels bei den Säugethieren. II. Ueber die Morphologie des Siebbeins und die Pneumaticität bei den Ungulaten und Probosciden. Morphologisches Jahrbuch 28: 179-251.

Paulli, S. 1900c. Ueber die Pneumaticität des Schädels bei den Säugethieren. III. Ueber die Morphologie des Siebbeins und die Pneumaticität bei den Insectivoren, Hyracoideen, Chiropteren, Carnivoren, Pinnipedien, Edentates, Rodentiern, Prosimien und Primaten. Morphologisches Jahrbuch 28: 483-564.

Penny, D., M.D. Hendy, and M.A. Steel. 1991. Testing the theory of descent. In M.M. Miyamoto and J. Cracraft (editors), Phylogenetic analysis of DNA sequences: 155-183. London: Oxford University Press.

Pfretzschner, H.U. 1988. Structural reinforcement and crack propagation in enamel. Mémoires du Muséum national d'Histoire Naturelle Série C 53: 133-143.

Poonkhum, R., S. Pongmayteegul, W. Meeratana, W. Pradidarcheep, S. Thongpila, T. Mingsakul, and R. Somana. 2000. Cerebral Microvascular architecture in the common tree shrew (Tupaia glis) revealed by plastic corrosion casts. Microscopy Research and Technique 50: 411-419.

Poran, N.S. 1998. Vomeronasal organ and its associated structures in the opossum Monodelphis domestica. Microscopy Research and Technique 43: 500-510.

Porter, C.A., M. Goodman, and M.J. Stanhope. 1996. Evidence on mammalian phylogeny from sequences of Exon 28 of the von Willebrand factor gene. Molecular Phylogenetics and Evolution 5: 89-101.

Presley. R. 1993. Preconception of adult structural pattern in the analysis of the developing skull. In J. Hanken and B.K. Hall (editors), The skull: patterns of structural and systematic diversity: 347-377. Chicago: University of Chicago Press.

Presley, R., and F.L.D. Steel. 1978. The pterygoid and ectopterygoid in mammals. Anatomy and Embryology 154: 95-110.

Radinsky, L. 1973. Evolution of the canid brain. Brain Behavior and Evolution 7: 169-202.

Radinsky, L. 1974. The fossil evidence of anthropoid brain evolution. American Journal of Physical Anthropology. 41: 15-28.

Radinsky, L. 1975. Evolution of the felid brain. Brain Behavior and Evolution 11: 214-254.

Radinsky, L. 1976. The brain of Mesonyx, a middle Eocene mesonychid condylarth. Fieldiana 33: 323-337.

Radinsky, L. 1977. Brain of early carnivores. Paleobiology 3: 333-349.

Radinsky, L. 1978. Evolution of brain size in carnivores and ungulates. The American Naturalist 112: 815-831.

Radinsky, L. 1981. Brain evolution in extinct South American ungulates. Brain Behavior and Evolution 18: 169-187.

Radinsky, L. 1985. Patterns in the evolution of ungulate jaw shape. American Zoologist 25: 303-314.

Remy, J.A. 1999. Un nouveau genre de ?Palaeotheriidae (Perissodactyla, Mammalia) decele dans les phosphorites du Quercy (Eocene superieur ou Oligocene) d'apres un arriere-crâ sans dents. Palaeovertebrata (Montpellier) 28: 75-87.

Rensberger, J.M. 1973. An occlusion model for mastication and dental wear in herbivorous mammals. Journal of Paleontology 47: 515528.

Rensberger, J.M. 1975. Function in the cheek tooth evolution of some hypsodont geomyoid rodents. Journal of Paleontology 49: 10-22.

Rensberger, J.M. 1978. Scanning electron microscopy of wear and occlusal events in some small herbivores. In P.M. Butler and K.A. Joysey (editors), Development, function, and evolution of teeth: 413-438. London: Academic Press.

Rensberger, J.M. 1982. Patterns of dental change in two locally persistent successions of fossil aplodontid rodents. In B. Kurten (editor), Teeth: form, function and evolution: 333-349. New York: Columbia University Press.

Rensberger, J.M. 1995. Determination of stresses in mammalian dental enamel and their relevance to their interpretation of feeding behavior in extinct taxa. In J. Thomason (editor), Functional morphology in vertebrate paleontology: 151-172. Cambridge: Cambridge University Press.

Rensberger, J.M. 2000. Pathways to functional differentiation in mammalian enamel. In M.F. Teaford, M.M. Smith, and M.W.J. Ferguson (editors), Development, function and evolution of teeth: 252-268. Cambridge: Cambridge University Press. 
Rensberger, J.M., and W.v. Koenigswald. 1980. Functional and phylogenetic interpretation of enamel microstructure in rhinoceroses. Paleobiology 6: 477-495.

Rich, T.H., P. Vickers-Rich, A. Constantine, T.F. Flannery, L. Kool, and N. van Klaveren. 1997. A tribosphenic mammal from the Mesozoic of Australia. Science 278: 1431-1438.

Rich, T.H., P. Vickers-Rich, A. Constantine, T.F. Flannery, L. Kool, and N. van Klaveren. 1999. Early Cretaceous mammals from Flat Rocks, Victoria, Australia. Records of Queen Victoria Museum 106: 1-30.

Rich, T.H., P. Vickers-Rich, P. Trusler, T.F. Flannery, R. Ciffelli, A. Constantine, L. Kool, and N. van Klaveren. 2001. Corroboration of the Garden of Eden hypothesis. In I. Metcalfe, J.M.B. Smith, M. Morwood, I. Davidson, and K. Hewison (editors), Faunal and floral migrations and evolution in SE Asia-Australia: 324332. Lisse: A.A. Balkema.

Rinker, G.C. 1954. The comparative myology of the mammalian genera Sigmodon, Oryzomys, Neotoma, and Peromyscus (Cricetinae), with remarks on their intergeneric relationships. Miscellaneous Publications Museum of Zoology University Michigan 83: 1-124.

Roberts, D. 1974. Structure and function of the primate scapula. In F.A. Jr. Jenkins (editor), Primate locomotion: 171-200. New York: Academic Press.

Rodriguez-Trelles F., R. Tarrío, and F.J. Ayala. 2001. Erratic overdispersion of three molecular clocks: GPDH, SOD, and XDH. Proceedings of the National Academy of Science of the United States of America 98: 11405-11410.

Rose, K.D. 1999. Postcranial skeleton of Eocene Leptictidae (Mammalia), and its implication for behavior and relationship. Journal of Vertebrate Paleontology, 19: 355-372.

Rose, K.D., and S.G. Lucas. 2000. An Early Paleocene palaeanodont (Mammalia, ?Pholidota) from New Mexico, and the origin of Palaeanodonta. Journal of Vertebrate Paleontology 20: 139-156.

Ross, C., B. Williams, and R.F. Kay. 1998. Phylogenetic analysis of anthropoid relationships. Journal of Human Evolution 35: 221-306.

Rougier, G.W., J.R Wible, and M.J. Novacek. 1998. Implications of Deltatheridium specimens for early marsupial history. Nature 396: 459-463.

Russell, D.E. 1964. Les mammifères paléocènes d'Europe. Mémoires du Muséum national d'Histoire Naturelle Série C 13: 1-324.

Russell, D.E., and R.-J. Zhai. 1987. The Palaeogene of Asia: mammals and Stratigraphy. Sciences de la Terre Series C 52: 1-488.
Russell, L.S. 1959. The dentition of rabbits and the origin of lagomorphs. National Museum of Canada Bulletin 166: 41-45.

Ryan, J.M. 1989. Comparative myology and phylogenetic systematics of the Heteromyidae (Mammalia, Rodentia). Miscellaneous Publications, Museum of Zoology University Michigan 176: 1-103.

Ryder, J.A. 1978. On the mechanical genesis of tooth-forms. Proceedings of the Academy of Natural Sciences of Philadelphia 79: 45-80.

Sahni, A. 1980. SEM studies of Eocene and Siwalik rodent enamels. Geoscience Journal 1/2: 21-30.

Sahni, A. 1985. Enamel structure of early mammals and its role in evaluating relationships among rodents. In W.P. Luckett and J.-L. Hartenberger (editors), Evolutionary relationships among rodents: a multidisciplinary analysis: 133-150. New York: Plenum.

Sánchez-Villagra, M.R. 2001. Ontogenetic and phylogenetic transformation of the vomeronasal complex and nasal floor elements in marsupial mammals. Zoological Journal of the Linnean Society 131: 459-479.

Sanderson, M.J. (1995). Objections to bootstrapping phylogenies: A critique. Systematic Biology 44: 299-320.

Sargis, E.J. 2001. The grasping behaviour, locomotion and substrate use of the tree shrews $\mathrm{Tu}$ paia minor and T. tana (Mammalia, Scandentia). Journal of Zoology (London) 253: 485490.

Sarich, V.M. 1985. Rodent macromolecular systematics. In W.P. Luckett, and J.-L. Hartenberger (editors), Evolutionary relationships among rodents-a multidisciplinary analysis: 423452. New York: Plenum.

Sarich, V.M. 1991. A molecular perspective on primate origins. American Journal of Primatology 12(Suppl. 1): 157.

Satoh, K. 1997. Comparative functional morphology of mandibular forward movement during mastication of two murid rodents, Apodemus speciosus (Murinae) and Clethrionomys rufocanus (Arvicolinae). Journal of Morphology 231: 131-142.

Satoh, K. 1998. Balancing function of the masticatory muscles during incisal biting in two murid rodents, Apodemus speciosus and Clethrionomys rufocanus. Journal of Morphology 236: 49-56.

Satoh, K. 1999. Mechanical advantage of area of origin for the external pterygoid muscle in two murid rodents, Apodemus speciosus and Clethrionomys rufocanus. Journal of Morphology 240: $1-14$

Saveliev, S.V., and A.V. Lavrov. 2001. A morpho- 
functional reconstruction of the brain of Neohyaenodon horridus (Hyaenodontidae, Creodonta) based on a natural endocranial cast. $\mathrm{Pa}$ leontological Journal 35: 75-83.

Scapino, R.P. 1972. Adaptive radiation of mammalian jaws. In G.-H. Schumacher (editor), Morphology of the maxillo-mandibular apparatus: 33-39. Leipzig: Thieme.

Schaeffer, B. 1947. Notes on the origin and function of the artiodactyls tarsus. American $\mathrm{Mu}-$ seum Novitates 1356: 1-24.

Schumacher G.-H. 1961. Funktionelle Morphologie der Kaumuskulatur. Jena: Gustav Fischer.

Schumacher, G.-H., D. Ivánkievicz, and J. Fanghänel. 1979. The role of function in the formation of the skull. Acta Morphologica Academiae Scientiarum Hungaricae 27: 53-56.

Scott, W.B. 1905. Mammalia of Santa Cruz beds. Reports of the Princeton University Expeditions to Patagonia 5: 1-449.

Shevyreva, N.S. 1989. New rodents (Ctenodactyloidea, Rodentia, Mammalia) from the Lower Eocene of Mongolia. Journal of Palaeontology 3: 60-72. [in Russian]

Shevyreva, N.S. 1995. The oldest lagomorphs (Lagomorpha, Mammalia) of the Eastern Hemisphere. Doklady Akademii Nauk SSSR 345: 377-379.

Shevyreva, N.S., V.M. Chkhikvadze, and V.I. Zhegallo. 1975. New data on the vertebrate fauna of the Gashato Formation, Mongolian People's Republic. Bulletin of the Georgian Academy of Science 77: 225-228.

Shevyreva, N.S., and L. Gabunia. 1986. The first discovery of Eurymylidae (Mixodontia, Mammalia) in USSR. Journal of Palaeontology 4: 77-82. [in Russian]

Shoshani, J., and M.C. McKenna. 1998. Higher taxonomic relationships among extant mammals based on morphology, with selected comparisons of results from molecular data. Molecular Phylogenetics and Evolution 9: 572-584.

Simmons, N.B. 1993. Phylogeny of Multituberculata. In F.S. Szalay, M.J. Novacek, and M.C. McKenna (editors), Mammal phylogeny: Mesozoic differentiation, multituberculates, monotremes, early therians, and marsupials: 146164. New York, Springer.

Simmons, N.B., and J.H. Geisler. 1998. Phylogenetic relationships of Icaronycteris, Archaeonycteris, Hassianycteris, and Palaeochiropteryx to extant bat lineages, with comments on the evolution of echolocation and foraging strategies in Microchiroptera. Bulletin of the American Museum of Natural History 235: 4182.

Simpson, G.G. 1926. Cretaceous mammal skull from Mongolia. American Museum Novitates 225: $1-20$.

Simpson, G.G. 1927. Mesozoic Mammalia. IX. The brain of Jurassic mammals. American Journal of Science XIV: 259-268.

Simpson, G.G. 1928a. Further notes on Mongolian Cretaceous mammals. American Museum Novitates 329: 1-9.

Simpson, G.G. 1928b. Affinities of the Mongolian Cretaceous insectivores. American Museum Novitates 330: 1-11.

Simpson, G.G. 1931. A new insectivore from the Oligocene, Ulan Gochu horizon, of Mongolia. American Museum Novitates 505: 1-22.

Simpson, G.G. 1945. The principles of classification and a classification of mammals. Bulletin of the American Museum of Natural History 85: $1-350$.

Simoens, P., H. Lauwers, W. Verraes, and A. Huysseune. On the homology of the incisor teeth in the rabbit. Belgian Journal of Zoology 125: 315-327.

Skogland, T. 1988. Tooth wear by food limitation and its life history consequences in wild reindeer. Oikos 51: 238-242.

Smith, A.G., D.G. Smith, and B.M. Funnell. 1994. Atlas of Mesozoic and Cenozoic Coastlines. Cambridge: Cambridge University Press.

Smith, M.J., and R.J.G. Savage. 1956. Some locomotory adaptations in mammals. Zoological Journal of the Linnean Society 42: 603-622.

Solounias, N., and L.-A.C. Hayek, 1993. New methods of tooth microwear and application to dietary determination of two extinct antelopes. Journal of Zoology (London) 229: 42-445.

Solounias, N., M. Teaford, and A. Walker. 1988. Interpreting the diet of extinct ruminants: the case of a nonbrowsing giraffid. Paleobiology 14: 287-300.

Spatz, W.B. 1964. Beitrag zur Kenntnis der Ontogenese des Cranium von Tupaia glis (Diard 1820). Morphologisches Jahrbuch 106: 321416.

Springer, M.S. 1997. Molecular clocks and the timing of the placental and marsupial radiations in relation to the Cretaceous-Tertiary boundary. Journal of Mammalian Evolution 4: 285-302.

Stanhope, M.J., W.J. Bailey, J. Czelusniak, M. Goodman, J.-S. Si, J. Nickerson, J.G. Sgouros, G.A.M. Singer, and T.K. Kleinschmidt. 1993. A molecular view of primate supraordinal relationships from the analysis of both nucleotide and amino acid sequences. In R.D.E. MacPhee (editor), Primates and their relatives in phylogenetic perspective: 251-292. New York: Plenum.

Stanhope, M.J., M.A. Smith, V.G. Waddell, C.A. Porter, M.S. Shivji, and M. Goodman. 1996. 
Mammalian evolution and the interphotoreceptor retinoid binding protein (IRPB) gene: convincing evidence for sereral superordinal clades. Journal of Molecular Evolution 43: 8392.

Stark, D., and H. Wehrli. 1935. Die Kaumuskulatur von Marmota marmota. Zeitschrift für Säugetierkunde 10: 33-38.

Stehlin, H.G. 1912. Die Säugetiere des schweizerischen Eocäens. Critischer Catalog der Materialen. Abhandlungen der Schweizerischen Paläontologischen Gesellschaft 38: 1165-1298.

Stucky, R.K. 1990. Evolution of land mammal diversity in North America during the Cenozoic. Current Mammalogy 2: 375-432.

Sulimski, A. 1968. Paleocene genus Pseudictops Matthew, Granger and Simpson 1929 (Mammalia) and its revision. Palaeontologia Polonica 19: 101-129.

Sullivan, J., and D.L. Swofford. 1997. Are guinea pigs rodents? The importance of adequate models in molecular phylogenetics. Journal of Mammalian Evolution 4: 77-86.

Swofford, D.L. 2000. PAUP*. Phylogenetic Analysis Using Parsimony (*and Other Methods). Version 4. Sunderland, MA: Sinauer.

Sych, L. 1971. Mixodontia, a new order of mammals from the Paleocene of Mongolia. Palaeontologia Polonica 25: 147-158.

Szalay, F.S. 1977. Phylogenetic relationships and a classification of the eutherian Mammalia. In M.K. Hecht, P.C. Goody, and B.M. Hecht (editors), Major pattern in vertebrate evolution: 317-374. New York: Plenum.

Szalay, F.S. 1984. Arboreality: is it homologous in metatherian and eutherian mammals? Evolutionary Biology 18: 215-258.

Szalay, F.S. 1985. Rodent and Lagomorph morphotype adaptations, origins, and relationships: some postcranial attributes analyzed. In W.P. Luckett, and J.-L. Hartenberger (editors), Evolutionary relationships among rodents-a multidisciplinary analysis: $83-132$. New York: Plenum.

Szalay, F.S. 1994. Evolutionary history of the marsupials and an analysis of osteological characters. New York: Cambridge University Press.

Szalay, F.S., and R.L. Decker. 1974. Origins, evolution, and function of the tarsus in late Cretaceous eutherians and Paleocene primates. In F. A. Jr. Jenkins (editor), Primate locomotion: 223-259. New York: Academic Press.

Szalay, F.S., and E. Delson. 1979. Evolutionary history of the primates. New York: Academic Press.

Szalay, F.S., and G. Drawhorn. 1980. Evolution and diversification of the Archonta in an arboreal milieu. In W.P. Luckett (editor), Compara- tive biology and evolutionary relationships of tree shrews: 35-93. New York: Plenum.

Szalay, F.S., and M.C. McKenna. 1971. Beginnings of the age of mammals in Asia: the late Paleocene Gashato fauna, Mongolia. Bulletin of the American Museum of Natural History 144: 269-318.

Szalay, F.S., and E.J. Sargis. 2001. Model-based analysis of postcranial osteology of marsupials from the Paleocene of Itaborai, Brazil, and the phylogenetics and biogeography of Metatheria. Geodiversitas 23: 139-302.

Szalay, F.S., I. Tattersall, and R.L. Decker. 1975. Phylogenetic relationships of Plesiadapis: postcranial evidence. Contributions to Primatology 5: 136-166.

Taylor, A.C., and E.C. Butcher, 1951. The regulation of eruption rate in the incisor teeth of the white rat. Journal of Experimental Zoology 117: $165-188$.

Taylor M.E. 1974. The functional anatomy of the forelimb of some African Viverridae (Carnivora). Journal of Morphology 143: 307-336.

Taylor M.E. 1976. The functional anatomy of the hindlimb of some African Viverridae (Carnivora). Journal of Morphology 148: 227-254.

Teaford, M.F. 1985. Molar microwear and diet in the genus Cebus. American Journal of Physical Anthropology 66: 363-370.

Teaford, M.F., and O.J. Oyen. 1989. In vivo and in vitro turnover in dental microwear. American Journal of Physical Anthropology 80: 447-460.

Teaford, M.F., and A. Walker. 1984. Quantitative differences in dental microwear between primate species with different diets and a comment on the presumed diet of Sivapithecus. American Journal of Physical Anthropology 64: 191-200.

Teilhard, de Chardin P. 1930. On the occurrence of a Mongolian Eocene perissodactyle in the red sandstone of Sichuan, Southwest Honan. Bulletin of the Geological Society of China (Peiping) 9: 331-332.

Thesleff, I., A. Vaahtokari, and A.-M. Partanen. 1995. Regulation of organogenesis. Common molecular mechanisms regulating the development of teeth and other tissues. The International Journal of Developmental Biology 39: $35-50$.

Thewissen, J.G.M. 1990. Evolution of Paleocene and Eocene Phenocadontidae (Mammalia, Condylarthra). University Michigan Papers on Paleontology 29: 1-107.

Thewissen, J.G.M., and D.P. Domning. 1992. The role of phenacodontids in the origin of the modern orders of ungulate mammals. Journal of Vertebrate Paleontology 12: 494-504.

Thorington, R.W. Jr., and K. Darrow. 1996. Jaw 
muscles of Old World squirrels. Journal of Morphology 230: 145-165.

Thorington, R.W. Jr., K. Darrow, and A.D.K. Betts. 1997. Comparative myology of the forelimb of squirrels (Sciuridae). Journal of Morphology 234: 155-182.

Ting, S.-Y., and C.-K. Li. 1984. The structure of the ear region of Rhombomylus (Anagalida, Mammalia). Vertebrate PalAsiatica 22: 92-102.

Ting, S.-Y., J. Meng, M.C. McKenna, and C.-K. Li. 2002. The osteology of Matutinia (Simplicidentata, Mammalia) and its relationship to Rhombomylus. American Museum Novitates 3371: 1-33.

Tong, Y.-S., and M.R. Dawson. 1995. Early Eocene rodents (Mammalia) from Shangdong Province, China. Annals of Carnegie Museum 64: 51-63.

Tullberg, T. 1899. Ueber das System der Nagethiere: eine Phylogenetische Studie. Nova Acta Regiae Societatis Scientiarum Upsaliensis 18: 1-514.

Turnbull, W.D. 1970. Mammalian masticatory apparatus. Fieldiana: Geology 18: 149-356.

Van Kampen, P.N. 1905. Die Tympanalgegend des Saugetierschadels. Morphologisches Jahrbuch 34: 321-722.

Van Valen, L. 1964. A possible origin for rabbits. Evolution 18: 484-491.

Van Valen, L. 1966. Deltatheridia, a new order of mammals. Bulletin of the American Museum of Natural History 132: 1-126.

Van Valen, L. 1971. Adaptive zones and the orders of mammals. Evolution 25: 420-428.

Velhagen, W.A., and V.L. Roth. 1997. Scaling of the mandible in squirrels. Journal of Morphology 232: 107-132.

Vinogradov, B. 1926. Materials for the systematics and the morphology of the rodents. IV. On the mechanism of gnawing and mastication in some fossorial rodents. Annals of the Museum of Zoology, Academy of Sciences of the USSR 27: 275-281.

Voss, R.S. 1988. Systematics and ecology of Ichthyomyine rodents (Muroidea): patterns of morphological evolution in a small adaptive radiation. Bulletin of the American Museum of Natural History 188: 260-493.

Wahlert, J.H. 1968. Variability of rodent incisor enamel as viewed in thin section, and the microstructure of the enamel in fossil and Recent rodent groups. Breviora of the Museum of Comparative Zoology 309: 1-18.

Wahlert, J.H. 1974. The cranial foramina of protrogomorphous rodents, an anatomical and phylogenetic study. Bulletin of the Museum of Comparative Zoology 146: 363-410.

Wahlert, J.H. 1978. Cranial foramina and relation- ships of the Eomyoidea (Rodentia, Geomorpha) skull and upper teeth of Kansasimys. American Museum Novitates 2645: 1-15.

Wahlert, J.H. 1983. Relationships of the Florentiamyidae (Rodentia, Geomyoidea) based on cranial and dental morphology. American Museum Novitates 2769: 1-23.

Wahlert, J.H. 1985. Skull morphologies and relationships of geomyoid rodents. American $\mathrm{Mu}-$ seum Novitates 2812: 1-20.

Wahlert, J.H. 1988. Skull morphology of Gregorymys and relationships of the Entoptychinae (Rodentia, Geomyidae). American Museum Novitates 2922: 1-13.

Wahlert, J.H. 1989. The three types of incisor enamel in rodents. In C.C. Black, and M. Dawson (editors), Papers on fossil rodents in honour of Albert Elmer Wood. Natural History Museum of Los Angeles County Science Series 33: 7-16.

Wahlert, J.H. 2000. Morphology of the auditory region in Paramys copei and other Eocene rodents from North America. American Museum Novitates. 3307: 1-16.

Wahlert, J.H., and W.v. Koenigswald. 1985. Specialized enamel in incisors of eomyid rodents. American Museum Novitates 2832: 1-12.

Wahlert, J.H., S.L. Sawitzke, and M.E. Holden. 1993. Cranial anatomy and relationships of dormice (Rodentia, Myoxidae). American Museum Novitates 3061: 1-32.

Walker, A., H.N. Hoeck, and L. Perez. 1978. Microwear of mammalian teeth as an indicator of diet. Science 201: 908-910.

Wang, B-Y. 1997. The mid-Tertiary Ctenodactylidae (Rodentia, Mammalia) of eastern and central Asia. Bulletin of the American Museum of Natural History, 234: 1-88.

Wang, B.-Y. 2001. On Tsaganomyidae (Rodentia, Mammalia) of Asia. American Museum Novitates 3317: 1-50.

Wang, B.-Y., and M.R. Dawson. 1994. A primitive cricetid (Mammalia: Rodentia) from the middle Eocene of Jiangsu Province, China. Annals of Carnegie Museum 63: 239-256.

Waters, N.E. 1980. Some mechanical and physical properties of teeth. In J.F.V. Vincent and J.D. Currey (editors), The mechanical properties of biological materials: 99-135. Cambridge: Cambridge University Press.

Weber, M. 1904. Die Säugetiere: Einführung in die Anatomie und Systematik der Recenten und Fossilen Mammalia. 8 vols. Jena.

Weidenreich, F. 1941. The brain and its role in the phylogenetic transformation of the human skull. American Philosophical Society Monograph 31: 321-442.

Weijs W.A. 1975. Mandibular movements of the 
albino rat during feeding. Journal of Morphology 145: 107-124.

Weijs, W.A. 1980. Biomechanical models and the analysis of form: a study of the mammalian masticatory apparatus. American Zoologist 20: 707-719.

Weijs, W.A., P. Brugman, and E.M. Klok. 1987. The growth of the skull and jaw muscles and its functional consequences in the New Zealand rabbit (Oryctolagus cuniculus). Journal of Morphology 194: 143-161.

Weijs, W.A., and R. Dantuma. 1975. Electromyography and mechanics of mastication in the albino rat. Journal of Morphology 146: 1-34.

Weijs, W.A., and R. Dantuma. 1981. Functional anatomy of the masticatory apparatus in the rabbit (Oryctolagus cuniculus L.). Netherlands Journal of Zoology 31: 99-147.

Wible, J.R. 1983. The internal carotid artery in early eutherians. Acta Palaeontologica Polonica 28: 281-293.

Wible, J.R. 1986. Transformations in the extracranial course of the internal carotid artery in mammalian phylogeny. Journal of Vertebrate Paleontology 6: 313-325.

Wible, J.R. 1987. The eutherian stapedial artery: character analysis and implications for superordinal relationships. Zoological Journal of the Linnean Society 91: 107-135.

Wible, J.R., and K.P. Bhatnagar. 1996. Chiropteran vomeronasal complex and the interfamilial relationships of bats. Journal of Mammalian Evolution 3: 285-314.

Wible, J.R., and J.R. Martin. 1993. Ontogeny of the tympanic floor and roof in archontans, In R.D.E. MacPhee (editor), Primates and their relatives in phylogenetic perspective: 111-148. New York: Plenum.

Wilkins, K.T. 1988. Prediction of direction of chewing from cranial and dental characters in Thomomys pocket gophers. Journal of Mammalogy 69: 46-56.

Wilkins, K.T., and L.L. Cunningham. 1993. Relationship of cranial and dental features to direction of mastication in tuco-tucos (Rodentia: Ctenomys). Journal of Mammalogy 74: 383-390.

Wilkins, K.T., and C.A. Woods. 1983. Modes of mastication in pocket gophers. Journal of Mammalogy 64: 636-641.

Wilson, R.W. 1949. Early Tertiary rodents of North America. Carnegie Institution of Washington Contribution to Palaeontology 584: 67-164.

Wilson, R.W. 1989. Rodent origin. In C.C. Black and M. Dawson (editors), Papers on fossil rodents in honour of Albert Elmer Wood. Natural History Museum of Los Angeles County Science Series 33: 3-6.

Wöhrmann-Repenning, A. 1984. Vergleichen an- atomische Untersuchungen am Vomeronasalkomplex und am rostralen Gaumen verschiedener Mammalia Teil I. Morphologisches Jahrbuch 130: 501-530.

Wöhrmann-Repenning, A. 1991. Functional aspects of the vomeronasal complex in mammals. Zoologisches Jahrbuch, Anatomie 121: 71-80.

Wöhrmann-Repenning, A. 1992. The anatomical characteristics of the vomeronasal complex in Chinchilla laniger (Molina, 1782) in comparison with other rodents. Zoologisches Jahrbuch, Anatomie 123: 13-21.

Wöhrmann-Repenning, A. 1993. The vomeronasal complex - a dual sensory system for olfaction and taste. Zoologisches Jahrbuch, Anatomie 123: 337-345.

Wolff-Exalto, A. de. 1951. On differences in the lower jaw of animalivorous and herbivorous mammals. Proceedings of the Koninklijke Nederlandse Akademie van Wetenschappen Series C Biological and Medical Sciences 54: 237246; 405-410.

Wood, A.E. 1936. Geomyoid rodents from the middle Tertiary. American Museum Novitates 866: $1-26$.

Wood, A.E. 1937. The mammalian fauna of the White River Oligocene. Part II. Rodentia. Transactions of the American Philosophical Society 28: 157-269.

Wood, A.E. 1942. Notes on the Paleocene lagomorph, Eurymylus. American Museum Novitates 1162: 1-7.

Wood, A.E. 1957. What, if anything, is a rabbit? Evolution 11: 417-427.

Wood, A.E. 1962. The early Tertiary rodents of the family Paramyidae. Transactions of the American Philosophical Society 52: 1-261.

Wood, A.E. 1965. Grades and clades among rodents. Evolution 19: 115-130.

Wood, A.E. 1968. The African Oligocene Rodentia. Part II of Early Cenozoic mammalian faunas, Fayum Province, Egypt. Peabody Museum of Natural History Yale University Bulletin 28: 23-105.

Wood, A.E. 1973. Eocene rodents, Pruett formation, South West Texas; their partinence to the origin of the south American caviomorpha. Pearce Sellards Series, Texas Memorial Museum 20: 1-39.

Wood, A.E. 1974. The evolution of the Old World and New World hystricomorphs. In I.W. Rowlands and B.J. Weir (editors), Symposium of the Zoological Society on The biology of hystricomorph rodents. Zoological Society of London, Symposium 34: 21-54.

Wood, A.E. 1977. The Rodentia as clues to the Cenozoic migrations between the Americas and Europe and Africa. In R.M. West (editor), Pa- 
leontology and plate tectonics with special reference to the history of the Atlantic Ocean. Special Publications of the Milwaukee Public Museum of Geology 2: 95-109.

Wood, A.E. 1980. The origin of the caviomorph rodents from a source in Middle America: a clue to the area of origin of the platyrrhine primates. In R.L. Ciochon, and A.B. Chiarelli (editors), Evolutionary biology of the New World monkeys and continental drift: 79-91. New York: Plenum.

Wood, A.E. 1984. Hystricognathy in the North American Oligocene rodent Cylindrodon and the origin of the Caviomorpha. In R.M. Mengel (editor), Papers in vertebrate paleontology honoring Robert Warren Wilson. Carnegie Museum of Natural History, Special Publication 9: 151160.

Wood, A.E. 1985. The relationships, origin and dispersal of the hystricognathous rodents. In W.P. Luckett, and J.-L. Hartenberger (editors), Evolutionary relationships among rodents-a multidisciplinary analysis: 475-513. New York: Plenum.

Wood, A.E., and B. Patterson. 1959. The Rodents of the Deseadan Oligocene of Patagonia and the beginnings of South American Rodent evolution. Bulletin of the Museum of Comparative Zoology 120: 282-428.

Wood, A.E., and B. Patterson. 1970. Relationships among Hystricognathous and Hystricomorphous rodents. Mammalia: 34: 628-639.

Wood, A.E., and R.W. Wilson. 1936. A suggested nomenclature for the cusps of the cheek teeth of rodents. Journal of Paleontology 10: 388-391.

Woodburne, M.O., and C.C. Swisher, III, 1995. Land mammal high-resolution geochronology, intercontinental overland dispersals, sea level, climate, and vicariance. Geochronology Time Scales and Global Stratigraphic Correlation, Society for Sedimentary Geology Special Publication No. 54: 335-363.

Woods, C.A. 1975. The hyoid, Laryngeal and pharyngeal regions of bathyergid and other selected rodents. Journal of Morphology 147: 229-250.

Woods, C.A. 1976. How hystricognath rodents chew. American Zoologist 16: 215.

Woods, C.A., and J.W. Hermanson. 1985. Myology of hystricognath rodents: an analysis of form, function, and phylogeny. In W.P. Luckett and J.-L. Hartenberger (editors), Evolutionary relationships among rodents: a multidisciplinary analysis: 515-548. New York: Plenum.

Woods, C.A., and E.B. Howland. 1979. Adaptive radiation of capromyid rodents: anatomy of the masticatory apparatus. Journal of Mammalogy 60: 95-116.

Wyss, A.R., J.J. Flynn, M.A. Norell, C.C. Swisher III, R. Charrier, M.J. Novacek, and M.C. McKenna. 1993. South America's earliest rodent and recognition of a new interval of mammalian evolution. Nature 365: 434-437.

Wyss, A.R., J.J. Flynn, M.A. Norell, C.C. Swisher III, M.J. Novacek, M.C. McKenna, and R. Charrier. 1994. Paleogene mammals from the Andes of central Chile: a preliminary taxonomic, biostratigraphic and geochronologic assessment. American Museum Novitates 3098: 1-31.

Wyss, A.R., and J. Meng. 1996. Application of phylogenetic taxonomy to poorly resolved crown clades: a stem-modified node-based definition of Rodentia. Systematic Biology 45: 559-568.

Xu, Y.-X., D.-F. Yan, S.-J. Zhou, S.-J. Han, and Y.-T. Zhong. 1979. Subdivision of the red beds of Li-Guan-Jiao Basin with description of fossil mammals therefrom. In Institute of Vertebrate Paleontology and Paleoanthropology and Nanking Institute of Geology and Paleontology (editors), Mesozoic and Cenozoic Red Beds of South China: 416-432. Beijing: Science Press. Zeller, U. 1987. Morphogenesis of the mammalian skull with special reference to Tupaia. In H.-J. Kuhn and U. Zeller (editors), Morphogenesis of the mammalian skull: 17-50. Berlin: Parey.

Zeller, U. 1989. Die Entwicklung und Morphologie des Schädels von Ornithorhynchus anatinus (Mammalia: Prototheria: Monotremata). Abhandlungen der Senckenbergischen Naturforschenden Gesellschaft 545: 1-188.

Zhai, R.-J. 1978. More fossil evidence favouring an Early Eocene connection between Asia and Neoarctic. Institute of Vertebrate Paleontology and Paleoanthropology Beijing Memoir 13: 107-115.

Zhai, R-J., Z.-G. Bi, and Z.-J. Yu. 1976. Stratigraphy of Eocene Zhanshanji Formation with note on a new species of eurymylid mammal. Vertebrata PalAsiatica 14: 100-103. 


\section{APPENDIX 1}

\section{Taxa Selected for Analysis and Source of Data $(\dagger=$ EXTinct $)$}

Outgroups

$\dagger$ Asioryctes (Kielan-Jaworowska, 1975, 1977 , 1981, 1984b)

$\dagger$ Kennalestes (Kielan-Jaworowska, 1969, 1977, $1981,1984 b)$

\section{Ingroup nongliroid taxa}

$\dagger$ Zalambdalestes (Simpson, 1928a, 1928b; Kielan-Jaworowska, 1969, 1978, 1984a, 1984b; Novacek et al., 1997)

$\dagger$ Barunlestes (Kielan-Jaworowska, 1975; KielanJaworowska and Trofimov 1980)

$\uparrow$ Hyopsodus (Gazin, 1968; Thewissen and Domning, 1992; NSM 02552)

$\dagger$ Phenacodus (Thewissen, 1990; Thewissen and Domning, 1992; AMNH 293, 296, and 4378)

$\uparrow$ Anagalopsis (Bohlin, 1951; Hu, 1993)

†Anagale (Simpson, 1931; McKenna, 1963; Hu, 1993; AMNH 16079, 26141)

$\dagger$ Leptictis (Novacek, 1986; AMNH 12230, 38829, 128787 [cast], and F:AM 96763)

Cynocephalus (AMNH 102704 [skull] and an unnumbered skeleton)

Tupaia (Luckett, 1980; AMNH 106505, 107597, 215174-8, and 113135)

Petrodromus (Evans, 1942; Corbet and Hanks, 1968; AMNH 187238)

Rhynchocyon (Evans, 1942; Corbet and Hanks, 1968; of AMNH 187232, 49429, 81430, 187231 , and 187233)

$\dagger$ Pseudictops (Sulimski, 1968; unpublished specimens from Inner Mongolia collected by Meng in 2000-2001)

$\dagger$ Plesiadapis (D.E. Russell, 1964; Gingerich, 1976, Szalay, 1977, 1985; Szalay et al., 1975; AMNH 17379, 17388, 17506, 17365, 17174, 17389, 17171, 33822, 88108, 100108, 33978, $86744,101500,111903$ [cast], 111909 [cast], 111922 [cast], 111915 [cast], and 111913 [cast])

$\uparrow$ Notharctus (Gregory, 1920; AMNH 12567 , 11983, 11997, 13134, 12576, 11984, 12588, 11982, 11985 [cast], 111992, 13133, 11997, $12575,12588,11483,12583,11477,12585$, 13024, 11469, 11457, 127167, 11454, 11475, 11474, 1727, 5009, 12001, 93617, and 11449)

$\uparrow$ Adapis (Stehlin, 1912; Gregory, 1920; Gingerich and Martin, 1981; AMNH109277, 109278, 109280-2, 113796, 111935-40, 127401-2, $55360,127393,127399,127400,127403-5$, 129055, 55364, 10004-18, 10408, 11045-7, 11097, 55352-9, 55361, 55362, 93841, 938422, 98203, 10408 [cast], 99979 [cast],
103402 [cast], 99980 [cast], 103403 [cast], 99982 [cast], 11045 [cast], and 99981 [cast])

Ingroup gliroid mammals

Ochotona (VP teaching collection 15519; four skulls with associated jaws of personal collection)

Prolagus (Dawson, 1969)

Lepus (VP teaching collection 5022)

Palaeolagus (Dawson, 1958; AMNH 1042, 5671, 5672, 5679, 6272, 5738, and uncataloged Frick collection)

$\uparrow$ Mimotona ( $\mathrm{Li}$ and Ting, 1985, 1993; IVPP V4327, V4324, V4325.1, V4325.2, V4326, and V4329)

$\uparrow$ Mimolagus (Bohlin, 1951; Bleefeld and McKenna, 1985; Szalay, 1985; IVPP RV51001.2 [returned specimens from Bohlin])

$\uparrow$ Eurymylus (Matthew and Granger, 1925; Matthew et al., 1929; Wood, 1942; Sych, 1971; Dashzeveg and Russell, 1988; AMNH 20422, 21737)

$\uparrow$ Rhombomylus (Zhai et al., 1976; Zhai, 1978; Li and Ting, 1985; Dashzeveg and Russull, 1988. See text for specimens studied.)

†Matutinia (Ting et al., 2002; IVPP V7443, V7444, V7445, V7446, V7447, V7448, V7449, V7450)

$\dagger$ Sinomylus (McKenna and Meng, 2001, IVPP $\mathrm{V} 12431)$

$\dagger$ Heomys (Li, 1977; Li and Ting, 1985; Dashzeveg and Russell, 1988; personal obs. of IVPP V4321, V4322, V4331)

$\dagger$ Tribosphenomys (Meng et al., 1994; Meng and Wyss, 1994; IVPP V10775, V10776, V10778, V10779, V10780a-c)

$\dagger$ Paramys (Wood, 1962; Szalay, 1985; AMNH $4755,4756,11594,12506,48595,17460$, 14726, 16969, 14718, 11577)

$\uparrow$ Reithroparamys (Wood, 1962; Meng, 1990; AMNH 12561, 17031)

$\dagger$ Paradjidaumo (Wilson, 1949; Black, 1965; Wahlert, 1978; AMNH 102153; the fragmentary postcranials [tibia and calcaneus] of this taxon were described by Cope [1883] but the specimens were not found by later worker [Wood, 1937]. Characters from the postcranials were based on the illustrations provided by Cope [1883])

$\dagger$ Florentiamys (Wahlert, 1983; F:AM 183381, 108882 102283, 103384, 103386, 103387, 103389)

†Gregorymys (Wood, 1936; Galbreath, 1967; AMNH 12887-9, 116846) 
$\uparrow$ Sciuravus (Dawson, 1961; personal obs.: AMNH $11614,11609,12548,5030,108241$ [cast]; USNM 64-59)

†Cocomys (Li et al., 1989; Dawson et al., 1984; IVPP V7401, V7399, V7403, V7404, V7405, V5347, V5348, V7409)

†Cricetops (Carrasco and Wahlert, 1999; AMNH 19046, 19051, 19054, 21660)

$\uparrow$ Tataromys (Bohlin, 1946; Wang, 1997; AMNH 19079, 22077, 84208, 85750, IVPP V10534.1)

$\dagger$ Tsaganomys (Bryant and McKenna, 1995; AMNH 19019, 19021, 19023, 19037, 21675, 82273, 83510; IVPP V11390)

$\dagger$ Incamys (Patterson and Wood, 1982)

$\dagger$ Paraphiomys (Lavocat, 1973; AMNH 5396, 114281 [cast], 114282 [cast]) Erethizon (University of Massachusetts, UMA 4001) $\uparrow$ Neoreomys (Scott, 1905; AMNH 9484, 9541-3, 9130, 98903)

Myocastor (AMNH VP teaching collection 1171)

Cavia (Cooper and Schiller, 1975; VP teaching collection 188)

Dasyprocta (AMNH 15669, 189 in teaching collection)

Sciurus (VP teaching collection 1249; AMNH 48093)

Marmota (VP teaching collection, 859)

Rattus (VP teaching collection 148)

Mus (VP teaching collection 203)

Note: Enamel data are from Wahlert (1968, 1989), Martin (1992, 1994, 1999), Flynn et al. (1987), Meng and Wyss (2001), and unpublished data. 


\section{APPENDIX 2}

DATA MATRIX

$\mathrm{A}=(0 / 1) ; \mathrm{B}=(0 / 2) ; \mathrm{C}=(1 / 2) ; \mathrm{D}=(2 / 3) ; \mathrm{E}=(0 / 3) ; \mathrm{F}=(3 / 4)$. Character numbers correspond to those described in the text. At the end of the sequence for each taxon are number of scored states (excluding question marks) and completeness (= number of scored states/227)

1111111111222222222233333333334444444444555555555566666666667777

Taxon/Node

Asioryctes

Kennalestes

zalambdalestes

Barunlestes

Hyopsodus

Phenacodus

Anagalopsis

Anagale

Leptictis

Cynocephalus

Tupaia

Petrodromus

Rhynchocyon

Pseudictops

Plesiadapis

Notharctus

Adapis

Ochotona

Prolagus

Lepus

Palaeolagus

Mimotona

Mimolagus

Eurymylus

Rhombomylus

Matutinia

Sinomylus

Heomys

Tribosphenomys

Paramys

Reithroparamys

Paradjidaumo

Florentiamys

Gregorymys

Sciuravus

Cocomys

Cricetops

Tataromys

Tsaganomys

Incamys

Paraphiomys

Erethizon

Neoreomys

Myocastor

Cavia

Dasyprocta

Sciurus

Marmota

Rattus

Mus 0 ?? $01000000000000001 ? 0000000000000000000000000000000000000000000000000000$ 0 ?? $111010000000001111 \mathrm{~A} 001100000000001000000000000000000004000000000000000$ $10 ? 1110100000000$ ?? $111 \mathrm{~A} 011101100000001000000000000000000004000000000000000$ 0??0110000000000001110111100002000001000000010000000100011000101000011001 0 ?? 0110000000000001110010100002010101010020000000000101011110101000011001

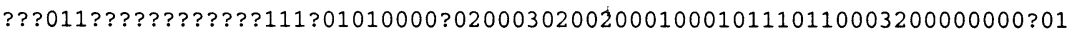
0??0110000000000001110010100002020003020000001000101110110003200000000001 0??011001?000000001110011100000000001010020000000001000000000001000000000 $0 ? ? 011001 ? 00000000111010101111 ? 0000010100200000010010000022001 \mathrm{~B} 0000000101$ 0??01100000000001-1110111111000000001000000000000000000000210020000000001 $0 ? ? 0110001000000001110111100000000403020020021110000300130001041000101011$ 0 ??011001?000000001110001100000000403020020021110000300130001041000A01011 $00 ? 0110000000000001110010100000020003020010001000101110110001210000000000$ 0 ?? $11101010000011 ? 1110 \mathrm{~B} 12111001000000000010000000001100010000031000000001$ $0 ? ? 01100000000011 ? 1110010100001000001000010000000001101011010031000000001$ 0??01100000000011?1110010100001000001000020010000001101011000031000000001 $1 C 12111$ ? $? 1111110211112 ? 2 ? 111030303030200 ? 022101 ? 302100444302361000301101$ 1C12111?1?1111110211112?2?111030303030200?022101?302100444302361000301101 1212111?1?1111110211112?2?111030303030200?0221002302100444302361000301101 $1212111 ? 1 ? 1111110211112$ ?2?111030303030200?0221002302100444302361000301101 $1212111 ? 1 ? 2121100111122$ ?2?111010201020000?01010001011001102A1111000000001

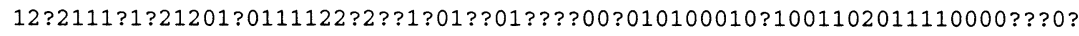
$1113111 ? 1 ? 2120111$ ?11122?2?1111?020102000010001000101100310201011000010001 1C13111?1?2120111?11122?2?1111?020102000010001000101100310201011000010001 $1 ? 13111 ? 1 ? 2120111 ? 11122 ? 2 ? 1111 ? 0201020000001010001011003102 \mathrm{~A} 1011000010001$

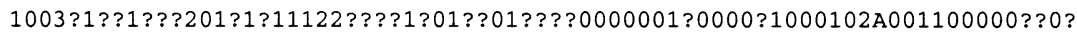
. $1113111 ? 1 ? 2120111 ? 11122 ? 2 ? 1111 ? 020102000010001000101100110201111000010001$ $100 ? 111$ ?1?21C0111?11122?2?1111?1?02020000B0000000001100002200111000000001 $1113111 ? 1 ? 2120111 ? 11122 ? 2 ? 1111$ ?1?0202010020001001001210010210111000010001 1113111 ??2120111????22?2?1111?1?0202010020001001001210010210111000010001

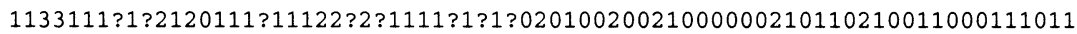
1133111 ?1?2120111?11122?2?1111?1?1?02110121021003000200220200001220?1?111 11?3111?1?212A111?11122?2?1111?1?1?02120121021003000200220201011220?1?111

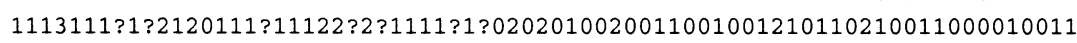
$1113111 ? 1 ? 2120111 ? 11122 ? 2$ ?111?1?02020000000010010012100A0210111000000001 1133111 ??2120111?11122?2?1111?1?1?1???1?????1003003310010200011000111011 1123111?1?2120111?11122?2?1111?1?1?0D0000000010011013101102013110002110C2 1123111?1?2130111?11132?2?1111?1?1?03020030201000101320110202310000000002 1123111 ?:2120111?11132?2?1111?1?1?03020030221000200C30110201351001111122 1123111?1?2120111?11122?2?1111?1?1?03020030221001100220113201311001111022 1123111?1?2120111?11122?2?1111?1?1?03020030221001A11230113201351001111022 1123111?1?2120111?11132?2?1111?1?1?03020030221000210230113201351001111022 1123111 ?:2120111?11122?2?1111?1?1?03020030221001211330113201351001111022 1123111?1?2120111?11122?2?1111?1?1?0302003022100121133011E201351001111122 1123111?1?2120111?11122?2?1111?1?1?0302003022100120133011320135100111??22 1133111 ?1?2120111?11122?2?1111?1?A202010020001001001210010200011000010001

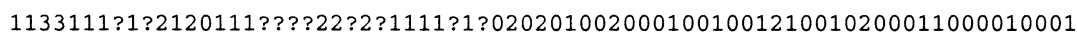
1133111 ?1?2120111?11122?2?1111?1?1?1???1?????1003003310020200031110?1?112 1133111 ?1?2120111?11122?2?1111?1?1?1???1????1003003310020200031110?1?112 
APPENDIX 2
(Continued)

\begin{tabular}{|c|c|}
\hline Taxon/Node & $\begin{array}{r}11111111111111111111111111111111111111111111111 \\
7777778888888888999999999900000000001111111111222222222233333333334444444 \\
4567890123456789012345678901234567890123456789012345678901234567890123456\end{array}$ \\
\hline Asioryctes & $0000000000000000000000 ? 000000000000000000000000000000000000 ? 0000000000000$ \\
\hline Kennalestes & $0000000000000000000 ? 00 ? 000000000 ? 00000000000000000000000000 ? 0000000000000$ \\
\hline Zalambdalestes & $0000000000000000000100000100 ? 00 ? 10 ? 0100000001 \mathrm{~A} 0001000000000 ? 00 ? ? ? 0 ? 000000$ \\
\hline Barunlestes & $0000000000000000000 ? 00 ? 0010000002020 ? 000 ? 0001 \mathrm{~A} 0001000000000000 ? ? ? 0 ? ? 00000$ \\
\hline Hyopsodus & $000100000200000000010 ? 000100 ? 00100001000 ? 000000000000000000 ? 0000000000000$ \\
\hline Phenacodus & $000100000201000000010 ? 100100000100001000 D 00000000000000000000000000000000$ \\
\hline Anagalopsis & $100 \mathrm{~A} 0000010001000001010000000001 ? 1 ? 01000 ? 010000000000000000 ? 001 ? 000000010$ \\
\hline Anagale & $10010000010000000001010000000001000010000010000000000000000 ? 0010000000010$ \\
\hline Leptictis & 0000000000000000000100010100000100011000201000002000000000000000000000000 \\
\hline Cynocephalus & 0000000000000010000101100500000001000002300000000000010000000000000100001 \\
\hline Tupaia & $000000000000001000010010010000000101100220000000000000000000000 ? 000020021$ \\
\hline Petrodromus & 1000000003010020000000010100000100021000201010002000000000000000000000000 \\
\hline Rhynchocyon & $100000000301002000000100010000010002 ? 001 D 01010002000000000000000000000001$ \\
\hline Pseudictops & 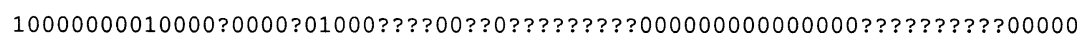 \\
\hline Plesiadapis & $0000000001 \mathrm{~A} 02100000101000000000100000000 ? 01020001000000000010001000020000$ \\
\hline Notharctus & $0000000001002 \mathrm{~A} 010001010000001001 ? 00000002000000010000010000 ? 0011100020021$ \\
\hline Adapis & $0000000001002 \mathrm{~A} 010001010000001001 ? 0000000200000001000011000010011100020021$ \\
\hline Ochotona & $10020040032210410102114103101102000011103 \mathrm{~A} 104101011101000101001 ? ? 20000100$ \\
\hline Prolagus & $10020040032210410102114103101102 ? 000111031104101011101000101001 ? ? 20000100$ \\
\hline Lepus & $100200400322104101021141031011020000111031404101011101011101001 ? ? 10000111$ \\
\hline Palaeolagus & $10020040032210410102014103101101 ? 0 ? 01 ? 10 ? 1404101011101011101001 ? ? 10000111$ \\
\hline Mimotona & 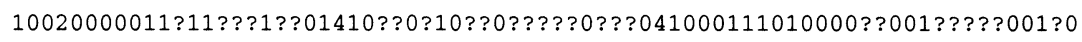 \\
\hline Mimolagus & 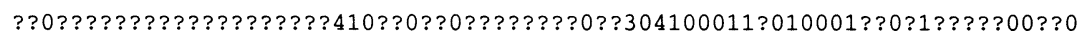 \\
\hline Eurymylus & 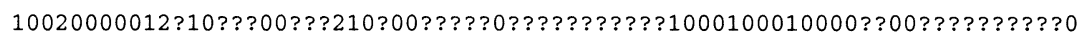 \\
\hline Rhombomylus & 1002000001211011010001210200110110101000102131000122010000010011000011010 \\
\hline Matutinia & 1002000001211011010001210200110110101000102131000122010000010011000011010 \\
\hline Sinomylus & 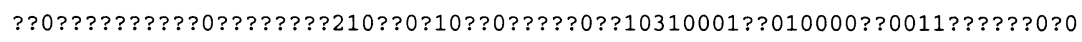 \\
\hline Heomys & 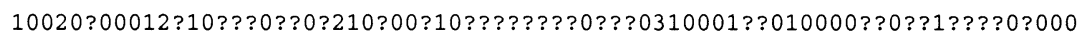 \\
\hline Tribosphenomys & 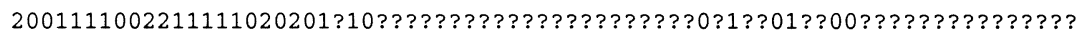 \\
\hline Paramys & $2002111 \mathrm{~A} 032111110202011104001101101010 ? 0 ? 020310002000100000 ? 0011000000000$ \\
\hline Reithroparamys & $20021011 ? 3211111120201210400 ? 10110 ? 010 ? 0 ? 020310002000100000 ? 0011000000000$ \\
\hline Paradjidaumo & $20020030132111111312015224001101102111 ? 0 ? 02031100200310000010011001000000$ \\
\hline Florentiamys & $20020020052111111312015224101102101111 ? 0002032100200310000011011 ? 0 ? 000000$ \\
\hline Gregorymys & $20020020052111111212015223001102101111 ? 0002032100200310000011011101000000$ \\
\hline Sciuravus & $200011 \mathrm{D} 013211 \mathrm{~A} 111202011104 ? 0 ? 101101010 ? 0202031000200010000010011000000000$ \\
\hline Cocomys & 2002111 A032110? $1110 ? ? ? 2104001101001010 ? 0 ? 02031000200010000010011-00000000$ \\
\hline Cricetops & $21120020032111111202012143012101101010 ? 0202031000200110000010011000000000$ \\
\hline Tataromys & $2002003014211111120201313 ? 00 ? 101 ? 0 ? 010 ? 0 ? 020310002-00100000 ? 0011-0 ?-00000$ \\
\hline Tsaganomys & $20020031132111112202016104001202101010 ? 0 ? 02021000200010000110010000100000$ \\
\hline Incamys & $2002103123211111230201313 ? 00 ? 20 ? ? 0 ? 011 ? 0303032000200020000110011100 ? 00010$ \\
\hline Paraphiomys & $2002103123211111230201313 ? 001201 ? 0 ? 01 ? ? 0302022000200020000110011100100000$ \\
\hline Erethizon & $2002203123211 \mathrm{~A} 11220201313300220230201110303032000200020000110111100100000$ \\
\hline Neoreomys & $2002203123211 \mathrm{~A} 11230201313 ? 00 ? 21 ? ? 0 ? 011 ? 0303032000200020000110 ? ? 1100100010$ \\
\hline Myocastor & 2002203123211131230201313300220130301110303032000200020000110111100100001 \\
\hline Cavia & 2002103123211131230201313300021130301110303032000200020000110001100100001 \\
\hline Dasyprocta & $20021031232111112302011133100211312 ? ? 112303032000200020000110001100100011$ \\
\hline Sciurus & $20021111032111111302011114001101101 \mathrm{~A} 1011202031000 \mathrm{C} 00210000010010000000011$ \\
\hline Marmota & $20021111 ? 3211111130201111400110100011011202031000 \mathrm{C} 00210000010010000000011$ \\
\hline Rattus & 2212102003211111130201214301210110101010202131000200110000010211001000000 \\
\hline Mus & 2212102003211111130201214301210110101010202131000200110000010211001000000 \\
\hline
\end{tabular}




\author{
APPENDIX 2 \\ (Continued)
}

\begin{tabular}{|c|c|c|}
\hline & 11111111111111111111111111111111111111111111111111111222222222222222222222222222 & \\
\hline & 4445555555555666666666677777777778888888888999999999990000000000111111111122222222 & \\
\hline Taxon/Node & 789012345678901234567890123456789012345678901234567890123456789012345678901234567 & \\
\hline Asioryctes & 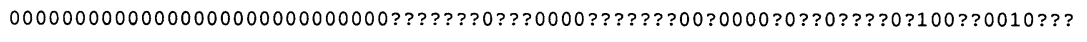 & 192,858 \\
\hline Kennalestes & 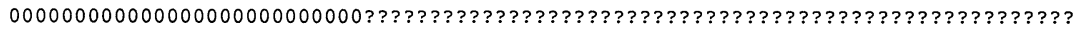 & 166,738 \\
\hline zalambdalestes & 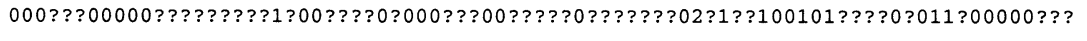 & 172,768 \\
\hline Barunlestes & 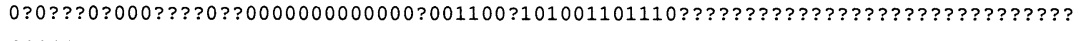 & 162,718 \\
\hline Hyopsodus & $00000000000 ? ? ? ? 02,000000000112000011001110111011000 ? 000001001011 ? ? 01 ? 10100000 ? ? ? ?$ & 208,928 \\
\hline Phenacodus & $00000000000 ? ? ? ? 0 ? ? 002 ? 00000112011011011110110101011 ? 000001001011 ? ? 00 ? 0010000 ? ? ? ? ? ?$ & 208,928 \\
\hline Anagalopsis & $000000 ?, 00 ? 10000200 ? ?, ? ? ?, ?, 11$ ? & 150,668 \\
\hline Anagale & $0100 ? 000000100001102102010011 ? 000010 ? 1 ? ? ? ? ? ? 1,1, ? 00 ? 0001010010100000 ? 1 ? ? 0 ? 0 ? 0 ? ? ? ?$ & 202， \\
\hline Leptictis & 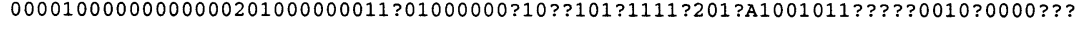 & 208,928 \\
\hline Cynocephalus & $0000110100011000110 \mathrm{~A} 2 ? 0000011200 ? 01111000101000110 ? 0001000 ? 0001101110001010000000$ & 219,968 \\
\hline Tupaia & $0000 ? 0000001100012021000000111000000000101010100000000100110011111010001010000000$ & 222,988 \\
\hline Petrodromus & $1100000000011001111100210001111 ? 10111001 ? ? ? 11111011121120110111010010113200010000$ & 221,978 \\
\hline Rhynchocyon & $1100100000011001111200010001110 ? 10110101100101011111211201 ? ? ? ? ? ? 10 ? ? 0113200010000$ & 214,948 \\
\hline Pseudictops & ??????????? & 146,648 \\
\hline Plesiadapis & $0000 ? ? 0000 ? 110000111 ? 000 ? 00 ? ? ? ? ? 000000000 ? ? 11 \mathrm{~A} 00100 ? 0 ? ? ? 01110000010000010 ? 0010 ? ? ?$ & 205,908 \\
\hline Notharctus & $00001000000110000022102000011 ? 000000000001010000010000000111000 ? 010100111100000 ? ?$ & 218,968 \\
\hline Adapis & 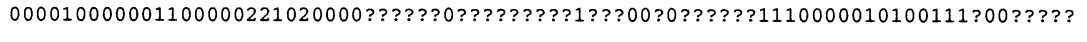 & 192,858 \\
\hline Ochotona & $421002010001103020042 ? 21000112111111011100010101011020110100111100111012111000100$ & 218,968 \\
\hline Prolagus & $421002000001101020032 ? ? 1 ? ? 0112111111011110 ? 1010101102011010011110011101211100 ? 100$ & 212,938 \\
\hline Lepus & $421002010001100020032 ? 01000112111111011110010100111020110100111100111012111100100$ & 219,968 \\
\hline Palaeolagus & $421002010001100020011001000 ? ? 20100100001 ? ? ? 10100111 ? 201101001111001110121110 ? 01 ? ?$ & 207, \\
\hline Mimotona & 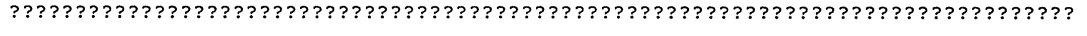 & 114,508 \\
\hline Mimolagus & ???????????? & 109,488 \\
\hline Eurymylus & 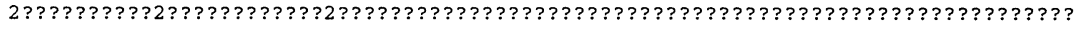 & 107,478 \\
\hline Rhombomylus & $221000101101100020042 ? C 1000 ? 110000100011 ? ? ? 11101111000001100101100100000000001100$ & 216,958 \\
\hline Matutinia & $22100010110110002003 \mathrm{~A} 0 \mathrm{C} 1000 ? ? 10 ? 0 ? ? ? 0 ? ? ?, 011 ? 001 ? 1100 ? 011100101 ? 000000 ? ? ? ? ? ? 0 ? 100$ & 199,888 \\
\hline Sinomylus & ????????????? & 79 , \\
\hline Heomys & 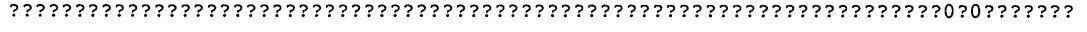 & 110,488 \\
\hline Tribosphenomys & 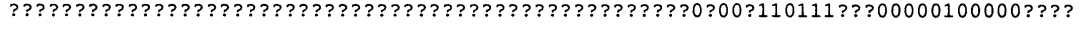 & 115,518 \\
\hline Paramys & $2210 ? 010000 ? ? 0 ? 0 ? 00100010001110 ? 0000000000011101000 ? 000011001111 ? ? 100001010001 ? ? ?$ & 205,908 \\
\hline Reithroparamys & $2210 ? ? ? 00001001020040101010 ? ? ? ? ? 001000 ? ? 01 ? 11011111 ? 000 ? 11001111 ? ? ? 000010 ? 000 ? ? ? ?$ & 190,848 \\
\hline Paradjidaumo & 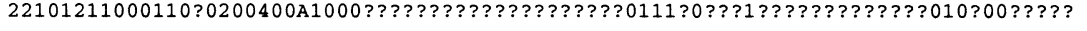 & 173,768 \\
\hline Florentiamys & 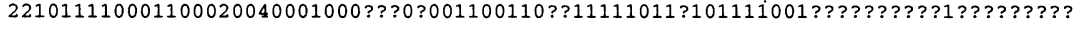 & 188,838 \\
\hline Gregorymys & 221012110001100020030001 ??????? & 164,728 \\
\hline Sciuravus & 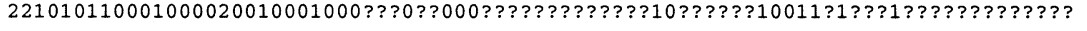 & 176,788 \\
\hline Cocomys & 22101A?100010000020010001000??????? & 159,708 \\
\hline Cricetops & 22101A110001?0?0200F0001000?????????? & 154,688 \\
\hline Tataromys & 221010?110?1?0?0200??? & 153,678 \\
\hline Tsaganomys & $22110 ? 1000$ ? 1102020042 ?21001?????? & 143,638 \\
\hline Incamys & 221102101001110020042,?1??? & 153,678 \\
\hline Paraphiomys & 22110211001111 ? $020042 ? ? 1$ ??:1????????????????? & 149,668 \\
\hline Erethizon & $321 ? ? 2100011100020042 ? 21101111000110010001010001100 \mathrm{~A} 01101110111100100001000001011$ & 216,958 \\
\hline Neoreomys & 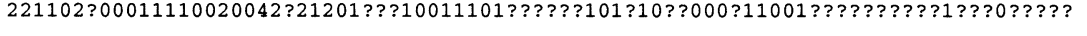 & 200,888 \\
\hline Myocastor & $321102000011100020042 ? 2110111110011100000101 ? 1 ? 1210000111110111101000001010001101$ & 216,958 \\
\hline Cavia & $321102000011110020042 ? 21201111111111001101011111111000111110111101000001010001111$ & 218, \\
\hline Dasyprocta & 321102000011110020042?2120111?11111101100101111111110011111010110A10000101000?111 & 212,938 \\
\hline Sciurus & $22101 \mathrm{~A} 100001101020041121110111000000000001011100000000111100111101000001010001000$ & 220, \\
\hline Marmota & 221011100001101020041121110111000110010001011101000000111100111100100001000001000 & 216,958 \\
\hline Rattus & $221012110001100020030011000111100100000001011111011 \mathrm{~A} 10111100111100100001000001000$ & 209,928 \\
\hline Mus & 221012110001100020030021000111110111000001011111011110111100111100110001000001000 & 209,928 \\
\hline
\end{tabular}




\section{APPENDIX 3}

\section{APOMORPHY LISTS}

These lists apply to the consensus cladogram illustrated in figure 74 . The numbers of nodes corresponds to those in figure 74 . The format is as follows: character number (description of the character), consistency index, and character transformation from one state to another. The double arrow $(\Rightarrow)$ represents an unequivocal transformation that occurs in both ACCTRAN and DELTRAN reconstruction. The single arrow $(\rightarrow)$ indicates an equivocal transformation that occurs in either ACCTRAN or DELTRAN reconstruction, but not in both. Character numbers correspond to those used in the text.

\section{ACCTRAN}

Asioryctes $\rightarrow$ node 93

5 (number of lower incisors), $1.000,0 \Rightarrow 1$

20 (i4 occurrence), $1.000,0 \Rightarrow 1$

21 (I5 occurrence), 1.000, $0 \rightarrow 1$

93 (mandibular condyle shape), 0.500, $0 \rightarrow 1$

188 (scaphoid and lunate fusion), 0.250, $0 \rightarrow 1$

201 (fibula size), $0.125,0 \rightarrow 1$

204 (astragalus trochlea), 0.500, $0 \rightarrow 1$

209 (astragalofibular facet), $0.500,0 \rightarrow 1$

216 (cuboid facet on astragalus), 0.200, $1 \rightarrow 0$

218 (calcaneofibular facet), $0.600,0 \rightarrow 1$

223 (cuboid length), 0.333, $1 \rightarrow 0$

\section{Node $93 \rightarrow$ node 92}

6 (number of upper incisors), $1.000,0 \Rightarrow 1$

19 (I4 occurrence), 1.000, $0 \Rightarrow 1$

24 (upper canine roots), 0.250, $0 \rightarrow 1$

25 (lower canine occurrence), $0.333,0 \Rightarrow 1$

26 (lower canine roots), $0.500,0 \Rightarrow 1$

37 (p4 trigonid shape), $0.500,0 \Rightarrow 1$

99 (postglenoid foramen), $0.500,0 \Rightarrow 1$

110 (alisphenoid canal), $0.333,0 \Rightarrow 1$

158 (bulla shape), $0.667,0 \rightarrow 1$

159 (external auditory canal), 0.167, $0 \rightarrow 1$

164 (entotympanic bulla), $0.333,0 \rightarrow 1$

\section{Node $92 \rightarrow$ node 51}

4 (upper diastema), $0.750,0 \Rightarrow 1$

8 (i1 size), $0.500,0 \Rightarrow 1$

18 (I3 position), $0.667,0 \rightarrow 1$

58 (upper molar trigon), $0.500,0 \Rightarrow 4$

106 (dorsal palatine foramen), $0.500,0 \rightarrow 1$

108 (sphenopalatine foramen), $0.429,0 \rightarrow 2$

118 (premaxilla size), $0.571,0 \Rightarrow 1$

123 (anterior zygomatic root), $0.667,0 \Rightarrow 1$

199 (distal tibia-fibula), $0.333,0 \rightarrow 2$

217 (calcaneus width), $0.250,0 \rightarrow 1$

\section{Node $51 \rightarrow$ Zalambdalestes}

24 (upper canine roots), 0.250, $1 \rightarrow 0$

167 (course of stapedial artery), $0.200,0 \Rightarrow 1$

\section{Node $51 \rightarrow$ Barunlestes}

1 (incisor enamel), $0.500,0 \Rightarrow 1$

28 (P1 occurrence), $0.250,0 \Rightarrow 1$

29 (p2 occurrence), $0.333,0 \Rightarrow 1$

106 (dorsal palatine foramen), 0.500, $1 \rightarrow 2$

\section{Node $92 \rightarrow$ node 91}

42 (P4 buccal cusp), $0.231,0 \Rightarrow 2$

64 (upper molar hypocone), $0.250,0 \Rightarrow 1$

73 (lower molar trigonid), $0.400,0 \rightarrow 1$

95 (mandibular foramen position), $0.250,0 \rightarrow 1$

105 (foramen ovale), $0.333,0 \Rightarrow 1$

114 (mastoid foramen), $0.429,0 \Rightarrow 2$

166 (distal internal carotid artery), 0.267, $0 \rightarrow 1$

174 (intercentrum and arches), $1.000,0 \Rightarrow 1$

175 (atlantal-axial joint), $1.000,0 \Rightarrow 1$

176 (number of sacral vertebrae), $0.500,0 \rightarrow 1$

190 (epipubic bone occurrence), $1.000,0 \Rightarrow 1$

197 (distal femur), $0.100,0 \rightarrow 1$

214 (astragalar neck length), $0.143,0 \rightarrow 1$

\section{Node $91 \rightarrow$ node 85}

53 (upper molar crown shape), $0.429,0 \Rightarrow 1$

57 (upper molar posteroloph), $0.571,0 \Rightarrow 1$

62 (upper molar conules), $0.300,0 \rightarrow 1$

70 (hypoflexid depth), 0.200, $0 \rightarrow 1$

83 (m3 hypoconulid), $0.556,0 \rightarrow 1$

85 (mandibular condyle height), $0.500,0 \rightarrow 1$

163 (ectotympanic contribution), $0.500,0 \Rightarrow 2$

187 (distal articulation surface), $0.333,0 \Rightarrow 1$

188 (scaphoid and lunate fusion), 0.250, $1 \rightarrow 0$

195 (third trochanter size), 0.182, $1 \rightarrow 0$

201 (fibula size), $0.125,1 \rightarrow 0$

\section{Node $85 \rightarrow$ node 52}

31 (P2 shape), $0.600,0 \Rightarrow 2$

58 (upper molar trigon), $0.500,0 \Rightarrow 1$

69 (mure occurrence), $0.200,0 \Rightarrow 1$

77 (lower molar paraconid), $0.400,0 \Rightarrow 1$

83 (m3 hypoconulid), $0.556,1 \rightarrow 2$

166 (distal internal carotid artery), 0.267, $1 \rightarrow 0$

176 (number of sacral vertebra), 0.500, $1 \rightarrow 2$

185 (central process of radial), $0.200,0 \Rightarrow 1$

189 (centrale occurrence), $0.500,0 \Rightarrow 1$

Node $52 \rightarrow$ Hyopsodus

23 (upper canine occurrence), $0.500,0 \Rightarrow 1$ 
42 (P4 buccal cusp), 0.231, $2 \Rightarrow 0$

45 (P4 hypocone), $0.286,0 \Rightarrow 1$

85 (mandibular condyle height), 0.500, $1 \rightarrow 0$

192 (great trochanter position), $0.167,1 \Rightarrow 0$

193 (femoral neck length), $0.167,0 \Rightarrow 1$

196 (patellar trochlea), $0.125,1 \Rightarrow 0$

197 (distal femur), $0.100,1 \rightarrow 0$

216 (cuboid facet on astragalus), $0.200,0 \Rightarrow 1$

\section{Node $52 \rightarrow$ Phenacodus}

25 (lower canine occurrence), $0.333,1 \Rightarrow 0$

33 (p3 shape), $1.000,0 \Rightarrow 1$

35 (P3 shape), $0.667,0 \Rightarrow 1$

39 (p4 talonid shape), $0.182,0 \Rightarrow 1$

55 (upper cheek tooth lingual), $0.500,0 \Rightarrow 1$

59 (upper molar centrocrsta), $0.750,0 \Rightarrow 1$

60 (upper molar mesostyle), $0.143,0 \Rightarrow 1$

96 (incisive foramen), $0.545,0 \Rightarrow 1$

167 (course of stapedial artery), $0.200,0 \Rightarrow 2$

178 (acromion), $0.200,0 \Rightarrow 1$

179 (greater tuberosity of hum), $0.250,0 \Rightarrow 1$

184 (posterior edge of proximal ulna), 0.125 ,

$0 \Rightarrow 1$

191 (articular surface on femurs), $0.167,1 \Rightarrow 0$ 214 (astragalar neck length), 0.143, $1 \rightarrow 0$

\section{Node $85 \rightarrow$ node 84}

37 (p4 trigonid shape), $0.500,1 \Rightarrow 3$

39 ( $\mathrm{p} 4$ talonid shape), $0.182,0 \rightarrow 2$

46 (molar labial cingulum), $0.500,0 \Rightarrow 1$

56 (upper molar protoloph), $0.571,0 \Rightarrow 1$

61 (upper molar protocone), $0.429,0 \Rightarrow 1$

63 (upper molar paracone and metacone), 0.667,

$0 \rightarrow 1$

74 (posterior wall of trigonid), $1.000,0 \Rightarrow 1$

116 (nasal), $0.444,0 \Rightarrow 1$

148 (postglenoid process) , 0.667, $0 \rightarrow 1$

170 (Squamosal to epitympanic) , $0.500,0 \rightarrow 1$

202 (tibia malleolus), $0.286,0 \rightarrow 1$

208 (lateral process of astragalus), $0.200,0 \Rightarrow 1$

210 (dorsal astragalar foramen), $0.250,1 \rightarrow 0$

217 (calcaneus width), $0.250,0 \Rightarrow 1$

\section{Node $84 \rightarrow$ node 82}

25 (lower canine occurrence), 0.333, $1 \rightarrow 0$

33 (p3 shape), $1.000,0 \Rightarrow 2$

50 (cheek teeth height), $0.500,0 \Rightarrow 1$

52 (lower tooth row size), $0.375,0 \Rightarrow 1$

70 (hypoflexid depth), 0.200, $1 \rightarrow 0$

99 (postglenoid foramen), 0.500, $1 \rightarrow 0$

133 (palatine-lacrimal contact), $0.500,0 \rightarrow 1$

136 (lacrimal facial process), $0.333,0 \Rightarrow 1$

164 (entotympanic bulla), $0.333,1 \rightarrow 0$

167 (course of stapedial artery), 0.200, $0 \rightarrow 1$

182 (supratrochlear foramen), 0.143, $1 \Rightarrow 0$

214 (astragalar neck length), $0.143,1 \rightarrow 0$

225 (plantar process of navicular), $0.333,0 \rightarrow 1$

\section{Node $82 \rightarrow$ node 54}

54 (upper cheek tooth precingulum), $0.600,0 \Rightarrow 1$

62 (upper molar conules), $0.300,1 \rightarrow 2$

64 (upper molar hypocone), $0.250,1 \Rightarrow 0$

85 (mandibular condyle height), 0.500, $1 \rightarrow 0$

114 (mastoid foramen), $0.429,2 \rightarrow 0$

159 (external auditory canal), 0.167, $1 \rightarrow 0$

166 (distal internal carotid artery), 0.267, $1 \rightarrow 2$

169 (facial nerve), $0.222,0 \rightarrow 2$

170 (squamosal to epitympanic), 0.500, $1 \rightarrow 0$

171 (tensor tympani muscle), $0.333,0 \rightarrow 1$

184 (posterior edge of proximal ulna), 0.125 ,

$0 \rightarrow 1$

193 (femoral neck length), $0.167,0 \Rightarrow 1$

220 (calcanealoastragalar position), $0.167,0 \rightarrow 1$

\section{Node $54 \rightarrow$ node 53}

31 (P2 shape), $0.600,0 \rightarrow 2$

61 (upper molar protocone), $0.429,1 \Rightarrow 3$

63 (upper molar paracone and metacone), 0.667, $1 \rightarrow 0$

77 (lower molar paraconid), 0.400, $0 \rightarrow 1$

145 (postorbital process of frontal), $0.222,0 \Rightarrow 1$

196 (patellar trochlea), 0.125, $1 \rightarrow 0$

197 (distal femur), 0.100, $1 \rightarrow 0$

208 (lateral process of astragalus), 0.200, $1 \rightarrow 0$

216 (cuboid facet on astragalus), $0.200,0 \Rightarrow 1$

\section{Node $53 \rightarrow$ Anagalopsis}

87 (number of mental foramen), $0.167,0 \Rightarrow 1$

107 (supraorbital foramen), $0.333,0 \Rightarrow 1$

148 (postglenoid process occur), $0.667,1 \rightarrow 0$

\section{Node $53 \rightarrow$ Anagale}

42 (P4 buccal cusp), $0.231,2 \Rightarrow 0$

163 (ectotympanic contribution), $0.500,2 \Rightarrow 1$

164 (entotympanic bulla), $0.333,0 \rightarrow 1$

\section{Node $54 \rightarrow$ Pseudictops}

42 (P4 buccal cusp), 0.231, $2 \Rightarrow 1$

73 (lower molar trigonid), $0.400,1 \Rightarrow 0$

218 (calcaneofibular facet), $0.600,1 \Rightarrow 2$

219 (calcaneoastragalar facet), $0.500,0 \Rightarrow 1$

221 (sustentaculum on calcaneus), $0.500,0 \Rightarrow 1$

\section{Node $82 \rightarrow$ node 81}

1 (incisor enamel), $0.500,0 \Rightarrow 1$

4 (upper diastema), 0.750, $0 \rightarrow 2$

7 (i1 occurrence), 1.000, $0 \Rightarrow 1$

9 (I1 occurrence), $0.250,0 \Rightarrow 1$

11 (di2 occurrence), 1.000, $0 \Rightarrow 2$

12 (i2 replacement), $1.000,0 \Rightarrow 1$

13 (DI2 size) $, 1.000,0 \Rightarrow 2$ 
15 (I2 replacement), $1.000,0 \Rightarrow 1$

16 (i3 occurrence), $0.333,0 \rightarrow 1$

18 (I3 position), $0.667,0 \rightarrow 1$

22 (incisor shape), $0.750,0 \Rightarrow 2$

23 (upper canine occurrence), $0.500,0 \Rightarrow 2$

25 (lower canine occurrence), $0.333,0 \rightarrow 2$

27 (p1 occurrence), $0.333,0 \Rightarrow 1$

28 (P1 occurrence), $0.250,0 \Rightarrow 1$

29 (p2 occurrence), $0.333,0 \Rightarrow 1$

31 (P2 shape), $0.600,0 \Rightarrow 1$

35 (P3 shape), 0.667, $0 \Rightarrow 1$

37 (p4 trigonid shape), $0.500,3 \rightarrow 2$

39 (p4 talonid shape), 0.182, $2 \rightarrow 0$

59 (upper molar centrocrsta), $0.750,0 \Rightarrow 2$

77 (lower molar paraconid), $0.400,0 \Rightarrow 2$

84 (low diastema), $1.000,0 \Rightarrow 2$

86 (horizontal ramus length), $1.000,0 \Rightarrow 1$

88 (coronoid process), $0.667,0 \rightarrow 1$

89 (angular process size), $0.500,0 \Rightarrow 1$

93 (mandibular condyle shape), $0.500,1 \Rightarrow 2$

96 (incisive foramen), $0.545,0 \rightarrow 2$

97 (infraorbital canal length), $0.500,0 \Rightarrow 1$

99 (postglenoid foramen), $0.500,0 \rightarrow 2$

102 (lacrimal foramen position), $0.400,0 \Rightarrow 1$

103 (nasolacrimal canal position), $1.000,0 \Rightarrow 1$

112 (sphenopalatine vacuity), $0.500,0 \rightarrow 1$

116 (nasal), $0.444,1 \rightarrow 2$

118 (premaxilla size), $0.571,0 \rightarrow 3$

119 (premaxilla on rostrum), $0.667,0 \Rightarrow 1$

123 (anterior zygomatic root), $0.667,0 \Rightarrow 1$

127 (orbit position), $0.500,0 \Rightarrow 1$

137 (lacrimal-jugal contact), $0.250,0 \rightarrow 1$

147 (glenoid fossa shape), $0.800,0 \rightarrow 2$

148 (postglenoid process), 0.667, $1 \Rightarrow 2$

149 (glenoid fossa position), $1.000,0 \Rightarrow 1$

195 (third trochanter size), $0.182,0 \rightarrow 1$

210 (dorsal astragalar foramen), 0.250, $0 \rightarrow 1$

213 (calcaneoastragalar and $\mathrm{Su}$ ), $0.143,0 \rightarrow 1$

\section{Node $81 \rightarrow$ node 58}

2 (enamel layer), $0.667,0 \Rightarrow 2$

14 (dI2 surface), $0.500,0 \rightarrow 1$

44 (P4 lophs), 0.500, $0 \Rightarrow 1$

85 (mandibular condyle height), $0.500,1 \rightarrow 2$

88 (coronoid process), 0.667, $1 \rightarrow 4$

91 (masseteric fossa), $0.300,0 \Rightarrow 1$

96 (incisive foramen), $0.545,2 \rightarrow 4$

99 (postglenoid foramen), 0.500, $2 \rightarrow 3$

100 (optic foramen), 0.333, $0 \rightarrow 1$

111 (sphenopterygoid canal), $0.333,0 \rightarrow 1$

114 (mastoid foramen), 0.429, $2 \rightarrow 3$

115 (skull shape), $1.000,0 \rightarrow 1$

116 (nasal), $0.444,2 \rightarrow 3$

118 (premaxilla size), $0.571,3 \rightarrow 4$

124 (ventral projection of maxilla), $1.000,0 \Rightarrow 1$

125 (zygomatic fossa), $1.000,0 \Rightarrow 1$

131 (hard palate length), $0.500,0 \rightarrow 1$
139 (posterior process of jugal), 1.000, $0 \rightarrow 1$

144 (anterior projection of frontal), $1.000,0 \Rightarrow 1$

145 (postorbital process of frontal), 0.222, $0 \rightarrow 1$

147 (glenoid fossa shape), 0.800, $2 \rightarrow 4$

152 (alisphenoid-parietal contact), $0.286,0 \rightarrow 2$

154 (interparietal occurrence), $0.143,0 \rightarrow 1$

176 (number of sacral vertebra), 0.500, $1 \rightarrow 2$

178 (acromion), 0.200, $0 \rightarrow 1$

191 (articular surface on femurs), 0.167, $1 \rightarrow 0$

194 (lesser trochanter orientation), 0.167, $1 \rightarrow 0$

215 (astragalonavicular facet), $1.000,0 \Rightarrow 1$

219 (calcaneoastragalar facet), $0.500,0 \Rightarrow 1$

221 (sustentaculum on calcaneus), $0.500,0 \Rightarrow 1$

\section{Node $58 \rightarrow$ node 57}

11 (di2 occurrence), $1.000,2 \Rightarrow 1$

13 (DI2 size), $1.000,2 \Rightarrow 1$

18 (I3 position), $0.667,1 \Rightarrow 2$

22 (incisor shape), $0.750,2 \Rightarrow 1$

31 (P2 shape), $0.600,1 \Rightarrow 3$

33 (p3 shape), $1.000,2 \Rightarrow 3$

35 (P3 shape), 0.667, $1 \Rightarrow 3$

37 (p4 trigonid shape), 0.500, $2 \rightarrow 3$

39 (p4 talonid shape), $0.182,0 \rightarrow 2$

44 (P4 lophs), $0.500,1 \Rightarrow 2$

45 (P4 hypocone), $0.286,0 \Rightarrow 2$

49 (upper tooth row size), $0.375,0 \Rightarrow 2$

50 (cheek teeth height), $0.500,1 \Rightarrow 3$

52 (lower tooth row size), $0.375,1 \Rightarrow 2$

56 (upper molar protoloph), $0.571,1 \Rightarrow 4$

57 (upper molar posteroloph), $0.571,1 \Rightarrow 4$

58 (upper molar trigon), $0.500,0 \Rightarrow 4$

59 (upper molar centrocrsta), $0.750,2 \Rightarrow 3$

61 (upper molar protocone), $0.429,1 \Rightarrow 2$

62 (upper molar conules), $0.300,1 \Rightarrow 3$

63 (upper molar paracone and metacone), 0.667, $1 \Rightarrow 6$

68 (hypoflexus), $0.600,0 \Rightarrow 3$

70 (hypoflexid depth), $0.200,0 \Rightarrow 1$

71 (ectolophid occurrence), $0.167,0 \Rightarrow 1$

80 (lower molar talonid basin), $0.800,0 \Rightarrow 4$

83 (m3 hypoconulid), $0.556,1 \rightarrow 3$

116 (nasal), $0.444,3 \rightarrow 4$

121 (fenestration of maxilla), $1.000,0 \Rightarrow 1$

129 (anteroventral rim of orbit), 0.500, $0 \rightarrow 1$

130 (orbit floor), $0.500,0 \rightarrow 1$

146 (supraorbital crest), $0.143,0 \rightarrow 1$

199 (distal tibia-fibula), $0.333,0 \Rightarrow 2$

201 (fibula size), $0.125,0 \Rightarrow 1$

214 (astragalar neck length), $0.143,0 \Rightarrow 1$

218 (calcaneofibular facet), $0.600,1 \Rightarrow 2$

220 (calcanealoastragalar position), $0.167,0 \Rightarrow 1$

\section{Node $57 \rightarrow$ node 56}

94 (coronoid canal occurrence), $1.000,0 \Rightarrow 1$

105 (foramen ovale), $0.333,1 \Rightarrow 2$ 
166 (distal internal carotid artery), 0.267, $1 \rightarrow 3$

167 (course of stapedial artery), 0.200, $1 \rightarrow 2$

177 (neck of scapula), $0.250,0 \Rightarrow 1$

179 (greater tuberosity of hum), $0.250,0 \Rightarrow 1$

180 (entepicondylar foramen), $0.333,0 \Rightarrow 1$

182 (supratrochlear foramen), $0.143,0 \Rightarrow 1$

184 (posterior edge of proximal ulna), 0.125 , $0 \Rightarrow 1$

185 (central process of radial), $0.200,0 \Rightarrow 1$

\section{Node $56 \rightarrow$ node 55}

48 (M3 occurrence), $0.500,0 \Rightarrow 1$

116 (nasal), $0.444,4 \rightarrow 1$

129 (anteroventral rim of orbit), 0.500, $1 \rightarrow 0$

130 (orbit floor), $0.500,1 \rightarrow 0$

139 (posterior process of jugal), $1.000,1 \Rightarrow 2$

145 (postorbital process of frontal), 0.222, $1 \rightarrow 0$

146 (supraorbital crest), 0.143, $1 \rightarrow 0$

161 (internal septa of bullae), $0.600,0 \rightarrow 1$

169 (facial nerve), $0.222,0 \rightarrow 2$

194 (less trochanter orientation), $0.167,0 \rightarrow 1$

195 (third trochanter size), 0.182, $1 \rightarrow 0$

\section{Node $55 \rightarrow$ Ochotona}

161 (internal septa of bullae), 0.600, $1 \rightarrow 3$

166 (distal internal carotid artery), 0.267, $3 \rightarrow 4$

187 (distal articulation surface), $0.333,1 \Rightarrow 0$

\section{Node $55 \rightarrow$ Prolagus}

154 (interparietal occurrence), $0.143,1 \Rightarrow 0$

\section{Node $56 \rightarrow$ Lepus}

222 (calcaneus-navicular contact), $0.500,0 \Rightarrow 1$

\section{Node $58 \rightarrow$ Mimotona}

16 (i3 occurrence), $0.333,1 \rightarrow 0$

84 (low diastema), $1.000,2 \Rightarrow 1$

87 (number of mental foramen), $0.167,0 \Rightarrow 1$

131 (hard palate length), $0.500,1 \rightarrow 0$

\section{Node $58 \rightarrow$ Mimolagus}

14 (dI2 surface), 0.500, $1 \rightarrow 0$

196 (patellar trochlea), $0.125,1 \Rightarrow 0$

197 (distal femur), 0.100, $1 \rightarrow 0$

210 (dorsal astragalar foramen), 0.250, $1 \rightarrow 0$

216 (cuboid facet on astragalus), $0.200,0 \Rightarrow 1$

222 (calcaneus-navicular contact), $0.500,0 \Rightarrow 1$

\section{Node $81 \rightarrow$ node 80}

4 (upper diastema), $0.750,2 \rightarrow 3$

17 (I3 occurrence), $0.333,0 \Rightarrow 1$
30 (P2 occurrence), $0.333,0 \Rightarrow 1$

106 (dorsal palatine foramen), $0.500,0 \Rightarrow 1$

108 (sphenopalatine foramen), $0.429,0 \Rightarrow 1$

153 (hamulus-bulla contact), $0.500,0 \Rightarrow 1$

166 (distal internal carotid artery), 0.267, $1 \rightarrow 4$

167 (course of stapedial artery), 0.200, $1 \rightarrow 0$

187 (distal articulation surface), 0.333, $1 \rightarrow 0$

203 (posterior process on distal tibia), 1.000,

$0 \Rightarrow 1$

217 (calcaneus width), $0.250,1 \Rightarrow 0$

224 (medial tarsal sesamoid bone), $1.000,0 \Rightarrow 1$

\section{Node $80 \rightarrow$ node 61}

2 (enamel layer), $0.667,0 \Rightarrow 1$

42 (P4 buccal cusp), 0.231, $2 \Rightarrow 1$

69 (mure occurrence), $0.200,0 \Rightarrow 1$

93 (mandibular condyle shape), 0.500, $2 \rightarrow 0$

112 (sphenopalatine vacuity), $0.500,1 \rightarrow 0$

114 (mastoid foramen), $0.429,2 \rightarrow 1$

143 (jugal ventral process), $1.000,0 \rightarrow 1$

155 (expansion of mastoid), $0.333,0 \rightarrow 1$

156 (mastoid in skull roof), $1.000,0 \rightarrow 1$

169 (facial nerve), $0.222,0 \rightarrow 1$

185 (central process of radial), $0.200,0 \rightarrow 1$

189 (centrale occurrence), $0.500,0 \rightarrow 1$

208 (lateral process of astragalus), 0.200, $1 \rightarrow 0$

218 (calcaneofibular facet), $0.600,1 \Rightarrow 0$

\section{Node $61 \rightarrow$ node 60}

56 (upper molar protoloph), $0.571,1 \Rightarrow 3$

62 (upper molar conules), $0.300,1 \Rightarrow 0$

117 (premaxilla-frontal suture), $0.500,0 \rightarrow 1$

142 (dorsal process of jugal), $0.667,0 \rightarrow 1$

145 (postorbital process of frontal), $0.222,0 \rightarrow 1$

\section{Node $60 \rightarrow$ Eurymylus}

158 (bulla shape), $0.667,1 \Rightarrow 2$

171 (tensor tympani muscle), $0.333,0 \Rightarrow 2$

\section{Node $60 \rightarrow$ node 59}

91 (masseteric fossa), $0.300,0 \Rightarrow 1$

124 (ventral projection of maxilla), $1.000,0 \Rightarrow 2$

125 (zygomatic fossa), 1.000, $0 \Rightarrow 2$

\section{Node $59 \rightarrow$ Rhombomylus}

167 (course of stapedial artery), $0.200,0 \Rightarrow 2$

202 (tibia malleolus), $0.286,1 \Rightarrow 0$

\section{Node $59 \rightarrow$ Matutinia}

42 (P4 buccal cusp), 0.231, $1 \Rightarrow 0$

44 (P4 lophs), 0.500, $0 \Rightarrow 1$

166 (distal internal carotid artery), 0.267, $4 \rightarrow 3$ 
192 (great trochanter position), $0.167,1 \Rightarrow 0$

213 (calcaneoastragalar), 0.143, $1 \rightarrow 0$

\section{Node $80 \rightarrow$ node 79}

3 (enamel type), $0.600,1 \rightarrow 0$

32 (p3 occurrence), $1.000,0 \Rightarrow 1$

35 (P3 shape), 0.667, $1 \rightarrow 2$

50 (cheek teeth height), $0.500,1 \Rightarrow 0$

56 (upper molar protoloph), 0.571, $1 \Rightarrow 0$

61 (upper molar protocone), $0.429,1 \Rightarrow 0$

74 (posterior wall of trigonid), $1.000,1 \Rightarrow 2$

78 (lower molar metaconid), $0.286,0 \Rightarrow 1$

79 (lower molar mesoconid), $0.333,0 \Rightarrow 1$

80 (lower molar talonid basin), $0.800,0 \Rightarrow 1$

83 (m3 hypoconulid), 0.556, $1 \rightarrow 2$

87 (number of mental foramen), $0.167,0 \rightarrow 1$

91 (masseteric fossa), $0.300,0 \Rightarrow 2$

99 (postglenoid foramen), 0.500, $2 \rightarrow 4$

151 (alisphenoid-frontal contact), 0.250, $0 \rightarrow 1$

159 (external auditory canal), $0.167,1 \rightarrow 0$

186 (ulnar facet of radial head), 0.200, $1 \rightarrow 0$

188 (scaphoid and lunate fusion), 0.250, $0 \rightarrow 1$

195 (third trochanter size), $0.182,1 \rightarrow 0$

197 (distal femur), 0.100, $1 \rightarrow 0$

225 (plantar process of navicular), 0.333, $1 \rightarrow 0$

\section{Node $79 \rightarrow$ Sinomylus}

30 (P2 occurrence), 0.333, $1 \Rightarrow 0$

35 (P3 shape), 0.667, $2 \rightarrow 1$

42 (P4 buccal cusp), 0.231, $2 \Rightarrow 0$

62 (upper molar conules), $0.300,1 \Rightarrow 0$

87 (number of mental foramen), 0.167, $1 \rightarrow 0$

116 (nasal), $0.444,2 \rightarrow 1$

\section{Node $79 \rightarrow$ Tribosphenomys}

46 (molar labial cingulum), $0.500,1 \Rightarrow 0$

57 (upper molar posteroloph), 0.571, $1 \Rightarrow 0$

58 (upper molar trigon), $0.500,0 \Rightarrow 2$

77 (lower molar paraconid), $0.400,2 \Rightarrow 1$

202 (tibia malleolus), $0.286,1 \Rightarrow 0$

205 (lateral astragalar trochlea), $0.200,0 \Rightarrow 1$

213 (calcaneoastragalar), $0.143,1 \rightarrow 0$

\section{Node $79 \rightarrow$ node 78}

2 (enamel layer), $0.667,0 \Rightarrow 1$

3 (enamel type), $0.600,0 \rightarrow 1$

39 (p4 talonid shape), $0.182,0 \Rightarrow 1$

49 (upper tooth row size), $0.375,0 \Rightarrow 1$

53 (upper molar crown shape), $0.429,1 \Rightarrow 2$

54 (upper cheek tooth precingulum), $0.600,0 \Rightarrow 1$

60 (upper molar mesostyle), $0.143,0 \Rightarrow 1$

69 (mure occurrence), $0.200,0 \Rightarrow 1$

81 (lower molar entoconid), $0.333,0 \rightarrow 1$

83 (m3 hypoconulid), $0.556,2 \rightarrow 3$
90 (angular process position), $0.667,0 \Rightarrow 1$

123 (anterior zygomatic root), $0.667,1 \Rightarrow 2$

\section{Node $78 \rightarrow$ Paramys}

90 (angular process position), $0.667,1 \Rightarrow 0$

96 (incisive foramen), $0.545,2 \rightarrow 1$

166 (distal internal carotid artery), $0.267,4 \Rightarrow 1$

181 (radial fossa size), $0.167,1 \Rightarrow 0$

188 (scaphoid and lunate fusion), $0.250,1 \Rightarrow 0$

196 (patellar trochlea), $0.125,1 \Rightarrow 0$

202 (tibia malleolus), $0.286,1 \Rightarrow 0$

220 (calcanealoastragalar position), $0.167,0 \rightarrow 1$

\section{Node $78 \rightarrow$ Reithroparamys}

79 (lower molar mesoconid), 0.333, $1 \Rightarrow 0$

161 (internal septa of bullae), $0.600,0 \Rightarrow 1$

168 (stapedial artery shielding), $0.500,0 \Rightarrow 1$

172 (meato-cochlear bridge), $0.500,0 \Rightarrow 1$

192 (great trochanter position), $0.167,1 \Rightarrow 0$

193 (femoral neck length), $0.167,0 \Rightarrow 1$

195 (third trochanter size), $0.182,0 \rightarrow 1$

197 (distal femur), $0.100,0 \rightarrow 1$

\section{Node $78 \rightarrow$ node 76}

56 (upper molar protoloph), $0.571,0 \Rightarrow 1$

62 (upper molar conules), $0.300,1 \Rightarrow 0$

72 (upper molar metaloph), $0.500,0 \Rightarrow 1$

80 (lower molar talonid basin), $0.800,1 \Rightarrow 2$

81 (lower molar entoconid), 0.333, $1 \rightarrow 0$

82 (lower molar hypolophid), 0.500, $0 \rightarrow 1$

154 (interparietal occurrence), $0.143,0 \Rightarrow 1$

193 (femoral neck length), 0.167, $0 \rightarrow 1$

201 (fibula size), $0.125,0 \rightarrow 1$

\section{Node $76 \rightarrow$ node 75}

3 (enamel type), $0.600,1 \Rightarrow 3$

34 (P3 occurrence), 1.000, $0 \Rightarrow 1$

49 (upper tooth row size), $0.375,1 \rightarrow 3$

60 (upper molar mesostyle), $0.143,1 \rightarrow 0$

68 (hypoflexus), $0.600,0 \Rightarrow 1$

70 (hypoflexid depth), $0.200,0 \Rightarrow 1$

78 (lower molar metaconid), $0.286,1 \Rightarrow 0$

79 (lower molar mesoconid), $0.333,1 \Rightarrow 0$

98 (infraorbital foramen diameter), $0.800,0 \rightarrow 2$

152 (alisphenoid-parietal contact), $0.286,0 \rightarrow 2$

159 (external auditory canal), 0.167, $0 \rightarrow 1$

182 (supratrochlear foramen), $0.143,0 \Rightarrow 1$

197 (distal femur), $0.100,0 \rightarrow 1$

\section{Node $75 \rightarrow$ node 63}

45 ( $\mathrm{P} 4$ hypocone), $0.286,0 \Rightarrow 2$

52 (lower tooth row size), $0.375,1 \Rightarrow 0$

91 (masseteric fossa), 0.300, $2 \rightarrow 3$ 
92 (incisor protuberance), $1.000,0 \Rightarrow 1$

96 (incisive foramen), $0.545,2 \Rightarrow 5$

97 (infraorbital canal length), $0.500,1 \Rightarrow 2$

109 (posterior maxillary notch), $0.400,0 \Rightarrow 1$

111 (sphenopterygoid canal), $0.333,0 \Rightarrow 1$

114 (mastoid foramen), 0.429, $2 \rightarrow 0$

120 (transverse constriction o), $1.000,0 \Rightarrow 1$

126 (Zygomatic plate), 1.000, $0 \Rightarrow 3$

140 (jugal size), $0.500,0 \Rightarrow 1$

185 (central process of radial), $0.200,0 \rightarrow 1$

186 (ulnar facet of radial head), $0.200,0 \rightarrow 1$

\section{Node $63 \rightarrow$ Paradjidaumo}

49 (upper tooth row size), $0.375,3 \rightarrow 0$

60 (upper molar mesostyle), $0.143,0 \rightarrow 1$

80 (lower molar talonid basin), 0.800, $2 \rightarrow 3$

108 (sphenopalatine foramen), $0.429,1 \Rightarrow 2$

194 (lesser trochanter orientation), $0.167,1 \Rightarrow 0$

195 (third trochanter size), $0.182,0 \rightarrow 1$

\section{Node $63 \rightarrow$ node 62}

38 (p4 protostylid occurrence), $1.000,0 \Rightarrow 1$

41 ( $\mathrm{P} 4$ size), $1.000,0 \Rightarrow 1$

43 (P4 styles), $1.000,0 \Rightarrow 1$

54 (upper cheek tooth precingulum), 0.600, $1 \Rightarrow 0$

56 (upper molar protoloph), $0.571,1 \Rightarrow 2$

57 (upper molar posteroloph), $0.571,1 \Rightarrow 2$

65 (lingual cusp to protocone), 1.000, $0 \Rightarrow 2$

66 (labial cusps to protoconid), 1.000, $0 \Rightarrow 2$

71 (ectolophid occurrence), $0.167,0 \Rightarrow 1$

82 (lower molar hypolophid), 0.500, $1 \rightarrow 0$

83 (m3 hypoconulid), $0.556,3 \Rightarrow 5$

105 (foramen ovale), $0.333,1 \Rightarrow 2$

119 (premaxilla on rostrum), $0.667,1 \Rightarrow 2$

134 (lacrimal projection), $1.000,0 \Rightarrow 1$

138 (jugal-maxilla contact), $0.333,0 \rightarrow 1$

199 (distal tibia-fibula), $0.333,0 \Rightarrow 1$

\section{Node $62 \rightarrow$ Florentiamys}

63 (upper molar paracone and metacone), 0.667, $1 \Rightarrow 0$

100 (optic foramen), $0.333,0 \Rightarrow 1$

152 (alisphenoid-parietal contact), $0.286,2 \Rightarrow 1$

\section{Node $62 \rightarrow$ Gregorymys}

39 (p4 talonid shape), $0.182,1 \Rightarrow 2$

61 (upper molar protocone), $0.429,0 \Rightarrow 1$

91 (masseteric fossa), $0.300,3 \rightarrow 2$

99 (postglenoid foramen), $0.500,4 \Rightarrow 3$

166 (distal internal carotid artery), $0.267,4 \Rightarrow 3$

Node $\mathbf{7 5} \rightarrow$ node 74

37 (p4 trigonid shape), $0.500,2 \rightarrow 3$
39 (p4 talonid shape), 0.182, $1 \rightarrow 0$

42 (P4 buccal cusp), 0.231, $2 \rightarrow 0$

53 (upper molar crown shape), $0.429,2 \Rightarrow 3$

73 (lower molar trigonid), $0.400,1 \rightarrow 2$

98 (infraorbital foramen diameter), $0.800,2 \rightarrow 3$

99 (postglenoid foramen), $0.500,4 \rightarrow 3$

169 (facial nerve), $0.222,0 \rightarrow 2$

177 (neck of scapula), $0.250,0 \Rightarrow 1$

180 (entepicondylar foramen), $0.333,0 \Rightarrow 1$

\section{Node $74 \rightarrow$ node 65}

36 ( $\mathrm{p} 4$ occurrence), 1.000, $0 \Rightarrow 1$

40 (P4 occurrence), 1.000, $0 \Rightarrow 1$

52 (lower tooth row size), $0.375,1 \Rightarrow 3$

56 (upper molar protoloph), $0.571,1 \Rightarrow 0$

75 (m1 anteroconid), 1.000, $0 \rightarrow 1$

76 (M1 anterocone), 1.000, $0 \Rightarrow 1$

82 (lower molar hypolophid), 0.500, $1 \rightarrow 0$

98 (infraorbital foramen diameter), $0.800,3 \rightarrow 4$

101 (nasolacrimal), $1.000,0 \Rightarrow 1$

102 (lacrimal foramen position), $0.400,1 \Rightarrow 2$

126 (zygomatic plate), $1.000,0 \Rightarrow 1$

166 (distal internal carotid artery), $0.267,4 \rightarrow 3$

198 (proximal tibia-fibula), $0.333,0 \rightarrow 1$

199 (distal tibia-fibula), $0.333,0 \rightarrow 1$

\section{Node $65 \rightarrow$ Cricetops}

73 (lower molar trigonid), 0.400, $2 \rightarrow 1$

152 (alisphenoid-parietal contact), $0.286,2 \rightarrow\{01\}$

169 (facial nerve), $0.222,2 \rightarrow 0$

\section{Node $65 \rightarrow$ node 64}

57 (upper molar posteroloph), $0.571,1 \Rightarrow 2$

63 (upper molar paracone and metacone), 0.667 , $1 \Rightarrow 3$

65 (lingual cusp to protocone), 1.000, $0 \Rightarrow 1$

66 (labial cusps to protoconid), 1.000, $0 \Rightarrow 1$

71 (ectolophid occurrence), $0.167,0 \Rightarrow 1$

75 ( $\mathrm{m} 1$ anteroconid), 1.000, $1 \rightarrow 2$

78 (lower molar metaconid), $0.286,0 \Rightarrow 1$

91 (masseteric fossa), $0.300,2 \Rightarrow 3$

117 (premaxilla-frontal suture), $0.500,0 \Rightarrow 1$

135 (lacrimal size), $0.667,0 \Rightarrow 2$

140 (jugal size), $0.500,0 \Rightarrow 1$

\section{Node $64 \rightarrow$ Rattus}

169 (facial nerve), 0.222, $2 \rightarrow 1$

181 (radial fossa size), $0.167,1 \Rightarrow 0$

182 (supratrochlear foramen), $0.143,1 \Rightarrow 0$

\section{Node $64 \rightarrow$ Mus}

178 (acromion), 0.200, $0 \Rightarrow 1$

214 (astragalar neck length), $0.143,0 \Rightarrow 1$ 


\section{Node $\mathbf{7 4} \rightarrow$ node $\mathbf{7 3}$}

3 (enamel type), $0.600,3 \Rightarrow 2$

49 (upper tooth row size), $0.375,3 \rightarrow 1$

50 (cheek teeth height), $0.500,0 \Rightarrow 1$

61 (upper molar protocone), $0.429,0 \Rightarrow 1$

62 (upper molar conules), $0.300,0 \Rightarrow 3$

72 (upper molar metaloph), 0.500, $1 \rightarrow 2$

80 (lower molar talonid basin), 0.800, $2 \rightarrow 3$

96 (incisive foramen), $0.545,2 \Rightarrow 3$

114 (mastoid foramen), $0.429,2 \rightarrow 3$

141 (jugal contribution to glenoid), $0.500,0 \rightarrow 1$

157 (mastoid exposure on occiput), $0.500,0 \rightarrow 1$

167 (course of stapedial artery), 0.200, $0 \rightarrow 2$

173 (round window position), 1.000, $0 \rightarrow 1$

195 (third trochanter size), $0.182,0 \rightarrow 1$

197 (distal femur), 0.100, $1 \rightarrow 0$

205 (lateral astragalar trochlea), 0.200, $0 \rightarrow 1$

226 (metatarsal IV occurrence), 0.500, $0 \rightarrow 1$

227 (metatarsal V occurrence), 1.000, $0 \rightarrow 1$

\section{Node $73 \rightarrow$ Tataromys}

68 (hypoflexus), $0.600,1 \Rightarrow 2$

83 (m3 hypoconulid), $0.556,3 \Rightarrow 4$

152 (alisphenoid-parietal contact), 0.286, $2 \rightarrow 0$

155 (expansion of mastoid), $0.333,0 \Rightarrow 1$

202 (tibia malleolus), $0.286,1 \Rightarrow 0$

\section{Node $\mathbf{7 3} \rightarrow$ node $\mathbf{7 2}$}

39 (p4 talonid shape), $0.182,0 \rightarrow 2$

42 (P4 buccal cusp), 0.231, $0 \rightarrow 3$

44 (P4 lophs), $0.500,0 \Rightarrow 2$

54 (upper cheek tooth precingulum), $0.600,1 \Rightarrow 2$

81 (lower molar entoconid), $0.333,0 \Rightarrow 1$

90 (angular process position), $0.667,1 \Rightarrow 2$

103 (nasolacrimal canal position), $1.000,1 \Rightarrow 2$

118 (premaxilla size), $0.571,3 \rightarrow 2$

132 (orbital wing of palatine), 1.000, $0 \Rightarrow 1$

150 (alisphenoid orbital process), 1.000, $0 \Rightarrow 1$

151 (alisphenoid-frontal contact), $0.250,1 \Rightarrow 0$

154 (interparietal occurrence), 0.143, $1 \rightarrow 0$

\section{Node $72 \rightarrow$ Tsaganomys}

13 (DI2 size), $1.000,2 \Rightarrow 3$

22 (incisor shape), $0.750,2 \Rightarrow 3$

49 (upper tooth row size), $0.375,1 \Rightarrow 0$

61 (upper molar protocone), $0.429,1 \Rightarrow 2$

64 (upper molar hypocone), $0.250,1 \Rightarrow 0$

68 (hypoflexus), $0.600,1 \Rightarrow 0$

69 (mure occurrence), $0.200,1 \Rightarrow 0$

70 (hypoflexid depth), $0.200,1 \Rightarrow 0$

72 (upper molar metaloph), $0.500,2 \rightarrow 0$

96 (incisive foramen), $0.545,3 \Rightarrow 6$

98 (infraorbital foramen diameter), $0.800,3 \rightarrow 0$

99 (postglenoid foramen), $0.500,3 \rightarrow 4$

105 (foramen ovale), $0.333,1 \Rightarrow 2$
137 (lacrimal-jugal contact), $0.250,1 \Rightarrow 0$

161 (internal septa of bullae), $0.600,0 \Rightarrow 2$

\section{Node $72 \rightarrow$ node 71}

45 ( $\mathrm{P} 4$ hypocone) $0.286,0 \Rightarrow 2$

53 (upper molar crown shape), $0.429,3 \rightarrow 2$

58 (upper molar trigon), $0.500,0 \Rightarrow 3$

67 (mesolophule occurrence), 1.000, $0 \Rightarrow 1$

78 (lower molar metaconid), $0.286,0 \rightarrow 1$

82 (lower molar hypolophid), $0.500,1 \Rightarrow 2$

91 (masseteric fossa), $0.300,2 \Rightarrow 3$

106 (dorsal palatine foramen), 0.500, $1 \rightarrow 3$

108 (sphenopalatine foramen), $0.429,1 \rightarrow 2$

111 (sphenopterygoid canal), $0.333,0 \rightarrow 1$

119 (premaxilla on rostrum), $0.667,1 \Rightarrow 2$

127 (orbit position), $0.500,1 \Rightarrow 2$

138 (jugal-maxilla contact), $0.333,0 \Rightarrow 1$

160 (opening of external audit), $0.333,0 \rightarrow 1$

171 (tensor tympani muscle), $0.333,0 \rightarrow 1$

\section{Node $71 \rightarrow$ node 70}

50 (cheek teeth height), 0.500, $1 \rightarrow 2$

51 (upper tooth row positions), $0.333,0 \Rightarrow 1$

54 (upper cheek tooth precingulum), $0.600,2 \Rightarrow 3$

63 (upper molar paracone and metacone), 0.667,

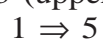

78 (lower molar metaconid), 0.286, $1 \rightarrow 2$

102 (lacrimal foramen position), $0.400,1 \Rightarrow 2$

116 (nasal), $0.444,2 \Rightarrow 3$

118 (premaxilla size), $0.571,2 \rightarrow 3$

147 (glenoid fossa shape), $0.800,2 \rightarrow 3$

\section{Node $70 \rightarrow$ node 67}

105 (foramen ovale), 0.333, $1 \rightarrow 2$

184 (posterior edge of proximal ulna), 0.125 , $0 \Rightarrow 1$

192 (great trochanter position), $0.167,1 \Rightarrow 0$

196 (patellar trochlea), $0.125,1 \Rightarrow 0$

202 (tibia malleolus), $0.286,1 \rightarrow 0$

\section{Node $67 \rightarrow$ node 66}

22 (incisor shape), 0.750, $2 \Rightarrow 3$

49 (upper tooth row size), $0.375,1 \Rightarrow 0$

52 (lower tooth row size), $0.375,1 \Rightarrow 0$

145 (postorbital process of frontal), $0.222,0 \Rightarrow 1$

147 (glenoid fossa shape), $0.800,3 \rightarrow 2$

171 (tensor tympani muscle), $0.333,1 \rightarrow 2$

201 (fibula size), $0.125,1 \rightarrow 0$

205 (lateral astragalar trochlea), 0.200, $1 \rightarrow 0$

\section{Node $66 \rightarrow$ Incamys}

51 (upper tooth rows positions), $0.333,1 \Rightarrow 0$

58 (upper molar trigon), $0.500,3 \Rightarrow 0$ 
71 (ectolophid occurrence), $0.167,0 \Rightarrow 1$

78 (lower molar metaconid), 0.286, $2 \rightarrow 1$

155 (expansion of mastoid), $0.333,0 \Rightarrow 1$

157 (mastoid exposure on occiput), $0.500,1 \Rightarrow 0$

Node $66 \rightarrow$ Neoreomys

104 (vacuity of nasolacrimal), $0.500,0 \Rightarrow 1$

\section{Node $67 \rightarrow$ Erethizon}

50 (cheek teeth height), $0.500,2 \rightarrow\{01\}$

91 (masseteric fossa), $0.300,3 \Rightarrow 2$

135 (lacrimal size), $0.667,0 \Rightarrow 1$

160 (opening of external audit), 0.333, $1 \rightarrow 0$

177 (neck of scapula), $0.250,1 \Rightarrow 0$

182 (supratrochlear foramen), $0.143,1 \Rightarrow 0$

191 (articular surface on femurs), $0.167,1 \Rightarrow 0$

193 (femoral neck length), $0.167,1 \Rightarrow 0$

200 (fibula-femur articulation), $0.500,0 \Rightarrow 1$

\section{Node $70 \rightarrow$ node 69}

53 (upper molar crown shape), $0.429,2 \rightarrow 3$

88 (coronoid process), $0.667,1 \rightarrow 3$

108 (sphenopalatine foramen), $0.429,2 \rightarrow 3$

146 (supraorbital crest), $0.143,0 \Rightarrow 1$

153 (hamulus-bulla contact), $0.500,1 \Rightarrow 0$

212 (sustentacular facet contact), $0.333,0 \Rightarrow 1$

213 (calcaneoastragalar), $0.143,1 \rightarrow 0$

220 (calcanealoastragalar position), $0.167,0 \Rightarrow 1$

225 (plantar process of navicular), $0.333,0 \Rightarrow 1$

\section{Node $69 \rightarrow$ Myocastor}

135 (lacrimal size), $0.667,0 \Rightarrow 1$

160 (opening of external audit), 0.333, $1 \rightarrow 0$

195 (third trochanter size), $0.182,1 \Rightarrow 2$

226 (metatarsal IV occurrence), 0.500, $1 \rightarrow 0$

\section{Node $69 \rightarrow$ node 68}

71 (ectolophid occurrence), $0.167,0 \rightarrow 1$

78 (lower molar metaconid), 0.286, $2 \rightarrow 1$

102 (lacrimal foramen position), $0.400,2 \Rightarrow 0$

104 (vacuity of nasolacrimal), $0.500,0 \Rightarrow 1$

136 (lacrimal facial process), $0.333,1 \Rightarrow 0$

171 (tensor tympani muscle), $0.333,1 \rightarrow 2$

178 (acromion), 0.200, $0 \Rightarrow 1$

179 (greater tuberosity), $0.250,0 \Rightarrow 1$

185 (central process of radial), $0.200,0 \Rightarrow 1$

197 (distal femur), 0.100, $0 \rightarrow 1$

\section{Node $68 \rightarrow$ Cavia}

186 (ulnar facet of radial head), $0.200,0 \Rightarrow 1$

\section{Node $68 \rightarrow$ Dasyprocta}

51 (upper tooth row positions), $0.333,1 \Rightarrow 0$

88 (coronoid process), $0.667,3 \rightarrow 1$

96 (incisive foramen), $0.545,3 \Rightarrow 1$

100 (optic foramen), $0.333,0 \Rightarrow 1$

107 (supraorbital foramen), $0.333,0 \Rightarrow 1$

108 (sphenopalatine foramen), $0.429,3 \rightarrow 2$

113 (supraorbital notch), $0.500,0 \Rightarrow 2$

145 (postorbital process of frontal), $0.222,0 \Rightarrow 1$

184 (posterior edge of proximal ulna), 0.125 , $0 \Rightarrow 1$

198 (proximal tibia-fibula), $0.333,0 \Rightarrow 1$

208 (lateral process of astragalus), $0.200,1 \Rightarrow 0$

213 (calcaneoastragalar), $0.143,0 \rightarrow 1$

\section{Node $71 \rightarrow$ Paraphiomys}

52 (lower tooth row size), $0.375,1 \Rightarrow 0$

154 (interparietal occurrence), $0.143,0 \rightarrow 1$

\section{Node $76 \rightarrow$ Sciuravus}

45 (P4 hypocone), 0.286, $0 \Rightarrow 1$

77 (lower molar paraconid), $0.400,2 \Rightarrow 0$

96 (incisive foramen), $0.545,2 \Rightarrow 1$

166 (distal internal carotid artery), 0.267, $4 \rightarrow 1$

181 (radial fossa size), $0.167,1 \Rightarrow 0$

214 (astragalar neck length), $0.143,0 \Rightarrow 1$

\section{Node $78 \rightarrow$ Cocomys}

39 (p4 talonid shape), $0.182,1 \Rightarrow 0$

42 (P4 buccal cusp), $0.231,2 \Rightarrow 0$

69 (mure occurrence), $0.200,1 \Rightarrow 0$

87 (number of mental foramen), $0.167,1 \Rightarrow 0$

91 (masseteric fossa), $0.300,2 \Rightarrow 1$

106 (dorsal palatine foramen), $0.500,1 \Rightarrow 0$

154 (interparietal occurrence), $0.143,0 \Rightarrow 1$

166 (distal internal carotid artery), $0.267,4 \rightarrow 1$

\section{Node $78 \rightarrow$ node 77}

3 (enamel type), $0.600,1 \Rightarrow 3$

60 (upper molar mesostyle), $0.143,1 \Rightarrow 0$

62 (upper molar conules), $0.300,1 \Rightarrow 0$

91 (masseteric fossa), $0.300,2 \Rightarrow 3$

96 (incisive foramen), $0.545,2 \Rightarrow 1$

98 (infraorbital foramen diameter), $0.800,0 \Rightarrow 1$

109 (posterior maxillary notch), $0.400,0 \rightarrow 1$

113 (supraorbital notch), $0.500,0 \Rightarrow 1$

126 (zygomatic plate), $1.000,0 \Rightarrow 2$

137 (lacrimal-jugal contact), $0.250,1 \Rightarrow 0$

145 (postorbital process of frontal), $0.222,0 \Rightarrow 1$

146 (supraorbital crest), $0.143,0 \Rightarrow 1$

152 (alisphenoid-parietal contact), $0.286,0 \rightarrow 1$

159 (external auditory canal), $0.167,0 \rightarrow 1$

161 (internal septa of bullae), $0.600,0 \Rightarrow 1$

167 (course of stapedial artery), $0.200,0 \Rightarrow 1$ 
168 (stapedial artery shielding), $0.500,0 \Rightarrow 1$

169 (facial nerve), $0.222,0 \Rightarrow 2$

171 (tensor tympani muscle), $0.333,0 \Rightarrow 1$

172 (meato-cochlear bridge), $0.500,0 \Rightarrow 1$

196 (patellar trochlea), $0.125,1 \Rightarrow 0$

201 (fibula size), $0.125,0 \Rightarrow 1$

\section{Node $77 \rightarrow$ Sciurus}

181 (radial fossa size), $0.167,1 \Rightarrow 0$

194 (less trochanter orientation), $0.167,1 \Rightarrow 0$

212 (sustentacular facet contact), $0.333,0 \Rightarrow 1$

213 (calcaneoastragalar), $0.143,1 \Rightarrow 0$

220 (calcanealoastragalar position), $0.167,0 \Rightarrow 1$

\section{Node $77 \rightarrow$ Marmota}

106 (dorsal palatine foramen), $0.500,1 \Rightarrow 0$

108 (sphenopalatine foramen), $0.429,1 \Rightarrow 0$

180 (entepicondylar foramen), $0.333,0 \Rightarrow 1$

184 (posterior edge of proximal ulna), 0.125 , $0 \Rightarrow 1$

\footnotetext{
Node $84 \rightarrow$ node 83

10 (I1 size), 0.500, $0 \rightarrow 1$

35 (P3 shape), $0.667,0 \Rightarrow 4$

45 ( $\mathrm{P} 4$ hypocone), $0.286,0 \Rightarrow 2$

47 (m3 occurrence), $1.000,0 \Rightarrow 1$

48 (M3 occurrence), $0.500,0 \Rightarrow 1$

53 (upper molar crown shape), $0.429,1 \Rightarrow 3$

57 (upper molar posteroloph), $0.571,1 \Rightarrow 3$

62 (upper molar conules), 0.300, $1 \rightarrow 0$

63 (upper molar paracone and metacone), 0.667,

$1 \rightarrow 4$

68 (hypoflexus), $0.600,0 \rightarrow 1$

72 (upper molar metaloph), $0.500,0 \Rightarrow 1$

83 (m3 hypoconulid), 0.556, $1 \rightarrow 3$

88 (coronoid process), $0.667,0 \Rightarrow 2$

93 (mandibular condyle shape), $0.500,1 \Rightarrow 0$

109 (posterior maxillary notch), $0.400,0 \Rightarrow 2$

118 (premaxilla size), $0.571,0 \Rightarrow 1$

122 (antorbital fossa), $0.667,0 \Rightarrow 2$

147 (glenoid fossa shape), $0.800,0 \Rightarrow 1$

162 (alisphenoid contribution) , $1.000,0 \Rightarrow 1$

163 (ectotympanic contribution), $0.500,2 \Rightarrow 1$

165 (petrosal contribution), $0.667,0 \Rightarrow 1$

179 (greater tuberosity), $0.250,0 \Rightarrow 1$

198 (proximal tibia-fibula), $0.333,0 \Rightarrow 1$

199 (distal tibia-fibula), $0.333,0 \Rightarrow 2$

200 (fibula-femur articulation), $0.500,0 \Rightarrow 1$

201 (fibula size), $0.125,0 \rightarrow 1$

202 (tibia malleolus), $0.286,1 \rightarrow 2$

205 (lateral astragalar trochlea), $0.200,0 \rightarrow 1$

211 (sustentacular facet), $0.500,0 \Rightarrow 1$

216 (cuboid facet on astragalus), $0.200,0 \Rightarrow 1$

218 (calcaneofibular facet), $0.600,1 \Rightarrow 3$

219 (calcaneoastragalar facet) $, 0.500,0 \Rightarrow 2$

223 (cuboid length), $0.333,0 \Rightarrow 1$
}

\section{Node $83 \rightarrow$ Petrodromus}

23 (upper canine occurrence), $0.500,0 \Rightarrow 1$

95 (mandibular foramen position), 0.250, $1 \rightarrow 0$

97 (infraorbital canal length), $0.500,0 \Rightarrow 1$

169 (facial nerve), $0.222,0 \Rightarrow 2$

177 (neck of scapula), $0.250,0 \Rightarrow 1$

183 (ulna-radia connection), $0.500,0 \Rightarrow 1$

193 (femoral neck length), $0.167,0 \Rightarrow 1$

\section{Node $83 \rightarrow$ Rhynchocyon}

9 (I1 occurrence), $0.250,0 \Rightarrow 1$

24 (upper canine roots), $0.250,1 \Rightarrow 0$

113 (supraorbital notch), $0.500,0 \Rightarrow 1$

146 (supraorbital crest), $0.143,0 \Rightarrow 1$

151 (alisphenoid-frontal contact), $0.250,0 \rightarrow 1$

159 (external auditory canal), 0.167, $1 \rightarrow 0$

186 (ulnar facet of radial head), 0.200, $1 \rightarrow 0$

188 (scaphoid and lunate fusion), $0.250,0 \rightarrow 1$

195 (third trochanter size), 0.182, $1 \rightarrow 0$

197 (distal femur), 0.100, $1 \rightarrow 0$

225 (plantar process of navicular), 0.333, $1 \rightarrow 0$

\section{Node $79 \rightarrow$ Sinomylus}

30 (P2 occurrence), 0.333, $1 \Rightarrow 0$

35 (P3 shape), 0.667, $2 \rightarrow 1$

42 (P4 buccal cusp), 0.231, $2 \Rightarrow 0$

62 (upper molar conules), $0.300,1 \Rightarrow 0$

87 (number of mental foramen), 0.167, $1 \rightarrow 0$

116 (nasal), $0.444,2 \rightarrow 1$

\section{Node $79 \rightarrow$ Tribosphenomys}

46 (molar labial cingulum), $0.500,1 \Rightarrow 0$

57 (upper molar posteroloph), $0.571,1 \Rightarrow 0$

58 (upper molar trigon), $0.500,0 \Rightarrow 2$

77 (lower molar paraconid), $0.400,2 \Rightarrow 1$

202 (tibia malleolus), $0.286,1 \Rightarrow 0$

205 (lateral astragalar trochlea), $0.200,0 \Rightarrow 1$

213 (calcaneoastragalar), $0.143,1 \rightarrow 0$

\section{Node $79 \rightarrow$ node 78}

2 (enamel layer), $0.667,0 \Rightarrow 1$

3 (enamel type), $0.600,0 \rightarrow 1$

39 (p4 talonid shape), $0.182,0 \Rightarrow 1$

49 (upper tooth row size), $0.375,0 \Rightarrow 1$

53 (upper molar crown shape), $0.429,1 \Rightarrow 2$

54 (upper cheek tooth precingulum), $0.600,0 \Rightarrow 1$

60 (upper molar mesostyle), $0.143,0 \Rightarrow 1$

69 (mure occurrence), $0.200,0 \Rightarrow 1$

81 (lower molar entoconid), $0.333,0 \rightarrow 1$

83 (m3 hypoconulid), $0.556,2 \rightarrow 3$

90 (angular process position), $0.667,0 \Rightarrow 1$

123 (anterior zygomatic root), $0.667,1 \Rightarrow 2$ 


\section{Node $78 \rightarrow$ Paramys}

90 (angular process position), $0.667,1 \Rightarrow 0$

96 (incisive foramen), $0.545,2 \rightarrow 1$

166 (distal internal carotid artery), $0.267,4 \Rightarrow 1$

181 (radial fossa size), $0.167,1 \Rightarrow 0$

188 (scaphoid and lunate fusion), $0.250,1 \Rightarrow 0$

196 (patellar trochlea), $0.125,1 \Rightarrow 0$

202 (tibia malleolus), $0.286,1 \Rightarrow 0$

220 (calcanealoastragalar position), $0.167,0 \rightarrow 1$

\section{Node $78 \rightarrow$ Reithroparamys}

79 (lower molar mesoconid), $0.333,1 \Rightarrow 0$

161 (internal septa of bullae), $0.600,0 \Rightarrow 1$

168 (stapedial artery shielding), $0.500,0 \Rightarrow 1$

172 (meato-cochlear bridge), $0.500,0 \Rightarrow 1$

192 (great trochanter position), $0.167,1 \Rightarrow 0$

193 (femoral neck length), $0.167,0 \Rightarrow 1$

195 (third trochanter size), $0.182,0 \rightarrow 1$

197 (distal femur), 0.100, $0 \rightarrow 1$

\section{Node $78 \rightarrow$ node 76}

56 (upper molar protoloph), $0.571,0 \Rightarrow 1$

62 (upper molar conules), $0.300,1 \Rightarrow 0$

72 (upper molar metaloph), $0.500,0 \Rightarrow 1$

80 (lower molar talonid basin), $0.800,1 \Rightarrow 2$

81 (lower molar entoconid), 0.333, $1 \rightarrow 0$

82 (lower molar hypolophid), $0.500,0 \rightarrow 1$

154 (interparietal occurrence), $0.143,0 \Rightarrow 1$

193 (femoral neck length), 0.167, $0 \rightarrow 1$

201 (fibula size), $0.125,0 \rightarrow 1$

\section{Node $76 \rightarrow$ node 75}

3 (enamel type), $0.600,1 \Rightarrow 3$

34 (P3 occurrence), 1.000, $0 \Rightarrow 1$

49 (upper tooth row size), $0.375,1 \rightarrow 3$

60 (upper molar mesostyle), 0.143, $1 \rightarrow 0$

68 (hypoflexus), $0.600,0 \Rightarrow 1$

70 (hypoflexid depth), $0.200,0 \Rightarrow 1$

78 (lower molar metaconid), $0.286,1 \Rightarrow 0$

79 (lower molar mesoconid), $0.333,1 \Rightarrow 0$

98 (infraorbital foramen diameter), $0.800,0 \rightarrow 2$

152 (alisphenoid-parietal contact), $0.286,0 \rightarrow 2$

159 (external auditory canal), $0.167,0 \rightarrow 1$

182 (supratrochlear foramen), $0.143,0 \Rightarrow 1$

197 (distal femur), 0.100, $0 \rightarrow 1$

\section{Node $75 \rightarrow$ node 63}

45 (P4 hypocone), $0.286,0 \Rightarrow 2$

52 (lower tooth row size), $0.375,1 \Rightarrow 0$

91 (masseteric fossa), 0.300, $2 \rightarrow 3$

92 (incisor protuberance), $1.000,0 \Rightarrow 1$

96 (incisive foramen), $0.545,2 \Rightarrow 5$

97 (infraorbital canal length), $0.500,1 \Rightarrow 2$

109 (posterior maxillary notch), $0.400,0 \Rightarrow 1$
111 (sphenopterygoid canal), $0.333,0 \Rightarrow 1$

114 (mastoid foramen), $0.429,2 \rightarrow 0$

120 (transverse constriction o), $1.000,0 \Rightarrow 1$

126 (Zygomatic plate), 1.000, $0 \Rightarrow 3$

140 (jugal size), $0.500,0 \Rightarrow 1$

185 (central process of radial), $0.200,0 \rightarrow 1$

186 (ulnar facet of radial head), $0.200,0 \rightarrow 1$

\section{Node $63 \rightarrow$ Paradjidaumo}

49 (upper tooth row size), $0.375,3 \rightarrow 0$

60 (upper molar mesostyle), $0.143,0 \rightarrow 1$

80 (lower molar talonid basin), 0.800, $2 \rightarrow 3$

108 (sphenopalatine foramen), $0.429,1 \Rightarrow 2$

194 (lesser trochanter orientation), $0.167,1 \Rightarrow 0$

195 (third trochanter size), $0.182,0 \rightarrow 1$

\section{Node $63 \rightarrow$ node 62}

38 (p4 protostylid occurrence), 1.000, $0 \Rightarrow 1$

41 ( $\mathrm{P} 4$ size), $1.000,0 \Rightarrow 1$

43 (P4 styles), $1.000,0 \Rightarrow 1$

54 (upper cheek tooth precingulum), $0.600,1 \Rightarrow 0$

56 (upper molar protoloph), $0.571,1 \Rightarrow 2$

57 (upper molar posteroloph), $0.571,1 \Rightarrow 2$

65 (lingual cusp to protocone), 1.000, $0 \Rightarrow 2$

66 (labial cusps to protoconid), 1.000, $0 \Rightarrow 2$

71 (ectolophid occurrence), $0.167,0 \Rightarrow 1$

82 (lower molar hypolophid), 0.500, $1 \rightarrow 0$

83 (m3 hypoconulid), $0.556,3 \Rightarrow 5$

105 (foramen ovale), $0.333,1 \Rightarrow 2$

119 (premaxilla on rostrum), $0.667,1 \Rightarrow 2$

134 (lacrimal projection), $1.000,0 \Rightarrow 1$

138 (jugal-maxilla contact), $0.333,0 \rightarrow 1$

199 (distal tibia-fibula), $0.333,0 \Rightarrow 1$

\section{Node $62 \rightarrow$ Florentiamys}

63 (upper molar paracone and metacone), 0.667, $1 \Rightarrow 0$

100 (optic foramen), $0.333,0 \Rightarrow 1$

152 (alisphenoid-parietal contact), $0.286,2 \Rightarrow 1$

\section{Node $62 \rightarrow$ Gregorymys}

39 (p4 talonid shape), $0.182,1 \Rightarrow 2$

61 (upper molar protocone), $0.429,0 \Rightarrow 1$

91 (masseteric fossa), $0.300,3 \rightarrow 2$

99 (postglenoid foramen), $0.500,4 \Rightarrow 3$

166 (distal internal carotid artery), $0.267,4 \Rightarrow 3$

\section{Node $75 \rightarrow$ node 74}

37 (p4 trigonid shape), 0.500, $2 \rightarrow 3$

39 (p4 talonid shape), 0.182, $1 \rightarrow 0$

42 (P4 buccal cusp), 0.231, $2 \rightarrow 0$

53 (upper molar crown shape), $0.429,2 \Rightarrow 3$

73 (lower molar trigonid), 0.400, $1 \rightarrow 2$ 
98 (infraorbital foramen diameter), 0.800, $2 \rightarrow 3$

99 (postglenoid foramen), 0.500, $4 \rightarrow 3$

169 (facial nerve), $0.222,0 \rightarrow 2$

177 (neck of scapula), $0.250,0 \Rightarrow 1$

180 (entepicondylar foramen), $0.333,0 \Rightarrow 1$

\section{Node $74 \rightarrow$ node 65}

36 (p4 occurrence), $1.000,0 \Rightarrow 1$

40 (P4 occurrence), $1.000,0 \Rightarrow 1$

52 (lower tooth row size), $0.375,1 \Rightarrow 3$

56 (upper molar protoloph), $0.571,1 \Rightarrow 0$

75 (m1 anteroconid), 1.000, $0 \rightarrow 1$

76 (M1 anterocone), $1.000,0 \Rightarrow 1$

82 (lower molar hypolophid), 0.500, $1 \rightarrow 0$

98 (infraorbital foramen diameter), 0.800, $3 \rightarrow 4$

101 (nasolacrimal), $1.000,0 \Rightarrow 1$

102 (lacrimal foramen position), $0.400,1 \Rightarrow 2$

126 (zygomatic plate), $1.000,0 \Rightarrow 1$

166 (distal internal carotid artery), $0.267,4 \rightarrow 3$

198 (proximal tibia-fibula), 0.333, $0 \rightarrow 1$

199 (distal tibia-fibula), 0.333, $0 \rightarrow 1$

\section{Node $65 \rightarrow$ Cricetops}

73 (lower molar trigonid), 0.400, $2 \rightarrow 1$

152 (alisphenoid-parietal contact), 0.286,

$2 \rightarrow\{01\}$

169 (facial nerve), $0.222,2 \rightarrow 0$

\section{Node $65 \rightarrow$ node 64}

57 (upper molar posteroloph), $0.571,1 \Rightarrow 2$

63 (upper molar paracone and metacone), 0.667 , $1 \Rightarrow 3$

65 (lingual cusp to protocone), $1.000,0 \Rightarrow 1$

66 (labial cusps to protoconid), $1.000,0 \Rightarrow 1$

71 (ectolophid occurrence), $0.167,0 \Rightarrow 1$

75 (m1 anteroconid), 1.000, $1 \rightarrow 2$

78 (lower molar metaconid), $0.286,0 \Rightarrow 1$

91 (masseteric fossa), $0.300,2 \Rightarrow 3$

117 (premaxilla-frontal suture), $0.500,0 \Rightarrow 1$

135 (lacrimal size), $0.667,0 \Rightarrow 2$

140 (jugal size), $0.500,0 \Rightarrow 1$

\section{Node $64 \rightarrow$ Rattus}

169 (facial nerve), $0.222,2 \rightarrow 1$

181 (radial fossa size), $0.167,1 \Rightarrow 0$

182 (supratrochlear foramen), $0.143,1 \Rightarrow 0$

\section{Node $64 \rightarrow$ Mus}

178 (acromion), 0.200, $0 \Rightarrow 1$

214 (astragalar neck length), $0.143,0 \Rightarrow 1$
Node $74 \rightarrow$ node 73

3 (enamel type), $0.600,3 \Rightarrow 2$

49 (upper tooth row size), $0.375,3 \rightarrow 1$

50 (cheek teeth height), $0.500,0 \Rightarrow 1$

61 (upper molar protocone), $0.429,0 \Rightarrow 1$

62 (upper molar conules), $0.300,0 \Rightarrow 3$

72 (upper molar metaloph), 0.500, $1 \rightarrow 2$

80 (lower molar talonid basin), 0.800, $2 \rightarrow 3$

96 (incisive foramen), $0.545,2 \Rightarrow 3$

114 (mastoid foramen), 0.429, $2 \rightarrow 3$

141 (jugal contribution to glenoid), 0.500, $0 \rightarrow 1$

157 (mastoid exposure on occiput), $0.500,0 \rightarrow 1$

167 (course of stapedial artery), $0.200,0 \rightarrow 2$

173 (round window position), $1.000,0 \rightarrow 1$

195 (third trochanter size), $0.182,0 \rightarrow 1$

197 (distal femur), 0.100, $1 \rightarrow 0$

205 (lateral astragalar trochlea), 0.200, $0 \rightarrow 1$

226 (metatarsal IV occurrence), $0.500,0 \rightarrow 1$

227 (metatarsal V occurrence), 1.000, $0 \rightarrow 1$

\section{Node $\mathbf{7 3} \rightarrow$ Tataromys}

68 (hypoflexus), $0.600,1 \Rightarrow 2$

83 (m3 hypoconulid), $0.556,3 \Rightarrow 4$

152 (alisphenoid-parietal contact), $0.286,2 \rightarrow 0$

155 (expansion of mastoid), $0.333,0 \Rightarrow 1$

202 (tibia malleolus), $0.286,1 \Rightarrow 0$

Node $73 \rightarrow$ node 72

39 (p4 talonid shape), $0.182,0 \rightarrow 2$

42 (P4 buccal cusp), 0.231, $0 \rightarrow 3$

44 (P4 lophs), 0.500, $0 \Rightarrow 2$

54 (upper cheek tooth precingulum), $0.600,1 \Rightarrow 2$

81 (lower molar entoconid), $0.333,0 \Rightarrow 1$

90 (angular process position), $0.667,1 \Rightarrow 2$

103 (nasolacrimal canal position), $1.000,1 \Rightarrow 2$

118 (premaxilla size), $0.571,3 \rightarrow 2$

132 (orbital wing of palatine), $1.000,0 \Rightarrow 1$

150 (alisphenoid orbital process), 1.000, $0 \Rightarrow 1$

151 (alisphenoid-frontal contact), $0.250,1 \Rightarrow 0$

154 (interparietal occurrence), 0.143, $1 \rightarrow 0$

\section{Node $72 \rightarrow$ Tsaganomys}

13 (DI2 size), $1.000,2 \Rightarrow 3$

22 (incisor shape), $0.750,2 \Rightarrow 3$

49 (upper tooth row size), $0.375,1 \Rightarrow 0$

61 (upper molar protocone), $0.429,1 \Rightarrow 2$

64 (upper molar hypocone), $0.250,1 \Rightarrow 0$

68 (hypoflexus), $0.600,1 \Rightarrow 0$

69 (mure occurrence), $0.200,1 \Rightarrow 0$

70 (hypoflexid depth), $0.200,1 \Rightarrow 0$

72 (upper molar metaloph), 0.500, $2 \rightarrow 0$

96 (incisive foramen), $0.545,3 \Rightarrow 6$

98 (infraorbital foramen diameter), 0.800, $3 \rightarrow 0$

99 (postglenoid foramen), $0.500,3 \rightarrow 4$

105 (foramen ovale), $0.333,1 \Rightarrow 2$ 
137 (lacrimal-jugal contact), $0.250,1 \Rightarrow 0$

161 (internal septa of bullae), $0.600,0 \Rightarrow 2$

\section{Node $72 \rightarrow$ node 71}

45 (P4 hypocone), $0.286,0 \Rightarrow 2$

53 (upper molar crown shape), $0.429,3 \rightarrow 2$

58 (upper molar trigon), $0.500,0 \Rightarrow 3$

67 (mesolophule occurrence), 1.000, $0 \Rightarrow 1$

78 (lower molar metaconid), 0.286, $0 \rightarrow 1$

82 (lower molar hypolophid), $0.500,1 \Rightarrow 2$

91 (masseteric fossa), $0.300,2 \Rightarrow 3$

106 (dorsal palatine foramen), 0.500, $1 \rightarrow 3$

108 (sphenopalatine foramen), 0.429, $1 \rightarrow 2$

111 (sphenopterygoid canal), $0.333,0 \rightarrow 1$

119 (premaxilla on rostrum), $0.667,1 \Rightarrow 2$

127 (orbit position), $0.500,1 \Rightarrow 2$

138 (jugal-maxilla contact), $0.333,0 \Rightarrow 1$

160 (opening of external audit), $0.333,0 \rightarrow 1$

171 (tensor tympani muscle), $0.333,0 \rightarrow 1$

\section{Node $71 \rightarrow$ node 70}

50 (cheek teeth height), $0.500,1 \rightarrow 2$

51 (upper tooth row positions), $0.333,0 \Rightarrow 1$

54 (upper cheek tooth precingulum), $0.600,2 \Rightarrow 3$

63 (upper molar paracone and metacone), 0.667,

$$
1 \Rightarrow 5
$$

78 (lower molar metaconid), 0.286, $1 \rightarrow 2$

102 (lacrimal foramen position), $0.400,1 \Rightarrow 2$

116 (nasal), $0.444,2 \Rightarrow 3$

118 (premaxilla size), $0.571,2 \rightarrow 3$

147 (glenoid fossa shape), 0.800, $2 \rightarrow 3$

\section{Node $70 \rightarrow$ node 67}

105 (foramen ovale), $0.333,1 \rightarrow 2$

184 (posterior edge of proximal ulna), 0.125 , $0 \Rightarrow 1$

192 (great trochanter position), $0.167,1 \Rightarrow 0$

196 (patellar trochlea), $0.125,1 \Rightarrow 0$

202 (tibia malleolus), 0.286, $1 \rightarrow 0$

\section{Node $67 \rightarrow$ node 66}

22 (incisor shape), $0.750,2 \Rightarrow 3$

49 (upper tooth row size), $0.375,1 \Rightarrow 0$

52 (lower tooth row size), $0.375,1 \Rightarrow 0$

145 (postorbital process of frontal), $0.222,0 \Rightarrow 1$

147 (glenoid fossa shape), $0.800,3 \rightarrow 2$

171 (tensor tympani muscle), $0.333,1 \rightarrow 2$

201 (fibula size), 0.125, $1 \rightarrow 0$

205 (lateral astragalar trochlea), 0.200, $1 \rightarrow 0$

\section{Node $66 \rightarrow$ Incamys}

51 (upper tooth rows positions), $0.333,1 \Rightarrow 0$

58 (upper molar trigon), $0.500,3 \Rightarrow 0$
71 (ectolophid occurrence), $0.167,0 \Rightarrow 1$

78 (lower molar metaconid), 0.286, $2 \rightarrow 1$

155 (expansion of mastoid), $0.333,0 \Rightarrow 1$

157 (mastoid exposure on occiput), $0.500,1 \Rightarrow 0$

Node $66 \rightarrow$ Neoreomys

104 (vacuity of nasolacrimal), $0.500,0 \Rightarrow 1$

\section{Node $67 \rightarrow$ Erethizon}

50 (cheek teeth height), 0.500, $2 \rightarrow\{01\}$

91 (masseteric fossa), $0.300,3 \Rightarrow 2$

135 (lacrimal size), $0.667,0 \Rightarrow 1$

160 (opening of external audit), $0.333,1 \rightarrow 0$

177 (neck of scapula), $0.250,1 \Rightarrow 0$

182 (supratrochlear foramen), $0.143,1 \Rightarrow 0$

191 (articular surface on femurs), $0.167,1 \Rightarrow 0$

193 (femoral neck length), $0.167,1 \Rightarrow 0$

200 (fibula-femur articulation), $0.500,0 \Rightarrow 1$

\section{Node $70 \rightarrow$ node 69}

53 (upper molar crown shape), $0.429,2 \rightarrow 3$

88 (coronoid process), 0.667, $1 \rightarrow 3$

108 (sphenopalatine foramen), $0.429,2 \rightarrow 3$

146 (supraorbital crest), $0.143,0 \Rightarrow 1$

153 (hamulus-bulla contact), $0.500,1 \Rightarrow 0$

212 (sustentacular facet contact), $0.333,0 \Rightarrow 1$

213 (calcaneoastragalar), $0.143,1 \rightarrow 0$

220 (calcanealoastragalar position), $0.167,0 \Rightarrow 1$

225 (plantar process of navicular), $0.333,0 \Rightarrow 1$

\section{Node $69 \rightarrow$ Myocastor}

135 (lacrimal size), $0.667,0 \Rightarrow 1$

160 (opening of external audit), $0.333,1 \rightarrow 0$

195 (third trochanter size), $0.182,1 \Rightarrow 2$

226 (metatarsal IV occurrence), 0.500, $1 \rightarrow 0$

\section{Node $69 \rightarrow$ node 68}

71 (ectolophid occurrence), $0.167,0 \rightarrow 1$

78 (lower molar metaconid), 0.286, $2 \rightarrow 1$

102 (lacrimal foramen position), $0.400,2 \Rightarrow 0$

104 (vacuity of nasolacrimal), $0.500,0 \Rightarrow 1$

136 (lacrimal facial process), $0.333,1 \Rightarrow 0$

171 (tensor tympani muscle), $0.333,1 \rightarrow 2$

178 (acromion), $0.200,0 \Rightarrow 1$

179 (greater tuberosity), $0.250,0 \Rightarrow 1$

185 (central process of radial), $0.200,0 \Rightarrow 1$

197 (distal femur), $0.100,0 \rightarrow 1$

Node $68 \rightarrow$ Cavia

186 (ulnar facet of radial head), $0.200,0 \Rightarrow 1$

Node $68 \rightarrow$ Dasyprocta

51 (upper tooth row positions), $0.333,1 \Rightarrow 0$ 
88 (coronoid process), 0.667, $3 \rightarrow 1$

96 (incisive foramen), $0.545,3 \Rightarrow 1$

100 (optic foramen), $0.333,0 \Rightarrow 1$

107 (supraorbital foramen), $0.333,0 \Rightarrow 1$

108 (sphenopalatine foramen), $0.429,3 \rightarrow 2$

113 (supraorbital notch), $0.500,0 \Rightarrow 2$

145 (postorbital process of frontal), $0.222,0 \Rightarrow 1$

184 (posterior edge of proximal ulna), 0.125 ,

$0 \Rightarrow 1$

198 (proximal tibia-fibula), $0.333,0 \Rightarrow 1$

208 (lateral process of astragalus), $0.200,1 \Rightarrow 0$

213 (calcaneoastragalar), $0.143,0 \rightarrow 1$

\section{Node $71 \rightarrow$ Paraphiomys}

52 (lower tooth row size), $0.375,1 \Rightarrow 0$

154 (interparietal occurrence), $0.143,0 \rightarrow 1$

\section{Node $76 \rightarrow$ Sciuravus}

45 (P4 hypocone), $0.286,0 \Rightarrow 1$

77 (lower molar paraconid), $0.400,2 \Rightarrow 0$

96 (incisive foramen), $0.545,2 \Rightarrow 1$

166 (distal internal carotid artery), $0.267,4 \rightarrow 1$

181 (radial fossa size), $0.167,1 \Rightarrow 0$

214 (astragalar neck length), $0.143,0 \Rightarrow 1$

\section{Node $78 \rightarrow$ Cocomys}

39 (p4 talonid shape), $0.182,1 \Rightarrow 0$

42 (P4 buccal cusp), 0.231, $2 \Rightarrow 0$

69 (mure occurrence), $0.200,1 \Rightarrow 0$

87 (number of mental foramen), $0.167,1 \Rightarrow 0$

91 (masseteric fossa), $0.300,2 \Rightarrow 1$

106 (dorsal palatine foramen), $0.500,1 \Rightarrow 0$

154 (interparietal occurrence), $0.143,0 \Rightarrow 1$

166 (distal internal carotid artery), $0.267,4 \rightarrow 1$

\section{Node $78 \rightarrow$ node 77}

3 (enamel type), $0.600,1 \Rightarrow 3$

60 (upper molar mesostyle), $0.143,1 \Rightarrow 0$

62 (upper molar conules), 0.300, $1 \Rightarrow 0$

91 (masseteric fossa), $0.300,2 \Rightarrow 3$

96 (incisive foramen), $0.545,2 \Rightarrow 1$

98 (infraorbital foramen diameter), $0.800,0 \Rightarrow 1$

109 (posterior maxillary notch), 0.400, $0 \rightarrow 1$

113 (supraorbital notch), $0.500,0 \Rightarrow 1$

126 (zygomatic plate), $1.000,0 \Rightarrow 2$

137 (lacrimal-jugal contact), $0.250,1 \Rightarrow 0$

145 (postorbital process of frontal), $0.222,0 \Rightarrow 1$

146 (supraorbital crest), $0.143,0 \Rightarrow 1$

152 (alisphenoid-parietal contact), 0.286, $0 \rightarrow 1$

159 (external auditory canal), $0.167,0 \rightarrow 1$

161 (internal septa of bullae), $0.600,0 \Rightarrow 1$

167 (course of stapedial artery), $0.200,0 \Rightarrow 1$

168 (stapedial artery shielding), $0.500,0 \Rightarrow 1$

169 (facial nerve), $0.222,0 \Rightarrow 2$
171 (tensor tympani muscle), $0.333,0 \Rightarrow 1$

172 (meato-cochlear bridge), $0.500,0 \Rightarrow 1$

196 (patellar trochlea), $0.125,1 \Rightarrow 0$

201 (fibula size), $0.125,0 \Rightarrow 1$

\section{Node $77 \rightarrow$ Sciurus}

181 (radial fossa size), $0.167,1 \Rightarrow 0$

194 (less trochanter orientation), $0.167,1 \Rightarrow 0$

212 (sustentacular facet contact), $0.333,0 \Rightarrow 1$

213 (calcaneoastragalar), $0.143,1 \Rightarrow 0$

220 (calcanealoastragalar position), $0.167,0 \Rightarrow 1$

\section{Node $77 \rightarrow$ Marmota}

106 (dorsal palatine foramen), $0.500,1 \Rightarrow 0$

108 (sphenopalatine foramen), $0.429,1 \Rightarrow 0$

180 (entepicondylar foramen), $0.333,0 \Rightarrow 1$

184 (posterior edge of proximal ulna), 0.125 ,

$$
0 \Rightarrow 1
$$

\section{Node $84 \rightarrow$ node 83}

10 (I1 size), 0.500, $0 \rightarrow 1$

35 (P3 shape), $0.667,0 \Rightarrow 4$

45 (P4 hypocone), $0.286,0 \Rightarrow 2$

47 (m3 occurrence), $1.000,0 \Rightarrow 1$

48 (M3 occurrence), $0.500,0 \Rightarrow 1$

53 (upper molar crown shape), $0.429,1 \Rightarrow 3$

57 (upper molar posteroloph), $0.571,1 \Rightarrow 3$

62 (upper molar conules), 0.300, $1 \rightarrow 0$

63 (upper molar paracone and metacone), 0.667,

$$
1 \rightarrow 4
$$

68 (hypoflexus), $0.600,0 \rightarrow 1$

72 (upper molar metaloph), $0.500,0 \Rightarrow 1$

83 (m3 hypoconulid), 0.556, $1 \rightarrow 3$

88 (coronoid process), $0.667,0 \Rightarrow 2$

93 (mandibular condyle shape), $0.500,1 \Rightarrow 0$

109 (posterior maxillary notch), $0.400,0 \Rightarrow 2$

118 (premaxilla size), $0.571,0 \Rightarrow 1$

122 (antorbital fossa), $0.667,0 \Rightarrow 2$

147 (glenoid fossa shape), $0.800,0 \Rightarrow 1$

162 (alisphenoid contribution) , $1.000,0 \Rightarrow 1$

163 (ectotympanic contribution), $0.500,2 \Rightarrow 1$

165 (petrosal contribution), $0.667,0 \Rightarrow 1$

179 (greater tuberosity), $0.250,0 \Rightarrow 1$

198 (proximal tibia-fibula), $0.333,0 \Rightarrow 1$

199 (distal tibia-fibula), $0.333,0 \Rightarrow 2$

200 (fibula-femur articulation), $0.500,0 \Rightarrow 1$

201 (fibula size), $0.125,0 \rightarrow 1$

202 (tibia malleolus), $0.286,1 \rightarrow 2$

205 (lateral astragalar trochlea), $0.200,0 \rightarrow 1$

211 (sustentacular facet), $0.500,0 \Rightarrow 1$

216 (cuboid facet on astragalus), $0.200,0 \Rightarrow 1$

218 (calcaneofibular facet), $0.600,1 \Rightarrow 3$

219 (calcaneoastragalar facet) $, 0.500,0 \Rightarrow 2$

223 (cuboid length), $0.333,0 \Rightarrow 1$ 


\section{Node $83 \rightarrow$ Petrodromus}

23 (upper canine occurrence), $0.500,0 \Rightarrow 1$

95 (mandibular foramen position), $0.250,1 \rightarrow 0$

97 (infraorbital canal length), $0.500,0 \Rightarrow 1$

169 (facial nerve), $0.222,0 \Rightarrow 2$

177 (neck of scapula), $0.250,0 \Rightarrow 1$

183 (ulna-radia connection), $0.500,0 \Rightarrow 1$

193 (femoral neck length), $0.167,0 \Rightarrow 1$

\section{Node $83 \rightarrow$ Rhynchocyon}

9 (I1 occurrence), $0.250,0 \Rightarrow 1$

24 (upper canine roots), $0.250,1 \Rightarrow 0$

113 (supraorbital notch), $0.500,0 \Rightarrow 1$

146 (supraorbital crest), $0.143,0 \Rightarrow 1$

151 (alisphenoid-frontal contact), $0.250,0 \Rightarrow 1$

166 (distal internal carotid artery), $0.267,1 \rightarrow 2$

184 (posterior edge of proximal ulna), 0.125 ,

$0 \Rightarrow 1$

191 (articular surface on femurs), $0.167,1 \Rightarrow 0$

195 (third trochanter size), $0.182,0 \rightarrow 1$

\section{Node $91 \rightarrow$ node 90}

52 (lower tooth row size), $0.375,0 \Rightarrow 1$

151 (alisphenoid-frontal contact), $0.250,0 \Rightarrow 1$

181 (radial fossa size), $0.167,1 \Rightarrow 0$

182 (supratrochlear foramen), $0.143,1 \Rightarrow 0$

191 (articular surface on femurs), $0.167,1 \Rightarrow 0$

212 (sustentacular facet contact), $0.333,0 \rightarrow 1$

220 (calcanealoastragalar position), $0.167,0 \rightarrow 1$

\section{Node $90 \rightarrow$ Leptictis}

9 (I1 occurrence), $0.250,0 \Rightarrow 1$

39 (p4 talonid shape), $0.182,0 \Rightarrow 1$

73 (lower molar trigonid), 0.400, $1 \rightarrow 0$

95 (mandibular foramen position), $0.250,1 \rightarrow 0$

97 (infraorbital canal length), $0.500,0 \Rightarrow 1$

109 (posterior maxillary notch), $0.400,0 \Rightarrow 1$

116 (nasal), $0.444,0 \Rightarrow 1$

122 (antorbital fossa), 0.667, $0 \Rightarrow 2$

158 (bulla shape), $0.667,1 \rightarrow 0$

159 (external auditory canal), 0.167, $1 \rightarrow 0$

164 (entotympanic bulla), $0.333,1 \rightarrow 2$

178 (acromion), $0.200,0 \Rightarrow 1$

199 (distal tibia-fibula), $0.333,0 \Rightarrow 2$

\section{Node $90 \rightarrow$ node 89}

17 (I3 occurrence), 0.333, $0 \rightarrow 1$

27 ( $\mathrm{p} 1$ occurrence), $0.333,0 \rightarrow 1$

28 (P1 occurrence), $0.250,0 \rightarrow 1$

63 (upper molar paracone and metacone), 0.667, $0 \rightarrow 2$

110 (alisphenoid canal), 0.333, $1 \rightarrow 0$

142 (dorsal process of jugal), $0.667,0 \rightarrow 2$

146 (supraorbital crest), $0.143,0 \rightarrow 1$
167 (course of stapedial artery), $0.200,0 \Rightarrow 1$

186 (ulnar facet of radial head), 0.200, $1 \rightarrow 0$

192 (greater trochanter position), $0.167,1 \rightarrow 0$

194 (lesser trochanter orientation), $0.167,1 \rightarrow 0$

196 (patellar trochlea), 0.125, $1 \rightarrow 0$

197 (distal femur), 0.100, $1 \rightarrow 0$

205 (lateral astragalar trochlea), $0.200,0 \Rightarrow 1$

207 (ventral limit of astragalus), 1.000, $1 \Rightarrow 0$

\section{Node $89 \rightarrow$ node 86}

23 (upper canine occurrence), $0.500,0 \Rightarrow 1$

59 (upper molar centrocrsta), 0.750, $0 \Rightarrow 2$

64 (upper molar hypocone), $0.250,1 \Rightarrow 0$

88 (coronoid process), $0.667,0 \Rightarrow 1$

96 (incisive foramen), $0.545,0 \Rightarrow 1$

105 (foramen ovale), $0.333,1 \Rightarrow 0$

107 (supraorbital foramen), $0.333,0 \Rightarrow 1$

113 (supraorbital notch), $0.500,0 \Rightarrow 2$

163 (ectotympanic contribution), $0.500,0 \Rightarrow 1$

\section{Node $86 \rightarrow$ Cynocephalus}

9 (I1 occurrence), $0.250,0 \Rightarrow 1$

17 (I3 occurrence), $0.333,1 \rightarrow 0$

24 (upper canine roots), $0.250,1 \Rightarrow 0$

26 (lower canine roots), $0.500,1 \Rightarrow 0$

29 (p2 occurrence), 0.333, $0 \Rightarrow 1$

30 (P2 occurrence), $0.333,0 \Rightarrow 1$

39 (p4 talonid shape), $0.182,0 \Rightarrow 1$

49 (upper tooth row size), $0.375,0 \Rightarrow 1$

58 (upper molar trigon), $0.500,0 \Rightarrow 2$

62 (upper molar conules), $0.300,0 \Rightarrow 1$

71 (ectolophid occurrence), $0.167,0 \Rightarrow 1$

99 (postglenoid foramen), $0.500,1 \Rightarrow 5$

114 (mastoid foramen), $0.429,2 \Rightarrow 3$

127 (orbit position), $0.500,0 \Rightarrow 1$

141 (jugal contribution to glenoid), $0.500,0 \Rightarrow 1$

142 (dorsal process of jugal), 0.667, $2 \rightarrow 0$

152 (alisphenoid-parietal contact), $0.286,0 \Rightarrow 1$

154 (interparietal occurrence), $0.143,0 \Rightarrow 1$

167 (course of stapedial artery), $0.200,1 \Rightarrow 2$

176 (number of sacral vertebrae), $0.500,1 \rightarrow 2$

181 (radial fossa size), $0.167,0 \Rightarrow 1$

182 (supratrochlear foramen), $0.143,0 \Rightarrow 1$

183 (ulna-radia connection), $0.500,0 \Rightarrow 1$

184 (posterior edge of proximal ulna), 0.125 ,

$0 \Rightarrow 1$

194 (lesser trochanter orientation), $0.167,0 \rightarrow 1$

204 (astragalus trochlea), $0.500,1 \Rightarrow 0$

213 (calcaneoastragalar), $0.143,0 \Rightarrow 1$

\section{Node $86 \rightarrow$ Tupaia}

42 (P4 buccal cusp), 0.231, $2 \Rightarrow 0$

52 (lower tooth row size), $0.375,1 \Rightarrow 0$

60 (upper molar mesostyle), $0.143,0 \Rightarrow 1$

95 (mandibular foramen position), $0.250,1 \rightarrow 0$ 
109 (posterior maxillary notch), $0.400,0 \Rightarrow 1$

110 (alisphenoid canal), 0.333, $0 \rightarrow 1$

145 (postorbital process of frontal), $0.222,0 \Rightarrow 2$

164 (entotympanic bulla), 0.333, $1 \rightarrow 2$

166 (distal internal carotid artery), $0.267,1 \Rightarrow 2$

186 (ulnar facet of radial head), $0.200,0 \rightarrow 1$

192 (greater trochanter position), $0.167,0 \rightarrow 1$

195 (third trochanter size), $0.182,1 \Rightarrow 0$

208 (lateral process of astragalus), $0.200,0 \Rightarrow 1$

211 (sustentacular facet orientation), $0.500,0 \Rightarrow 1$

\section{Node $89 \rightarrow$ node 88}

16 (i3 occurrence), $0.333,0 \Rightarrow 1$

25 (lower canine occurrence), $0.333,1 \rightarrow 0$

31 (P2 shape), $0.600,0 \Rightarrow 1$

42 (P4 buccal cusp), 0.231, $2 \rightarrow 1$

53 (upper molar crown shape), $0.429,0 \Rightarrow 1$

57 (upper molar posteroloph), $0.571,0 \Rightarrow 1$

63 (upper molar paracone and metacone), 0.667, $2 \rightarrow 3$

83 (m3 hypoconulid), $0.556,0 \Rightarrow 1$

86 (horizontal ramus length), $1.000,0 \Rightarrow 2$

87 (number of mental foramen), $0.167,0 \rightarrow 1$

99 (postglenoid foramen), $0.500,1 \Rightarrow 0$

122 (antorbital fossa), $0.667,0 \Rightarrow 1$

133 (palatine-lacrimal contact), $0.500,0 \Rightarrow 1$

137 (lacrimal-jugal contact), $0.250,0 \Rightarrow 1$

165 (petrosal contribution), $0.667,0 \rightarrow 1$

201 (fibula size), 0.125, $1 \rightarrow 0$

206 (astragalar posterior trochlea), 1.000, $0 \Rightarrow 1$

209 (astragalofibular facet), $0.500,1 \Rightarrow 0$

210 (dorsal astragalar foramen), $0.250,1 \Rightarrow 0$

\section{Node $88 \rightarrow$ Plesiadapids}

4 (upper diastema), $0.750,0 \Rightarrow 1$

8 (i1 size), $0.500,0 \Rightarrow 1$

10 (I1 size), $0.500,0 \Rightarrow 1$

25 (lower canine occurrence), 0.333, $0 \rightarrow 2$

37 (p4 trigonid shape), $0.500,1 \Rightarrow 0$

116 (nasal), $0.444,0 \Rightarrow 1$

118 (premaxilla size), $0.571,0 \Rightarrow 2$

146 (supraorbital crest), $0.143,1 \rightarrow 0$

191 (articular surface on femurs), $0.167,0 \Rightarrow 1$

214 (astragalar neck length), 0.143, $1 \rightarrow 0$

223 (cuboid length), $0.333,0 \Rightarrow 1$

\section{Node $88 \rightarrow$ node 87}

27 (p1 occurrence), $0.333,1 \rightarrow 0$

28 (P1 occurrence), 0.250, $1 \rightarrow 0$

55 (upper cheek tooth), $0.500,0 \Rightarrow 1$

58 (upper molar trigon), $0.500,0 \Rightarrow 1$

89 (angular process size), $0.500,0 \Rightarrow 1$

102 (lacrimal foramen position), $0.400,0 \Rightarrow 1$

128 (orbit orientation), $1.000,0 \Rightarrow 1$

136 (lacrimal facial process), $0.333,0 \Rightarrow 1$
138 (jugal-maxilla contact), $0.333,0 \Rightarrow 1$

145 (postorbital process of frontal), $0.222,0 \Rightarrow 2$

164 (entotympanic bulla), 0.333, $1 \rightarrow 0$

165 (petrosal contribution), $0.667,1 \rightarrow 2$

166 (distal internal carotid artery), $0.267,1 \Rightarrow 2$

169 (facial nerve), $0.222,0 \Rightarrow 2$

195 (third trochanter size), $0.182,1 \Rightarrow 0$

196 (patellar trochlea), $0.125,0 \rightarrow 1$

217 (calcaneus width), 0.250, $0 \rightarrow 1$

219 (calcaneoastragalar facet), $0.500,0 \Rightarrow 1$

\section{Node $87 \rightarrow$ Notharctus}

60 (upper molar mesostyle), $0.143,0 \Rightarrow 1$

Node $87 \rightarrow$ Adapis

42 (P4 buccal cusp), 0.231, $1 \rightarrow 2$

45 (P4 hypocone), $0.286,0 \Rightarrow 1$

127 (orbit position), $0.500,0 \Rightarrow 1$

\section{DELTRAN}

Asioryctes $\rightarrow$ node 93

5 (number of lower incisors), $1.000,0 \Rightarrow 1$

20 (i4 occurrence), $1.000,0 \Rightarrow 1$

\section{Node $93 \rightarrow$ node 92}

6 (number of upper incisors), $1.000,0 \Rightarrow 1$

19 (I4 occurrence), $1.000,0 \Rightarrow 1$

21 (I5 occurrence), 1.000, $0 \rightarrow 1$

25 (lower canine occurrence), $0.333,0 \Rightarrow 1$

26 (lower canine roots), $0.500,0 \Rightarrow 1$

37 (p4 trigonid shape), $0.500,0 \Rightarrow 1$

93 (mandibular condyle shape), $0.500,0 \rightarrow 1$

99 (postglenoid foramen), $0.500,0 \Rightarrow 1$

110 (alisphenoid canal), $0.333,0 \Rightarrow 1$

204 (astragalus trochlea), 0.500, $0 \rightarrow 1$

209 (astragalofibular facet), $0.500,0 \rightarrow 1$

216 (cuboid facet on astragalus), 0.200, $1 \rightarrow 0$

218 (calcaneofibular facet), $0.600,0 \rightarrow 1$

223 (cuboid length), 0.333, $1 \rightarrow 0$

\section{Node $92 \rightarrow$ node 51}

4 (upper diastema), 0.750, $0 \Rightarrow 1$

8 (i1 size), $0.500,0 \Rightarrow 1$

58 (upper molar trigon), $0.500,0 \Rightarrow 4$

118 (premaxilla size), $0.571,0 \Rightarrow 1$

123 (anterior zygomatic root), $0.667,0 \Rightarrow 1$

\section{Node $\mathbf{5 1} \rightarrow$ Zalambdalestes}

18 (I3 position), $0.667,0 \rightarrow 1$

106 (dorsal palatine foramen), $0.500,0 \rightarrow 1$

167 (course of stapedial artery), $0.200,0 \Rightarrow 1$

199 (distal tibia-fibula), 0.333, $0 \rightarrow 2$

201 (fibula size), $0.125,0 \rightarrow 1$

217 (calcaneus width), $0.250,0 \rightarrow 1$ 


\section{Node $51 \rightarrow$ Barunlestes}

1 (incisor enamel), 0.500, $0 \Rightarrow 1$

24 (upper canine roots), $0.250,0 \rightarrow 1$

28 ( $\mathrm{P} 1$ occurrence), $0.250,0 \Rightarrow 1$

29 (p2 occurrence), $0.333,0 \Rightarrow 1$

106 (dorsal palatine foramen), $0.500,0 \rightarrow 2$

108 (sphenopalatine foramen), $0.429,0 \rightarrow 2$

188 (scaphoid and lunate fusion), $0.250,0 \rightarrow 1$

\section{Node $92 \rightarrow$ node 91}

24 (upper canine roots), $0.250,0 \rightarrow 1$

42 (P4 buccal cusp), 0.231, $0 \Rightarrow 2$

64 (upper molar hypocone), $0.250,0 \Rightarrow 1$

105 (foramen ovale), $0.333,0 \Rightarrow 1$

114 (mastoid foramen), $0.429,0 \Rightarrow 2$

174 (intercentrum and arches), 1.000, $0 \Rightarrow 1$

175 (atlantal-axial joint), 1.000, $0 \Rightarrow 1$

176 (number of sacral vertebrae), $0.500,0 \rightarrow 1$

190 (epipubic bone occurrence), $1.000,0 \Rightarrow 1$

\section{Node $91 \rightarrow$ node 85}

53 (upper molar crown shape), $0.429,0 \Rightarrow 1$

57 (upper molar posteroloph), $0.571,0 \Rightarrow 1$

73 (lower molar trigonid), $0.400,0 \rightarrow 1$

163 (ectotympanic contribution), $0.500,0 \Rightarrow 2$

187 (distal articulation surface), $0.333,0 \Rightarrow 1$

\section{Node $85 \rightarrow$ node 52}

31 (P2 shape), $0.600,0 \Rightarrow 2$

58 (upper molar trigon), $0.500,0 \Rightarrow 1$

62 (upper molar conules), 0.300, $0 \rightarrow 1$

69 (mure occurrence), $0.200,0 \Rightarrow 1$

70 (hypoflexid depth), 0.200, $0 \rightarrow 1$

77 (lower molar paraconid), $0.400,0 \Rightarrow 1$

83 (m3 hypoconulid), $0.556,0 \rightarrow 2$

176 (number of sacral vertebrae), $0.500,1 \rightarrow 2$

185 (central process of radial), $0.200,0 \Rightarrow 1$

189 (centrale occurrence), $0.500,0 \Rightarrow 1$

195 (third trochanter size), $0.182,1 \rightarrow 0$

\section{Node $52 \rightarrow$ Hyopsodus}

23 (upper canine occurrence), $0.500,0 \Rightarrow 1$

42 ( $\mathrm{P} 4$ buccal cusp), $0.231,2 \Rightarrow 0$

45 ( $\mathrm{P} 4$ hypocone), $0.286,0 \Rightarrow 1$

192 (greater trochanter position), $0.167,1 \Rightarrow 0$

193 (femoral neck length), $0.167,0 \Rightarrow 1$

196 (patellar trochlea), $0.125,1 \Rightarrow 0$

214 (astragalar neck length), $0.143,0 \rightarrow 1$

216 (cuboid facet on astragalus), $0.200,0 \Rightarrow 1$

\section{Node $52 \rightarrow$ Phenacodus}

25 (lower canine occurrence), $0.333,1 \Rightarrow 0$

33 (p3 shape), 1.000, $0 \Rightarrow 1$
35 (P3 shape), 0.667, $0 \Rightarrow 1$

39 (p4 talonid shape), $0.182,0 \Rightarrow 1$

55 (upper cheek tooth lingual) $0.500,0 \Rightarrow 1$

59 (upper molar centrocrsta), $0.750,0 \Rightarrow 1$

60 (upper molar mesostyle), $0.143,0 \Rightarrow 1$

85 (mandibular condyle height), $0.500,0 \rightarrow 1$

96 (incisive foramen), $0.545,0 \Rightarrow 1$

167 (course of stapedial artery), $0.200,0 \Rightarrow 2$

178 (acromion), 0.200, $0 \Rightarrow 1$

179 (greater tuberosity), $0.250,0 \Rightarrow 1$

184 (posterior edge of proximal ulna), 0.125 , $0 \Rightarrow 1$

191 (articular surface on femurs), $0.167,1 \Rightarrow 0$

197 (distal femur), 0.100, $0 \rightarrow 1$

\section{Node $85 \rightarrow$ node 84}

37 (p4 trigonid shape), $0.500,1 \Rightarrow 3$

46 (molar labial cingulum), $0.500,0 \Rightarrow 1$

56 (upper molar protoloph), $0.571,0 \Rightarrow 1$

61 (upper molar protocone), $0.429,0 \Rightarrow 1$

74 (posterior wall of trigonid), 1.000, $0 \Rightarrow 1$

116 (nasal), $0.444,0 \Rightarrow 1$

158 (bulla shape), $0.667,0 \rightarrow 1$

166 (distal internal carotid artery), $0.267,0 \rightarrow 1$

208 (lateral process of astragalus), $0.200,0 \Rightarrow 1$

217 (calcaneus width), $0.250,0 \Rightarrow 1$

\section{Node $84 \rightarrow$ node 82}

33 (p3 shape), 1.000, $0 \Rightarrow 2$

50 (cheek teeth height), $0.500,0 \Rightarrow 1$

52 (lower tooth row size), $0.375,0 \Rightarrow 1$

83 ( $\mathrm{m} 3$ hypoconulid), 0.556, $0 \rightarrow 1$

95 (mandibular foramen position), $0.250,0 \rightarrow 1$

136 (Lacrimal facial process), $0.333,0 \Rightarrow 1$

182 (supratrochlear foramen), $0.143,1 \Rightarrow 0$

202 (tibia malleolus), $0.286,0 \rightarrow 1$

\section{Node $82 \rightarrow$ node 54}

25 (lower canine occurrence), 0.333, $1 \rightarrow 0$

39 (p4 talonid shape), $0.182,0 \rightarrow 2$

54 (upper cheek tooth precingulum), $0.600,0 \Rightarrow 1$

62 (upper molar conules), $0.300,0 \rightarrow 2$

64 (upper molar hypocone), $0.250,1 \Rightarrow 0$

193 (femoral neck length), $0.167,0 \Rightarrow 1$

195 (third trochanter size), $0.182,1 \rightarrow 0$

210 (dorsal astragalar foramen), 0.250, $1 \rightarrow 0$

\section{Node $54 \rightarrow$ node 53}

61 (upper molar protocone), $0.429,1 \Rightarrow 3$

99 (postglenoid foramen), $0.500,1 \rightarrow 0$

145 (postorbital process of frontal), $0.222,0 \Rightarrow 1$

216 (cuboid facet on astragalus), $0.200,0 \Rightarrow 1$ 


\section{Node $53 \rightarrow$ Anagalopsis}

87 (number of mental foramen), $0.167,0 \Rightarrow 1$

107 (supraorbital foramen), $0.333,0 \Rightarrow 1$

220 (calcanealoastragalar position), $0.167,0 \rightarrow 1$

\section{Node $53 \rightarrow$ Anagale}

31 (P2 shape), 0.600, $0 \rightarrow 2$

42 (P4 buccal cusp), 0.231, $2 \Rightarrow 0$

77 (lower molar paraconid), $0.400,0 \rightarrow 1$

114 (mastoid foramen), 0.429, $2 \rightarrow 0$

148 (postglenoid process), 0.667, $0 \rightarrow 1$

163 (ectotympanic contribution), $0.500,2 \Rightarrow 1$

164 (entotympanic bulla), $0.333,0 \rightarrow 1$

166 (distal internal carotid artery), $0.267,1 \rightarrow 2$

167 (course of stapedial artery), $0.200,0 \rightarrow 1$

169 (facial nerve), $0.222,0 \rightarrow 2$

171 (tensor tympani muscle), $0.333,0 \rightarrow 1$

184 (posterior edge of proximal ulna), 0.125 ,

$0 \rightarrow 1$

196 (patellar trochlea), $0.125,1 \rightarrow 0$

208 (lateral process of astragalus), 0.200, $1 \rightarrow 0$

\section{Node $54 \rightarrow$ Pseudictops}

42 (P4 buccal cusp), 0.231, $2 \Rightarrow 1$

63 (upper molar paracone and metacone), 0.667, $0 \rightarrow 1$

73 (lower molar trigonid), $0.400,1 \Rightarrow 0$

197 (distal femur), $0.100,0 \rightarrow 1$

218 (calcaneofibular facet), $0.600,1 \Rightarrow 2$

219 (calcaneoastragalar facet), $0.500,0 \Rightarrow 1$

221 (sustentaculum on calcaneus), $0.500,0 \Rightarrow 1$

\section{Node $82 \rightarrow$ node 81}

1 (incisor enamel), 0.500, $0 \Rightarrow 1$

7 (i1 occurrence), 1.000, $0 \Rightarrow 1$

9 (I1 occurrence), $0.250,0 \Rightarrow 1$

11 (di2 occurrence), $1.000,0 \Rightarrow 2$

12 (i2 replacement), 1.000, $0 \Rightarrow 1$

13 (DI2 size), $1.000,0 \Rightarrow 2$

15 (I2 replacement), 1.000, $0 \Rightarrow 1$

22 (incisor shape), $0.750,0 \Rightarrow 2$

23 (upper canine occurrence), $0.500,0 \Rightarrow 2$

25 (lower canine occurrence), $0.333,1 \rightarrow 2$

27 ( $\mathrm{p} 1$ occurrence), $0.333,0 \Rightarrow 1$

28 (P1 occurrence), $0.250,0 \Rightarrow 1$

29 (p2 occurrence), $0.333,0 \Rightarrow 1$

31 (P2 shape), $0.600,0 \Rightarrow 1$

35 (P3 shape), 0.667, $0 \Rightarrow 1$

59 (upper molar centrocrsta), $0.750,0 \Rightarrow 2$

62 (upper molar conules), $0.300,0 \rightarrow 1$

63 (upper molar paracone and metacone), 0.667, $0 \rightarrow 1$

77 (lower molar paraconid), $0.400,0 \Rightarrow 2$

84 (lower diastema), $1.000,0 \Rightarrow 2$

86 (horizontal ramus length), $1.000,0 \Rightarrow 1$
89 (angular process size), $0.500,0 \Rightarrow 1$

93 (mandibular condyle shape), $0.500,1 \Rightarrow 2$

97 (infraorbital canal length), $0.500,0 \Rightarrow 1$

102 (lacrimal foramen position), $0.400,0 \Rightarrow 1$

103 (nasolacrimal canal position), $1.000,0 \Rightarrow 1$

119 (premaxilla on rostrum), $0.667,0 \Rightarrow 1$

123 (anterior zygomatic root), $0.667,0 \Rightarrow 1$

127 (orbit position), $0.500,0 \Rightarrow 1$

133 (palatine-lacrimal contact), 0.500, $0 \rightarrow 1$

148 (postglenoid process), $0.667,0 \Rightarrow 2$

149 (glenoid fossa position), $1.000,0 \Rightarrow 1$

170 (squamosal to epitympanic), $0.500,0 \rightarrow 1$

\section{Node $81 \rightarrow$ node 58}

2 (enamel layer), $0.667,0 \Rightarrow 2$

4 (upper diastema), 0.750, $0 \rightarrow 2$

18 (I3 position), $0.667,0 \rightarrow 1$

44 ( $\mathrm{P} 4$ anterior and posterior), $0.500,0 \Rightarrow 1$

91 (masseteric fossa), $0.300,0 \Rightarrow 1$

96 (incisive foramen), $0.545,0 \rightarrow 4$

118 (premaxilla size), $0.571,0 \rightarrow 4$

124 (ventral projection of maxilla), $1.000,0 \Rightarrow 1$

125 (zygomatic fossa), 1.000, $0 \Rightarrow 1$

144 (anterior projection of frontal), 1.000, $0 \Rightarrow 1$

213 (calcaneoastragalar axis), $0.143,0 \rightarrow 1$

215 (astragalonavicular facet), 1.000, $0 \Rightarrow 1$

219 (calcaneoastragalar facet), $0.500,0 \Rightarrow 1$

221 (sustentaculum on calcaneus), $0.500,0 \Rightarrow 1$

225 (plantar process of navicular), $0.333,0 \rightarrow 1$

\section{Node $\mathbf{5 8} \rightarrow$ node 57}

11 (di2 occurrence), 1.000, $2 \Rightarrow 1$

13 (DI2 size), $1.000,2 \Rightarrow 1$

14 (dI2 surface), $0.500,0 \rightarrow 1$

16 (i3 occurrence), $0.333,0 \rightarrow 1$

18 (I3 position), $0.667,1 \Rightarrow 2$

22 (incisor shape), $0.750,2 \Rightarrow 1$

31 (P2 shape), $0.600,1 \Rightarrow 3$

33 (p3 shape), $1.000,2 \Rightarrow 3$

35 (P3 shape), 0.667, $1 \Rightarrow 3$

39 (p4 talonid shape), $0.182,0 \rightarrow 2$

44 (P4 lophs), 0.500, $1 \Rightarrow 2$

45 (P4 hypocone), $0.286,0 \Rightarrow 2$

49 (upper tooth row size), $0.375,0 \Rightarrow 2$

50 (cheek teeth height), $0.500,1 \Rightarrow 3$

52 (lower tooth row size), $0.375,1 \Rightarrow 2$

56 (upper molar protoloph), $0.571,1 \Rightarrow 4$

57 (upper molar posteroloph), $0.571,1 \Rightarrow 4$

58 (upper molar trigon), $0.500,0 \Rightarrow 4$

59 (upper molar centrocrsta), $0.750,2 \Rightarrow 3$

61 (upper molar protocone), $0.429,1 \Rightarrow 2$

62 (upper molar conules), $0.300,1 \Rightarrow 3$

63 (upper molar paracone and metacone), 0.667,

$1 \Rightarrow 6$

68 (hypoflexus), $0.600,0 \Rightarrow 3$

70 (hypoflexid depth), $0.200,0 \Rightarrow 1$ 
71 (ectolophid occurrence), $0.167,0 \Rightarrow 1$

80 (lower molar talonid basin), $0.800,0 \Rightarrow 4$ 83 (m3 hypoconulid), 0.556, $1 \rightarrow 3$

85 (mandibular condyle height), $0.500,0 \rightarrow 2$

88 (coronoid process), 0.667, $0 \rightarrow 4$

99 (postglenoid foramen), 0.500, $1 \rightarrow 3$

100 (optic foramen), 0.333, $0 \rightarrow 1$

112 (sphenopalatine vacuity), $0.500,0 \rightarrow 1$

115 (skull shape), 1.000, $0 \rightarrow 1$

121 (fenestration of maxilla), $1.000,0 \Rightarrow 1$

131 (hard palate length), $0.500,0 \rightarrow 1$

139 (posterior process of jugal), 1.000, $0 \rightarrow 1$

147 (glenoid fossa shape), 0.800, $0 \rightarrow 4$

152 (alisphenoid-parietal contact), $0.286,0 \rightarrow 2$

154 (interparietal occurrence), $0.143,0 \rightarrow 1$

159 (external auditory canal), $0.167,0 \rightarrow 1$

176 (number of sacral vertebrae), $0.500,1 \rightarrow 2$

178 (acromion), 0.200, $0 \rightarrow 1$

191 (articular surface on femurs), $0.1671 \rightarrow 0$

197 (distal femur), $0.100,0 \rightarrow 1$

199 (distal tibia-fibula), $0.333,0 \Rightarrow 2$

201 (fibula size), $0.125,0 \Rightarrow 1$

214 (astragalar neck length), $0.143,0 \Rightarrow 1$

218 (calcaneofibular facet), $0.600,1 \Rightarrow 2$

220 (calcanealoastragalar position), $0.167,0 \Rightarrow 1$

\section{Node $57 \rightarrow$ node 56}

94 (coronoid canal occurrence), $1.000,0 \Rightarrow 1$

105 (foramen ovale), $0.333,1 \Rightarrow 2$

111 (sphenopterygoid canal), $0.333,0 \rightarrow 1$

114 (mastoid foramen), 0.429, $2 \rightarrow 3$

166 (distal internal carotid artery), 0.267, $1 \rightarrow 3$

167 (course of stapedial artery), 0.200, $0 \rightarrow 2$

177 (neck of scapula), $0.250,0 \Rightarrow 1$

179 (greater tuberosity), $0.250,0 \Rightarrow 1$

180 (entepicondylar foramen), $0.333,0 \Rightarrow 1$

182 (supratrochlear foramen), $0.143,0 \Rightarrow 1$

184 (posterior edge of proximal ulna), 0.125 , $0 \Rightarrow 1$

185 (central process of radial), $0.200,0 \Rightarrow 1$

\section{Node $56 \rightarrow$ node 55}

48 (M3 occurrence), $0.500,0 \Rightarrow 1$

139 (posterior process of jugal), 1.000, $1 \Rightarrow 2$

195 (third trochanter size), 0.182, $1 \rightarrow 0$

\section{Node $55 \rightarrow$ Ochotona}

161 (internal septa of bullae), $0.600,0 \rightarrow 3$

166 (distal internal carotid artery), 0.267, $3 \rightarrow 4$

169 (facial nerve), $0.222,0 \rightarrow 2$

187 (distal articulation surface), $0.333,1 \Rightarrow 0$

\section{Node $55 \rightarrow$ Prolagus}

154 (interparietal occurrence), $0.143,1 \Rightarrow 0$

161 (internal septa of bullae), $0.600,0 \rightarrow 1$

\section{Node $56 \rightarrow$ Lepus}

116 (nasal), 0.444, $1 \rightarrow 4$

129 (anteroventral rim of orbit), $0.500,0 \rightarrow 1$

130 (orbit floor), $0.500,0 \rightarrow 1$

145 (postorbital process of frontal), $0.222,0 \rightarrow 1$

146 (supraorbital crest), $0.143,0 \rightarrow 1$

194 (lesser trochanter orientation), $0.167,1 \rightarrow 0$

222 (calcaneus-navicular contact), $0.500,0 \Rightarrow 1$

\section{Node $57 \rightarrow$ Palaeolagus}

116 (nasal), 0.444, $1 \rightarrow 4$

129 (anteroventral rim of orbit), $0.500,0 \rightarrow 1$

130 (orbit floor), $0.500,0 \rightarrow 1$

145 (postorbital process of frontal), $0.222,0 \rightarrow 1$

146 (supraorbital crest), $0.143,0 \rightarrow 1$

167 (course of stapedial artery), $0.200,0 \rightarrow 1$

194 (less trochanter orientation), 0.167, $1 \rightarrow 0$

\section{Node $58 \rightarrow$ Mimotona}

14 (dI2 surface), $0.500,0 \rightarrow 1$

37 (p4 trigonid shape), $0.500,3 \rightarrow 2$

84 (lower diastema), $1.000,2 \Rightarrow 1$

87 (number of mental foramen), $0.167,0 \Rightarrow 1$

\section{Node $58 \rightarrow$ Mimolagus}

116 (nasal), $0.444,1 \rightarrow 3$

131 (hard palate length), $0.500,0 \rightarrow 1$

196 (patellar trochlea), $0.125,1 \Rightarrow 0$

210 (dorsal astragalar foramen), 0.250, $1 \rightarrow 0$

216 (cuboid facet on astragalus), $0.200,0 \Rightarrow 1$

222 (calcaneus-navicular contact), $0.500,0 \Rightarrow 1$

\section{Node $81 \rightarrow$ node 80}

4 (upper diastema), $0.750,0 \rightarrow 3$

16 (i3 occurrence), $0.333,0 \rightarrow 1$

17 (I3 occurrence), $0.333,0 \Rightarrow 1$

30 (P2 occurrence), $0.333,0 \Rightarrow 1$

37 (p4 trigonid shape), 0.500, $3 \rightarrow 2$

85 (mandibular condyle height), $0.500,0 \rightarrow 1$

88 (coronoid process), $0.667,0 \rightarrow 1$

96 (incisive foramen), $0.545,0 \rightarrow 2$

106 (dorsal palatine foramen), $0.500,0 \Rightarrow 1$

108 (sphenopalatine foramen), $0.429,0 \Rightarrow 1$

118 (premaxilla size), $0.571,0 \rightarrow 3$

137 (lacrimal-jugal contact), $0.250,0 \rightarrow 1$

147 (glenoid fossa shape), $0.800,0 \rightarrow 2$

153 (hamulus-bulla contact), $0.500,0 \Rightarrow 1$

203 (posterior process on distal tibia), 1.000, $0 \Rightarrow 1$

217 (calcaneus width), $0.250,1 \Rightarrow 0$

224 (medial tarsal sesamoid bone), $1.000,0 \Rightarrow 1$

\section{Node $80 \rightarrow$ node 61}

2 (enamel layer), $0.667,0 \Rightarrow 1$

42 (P4 buccal cusp), $0.231,2 \Rightarrow 1$ 
69 (mure occurrence), 0.200, $0 \Rightarrow 1$

218 (calcaneofibular facet), $0.600,1 \Rightarrow 0$

\section{Node $61 \rightarrow$ node 60}

56 (upper molar protoloph), $0.571,1 \Rightarrow 3$

62 (upper molar conules), $0.300,1 \Rightarrow 0$

\section{Node $60 \rightarrow$ Eurymylus}

158 (bulla shape), $0.667,1 \Rightarrow 2$

171 (tensor tympani muscle), $0.333,0 \Rightarrow 2$

\section{Node $60 \rightarrow$ node 59}

91 (masseteric fossa), $0.300,0 \Rightarrow 1$

93 (mandibular condyle shape), 0.500, $2 \rightarrow 0$

99 (postglenoid foramen), 0.500, $1 \rightarrow 2$

114 (mastoid foramen), 0.429, $2 \rightarrow 1$

116 (nasal), $0.444,1 \rightarrow 2$

117 (premaxilla-frontal suture), $0.500,0 \rightarrow 1$

124 (ventral projection of maxilla), $1.000,0 \Rightarrow 2$

125 (zygomatic fossa), $1.000,0 \Rightarrow 2$

142 (dorsal process of jugal), $0.667,0 \rightarrow 1$

143 (jugal ventral process), $1.000,0 \rightarrow 1$

145 (postorbital process of frontal), $0.222,0 \rightarrow 1$

155 (expansion of mastoid), $0.333,0 \rightarrow 1$

156 (mastoid in skull roof), 1.000, $0 \rightarrow 1$

159 (external auditory canal), 0.167, $0 \rightarrow 1$

169 (facial nerve), $0.222,0 \rightarrow 1$

197 (distal femur), $0.100,0 \rightarrow 1$

208 (lateral process of astragalus), 0.200, $1 \rightarrow 0$

225 (plantar process of navicular), 0.333, $0 \rightarrow 1$

\section{Node $59 \rightarrow$ Rhombomylus}

166 (distal internal carotid artery), 0.267, $1 \rightarrow 4$

167 (course of stapedial artery), $0.200,0 \Rightarrow 2$

185 (central process of radial), $0.200,0 \rightarrow 1$

202 (tibia malleolus), $0.286,1 \Rightarrow 0$

213 (calcaneoastragalar), $0.143,0 \rightarrow 1$

\section{Node $59 \rightarrow$ Matutinia}

42 (P4 buccal cusp), 0.231, $1 \Rightarrow 0$

44 (P4 lophs), 0.500, $0 \Rightarrow 1$

166 (distal internal carotid artery), 0.267, $1 \rightarrow 3$

189 (centrale occurrence), $0.500,0 \rightarrow 1$

192 (greater trochanter position), $0.167,1 \Rightarrow 0$

\section{Node $80 \rightarrow$ node 79}

32 (p3 occurrence), $1.000,0 \Rightarrow 1$

50 (cheek teeth height), $0.500,1 \Rightarrow 0$

56 (upper molar protoloph), $0.571,1 \Rightarrow 0$

61 (upper molar protocone), $0.429,1 \Rightarrow 0$

74 (posterior wall of trigonid), $1.000,1 \Rightarrow 2$

78 (lower molar metaconid), $0.286,0 \Rightarrow 1$
79 (lower molar mesoconid), $0.333,0 \Rightarrow 1$

80 (lower molar talonid basin), $0.800,0 \Rightarrow 1$

91 (masseteric fossa), $0.300,0 \Rightarrow 2$

\section{Node $79 \rightarrow$ Sinomylus}

3 (enamel type), 0.600, $1 \rightarrow 0$

30 (P2 occurrence), 0.333, $1 \Rightarrow 0$

42 (P4 buccal cusp), 0.231, $2 \Rightarrow 0$

62 (upper molar conules), $0.300,1 \Rightarrow 0$

\section{Node $79 \rightarrow$ Tribosphenomys}

3 (enamel type), 0.600, $1 \rightarrow 0$

35 (P3 shape), 0.667, $1 \rightarrow 2$

46 (molar labial cingulum), $0.500,1 \Rightarrow 0$

57 (upper molar posteroloph), $0.571,1 \Rightarrow 0$

58 (upper molar trigon), $0.500,0 \Rightarrow 2$

77 (lower molar paraconid), $0.400,2 \Rightarrow 1$

83 (m3 hypoconulid), $0.556,1 \rightarrow 2$

87 (number of mental foramen), $0.167,0 \rightarrow 1$

202 (tibia malleolus), $0.286,1 \Rightarrow 0$

205 (lateral astragalar trochlea), $0.200,0 \Rightarrow 1$

\section{Node $79 \rightarrow$ node 78}

2 (enamel layer), $0.667,0 \Rightarrow 1$

35 (P3 shape), 0.667, $1 \rightarrow 2$

39 (p4 talonid shape), $0.182,0 \Rightarrow 1$

49 (upper tooth row size), $0.375,0 \Rightarrow 1$

53 (upper molar crown shape), $0.429,1 \Rightarrow 2$

54 (upper cheek tooth precingulum), $0.600,0 \Rightarrow 1$

60 (upper molar mesostyle), $0.143,0 \Rightarrow 1$

69 (mure occurrence), $0.200,0 \Rightarrow 1$

83 (m3 hypoconulid), $0.556,1 \rightarrow 3$

87 (number of mental foramen), 0.167, $0 \rightarrow 1$

90 (angular process position), $0.667,0 \Rightarrow 1$

99 (postglenoid foramen), 0.500, $1 \rightarrow 4$

112 (sphenopalatine vacuity), $0.500,0 \rightarrow 1$

116 (nasal), $0.444,1 \rightarrow 2$

123 (anterior zygomatic root), 0.667, $1 \Rightarrow 2$

151 (alisphenoid-frontal contact), 0.250, $0 \rightarrow 1$

186 (ulnar facet of radial head), 0.200, $1 \rightarrow 0$

187 (distal articulation surface), 0.333, $1 \rightarrow 0$

188 (scaphoid and lunate fusion), 0.250, $0 \rightarrow 1$

213 (calcaneoastragalar and $\mathrm{Su}$ ), $0.143,0 \rightarrow 1$

\section{Node $78 \rightarrow$ Paramys}

90 (angular process position), 0.667, $1 \Rightarrow 0$

96 (incisive foramen), $0.545,2 \Rightarrow 1$

181 (radial fossa size), $0.167,1 \Rightarrow 0$

188 (scaphoid and lunate fusion), $0.250,1 \Rightarrow 0$

195 (third trochanter size), 0.182, $1 \rightarrow 0$

196 (patellar trochlea), $0.125,1 \Rightarrow 0$

202 (tibia malleolus), $0.286,1 \Rightarrow 0$

220 (calcanealoastragalar position), $0.167,0 \Rightarrow 1$ 


\section{Node $78 \rightarrow$ Reithroparamys}

79 (lower molar mesoconid), 0.333, $1 \Rightarrow 0$ 81 (lower molar entoconid), $0.333,0 \rightarrow 1$ 161 (internal septa of bullae), $0.600,0 \Rightarrow 1$ 166 (distal internal carotid artery), $0.267,1 \rightarrow 4$ 168 (stapedial artery shielding), $0.500,0 \Rightarrow 1$ 172 (meato-cochlear bridge), $0.500,0 \Rightarrow 1$ 192 (greater trochanter position), $0.167,1 \Rightarrow 0$ 193 (femoral neck length), $0.167,0 \Rightarrow 1$ 197 (distal femur), 0.100, $0 \rightarrow 1$

\section{Node $78 \rightarrow$ node 76}

56 (upper molar protoloph), $0.571,0 \Rightarrow 1$ 62 (upper molar conules), $0.300,1 \Rightarrow 0$

72 (upper molar metaloph), $0.500,0 \Rightarrow 1$

80 (lower molar talonid basin), $0.800,1 \Rightarrow 2$

154 (interparietal occurrence), $0.143,0 \Rightarrow 1$

\section{Node $76 \rightarrow$ node 75}

3 (enamel type), $0.600,1 \Rightarrow 3$

34 (P3 occurrence), 1.000, $0 \Rightarrow 1$

68 (hypoflexus), $0.600,0 \Rightarrow 1$

70 (hypoflexid depth), $0.200,0 \Rightarrow 1$

78 (lower molar metaconid), $0.286,1 \Rightarrow 0$

79 (lower molar mesoconid), $0.333,1 \Rightarrow 0$

159 (external auditory canal), $0.167,0 \rightarrow 1$

166 (distal internal carotid artery), 0.267, $1 \rightarrow 4$

182 (supratrochlear foramen), $0.143,0 \Rightarrow 1$

193 (femoral neck length), 0.167, $0 \rightarrow 1$

201 (fibula size), $0.125,0 \rightarrow 1$

\section{Node $75 \rightarrow$ node 63}

45 ( $\mathrm{P} 4$ hypocone), $0.286,0 \Rightarrow 2$

52 (lower tooth row size), $0.375,1 \Rightarrow 0$

92 (incisor protuberance), $1.000,0 \Rightarrow 1$

96 (incisive foramen), $0.545,2 \Rightarrow 5$

97 (infraorbital canal length), $0.500,1 \Rightarrow 2$

98 (infraorbital foramen diameter), $0.800,0 \rightarrow 2$

109 (posterior maxillary notch), $0.400,0 \Rightarrow 1$

111 (sphenopterygoid canal), $0.333,0 \Rightarrow 1$

120 (transverse constriction), $1.000,0 \Rightarrow 1$

126 (zygomatic plate), $1.000,0 \Rightarrow 3$

140 (jugal size), $0.500,0 \Rightarrow 1$

152 (alisphenoid-parietal contact), $0.286,0 \rightarrow 2$

197 (distal femur), 0.100, $0 \rightarrow 1$

\section{Node $63 \rightarrow$ Paradjidaumo}

49 (upper tooth row size), $0.375,1 \rightarrow 0$

80 (lower molar talonid basin), 0.800, $2 \rightarrow 3$

82 (lower molar hypolophid), $0.500,0 \rightarrow 1$

91 (masseteric fossa), 0.300, $2 \rightarrow 3$

108 (sphenopalatine foramen), $0.429,1 \Rightarrow 2$

194 (lesser trochanter orientation), $0.167,1 \Rightarrow 0$
Node $63 \rightarrow$ node 62

38 (p4 protostylid occurrence), 1.000, $0 \Rightarrow 1$

41 ( $\mathrm{P} 4$ size), $1.000,0 \Rightarrow 1$

43 (P4 styles), 1.000, $0 \Rightarrow 1$

49 (upper tooth row size), $0.375,1 \rightarrow 3$

54 (upper cheek tooth precingulum), $0.600,1 \Rightarrow 0$

56 (upper molar protoloph), $0.571,1 \Rightarrow 2$

57 (upper molar posteroloph), $0.571,1 \Rightarrow 2$

60 (upper molar mesostyle), 0.143, $1 \rightarrow 0$

65 (lingual cusp to protocone), 1.000, $0 \Rightarrow 2$

66 (labial cusps to protoconid), 1.000, $0 \Rightarrow 2$

71 (ectolophid occurrence), $0.167,0 \Rightarrow 1$

83 ( $\mathrm{m} 3$ hypoconulid), $0.556,3 \Rightarrow 5$

105 (foramen ovale), $0.333,1 \Rightarrow 2$

114 (mastoid foramen), $0.429,2 \rightarrow 0$

119 (premaxilla on rostrum), $0.667,1 \Rightarrow 2$

134 (lacrimal projection), $1.000,0 \Rightarrow 1$

199 (distal tibia-fibula), $0.333,0 \Rightarrow 1$

\section{Node $62 \rightarrow$ Florentiamys}

63 (upper molar paracone and metacone), 0.667,

$$
1 \stackrel{\Rightarrow}{\Rightarrow}
$$

91 (masseteric fossa), $0.300,2 \rightarrow 3$

100 (optic foramen), $0.333,0 \Rightarrow 1$

152 (alisphenoid-parietal contact), $0.286,2 \Rightarrow 1$

185 (central process of radial), $0.200,0 \rightarrow 1$

186 (ulnar facet of radial head), $0.200,0 \rightarrow 1$

195 (third trochanter size), 0.182, $1 \rightarrow 0$

\section{Node $62 \rightarrow$ Gregorymys}

39 (p4 talonid shape), $0.182,1 \Rightarrow 2$

61 (upper molar protocone), $0.429,0 \Rightarrow 1$

99 (postglenoid foramen), $0.500,4 \Rightarrow 3$

138 (jugal-maxilla contact), $0.333,0 \rightarrow 1$

166 (distal internal carotid artery), $0.267,4 \Rightarrow 3$

\section{Node $75 \rightarrow$ node 74}

53 (upper molar crown shape), $0.429,2 \Rightarrow 3$

60 (upper molar mesostyle), $0.143,1 \rightarrow 0$

177 (neck of scapula), $0.250,0 \Rightarrow 1$

180 (entepicondylar foramen), $0.333,0 \Rightarrow 1$

\section{Node $74 \rightarrow$ node 65}

36 ( $\mathrm{p} 4$ occurrence), 1.000, $0 \Rightarrow 1$

40 (P4 occurrence), 1.000, $0 \Rightarrow 1$

49 (upper tooth row size), $0.375,1 \rightarrow 3$

52 (lower tooth row size), $0.375,1 \Rightarrow 3$

56 (upper molar protoloph), $0.571,1 \Rightarrow 0$

76 (M1 anterocone), 1.000, $0 \Rightarrow 1$

98 (infraorbital foramen diameter), $0.800,0 \rightarrow 4$

99 (postglenoid foramen), $0.500,4 \rightarrow 3$

101 (nasolacrimal), $1.000,0 \Rightarrow 1$

102 (lacrimal foramen position), $0.400,1 \Rightarrow 2$

126 (zygomatic plate), $1.000,0 \Rightarrow 1$ 


\section{Node $65 \rightarrow$ Cricetops}

75 (m1 anteroconid), 1.000, $0 \rightarrow 1$

\section{Node $65 \rightarrow$ node 64}

57 (upper molar posteroloph), $0.571,1 \Rightarrow 2$

63 (upper molar paracone and metacone), 0.667, $1 \Rightarrow 3$

65 (lingual cusp to protocone), 1.000, $0 \Rightarrow 1$

66 (labial cusps to protoconid), 1.000, $0 \Rightarrow 1$

71 (ectolophid occurrence), $0.167,0 \Rightarrow 1$

73 (lower molar trigonid), 0.400, $1 \rightarrow 2$

75 ( $\mathrm{m} 1$ anteroconid), 1.000, $0 \rightarrow 2$

78 (lower molar metaconid), $0.286,0 \Rightarrow 1$

91 (masseteric fossa), $0.300,2 \Rightarrow 3$

117 (premaxilla-frontal suture), $0.500,0 \Rightarrow 1$

135 (lacrimal size), $0.667,0 \Rightarrow 2$

140 (jugal size), $0.500,0 \Rightarrow 1$

152 (alisphenoid-parietal contact), $0.286,0 \rightarrow 2$

166 (distal internal carotid artery), $0.267,4 \rightarrow 3$

195 (third trochanter size), $0.182,1 \rightarrow 0$

197 (distal femur), 0.100, $0 \rightarrow 1$

199 (distal tibia-fibula), $0.333,0 \rightarrow 1$

\section{Node $64 \rightarrow$ Rattus}

169 (facial nerve), $0.222,0 \rightarrow 1$

181 (radial fossa size), $0.167,1 \Rightarrow 0$

182 (supratrochlear foramen), $0.143,1 \Rightarrow 0$

\section{Node $64 \rightarrow$ Mus}

169 (facial nerve), $0.222,0 \rightarrow 2$

178 (acromion), $0.200,0 \Rightarrow 1$

198 (proximal tibia-fibula), $0.333,0 \rightarrow 1$

214 (astragalar neck length), $0.143,0 \Rightarrow 1$

\section{Node $74 \rightarrow$ node 73}

3 (enamel type), $0.600,3 \Rightarrow 2$

50 (cheek teeth height), $0.500,0 \Rightarrow 1$

61 (upper molar protocone), $0.429,0 \Rightarrow 1$

62 (upper molar conules), $0.300,0 \Rightarrow 3$

73 (lower molar trigonid), $0.400,1 \rightarrow 2$

80 (lower molar talonid basin), $0.8002 \rightarrow 3$

82 (lower molar hypolophid), $0.500,0 \rightarrow 1$

96 (incisive foramen), $0.545,2 \Rightarrow 3$

\author{
Node $73 \rightarrow$ Tataromys \\ 39 (p4 talonid shape), 0.182, $1 \rightarrow 0$ \\ 42 (P4 buccal cusp), 0.231, $2 \rightarrow 0$ \\ 68 (hypoflexus), $0.600,1 \Rightarrow 2$ \\ 83 (m3 hypoconulid), $0.5563 \Rightarrow 4$ \\ 98 (infraorbital foramen diameter), $0.800,0 \rightarrow 3$ \\ 155 (expansion of mastoid), $0.333,0 \Rightarrow 1$ \\ 202 (tibia malleolus), $0.286,1 \Rightarrow 0$
}

Node $73 \rightarrow$ node 72

37 (p4 trigonid shape), $0.500,2 \rightarrow 3$

39 (p4 talonid shape), $0.182,1 \rightarrow 2$

42 (P4 buccal cusp), 0.231, $2 \rightarrow 3$

44 (P4 lophs), 0.500, $0 \Rightarrow 2$

54 (upper cheek tooth precingulum), $0.600,1 \Rightarrow 2$

81 (lower molar entoconid), $0.333,0 \Rightarrow 1$

90 (angular process position), $0.667,1 \Rightarrow 2$

103 (nasolacrimal canal position), $1.000,1 \Rightarrow 2$

132 (orbital wing of palatine), $1.000,0 \Rightarrow 1$

141 (jugal contribution to glenoid), $0.500,0 \rightarrow 1$

150 (orbital process of alisphenoid), 1.000, $0 \Rightarrow 1$

151 (alisphenoid-frontal contact), 0.250, $1 \Rightarrow 0$

167 (course of stapedial artery), 0.200, $0 \rightarrow 2$

169 (facial nerve), $0.222,0 \rightarrow 2$

173 (round window position), 1.000, $0 \rightarrow 1$

\section{Node $72 \rightarrow$ Tsaganomys}

13 (DI2 size), $1.000,2 \Rightarrow 3$

22 (incisor shape), $0.750,2 \Rightarrow 3$

49 (upper tooth row size), $0.375,1 \Rightarrow 0$

61 (upper molar protocone), $0.429,1 \Rightarrow 2$

64 (upper molar hypocone), $0.250,1 \Rightarrow 0$

68 (hypoflexus), $0.600,1 \Rightarrow 0$

69 (mure occurrence), $0.200,1 \Rightarrow 0$

70 (hypoflexid depth), $0.200,1 \Rightarrow 0$

72 (upper molar metaloph), 0.500, $1 \rightarrow 0$

96 (incisive foramen), $0.545,3 \Rightarrow 6$

105 (foramen ovale), $0.333,1 \Rightarrow 2$

118 (premaxilla size), $0.571,3 \rightarrow 2$

137 (lacrimal-jugal contact), $0.250,1 \Rightarrow 0$

154 (interparietal occurrence), $0.143,1 \rightarrow 0$

161 (internal septa of bullae), $0.600,0 \Rightarrow 2$

\section{Node $72 \rightarrow$ node 71}

45 (P4 hypocone) $0.286,0 \Rightarrow 2$

58 (upper molar trigon), $0.500,0 \Rightarrow 3$

67 (mesolophule occurrence), $1.000,0 \Rightarrow 1$

72 (upper molar metaloph), $0.500,1 \rightarrow 2$

82 (lower molar hypolophid), $0.500,1 \Rightarrow 2$

91 (masseteric fossa), $0.300,2 \Rightarrow 3$

98 (infraorbital foramen diameter), $0.800,0 \rightarrow 3$

114 (mastoid foramen), $0.429,2 \rightarrow 3$

119 (premaxilla on rostrum), $0.667,1 \Rightarrow 2$

127 (orbit position), $0.500,1 \Rightarrow 2$

138 (jugal-maxilla contact), $0.333,0 \Rightarrow 1$

152 (alisphenoid-parietal contact), $0.286,0 \rightarrow 2$

157 (mastoid exposure on occiput), $0.500,0 \rightarrow 1$

Node $71 \rightarrow$ node 70

51 (upper tooth row positions), $0.333,0 \Rightarrow 1$

54 (upper cheek tooth precingulum), $0.600,2 \Rightarrow 3$

63 (upper molar paracone and metacone), 0.667 , $1 \Rightarrow 5$

78 (lower molar metaconid), $0.286,0 \rightarrow 2$ 
99 (postglenoid foramen), 0.500, $4 \rightarrow 3$

102 (lacrimal foramen position), $0.400,1 \Rightarrow 2$

106 (dorsal palatine foramen), 0.500, $1 \rightarrow 3$

111 (sphenopterygoid canal), $0.333,0 \rightarrow 1$

116 (nasal), $0.444,2 \Rightarrow 3$

154 (interparietal occurrence), $0.143,1 \rightarrow 0$

171 (tensor tympani muscle) $, 0.333,0 \rightarrow 1$

227 (metatarsal V occurrence), 1.000, $0 \rightarrow 1$

\section{Node $70 \rightarrow$ node 67}

53 (upper molar crown shape), $0.429,3 \rightarrow 2$

184 (posterior edge of proximal ulna), 0.125 , $0 \Rightarrow 1$

192 (great trochanter position), $0.167,1 \Rightarrow 0$

196 (patellar trochlea), $0.125,1 \Rightarrow 0$

\section{Node $67 \rightarrow$ node 66}

22 (incisor shape), $0.750,2 \Rightarrow 3$

49 (upper tooth row size), $0.375,1 \Rightarrow 0$

50 (cheek teeth height), $0.500,1 \rightarrow 2$

52 (lower tooth row size), $0.375,1 \Rightarrow 0$

145 (postorbital process of frontal), $0.222,0 \Rightarrow 1$

160 (opening of external audit), $0.333,0 \rightarrow 1$

\section{Node $66 \rightarrow$ Incamys}

51 (upper tooth row positions), $0.333,1 \Rightarrow 0$

58 (upper molar trigon), $0.500,3 \Rightarrow 0$

71 (ectolophid occurrence), $0.167,0 \Rightarrow 1$

78 (lower molar metaconid), 0.286, $2 \rightarrow 1$

155 (expansion of mastoid), $0.333,0 \Rightarrow 1$

157 (mastoid exposure on occiput), $0.500,1 \Rightarrow 0$

\section{Node $66 \rightarrow$ Neoreomys}

104 (vacuity of nasolacrimal), $0.500,0 \Rightarrow 1$

171 (Tensor tympani muscle), $0.333,1 \rightarrow 2$

201 (fibula size), $0.125,1 \rightarrow 0$

\section{Node $67 \rightarrow$ Erethizon}

91 (masseteric fossa), $0.300,3 \Rightarrow 2$

105 (foramen ovale), $0.333,1 \rightarrow 2$

108 (sphenopalatine foramen), 0.429, $1 \rightarrow 2$

135 (lacrimal size), $0.667,0 \Rightarrow 1$

147 (glenoid fossa shape), 0.800, $2 \rightarrow 3$

177 (neck of scapula), $0.250,1 \Rightarrow 0$

182 (supratrochlear foramen), $0.143,1 \Rightarrow 0$

191 (articular surface on femurs), $0.167,1 \Rightarrow 0$

193 (femoral neck length), $0.167,1 \Rightarrow 0$

200 (fibula-femur articulation), $0.500,0 \Rightarrow 1$

202 (tibia malleolus), 0.286, $1 \rightarrow 0$

205 (lateral astragalar trochlea), $0.200,0 \rightarrow 1$

226 (metatarsal IV occurrence), $0.500,0 \rightarrow 1$

\section{Node $70 \rightarrow$ node 69}

50 (cheek teeth height), 0.500, $1 \rightarrow 2$

108 (sphenopalatine foramen), 0.429, $1 \rightarrow 3$

146 (supraorbital crest), $0.143,0 \Rightarrow 1$

147 (glenoid fossa shape), $0.8002 \rightarrow 3$

153 (hamulus-bulla contact), $0.500,1 \Rightarrow 0$

205 (lateral astragalar trochlea), 0.200, $0 \rightarrow 1$

212 (sustentacular facet contact), $0.333,0 \Rightarrow 1$

220 (calcanealoastragalar position), $0.167,0 \Rightarrow 1$

225 (plantar process of navicular), $0.333,0 \Rightarrow 1$

\section{Node $69 \rightarrow$ Myocastor}

88 (coronoid process), 0.667, $1 \rightarrow 3$

135 (lacrimal size), $0.667,0 \Rightarrow 1$

195 (third trochanter size), $0.182,1 \Rightarrow 2$

213 (calcaneoastragalar), $0.143,1 \rightarrow 0$

\section{Node $69 \rightarrow$ node 68}

78 (lower molar metaconid), 0.286, $2 \rightarrow 1$

102 (lacrimal foramen position), $0.400,2 \Rightarrow 0$

104 (vacuity of nasolacrimal), $0.500,0 \Rightarrow 1$

136 (lacrimal facial process), $0.333,1 \Rightarrow 0$

160 (opening of external audit), 0.333, $0 \rightarrow 1$

171 (tensor tympani muscle), $0.333,1 \rightarrow 2$

178 (acromion), 0.200, $0 \Rightarrow 1$

179 (greater tuberosity), $0.250,0 \Rightarrow 1$

185 (central process of radial), $0.200,0 \Rightarrow 1$

197 (distal femur), $0.100,0 \rightarrow 1$

226 (metatarsal IV occurrence), $0.500,0 \rightarrow 1$

\section{Node $68 \rightarrow$ Cavia}

71 (ectolophid occurrence), $0.167,0 \rightarrow 1$

88 (coronoid process), $0.667,1 \rightarrow 3$

186 (ulnar facet of radial head), $0.200,0 \Rightarrow 1$

213 (calcaneoastragalar), $0.143,1 \rightarrow 0$

\section{Node $68 \rightarrow$ Dasyprocta}

51 (upper tooth row positions), $0.333,1 \Rightarrow 0$

96 (incisive foramen), $0.545,3 \Rightarrow 1$

100 (optic foramen), $0.333,0 \Rightarrow 1$

107 (supraorbital foramen), $0.333,0 \Rightarrow 1$

108 (sphenopalatine foramen), 0.429, $3 \rightarrow 2$

113 (supraorbital notch), $0.500,0 \Rightarrow 2$

145 (postorbital process of frontal), $0.222,0 \Rightarrow 1$

184 (posterior edge of proximal ulna), 0.125 , $0 \Rightarrow 1$

198 (proximal tibia-fibula), $0.333,0 \Rightarrow 1$

208 (lateral process of astragalus), $0.200,1 \Rightarrow 0$

\section{Node $71 \rightarrow$ Paraphiomys}

52 (lower tooth row size), $0.375,1 \Rightarrow 0$

53 (upper molar crown shape), $0.429,3 \rightarrow 2$

78 (lower molar metaconid), $0.286,0 \rightarrow 1$ 
118 (premaxilla size), $0.571,3 \rightarrow 2$

160 (opening of external audit), $0.333,0 \rightarrow 1$

\section{Node $76 \rightarrow$ Sciuravus}

45 (P4 hypocone), $0.286,0 \Rightarrow 1$

77 (lower molar paraconid), 0.400, $2 \Rightarrow 0$

82 (lower molar hypolophid), $0.500,0 \rightarrow 1$

96 (incisive foramen), $0.5452 \Rightarrow 1$

181 (radial fossa size), $0.167,1 \Rightarrow 0$

214 (astragalar neck length), $0.143,0 \Rightarrow 1$

\section{Node $78 \rightarrow$ Cocomys}

39 (p4 talonid shape), $0.182,1 \Rightarrow 0$

42 (P4 buccal cusp), 0.231, $2 \Rightarrow 0$

69 (mure occurrence), 0.200, $1 \Rightarrow 0$

87 (number of mental foramina), $0.167,1 \Rightarrow 0$

91 (masseteric fossa), $0.300,2 \Rightarrow 1$

106 (dorsal palatine foramen), $0.500,1 \Rightarrow 0$

154 (interparietal occurrence), $0.143,0 \Rightarrow 1$

\section{Node $78 \rightarrow$ node 77}

3 (enamel type), $0.600,1 \Rightarrow 3$

60 (upper molar mesostyle), $0.143,1 \Rightarrow 0$

62 (upper molar conules), $0.300,1 \Rightarrow 0$

81 (lower molar entoconid), $0.333,0 \rightarrow 1$

91 (masseteric fossa), $0.300,2 \Rightarrow 3$

96 (incisive foramen), $0.545,2 \Rightarrow 1$

98 (infraorbital foramen diameter), $0.800,0 \Rightarrow 1$

113 (supraorbital notch), $0.500,0 \Rightarrow 1$

126 (zygomatic plate), $1.000,0 \Rightarrow 2$

137 (lacrimal-jugal contact), $0.250,1 \Rightarrow 0$

145 (postorbital process of frontal), $0.222,0 \Rightarrow 1$

146 (supraorbital crest), $0.143,0 \Rightarrow 1$

159 (external auditory canal), 0.167, $0 \rightarrow 1$

161 (internal septa of bullae), $0.600,0 \Rightarrow 1$

166 (distal internal carotid artery), 0.267, $1 \rightarrow 4$

167 (course of stapedial artery), $0.200,0 \Rightarrow 1$

168 (stapedial artery shielding), $0.500,0 \Rightarrow 1$

169 (facial nerve), $0.222,0 \Rightarrow 2$

171 (tensor tympani muscle), $0.333,0 \Rightarrow 1$

172 (meato-cochlear bridge), $0.500,0 \Rightarrow 1$

195 (third trochanter size), 0.182, $1 \rightarrow 0$

196 (patellar trochlea), $0.125,1 \Rightarrow 0$

201 (fibula size), $0.125,0 \Rightarrow 1$

\section{Node $77 \rightarrow$ Sciurus}

181 (radial fossa size), $0.167,1 \Rightarrow 0$

194 (lesser trochanter orientation), $0.167,1 \Rightarrow 0$

212 (sustentacular facet contact), $0.333,0 \Rightarrow 1$

213 (calcaneoastragalar and Su), $0.143,1 \Rightarrow 0$

220 (calcanealoastragalar position), $0.167,0 \Rightarrow 1$

\section{Node $77 \rightarrow$ Marmota}

106 (dorsal palatine foramen), $0.500,1 \Rightarrow 0$

108 (sphenopalatine foramen), $0.429,1 \Rightarrow 0$
109 (posterior maxillary notch), 0.400, $0 \rightarrow 1$

152 (alisphenoid-parietal contact), $0.286,0 \rightarrow 1$

180 (entepicondylar foramen), $0.333,0 \Rightarrow 1$

184 (posterior edge of proximal ulna), 0.125 ,

$$
0 \Rightarrow 1
$$

\section{Node $84 \rightarrow$ node 83}

35 (P3 shape), $0.667,0 \Rightarrow 4$

39 (p4 talonid shape), $0.182,0 \rightarrow 2$

45 (P4 hypocone), $0.286,0 \Rightarrow 2$

47 (m3 occurrence), $1.000,0 \Rightarrow 1$

48 (M3 occurrence), $0.500,0 \Rightarrow 1$

53 (upper molar crown shape), $0.429,1 \Rightarrow 3$

57 (upper molar posteroloph), $0.571,1 \Rightarrow 3$

63 (upper molar paracone and metacone), 0.667, $0 \rightarrow 4$

70 (hypoflexid depth), $0.200,0 \rightarrow 1$

72 (upper molar metaloph), $0.500,0 \Rightarrow 1$

83 (m3 hypoconulid), $0.556,0 \rightarrow 3$

85 (mandibular condyle height), $0.500,0 \rightarrow 1$

88 (coronoid process), $0.667,0 \Rightarrow 2$

93 (mandibular condyle shape), $0.500,1 \Rightarrow 0$

109 (posterior maxillary notch), $0.400,0 \Rightarrow 2$

118 (premaxilla size), $0.571,0 \Rightarrow 1$

122 (antorbital fossa), $0.667,0 \Rightarrow 2$

147 (glenoid fossa shape), $0.800,0 \Rightarrow 1$

148 (postglenoid process occur), $0.667,0 \rightarrow 1$

159 (external auditory canal), $0.167,0 \rightarrow 1$

162 (alisphenoid contribution), 1.000, $0 \Rightarrow 1$

163 (ectotympanic contribution), $0.500,2 \Rightarrow 1$

164 (entotympanic bulla), $0.333,0 \rightarrow 1$

165 (petrosal contribution), $0.667,0 \Rightarrow 1$

170 (squamosal to epitympanic), $0.500,0 \rightarrow 1$

179 (greater tuberosity), $0.250,0 \Rightarrow 1$

197 (distal femur), $0.100,0 \rightarrow 1$

198 (proximal tibia-fibula), $0.333,0 \Rightarrow 1$

199 (distal tibia-fibula), $0.333,0 \Rightarrow 2$

200 (fibula-femur articulation), $0.500,0 \Rightarrow 1$

201 (fibula size), $0.125,0 \rightarrow 1$

202 (tibia malleolus), $0.286,0 \rightarrow 2$

211 (sustentacular facet orientation), $0.500,0 \Rightarrow 1$

216 (cuboid facet on astragalus), $0.200,0 \Rightarrow 1$

218 (calcaneofibular facet), $0.600,1 \Rightarrow 3$

219 (calcaneoastragalar facet), $0.500,0 \Rightarrow 2$

223 (cuboid length), $0.333,0 \Rightarrow 1$

\section{Node $83 \rightarrow$ Petrodromus}

10 (I1 size), $0.500,0 \rightarrow 1$

23 (upper canine occurrence), $0.500,0 \Rightarrow 1$

68 (hypoflexus), $0.600,0 \rightarrow 1$

97 (infraorbital canal length), $0.500,0 \Rightarrow 1$

169 (facial nerve), $0.222,0 \Rightarrow 2$

177 (neck of scapula), $0.250,0 \Rightarrow 1$

183 (ulna-radia connection), $0.500,0 \Rightarrow 1$

193 (femoral neck length), $0.167,0 \Rightarrow 1$

195 (third trochanter size), 0.182, $1 \rightarrow 0$ 
205 (lateral astragalar trochlea), 0.200, $0 \rightarrow 1$

210 (dorsal astragalar foramen), 0.250, $1 \rightarrow 0$

214 (astragalar neck length), $0.143,0 \rightarrow 1$

\section{Node $83 \rightarrow$ Rhynchocyon}

9 (I1 occurrence), $0.250,0 \Rightarrow 1$

24 (upper canine roots), 0.250, $1 \Rightarrow 0$

95 (mandibular foramen position), 0.250, $0 \rightarrow 1$

113 (supraorbital notch), $0.500,0 \Rightarrow 1$

146 (supraorbital crest), $0.143,0 \Rightarrow 1$

151 (alisphenoid-frontal contact), $0.250,0 \Rightarrow 1$

166 (distal internal carotid artery), 0.267, $1 \rightarrow 2$

184 (posterior edge of proximal ulna), 0.125 ,

$0 \Rightarrow 1$

191 (articular surface on femurs), $0.167,1 \Rightarrow 0$

\section{Node $91 \rightarrow$ node 90}

52 (lower tooth row size), $0.375,0 \Rightarrow 1$

151 (alisphenoid-frontal contact), $0.250,0 \Rightarrow 1$

166 (distal internal carotid artery), $0.267,0 \rightarrow 1$

181 (radial fossa size), $0.167,1 \Rightarrow 0$

182 (supratrochlear foramen), $0.143,1 \Rightarrow 0$

191 (articular surface on femurs), $0.167,1 \Rightarrow 0$

\section{Node $90 \rightarrow$ Leptictis}

9 (I1 occurrence), $0.250,0 \Rightarrow 1$

39 (p4 talonid shape), $0.182,0 \Rightarrow 1$

97 (infraorbital canal length), $0.500,0 \Rightarrow 1$

109 (posterior maxillary notch), $0.400,0 \Rightarrow 1$

116 (nasal), $0.444,0 \Rightarrow 1$

122 (antorbital fossa), $0.667,0 \Rightarrow 2$

164 (entotympanic bulla), $0.333,0 \rightarrow 2$

178 (acromion), $0.200,0 \Rightarrow 1$

197 (distal femur), $0.100,0 \rightarrow 1$

199 (distal tibia-fibula), $0.333,0 \Rightarrow 2$

201 (fibula size), $0.125,0 \rightarrow 1$

\section{Node $90 \rightarrow$ node 89}

73 (lower molar trigonid), 0.400, $0 \rightarrow 1$

158 (bulla shape), $0.667,0 \rightarrow 1$

159 (external auditory canal), $0.167,0 \rightarrow 1$

167 (course of stapedial artery), $0.200,0 \Rightarrow 1$

188 (scaphoid and lunate fusion), $0.250,0 \rightarrow 1$

205 (lateral astragalar trochlea), $0.200,0 \Rightarrow 1$

207 (ventral limit of astragalus), 1.000, $1 \Rightarrow 0$

212 (sustentacular facet contact), $0.333,0 \rightarrow 1$

220 (calcanealoastragalar position), $0.167,0 \rightarrow 1$

\section{Node $89 \rightarrow$ node 86}

23 (upper canine occurrence), $0.500,0 \Rightarrow 1$

27 ( $\mathrm{p} 1$ occurrence) $, 0.333,0 \rightarrow 1$

28 (P1 occurrence), $0.250,0 \rightarrow 1$

59 (upper molar centrocrsta), $0.750,0 \Rightarrow 2$
64 (upper molar hypocone), $0.250,1 \Rightarrow 0$

88 (coronoid process), $0.667,0 \Rightarrow 1$

96 (incisive foramen), $0.545,0 \Rightarrow 1$

105 (foramen ovale), $0.333,1 \Rightarrow 0$

107 (supraorbital foramen), $0.333,0 \Rightarrow 1$

113 (supraorbital notch), $0.500,0 \Rightarrow 2$

146 (supraorbital crest), 0.143, $0 \rightarrow 1$

163 (ectotympanic contribution), $0.500,0 \Rightarrow 1$

196 (patellar trochlea), 0.125, $1 \rightarrow 0$

201 (fibula size), $0.125,0 \rightarrow 1$

214 (astragalar neck length), 0.143, $0 \rightarrow 1$

\section{Node $86 \rightarrow$ Cynocephalus}

9 (I1 occurrence), $0.250,0 \Rightarrow 1$

24 (upper canine roots), $0.250,1 \Rightarrow 0$

26 (lower canine roots), $0.500,1 \Rightarrow 0$

29 (p2 occurrence), $0.333,0 \Rightarrow 1$

30 (P2 occurrence), $0.333,0 \Rightarrow 1$

39 (p4 talonid shape), $0.182,0 \Rightarrow 1$

49 (upper tooth row size), $0.375,0 \Rightarrow 1$

58 (upper molar trigon), $0.500,0 \Rightarrow 2$

62 (upper molar conules), $0.300,0 \Rightarrow 1$

71 (ectolophid occurrence), $0.167,0 \Rightarrow 1$

95 (mandibular foramen position), $0.250,0 \rightarrow 1$

99 (postglenoid foramen), $0.500,1 \Rightarrow 5$

110 (alisphenoid canal), 0.333, $1 \rightarrow 0$

114 (mastoid foramen), $0.429,2 \Rightarrow 3$

127 (orbit position), $0.500,0 \Rightarrow 1$

141 (jugal contribution to glenoid), $0.500,0 \Rightarrow 1$

152 (alisphenoid-parietal contact), $0.286,0 \Rightarrow 1$

154 (interparietal occurrence), $0.143,0 \Rightarrow 1$

164 (entotympanic bulla), $0.333,0 \rightarrow 1$

167 (course of stapedial artery), $0.200,1 \Rightarrow 2$

176 (number of sacral vertebra), $0.500,1 \rightarrow 2$

181 (radial fossa size), $0.167,0 \Rightarrow 1$

182 (supratrochlear foramen), $0.143,0 \Rightarrow 1$

183 (ulna-radia connection), $0.500,0 \Rightarrow 1$

184 (posterior edge of proximal ulna), 0.125 , $0 \Rightarrow 1$

186 (ulnar facet of radial head), 0.200, $1 \rightarrow 0$

192 (greater trochanter position), $0.167,1 \rightarrow 0$

204 (astragalus trochlea), 0.500, $1 \Rightarrow 0$

213 (calcaneoastragalar), $0.143,0 \Rightarrow 1$

\section{Node $86 \rightarrow$ Tupaia}

17 (I3 occurrence), 0.333, $0 \rightarrow 1$

42 (P4 buccal cusp), 0.231, $2 \Rightarrow 0$

52 (lower tooth row size), $0.375,1 \Rightarrow 0$

60 (upper molar mesostyle), $0.143,0 \Rightarrow 1$

63 (upper molar paracone and metacone), 0.667, $0 \rightarrow 2$

109 (posterior maxillary notch), $0.400,0 \Rightarrow 1$

142 (dorsal process of jugal), $0.667,0 \rightarrow 2$

145 (postorbital process of frontal), $0.222,0 \Rightarrow 2$

164 (entotympanic bulla), $0.333,0 \rightarrow 2$

166 (distal internal carotid artery), $0.267,1 \Rightarrow 2$ 
194 (lesser trochanter orientation), $0.1671 \rightarrow 0$

195 (third trochanter size), $0.182,1 \Rightarrow 0$

208 (lateral process of astragalus), $0.200,0 \Rightarrow 1$

211 (sustentacular facet orientation), $0.500,0 \Rightarrow 1$

\section{Node $89 \rightarrow$ node 88}

16 (i3 occurrence), $0.333,0 \Rightarrow 1$

17 (I3 occurrence), 0.333, $0 \rightarrow 1$

31 (P2 shape), $0.600,0 \Rightarrow 1$

53 (upper molar crown shape), $0.429,0 \Rightarrow 1$

57 (upper molar posteroloph), $0.571,0 \Rightarrow 1$

63 (upper molar paracone and metacone), 0.667, $0 \rightarrow 3$

83 ( $\mathrm{m} 3$ hypoconulid), $0.556,0 \Rightarrow 1$

86 (horizontal ramus length), $1.000,0 \Rightarrow 2$

95 (mandibular foramen position), $0.250,0 \rightarrow 1$

99 (postglenoid foramen), $0.500,1 \Rightarrow 0$

110 (alisphenoid canal), $0.333,1 \rightarrow 0$

122 (antorbital fossa), $0.667,0 \Rightarrow 1$

133 (palatine-lacrimal contact), $0.500,0 \Rightarrow 1$

137 (lacrimal-jugal contact), $0.250,0 \Rightarrow 1$

142 (dorsal process of jugal), 0.667, $0 \rightarrow 2$

186 (ulnar facet of radial head), $0.200,1 \rightarrow 0$

194 (lesser trochanter orientation), 0.167, $1 \rightarrow 0$

206 (astragalar posterior trochlea), 1.000, $0 \Rightarrow 1$

209 (astragalofibular facet), $0.500,1 \Rightarrow 0$

210 (dorsal astragalar foramen), $0.250,1 \Rightarrow 0$

\section{Node $88 \rightarrow$ Plesiadapids}

4 (upper diastema), $0.750,0 \Rightarrow 1$

8 (i1 size), $0.500,0 \Rightarrow 1$

10 (I1 size), $0.500,0 \Rightarrow 1$

25 (lower canine occurrence), 0.333, $1 \rightarrow 2$

27 ( $\mathrm{p} 1$ occurrence), $0.333,0 \rightarrow 1$

28 (P1 occurrence), $0.250,0 \rightarrow 1$

37 ( $\mathrm{p} 4$ trigonid shape), $0.500,1 \Rightarrow 0$

42 (P4 buccal cusp), 0.231, $2 \rightarrow 1$
87 (number of mental foramen), $0.167,0 \rightarrow 1$

116 (nasal), $0.444,0 \Rightarrow 1$

118 (premaxilla size), $0.571,0 \Rightarrow 2$

164 (entotympanic bulla), 0.333, $0 \rightarrow 1$

165 (petrosal contribution), $0.667,0 \rightarrow 1$

191 (articular surface on femurs), $0.167,0 \Rightarrow 1$

196 (patellar trochlea), 0.125, $1 \rightarrow 0$

223 (cuboid length), $0.333,0 \Rightarrow 1$

\section{Node $88 \rightarrow$ node 87}

25 (lower canine occurrence), $0.3331 \rightarrow 0$

55 (upper cheek tooth lingual), $0.500,0 \Rightarrow 1$

58 (upper molar trigon), $0.500,0 \Rightarrow 1$

89 (angular process size), $0.500,0 \Rightarrow 1$

102 (lacrimal foramen position), $0.400,0 \Rightarrow 1$

128 (orbit orientation), 1.000, $0 \Rightarrow 1$

136 (lacrimal facial process), $0.333,0 \Rightarrow 1$

138 (jugal-maxilla contact), $0.333,0 \Rightarrow 1$

145 (postorbital process of frontal), $0.222,0 \Rightarrow 2$

146 (supraorbital crest), $0.143,0 \rightarrow 1$

165 (petrosal contribution), $0.667,0 \rightarrow 2$

166 (distal internal carotid artery), $0.267,1 \Rightarrow 2$

169 (facial nerve), $0.222,0 \Rightarrow 2$

195 (third trochanter size), $0.182,1 \Rightarrow 0$

214 (astragalar neck length), $0.143,0 \rightarrow 1$

217 (calcaneus width), $0.250,0 \Rightarrow 1$

219 (calcaneoastragalar facet), $0.500,0 \Rightarrow 1$

\section{Node $87 \rightarrow$ Notharctus}

42 (P4 buccal cusp), 0.231, $2 \rightarrow 1$

60 (upper molar mesostyle), $0.143,0 \Rightarrow 1$

192 (greater trochanter position), $0.1671 \rightarrow 0$

Node $87 \rightarrow$ Adapis

45 (P4 hypocone), $0.286,0 \Rightarrow 1$

127 (orbit position), $0.500,0 \Rightarrow 1$ 


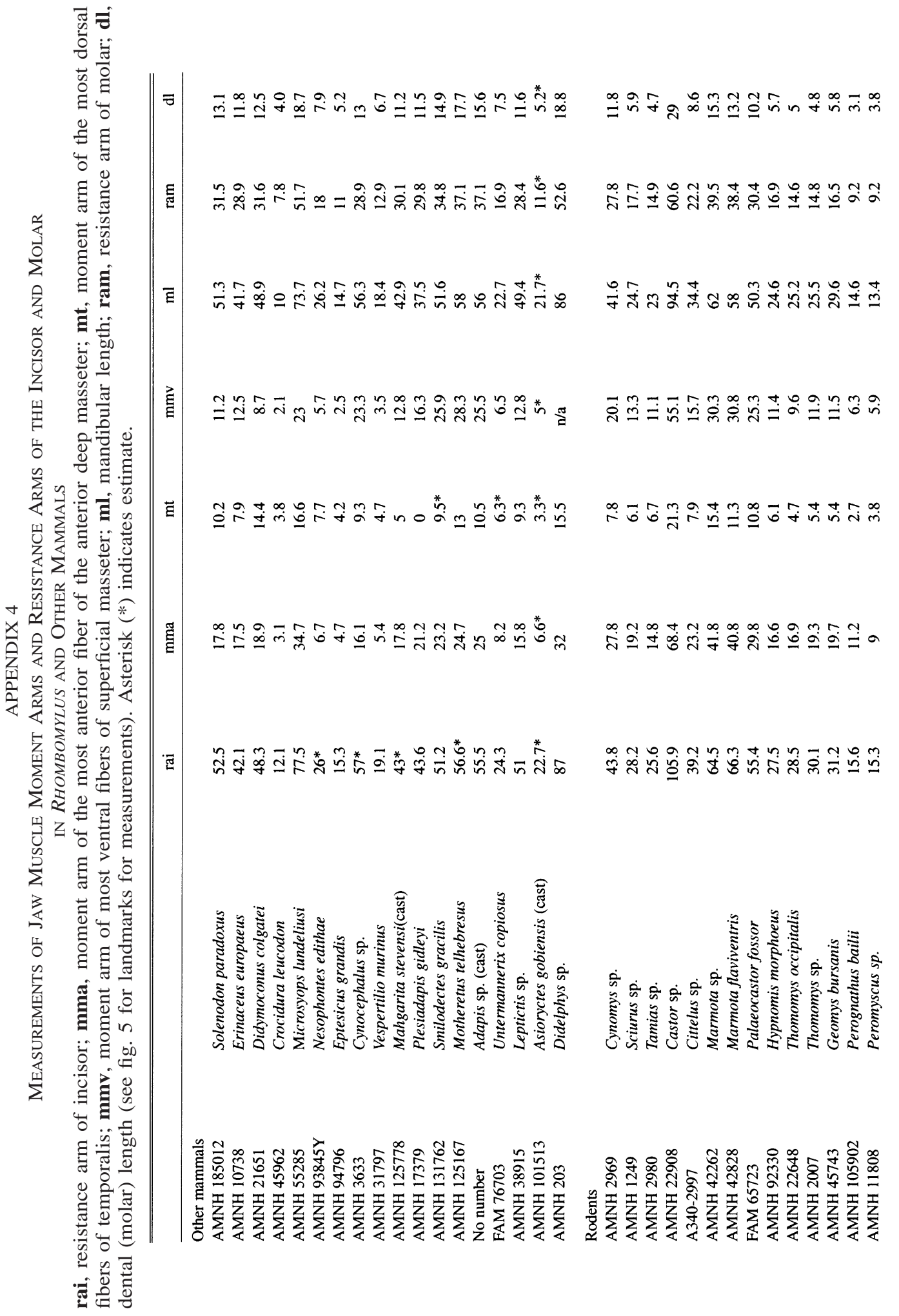




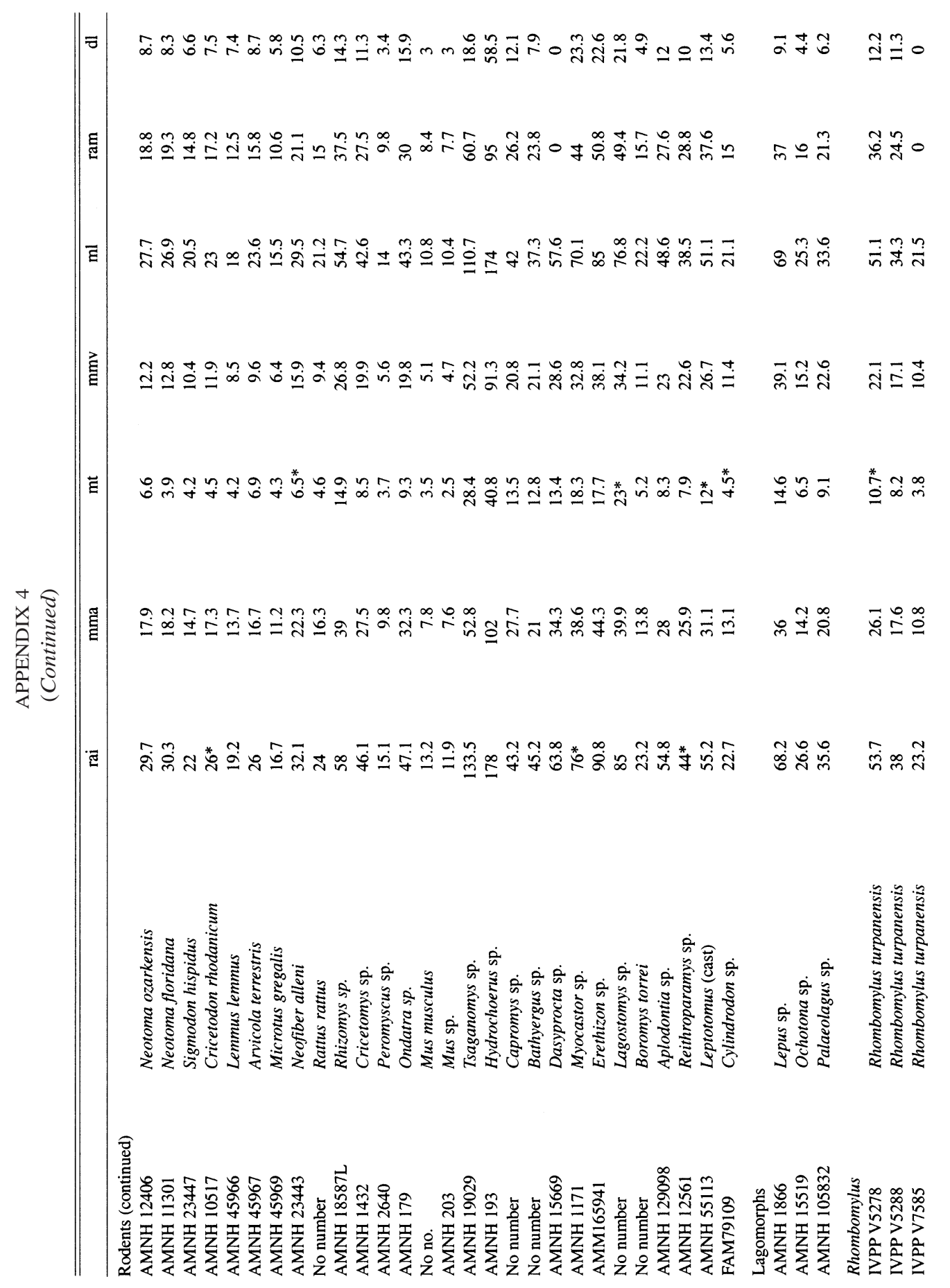




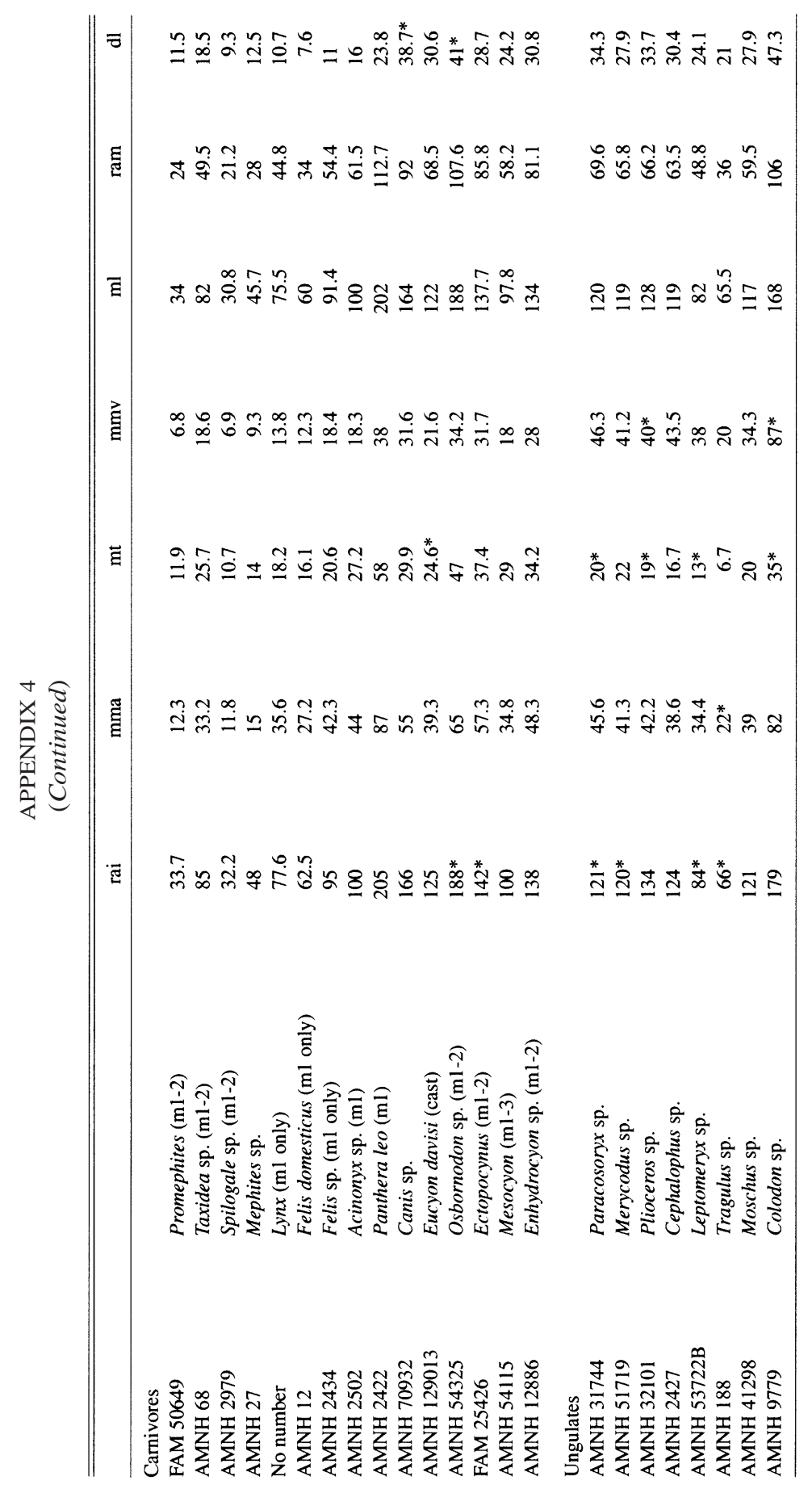




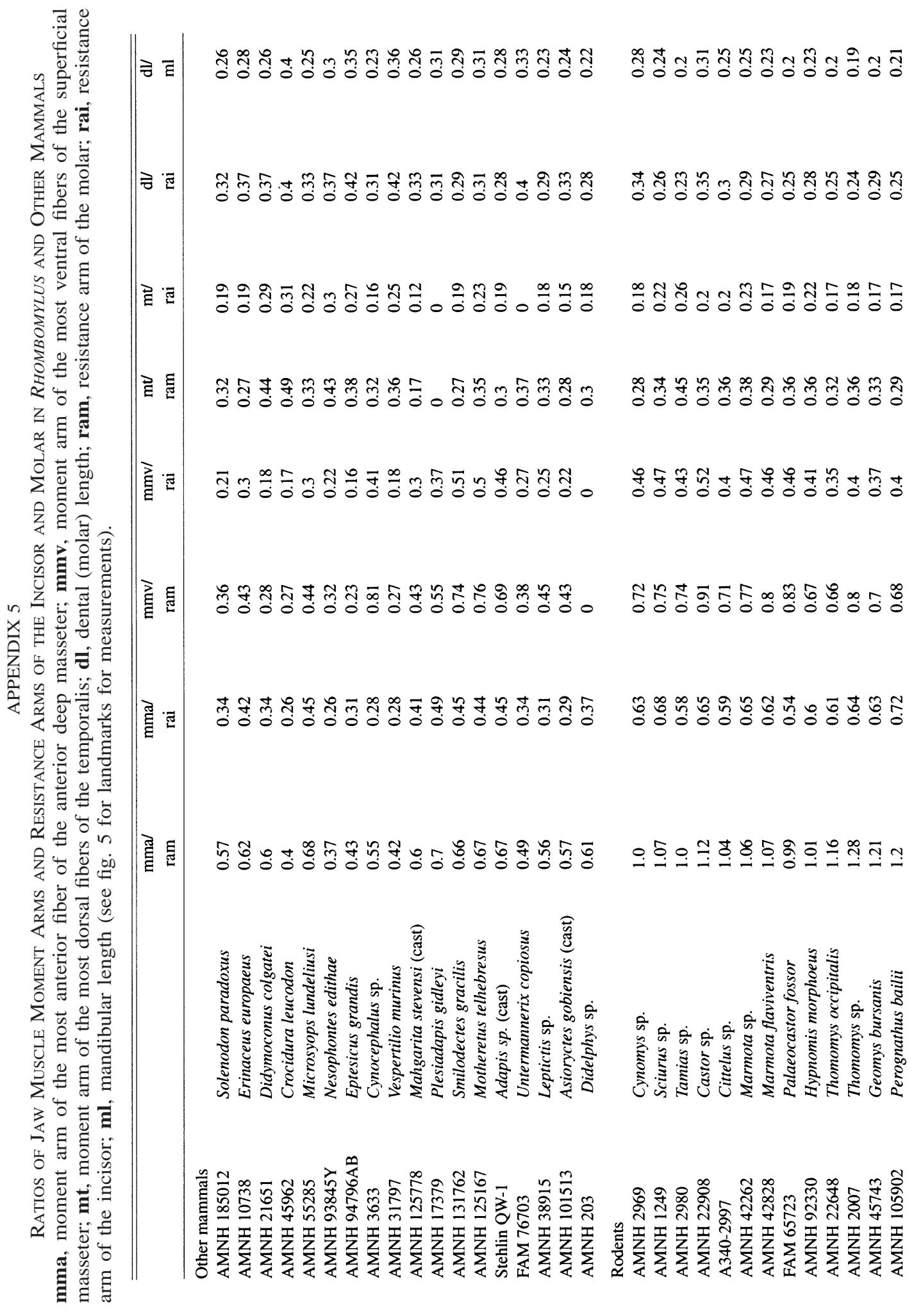




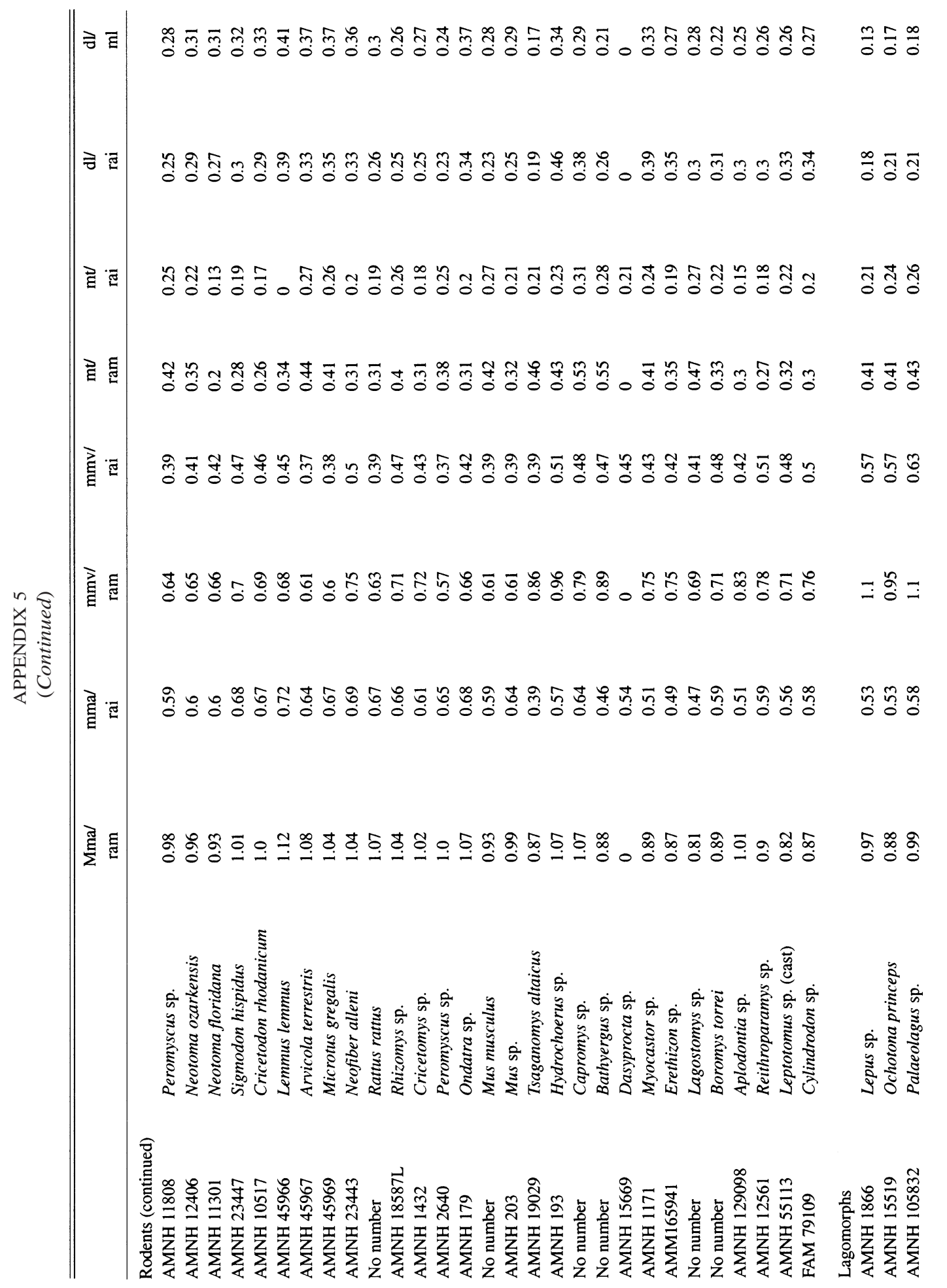




\begin{tabular}{|c|c|c|c|}
\hline क $\overline{\mathrm{E}}$ & 范骨。 & 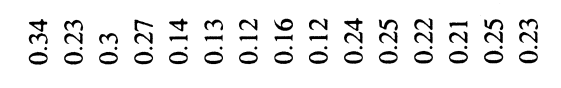 & 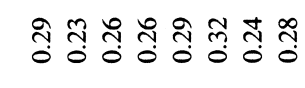 \\
\hline 亏 ฐ & $\tilde{\tilde{c}}$ & 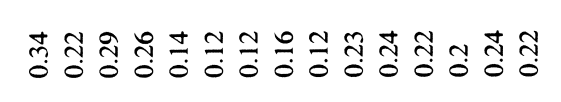 & 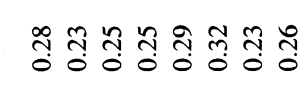 \\
\hline 곱 & ำํำำㅇำ & 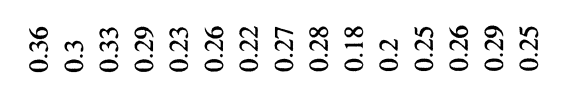 & 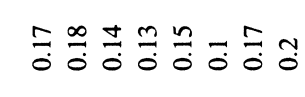 \\
\hline 兰焉 & : ल & 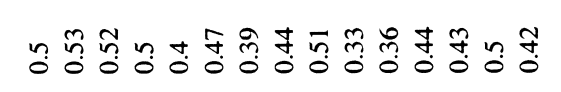 & 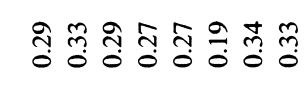 \\
\hline 啇要 & 专影路 & 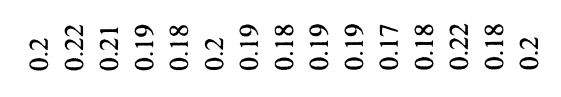 & 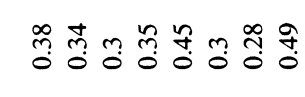 \\
\hline 顡豆 & $\overline{0}: 0$ & 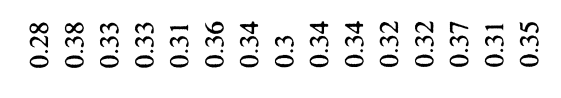 & 与ั \\
\hline 置 & 舟呫年 & 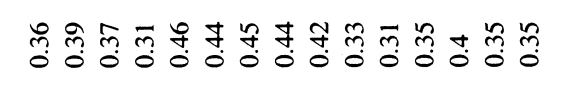 & 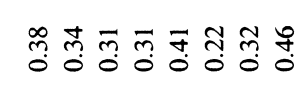 \\
\hline$\frac{7}{2}$ & กับ & 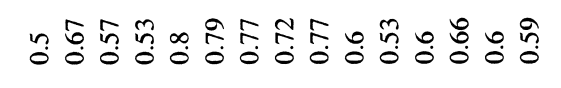 & 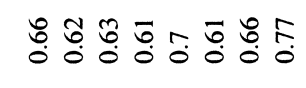 \\
\hline & 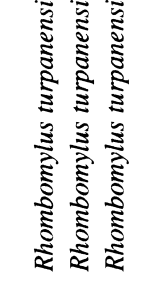 & 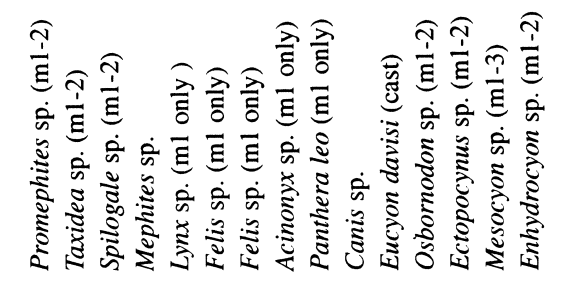 & 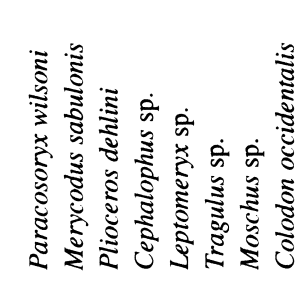 \\
\hline & 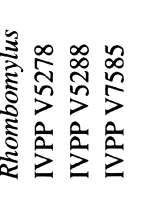 & ồे & 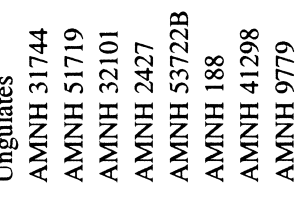 \\
\hline
\end{tabular}

